Universidade de São Paulo

Instituto de Astronomia, Geofísica e Ciências Atmosféricas

Departamento de Astronomia

Leandro Rocha Rímulo

\title{
The life cycles of viscous decretion disks around Be stars: fundamental disk parameters in the $\mathrm{SMC}$
}

(Os ciclos de vida dos discos viscosos de decreção de estrelas Be: parâmetros fundamentais de discos na Pequena Nuvem de Magalhães)

São Paulo 

Leandro Rocha Rímulo

\section{The life cycles of viscous decretion disks around Be stars: fundamental disk parameters in the $\mathrm{SMC}$}

(Os ciclos de vida dos discos viscosos de decreção de estrelas Be: parâmetros fundamentais de discos na Pequena Nuvem de Magalhães)

Tese apresentada ao Departamento de Astronomia do Instituto de Astronomia, Geofísica e Ciências Atmosféricas da Universidade de São Paulo como requisito parcial para a obtenção do título de Doutor em Ciências.

Área de Concentração: Astronomia

Orientador: Prof. Dr.

Alex Cavaliéri Carciofi

Versão Corrigida. O original encontra-se disponível na Unidade.

São Paulo 

To the $\mathbf{B e A C o N}^{1} s !$

\footnotetext{
1 "Be Aficionados Collaborative Network" (http://beacon.iag.usp.br/).
} And if you are interested in this thesis, you are one of us! 



\section{Agradecimentos/Acknowledgements}

Muitas pessoas contribuíram para que este trabalho chegasse ao ponto em que chegou. Para mim, este trabalho representa apenas uma parte de uma conquista pessoal muito maior que eu tive desde que vim para São Paulo, já quase em abril de 2011, e que envolve muitas coisas íntimas às quais eu me darei o direito de não expor aqui.

Eu primeiramente agradeço imensamente ao Alex, meu orientador durante esses pouco mais de quatro anos em que trabalhamos juntos. Nesse período, desenvolvemos uma relação muito mais que profissional. Desenvolvemos uma grande amizade que eu espero que dure para sempre! Não deve ter sido fácil me orientar, já desde o começo, quando fui admitido como seu orientando de uma forma não usual. E, depois, teve o meu jeito peculiar de perder o foco e ir a fundo em algumas coisas e, muitas vezes, me dispersar por muito tempo por caminhos desnecessários! E muito obrigado pela paciência durante as minhas fases de baixa produtividade que ocorreram principalmente em 2014 e 2015, e por ainda confiar em mim, apesar daqueles dias! Este trabalho científico, brevemente apresentado nesta tese, é dedicado principalmente a você, e, em seguida, ao nosso grupo BeACoN, que espero poder se beneficiar com ele. Eu espero poder continuar colaborando profissionalmente com você por mais bastante tempo!

Eu agradeço em seguida à professora Sílvia Rossi, que se mostrou verdadeiramente uma grande amiga durante o final do meu doutorado. Agradeço pela grande empatia. Também vou guardar uma boa recordação de você para sempre!

Muito obrigado, também, professor Eduardo Janot, por me entender quando eu disse que estava descontente com o nosso projeto, estava infeliz com ele, e decidi mudar de projeto e de orientador, lá pela segunda metade de 2012. Obrigado por aceitar a minha escolha e pelos calorosos e descontraídos cumprimentos quando passamos a nos encontrar ocasionalmente pelos corredores do IAG desde então. Te desejo tudo de bom!

Agradeço imensamente à minha família por terem me dado tanto amor e compreensão, e por terem trabalhado muito para que eu conseguisse chegar até aqui. Agradeço à minha mãe por se dedicar de corpo e alma pela nossa formação - de meus irmãos Alexandre e Daniel e minha. E grande foi a sua preocupação comigo aqui em São Paulo! Obrigado por 
ter me ajudado com sua presença, conselhos e até o ombro amigo nessas últimas semanas em que escrevi esta tese! Igualmente agradeço ao meu pai, também pela dedicação ao longo de nossas vidas, e por ser um grande exemplo para mim! Eu te admiro muito! Tanto que estou trilhando um caminho semelhante ao seu. Me recordei, agora, inclusive, de quando falávamos de átomos e de estrelas quando eu era pequeno. Eu suspeito que você foi essencial na minha paixão pelo entendimento da natureza. E espero um dia me tornar um profissional tão bom quanto você!

Obrigado Alexandre e Daniel, meus irmãos, pela amizade e companheirismo ao crescermos juntos. E obrigado por estarem à disposição em momentos que eu precisei, e pelas boas recepções quando eu voltava para BH. Agradeço também às minhas sobrinhas Raquel e Joana, simplesmente por serem uma alegria em nossas vidas! Vocês realmente coloriram a nossa família! Desculpem-me por eu ficar longe de vocês na maior parte do tempo!

Obrigado, tia Neusa, por também estar sempre presente em nossas vidas, e sempre disposta a ajudar. Obrigado por considerar aos meus irmãos e a mim como se fôssemos seus filhos e por nos acompanhar desde sempre! Obrigado, também, Bigode (o meu fii...). Agora, você também inventou de morar em São Paulo! Obrigado por ser sempre um primo companheiro e amigo de todos nós! Um amber!... Obrigado, também, Izabela, tia Marisa e tio Zé, igualmente pela amizade e pelo companheirismo, e pelas risadas!

E um obrigado à Pedrita, à Babi e à Bianca, que já se foram, e à Sisi que está bem velhinha!

Agradeço imensamente aos meus amigos do grupo BeACoN e demais amigos do IAG e daqui de São Paulo. Eu realmente descobri pessoas maravilhosas nesta cidade! Primeiramente, muito obrigado, Daniel Moser, pela amizade e por me aconselhar em minhas dificuldades e incertezas pessoais (pessoalmente, ou por Skype, de Nice ou de Santiago). Obrigado a você e aos seus pais pela recepção lá em Santa Catarina. Obrigado também pela ajuda computacional com o seu pacote pyhdust (que agora é de todos nós), por me introduzir ao emcee e por me auxiliar na administração da minha grade de modelos que foi gerada em semanas, pela máquina Alphacrucis, também com a ajuda de vários BeACoNs.

Muito obrigado, Bruno, pela imensa amizade desde que cheguei em SP, por se preocupar comigo e por me aconselhar nos meus momentos de crise! Acho que você teve muita sensibilidade de me perceber nesses momentos e me oferecer a sua amizade! Foi também muito bom aquele tempo em que moramos juntos no apartamento! E obrigado a você e sua família pela calorosa recepção e passeios, me fazendo me sentir parte da família!

Agradeço imensamente também à Daiane e ao Marcelo, pela descontração e pela maior intimidade que desenvolvemos especialmente nestes últimos meses de meu doutorado! Obrigado, também, André, pelo acolhimento nas recepções em sua casa e em seu casa- 
mento e pelo voto de confiança recente de querer trabalhar junto comigo na continuação deste trabalho! Agradeço, enfim, a todos os BeACoNs daqui do IAG, pela ajuda mútua que damos uns aos outros e por sermos amigos, além de colaboradores. Muito obrigado, Rodrigo (Chuchu), Mohammad, Daniel Bednarski, Fellipy, Despo, Amanda, Artur, Lucas, Cyril e ao BeACoN a quem eu infelizmente tenha me esquecido de citar!

I extend my thanks to some BeACoNs that live far away. Thanks, Thomas Rivinius, for providing me the data for the execution of this work and for the nice personal meetings we had here in São Paulo, at IAG and at Alex' house, or in Canada. Thanks, Jon Bjorkman and Atsuo Okazaki, for the fruitful theoretical discussions on the physics involved in all what we were doing! And thanks, Robert Klement, for being a nice friend and collaborator from abroad! Unfortunately, I could not participate in that project Alex set for both of us, because I was too "lost" by that time! But we had nice times together here in São Paulo!

Obrigado, Fábio Mendes, dono da agência de viagens FábioTur, pela amizade que desenvolvemos, e, especialmente, por me aturar durante os vários meses que convivemos juntos no apartamento! Obrigado por me ensinar muitas coisas da "vida prática" e também pelos conselhos. Agradeço também a alegria e a descontração dos vários amigos da FábioTur: Thamirys, Luiz, Vanessa, o senhor Big, Daniel May, Arianna e demais malucos envolvidos!

Obrigado, Mille, pela amizade distante e pela imensa intimidade que tivemos, lá pelo final de 2013! Foi uma uma experiência muito bonita aquela, que eu vou guardar comigo para sempre! Te desejo muitas felicidades em seu casamento e em sua vida!

Thanks, Neenie, for the distant friendship in the last year and for listening to me in my times of solitude!

Muito obrigado, também, Ercília Zilli e Alfredo Simonetti, profissionais que têm trabalhado para me ajudar em meus conflitos internos desde 2012! Muito obrigado, seu Geraldo, dona Mariazinha e Joana, por me acolherem em momentos de grande crise e por darem muito de si, gratuitamente, no intuito de me ajudar! Desculpem-me, hoje, por estar mais distante. Sinto que esse meu distanciamento foi necessário para um apaziguamento interno nas minhas reflexões existenciais. Mas quero mandar um salve a todos os "jovens do Maria", incluindo o João e o Francisco, filhos do meu orientador Alex! Saudades de todos!

Tenho saudades, enfim, dos meus amigos da AECX, lá de Belo Horizonte! Nos vimos tão poucas vezes nesses últimos seis anos, e peço desculpas pelo meu sumisso! Vocês estão no meu coração e quero que nossa amizade dure para sempre!

Finalmente, agradeço à FAPESP (processo 2012/21518-5) e ao CNPq (processo 142411/20116) pelo apoio financeiro. 
Esta tese/dissertação foi escrita em $\mathrm{H}_{\mathrm{E}} \mathrm{EX}$ com a classe IAGTESE, para teses e dissertações do IAG. 
"In principle, we can know all of mathematics. It is given to us in its entirety and does not change. ... That part of it of which we have a perfect view seems beautiful, suggesting harmony; that is that all the parts fit together although we see fragments of them only. ...Mathematics is applied to the real world and has proved fruitful. This suggests that the mathematical parts and the empirical parts are in harmony and the real world is also beautiful."

"(...) Our total reality and total existence are beautiful and meaningful. ... We should judge reality by the little which we truly know of it. Since that part which conceptually we know fully turns out to be so beautiful, the real world of which we know so little should also be beautiful. Life may be miserable for seventy years and happy for a million years: the short period of misery may even be necessary for the whole."

Kurt Gödel, in A Logical Journey, MIT Press, 1996

"Two things fill the mind with ever new and increasing wonder and awe - the starry heavens above me and the moral law within me."

Immanuel Kant, in Critique of Practical Reason, 1788.

"One of the most important societal functions of modern astronomy is as a tool for education in the broadest sense. Because it is one of the most approachable of sciences, and one that consistently fascinates young people, astronomy is an excellent vehicle for introducing science and technology to children. The accessibility of the sky, the beauty of cosmic objects and the immensity of the Universe are inspirational and provide a perspective that encourages internationalism and tolerance. The excitement of astronomy has stimulated large numbers of young people to choose a career in science and technology, thereby contributing to the 'knowledge economy' of many countries."

International Astronomical Union, Astronomy for the Developing World: Strategic Plan 2010 - 2020 



\section{Resumo}

Estrelas Be são estrelas massivas na sequência principal, com emissões em seus espectros que se originam de um disco circunstelar gasoso. Embora o modelo de disco viscoso de decreção ("viscous decretion disk" - VDD) seja capaz de explicar satisfatoriamente a maior parte das observações, dois ingredientes físicos: a magnitude da viscosidade $(\alpha)$ e a taxa de injeção de massa no disco, ainda são muito pouco conhecidos. Além disso, muito trabalho ainda precisa ser feito para que entendamos os ciclos de vida desses discos: quão rápido eles crescem e dissipam, quanto tempo eles duram, etc. As curvas de luz de estrelas Be que produzem eventos de formação e dissipação de disco oferecem uma oportunidade de se estudar os seus ciclos de vida e de estimar as propriedades fundamentais dos discos. Uma lista de procedimentos (um "pipeline") foi desenvolvida para modelar esses eventos. Ela usa uma grade de curvas de luz sintéticas, computadas a partir de simulações hidrodinâmicas detalhadas combinadas com cálculos de transferência radiativa. A comparação entre modelos e dados foi possível utilizando-se de duas leis empíricas que descobrimos, que imitam bastante o perfil fotométrico dos eventos. Uma amostra de 54 estrelas Be do "survey" OGLE na direção da Pequena Nuvem de Magalhães ("Small Magellanic Cloud" - SMC) foi selecionada para este estudo. As taxas típicas de perda de massa e momento angular associadas aos eventos são da ordem de $\sim 10^{-10} M_{\odot} \mathrm{yr}^{-1} \mathrm{e} \sim 5 \times 10^{36} \mathrm{~g} \mathrm{~cm}^{2} \mathrm{~s}^{-2}$, respectivamente. Nós demonstramos que o momento angular perdido pela estrela, mesmo para os eventos associados aos discos mais densos, ainda está abaixo que o requerido pelos melhores modelos de evolução estelar, de tal forma que as estrelas não alcancem suas velocidades de ruptura ("break-up velocities"). Nossos números oferecem, pela primeira vez, restrições aos mecanismos internos de transporte de momento angular de estrelas massivas em alta rotação. Este trabalho também aumentou em 54 vezes o número de estrelas Be cujos parâmetros $\alpha$ foram determinados, e ele representa a primeira determinação estatística do parâmetro $\alpha$ para estrelas Be. Os valores de $\alpha$ encontrados são, tipicamente, da ordem de alguns décimos, consistentes com os resultados recentes na literatura e com os valores encontrados para as novas anãs, mas maiores que os preditos pela teoria atual. Considerando a amostra como um todo, o parâmetro de viscosidade é aproximadamente duas vezes maior na formação $\left(\left\langle\alpha_{\mathrm{bu}}\right\rangle=0.63\right)$ do que na dissipação $\left(\left\langle\alpha_{\mathrm{d}}\right\rangle=0.29\right)$. Pes- 
quisa futura é necessária para verificar se essa tendência é real ou um resultado de nossas considerações feitas nos modelos. Se real, este fenômeno merece ser melhor investigado, já que ele pode fornecer indícios da origem da viscosidade anômala de discos astrofísicos. No futuro próximo, nós estenderemos o nosso trabalho para estrelas Be da Grande Nuvem de Magalhães ("Large Magellanic Cloud" - LMC) e da Galáxia, através do uso dos muitos "surveys" fotométricos disponíveis que contêm coberturas temporais de anos. 


\section{Abstract}

Be stars are main-sequence massive stars with emission features in their spectrum, which originates from a circumstellar gaseous disk. Even though the viscous decretion disk (VDD) model can satisfactorily explain most observations, two important physical ingredients, namely the magnitude of the viscosity $(\alpha)$ and the disk mass injection rate, remain poorly constrained. In addition, substantial work remains to be done in order to fully understand the life cycles of these disks: how fast they grow and dissipate, for how long they last, etc. The light curves of Be stars that undergo events of disk formation and dissipation offer an opportunity to study their life cycles and to constrain the disks' fundamental properties. A pipeline was developed to model these events that uses a grid of synthetic light curves, computed from detailed hydrodynamic simulations combined with radiative transfer calculations. Comparison between model and data was made possible by two empirical laws we discovered, which closely match the photometric behaviour of the events. A sample of 54 Be stars from the OGLE survey of the Small Magellanic Cloud (SMC) was selected for this study. The typical mass and angular momentum loss rates associated with the disk events are of the order of $\sim 10^{-10} M_{\odot} \mathrm{yr}^{-1}$ and $\sim$ $5 \times 10^{36} \mathrm{~g} \mathrm{~cm}^{2} \mathrm{~s}^{-2}$, respectively. We showed that the angular momentum lost by the star, even for the events with the densest disks, was still smaller than the required by the best evolutionary models so that the stars do not reach their break-up velocities. These numbers offer, for the first time, constraints on the internal angular momentum transport mechanisms of fast rotating massive stars. This work also increased the number of Be stars whose $\alpha$ parameters have been determined by 54 times, and it represents the first statistically significant determination of $\alpha$ for Be stars. The values of $\alpha$ found are typically of a few tenths, consistent with recent results in the literature and with the ones found in dwarf novae, but larger than the current theory predicts. Considering the sample as a whole, the viscosity parameter is roughly two times larger at build-up $\left(\left\langle\alpha_{\text {bu }}\right\rangle=0.63\right)$ than at dissipation $\left(\left\langle\alpha_{\mathrm{d}}\right\rangle=0.29\right)$. Further work is necessary to verify whether this trend is real or a result of some of the model assumptions. If real, this is a phenomenon worth further investigation, as it may lead to clues as to the origin of anomalous viscosity in astrophysical disks. In the near future, we intend to extend our work to Be stars from 
the Large Magellanic Cloud (LMC) and from the Galaxy, by making use of the many photometric surveys, with years of coverage, available. 


\section{List of Figures}

$1.1 \mathrm{H}-\mathrm{R}$ diagram of nearby stars. . . . . . . . . . . . . . 26

1.2 Amateur image of the Pleiades open cluster, also showing $\mathrm{H} \alpha$ line profile of the seven main stars. . . . . . . . . . . . . . . . . . . . . . . . . 28

1.3 Left: Schematic view of a Be star, with examples of spectral profiles from pole-on to shell. Right: Image reconstruction of the Be star $\zeta$ Tauri in $\mathrm{H} \alpha$, based on optical interferometry. . . . . . . . . . . . . . . . . . . . . . . . . 29

1.4 Left: $\mathrm{V} / \mathrm{R}$ variations in $\mathrm{H} \alpha$ of the Be star $\zeta$ Tauri. Right: Schematic representation of an one-armed spiral pattern. . . . . . . . . . . . . . . 31

1.5 Variability of the Be star $\delta$ Scorpii over more than 11 years. . . . . . . . . 32

1.6 Appearance of a gravity darkened star. . . . . . . . . . . . . . . . . . 34

1.7 Evolutionary tracks for A-B type stars for $Z=0.014$ (left) and $Z=0.002$ (right). . . . . . . . . . . . . . . . . 35

1.8 Wind streamlines and disk formation for three disk models: WCD, MWCD and VDD. . . . . . . . . . . . . . . . . . 37

1.9 A VDD model of the Be star $\zeta$ Tauri that includes global density waves. $\quad 39$

1.10 Theoretical non-LTE $\mathrm{H} \alpha$ line profiles for a B0e star with a Keplerian disk. 40

1.11 The formation loci of continuum emission. . . . . . . . . . . . . . . . . 41

2.1 Roche shapes of the stellar configurations, with critical ratios $W$ varing

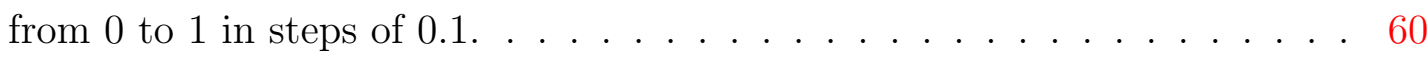

2.2 For the same stellar models of Fig. 2.1: Upper-left: Flux at the surface of the stars compared to the non-rotating case as a function of the polar angle $\theta$. Upper-right: Effective temperature compared to the non-rotating case as a function of $\theta$. In dotted straight lines, values of $0.6\left(L / S_{W} \sigma\right)^{\frac{1}{4}}$, where $S_{W}$ is the surface area of the star with parameter $W$. Lower-left: $\log g_{\text {eff }}$ compared to the non-rotating case as a function of $\theta$. Lower-right: $\log T_{\text {eff }}$ versus $\log g_{\text {eff. }}$ The inclinations of these curves give the $\beta$ coefficient. . . . . 62 
3.1 Two light curves, in photometric bands $V$ (green) and $I$ (red), selected from the OGLE-II and OGLE-III photometric surveys. Above: light curve of SMC_SC1 75701 (OGLE-II ID). Below: light curve of SMC_SC6 128831 (OGLE-II ID).

3.2 Simulation of a constant build up phase $\left(\Sigma_{0}=1.549 \mathrm{~g} \mathrm{~cm}^{-2}\right)$, during $\tilde{\tau}_{\mathrm{bu}}=$ 30 , followed by a complete dissipation $\left(\Sigma_{0}=0\right)$. Three injection radii were used: $\tilde{R}_{\text {inj }}=1.017$ (dotted), 1.169 (dashed), 1.319 (solid) . . . . . . . . 75

3.3 Dynamical bump model with $\tilde{\tau}_{\text {bu }}=30 \ldots \ldots$. . . . . . . . . . . 77

3.4 Simulation of a constant build up phase $\left(\Sigma_{0}=1.549 \mathrm{~g} \mathrm{~cm}^{-2}\right)$, during $\tilde{\tau}_{\mathrm{bu}}=$ 30 , followed by a complete dissipation $\left(\Sigma_{0}=0\right)$. The different colors corresponds to different instants after beginning of the build-up phase: $\tilde{\tau}+30 \sim 0$ (red), $\tilde{\tau}+30=0.2$ (orange), $\tilde{\tau}+30=1$ (green), $\tilde{\tau}+30=6$ (blue), $\tilde{\tau}+30=25$ (purple).

3.5 Simulation of two constant build-up phases $\left(\Sigma_{0}=1.549 \mathrm{~g} \mathrm{~cm}^{-2}\right)$, one during $\tilde{\tau}_{\mathrm{bu}}=30$ (solid line), and another during $\tilde{\tau}_{\mathrm{bu}}=30$ (dashed line). Both were followed by a complete dissipation $\left(\Sigma_{0}=0\right)$. The different colors corresponds to different instants after beginning of the build-up phase: $\tilde{\tau} \sim 0$ (red), $\tilde{\tau}=0.2$ (orange), $\tilde{\tau}=1$ (green), $\tilde{\tau}=6$ (blue), $\tilde{\tau}=25$ (purple).

3.6 M. Ghoreyshi et al.'s current best results for the photometric modeling of the light curve of $28 \mathrm{CMa}$.

4.1 Temperatures in the cells and on the stellar surface in a simulation of nearKeplerian disk of a Be star made by HDUST. . . . . . . . . . . . . . . . . . 90

4.2 HDUST's current ellipsoid stars (black) versus Roche-shaped stars with $W=$ 0.7 and $W=0.9$ (red).

4.3 UBVRI Johnson-Cousins passbands and the limits in frequency of the SEDs generated by HDUST in this work. . . . . . . . . . . . . . 92

4.4 Left: CMD in bands $V$ and $I$ of the diskless stars of Table 4.3. Right: diagram showing $M_{I}$ (the same vertical axis of the upper-left one) versus $\alpha \tau$ (in days) for the diskless stars. . . . . . . . . . . . . . . . 95

4.5 Examples of model $I$-band light curves, for several inclination angles. . . . 97

4.6 The values of $\Delta I_{\mathrm{bu}}^{\infty}$ versus $\cos i$ (left) and $\Delta V_{\mathrm{bu}}^{\infty}$ versus $\cos i$ (right) for our grid. . . . . . . . . . . . . . . . . . . . . . . . . 98

4.7 Examples of the $\log -\log$ derivative of $1-\Delta I_{\mathrm{bu}}(\tilde{\tau}) / \Delta I_{\mathrm{bu}}^{\infty}$ vs. $\Delta I_{\mathrm{bu}}(\tilde{\tau}) / \Delta I_{\mathrm{bu}}^{\infty}$ (top four panels) and the log - log derivative of $\Delta I_{\mathrm{d}} / \Delta I_{\mathrm{d}}^{0}$ vs. $1-\Delta I_{\mathrm{d}} / \Delta I_{\mathrm{d}}^{0}$, for values of $\tilde{\tau}_{\mathrm{bu}}$ equal to $0.45,1.5,6$ and 30 (bottom four panels). . . . . . 99

4.8 The $I$ - and $V$-band values of $\xi_{\text {bu }}$ for our grid vs. $\cos i$. . . . . . . . . . . 101

4.9 Selected $I$ and $V$-band values of $\xi_{\mathrm{d}}$ for our grid vs. $\cos i$. The scaled build-up time was fixed to $\tilde{\tau}_{\text {bu }}=2.25 \ldots \ldots$. . . . . . . . . . 102 
4.10 Selected $I$ and $V$-band values of $\xi_{\mathrm{d}}$ for our grid vs. $\tilde{\tau}_{\mathrm{bu}}$.

5.1 $V$ light curves and $V-R$ color curves for examples of the five modes of variability of Keller et al. (2002). . . . . . . . . . . . . . . . . . . . 113

5.2 Examples of OGLE II light curves selected as Be star candidates: type-1 and type-1/type-2 light curves. . . . . . . . . . . . . . . . . . . . . 114

5.3 Examples of OGLE II light curves selected as Be star candidates: type-2, type-3 and type-4 light curves. . . . . . . . . . . . . . . . . . . . . 115

5.4 Left: CMD diagram for the selected stars from the SMC. Right: CMD diagram for the selected stars from the LMC. . . . . . . . . . . . . . . . 115

5.5 Examples of SMC light curves showing clear zero point shifts between the OGLE-II and OGLE-III components. . . . . . . . . . . . . . . 117

5.6 Examples of SMC light curves found to have no clear inactive phase (e.g., above: SMC_SC4 60186) or to clearly be associated with a more complicated scenario of mass injection than the one described in the Chapter 3 (e.g., below: SMC_SC4 116957). . . . . . . . . . . . . . . . . . 118

5.7 Color-magnitude diagram of simulated diskless stars (Table 4.3). Also shown, as errorbars, is the position of our selected stars (see eighth and ninth columns of Table 5.1). . . . . . . . . . . . . . . . . . . . . . 119

6.1 Results for the selected bump of SMC_SC1 75701. . . . . . . . . . . . . . 124

6.2 Results for the selected bump of SMC_SC6 128831. . . . . . . . . . . . . . 125

6.3 Solid line: Histogram of the sum of the posterior probabilities of parameter $M$ for all stars in our sample. Dashed line: IMF of Kroupa (2001) weighed by the fraction of Be stars over B stars of Martayan et al. (2007), given by the factor $M^{-2.3} f_{\mathrm{Be}}(M)$ in Eq. (4.5.8). . . . . . . . . . . . . . . . 128

6.4 Mean error and rms for a sample of stars from one of the fields of the OGLE-II survey. . . . . . . . . . . . . . . . . . . . 129

6.5 Box and whisker plots of $\Sigma_{0}$ (above) and $t_{2}-t_{1}$ (below) for the summed posterior probabilities of our sample of bumps, separated in six equal intervals of mass, ranging from 5 to 17 solar masses. . . . . . . . . . . . . . . . . . 130

6.6 Distribution of the parameters $M$ and $\Sigma_{0}$ for our sample. . . . . . . . . . 131

6.7 Same as Fig. 6.5 for $\alpha_{\mathrm{bu}}$ (above) and $\alpha_{\mathrm{d}}$ (below). . . . . . . . . . . . . . . 131

6.8 Distribution of the two viscosity parameters $\alpha_{\mathrm{bu}}$ and $\alpha_{\mathrm{d}}$. . . . . . . . . 132

6.9 Evolution of the disk mass and mean disk temperature of the Be star 28 CMa, complementing the panels of Fig. 3.6. . . . . . . . . . . . . . . 133

6.10 Distributions of the steady-state mass (above) and angular momentum (below) loss rates for our sample. . . . . . . . . . . . . . . . . . . 135

B.1 $\left(\operatorname{Star}, i, \Sigma_{0}, \tilde{\tau}_{\mathrm{bu}}\right)=\left(\operatorname{Star} 2,90 \mathrm{deg}, 1.37 \mathrm{~g} \mathrm{~cm}^{-2}, \mathbf{b u}\right) \ldots \ldots \ldots \ldots$ 
B.2 (Star, $\left.i, \Sigma_{0}, \tilde{\tau}_{\text {bu }}\right)=\left(\operatorname{Star} 2,90 \mathrm{deg}, 1.37 \mathrm{~g} \mathrm{~cm}^{-2}, \mathbf{1 . 5}\right) \ldots \ldots \ldots \ldots \ldots \ldots$

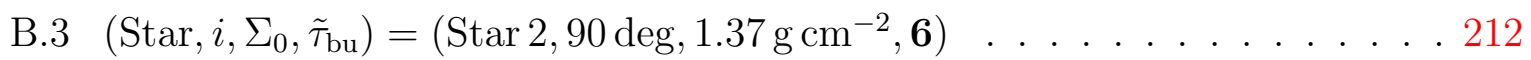

B.4 $\left(\operatorname{Star}, i, \Sigma_{0}, \tilde{\tau}_{\text {bu }}\right)=\left(\operatorname{Star} 2,69.1 \mathrm{deg}, 1.37 \mathrm{~g} \mathrm{~cm}^{-2}, \mathrm{bu}\right) \ldots \ldots . \ldots 213$

B.5 $\left(\operatorname{Star}, i, \Sigma_{0}, \tilde{\tau}_{\text {bu }}\right)=\left(\operatorname{Star} 2,69.1 \mathrm{deg}, 1.37 \mathrm{~g} \mathrm{~cm}^{-2}, \mathbf{1 . 5}\right) \ldots \ldots . \ldots 214$

B.6 $\left(\right.$ Star $\left., i, \Sigma_{0}, \tilde{\tau}_{\text {bu }}\right)=\left(\operatorname{Star} 2,69.1 \mathrm{deg}, 1.37 \mathrm{~g} \mathrm{~cm}^{-2}, 6\right) \ldots \ldots \ldots . \ldots \ldots$

B.7 $\left(\operatorname{Star}, i, \Sigma_{0}, \tilde{\tau}_{\text {bu }}\right)=\left(\operatorname{Star} 2,0 \mathrm{deg}, 1.37 \mathrm{~g} \mathrm{~cm}^{-2}, \mathbf{b u}\right) \ldots \ldots \ldots \ldots$

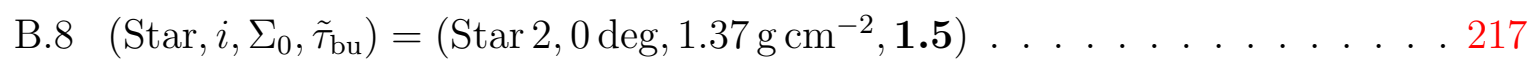

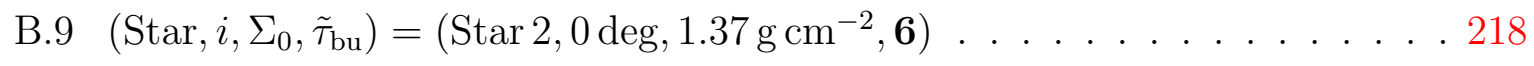

B.10 $\left(\right.$ Star $\left., i, \Sigma_{0}, \tilde{\tau}_{\text {bu }}\right)=\left(\operatorname{Star} 2,38.2 \mathrm{deg}, 2.50 \mathrm{~g} \mathrm{~cm}^{-2}\right.$, bu $) \ldots \ldots . \ldots 220$

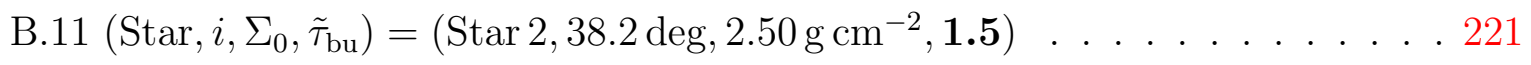

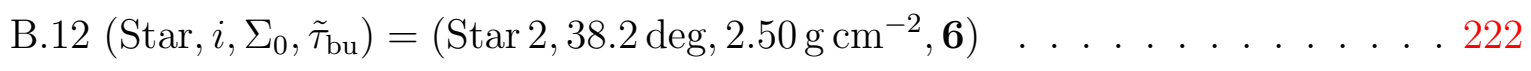

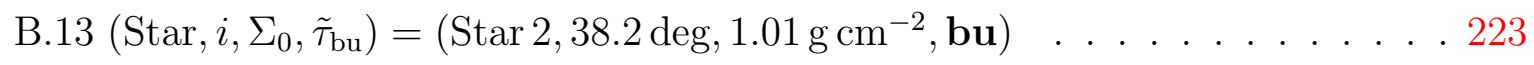

B.14 $\left(\right.$ Star $\left., i, \Sigma_{0}, \tilde{\tau}_{\text {bu }}\right)=\left(\operatorname{Star} 2,38.2 \mathrm{deg}, 1.01 \mathrm{~g} \mathrm{~cm}^{-2}, \mathbf{1 . 5}\right) \ldots \ldots . \ldots . \ldots 224$

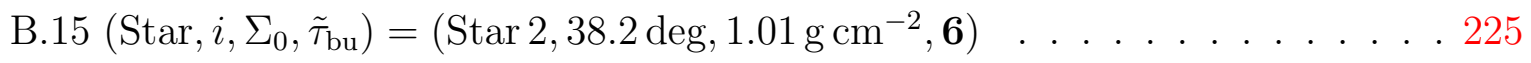

B.16 $\left(\right.$ Star, $\left.i, \Sigma_{0}, \tilde{\tau}_{\text {bu }}\right)=\left(\operatorname{Star} 2,38.2 \mathrm{deg}, 0.41 \mathrm{~g} \mathrm{~cm}^{-2}, \mathbf{b u}\right) \ldots \ldots . \ldots 226$

B.17 $\left(\operatorname{Star}, i, \Sigma_{0}, \tilde{\tau}_{\mathrm{bu}}\right)=\left(\operatorname{Star} 2,38.2 \mathrm{deg}, 0.41 \mathrm{~g} \mathrm{~cm}^{-2}, \mathbf{1 . 5}\right) \ldots \ldots \ldots 227$

B.18 $\left(\right.$ Star, $\left.i, \Sigma_{0}, \tilde{\tau}_{\text {bu }}\right)=\left(\operatorname{Star} 2,38.2 \mathrm{deg}, 0.41 \mathrm{~g} \mathrm{~cm}^{-2}, 6\right) \ldots \ldots . \ldots 228$ 


\section{List of Tables}

2.1 Conversion table between $W, \omega, R_{\text {eq }} / R_{\text {pole }}$ and $\Upsilon \ldots \ldots \ldots$. . . . . 61

4.1 Parameters of the grid of photometric models of bumps . . . . . . . . . 93

4.2 Parameters of the stellar models of Table $4.1 \ldots \ldots$. . . . . . . . . . . 93

4.3 Parameters of the grid of photometric models of diskless stars . . . . . . . 94

4.4 Values of $\eta$ 's. . . . . . . . . . . . . . . . . . . . . . . . . 101

5.1 List of Be stars and their respective bumps selected for this study . . . . . 120

6.1 Results of the pipeline for each star and bump of the sample . . . . . . 138

A.1 Empirical law parameters for band B. . . . . . . . . . . . . . . . . . 164

A.2 Empirical law parameters for band $V \ldots \ldots \ldots$. . . . . . . . . . . . . . 175

A.3 Empirical law parameters for band $R$. . . . . . . . . . . . . . . . 186

A.4 Empirical law parameters for band $I \ldots \ldots$. . . . . . . . . . . . 197 



\section{Contents}

1. Introduction . . . . . . . . . . . . . . . . . . 25

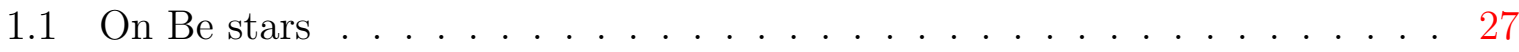

1.1.1 The fastest rotators among non-degenerate stars . . . . . . . . . . 33

1.1.2 The Viscous Decretion Disk (VDD) model . . . . . . . . . . . . . . 37

1.1.2.1 Wind Compressed Disk _. . . . . . . . . . . . 38

1.1.2.2 Magnetically Wind Compressed Disk . . . . . . . . . . 38

1.1.2.3 Viscous Decretion Disk . . . . . . . . . . . . . . . 38

1.2 Dynamical VDDs . . . . . . . . . . . . . . . . . . . . . 42

1.2.1 The origin of the viscosity of VDDs . . . . . . . . . . . . . . . . . . . . . . . . . 43

1.2.2 Mass and angular momentum loss rates . . . . . . . . . . . . . . . . 44

1.3 Outline of this thesis . . . . . . . . . . . . . . . . . 45

2. Dynamical Principles of VDDs and rotating stars . . . . . . . . . . . . . . 47

2.1 Hydrodynamical considerations . . . . . . . . . . . . . . . 47

2.2 Thin and axisymmetric disks . . . . . . . . . . . . . . . . . 49

2.2.1 An inviscid and near-Keplerian disk . . . . . . . . . . . . . . . . . . 49

2.2.2 On the vertical profile of thin disks . . . . . . . . . . . . 51

2.2.2.1 Vertically integrated quantities and the thin disk approximation . . . . . . . . . . . . . . 52

2.2.3 The alpha-disk approach to thin VDDs . . . . . . . . . . 53

2.3 The fast rotating star . . . . . . . . . . . . . . . . 56

2.3.1 Espinosa-Lara \& Rieutord's flux prescription . . . . . . . . . . . . . 60

3. Viscous decretion disks around Be stars . . . . . . . . . . . . . . . . . . 65

3.1 Main considerations . . . . . . . . . . . . . . . . 66

3.1.1 The star-disk connection . . . . . . . . . . . . . 67

3.1.2 The alpha-disk code SINGLEBE . . . . . . . . . . . . . . 68

3.1.3 The timescale parameter $\tau$ and the time parameter $\tilde{\tau} \ldots$. . . . . 70

3.2 Dynamical disk parameters . . . . . . . . . . . . . . . . . . . . 71 
3.2.1 The steady-state solution of the VDD . . . . . . . . . . . . . 71

3.2.2 Rates of mass and angular momentum loss . . . . . . . . . . . . 73

3.2.3 On the importance of the angular momentum loss rate . . . . . . . 77

3.3 The mass reservoir effect . . . . . . . . . . . . . . . . . . . . . . . 80

4. A model grid of disk formation and dissipation events . . . . . . . . . . . . . . . 87

4.1 The dynamical model grid . . . . . . . . . . . . . . . . . 87

4.2 Radiative transfer models . . . . . . . . . . . . . . . . . . . . 88

4.2.1 The Monte Carlo Radiative Transfer code HDUST . . . . . . . . . . . 89

4.2.2 Radiative transfer models . . . . . . . . . . . . . . . . . . 90

4.3 Empirical law . . . . . . . . . . . . . . . . . . . . . . 95

4.4 Fitting pipeline . . . . . . . . . . . . . . . . . . 103

4.5 Fitting using MCMC sampling . . . . . . . . . . . . . . . 105

4.5.1 Statistical considerations . . . . . . . . . . . . . . . . 105

4.5.1.1 MCMC sampling with emcee . . . . . . . . . . . . 107

4.5.2 The likelihood and prior distributions of our problem . . . . . . . 108

5. The remarkable light curves of Be stars from the Magellanic Clouds . . . . . . . 111

5.1 Selections of Be stars from the SMC and LMC . . . . . . . . . . . . . . . 112

5.2 OGLE light curves of Be star candidates . . . . . . . . . . . . . 116

5.2 .1 Our selection of bumps from the SMC . . . . . . . . . 116

6. Results . . . . . . . . . . . . . . . . . . . . . 123

6.1 SMC_SC1 75701 and SMC_SC6 $128831 \ldots \ldots$. . . . . . . . . 123

6.2 Results for the whole sample . . . . . . . . . . . . . . 127

6.2.1 Mass distribution . . . . . . . . . . . . . . . . . . . 128

6.2.2 Asymptotic surface density . . . . . . . . . . . . . . . . . . 129

6.2 .3 Disk life cycles . . . . . . . . . . . . . . . . . . . . 130

6.2.4 Viscosity parameter . . . . . . . . . . . . . . . . . . 132

6.2.5 Mass and angular momentum loss . . . . . . . . . . . . 135

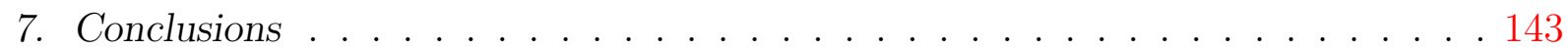

8. Prospectives . . . . . . . . . . . . . . . . . . 147

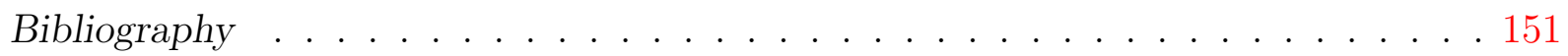

$\begin{array}{ll}\text { Appendix } & 161\end{array}$

A. Long tables . . . . . . . . . . . . . . . . . . . . . . . . . 163 
B. Examples of fittings of the empirical laws . . . . . . . . . . . . . . . . 209

B.1 $\left(\operatorname{Star}, i, \Sigma_{0}, \tilde{\tau}_{\mathrm{bu}}\right)=\left(\operatorname{Star} \mathbf{2}, *, \mathbf{1 . 3 7} \mathbf{g ~ c m}^{-\mathbf{2}}, *\right) \ldots \ldots \ldots \ldots 20 . \ldots \ldots$

B.1.1 $\left(\operatorname{Star}, i, \Sigma_{0}, \tilde{\tau}_{\mathrm{bu}}\right)=\left(\operatorname{Star} 2, \mathbf{9 0} \mathrm{deg}, 1.37 \mathrm{~g} \mathrm{~cm}^{-2}, *\right) \ldots \ldots 210$

B.1.2 (Star, $\left.i, \Sigma_{0}, \tilde{\tau}_{\mathrm{bu}}\right)=\left(\operatorname{Star} 2,69.1 \mathrm{deg}, 1.37 \mathrm{~g} \mathrm{~cm}^{-2}, *\right) \ldots \ldots .213$

B.1.3 (Star, $\left.i, \Sigma_{0}, \tilde{\tau}_{\mathrm{bu}}\right)=\left(\operatorname{Star} 2,0 \mathrm{deg}, 1.37 \mathrm{~g} \mathrm{~cm}^{-2}, *\right) \ldots \ldots 216$

B.2 $\left(\operatorname{Star}, i, \Sigma_{0}, \tilde{\mathrm{t}}_{\mathrm{bu}}\right)=(\operatorname{Star} \mathbf{2}, \mathbf{3 8 . 2} \mathbf{d e g}, *, *) \ldots \ldots \ldots \ldots \ldots \ldots \ldots \ldots \ldots$

B.2.1 $\left(\operatorname{Star}, i, \Sigma_{0}, \tilde{\tau}_{\text {bu }}\right)=\left(\operatorname{Star} 2,38.2 \mathrm{deg}, \mathbf{2 . 5 0} \mathbf{g ~ c m}^{-2}, *\right) \ldots \ldots 220$

B.2.2 $\left(\operatorname{Star}, i, \Sigma_{0}, \tilde{\tau}_{\mathrm{bu}}\right)=\left(\operatorname{Star} 2,38.2 \mathrm{deg}, \mathbf{1 . 0 1} \mathbf{g ~ c m}^{-\mathbf{2}}, *\right) \ldots \ldots 223$

B.2.3 $\left(\operatorname{Star}, i, \Sigma_{0}, \tilde{\tau}_{\mathrm{bu}}\right)=\left(\operatorname{Star} 2,38.2 \mathrm{deg}, \mathbf{0 . 4 1} \mathbf{g} \mathbf{~ c m}^{-\mathbf{2}}, *\right) \ldots \ldots 226$

C. Scientific paper . . . . . . . . . . . . . . . . . . . . . . . . . 2229 

Chapter 1

\section{Introduction}

Albert: “(...) Phosphorescence and all other organisms are made of elements that were once blended together in a star." Hilde: "Us too?" Albert: "Yes, we too are stardust."

Jostein Gaarder, in Sophie's World

The stars are the main components of the visible Universe and our main sources of information about it. The study of stars, therefore, is crucial for the development of all other fields of modern astronomy.

A collection of 23000 nearby stars is summarized in the H-R diagram ${ }^{1}$ shown in Fig. 1.1, composed of 22000 stars from the Hipparcos Catalogue together with 1000 low-luminosity stars (red and white dwarfs) from the Gliese Catalogue of Nearby Stars. The figure also shows the locations of the luminosity classes, according to the Morgan-Keenan classification, and, in the upper horizontal axis, it shows the effective temperature and modern spectral classification of the stars.

From the figure, we see that the most populated luminosity class is the Main Sequence. The main sequence is the locus of the stars that are burning hydrogen in their cores. The hydrogen burning phase is the longest phase in the life of the star, which explains why most of the randomly selected stars are found in the main sequence.

The least massive viable star (capable of hydrogen burning) has about $0.08 M \odot$, and its absolute magnitude is about +19 . Such stars are at the bottom of the H-R diagram of Fig. 1.1. The absolute magnitude of the Sun, a G2V star, is +4.8 . The heaviest stars begin their lives with masses of roughly $100 M_{\odot}$ (or possibly more), and they are the Galaxy's beacons, with absolute magnitudes above -8 , almost on the top of the H-R diagram of

\footnotetext{
1 The most famous diagram in astronomy is the Hertzsprung-Russell diagram, or simply H-R diagram, idealized by Ejnar Hertzsprung in 1911 and, independently, by Henry Norris Russell in 1913. This diagram is a plot of luminosity (or absolute magnitude) against the colour of the stars.
} 


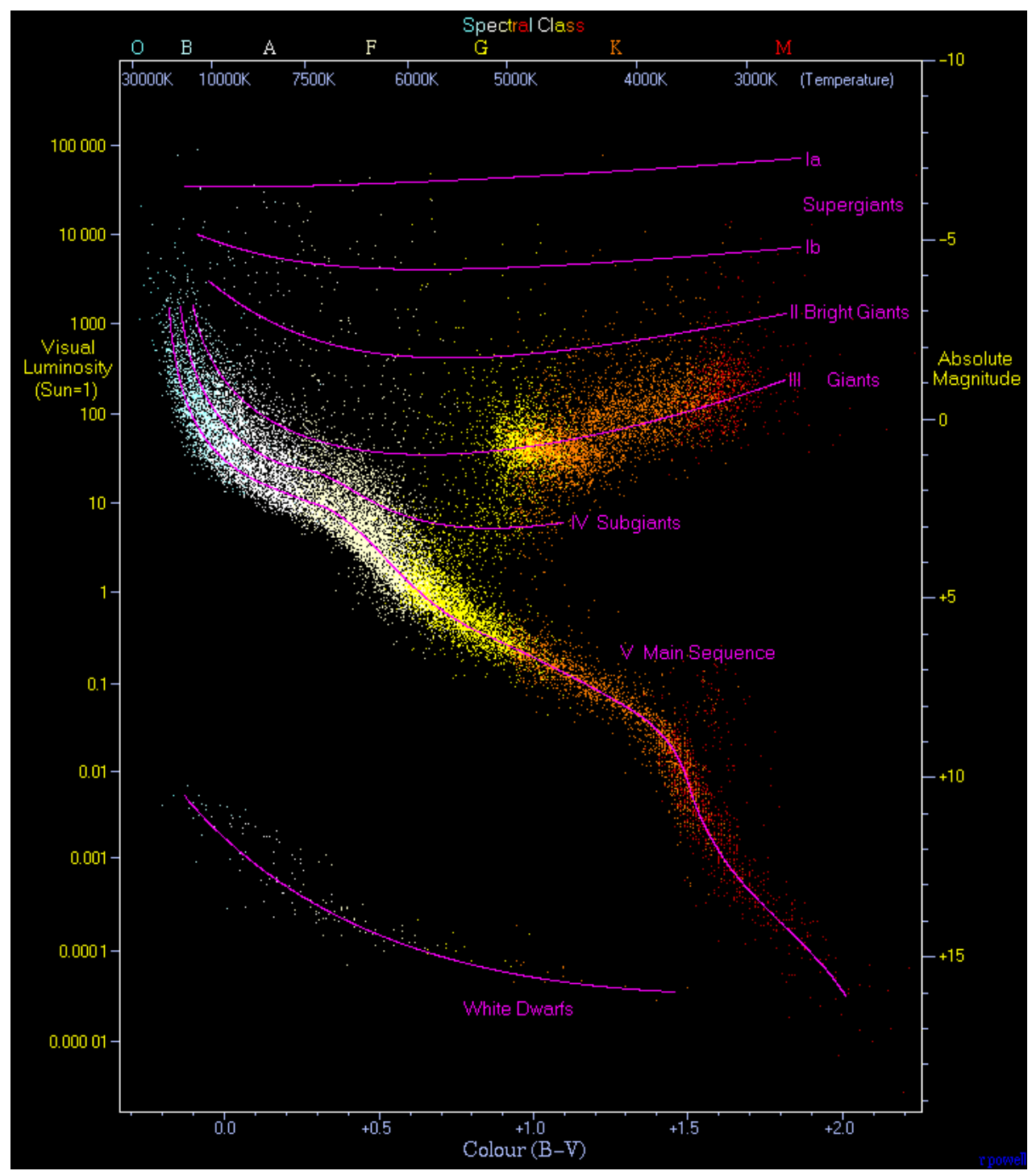

Figure 1.1: A plot of 22000 stars from the Hipparcos Catalogue together with 1000 low-luminosity stars (red and white dwarfs) from the Gliese Catalogue of Nearby Stars. (Credits: http://www. atlasoftheuniverse.com)

Fig. 1.1. The range of absolute magnitudes found in the stars, therefore, occupies an interval of $\sim 27$ magnitudes, or a factor of $\sim 6 \times 10^{10}$ in luminosity (Ledrew, 2001).

It is found that the more massive the star, the rarer it is. Nature has a preference for forming less massive stars. For stars more massive than the Sun, the work of Kroupa (2001) finds that the probability density $\xi$ (commonly referred to as the Initial Mass Function - IMF) of a Galactic star to be formed with mass $M$ is $\xi(M) \propto M^{-2.3}$. Consequently, OB main sequence stars, for instance, are only approximately $0.1 \%$ of the stars in the Solar neighborhood (Ledrew, 2001). Also, the more massive the star, the shorter is its life. A star with 10 times the mass of the Sun, for instance, has a lifespan $\sim 120$ times shorter than the life of the Sun (Granada et al., 2013).

Despite the fact that most of the stellar mass in the Galaxy is found in low-mass, dim 
stars, nearly all of the light in the galaxies is emitted by the brightest stars, in such a way that our naked-eye view of the sky is strongly biased towards the brighter stars (mostly B and A typed main sequence stars, Ledrew, 2001).

Massive stars are generally defined as those with masses higher than around $8 M_{\odot}$, which are the stars that will end up their lives as supernovae. Because, they are shortlived, they are usually found in or not too far away from their birth places. Massive stars are, then, typically located in regions of active star formation, such as the spiral arms of spiral galaxies.

Despite their rarity, these stars have a fundamental influence over the interstellar medium (ISM), because they are responsible for the ionization of the surrounding gas. They generate most of the ultraviolet radiation of the galaxies, which is the radiation usually detected in the observations of the farthest and most redshifted galaxies. Also, they power the far infrared of the galaxies through the heating of dust.

The usually strong stellar winds of massive stars and, later on, their supernovae, inject mechanical energy into the ISM and, also, nuclear processed material generated by their cores, during their lifetime. These stars, thus, supply the medium with new material and energy for the birth of new generations of stars.

In this work, we will focus on a group of main sequence, usually massive stars, known as "Be stars". Be stars are found in the lower end of the group of massive stars and also a little below it. In Fig. 1.1, they are found in the upper end of the main sequence (roughly between $\sim 300 L_{\odot}$ and $\left.\sim 30000 L_{\odot}\right)$. We will describe this group of peculiar stars in more detail in the next section.

\subsection{On Be stars}

(...) une particularité curieuse (...) une ligne lumineuse très belle et bien plus brillante que tout le reste du spectre.

Father A. Secchi, 1866

In a classical, observational, and quite broad definition, a Be star is a hot star, with a B spectral type (mass ranging roughly from 3 to 17 solar masses), non-supergiant, whose spectrum has, or had at some time, one or more Balmer lines in emission (Jaschek et al., 1981; Collins, 1987). In a more modern and theoretically-oriented definition, a Be star is a very rapidly rotating B star, tipically with non-radial pulsations, that forms a geometrically thin viscous decretion disk (VDD) composed of an outwardly diffusing gaseous Keplerian disk that is fed by mass ejected from the central star and is governed by viscosity (Rivinius et al., 2013) and a possibly non-negligible line-driven wind (Kee et al., 2016). 


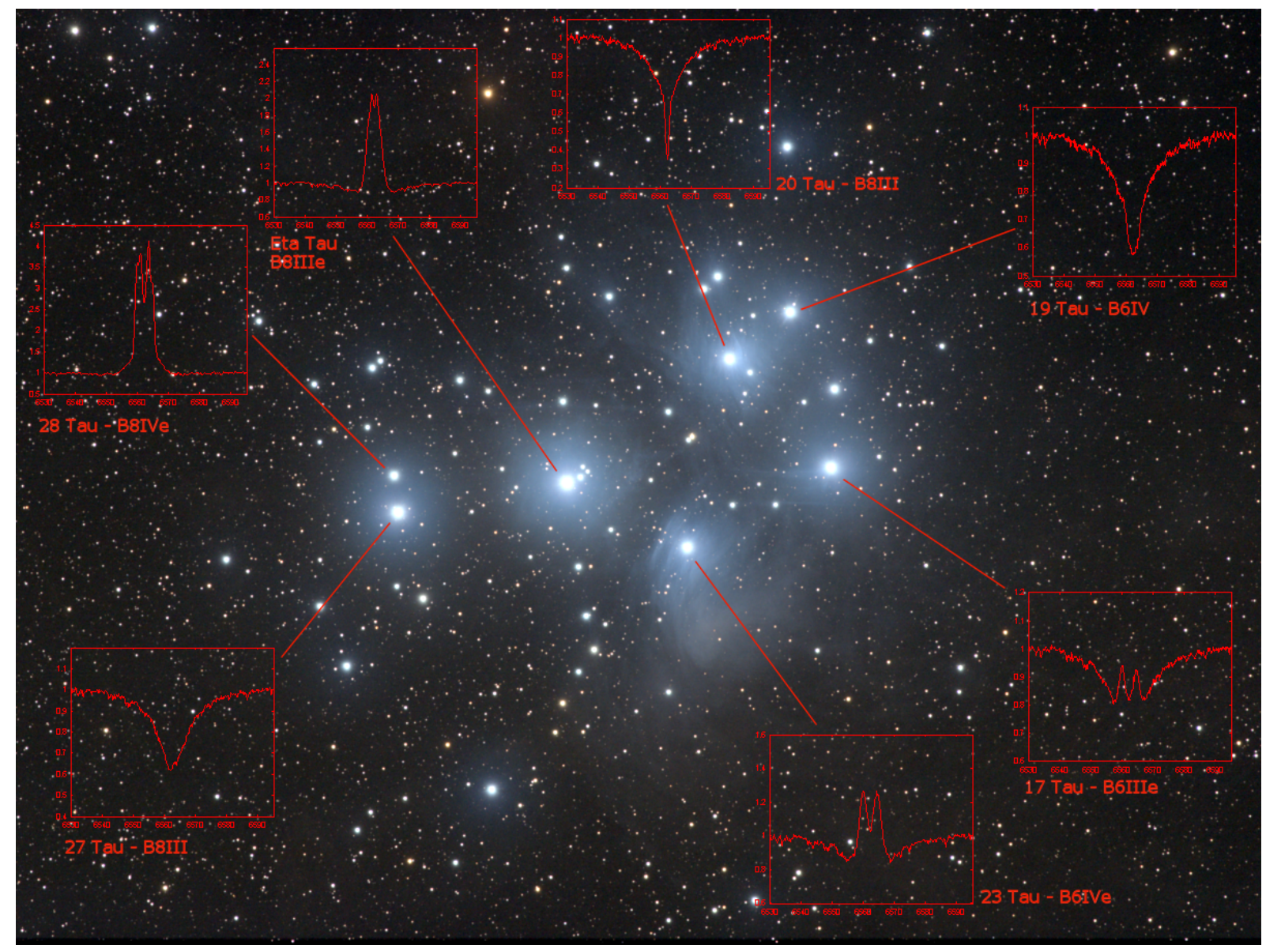

Figure 1.2: Amateur image of the Pleiades open cluster, also showing $\mathrm{H} \alpha$ line profile of the seven main stars. Four of them (17 Tau, $23 \mathrm{Tau}$, Eta Tau and $28 \mathrm{Tau}$ ) are Be stars, which is evidenced from the emission features in their line profiles. Credits: http://www.astrosurf.com/buil/us/bestar.htm

Be stars were discovered roughly 150 years ago, in the early years of astronomical spectroscopy, by Secchi (1866), who noticed the $\mathrm{H} \beta$ line in emission in the Be star $\gamma$ Cassiopeiae. Like, $\gamma$ Cas, some Be stars are among the brightest stars in the visible sky, which makes them interesting also for amateur astronomers (see, e.g., Fig. 1.2). It was only in the last two to three decades, however, that the understanding of Be stars has advanced from simple classifications and toy-models to more sophisticated views based on physical principles. This happened, both due to the advances in observing techniques, including the now available optical an near-infrared (near-IR) interferometry, and due to computational and theoretical advances, which allowed the construction of much more detailed and complex physical models for these stars.

Be stars are not rare. Approximately $10 \%$ of the main-sequence B stars in the Galaxy are in fact Be stars (Rivinius et al., 2013). This percentage can grow to roughly 35\% when we go to the Small Magellanic Cloud and can be even higher in some young clusters (Martayan et al., 2011).

The "e" letter in the spectral classification of Be stars comes from "emission". Indeed, 

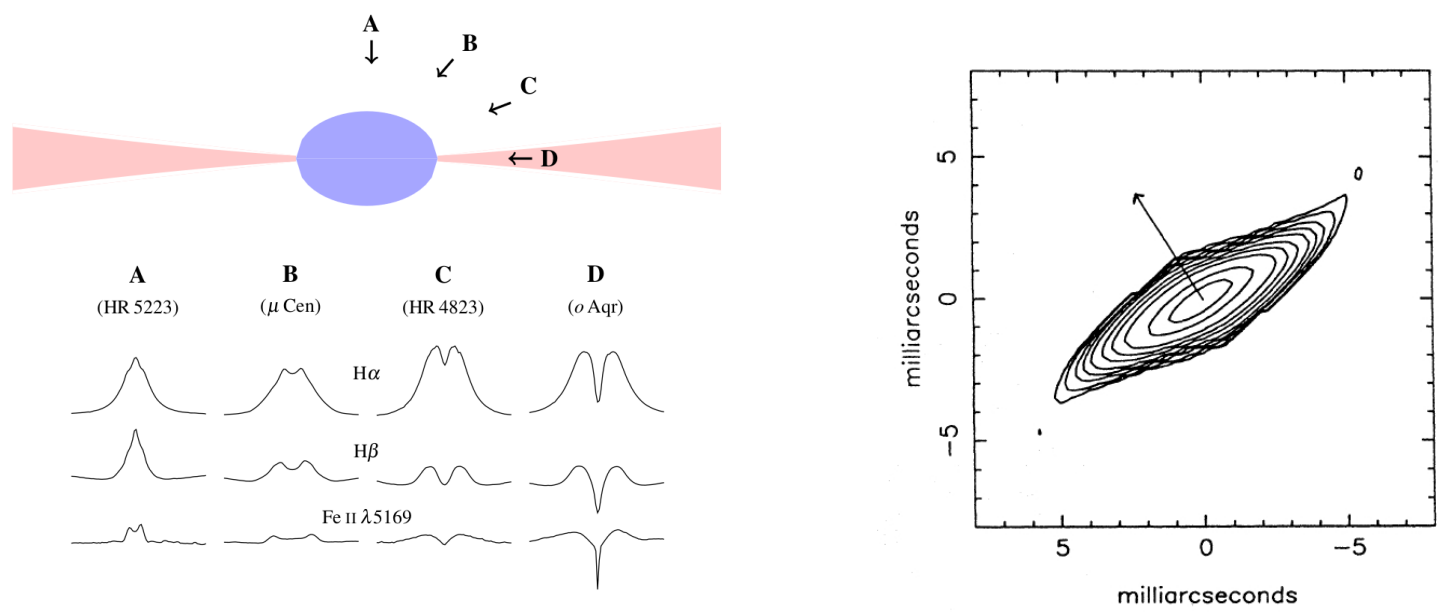

Figure 1.3: Left: Schematic view of a Be star with a flared disk (and an oblate fast rotating central star). Below, examples of spectral profiles from pole-on to shell Be stars are shown (Credits: Rivinius et al., 2013). Right: Image reconstruction of the Be star $\zeta$ Tauri in $\mathrm{H} \alpha$, based on optical interferometry. The arrow indicates the direction of linear polarization. (Credits: Quirrenbach et al., 1994)

they are easiest identified by the emission features in their spectra, examples of which are seen in Fig. 1. This extra emission comes from circumstellar material around the star. Further evidence of this material comes from the excess IR emission (e.g., Gehrz et al., 1974) and polarization (e.g., Wood et al., 1997). Furthermore, there is ample evidence that this material is not spherical, but rather distributed in a disk-like structure (Quirrenbach et al., 1994). One important example is the double-peaked profile of emission lines. The simplest explanation for this feature is a rotating and geometrically thin gaseous disk of material, as the schematic Fig. 1.3 (left panel) illustrates. The two peaks are the blueshifted (left) and redshifted (right) emission from the two oposing sides of the rotating disk. Be stars whose disks are seen nearly edge-on, which present intense absorption at the center of the line, are commonly referred to as shell stars.

Incidentaly, the double-peaked shape in the Balmer lines also means that the radial velocity of the disk-like material is much smaller than the rotation velocity. If that were not the case, the significant outward radial motion would produce asymmetric line profiles with redshifted emission and blueshifted absorption that would resemble the classical $\mathrm{P}$ Cygni profiles for winds of high radial velocities (Porter and Rivinius, 2003). Since such profiles are not observed in the $\mathrm{H}$ recombination lines, the radial velocity is observationally constrained to upper limits of the order of $\sim 10-20 \mathrm{~km} \mathrm{~s}^{-1}$ (Owocki and Ud-Doula, 2003).

Despite the evidence, there was still some dispute over the non-sphericity of the circumstellar material of Be stars during the 70s and 80s (Rivinius et al., 2013). The final confirmation of the disk-like nature of the circumstellar material came from optical interferometry, beginning with the work of Quirrenbach et al. (1994), who produced the first 
interferometric image of a Be star ( $\zeta$ Tauri, see the right panel of Fig. 1.3), showing its highly flattened appearance.

Be stars are sometimes confused with Herbig Ae/Be stars, which are pre-main-sequence objects. These stars are still embedded in clouds of gas and dust and are accreting material, usually in the form of circumstellar disks. Be stars, however, are not pre-mainsequence objects. Their circumstellar disks originate from outbursts from the star and not from an external cloud.

An important feature of Be stars is that, due to the high effective temperatures of their central B type stars, their disks are completely ionized and devoid of dust. Therefore, their opacity comes primarily from hydrogen and free electrons (Carciofi, 2011). These opacities are much better understood than the opacities of the disks of young stellar objects (YSO), which are dominated by dust. This is an excellent advantage for using Be stars with the aim of understanding the physics of circumstellar disks of astronomical objects.

The regions above and below the equatorial disk are similar to those around a normal B-type star. They present a weak radiatively driven wind. When viewed at near poleon angles, Be stars show weak asymetric blueshifted UV absorption lines (weak P Cygni profiles), most typically of Si IV and C IV (Snow, 1981), only accessible from observations from space. From these lines, wind velocities and densities can be derived (Puls et al., 2008). Typical wind mass loss rates of Be stars are $\sim 10^{-11}-10^{-9} M_{\odot} \mathrm{yr}^{-1}$ and typical terminal velocities are $\sim 500-1500 \mathrm{~km} \mathrm{~s}^{-1}$ (Snow, 1981).

The presence of the circumstellar disk causes scattering of part of the stellar radiation by free electrons. This, together with absorption by $\mathrm{H}$ atoms, is the cause of the observed polarized spectrum of Be stars, which is described in details by Haubois et al. (2014). The detailed shape of the polarized continuum is very sensitive to the density of the disk material, and has been used as a density diagnostic in the literature (an example will be provided below). Furthermore, in a disk-like configuration iluminated by a central star, the net polarization will be perpendicular to the disk. This is illustrated in Fig. 1.3, which shows that the direction of the linear polarization is perpendicular to the main elongation axis determined by interferometry. Almost all Be stars emit polarized continuous light (Rivinius et al., 2013), and the percentage of linear polarization is found to be as high as $2 \%$ for some denser disks.

As mentioned above, additional evidence of the presence of the disk is the flux excess observed from visible to radio wavelenths. This excess is caused by continuum emission from the disk by both the free-free process, involving free electrons, and the free-bound recombination, involving protons and electrons.

In addition to the fact that the radial velocity of the disks of Be stars must be much smaller than the azimutal velocity $v_{\phi}$, as imposed by the symmetric double-peaked shape with a central absorption, there is another feature observed in the line profiles that sug- 

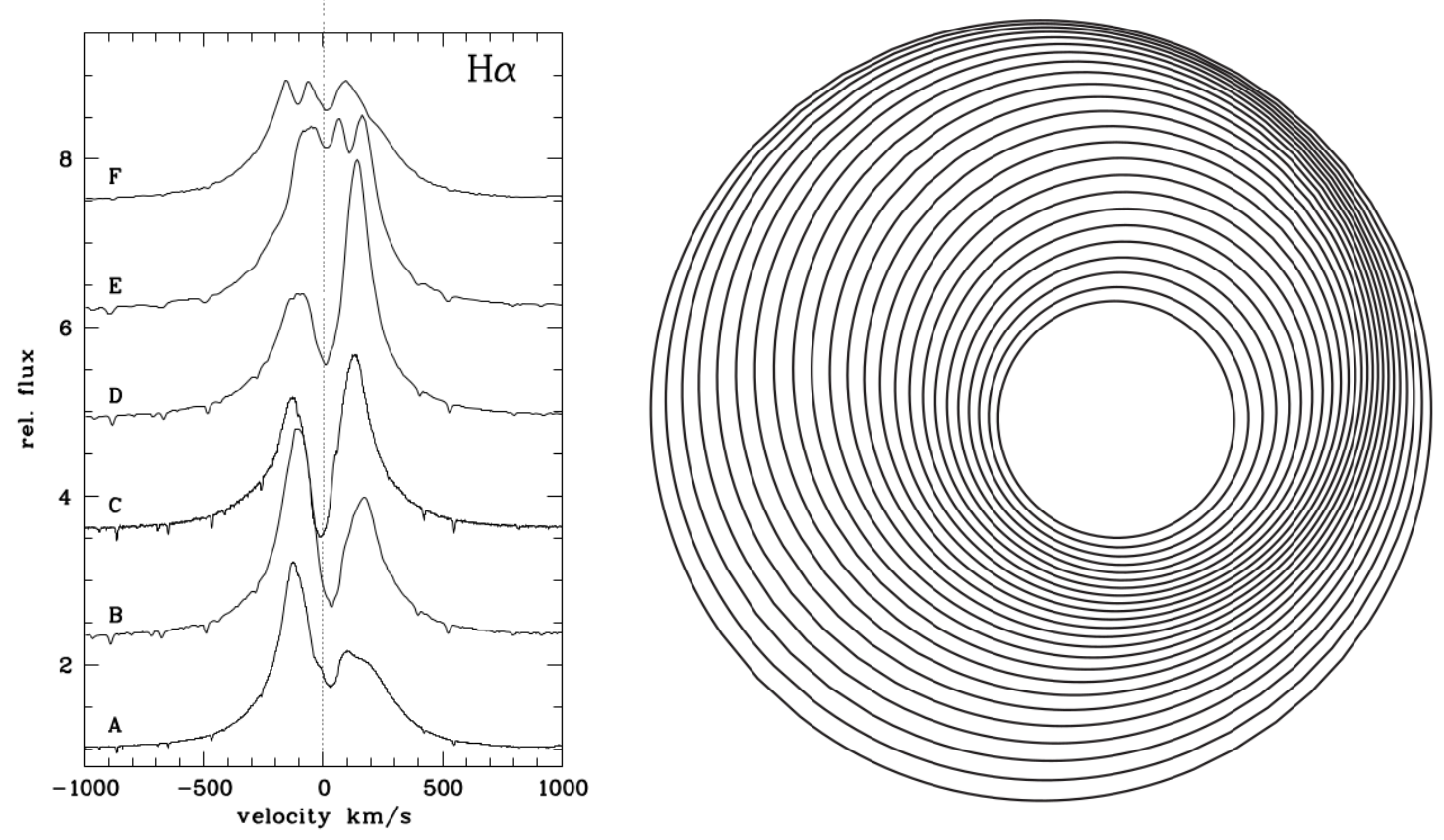

Figure 1.4: Left: $\mathrm{V} / \mathrm{R}$ variations in $\mathrm{H} \alpha$ of the Be star $\zeta$ Tauri. The period of the variations is $\sim 3.8$ years. (Credits: Štefl et al., 2009). Right: Schematic representation of an one-armed spiral pattern generated by elliptical (perturbed) orbits. (Credits: Sparke and Gallagher, 2007)

gests that the azimutal flow must be near-Keplerian (i.e., $v_{\phi} \propto 1 / R^{\frac{1}{2}}$ ), namely the $\mathrm{V} / \mathrm{R}$ variations, which are near-periodic asymmetries in the line profiles observed in several Be stars (Okazaki, 1997). In the left panel of Fig. 1.4, V/R variations of the $\mathrm{H} \alpha$ profile of $\zeta$ Tau are shown. The period of the variations for that Be star is $\sim 3.8$ years (Štefl et al., 2009).

The $V / R$ variations are easily explained in the context of a Keplerian disk. In an exactly Keplerian disk, which would be the case if the fluid elements were only under a radial acceleration from a point-like central star, then the orbits of the fluid elements would be circular, with azimutal velocities following $v_{\phi} \propto 1 / R^{\frac{1}{2}}$. A global perturbation on the disk would make these orbits elliptical, forming an one-armed spiral pattern composed by the clumping of the elliptical orbits like the one sketched in Fig. 1.4 (right). For a fluid under a radial acceleration $\propto 1 / r^{2}$, that configuration would be static (Sparke and Gallagher, 2007). The density of the disk would be greater near the clumping of the orbits and smaller away from them. However, due to several deviations from the $1 / r^{2}$ law - e.g., from deformities of the central star from the spherical shape, or the radiative wind from the central hot star - the spiral pattern shown in the figure will precess around the star, usually in timescales much longer than the orbital periods of the orbits. The secular winding of the spiral pattern, produced by a near-Keplerian disk, explains the near periodic $\mathrm{V} / \mathrm{R}$ variations, as first demonstrated by (Carciofi et al., 2009).

Finally, apart from the $\mathrm{V} / \mathrm{R}$ variations, which are perturbations of a steady-state disk, 

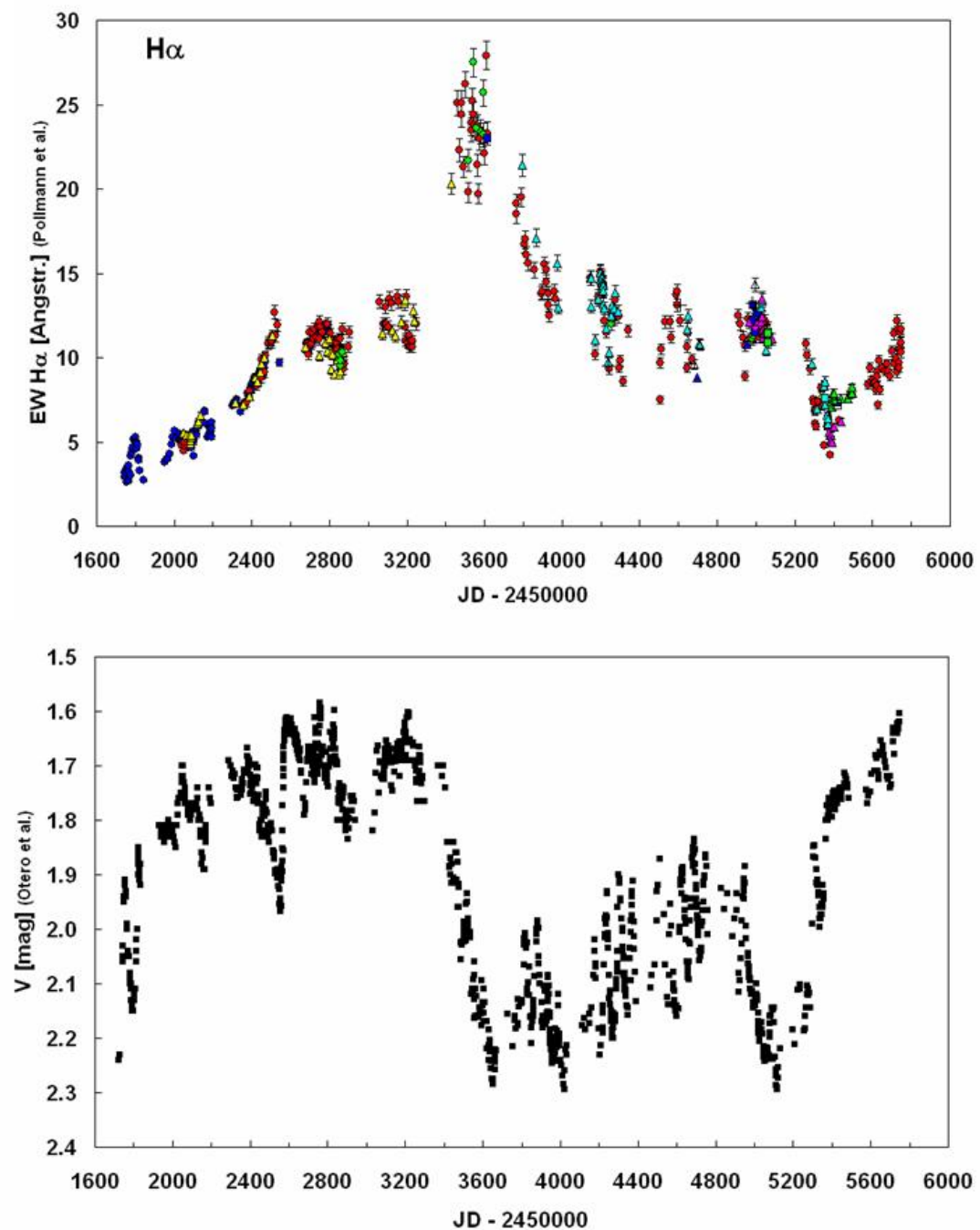

Figure 1.5: Variability of the Be star $\delta$ Scorpii over more than 11 years. Above: Equivalent width of the $\mathrm{H} \alpha$ emission line. Below: $V$-band light curve. (Credits: https://www.aavso.org/vsots_delsco)

another striking feature of Be stars is that most are usually variable in all observables and in several timescales (days, weeks, months or even years). This variability indicates that the injection of matter and angular momentum from the star into the disk is variable, with sudden outbursts of mass injection and periods of no or negligible mass injection. As a result of that, many Be stars are known to alternate phases in which a disk is present with diskless phases, during which their spectroscopic appearance is that of a normal $\mathrm{B}$ star. Rivinius et al. (2016) proposes a terminology, which will be used here, in which a Be star that possesses a disk is said to be active and, conversely, when there is no detectable disk, the Be star is inactive. Two aditional terms are used to distinguish the phases of active disk formation (outbursting Be star) and dissipation (dissipating Be star).

As an example of variability, in Fig. 1.5 we show measurements of the equivalent width 
(EW) of the $\mathrm{H} \alpha$ line (above) and the visual photometric $V$ band (below) of the Be star $\delta$ Sco over the course of more than 11 years, during which the $V$ magnitude varied between its inactive value $(V=2.3)$ and its maximum excess $(V=1.59)$.

From the panels, we see that a disk started forming around RJD $=1700^{2}$. The continuum $V$-band excess, which comes from the inner regions of the disk (Carciofi, 2011), rapidly increased until around $\mathrm{RJD}=2000$. From then on, there was still a general trend of increasing, but at a slower rate, as if a plateau were being reached. The equivalent width of $\mathrm{H} \alpha$, which originates from a much larger region than the visual $V$ band (Carciofi, 2011), increased during this time at a much slower pace. The panels also show that, apart from this general description, the evolution of the observables was filled with small-scale variability, very likely indicating that the rate of disk feeding from the star was not constant but rather variable.

Nearly around RJD $=3400$, however, there was a fast decrease in the $V$-band excess, indicating the disappearance of the inner disk. This happened because the disk stopped being fed by the star. That resulted in an initial increase in the $\mathrm{H} \alpha \mathrm{EW}$ line due to the fact that the continuum around the line decreased just like the $V$-band excess. However, after the peak, the EW also started to decrease, showing that the extended disk also gradually disappeared. The light curve of $\delta$ Sco is a very irregular one. Several Be stars show light curves more well behaved, even with some periodicity in the outbursts (see, e.g., Mennickent et al., 2002; Sabogal et al., 2005).

\subsubsection{The fastest rotators among non-degenerate stars}

Another peculiarity of Be stars is their fast rotation, which is easily seen by their broad photospheric lines. A convenient parameter for specifying the rotational rate of a star is the ratio of the rotational velocity at the equator to the orbital velocity at the equator:

$$
W=\frac{v_{\text {eq }}}{v_{\text {orb }}},
$$

where

$$
v_{\mathrm{orb}}=\left(\frac{G M}{R_{\mathrm{eq}}}\right)^{\frac{1}{2}},
$$

where $M$ is the mass of the star and $R_{\text {eq }}$ is its equatorial radius. The parameter $W$, therefore, is a number between zero (for a non-rotating star) and one (for a star rotating at its critical limit).

Another commonly used parameter for specifying the rotational rate of a star is the ratio of the surface angular velocity to the critical surface angular velocity (assuming that the star's shape is given by the a Roche equipotential, see Sect. 2.3):

$$
\omega=\frac{\Omega}{\Omega_{0}},
$$

\footnotetext{
${ }^{2}$ The reduced Julian Date (RJD) is given by RJD $=\mathrm{JD}-2450000$.
} 

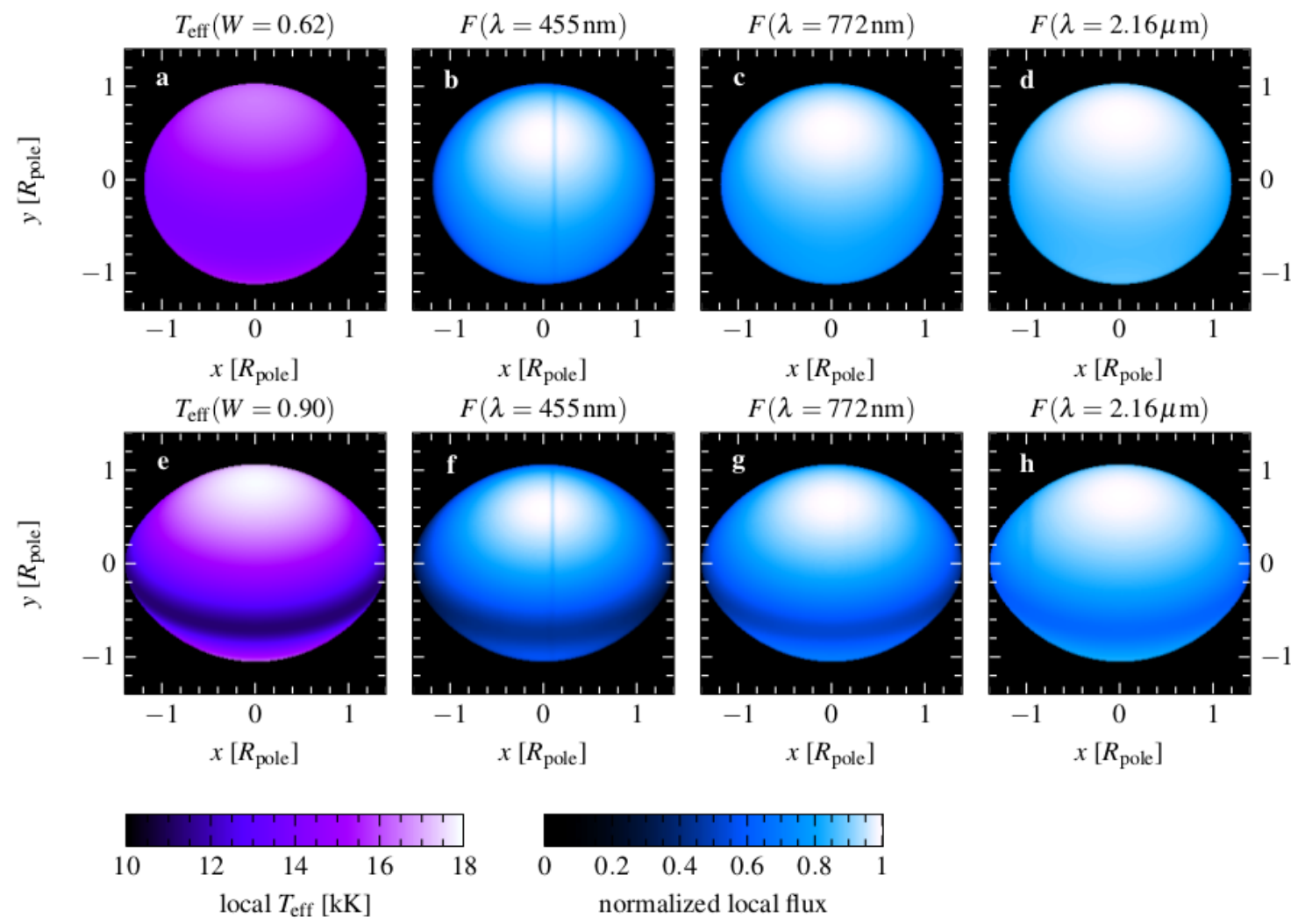

$x\left[R_{\text {pole }}\right]$

$x\left[R_{\text {pole }}\right]$

$x\left[R_{\text {pole }}\right]$

$F(\lambda=455 \mathrm{~nm})$

$F(\lambda=772 \mathrm{~nm})$

$F(\lambda=2.16 \mu \mathrm{m})$
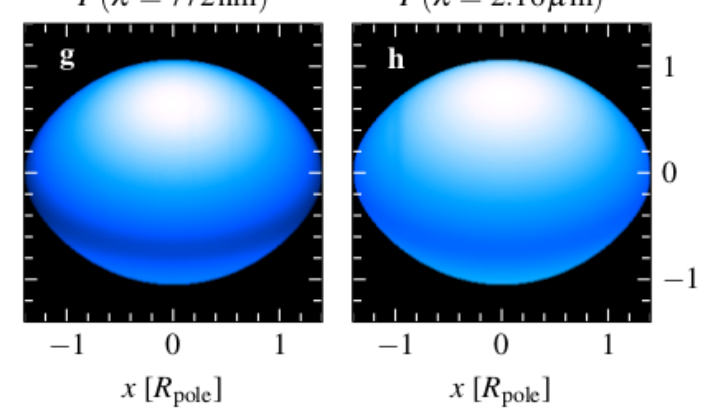

$x\left[R_{\text {pole }}\right]$

$x\left[R_{\text {pole }}\right]$

$x\left[R_{\text {pole }}\right]$

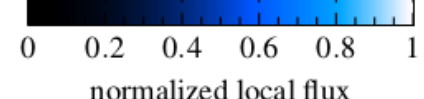

normalized local flux

Figure 1.6: Appearance of a gravity darkened star. The temperatures shown follow the law $T_{\text {eff }} \propto g_{\text {eff }}^{\beta}$, with $\beta=0.20$. Upper row: Surface appearance of a star rotating at $W=0.62$. From left to right: Temperature and flux distributions in line free regions at $\lambda=455 \mathrm{~nm}, \lambda=772 \mathrm{~nm}$, and $\lambda=2.16 \mathrm{~mm}$. Lower row: Same as upper row, but for $W=0.90$. (Credits: Rivinius et al., 2013)

where

$$
\Omega_{0}=\left(\frac{G M}{\left(\frac{3}{2} R_{\text {pole }}\right)^{3}}\right)^{\frac{1}{2}},
$$

where $R_{\text {pole }}$ is the polar radius of the star.

From the compilation of the values of $W$ of several Be stars, from several studies ranging from spectroscopic modeling, interferometric imaging and measurements of $v_{\text {eq }}$ from Be shell stars, it was concluded that all well-studied Be stars present $W \gtrsim 0.7$, which offers a loose estimate for the minimum rotation rate necessary for a B star to become a Be star (Rivinius et al., 2013). So, fast stellar rotation is a property fundamentally linked to the so-called Be phenomenon.

The role of fast rotation for the Be phenonemon is that it reduces the effective gravity mainly at the stellar equator and facilitates mass ejection into an equatorial disk. One important effect of rotation is that the centrifugal acceleration forces the outer layers of the star, specially at lower latitudes, into an oblate shape. Furthermore, the lower 

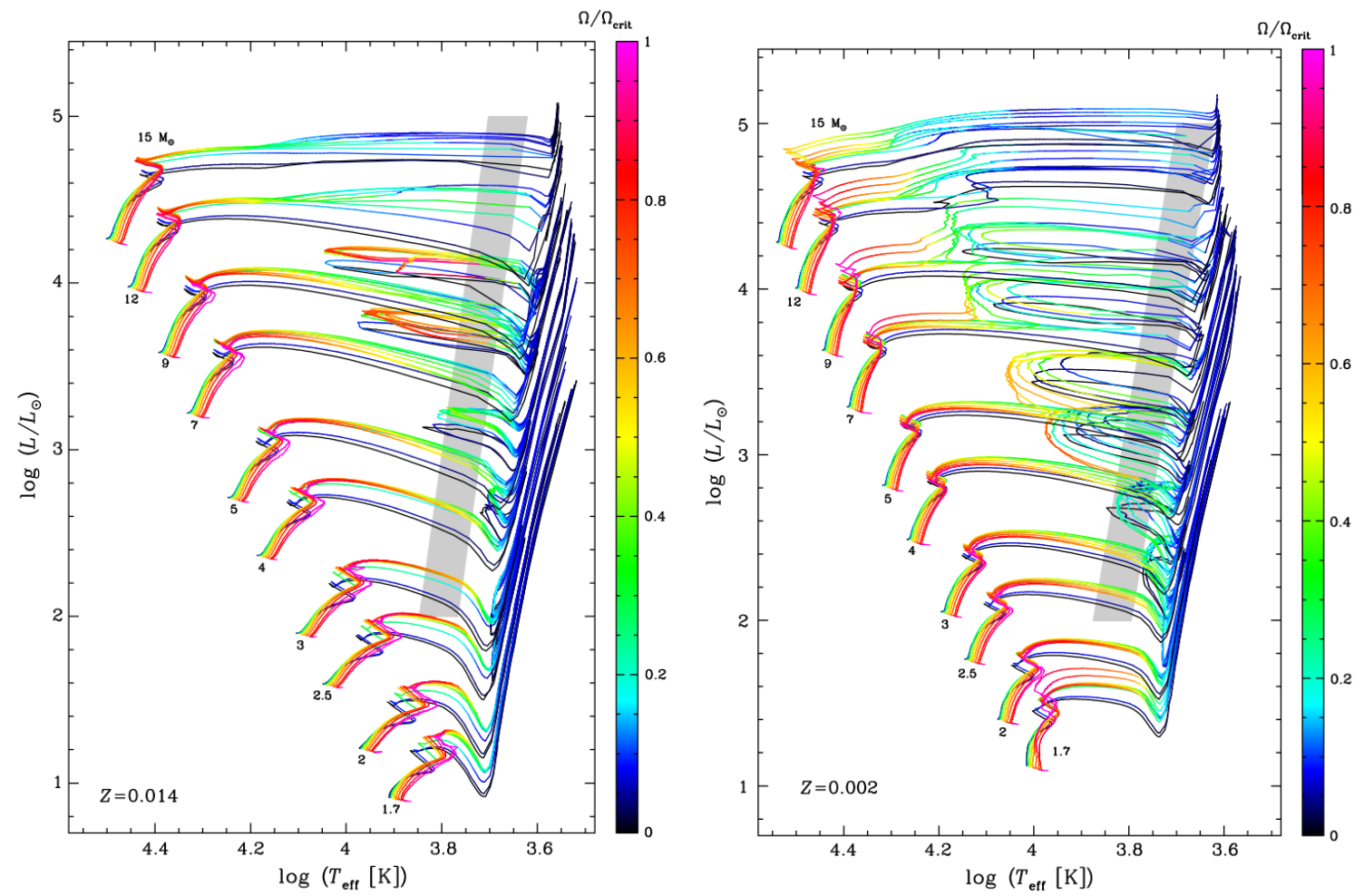

Figure 1.7: Evolutionary tracks for A-B type stars for $Z=0.014$ (left) and $Z=0.002$ (right), from the ZAMS to very late stages of the evolution of the stars. The colour code represents the rotational parameter $\omega$ (scales on the right of each panel). Be stars are usually found with $\omega \gtrsim 0.9$. The gray strips are the classical Cepheid instability strips. (Adapted from: Georgy et al., 2013)

effective gravity near the stellar equator increases the scale height, diverting the internal flux towards the poles (Espinosa Lara and Rieutord, 2011), making them hotter while the equatorial regions become colder. Fig. 1.6 shows the shape, the temperature and the flux in three continuum bands of two stars with rotation rates given by $W=0.62$ (above) and $W=0.90$ (below), and with the surface temperature given by the law $T_{\text {eff }} \propto g_{\text {eff }}^{\beta}$, with $\beta=0.20$. The distribution of the rotation rate $W$ obtained from measurements of Galactic shell Be stars peaks around $W=0.81$ with a standard deviation of 0.12 (Rivinius et al., 2006). Therefore, the top panels represent rotating B stars usually rotating too slowly to be Be stars, and the lower panels likely represent the rapidly rotating Be stars.

There are two proposed evolutionary channels to explain why Be stars rotate so fast. The first channel involves only an isolated star. As the rotating B star evolves on the main sequence, core contraction and internal angular momentum redistribution generally tends to enhance surface angular rotation (Ekström et al., 2008; Granada et al., 2013). The near critical limit is, therefore, reached as a natural evolution of the rotating star (Ekström et al., 2008). The best rotating evolutionary models, however, require that such stars must already start as quite fast rotators already in the zero age main sequence (ZAMS). In Fig. 1.7, we show evolutionary tracks of $\mathrm{A}$ and $\mathrm{B}$ type stars for metallicities $Z=0.014$ 
(left) and $Z=0.002$ (right), from the ZAMS to very late stages of the evolution of the stars. The colors of the tracks give information of the rotational rate of the stars. The condition that Be stars are found with $W \gtrsim 0.7$ corresponds to the condition $\omega \gtrsim 0.9$ in their scale, and, therefore, the red to pink portions of the tracks are the predicted locii of Be stars in the HR-diagram.

Not much after the end of the main sequence phase, the expansion of the outer layers of the stars reduce $W$, which must inhibit the Be phenomenon from then on. Be stars might, thus, be a natural outcome of stars with an initial rotational velocity in the upper extreme of the initial velocity distribution.

The other proposed channel for the fast rotation of Be stars is interaction with a binary companion. The most massive stars $\left(M \geq 8 M_{\odot}\right)$ either are binaries (about $75 \%$ ) or were so at some point of their evolution (Sana et al., 2012). It is possible that at least some fraction of the fast rotating Be stars were spun-up due to mass transfer from its binary companion (Ekström et al., 2008).

Is rotation the only key parameter to control whether a B star becomes a Be star? The answer to that is: probably no. Since several Be stars rotate still quite below the critical limit $(W \approx 1)$, and since old theories of disk formation as a consequence of the winds (see Sect. 1.1.2 below) have failed to predict the general properties of the observed disks, it follows that an additional mechanism, referred to in the literature as the "Be mechanism" must operate in order to give the additional push to the near equatorial matter, in order to put it in orbit around the star. There is growing evidence that this mechanism must be stellar pulsation (Rivinius et al., 1998; Baade et al., 2016; Rivinius et al., 2016).

Why is the Be phenomenon mainly restricted to B type stars? Ekström et al. (2008) especulate that, for stars with spectral type earlier than B (O-type) stars, the intense mass and momentum loss through radiative winds prevents these stars from reaching near critical limits of surface rotation. These massive stars actually have their surfaces decelerated during their evolution in the main sequence. Following Kee et al. (2016), we would add that, even if the stars were rotating near critically, the strong radiative winds would ablate any forming disk too fast to be detected as a Be disk. Conversely, Ekström et al. (2008) also argue that, for stars with spectral type later than B (A-type stars), the meridional currents are not strong enough to accelerate the outer envelope.

Following the argument of Ekström et al. (2008), in environments of lower metallicity, the line-driven radiative mass and angular momentum loss must diminish, further extending the range of appearance of the Be phenomenon to stars of earlier type in those environments. Also, the minimum initial mass for the meridional currents become efficient enough to accelerate the surface would raise. That would also move the minimum mass for the appearance of Be stars upwards (Ekström et al., 2008).

Therefore, with their gaseous disks and fast rotation, Be stars, which some decades ago were considered to be of little relevance to the main field of hot and massive stars, 

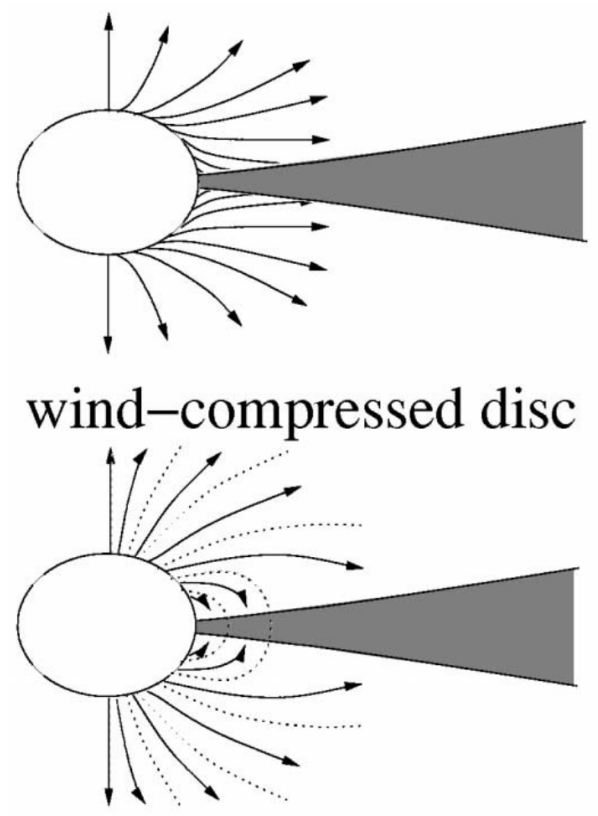

magnetic WCD

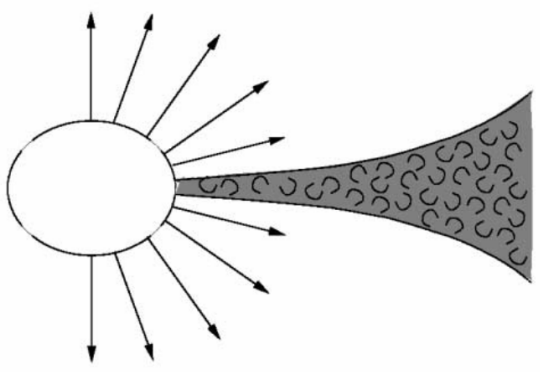

viscous disc

Figure 1.8: Wind streamlines and disk formation for three disk models. In the WCD, the streamlines are deflected towards the equator, creating the disk. In the MWCD, the wind is channeled by a magnetic field towards the equator, creating the disk. In the VDD, turbulence generates the disk and the wind is not important in the formation of the disk. (Credits: Porter and Rivinius, 2003)

turned out to be fundamental laboratories to test the physical mechanisms relevant for the evolution of the upper main sequence stars!

\subsubsection{The Viscous Decretion Disk (VDD) model}

Several theories were put forward to try to explain the properties of the disks of Be stars (even before they were actually confirmed interferometrically as disks in the mid nineties). As a first example, there is the toy-model used by Waters et al. (1987) for estimating of the mass loss rates of Be stars, using the Infrared Astronomical Satellite (IRAS) data. Their model was that of a mass conserving outflowing equatorial wind, with no other hydrodynamics involved. Coupled with a simple treatment of radiative transfer, this model offered some insight into the circumstellar properties of Be stars. 
Some authors, however, still mistakenly cite their mass loss rate determinations for Be stars, which are two orders of magnitude above the now accepted values, as Vieira et al. (2015, 2017) have recently shown, and this work will also confirm.

Fig. 1.8, schematically shows three hydrodynamically sophisticated disk models proposed to explain the formation of Be star disks.

\subsubsection{Wind Compressed Disk}

Bjorkman and Cassinelli (1993) proposed a model in which the rotation of the radiationdriven wind of the fast rotating star B star would bend the flow towards the equatorial plane (due to the centrifugal and Coriolis forces). The streamlines from opposing hemispheres would, then, produce a shock at the equatorial plane, which would result in a dense region in the equatorial plane, representing the disk. This is the Wind Compressed Disk (WCD) model.

The WCD model was ruled out, however, by dynamical simulations (Cranmer and Owocki, 1995; Owocki et al., 1996) that showed that the nonradial components of the linedriving force and the gravity darkening of the fast rotating star would actually inhibit the equatorial flow required by the WCD model. While ruled out from dynamical arguments, WCDs also failed to reproduce the observed IR excess and the near-Keplerian azimutal flow that agrees with observations (Porter and Rivinius, 2003), since, in this model, the azimuthal velocity is close to angular momentum conserving $\left(v_{\phi} \propto 1 / R\right)$ and the radial outflow is too large also.

\subsubsection{Magnetically Wind Compressed Disk}

Soon later, the spirit of the WCD model was revived with the addition of magnetic fields, in the magnetically wind compressed disk (MWCD, Cassinelli et al., 2002). A key issue was the role of magnetic fields in channeling the line-driven wind of the star into the equatorial plane.

The magnetic fields that are required to significantly affect the B star wind flow are on the order of hundreds of gauss. Currently, however, there is no detection of large scale magnetic fields in Be stars, setting a lower limit of a few hundred gauss to possible large scale fields in Be stars (Wade et al., 2012). Also, simulations show that MWCDs present time dependent configurations more complicated than the expectation that the azimutal flow must be near Keplerian. Actually, these simulations put serious doubts on the feasibility of these disks (Owocki and Ud-Doula, 2003).

\subsubsection{Viscous Decretion Disk}

In the Viscous Decretion Disk (VDD) model, the azimutal motion is near Keplerian and a viscous mechanism produces the torque that causes the outward diffusion of mate- 

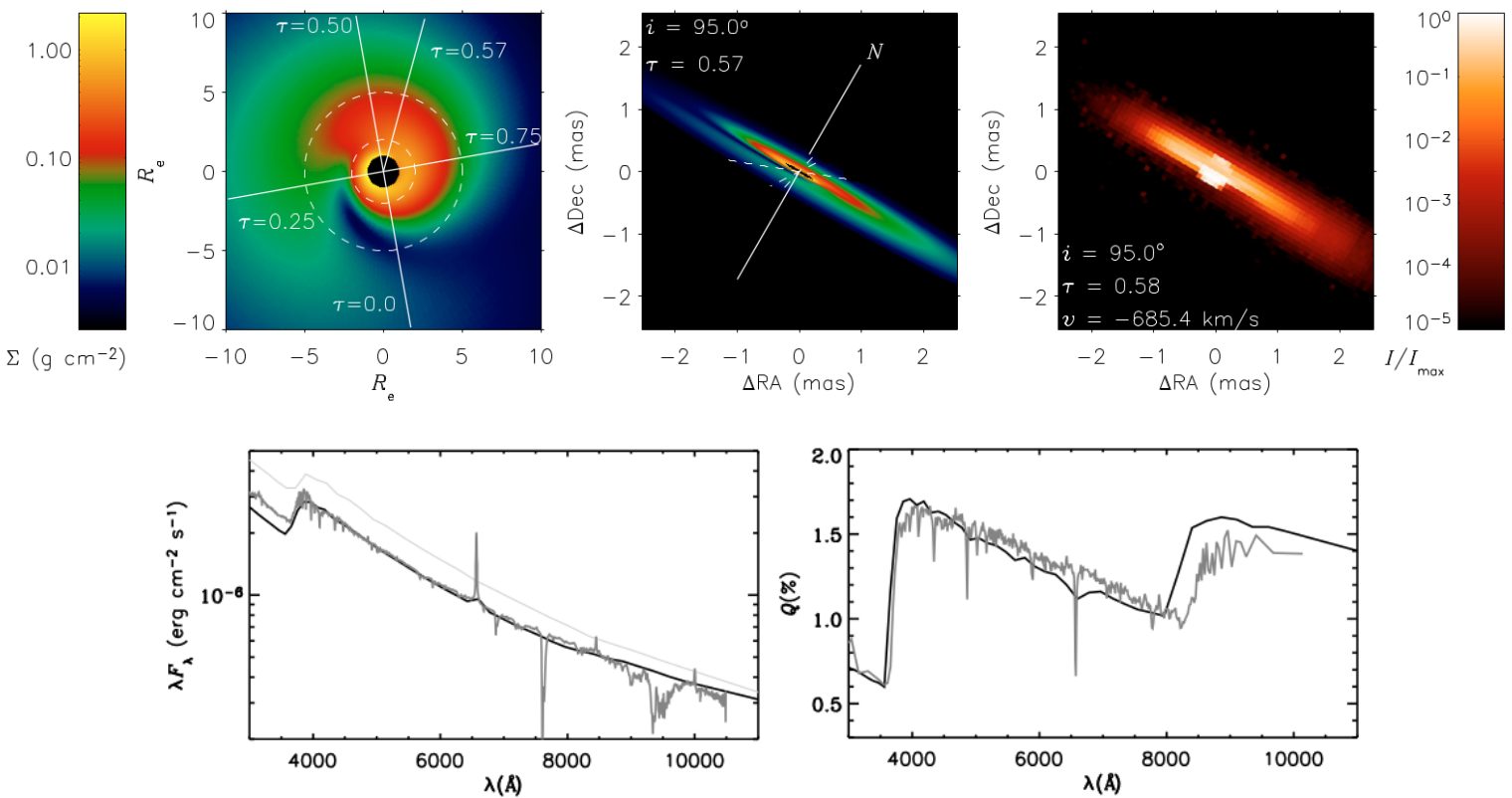

Figure 1.9: A VDD model of the Be star $\zeta$ Tauri that includes global density waves. Above, left: Density perturbation pattern as seen from above the disk. Above, center: Density perturbation pattern projected onto the plane of the sky. Above, right: continuum synthetic image generated by HDUST at $2.16 \mu \mathrm{m}$, and used for interferometric fitting. Below: The dark grey lines are the observations of the optical spectrum (left) and optical spectropolarization (right), and the black lines represent the perturbed VDD model results. (Credits: Carciofi et al., 2009)

rial. The VDD model was first proposed by Lee et al. (1991) and further developed by Porter (1999); Okazaki (2001); Bjorkman and Carciofi (2005), and it is nearly identical, physically, to the accretion disks of pre-main-sequence objects, which are also viscous disks.

In the VDD model, the radiative wind from the star is believed not to participate in the formation of the disk. Rather, the model requires that some mechanism must put the stellar matter into orbit in the inner disk. From that point on, viscosity takes over as the mechanism governing the fate of the material, diffusing matter and angular momentum outwards, thus making the disk grow.

The VDD is the only theory to date that satisfies the whole set of observational facts. It has been demonstrated (e.g., Haubois et al., 2012) that a VDD fed roughly at a constant rate, and for a sufficiently long time (a few to several years, depending on the value of the viscosity), reaches a quasi-steady state in which the density is nearly constant in time. If the gas temperature is properly taken into consideration, the density profile is typically a complicated function of the distance from the star (e.g., Carciofi and Bjorkman, 2008). However, a usual approximation is to consider the temperature of the gas to follow a power law with the radial distance, in which case the density profile assumes a power-law 

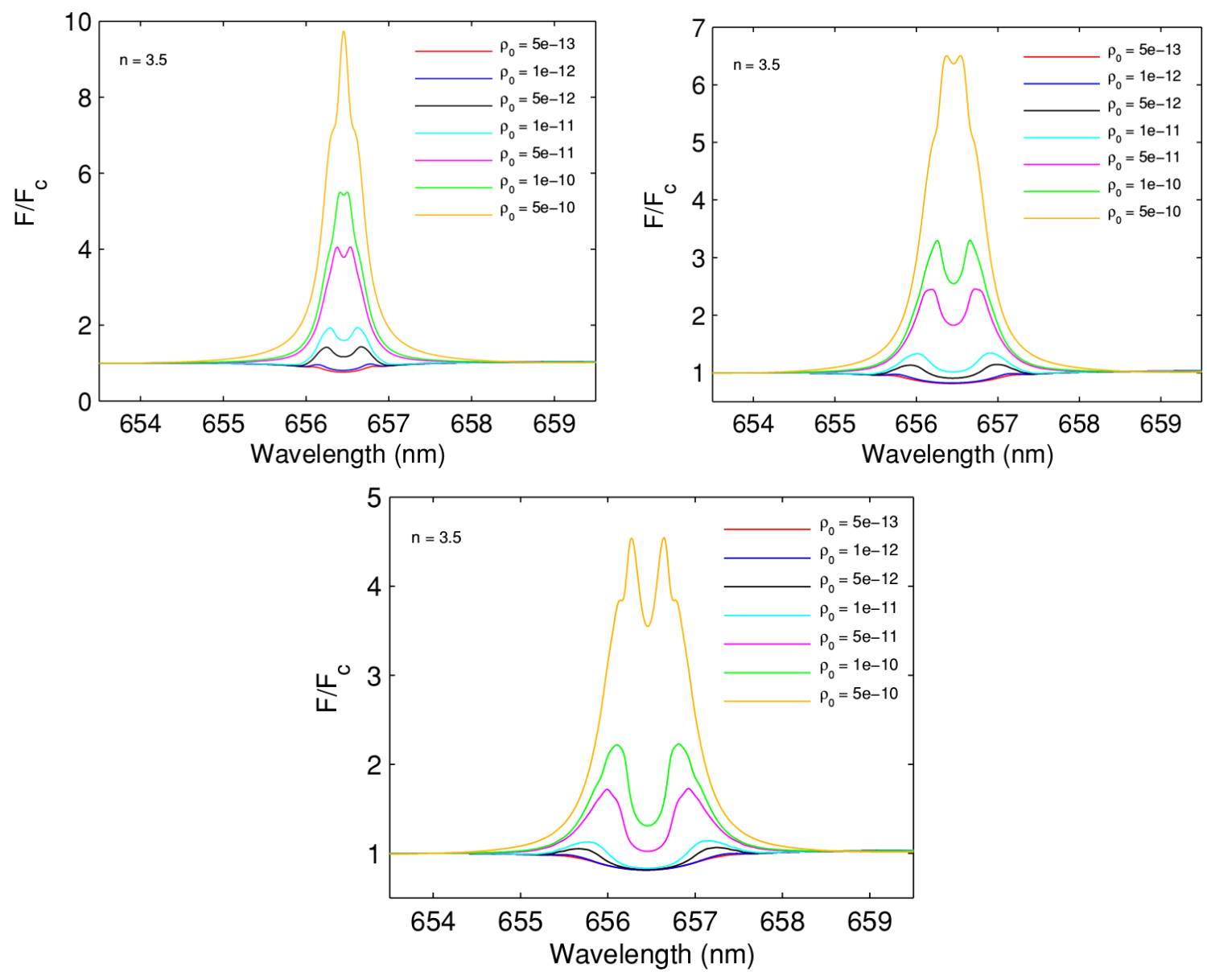

Figure 1.10: Theoretical non-LTE H $\alpha$ line profiles for a B0e star with a Keplerian disk and with several base densities $\rho_{0}$, viewed at $i=20 \mathrm{deg}$ (upper-left), $i=45 \mathrm{deg}$ (upper-right) and $i=70 \mathrm{deg}$ (below). (Credits: Silaj et al., 2010)

form given by

$$
\rho(R, z)=\rho_{0}\left(\frac{R}{R_{\mathrm{eq}}}\right)^{-n} e^{-\frac{z^{2}}{2 H^{2}}},
$$

where $H=\left(c_{s} / v_{\text {orb }}\right) R^{\frac{3}{2}}$ is the disk scale height, with $c_{s}=\left(k T_{\text {disk }} / \mu m_{H}\right)^{\frac{1}{2}}$ and $v_{\text {orb }}=$ $\left(G M / R_{\mathrm{eq}}\right)^{\frac{1}{2}}$ being respectively the isothermal sound speed and the orbital velocity at the stellar equator. For an isothermal disk, we must have $n=7 / 2$ (e.g., Carciofi, 2011).

In the last decade, this simple steady-state VDD has been successful in describing the main observed features of individual Be disks (Carciofi et al., 2006, 2007; Jones et al., 2008; Carciofi et al., 2009; Klement et al., 2015) and samples of Be stars (Silaj et al., 2010; Touhami et al., 2011; Vieira et al., 2017). In Fig. 1.9, in particular, we show a fitting of a steady-state VDD with a density wave to several observables of the Be star $\zeta$ Tau (Carciofi et al., 2009), a Be star well known for its V/R variations, using the Monte Carlo radiative transfer code HDUST (Carciofi et al., 2004; Carciofi and Bjorkman, 2006, 2008).

In Fig. 1.10, we show theoretical $\mathrm{H} \alpha$ line profiles of an early-type Be star made by Silaj et al. (2010), using a non-LTE radiative transfer code. For these calculations, they 

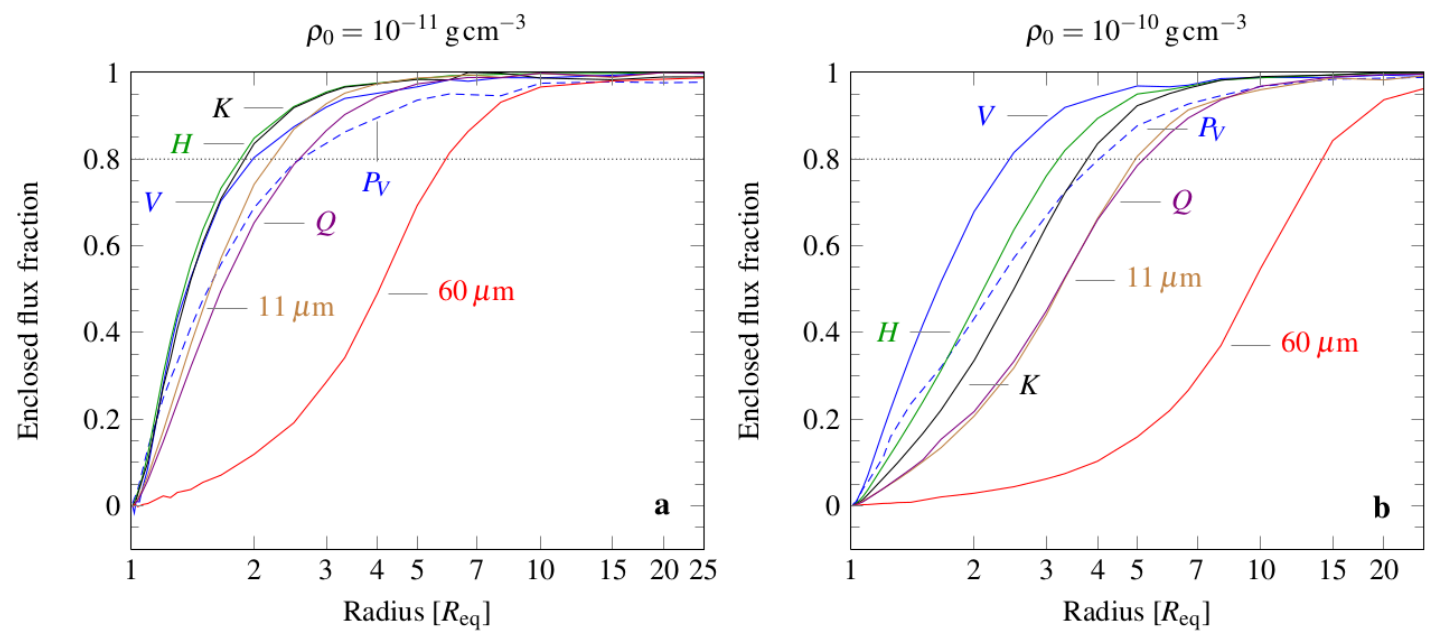

Figure 1.11: The formation loci of continuum emission for various wavelength bands for the base densities $\rho_{0}=10^{-11} \mathrm{~g} \mathrm{~cm}^{-3}$ (left) and $\rho_{0}=10^{-10} \mathrm{~g} \mathrm{~cm}^{-3}$ (right). Data were computed with HDUST, for a steady-state VDD seen at $i=30 \mathrm{deg}$. (Credits: Rivinius et al., 2013)

assumed Keplerian disk with a surface density law given by Eq. (1.1.5), with $n=7 / 2$. From these panels, we may infer that the base density $\rho_{0}=5 \times 10^{-13} \mathrm{~g} \mathrm{~cm}^{-3}$ is roughly the threshold density for the detection of a disk in a Be star.

Fig. 1.11, generated by HDUST, shows the locii of formation of several observables in a VDD, for two density scales. From the figures, we see that continuum visible and nearinfrared observations generally probe the inner disk (between $1 R_{\text {eq }}$ and $\sim 3-4 R_{\text {eq }}$ at most). Mid to far infrared (e.g., $60 \mu \mathrm{m}$ ), on the other hand, probe a much more extended region. The $\mathrm{H} \alpha$ line (not shown in the panels) probe an area more or less similar to the $60 \mu \mathrm{m}$ band (Carciofi, 2011).

I conclude this section reflecting on the fact that the idea of the Keplerian disk existed before the two alternative proposed disks (the WCD and the MWCD). The reason it faced skepticism due to the fact (since $W \sim 0.8$ ) that it required an unidentified mechanism for injecting matter at its base. Mass injection would be much easier if all Be stars were nearly critically rotating $(W \approx 1)$. In that case, a simple convective layer at the (colder) equator would have the energy to produce the observed outbursts. Observations show, however that critical rotation is not the general case of Be stars. Thus, the VDD requires the "Be mechanism": a physical mechanism able to give the additional push to the near equatorial stellar matter in order to put it into orbit. Also, the VDD requires a viscous mechanism, which needs a physical description. What is the source of this viscosity? (Since the circumstellar matter is a hot plasma, it must contain small scale magnetic fields, which must be a clue.) What is the required magnitude of this viscous mechanism? These fundamental questions are partly adressed in this thesis. 


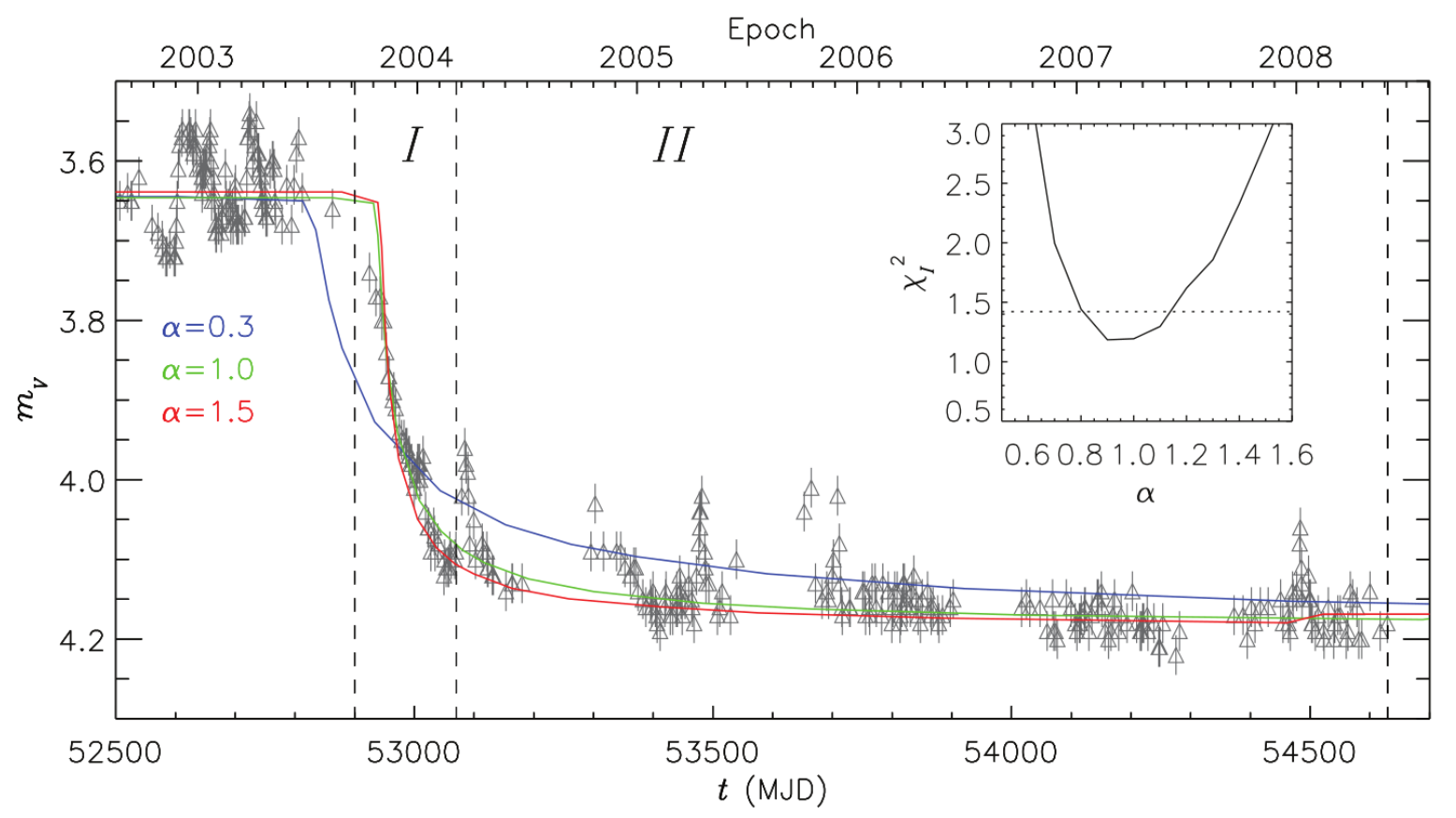

Figure 1.12: $\quad V$-band light curve of the Be star $28 \mathrm{CMa}$, together with synthetic light curves of the dissipation of a VDD for different values of the viscosity parameter $\alpha$. (Credits: Carciofi et al., 2012)

\subsection{Dynamical VDDs}

In this work, as in the most of the recent works on Be stars, the VDD model offers the conceptual framework with which Be stars disks are analyzed. Despite the great success of the VDD model, there are several open and intriguing theoretical questions about it that remain unexplained. The main ones are the identification of the "Be mechanism" producing the Be phenomenom and the proper description of the viscous mechanism. Explaining both mechanisms are the key to understand the disk variability of these stars, and may hold the key to understand astrophysical disks in general.

The VDD model was always formulated in the alpha-disk formalism, in which the kinematic viscosity is scaled by the $\alpha$ parameter, defined such that the $R \phi$ component of the stress tensor is proportional to the gas pressure: $W_{R \phi}=-\alpha P$ (Shakura and Sunyaev, 1973).

Dynamical studies of the VDD model are recent in the literature, and still quite scarse. Jones et al. (2008), using a 1-D time-dependent treatment of the alpha-disk and a nonLTE radiative transfer code, studied the temperature and density profiles of a dynamical disk and their respective $\mathrm{H} \alpha$ line profiles. Haubois et al. (2012) studied the theoretical photometric effects of time variable mass injection rates on the structure of the disk, also using an 1-D time-dependent treatment of the alpha-disk, and the radiative transfer code HDUST. 
Carciofi et al. (2012) used these the time-dependent calculations of the disk surface density and the emergent flux at selected times, in order to estimate, for the first time, the $\alpha$ parameter for a Be circumstellar disk. The chosen star (28 CMa) passed from an active phase, that lasted from 2001 to 2003, to a dissipating phase at the end of 2003. The modeling of the dissipating phase allowed them to determine the value $\alpha=1.0 \pm 0.2$.

Later, however, it was realized that a proper consideration of the previous history of the disk must be taken into consideration when fitting the dissipating portion of a lightcurve. This is a consequence of an effect identified during this thesis work, which we dubbed "mass reservoir effect". The discovery of this effect was fundamental for the success of the PhD work of M. Ghoreyshi (IAG/USP) and my own. As described in Sect. 3.3, the discovery of mass reservoir effect resulted in a revision of the $\alpha$ parameter of the 2003 disk dissipation of $28 \mathrm{CMa}$ to $0.21 \pm 0.05$.

\subsubsection{The origin of the viscosity of VDDs}

It has been argued (Shakura and Sunyaev, 1973) that magnetic fields are the likely way in which a plasma shearing disk flow transports angular momentum from the inner rapidly rotating fluid to the outer slowly rotating fluid. Balbus and Hawley (1991) realized that the magnetorotational instability (MRI) could provide the necessary feedback to maintain a magnetic dynamo in the plasma disks (King et al., 2007). Since then, the MRI is the main theoretical bet for the mechanism that generates the necessary viscosity of alpha-disks. The reason is that simulations involving the MRI do demonstrate that a self-maintaining process which transports angular momentum in the required manner implied by the observations of such disks is feasible.

The most recent computations, however, still obtain values of $\alpha$ smaller than those required by observations of hot alpha disks (see below) by at least an order of magnitude, and often more (unless quite unlikely assumptions are made on the geometry of the magnetic fields). King et al. (2007) point out, however, that most of the computational limitations are likely to act in the direction of reducing $\alpha$.

On the observational side, currently the largest body of evidence of values of $\alpha$ for hot disks comes from the light curves of dwarf novae which represent a subclass of cataclismic variables: binaries with white dwarf primaries and usually main sequence companions, which undergo outbursts at irregular intervals. In the currently most developed theory, these outbursts are due to an instability in the disk. The outbursts are episodes of enhanced mass transfer of the disk onto the primary, involving a significant part of the whole disk. The decay time of the burst is thus a measure of the viscous time scale of the disk. In the most clear-cut cases there appears to be evidence that values of $\alpha \sim 0.1-0.4$ are required to provide a good description of the behaviour of fully ionized, thin accretion discs (King et al., 2007).

On the opposite direction, there are observational indications from the inferred lifeti- 


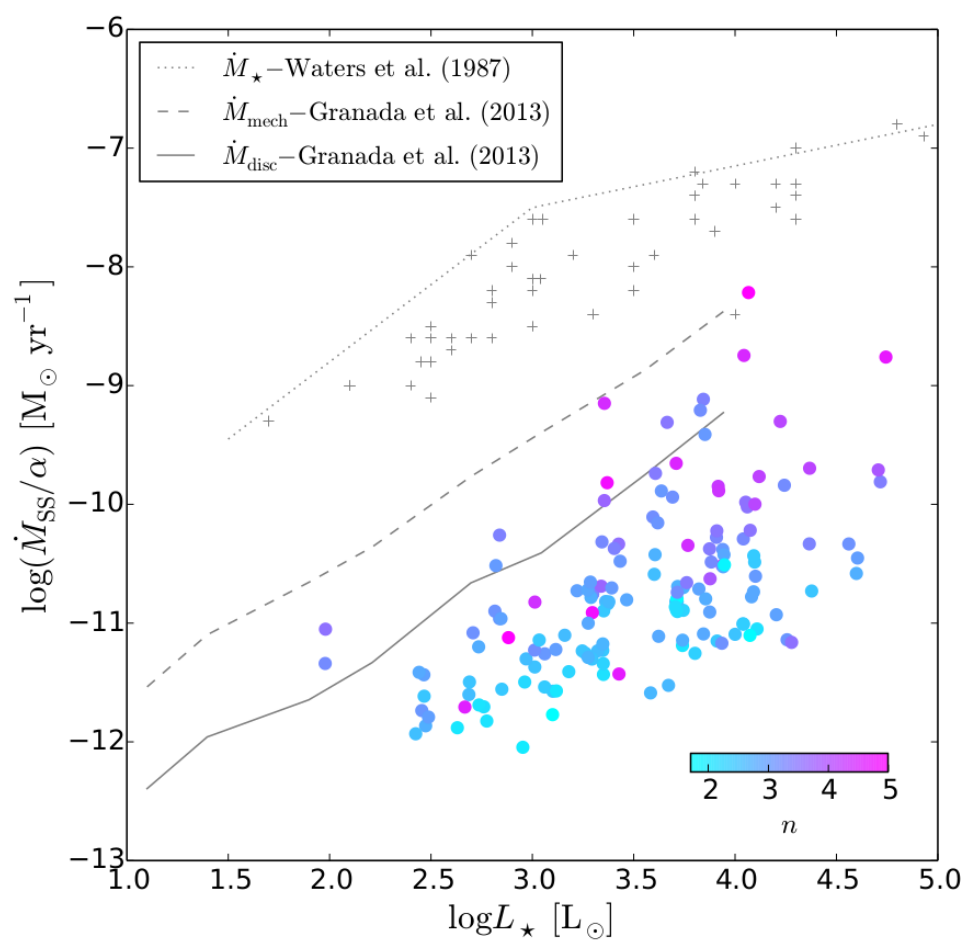

Figure 1.13: Left: Steady-state mass loss rate as a function of stellar luminosity. The circles are determinations of Vieira et al. (2017) The circle colors indicate the fitted $n$ value. The solid line correspond to the mass loss rate computed by Granada et al. (2013). The gray crosses indicate the values computed using an old toy-model by Waters et al. (1987). (Credits: Vieira et al., 2017)

mes and sizes of the much cooler and denser protostellar disks (Strom et al., 1993) that $\alpha$ may be rather small there, $\sim 10^{-3}$.

The recent determinations of the $\alpha$ parameter for $28 \mathrm{CMa}$ (Sect. 3.3) are of the order of the ones estimated for the dwarf novae. The realization that the light curves of Be stars offer the key to measuring alpha directly (or as directly as possible) provided the primary motivation for this thesis work. This method of light curve fitting has brought Be disks to the small group of astrophysical objects with disk for which viscosity can be determined!

\subsubsection{Mass and angular momentum loss rates}

It was proposed (e.g., Ekström et al., 2008; Krtička et al., 2011) that the disks of Be stars may provide natural mechanisms for removing large quantities of angular momentum from the fast rotating stars, preventing them from reaching the critical limit of rotation, during their main sequence evolution. The evolutionary models of Granada et al. (2013), in particular, adopted the prescription outlined by Krtička et al. (2011): they assumed the appearance of completely formed VDDs every time their models reached a near-critical limit of rotation. The predicted mass density and the rate of angular momentum loss of 
their disks were roughly similar to the ones found by Vieira et al. (2017), who modeled the spectral energy distribution (SED) of 80 Be stars using the VDD model (provided $\alpha$ a few tenths). This is illustrated in Fig. 1.13.

\subsection{Outline of this thesis}

In this chapter we introduced the basic concepts of Be stars, and outlined the current status of the research on those objects.

The development of the VDD theory brought Be star research to a whole new level, allowing for the first time the establishment of a quantitative research where models and observations could be compared to extract meaningful information from the observations.

Recently, dynamical VDD studies demonstrated that, by modeling the temporal variability of Be stars (e.g., their light curves), it is possible to measure fundamental quantities of their disks (mass loss rates, viscosity parameters, etc.). However, to date these dynamical studies have been performed only on a single star (28 CMa).

The goal of this thesis work is to greatly increase the sample of variable Be stars studied with the dynamical VDD.

In Chapter 2, we describe the main hydrodynamical equations of the VDD model in the alpha-disk formalism (Shakura and Sunyaev, 1973) and the main equations of the fast rotating star used in this work. We show how these equations are derived from the continuity principles of hydrodynamics.

In Chapter 3, we describe how the star-disk connection is described in the VDD equations and we study dynamical VDDs. We show how important physical parameters of the dynamical disks are related to their steady-state counterparts, and we also link them to the mass and angular loss rates of the star. We also discuss how the history of mass injection of the disk affects its evolution, in what has been dubbed "the mass reservoir effect".

Armed with the conclusions of the studies of dynamical disks, in Chapter 4 we develop a grid of dynamical timescaled models representative of bumps usually found in the light curves of Be stars. This grid of dynamical models is expanded to include different possibilities of central stars and viewing angles in a grid of timescaled light curves, generated by a non-LTE radiative transfer treatment of the dynamical models. It is found that, for most of the viewing angles, the synthetic light curves can be approximated by empirical formulae, which can be used to fit the photometric bumps. We, therefore, describe a chain of procedures (a pipeline) for fitting the observed bumps in the light curves and extracting important parameters of the star and the disk. From these, other physical parameters described in Chapter 3 can be derived.

We, then, advance to Chapter 5, where we select very good examples of bumps from the catalog of Be star candidates from the SMC. We identify the bumps in the light curves 
and the intervals of inactivity (with no detectable disk).

This selection of light curves and bumps is used in Chapter 6, where we fit them using the Markov Chain Monte Carlo (MCMC) technique, which is a powerful technique that allows us to see the probability distributions of the stellar and disk parameters, enabling us to derive quantities of interest and their uncertainties.

The main conclusions and perspectives of this work are outlined in Chapters 7 and 8 . 


\section{Chapter 2}

\section{Dynamical Principles of VDDs and rotating stars}

If you wish to make an apple pie from scratch, you must first invent the

Universe.

Carl Sagan, Cosmos TV series, 1980.

\subsection{Hydrodynamical considerations}

The equations that govern the evolution of a fluid's properties and its motion are conservation equations, from the laws of physics. The first conservation equation we enunciate is mass continuity, given by

$$
\frac{\partial \rho}{\partial t}+\nabla \cdot(\rho \boldsymbol{v})=\mathcal{S}
$$

where $\rho$ is the volume density $\left[\mathrm{g} \mathrm{cm}^{-3}\right]$, the vector $\rho \boldsymbol{v}$ is the mass flux, and the term $\mathcal{S}$ represents any source (or sink) of mass $\left[\mathrm{g} \mathrm{cm}^{-3} \mathrm{~s}^{-1}\right]$.

When we write the Eq. (2.1.1) as $\partial \rho / \partial t=\mathcal{S}-\nabla \cdot(\rho \boldsymbol{v})$, we clearly see that the equation says that, "in an infinitesimal element of fluid $\delta V$, the variation of the mass inside that volume, $\int_{\delta V}(\partial \rho / \partial t) \mathrm{d} V$, is given by the mass that is added (or removed) there, $\int_{\delta V} \mathcal{S} \mathrm{d} V$, minus the mass that flows away from there, $\oint_{\delta V} \rho \boldsymbol{v} \cdot \mathrm{d} \boldsymbol{A}$ ".

We use Eq. (2.1.1) to describe the fluid that composes the disk of the Be star. This fluid is known to be supplied by the star. So, we use the source term $\mathcal{S}$ for describing the connection between the disk and the star and the disk and its outer medium, which is empty space.

The mass flux, $\rho \boldsymbol{v}$, is also equal to the volume density of linear momentum of the fluid $\left[\mathrm{g} \mathrm{cm}^{-3} \mathrm{~cm} \mathrm{~s}^{-1}\right]$. So, the second conservation equation we enunciate is that of linear momentum, as follows

$$
\frac{\partial \rho \boldsymbol{v}}{\partial t}+\nabla \cdot \boldsymbol{\Pi}=\mathcal{F},
$$

where $\boldsymbol{\Pi}$ is the momentum flux tensor (Landau and Lifshitz, 2013) and $\mathcal{F}$ represents the sources (or sinks) of linear momentum (usually, external forces acting on the fluid). 
The momentum flux tensor, for convenience, is separated in two components given by $\boldsymbol{\Pi}=\rho \boldsymbol{v} \boldsymbol{v}-\boldsymbol{\sigma}$, where $\rho \boldsymbol{v} \boldsymbol{v}$ is the Reynolds tensor, related to the advective transport of momentum through the fluid and $\boldsymbol{\sigma}$ is Cauchy's stress tensor, related to non-advective mechanisms of transport of momentum (e.g., internal forces, like that resulting from the gradient of pressure).

Since the addition or removal of mass (with velocity $\boldsymbol{v}$ ) will also result in addition or removal of linear momentum, we subtract this contribution from $\mathcal{F}$ and define the new force term $\mathcal{F}^{*}$, given by

$$
\mathcal{F}^{*}=\mathcal{F}-\mathcal{S} \boldsymbol{v}
$$

Substitution of the above definitions into the equation of continuity of linear momentum leads us to

$$
\begin{array}{r}
\frac{\partial \rho \boldsymbol{v}}{\partial t}+\nabla \cdot(\rho \boldsymbol{v} \boldsymbol{v}-\boldsymbol{\sigma})=\mathcal{F}^{*}+\mathcal{S} \boldsymbol{v} \\
{\left[\boldsymbol{v} \frac{\partial \rho}{\partial t}+\rho \frac{\partial \boldsymbol{v}}{\partial t}\right]+[(\nabla \cdot \boldsymbol{v}) \rho \boldsymbol{v}+\rho \boldsymbol{v} \cdot \nabla \boldsymbol{v}+(\nabla \rho \cdot \boldsymbol{v}) \boldsymbol{v}-\nabla \cdot \boldsymbol{\sigma}]=\mathcal{F}^{*}+\mathcal{S} \boldsymbol{v}} \\
{\left[\boldsymbol{v} \frac{\partial \rho}{\partial t}+\rho \frac{\partial \boldsymbol{v}}{\partial t}\right]+\rho \boldsymbol{v} \cdot \nabla \boldsymbol{v}+(\rho \nabla \cdot \boldsymbol{v}) \boldsymbol{v}+(\nabla \rho \cdot \boldsymbol{v}) \boldsymbol{v}=\mathcal{F}^{*}+\mathcal{S} \boldsymbol{v}+\nabla \cdot \boldsymbol{\sigma}} \\
\boldsymbol{v}\left(\frac{\partial \rho}{\partial t}+\nabla \cdot(\rho \boldsymbol{v})\right)+\rho\left(\frac{\partial \boldsymbol{v}}{\partial t}+\boldsymbol{v} \cdot \nabla \boldsymbol{v}\right)=\mathcal{F}^{*}+\mathcal{S} \boldsymbol{v}+\nabla \cdot \boldsymbol{\sigma}
\end{array}
$$

from which we see that the first term in parenthesis is just the first member of the mass continuity equation, which will cancel with $\mathcal{S} \boldsymbol{v}$. Therefore, we end up with

$$
\rho\left(\frac{\partial \boldsymbol{v}}{\partial t}+\boldsymbol{v} \cdot \nabla \boldsymbol{v}\right)=\mathcal{F}^{*}+\nabla \cdot \boldsymbol{\sigma}
$$

which is the Navier-Stokes equation - the analog of Newton's second law of motion for hydrodynamics (Landau and Lifshitz, 2013).

The equation of motion of the fluid (Eq. 2.1.4) says that the elements of the fluid will be accelerated due to external forces (like the gravitational force, per volume, from a point mass: $\left.\mathcal{F}^{*}=-\rho\left(G M / r^{2}\right) \hat{\boldsymbol{r}}\right)$ and internal stresses of the fluid.

The third conservation equation we enunciate is that of angular momentum (Landau and Lifshitz, 2013), given by

$$
\frac{\partial \boldsymbol{r} \times \rho \boldsymbol{v}}{\partial t}+\nabla \cdot(\boldsymbol{r} \times \boldsymbol{\Pi})=\boldsymbol{r} \times \mathcal{F},
$$

which, after some manipulation and taking the continuity of linear momentum equations into consideration (Eq. 2.1.2), results in the condition $\nabla \cdot(\boldsymbol{r} \times \boldsymbol{\sigma})=\boldsymbol{r} \times \nabla \cdot \boldsymbol{\sigma}$, from which it follows that Cauchy's stress tensor must be symmetric:

$$
\boldsymbol{\sigma}=\boldsymbol{\sigma}^{T} \text { or } \sigma_{i j}=\sigma_{j i}
$$

In a fluid at rest, Cauchy's stress tensor becomes "hydrostatic". Therefore, it is given by $\boldsymbol{\sigma}=-P \mathbf{1}$, where $\mathbf{1}$ is the identity tensor and $P$ is the hydrostatic pressure, which, 
according to thermodynamics, is defined by an equation of state. We therefore isolate the thermodynamic pressure component from $\boldsymbol{\sigma}$, which leads us to

$$
\boldsymbol{\sigma}=-P 1+\boldsymbol{W}
$$

where the stress tensor $\boldsymbol{W}$ contains the other body forces apart from the thermodinamic pressure (e.g., viscous forces).

The set of equations that represent the elements of the stress tensor $\boldsymbol{W}$ are called constitutive equations (Heinbockel, 2001). When, in particular, the stress tensor $\boldsymbol{W}$ is zero, the fluid is said to be an ideal fluid (or an inviscid fluid). In that case, the NavierStokes (Eq. 2.1.9) is also called the Euler equation. Another common type of fluid is the newtonian fluid (Heinbockel, 2001), which is a special case of the general fluid in which the stress tensor is linear with respect to the velocity gradient tensor $\nabla \boldsymbol{v}$. For a newtonian fluid, $\boldsymbol{W}$ is given by

$$
\boldsymbol{W}=\rho \nu^{\prime}(\nabla \cdot \boldsymbol{v}) \mathbf{1}+2 \rho \nu \boldsymbol{\epsilon},
$$

where $\boldsymbol{\epsilon}=\left[(\nabla \boldsymbol{v})+(\nabla \boldsymbol{v})^{T}\right] / 2$ is the strain tensor, and $\nu$ and $\nu^{\prime}$ are, respectively, the kinematic and bulk viscosities.

From Eq. (2.1.7), the Navier-Stokes equation is finally written as follows

$$
\rho\left(\frac{\partial \boldsymbol{v}}{\partial t}+\boldsymbol{v} \cdot \nabla \boldsymbol{v}\right)=\mathcal{F}^{*}-\nabla P+\nabla \cdot \boldsymbol{W}
$$

\subsection{Thin and axisymmetric disks}

Theorem 1. "Assume that a cow is a uniform sphere with mass $m$ and radius $r . . . "$

Unknown author

In this work, we will assume that the disk is azimutally symmetric. From the assumption of azimuthal symmetry, it follows that any derivative with respect to $\phi$ is zero.

We will also assume that the disk is symmetric with respect to the plane $z=0$. From this assumption, it follows that, in our description, scalar functions are even in $z$. So, $\rho(z)=\rho(-z), \mathcal{S}(z)=\mathcal{S}(-z)$, etc. Also, the $z$ component of vectorial functions are odd in $z: v_{z}(z)=-v_{z}(-z), \mathcal{F}_{z}(z)=-\mathcal{F}_{z}(-z)$, etc.; and tensorial functions follow the rule $\hat{\boldsymbol{x}}_{i} \cdot \boldsymbol{W} \cdot \hat{\boldsymbol{x}}_{j}(z)=(-1)^{\delta_{i 3}+\delta_{j 3}} \hat{\boldsymbol{x}}_{i} \cdot \boldsymbol{W} \cdot \hat{\boldsymbol{x}}_{j}(-z)$.

\subsubsection{An inviscid and near-Keplerian disk}

As a first instructive example of a disk, let us consider an axisymmetric and timeindependent rotational flow given by $\boldsymbol{v}=\Omega \hat{\boldsymbol{z}} \times \boldsymbol{r}$ with $\partial \Omega / \partial t=0$. Let us assume also that there is no viscosity, such that the stress tensor $\boldsymbol{W}$ is zero. 
Therefore, it follows that "material derivative" term in the Navier-Stokes equation becomes

$$
\begin{aligned}
\frac{\partial \boldsymbol{v}}{\partial t}+\boldsymbol{v} \cdot \nabla \boldsymbol{v} & =(\boldsymbol{\Omega} \times \boldsymbol{r}) \cdot \nabla(\boldsymbol{\Omega} \times \boldsymbol{r}) \\
& =((\boldsymbol{\Omega} \times \boldsymbol{r}) \cdot \nabla \boldsymbol{\Omega}) \times \boldsymbol{r}+\boldsymbol{\Omega} \times\{(\boldsymbol{\Omega} \times \boldsymbol{r}) \cdot \nabla \boldsymbol{r}\} \\
& =((\boldsymbol{\Omega} \times \boldsymbol{r}) \cdot \nabla \boldsymbol{\Omega}) \times \boldsymbol{r}+\boldsymbol{\Omega} \times\{(\boldsymbol{\Omega} \times \boldsymbol{r}) \cdot \mathbf{1}\} \\
& =((\boldsymbol{\Omega} \times \boldsymbol{r}) \cdot \nabla \boldsymbol{\Omega}) \times \boldsymbol{r}+\boldsymbol{\Omega} \times(\boldsymbol{\Omega} \times \boldsymbol{r}) \\
\frac{\partial \boldsymbol{v}}{\partial t}+\boldsymbol{v} \cdot \nabla \boldsymbol{v} & =((\boldsymbol{\Omega} \times \boldsymbol{r}) \cdot \nabla \boldsymbol{\Omega}) \times \boldsymbol{r}-\Omega^{2} r(\hat{\boldsymbol{r}}-(\hat{\boldsymbol{z}} \cdot \hat{\boldsymbol{r}}) \hat{\boldsymbol{z}}),
\end{aligned}
$$

from which we also see that the first term of the second member vanishes, because we are considering an azimutally symmetric flow: $((\boldsymbol{\Omega} \times \boldsymbol{r}) \cdot \nabla \boldsymbol{\Omega}) \times \boldsymbol{r}=\Omega R(\partial \Omega / \partial \phi) \hat{\boldsymbol{\phi}}=\mathbf{0}$.

The equation of motion is then given by

$$
\frac{1}{\rho} \nabla P=\frac{1}{\rho} \mathcal{F}^{*}+\Omega^{2} R \hat{\boldsymbol{R}} .
$$

Taking the curl of the above equation

$$
\nabla \times\left(\frac{1}{\rho} \nabla P\right)=\nabla \times\left(\frac{1}{\rho} \mathcal{F}^{*}\right)+\nabla \times\left(\Omega^{2} R \hat{\boldsymbol{R}}\right)
$$

results in

$$
\nabla c_{s}^{2} \times \nabla \ln \rho=\nabla \times\left(\frac{1}{\rho} \mathcal{F}^{*}\right)+\frac{\partial}{\partial z}\left(\Omega^{2} R\right) \hat{\boldsymbol{\phi}}
$$

where we have used the equation of state of the ideal gas, $P=\rho c_{s}^{2}$, and the assumption of axisymmetry.

Therefore, if the external force is radial (e.g., the gravitational force from a point mass, $\left.\mathcal{F}^{*}=-\left(G M / r^{2}\right) \hat{\boldsymbol{r}}\right)$, then $\nabla \times\left(\mathcal{F}^{*} / \rho\right)=\mathbf{0}$. Then, if the term on the left in (Eq. 2.2.2) is zero (e.g., if the disk is isothermal or polytropic), then we have $\partial \Omega / \partial z=0$, which means that the rotational velocity is constant in cylinders.

Therefore, returning to Eq. (2.2.1), let us look only to its radial component. It is given by

$$
\begin{aligned}
\Omega^{2} R & =-\frac{1}{\rho} \mathcal{F}_{R}^{*}+\frac{1}{\rho} \frac{\partial P}{\partial R} \\
& =-\frac{1}{\rho} \mathcal{F}_{R}^{*}\left(1+\left(-\frac{\rho c_{s}^{2}}{\mathcal{F}_{R}^{*}}\right)\left(\frac{\partial \ln \rho}{\partial R}+\frac{\partial \ln c_{s}^{2}}{\partial R}\right)\right),
\end{aligned}
$$

where, in the last step, we have used the relation $P=\rho c_{s}^{2}$.

Then, in the approximation that the azimutal motion is constant on cylinders, we may evaluate the above equation for $\Omega$ at the plane of the disk $(z=0)$. Assuming the gravitational acceleration of a point star, $\left.\mathcal{F}^{*}\right|_{z=0}=-\left(G M / R^{2}\right) \hat{\boldsymbol{R}}$, we conclude that

$$
\Omega^{2} R=\frac{G M}{R^{2}}\left(1+\left(\frac{R c_{s}^{2}}{G M}\right)\left(\frac{\partial \ln \rho}{\partial \ln R}+\frac{\partial \ln c_{s}^{2}}{\partial \ln R}\right)\right) .
$$


Since $v_{\phi}=\Omega R$, the above equation becomes

$$
v_{\phi}^{2}=v_{K}^{2}\left(1+\left(\frac{c_{s}^{2}}{v_{K}^{2}}\right)\left(\frac{\partial \ln \rho}{\partial \ln R}+\frac{\partial \ln c_{s}^{2}}{\partial \ln R}\right)\right),
$$

where $v_{K}=v_{\text {orb }} \tilde{R}^{-\frac{1}{2}}$ is the Keplerian azimutal velocity.

Therefore, for an inviscid isothermal/polytropic circumstellar disk whose stream lines are circular orbits, the near-Keplerian azimutal motion is a solution. The deviations from the Keplerian motion come from the density $\rho$ and temperature distributions, and they start to be non-negligible when the gradient of pressure becomes non negligible compared to the force of gravity.

We will see later that an isothermal VDD in steady-state has $\partial \ln \rho / \partial \ln R \approx-7 / 2$. Also, near the stellar equator $\left(R=R_{\text {eq }}\right)$, the ratio between the isothermal sound speed and the Keplerian velocity is very small. For Be stars, we typically have $c_{s} / v_{\text {orb }} \approx 0.02$. However, at $R \sim 300 R_{\text {eq }}$, Eq. (2.2.5) already typically predicts $v_{\phi} \approx 0.5 v_{K}$.

\subsubsection{On the vertical profile of thin disks}

Interferometric observations of Be stars reveal that their disks are geometrically thin, with opening angles of a few degrees (Rivinius et al., 2013). Let us explore the properties of a thin disk, by using again the Navier-Stokes equation (Eq. 2.1.9). Isolating the gradient of pressure, we have

$$
\nabla P=\mathcal{F}^{*}-\rho\left(\frac{\partial \boldsymbol{v}}{\partial t}+\boldsymbol{v} \cdot \nabla \boldsymbol{v}\right)+\nabla \cdot \boldsymbol{W}
$$

We will study the $z$ component of that equation. Hence, multiplying by $\cdot \hat{z}$ to see the vertical component of the motion, and dividing by $P$, we have

$$
\begin{aligned}
\frac{\partial \ln P}{\partial z} & =\frac{1}{P}\left(\mathcal{F}_{z}^{*}+\left(-\rho\left(\frac{\partial \boldsymbol{v}}{\partial t}+\boldsymbol{v} \cdot \nabla \boldsymbol{v}\right) \cdot \hat{\boldsymbol{z}}+\nabla \cdot \boldsymbol{W} \cdot \hat{\boldsymbol{z}}\right)\right) \\
& =\frac{1}{c_{s}^{2}}\left(\frac{1}{\rho} \mathcal{F}_{z}^{*}+\left(-\left(\frac{\partial \boldsymbol{v}}{\partial t}+\boldsymbol{v} \cdot \nabla \boldsymbol{v}\right) \cdot \hat{\boldsymbol{z}}+\frac{1}{\rho} \nabla \cdot \boldsymbol{W} \cdot \hat{\boldsymbol{z}}\right)\right) .
\end{aligned}
$$

In general (e.g., Shakura and Sunyaev, 1973), it is assumed that the acceleration in the $z$ direction is small, at least close to the denser regions of the disk, near the plane $z=0$. Therefore, we will ignore the vertical velocity $v_{z}$ and the forces due to stresses in that direction. We, therefore, have

$$
\frac{\partial \ln P}{\partial z} \approx \frac{1}{c_{s}^{2}} \frac{1}{\rho} \mathcal{F}_{z}^{*}
$$

Eq. (2.2.8) says that the vertical component of the external force over the disk is counter balanced by the vertical gradient of the hydrostatic pressure. The scale height $H$ of a thin disk is defined (e.g., Shakura and Sunyaev, 1973) by the gradient of pressure 
with vertical height $\left(\partial \ln P / \partial \ln z=-z^{2} / H^{2}\right)$. Therefore, with the external force being the gravitational force of the central star $\left(\mathcal{F}^{*}=-\rho\left(G M / r^{2}\right) \hat{\boldsymbol{r}}\right)$, we rewrite Eq. (2.2.8) as

$$
\frac{\partial \ln \rho}{\partial z}+\frac{\partial \ln c_{s}^{2}}{\partial z} \approx-\frac{z}{H^{2}} \text { where } \frac{H^{2}}{R^{2}}=\frac{c_{s}^{2}}{v_{K}^{2}}\left(1+\left(\frac{z}{R}\right)^{2}\right)^{\frac{3}{2}},
$$

which can be integrated in $z$ to obtain $\rho$ :

$$
\rho(R, z, t)=\rho(R, 0, t)\left(\frac{c_{s}^{2}(z=0)}{c_{s}^{2}}\right) e^{-\int_{0}^{z} \frac{z^{\prime}}{H^{2}} \mathrm{~d} z^{\prime}}
$$

In particular, for a disk that is isothermal in height, it follows that

$$
\rho(R, z, t) \approx \rho(R, 0, t) e^{-\frac{z^{2}}{2 H^{2}}}
$$

where we have also approximated the scale height by

$$
\frac{H}{R} \approx \frac{c_{s}}{v_{K}},
$$

which, as we have already seen in Sect. 2.2.1, is of the order of $\sim 0.02$ near the stellar equator (but, e.g., $\sim 0.35$ at $R \sim 300 R_{\text {eq }}$ ).

Therefore, the rotating circumstellar disks with temperatures of the order of $\sim 60 \%$ of the mean effective temperature of the Be star (Carciofi and Bjorkman, 2006) are thin disks, vertically sustained by hydrostatic pressure. The condition in which $c_{s}^{2} / v_{K}^{2} \ll 1$ will be referred in this work as the "thin disk approximation".

\subsubsection{Vertically integrated quantities and the thin disk approximation}

The separation of the $R$ and $z$ components of the density of the disk (Eq. 2.2.11) suggests the usage of vertically integrated quantities for the description of the thin disk. Hence, the surface density of the disk is defined by

$$
\Sigma(R, t)=\int_{-\infty}^{\infty} \rho(R, z, t) \mathrm{d} z
$$

By substituting Eq. 2.2.11 into Eq. (2.2.13), we conclude that the surface density of the rotating circumstellar disk is $\Sigma(R, t)=(2 \pi)^{\frac{1}{2}} H \rho(R, 0, t)$, which can be used to rewrite Eq. 2.2.11 as the relation between $\Sigma$ and $\rho$, given by

$$
\rho(R, z, t) \approx \frac{\Sigma(R, t)}{(2 \pi)^{\frac{1}{2}} H} e^{-\frac{z^{2}}{2 H^{2}}}, \text { where } \frac{H}{R} \approx \frac{c_{s}}{v_{K}} .
$$

Any other physical quantity $Q$, after being vertically integrated, is going to be given in terms of its averaged value $\langle Q\rangle$, as defined by the following equation:

$$
\langle Q\rangle \Sigma=\int_{-\infty}^{\infty} \rho Q \mathrm{~d} z
$$


Since our system is symmetric with respect to the plane $z=0$, it follows that the physical quantities $Q$ are odd or even functions of $z$. If the physical quantity $Q$ is odd in $z$ (e.g., $\left.v_{z}, \mathcal{F}_{z}^{*}\right)$, then its vertically averaged quantity will be zero $\left(\int_{-\infty}^{\infty} \rho Q^{\text {odd }} \mathrm{d} z=0\right)$, since $\rho$ is an even function. If, however, $Q$ is an even quantity in $z$ (e.g., $v_{R}, P, c_{s}$ ), its vertically averaged quantity will not be zero.

\subsubsection{The alpha-disk approach to thin VDDs}

For describing a thin, axisymmetric and viscous disk (with $\boldsymbol{W} \neq \mathbf{0}$ ), we first start with the mass continuity equation:

$$
\begin{array}{r}
\frac{\partial \rho}{\partial t}+\nabla \cdot(\rho \boldsymbol{v})=\mathcal{S} \\
\frac{\partial \rho}{\partial t}+\frac{1}{R} \frac{\partial}{\partial R}\left(R \rho v_{R}\right)+\frac{1}{R} \frac{\partial \rho v_{\phi}}{\partial \phi}+\frac{\partial \rho v_{z}}{\partial z}=\mathcal{S} \\
\frac{\partial \rho}{\partial t}+\frac{1}{R} \frac{\partial}{\partial R}\left(R \rho v_{R}\right)+\frac{\partial \rho v_{z}}{\partial z}=\rho \frac{\mathcal{S}}{\rho}
\end{array}
$$

where, in the last step, we used the axisymmetric condition.

The vertically-integrated mass continuity equation is, then, given by

$$
\frac{\partial \Sigma}{\partial t}+\frac{1}{R} \frac{\partial\left(R \Sigma\left\langle v_{R}\right\rangle\right)}{\partial R}=\Sigma\left\langle\frac{\mathcal{S}}{\rho}\right\rangle-\left.2\left(\rho v_{z}\right)\right|_{\infty}
$$

where, besides the term containing the mass source $\mathcal{S}$, we see a new term, $\left.2\left(\rho v_{z}\right)\right|_{\infty}$, which is the upward mass flux, at infinity. This term may represent a disk wind, such as the ablating line-driven wind described by (Krtička et al., 2011) in their radially extended steady-state VDD irradiated by the central star. The physical quantity $2 \pi R \Sigma\left\langle v_{R}\right\rangle$ is the mass flux crossing the cylinder within radius $R$.

We now procede to the Navier-Stokes equation (Eq. 2.1.9). It contains the advective term $\rho \boldsymbol{v} \cdot \nabla \boldsymbol{v}$. In cylindrical coordinates, it is given by

$$
\begin{aligned}
\rho \boldsymbol{v} \cdot \nabla \boldsymbol{v}= & \left(\begin{array}{ccc}
\rho v_{R} & \rho v_{\phi} & \rho v_{z}
\end{array}\right)\left(\begin{array}{ccc}
\frac{\partial v_{R}}{\partial R} & \frac{\partial v_{\phi}}{\partial R} & \frac{\partial v_{z}}{\partial R} \\
\frac{1}{R}\left(\frac{\partial v_{R}}{\partial \phi}-v_{\phi}\right) & \frac{1}{R}\left(\begin{array}{c}
\frac{\partial v_{\phi}}{\partial \phi}+v_{R} \\
\frac{\partial v_{R}}{\partial z}
\end{array}\right. & \frac{1}{R} \frac{\partial v_{z}}{\partial \phi} \\
\frac{\partial v_{\phi}}{\partial z} & \frac{\partial v_{z}}{\partial z}
\end{array}\right) \\
\rho \boldsymbol{v} \cdot \nabla \boldsymbol{v}= & \left(\rho v_{R} \frac{\partial v_{R}}{\partial R}+\frac{\rho v_{\phi}}{R}\left(\frac{\partial v_{R}}{\partial \phi}-v_{\phi}\right)+\rho v_{z} \frac{\partial v_{R}}{\partial z}\right) \hat{\boldsymbol{R}}+ \\
& \left(\rho v_{R} \frac{\partial v_{\phi}}{\partial R}+\frac{\rho v_{\phi}}{R}\left(\frac{\partial v_{\phi}}{\partial \phi}+v_{R}\right)+\rho v_{z} \frac{\partial v_{\phi}}{\partial z}\right) \hat{\boldsymbol{\phi}}+ \\
& \left(\rho v_{R} \frac{\partial v_{z}}{\partial R}+\frac{\rho v_{\phi}}{R} \frac{\partial v_{z}}{\partial \phi}+\rho v_{z} \frac{\partial v_{z}}{\partial z}\right) \hat{\boldsymbol{z}}
\end{aligned}
$$


which, owing to the axisymmetry of our problem, simplifies to

$$
\begin{aligned}
\rho \boldsymbol{v} \cdot \nabla \boldsymbol{v}= & \left(\rho v_{R} \frac{\partial v_{R}}{\partial R}-\frac{\rho v_{\phi}^{2}}{R}+\rho v_{z} \frac{\partial v_{R}}{\partial z}\right) \hat{\boldsymbol{R}}+ \\
& \left(\rho v_{R} \frac{\partial v_{\phi}}{\partial R}+\frac{\rho v_{\phi} v_{R}}{R}+\rho v_{z} \frac{\partial v_{\phi}}{\partial z}\right) \hat{\boldsymbol{\phi}}+ \\
& \left(\rho v_{R} \frac{\partial v_{z}}{\partial R}+\rho v_{z} \frac{\partial v_{z}}{\partial z}\right) \hat{\boldsymbol{z}}
\end{aligned}
$$

The Navier-Stokes equation also contains the term $\nabla \cdot \boldsymbol{W}$, the force per unit of volume over the fluid due to the stress tensor. In cylindrical coordinates, it is given by

$$
\begin{aligned}
\nabla \cdot \boldsymbol{W}= & \left(\frac{1}{R} \frac{\partial}{\partial R}\left(R W_{R R}\right)+\frac{1}{R} \frac{\partial W_{\phi R}}{\partial \phi}-\frac{W_{\phi \phi}}{R}+\frac{\partial W_{z \phi}}{\partial z}\right) \hat{\boldsymbol{R}}+ \\
& \left(\frac{\partial W_{R \phi}}{\partial R}+\frac{1}{R} \frac{\partial W_{\phi \phi}}{\partial \phi}+\frac{W_{\phi R}+W_{R \phi}}{R}+\frac{\partial W_{z \phi}}{\partial z}\right) \hat{\boldsymbol{\phi}}+ \\
& \left(\frac{1}{R} \frac{\partial}{\partial R}\left(R W_{R z}\right)+\frac{1}{R} \frac{\partial W_{\phi z}}{\partial \phi}+\frac{\partial W_{z z}}{\partial z}\right) \hat{\boldsymbol{z}}
\end{aligned}
$$

which, after involking the axisymmetry of the problem and the fact that stress tensor $\boldsymbol{W}$ is symmetric (meaning that $W_{\phi R}+W_{R \phi}=2 W_{R \phi}$ ), the above equation simplifies to

$$
\begin{aligned}
\nabla \cdot \boldsymbol{W}= & \left(\frac{1}{R} \frac{\partial}{\partial R}\left(R W_{R R}\right)-\frac{W_{\phi \phi}}{R}+\frac{\partial W_{z \phi}}{\partial z}\right) \hat{\boldsymbol{R}}+ \\
& \left(\frac{1}{R^{2}} \frac{\partial}{\partial R}\left(R^{2} W_{R \phi}\right)+\frac{\partial W_{z \phi}}{\partial z}\right) \hat{\boldsymbol{\phi}}+ \\
& \left(\frac{1}{R} \frac{\partial}{\partial R}\left(R W_{R z}\right)+\frac{\partial W_{z z}}{\partial z}\right) \hat{\boldsymbol{z}}
\end{aligned}
$$

The $z$ component of the Navier-Stokes was already adressed in Sect. 2.2.2, and resulted in the thin disk approximation. We here procede to the $\phi$ component of the Navier-Stokes equation. It is given by

$$
\begin{gathered}
\rho\left(\frac{\partial v_{\phi}}{\partial t}+v_{R} \frac{\partial v_{\phi}}{\partial R}+\frac{v_{\phi} v_{R}}{R}+v_{z} \frac{\partial v_{\phi}}{\partial z}\right)=\mathcal{F}_{\phi}^{*}-\frac{1}{R} \frac{\partial P}{\partial \phi}+\nabla \cdot \boldsymbol{W} \cdot \hat{\boldsymbol{\phi}} \\
\rho\left(\frac{\partial v_{\phi}}{\partial t}+v_{R} \frac{\partial v_{\phi}}{\partial R}+\frac{v_{\phi} v_{R}}{R}\right)=\mathcal{F}_{\phi}^{*}+\frac{1}{R^{2}} \frac{\partial}{\partial R}\left(R^{2} W_{R \phi}\right)+\frac{\partial W_{z \phi}}{\partial z}
\end{gathered}
$$

where in the last step we have used the axisymmetry of the problem (to make $\partial P / \partial \phi=0$ ) and the approximation that $\partial v_{\phi} / \partial z \approx 0$, as is the case for the azimutal motion for the polytropic inviscid disk (see Sect. 2.2.1). Another argument for eliminating that term would be the assumption that $v_{z}$ is much smaller than the other velocity components in the regions where the disk is dense, as was done in Sect 2.2.2. 
By multiplying the Eq. (2.2.21) by $R$ and integrating vertically, we obtain the following

$$
\Sigma \frac{\partial}{\partial t}\left(R v_{\phi}\right)+R \Sigma\left\langle v_{R}\right\rangle \frac{1}{R} \frac{\partial}{\partial R}\left(R v_{\phi}\right)=R \Sigma\left\langle\frac{\mathcal{F}_{\phi}^{*}}{\rho}\right\rangle+\frac{1}{R} \frac{\partial}{\partial R}\left(R^{2} \Sigma\left\langle\frac{W_{R \phi}}{\rho}\right\rangle\right)+\left.2 R W_{z \phi}\right|_{\infty} .
$$

The above equation contains the term $R \Sigma\left\langle v_{R}\right\rangle$, which is proportional to mass flux through the thin disk. Solving for this term, we obtain the following equation

$$
R \Sigma\left\langle v_{R}\right\rangle=\frac{R \Sigma\left\langle\frac{\mathcal{F}_{\phi}^{*}}{\rho}\right\rangle+\frac{1}{R} \frac{\partial}{\partial R}\left(R^{2} \Sigma\left\langle\frac{W_{R \phi}}{\rho}\right\rangle\right)+\left.2 R W_{z \phi}\right|_{\infty}-\Sigma \frac{\partial}{\partial t}\left(R v_{\phi}\right)}{\frac{1}{R} \frac{\partial}{\partial R}\left(R v_{\phi}\right)} .
$$

Now, it is expected from the observations of the disks of Be stars that the radial velocity must be much smaller than the azimutal velocity: $\left\langle v_{R}\right\rangle \ll v_{\phi}$. So, for an external force whose radial component is dominated by gravity, i.e.,

$$
\mathcal{F}_{R}^{*}=-\rho\left(G M / R^{2}\right)+\text { other small forces, }
$$

we will have a similar situation as the one described in Sect. 2.2.1 for the azimutal velocity $v_{\phi}$. It follows, therefore, that the latter is approximatelly given by the Keplerian orbital velocity, at least in the thin disk approximation.

Therefore, assuming that $v_{\phi} \approx v_{K}$, the denominator of Eq. (2.2.23) will be given by $R^{-1} \partial\left(R v_{\phi}\right) / \partial R \approx \frac{1}{2}\left(G M / R^{3}\right)^{\frac{1}{2}}$ and the time dependent term $\partial\left(R v_{\phi}\right) / \partial t$ will approximately vanish. The mass flux is, thus, given by

$$
2 \pi R \Sigma\left\langle v_{R}\right\rangle=4 \pi\left(\frac{R^{3}}{G M}\right)^{\frac{1}{2}}\left(R \Sigma\left\langle\frac{\mathcal{F}_{\phi}^{*}}{\rho}\right\rangle+\frac{1}{R} \frac{\partial}{\partial R}\left(R^{2} \Sigma\left\langle\frac{W_{R \phi}}{\rho}\right\rangle\right)+\left.2 R W_{z \phi}\right|_{\infty}\right)
$$

The above equation shows that a force in the positive $\phi$ direction due to the component $W_{R \phi}$ of the stress tensor or a positive component of an external force in the $\phi$ direction (the term containing $\mathcal{F}_{\phi}^{*}$ ) can produce an outward mass flux, by transfering angular momentum to the fluid. (Also, a torque exerted by the outer boundary in infinity, given by the term $\left.2 R W_{z \phi}\right|_{\infty}$, can produce a mass flux.)

If the fluid of the disk is a viscous one of the newtonian kind, then, by Eq. (2.1.8), it follows that $W_{R \phi}=\rho R \nu \partial\left(v_{\phi} / R\right) / \partial R$, where $\nu$ is the kinematic viscosity. In the approximation that $v_{\phi} \approx v_{K}$, it follows that the $R \phi$ component of the stress tensor of the newtonian fluid is given by

$$
W_{R \phi}=-\frac{3}{2} \rho \nu\left(\frac{G M}{R^{3}}\right)^{\frac{1}{2}},
$$

Instead of assuming that the fluid is newtonian, however, it is customary to scale the stress $W_{R \phi}$, which has unit of pressure, with the thermodynamic pressure $P$, through the dimensionless parameter $\alpha$, as follows

$$
W_{R \phi}=-\alpha P .
$$


From Eqs. (2.2.26) and (2.2.27), it follows that the kinematic viscosity is related to the $\alpha$ parameter by the equation $\nu=(2 / 3) \alpha c_{s} H$, where $H$ is the scale height (Eq. 2.2.12).

In this work, we will follow the definition of the $\alpha$ parameter given by (e.g., Eq. (2.2.27) (following, e.g., Okazaki, 2001). In this definition of $\alpha$, there is no mention to the newtonian stress tensor. However, several other works in the literature employ an alternative definition of the viscosity parameter. It is argued that the kinematic viscosity must be $\nu \leq c_{s} H$, since it results from momentum transport via eddies whose speed cannot be faster than the isothermal sound speed $c_{s}$ and whose size cannot be bigger than the scale height $H$ of the disk. Therefore, an alternative parameter $\alpha^{*}$ is defined by $\nu=\alpha^{*} c_{s} H$, and it is argued that $0 \leq \alpha^{*} \leq 1$. This alternative parameter relates to our viscosity parameter by $\alpha=(3 / 2) \alpha^{*}$.

Substitution of Eq. 2.2.27 into Eq. 2.2.25, gives

$$
2 \pi R \Sigma\left\langle v_{R}\right\rangle=4 \pi\left(\frac{R^{3}}{G M}\right)^{\frac{1}{2}}\left(R \Sigma\left\langle\frac{\mathcal{F}_{\phi}^{*}}{\rho}\right\rangle-\frac{1}{R} \frac{\partial}{\partial R}\left(R^{2} \Sigma\left\langle\alpha c_{s}^{2}\right\rangle\right)+\left.2 R W_{z \phi}\right|_{\infty}\right) .
$$

In this work, we will consider that $\left.2 R W_{z \phi}\right|_{\infty}=0$, because the outside medium of our disk system is empty space. (Gayley et al., 2001) has pointed out, however, that the nonnegligible size of the aparent disk of the star together with a non-zero radial velocity may lead to the appearance of a $\phi$ component in the radiative line-driven force. That force can take the place of our unspecified external force $\mathcal{F}_{\phi}^{*}$. We don't know if the common assumptions related to the line-force formalism apply to the generally optically thick inner portions of the disks of the Be stars. In this work, therefore, we will assume that there is no external force with a $\phi$ component.

From the above equation, we see that the effective viscous force in the $\phi$ direction is positive or negative, if the gradient of $R^{2} \Sigma\left\langle\alpha c_{s}^{2}\right\rangle$ is negative or positive, respectively. For an isothermal disk with $\alpha$ constant, if we assume a surface density profile $\Sigma \propto R^{-m}$, then the viscous force is positive (producing a positive mass flux - decretion) if $m>2$, and negative (producing a negative mass flux - accretion) if $m<2$.

\subsection{The fast rotating star}

In this section, we detail the main features of our stellar model used for the central star of the Be system. This stellar model will enter as input into the radiative transfer code HDUST.

The fast rotating star is a spinning fluid, under its own gravity, for which we will assume a negligible viscosity (at least for movements on timescales smaller than the evolutionary ones - Kippenhahn et al., 2012). Therefore, from the Navier-Stokes equation (Eq. 2.1.4), we write

$$
\frac{1}{\rho} \nabla P=\frac{1}{\rho} \mathcal{F}^{*}-\frac{\partial \boldsymbol{v}}{\partial t}-\boldsymbol{v} \cdot \nabla \boldsymbol{v}
$$


where the vector $\nabla P / \rho$ is the effective gravity of the stellar configuration. The surface of a hydrostatic star, therefore, must be normal to this vector.

We will assume that the motion of the stellar fluid is axisymmetric and, at all places, circular around the axis of the star. We will, therefore, ignore complications such as convective motion or meridional circulations or stellar pulsations in our simple model. Let us, therefore, consider that the circular streamlines are given by $\boldsymbol{v}=\boldsymbol{\Omega} \times \boldsymbol{r}$ where $\Omega=\Omega \hat{\boldsymbol{z}}$ is the angular velocity. As already shown in Sect. 2.2.1, it follows that

$$
\frac{\partial \boldsymbol{v}}{\partial t}+\boldsymbol{v} \cdot \nabla \boldsymbol{v}=-\Omega^{2} r(\hat{\boldsymbol{r}}-(\hat{\boldsymbol{z}} \cdot \hat{\boldsymbol{r}}) \hat{\boldsymbol{z}})
$$

Hence, the effective gravity of the star is given by

$$
\boldsymbol{g}_{\mathrm{eff}}=\frac{1}{\rho} \nabla P=-\frac{G M}{r^{2}} \hat{\boldsymbol{r}}+\Omega^{2} r(\hat{\boldsymbol{r}}-(\hat{\boldsymbol{z}} \cdot \hat{\boldsymbol{r}}) \hat{\boldsymbol{z}}),
$$

where have assumed that the majority of the stellar mass is near its center, so $\mathcal{F}^{*} / \rho=$ $-\left(G M / r^{2}\right) \hat{\boldsymbol{r}}$ is a good approximation for the gravitational acceleration acting at every point of the star. This acceleration can also be expressed as the gradient of the gravitational potential $\Phi=-G M / r$.

The last term in Eq. (2.3.2) is the centrifugal acceleration. In cylindrical coordinates, which will be very useful later in this work, this acceleration is given by $\Omega^{2} R \hat{\boldsymbol{R}}$, from which we see that it increases linearly with the distance from the axis of rotation of the star (for a star rotating as a solid-body). Therefore, the closer a point is to the stellar equator, the more it will feel the centrifugal acceleration.

We must remember that the angular velocity in Eq. 2.3.2 may depend on the position of the fluid inside the star. It is thus a function of $r$ and $\theta$ (in spherical coordinates) and of $R$ and $z$ in cylindrical coordinates. By this fact, the centrifugal acceleration cannot, in general, be defined in terms of a potential, like: $-\nabla V=\Omega^{2} R \hat{\boldsymbol{R}}$. The centrifugal acceleration will be expressed in terms of a potential, if its curl is zero (because of the vector property: $\nabla \times \nabla V=\mathbf{0}$ ). Therefore, taking the curl $\nabla \times \Omega^{2} R \hat{\boldsymbol{R}}$, results in the requirement that $\partial \Omega / \partial z=0$, which means that the streamlines of the stellar fluid must be constant in cylinders. This is, obviously a serious restriction. An even greater restriction is that in which the stellar fluid rotates as solid body, meaning that, not even $\partial \Omega / \partial z=0$, but $\partial \Omega / \partial R=0$ also. In the case of a solid body rotation, one can easily show that $V=$ $(1 / 2) \Omega^{2} r^{2} \sin ^{2} \theta$ is an appropriate potential that results in the centrifugal acceleration: $-\nabla V=\Omega^{2} R \hat{\boldsymbol{R}}$. We therefore, rewrite Eq. (2.3.2) as follows

$$
\frac{1}{\rho} \nabla P=-\nabla \Psi
$$

where

$$
\Psi=-\frac{G M}{r}-\frac{1}{2} \Omega^{2} r^{2} \sin ^{2} \theta
$$


is the Roche potential.

Now that the effective gravity of the star is given as the gradient of the potential $\Psi$, it follows that the surface of the star is defined by an equipotential $\Psi=$ constant. It must be remembered, however, as explained above, that Eq. (2.3.2) is more general than Eqs. (2.3.3) and (2.3.4), and stars do have differential rotation. Therefore, their shapes might not be well described by the equipotentials of the Roche potential. Since the internal rotation of fast rotating stars is still poorly constrained, this effect can be potentially serious when determinations of the critical fraction $W$ of a star is made, e.g., interferometrically (based solely on its shape).

Let us, then, follow with the determination of the shape of the star, assumed to be given by a Roche equipotential. First, we define the parameter $r_{0}$, which has dimension of distance, and the dimensionless variable $s$ as

$$
s=\frac{r}{r_{0}} .
$$

Let us assume that $r_{0}$ is the value of the polar radius of a star rotating at critical velocity. The radius of the stellar equator of that star is $r_{0}^{\prime}$. The form of this star is given by the equipotential $\Psi=\Psi_{0}$, and it is rotating with angular velocity $\Omega_{0}$.

Since, the star is rotating at critical velocity, it follows that the effective gravity at its equator is zero, and, therefore, $-G M / r_{0}^{\prime 2}+\Omega_{0}^{2} r_{0}^{\prime}=0$, from which follows that $\Omega_{0}^{2}=G M / r_{0}^{\prime 3}$.

Now, since both the polar radius (measuring $r_{0}$ ) and the equatorial radius (measuring $r_{0}^{\prime}$ ) are on the same surface $\Psi=\Psi_{0}$, we can eliminate $r_{0}^{\prime}$ from the equality: $-G M / r_{0}=$ $-G M / r_{0}^{\prime}-(1 / 2) \Omega_{0}^{2} r_{0}^{\prime 2}$, from which it results that $r_{0}^{\prime}=(3 / 2) r_{0}$ (the ratio between the equatorial radius and the polar radius of a critically rotating star is 1.5), and we end up with the other two important parameters defined as

$$
\Psi_{0}=-\frac{G M}{r_{0}}
$$

and

$$
\Omega_{0}^{2}=\frac{8}{27} \frac{G M}{r_{0}^{3}} .
$$

Now, let us define the scaled Roche potential by

$$
\psi=\frac{\Psi}{\Psi_{0}},
$$

and the dimensionless angular velocity by

$$
\omega=\frac{\Omega}{\Omega_{0} \psi^{\frac{3}{2}}} .
$$


By substitution of all definitions into the Roche potential (Eq. 2.3.4), it follows that

$$
\begin{aligned}
\frac{\Psi}{\Psi_{0}}=\psi & =+\frac{G M}{r} \frac{r_{0}}{G M}+\frac{r_{0}}{G M} \frac{1}{2} \Omega^{2} r^{2} \sin ^{2} \theta \\
& =+\frac{G M}{r} \frac{r_{0}}{G M}+\frac{r_{0}^{3}}{G M} \frac{1}{2} \Omega^{2}\left(\frac{r}{r_{0}}\right)^{2} \sin ^{2} \theta \\
& =+\frac{G M}{r} \frac{r_{0}}{G M}+\frac{8}{27} \frac{1}{\Omega_{0}^{2}} \frac{1}{2} \Omega^{2}\left(\frac{r}{r_{0}}\right)^{2} \sin ^{2} \theta \\
\psi & =\frac{1}{s}+\frac{4}{27} \psi^{3} \omega^{2} s^{2} \sin ^{2} \theta
\end{aligned}
$$

from which it follows that the surface of the star, $s_{\psi}(\omega, \theta)$, is given by the following third order equation

$$
\begin{aligned}
s^{3}-\frac{27}{4} \frac{1}{\psi^{2} \omega^{2} \sin ^{2} \theta} s+\frac{27}{4} \frac{1}{\psi^{3} \omega^{2} \sin ^{2} \theta} & =0, \quad \theta \neq 0, \pi \\
s=\frac{1}{\psi}, & \theta=0, \pi
\end{aligned}
$$

which, for the case of $0 \leq \omega \leq 1$, results in closed surfaces given by

$$
\begin{array}{r}
s_{\psi}=\frac{1}{\psi}, \quad \theta=0, \pi \\
s_{\psi}=\frac{3}{\psi} \frac{1}{\omega \sin \theta} \cos \left[\frac{\pi}{3}+\frac{1}{3} \arccos (\omega \sin \theta)\right], \quad \theta \neq 0, \pi
\end{array}
$$

Eq. (2.3.13) gives the analytical form of the surface of stellar configuration rotating as a solid body.

Observationally, the rotation rate of the star is better stated in terms of the critical ratio $W=\Omega R_{\text {eq }} / v_{\text {orb }}$, where $v_{\text {orb }}=\left(G M / R_{\text {eq }}\right)^{\frac{1}{2}}$. (The equatorial and polar radius are given by $R_{\text {eq }}=r_{0} s_{\psi, \text { eq }}$ and $R_{\text {pole }}=r_{0} s_{\psi, \text { pole. }}$ ) Just like $\omega$, the parameter $W$ is also a number between 0 and 1 . It is, however, better suited for comparison with observations, since $v_{\text {eq }}=\Omega R_{\text {eq }}$ can be easily estimated from them.

In Fig. 2.1, we show the forms of Roche stars with increasing values of $W$, from 0 to 1 , in steps of 0.1 . The blue shape correspond to $W=0.8$ and the red shapes correspond to $W=0.7$ and $W=0.9$. The red shapes more or less correspond to the limits (within $\pm 1 \sigma$ ) of the distribution of values of $W$ found by Rivinius et al. (2006), by studing shell Be stars.

In some works in the literature, however, it is also common to find the $\Upsilon$ parameter, defined as the ratio of the equatorial velocity and the critical velocity defined by $v_{\text {crit }}=$ $\left(G M / 1.5 R_{\text {pole }}\right)^{\frac{1}{2}}$, which, for our Roche model, is also a number between 0 and 1 . If the star, on the other hand, does not follow a Roche potential, it would be possible to obtain values beyond 1 for $\Upsilon$. Therefore, the usage of this parameter should be discouraged as Rivinius et al. (2013) pointed out. The practical problem, however, with the three definitions of $W, \omega$ and $\Upsilon$ is that they are only equal for a specific star if they are equal to 


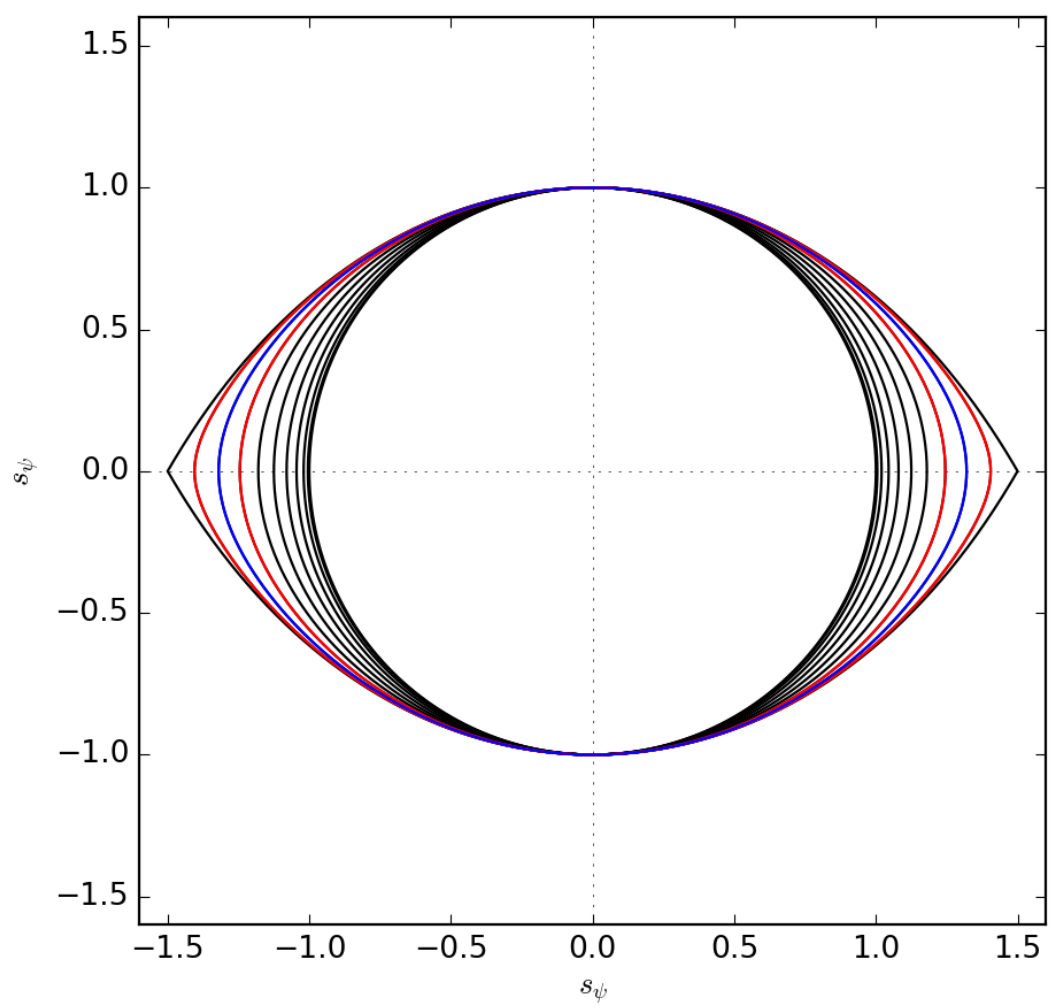

Figure 2.1: Shapes of the stellar configurations, rotating as solid bodies, with critical ratios $W$ varing from 0 to 1 in steps of 0.1 . The blue shape correspond to $W=0.8$ and the red shapes correspond to $W=0.7$ and $W=0.9$. For all stars, it was chosen $\psi=1$.

0 or 1 . They differ in all other cases and that can lead to a gread deal of confusion. (In this work, in particular, it was necessary to perform conversions between these parameters.) Finally, interferometrists might define the stellar rotation rate by the ratio between the equatorial radius and the polar radius, known as the oblateness of the star. For our Roche model, it is a number between 1 and 1.5.

In Table 2.3, we list the set of formulas required to convert between the four parameters: $W, \omega, R_{\text {eq }} / R_{\text {pole }}$ and $\Upsilon$.

\subsubsection{Espinosa-Lara \& Rieutord's flux prescription}

Inside the star, energy is in the stellar core as the result of nuclear reactions. Outside the core, energy is not generated. It diffuses outwards until it leaves through the stellar surface. Therefore, outside the core, the energy flux $\boldsymbol{F}$ must be divergenceless: $\nabla \cdot \boldsymbol{F}=0$, meaning that there is no source of energy in the envelope.

For a radiative envelope, the radiative flux is given by the diffusion equation $\boldsymbol{F}=$ $-\chi \nabla T$. The temperature is a function of $\rho$ and $P$. If the star is barotropic, meaning, in our case, that the $\rho$ and $P$ are constants on an equipotential $\Psi$, then, it follows the 


\begin{tabular}{|c|c|c|c|c|}
\hline$W$ & $W$ & $\frac{R_{\mathrm{eq}}}{R_{\mathrm{pole}}}$ & $\Upsilon$ \\
\hline$W$ & - & $\left(\frac{8}{\omega} \cos ^{3}\left(\frac{\pi}{3}+\frac{1}{3} \arccos \omega\right)\right)^{\frac{1}{2}}$ & $\left(2 \frac{R_{\mathrm{eq}}}{R_{\mathrm{pole}}}-2\right)^{\frac{1}{2}}$ & $\left(\frac{3 \Upsilon}{\cos \left(3 \arccos \left(\frac{\Upsilon}{2}\right)-\pi\right)}-2\right)^{\frac{1}{2}}$ \\
\hline$\omega$ & $\left(\frac{27}{8} \frac{W^{2}}{\left(1+\frac{1}{2} W^{2}\right)^{3}}\right)^{\frac{1}{2}}$ & - & $\left(\frac{27}{4} \frac{\left(\frac{R_{\mathrm{eq}}}{R_{\mathrm{pole}}}-1\right)}{\left(\frac{R_{\mathrm{eq}}}{R_{\mathrm{pole}}}\right)^{3}}\right)^{\frac{1}{2}}$ & $\cos \left(3 \arccos \left(\frac{\Upsilon}{2}\right)-\pi\right)$ \\
\hline$\frac{R_{\mathrm{eq}}}{R_{\mathrm{pole}}}$ & $1+\frac{1}{2} W^{2}$ & $\frac{3}{\omega} \cos \left(\frac{\pi}{3}+\frac{1}{3} \arccos \omega\right)$ & - & $\frac{3 \Upsilon}{2 \cos \left(3 \arccos \left(\frac{\Upsilon}{2}\right)-\pi\right)}$ \\
\hline$\Upsilon$ & $\left(\frac{3}{2} \frac{W^{2}}{1+\frac{1}{2} W^{2}}\right)^{\frac{1}{2}}$ & $2 \cos \left(\frac{\pi}{3}+\frac{1}{3} \arccos \omega\right)$ & $\left(3 \frac{\frac{R_{\mathrm{eq}}}{R_{\mathrm{pole}}}-1}{\frac{R_{\mathrm{eq}}}{R_{\mathrm{pole}}}}\right)^{\frac{1}{2}}$ & - \\
\hline
\end{tabular}

Table 2.1 - Conversion table between $W, \omega, R_{\text {eq }} / R_{\text {pole }}$ and $\Upsilon$.

temperature is also constant in the equipotential $\Psi$ and $\nabla T=(\partial T / \partial \Psi) \nabla \psi$, which means that $\boldsymbol{F}=\chi(\partial T / \partial \Psi) \boldsymbol{g}_{\text {eff }}$. Then, at the surface of the star, the flux must be given by $\sigma T_{\text {eff }}^{4}$, from which the "von Zeipel relation" $T_{\text {eff }} \propto g_{\text {eff }}^{\frac{1}{4}}$ is obtained. However, there is a contradiction, since the effective gravity is not constant along the equipotential $\Psi$, in contradiction with our assumption that the temperature is constant on an equipotential. This contradiction was known since von Zeipel (1924), and it implies that a rotating star that is isothermal on equipotentials cannot be in radiative equilibrium.

Nevertheless, in the convective envelopes, heat is transported by buoyance, essentially following the oposing direction of the effective gravity (Espinosa Lara and Rieutord, 2011). Therefore, inspired by this fact and by the "von Zeipel" law, which also prescribed a flux in the direction of the effective gravity, Espinosa Lara and Rieutord (2011) suggested the following prescription for the flux transport inside the rotating star:

$$
\nabla \cdot \boldsymbol{F}=L \delta^{3}(\boldsymbol{r}), \text { such that } \boldsymbol{F} \| \boldsymbol{g}_{\mathrm{eff}},
$$

that is: all the stellar luminosity is generated at the center of the star (roughly the location of the near spherical core) and, in every point, the flux follows parallel to the effective gravity. Interestingly, this prescription matches quite well with the predictions of their more detailed rotating stellar interior code ESTER (Espinosa Lara and Rieutord, 2011).

We therefore adopt their prescription in this work. The analytical solution of the problem given by Eq. (2.3.14) for a Roche star is lengthy and well described in Espinosa Lara and Rieutord (2011). We will only state their results here.

The flux at every point inside the star, and at the surface in particular, is given by the following equation

$$
\boldsymbol{F}=-\frac{L}{4 \pi G M} F_{\omega}(s, \theta) \boldsymbol{g}_{\mathrm{eff}},
$$



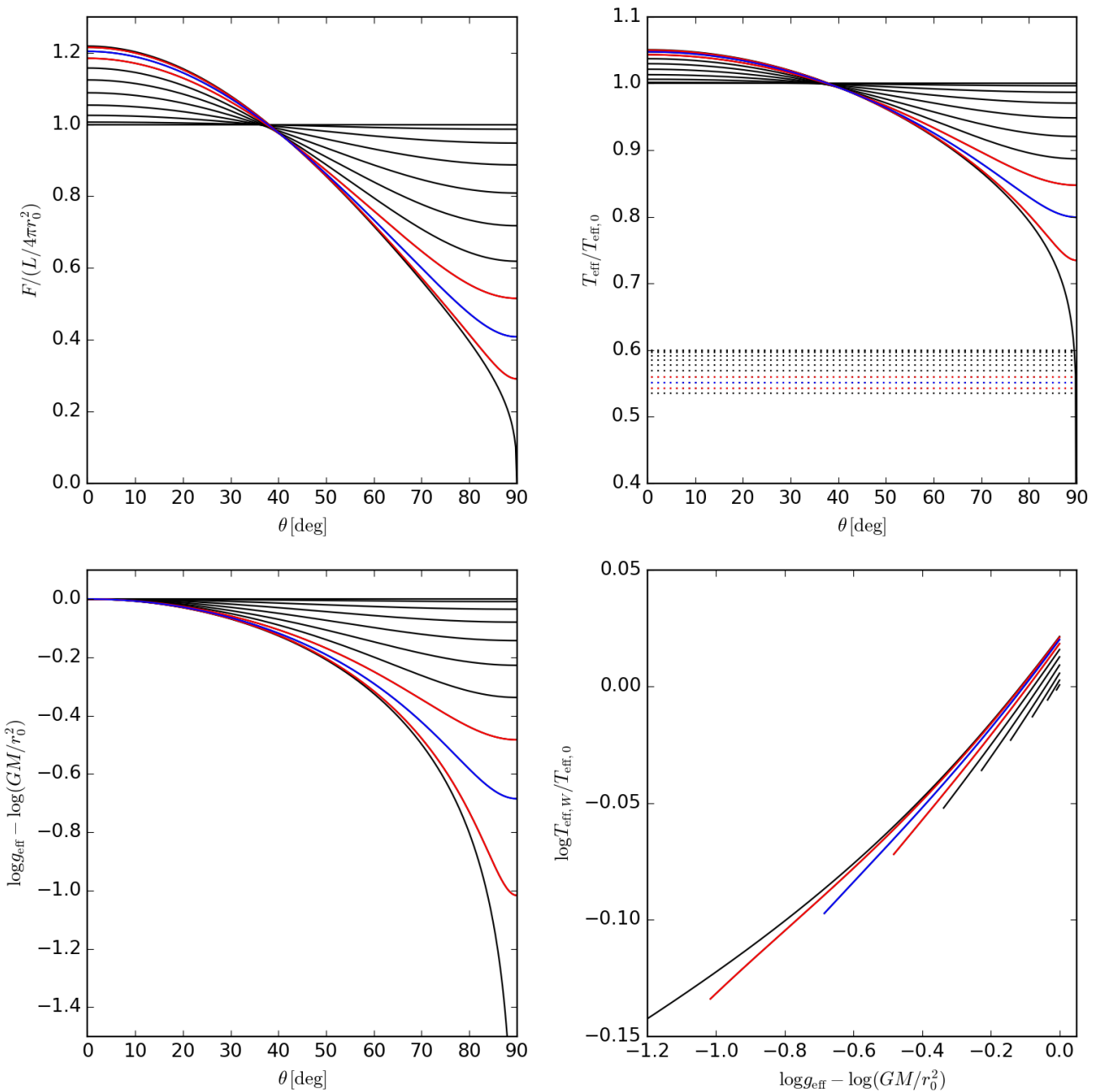

Figure 2.2: For the same stellar models of Fig. 2.1: Upper-left: Flux at the surface of the stars compared to the non-rotating case as a function of the polar angle $\theta$. Upper-right: Effective temperature compared to the non-rotating case as a function of $\theta$. In dotted straight lines, values of $0.6\left(L / S_{W} \sigma\right)^{\frac{1}{4}}$, where $S_{W}$ is the surface area of the star with parameter $W$. Lower-left: $\log g_{\text {eff }}$ compared to the non-rotating case as a function of $\theta$. Lower-right: $\log T_{\text {eff }}$ versus $\log g_{\text {eff }}$. The inclinations of these curves give the $\beta$ coefficient.

where, the function $F_{\omega}(s, \theta)$ is given by

$$
F_{\omega}(s, \theta)= \begin{cases}\frac{\tan ^{2} \vartheta(s, \theta)}{\tan ^{2} \theta} & , \quad \theta \neq 0, \frac{\pi}{2} \\ \exp \left(\frac{2^{4}}{3^{4}} \psi^{3} \omega^{2} s^{3}\right) & , \quad \theta=0 \\ \left(1-\frac{2^{3}}{3^{3}} \psi^{3} \omega^{2} s^{3}\right)^{-\frac{2}{3}} & , \quad \theta=\frac{\pi}{2}\end{cases}
$$

where the function $\vartheta(s, \theta)$ is defined implicitly by the relation

$$
\frac{2^{3}}{3^{4}} \psi^{3} \omega^{2} s^{3} \cos ^{3} \theta+\cos \theta+\ln \tan \frac{\theta}{2}=\cos \vartheta+\ln \tan \frac{\vartheta}{2} .
$$

In order to obtain the function $\vartheta(s, \theta)$ at any point $s$ and $\theta$, in this work, we applied 
the following iterative method (as suggested in Espinosa Lara, 2014):

$$
\vartheta_{N+1}=\vartheta_{N}-\frac{y_{N}}{\left(\frac{\mathrm{d} y}{\mathrm{~d} \vartheta}\right)_{N}}, \text { where } \vartheta_{0}=\theta
$$

where

$$
y_{N}=\cos \vartheta_{N}+\ln \tan \frac{\vartheta_{N}}{2}-\frac{2^{3}}{3^{4}} \psi^{3} \omega^{2} s^{3} \cos ^{3} \theta-\cos \theta-\ln \tan \frac{\theta}{2}
$$

and

$$
\left(\frac{\mathrm{d} y}{\mathrm{~d} \vartheta}\right)_{N}=-\sin \vartheta_{N}+\frac{1}{\sin \vartheta_{N}} .
$$

In Fig. 2.2, for the same stars of Fig. 2.1, we show the values of the flux (upperleft), temperature (upper-right), $\log g_{\text {eff }}$ (lower-left), relative to the non-rotating case, as a function of the polar angle $\theta$. Clearly, rotation creates hotter polar regions and colder equatorial regions. Rotation also lowered the effective gravity at the surface of the star, specially near the equator. At the poles, however, the effective gravity remains the same as for the non-rotating case.

Also, in the lower-right panel, we show the curves of $\log T_{\text {eff }}$ versus $\log g_{\text {eff }}$. The curves are almost straight lines, meaning that, in Espinosa-Lara \& Rieutord's prescription, we can still adopt the common approach $T_{\text {eff }} \propto g_{\text {eff }}^{\beta}$, where $\beta$ is the slope of the curves shown in the lower-right panel.

The $T_{\text {eff }} \propto g_{\text {eff }}^{\beta}$ is already implemented in the radiative transfer code HDUST. Therefore, for a rotating star with parameter $W$, we adopted the coefficient $\beta(W)$ obtained by fitting a straight line to the $\log T_{\text {eff }}$ versus $\log g_{\text {eff }}$ curve. 
Chapter 3

\section{Viscous decretion disks around Be stars}

Every formula which expresses a law of nature is a hymn of praise to God.

Maria Mitchell, Life, Letters and Journals (1896)

The optical light curves of early-type Be stars (with spectral type ranging roughly from B0 to B4) are usually quite variable in timescales of days to years, with amplitudes of up to tenths of a magnitude (Rivinius et al., 2013). The majority of them show very irregular variability. Most show clear single bump-like features, characterized by a fast rise in brightness followed by a slower fading. Frequently, between the brightening and the fading phases some sort of plateau of nearly constant brightness is seen. Sometimes, a dip is seen, such that an initial rapid fading is followed by a slow recovery of the stellar brightness. Two examples of light curves showing these features are given in Fig. 3.1, taken from OGLE-II (Udalski et al., 1997) and OGLE-III (Udalski et al., 2008) data from two Be star candidates from the SMC, based on the selection made by Mennickent et al. (2002). Object SMC_SC1 75701 shows two brightening bumps, while object SMC_SC6 128831 shows a dip.

These bumps and dips resemble the photometric features shown in Haubois et al. (2012, e.g., their Fig. 14) of a circumstellar viscous disk that builds-up as a result of a constant rate of mass injection to the disk and, later, is left to dissipate after the cessation of the mass injection. Haubois et al. (2012) studied several disk feeding scenarios (constant vs. cyclic vs. outburst), calculated their photometric observables, and demonstrated that the bumps are disk formation/dissipation events of active Be stars seen at near pole-on inclination angles $(i \lesssim 70 \mathrm{deg})$ while the dips are associated with near edge-on Be stars (shell stars, $i \gtrsim 70 \mathrm{deg}$ ). The inclination angle plays an important role in how the stellar brightness is modified by the presence of a disk because, in the second case (edge-on), the disk is seen projected against the star and, thus, causes an attenuation of the stellar radiation. This attenuation does not happen for the pole-on case, where the net effect of the disk is to increase the optical brightness as a result of free-bound and free-free 

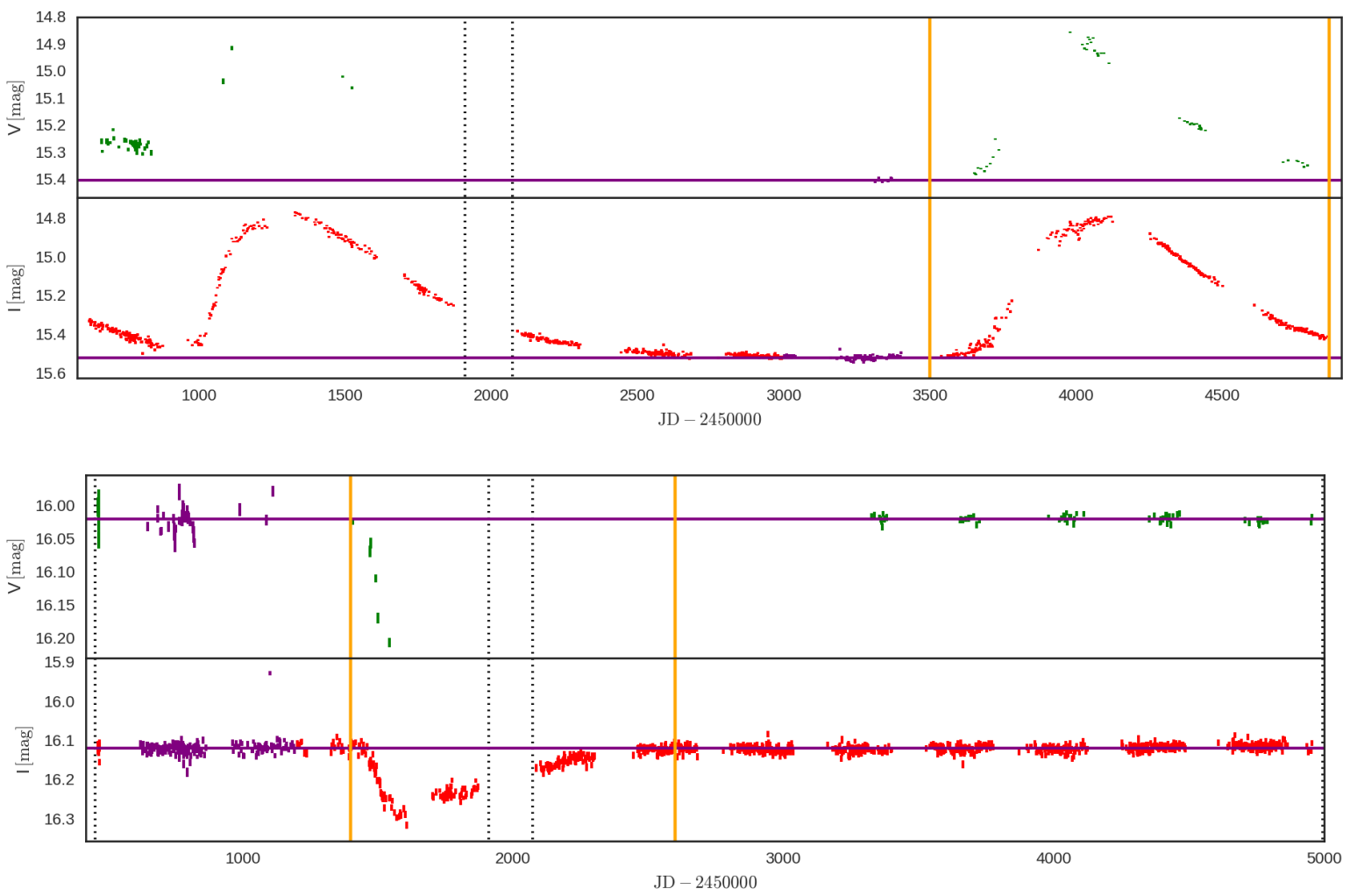

Figure 3.1: Two light curves, in photometric bands $V$ (green) and $I$ (red), selected from the OGLE-II and OGLE-III photometric surveys. Above: light curve of SMC_SC1 75701 (OGLE-II ID). Below: light curve of SMC_SC6 128831 (OGLE-II ID). The pair of vertical dotted straight lines near JD - $2450000=2000$ separates OGLE-II from OGLE-III data. The measurements shown in purple are assumed to represent the inactive (diskless) brightness level of the Be star. Their mean is given by the horizontal purple straight lines. The pairs of vertical orange straight lines bracket our visually selected bumps.

radiation from the gas (Gehrz et al., 1974; Vieira et al., 2015)

In this chapter, we describe the basic hydrodynamical concepts of gaseous viscous Keplerian disks, with a focus on how to model the aforementioned events of disk construction and dissipation in the light curves of active Be stars.

\subsection{Main considerations}

The evolution of the surface density of the thin circumstellar axisymmetric disks of Be stars is described by Eq. (2.2.17), which we rewrite here:

$$
\frac{\partial \Sigma}{\partial t}+\frac{1}{R} \frac{\partial}{\partial R}\left(R \Sigma\left\langle v_{R}\right\rangle\right)=\Sigma\left\langle\frac{\mathcal{S}}{\rho}\right\rangle-\left.2\left(\rho v_{z}\right)\right|_{\infty},
$$

where, in the circumstellar alpha-disk approach, the mass flux, $2 \pi R \Sigma\left\langle v_{R}\right\rangle$ is given by Eq. (2.2.28). 
We assume here that $\left.2 R W_{z \phi}\right|_{\infty}$ vanishes, because the external medium beyond the disk is empty space. We also, assume that possible azimutal forces (e.g., the azimutal component of a radiative line-driven force) are negligible, meaning that $\left\langle\mathcal{F}_{\phi}^{*} / \rho\right\rangle \approx 0$. Therefore, according to Eq. (2.2.28), the mass flux is given by

$$
2 \pi R \Sigma\left\langle v_{R}\right\rangle=-4 \pi\left(\frac{R^{3}}{G M}\right)^{\frac{1}{2}} \frac{1}{R} \frac{\partial}{\partial R}\left(R^{2} \Sigma\left\langle\alpha c_{s}^{2}\right\rangle\right)
$$

where $c_{s}^{2}=k T_{\text {disk }} / \mu m_{H}$.

Substitution of Eqs. (3.1.2) into (3.1.1) leads to the following partial differential equation for the evolution of the surface density of the thin VDD

$$
\frac{\partial \Sigma}{\partial t}=\frac{2}{R} \frac{\partial}{\partial R}\left(\left(\frac{R}{G M}\right)^{\frac{1}{2}} \frac{\partial}{\partial R}\left(R^{2} \alpha c_{s}^{2} \Sigma\right)\right)+\Sigma\left\langle\frac{\mathcal{S}}{\rho}\right\rangle-\left.2\left(\rho v_{z}\right)\right|_{\infty},
$$

where we have also considered $\left\langle\alpha c_{s}^{2}\right\rangle \approx \alpha c_{s}^{2}$, for simplicity.

\subsubsection{The star-disk connection}

The source terms $\Sigma\langle\mathcal{S} / \rho\rangle$ and $-\left.2\left(\rho v_{z}\right)\right|_{\infty}$ represent the rate of mass injected into (or removed from) the disk per unit of area. In this work, we further assume that the vertical losses of mass from the disk (see, e.g., the disk wind of Krtička et al., 2011) are negligible $\left(\left.\left(\rho v_{z}\right)\right|_{\infty} \approx 0\right)$. The term $\Sigma\langle\mathcal{S} / \rho\rangle$, on the other hand, represents the variable mass exchange between the star and the disk and the disk and the outer medium through an outer boundary.

In this work, we assume that mass is injected from the star into a ring with radius $R_{\text {inj }}$ and negligible thickness. Therefore, the rate of mass injection per unit of area is given by $\dot{M}_{\text {inj }}(t) / 2 \pi R_{\text {inj }} \delta R$, where $\delta R \rightarrow 0^{+}$. In addition, mass can flow away from the disk through its boundaries. Mass can fall back into the star through the inner boundary at $R_{\text {eq }}$, or it can leave the system at an outer boundary, $R_{\text {out }}$.

Therefore, the source function is given by

$$
\Sigma\left\langle\frac{\mathcal{S}}{\rho}\right\rangle=\dot{M}_{\mathrm{inj}}(t) \frac{\delta\left(R-R_{\mathrm{inj}}\right)}{2 \pi R}+\text { boundaries },
$$

where $\dot{M}_{\text {inj }}(t)$ is the mass injection rate from the star into the disk at $R_{\text {inj }}$.

We assume that all mass that eventually reaches the stellar equator $R_{\text {eq }}$ is totally absorbed by the star. Therefore, the inner boundary consists in setting $\Sigma\left(R_{\text {eq }}, t\right)=0$. The outer boundary, on the other hand, can be interpreted as the limiting radius of the disk due to a binary companion (e.g., Okazaki et al., 2002) or due to the photoevaporation of the disk (e.g., Okazaki, 2001). Therefore, we also assume that all mass that eventually reaches the outer boundary at the radius $R_{\text {out }}$ is totally absorbed. Therefore, at the outer boundary, as at the inner boundary, we set $\Sigma\left(R_{\text {out }}, t\right)=0$. 
The source terms contain explicit reference to the boundaries because the boundaries are just like additional rings, where mass is removed from the disk, limiting the existence of the disk to the region $R_{\text {eq }} \leq R \leq R_{\text {out }}$. Therefore, Eq. (3.1.4) with our assumptions of boundaries are completely defined by the following equation

$$
\Sigma\left\langle\frac{\mathcal{S}}{\rho}\right\rangle=\dot{M}_{\text {eq }}(t) \frac{\delta\left(R-R_{\text {eq }}\right)}{2 \pi R}+\dot{M}_{\text {inj }}(t) \frac{\delta\left(R-R_{\text {inj }}\right)}{2 \pi R}+\dot{M}_{\text {out }}(t) \frac{\delta\left(R-R_{\text {out }}\right)}{2 \pi R} .
$$

In the above equation, $\dot{M}_{\text {eq }}(t)$ is the rate of mass removal (since $\dot{M}_{\text {eq }}(t) \leq 0$ always) at the stellar equator. It is given by

$$
\dot{M}_{\mathrm{eq}}(t)=-\left.4 \pi\left(\frac{R_{\mathrm{eq}}}{G M}\right)^{\frac{1}{2}} \frac{\partial}{\partial R}\left(\alpha c_{s}^{2} R^{2} \Sigma\right)\right|_{R_{\mathrm{eq}}^{+}} .
$$

Similarly, $\dot{M}_{\text {out }}(t)$ is the rate of mass removal (since $\dot{M}_{\text {out }}(t) \leq 0$ always) at the outer radius $R_{\text {out }}$. It is given by

$$
\dot{M}_{\text {out }}(t)=\left.4 \pi\left(\frac{R_{\text {out }}}{G M}\right)^{\frac{1}{2}} \frac{\partial}{\partial R}\left(\alpha c_{s}^{2} R^{2} \Sigma\right)\right|_{R_{\text {out }}^{-}} .
$$

In Eq. (3.1.5), the first two terms represent the star-disk connection. The third term represents the relationship between the disk and the outer space.

The dynamical problem described by Eq. (3.1.3) was solved, in this work, using the numerical code SINGLEBE (Okazaki, 2007), which I studied in the beginning of this thesis and slightly modified. We address the main features of this code in Sect. 3.1.2 below. Later, we return to the modeling of dynamical disks, responsible for the observed bumps and dips (and other often more complex shapes) seen in the light curves of Be stars.

\subsubsection{The alpha-disk code SINGLEBE}

Eq. (3.1.3) can be multiplied by $R^{2}$ and written in the following way

$$
\frac{\partial R^{2} \Sigma}{\partial t}=2 \frac{\partial}{\partial \ln R}\left(\left(\frac{1}{G M R}\right)^{\frac{1}{2}} \frac{\partial}{\partial \ln R}\left(\alpha c_{s}^{2} R^{2} \Sigma\right)\right)+\operatorname{sources}(R) .
$$

Eq. (3.1.8) is the partial differential equation solved by SINGLEBE (Okazaki, 2007). SINGLEBE is a very simple code, if compared to other available general 3D hydrodynamical solvers. It was, nevertheless, very carefully written for the purpose of simulating the time-dependent evolution of an axisymmetric alpha-disk. It solves Eq. (3.1.8) by the FTCS (Forward-Time Central-Space) scheme. Eq. (3.1.8) is a parabolic partial differential equation, and the FTCS is known to be stable for that class of equations (Pletcher et al., 1997). The integration, therefore, is given by the following finite difference method

$$
f_{i}^{n+1} \approx f_{i}^{n}+\left(\frac{\partial f}{\partial t}\right)_{i}^{n} \mathrm{~d} t
$$


where $f=R^{2} \Sigma$ and $(\partial f / \partial t)_{i}^{n}$ represents the second member of Eq. (3.1.8). The derivatives contained in that member (e.g., of some function $g(R)$ ) are evaluated as follows:

$$
\left(\frac{\partial g}{\partial \ln R}\right)_{i}^{n} \approx \frac{g_{i+\frac{1}{2}}^{n}-g_{i-\frac{1}{2}}^{n}}{\ln R_{i+\frac{1}{2}}-\ln R_{i-\frac{1}{2}}}
$$

where $\ln R_{i+\frac{1}{2}}=\left(\ln R_{i}+\ln R_{i+1}\right) / 2, g_{i-\frac{1}{2}}=\left(g_{i-1}+g_{i}\right) / 2$, and so on...

The time increment $\mathrm{d} t$ is defined only once at the beginning of each run, following the classic Courant-Friedrichs-Lewy (CFL) numerical stability condition: $\mathrm{d} t=C \min \left(\left|\frac{\mathrm{d} R}{v_{R}}\right|\right)$ (with the choice $C=0.3$ ), calculated assuming a shock configuration. This time interval is usually several times smaller than the usual time intervals (chosen by the user) between consecutive prints of the calculations to an external file.

Following Eq. (3.1.8), SINGLEBE's radial grid was defined as a logarithmic one. At the beginning of my work, I changed it to a more general logarithm grid defined by

$$
\ln \tilde{R}[i]=\ln \tilde{R}_{1}+\left(i-i_{1}\right) \frac{\ln \tilde{R}_{2}-\ln \tilde{R}_{1}}{i_{21}}, \text { for } i=1,2,3,4, \ldots, N,
$$

where $\tilde{R}=R / R_{\text {eq }}$ and $N=i_{1}+i_{21}+n_{2}$, and the three natural numbers $i_{1}>0, i_{21}>0$ and $n_{2} \geq 0$ are chosen by the user.

So, in this grid, we freely choose two radii (two real numbers) $\tilde{R}_{1}$ and $\tilde{R}_{2}$ and their indices in the grid, respectively given by $i_{1}$ and $i_{1}+i_{21}$. Therefore, we in summary define that $\tilde{R}_{1}=\tilde{R}\left[i_{1}\right]$ and $\tilde{R}_{2}=\tilde{R}\left[i_{1}+i_{21}\right]$. (Notice, therefore, that if $i_{1}>1$, then there are $i_{1}-1$ radii before $R_{1}$.) Finally, we may extend the grid to $n_{2}$ radii after $R_{2}$. Therefore, the total number of radii in the grid is given by $N=i_{1}+i_{21}+n_{2}$. In any case, the problem has an inner boundary condition that is always in the first radius $(\tilde{R}[1])$ and an outer boundary condition that is always in the last radius $(\tilde{R}[N])$.

When the code is run, since the definition of the grid is done before the time increment $\mathrm{d} t$ is calculated by the CFL condition, our redefinition of the grid does not alter the stability of the calculation. (It affects the size of the timestep $\mathrm{d} t$ and, thus, the amount of time taken to perform the calculation.)

It is important to note here that, in the work of Carciofi et al. (2012), where the parameter $\alpha$ was first determined for a dissipating portion of the light curve of the Be star $28 \mathrm{CMa}$, we found that the grid was set with the following definition (using the notation of my new grid): " $\tilde{R}_{1}[10]=1$ and $\tilde{R}_{2}[400]=1000$, with the radius where mass was being injected set to $\tilde{R}_{\text {inj }}=\tilde{R}_{1}$ ". Therefore, the mass was being put into orbit exactly at the stellar equator, but there were 9 radial cells before it: the inner boundary was inside the star, at $\tilde{R}[1]=0.8526$. This is clearly a physical inconsistency. The absorption of the disk matter should happen at the surface of the star. The code was in effect treating the star to be smaller, with $85.26 \%$ of its size. The bigger distance between the inner boundary and the injection radius was one of the causes that made it necessary to use a 
bigger value of $\alpha$ to fit the dissipation of $28 \mathrm{CMa}$ 's light curve. (The other major cause was the fact that the authors didn't take into account the previous history of the disk, as we shall see later in Sect. 3.3)

In Ghoreyshi et al. (2016) and in this work, we defined the grid by " $\tilde{R}_{1}[1]=1$ and $\tilde{R}_{2}[400]=1000$, with the injection radius set to $\tilde{R}_{\text {inj }}=\tilde{R}[2]$ (with these definitions, Eq. 3.1.11 dictates that $\tilde{R}[2]=1.017)$ ". Therefore, the inner boundary condition is at the stellar equator, as we think it should be, and mass is injected into the disk just a little distance above the equator.

\subsubsection{The timescale parameter $\tau$ and the time parameter $\tilde{\tau}$}

In this work, we assume that the disk is isothermal with $T_{\text {disk }}=0.6 T_{\text {eff }}$, following Carciofi and Bjorkman (2006), and that the $\alpha$ parameter is constant with $R$, but we allow it to possibly be time-dependent, as there is evidence for changing $\alpha$ in the disks of $\mathrm{Be}$ stars (see Sect. 3.3 below). Eqs. (3.1.3) and (3.1.5) can, then, be scaled in the following way

$$
\frac{\partial \Sigma}{\partial t}=\frac{1}{\tau}\left\{\frac{2}{\tilde{R}} \frac{\partial}{\partial \tilde{R}}\left[\tilde{R}^{\frac{1}{2}} \frac{\partial}{\partial \tilde{R}}\left(\tilde{R}^{2} \Sigma\right)\right]+\tau \Sigma\left\langle\frac{\mathcal{S}}{\rho}\right\rangle\right\}
$$

where we introduce the timescale parameter, $\tau(t)$, given by

$$
\tau(t)=\frac{1}{\alpha(t)}\left(\frac{R_{\mathrm{eq}}^{3}}{G M}\right)^{\frac{1}{2}} \frac{v_{\mathrm{orb}}^{2}}{c_{s}^{2}} .
$$

The timescale parameter is proportional to the viscous timescale at the stellar equator. The viscous timescale is given by $t_{\mathrm{vis}}=R^{2} / \nu$, where the viscosity $\nu$ is given by $\nu=$ $(2 / 3) \alpha c_{s}^{2} R / v_{K}$. Since we are in the thin disk limit $\left(c_{s}^{2} / v_{K}^{2} \ll 1\right)$, we see that $\tau$ is much larger than the orbital period at the stellar equator, given by $2 \pi\left(R_{\mathrm{eq}}^{3} / G M\right)^{\frac{1}{2}}$.

The solution of Eq. 3.1.12 is scaled in time by the timescale parameter, which controls how fast matter is redistributed throughout the disk and, consequently, its observational counterparts. It follows that by fitting observed light curves of Be stars, this parameter can be estimated, and once other parameters are known $\left(R_{\mathrm{eq}}, M, T_{\text {disk }}\right)$, the $\alpha$ value can be determined (e.g., Carciofi et al., 2012). This scaling relation is fundamental to this $\mathrm{PhD}$ thesis.

In order to generate models that do not depend on the time-dependent form of $\alpha(t)$ and also on the parameters $R_{\text {eq }}, M$ and $T_{\text {disk }}$, which are related to the central star, we will define a dimensionless time parameter, $\tilde{\tau}(t)$, such that

$$
\mathrm{d} \tilde{\tau}=\frac{\mathrm{d} t}{\tau(t)},
$$

which allows us to solve Eq. (3.1.12) in terms of $\tilde{\tau}$ instead of the physical time $t$. The advantage of using this new parameter is that it removes the problem of the time dependency of $\alpha(t)$ and separates it from the problem of solving Eq. (3.1.12). Consequently, it 
allows us to create a grid of solutions of Eq. (3.1.12) that is independent of $\alpha(t), R_{\text {eq }}, M$ and $T_{\text {disk }}$ (see Chapter 4 ).

The source term, now multiplied by $\tau$, in Eq. (3.1.12), is given by

$$
\tau \Sigma\left\langle\frac{\mathcal{S}}{\rho}\right\rangle=\left(\frac{\tau \dot{M}_{\mathrm{inj}}}{2 \pi R_{\mathrm{eq}}^{2}}\right) \frac{\delta\left(\tilde{R}-\tilde{R}_{\mathrm{inj}}\right)}{\tilde{R}}+\text { boundaries }
$$

where the term in parentesis is a measure of the mass injection rate in terms of the time parameter, instead of the physical time. This term has the dimension of a surface density.

Before moving on to the modeling of bump-like events like the ones in Fig. 3.1, we introduce in the next section some important parameters of the dynamical disks implied in the equations shown above.

\subsection{Dynamical disk parameters}

The steady-state solution of the viscous disk corresponds to the limiting case of a disk that has been fed at a constant rate for an infinitely long time. It is obtained by setting $\partial \Sigma / \partial t=0$ (in Eq. 3.1.12 or 3.1.1) and assuming that $\alpha$ and $\dot{M}_{\text {inj }}$ are time-independent.

Probably the best way to describe the fundamental parameters of a dynamical disk is by comparing their dynamical properties with their corresponding steady-state properties. We will, consequently, dedicate some effort in this section to the study of the steady-state solution of the dynamical problem of the viscous disk.

\subsubsection{The steady-state solution of the VDD}

Eq. (3.1.5) shows that the radius of mass injection divides the disk in two regions: a narrow region between the inner boundary and the radius of mass injection $\left(1 \leq \tilde{R} \leq \tilde{R}_{\text {inj }}\right)$, and the much wider region between the radius of mass injection and the outer boundary $\left(\tilde{R}_{\text {inj }} \leq \tilde{R} \leq \tilde{R}_{\text {out }}\right)$, where we have defined $\tilde{R}_{\text {inj }}=R_{\text {inj }} / R_{\text {eq }}$ and $\tilde{R}_{\text {out }}=R_{\text {out }} / R_{\text {eq }}$.

By setting $\partial \Sigma / \partial t=0$ in Eq. (3.1.1), it follows that the steady-state disk is given by the solution of the following equation

$$
\frac{\partial}{\partial R}\left(2 \pi R \Sigma\left\langle v_{R}\right\rangle\right)=\dot{M}_{\text {inj }} \delta\left(R-R_{\text {inj }}\right), 1<\tilde{R}<\tilde{R}_{\text {out }},
$$

where we have used the mass source defined by Eq. (3.1.5).

The above equation says that, in steady-state, the mass flux is a constant in the region $1<\tilde{R}<\tilde{R}_{\text {inj }}$ and another constant in the region $\tilde{R}_{\text {inj }}<\tilde{R}<\tilde{R}_{\text {out }}$. Integrating from just before $R_{\text {inj }}$ (from $R_{\text {inj }}^{-}$) to just after $R_{\text {inj }}$ (to $R_{\text {inj }}^{+}$) results in the following discontinuity condition

$$
\left.\left(2 \pi R \Sigma\left\langle v_{R}\right\rangle\right)\right|_{R_{\mathrm{inj}}^{+}}-\left.\left(2 \pi R \Sigma\left\langle v_{R}\right\rangle\right)\right|_{R_{\mathrm{inj}}^{-}}=\dot{M}_{\mathrm{inj}},
$$

which just means that the mass being injected at $R_{\text {inj }}$ is flowing in both directions: inwards and outwards. 
Consequently, the steady-state mass flux in the narrow region $1<\tilde{R}<\tilde{R}_{\text {inj }}$ is given by the following constant

$$
2 \pi R \Sigma\left\langle v_{R}\right\rangle=-\dot{M}_{\mathrm{inj}} \Xi, 1<\tilde{R}<\tilde{R}_{\mathrm{inj}}
$$

where $\Xi$ is some number between 0 and 1 , representing the fraction of the injected mass that is flowing back to the star. Then, in order to satisfy the discontinuity condition given by Eq. (3.2.2), the steady-state mass flux in the wider region $\tilde{R}_{\text {inj }}<\tilde{R}<\tilde{R}_{\text {out }}$ is given by

$$
2 \pi R \Sigma\left\langle v_{R}\right\rangle=\dot{M}_{\text {inj }}(1-\Xi), \tilde{R}_{\text {inj }}<\tilde{R}<\tilde{R}_{\text {out }} .
$$

Let us first look into the mass flux in the outer region $\tilde{R}_{\text {inj }}<\tilde{R}<\tilde{R}_{\text {out }}$. Since, in steady-state, the mass flux is constant in the whole region, it results in the following equation

$$
-4 \pi\left(\frac{R^{3}}{G M}\right)^{\frac{1}{2}} \frac{1}{R} \frac{\partial}{\partial R}\left(\alpha c_{s}^{2} R^{2} \Sigma\right)=\dot{M}_{\text {inj }}(1-\Xi), \tilde{R}_{\text {inj }}<\tilde{R}<\tilde{R}_{\text {out }},
$$

which, by isolating the term with the derivative on the left and, then, integrating from $R_{\text {inj }}$ to $R$, results in the following steady-state profile for $\Sigma$

$$
\alpha c_{s}^{2} R^{2} \Sigma-\alpha c_{s}^{2} R_{\mathrm{inj}}^{2} \Sigma_{\mathrm{inj}}=-\frac{(G M)^{\frac{1}{2}}}{2 \pi} \dot{M}_{\mathrm{inj}}(1-\Xi)\left(R^{\frac{1}{2}}-R_{\mathrm{inj}}^{\frac{1}{2}}\right), \tilde{R}_{\mathrm{inj}}<\tilde{R}<\tilde{R}_{\text {out }} .
$$

Now, the outer boundary condition comes into play. Setting $\Sigma\left(R_{\text {out }}, t\right)=0$ gives

$$
\alpha c_{s}^{2} R_{\mathrm{inj}}^{2} \Sigma_{\mathrm{inj}}=\frac{(G M)^{\frac{1}{2}}}{2 \pi} \dot{M}_{\mathrm{inj}}(1-\Xi)\left(R_{\mathrm{out}}^{\frac{1}{2}}-R_{\mathrm{inj}}^{\frac{1}{2}}\right)
$$

which, substituting in Eq. (3.2.6) results in the following formula for the steady-state surface density in the region $\tilde{R}_{\text {inj }}<\tilde{R}<\tilde{R}_{\text {out }}$ :

$$
\Sigma=\frac{(G M)^{\frac{1}{2}} \dot{M}_{\text {inj }}(1-\Xi)}{2 \pi \alpha c_{s}^{2}} \frac{1}{R^{2}}\left(R_{\text {out }}^{\frac{1}{2}}-R^{\frac{1}{2}}\right), \tilde{R}_{\text {inj }}<\tilde{R}<\tilde{R}_{\text {out }} .
$$

We recognize the above equation as the equation number 24 of Carciofi and Bjorkman (2008). Notice, however, that their $\alpha$ parameter corresponds to $2 / 3$ times our $\alpha$ (see the discussion on the variation in the definitions of $\alpha$ in Sect. 2.2.3). That is the reason the number $3 \pi$ appears in their equation while the number $2 \pi$ appears in our Eq. (3.2.8). Also notice that their $\dot{M}$ corresponds to our $\dot{M}_{\text {inj }}(1-\Xi)$. This latter quantity is the steady-state decretion rate.

In order to find the value of $\Xi$, we must complement our analysis with the study of the steady-state in the narrow region $1 \leq \tilde{R} \leq \tilde{R}_{\text {inj }}$. Eq. (3.2.3) implies in the following equation

$$
-4 \pi\left(\frac{R^{3}}{G M}\right)^{\frac{1}{2}} \frac{1}{R} \frac{\partial}{\partial R}\left(\alpha c_{s}^{2} R^{2} \Sigma\right)=-\dot{M}_{\mathrm{inj}} \Xi, 1 \leq \tilde{R} \leq \tilde{R}_{\mathrm{inj}}
$$


which, by isolating the term with the derivative on the left and, then, integrating from $R$ to $R_{\text {inj }}$, results in the following steady-state profile for $\Sigma$

$$
\alpha c_{s}^{2} R^{2} \Sigma-\alpha c_{s}^{2} R_{\mathrm{inj}}^{2} \Sigma_{\mathrm{inj}}=-\frac{(G M)^{\frac{1}{2}}}{2 \pi} \dot{M}_{\mathrm{inj}} \Xi\left(R_{\mathrm{inj}}^{\frac{1}{2}}-R^{\frac{1}{2}}\right), 1 \leq \tilde{R} \leq \tilde{R}_{\mathrm{inj}}
$$

We are using the inner boundary condition that $\Sigma\left(R_{\text {eq }}, t\right)=0$. Therefore, from Eq. (3.2.10), it follows that we must have

$$
\alpha c_{s}^{2} R_{\mathrm{inj}}^{2} \Sigma_{\mathrm{inj}}=\frac{(G M)^{\frac{1}{2}}}{2 \pi} \dot{M}_{\mathrm{inj}} \Xi\left(R_{\mathrm{inj}}^{\frac{1}{2}}-R_{\mathrm{eq}}^{\frac{1}{2}}\right)
$$

From Eqs. (3.2.7) and (3.2.11), we can now obtain the value of $\Xi$. It is given by

$$
\Xi=\frac{R_{\text {out }}^{\frac{1}{2}}-R_{\mathrm{inj}}^{\frac{1}{2}}}{R_{\text {out }}^{\frac{1}{2}}-R_{\mathrm{eq}}^{\frac{1}{2}}}
$$

from which we see that, since $R_{\text {inj }}$ must be very close to $R_{\text {eq }}$ and $R_{\text {out }}$ must be much further away, it follows that $\Xi$ is usually a number just a little smaller than 1 for every Be star. Therefore, in steady-state, most of the injected mass comes back to the star and is absorbed.

Substitution of Eq. (3.2.11) into Eq. (3.2.10) and of Eq. (3.2.7) into Eq. (3.2.6) finally results in the steady-state surface density of the disk:

$$
\Sigma_{\text {steady }}(\tilde{R})= \begin{cases}\frac{\Sigma_{0}}{\tilde{R}^{2}} \Xi\left(\frac{\tilde{R}^{\frac{1}{2}}-1}{\tilde{R}_{\text {inj }}^{\frac{1}{2}}-1}\right), & 1 \leq \tilde{R}<\tilde{R}_{\text {inj }} \\ \frac{\Sigma_{0}}{\tilde{R}^{2}}\left(\frac{\tilde{R}_{\text {out }}^{\frac{1}{2}}-\tilde{R}^{\frac{1}{2}}}{\tilde{R}_{\text {out }}^{\frac{1}{2}}-1}\right), \quad \tilde{R}_{\text {inj }} \leq \tilde{R} \leq \tilde{R}_{\text {out }}\end{cases}
$$

where we have defined the physical quantity $\Sigma_{0}$ by the following equation

$$
\Sigma_{0}=\frac{1}{\Xi} \tilde{R}_{\mathrm{inj}}^{2} \Sigma_{\mathrm{inj}}
$$

The physical quantity $\Sigma_{0}$ represents the surface density at $\tilde{R}=1$, obtained by extrapolating $\Sigma_{\text {steady }}$ in the domain $\tilde{R}_{\text {inj }} \leq \tilde{R} \leq \tilde{R}_{\text {out }}$ to $\tilde{R}=1$. We will refer to this physical quantity as the asymptotic surface density. It is the asymptotic value reached after an infinitely long disk build-up under a constant $\dot{M}_{\text {inj }}$.

\subsubsection{Rates of mass and angular momentum loss}

Substitution of Eq. (3.2.14) into Eq. (3.2.11) shows that $\Sigma_{0}$ is related to $\dot{M}_{\text {inj }}$ by the following equation

$$
2 \pi R_{\mathrm{eq}} \Sigma_{0}\left(\frac{R_{\mathrm{eq}}}{\tau}\right)=\dot{M}_{\mathrm{inj}}\left(\tilde{R}_{\mathrm{inj}}^{\frac{1}{2}}-1\right) \equiv\left(-\frac{\partial M}{\partial t}\right)_{\mathrm{typ}} .
$$


We may extend Eq. (3.2.15) to the general case of a time-dependent $\dot{M}_{\text {inj }}(\tilde{\tau})$, which would define, by the same equation, a time dependent asymptotic surface density, $\Sigma_{0}(\tilde{\tau})$.

Applying Eq. (3.2.15) to Eq. (3.1.15), it follows that

$$
\tau \Sigma\left\langle\frac{\mathcal{S}}{\rho}\right\rangle=\frac{\Sigma_{0}(\tilde{\tau})}{\tilde{R}_{\mathrm{inj}}^{\frac{1}{2}}-1} \frac{\delta\left(\tilde{R}-\tilde{R}_{\mathrm{inj}}\right)}{\tilde{R}}+\text { boundaries . }
$$

The function $\Sigma_{0}(\tilde{\tau})$, therefore, is just another way of specifying the history of mass injection from the star into the disk. It has, however, the advantage of being a surface density, which is a quantity that may be determined from, e.g, SED analyses, in contrast to the mass injection rate and the radius of mass injection, which are parameters that cannot be directly determined.

The steady-state solution (Eq. 3.2.13) shows that, in the outer domain $\tilde{R}_{\text {inj }} \leq \tilde{R} \leq$ $\tilde{R}_{\text {out }}$, the density profile of the disk is not altered if $\tilde{R}_{\text {inj }}$ is changed, provided that $\dot{M}_{\text {inj }}$ is also changed in order to maintain $\Sigma_{0}$ fixed, according to Eq. (3.2.15). In fact, we verified that the time-dependent solutions of Eq. (3.1.12) in the domain $\tilde{R}_{\text {inj }} \leq \tilde{R} \leq \tilde{R}_{\text {out }}$ are negligibly affected by the particular choice of $\tilde{R}_{\text {inj }}$ and $\dot{M}_{\text {inj }}(\tilde{\tau})$, as long as the quantity $\Sigma_{0}(\tilde{\tau})$ is kept fixed, as Fig. 3.2 shows.

In Fig. 3.2, we show the evolution of three disk models that started from the diskless state, received a constant mass injection rate $\Sigma_{0}=1.549 \mathrm{~g} \mathrm{~cm}^{-2}$ for a period $\tilde{\tau}_{\mathrm{bu}}=30$, and, after that, were left to dissipate (with $\Sigma_{0}=0$ ). The three disk models had different values of $\tilde{R}_{\text {inj }}$ (and of $\dot{M}_{\text {inj }}$, consequently, so that $\Sigma_{0}$ is fixed). The upper-left panel shows the surface density profile at 5 times during the build-up phase and the upper-right panel shows the surface density profiles at 5 times during the dissipation phase. The dotted, dashed and solid curves show the models with $\tilde{R}_{\text {inj }}$ given by $1.017,1.169$ and 1.319, respectively. It is easy to see the positions of the injection radii in the curves by the positions of the discontinuities in their derivatives, as a consequence of the delta function in Eq. 3.1.5. The upper-left and upper-right panels illustrate the fact that the evolution of the disks with the same $\Sigma_{0}(\tilde{\tau})$, but different $\tilde{R}_{\text {inj }}$ and $\dot{M}_{\text {inj }}$ are nearly indistinguishable in their domain $\tilde{R}_{\text {inj }} \leq \tilde{R} \leq \tilde{R}_{\text {out }}$. This is, we believe, in part a consequence of the fact that the dynamical solutions reach a near steady-state very quickly in the vicinity of the injection radius (Haubois et al., 2012). See, however, further discussions in Sect. 3.2.3.

Provided that mass is injected not too far from the stellar photosphere (i.e., assuming $\tilde{R}_{\text {inj }} \approx 1$ ), the domain $1 \leq \tilde{R} \leq \tilde{R}_{\text {inj }}$ is much narrower than the region where the continuum visual flux of Be stars is generated (see Carciofi, 2011, and Fig. 1.11), which means that the emission from this region can be ignored. Consequently, we conclude that $\Sigma_{0}(\tilde{\tau})$ (with the assumption that $\tilde{R}_{\text {inj }} \approx 1$ ) is a much better parameter for describing the mass injection history of the disk than the pair of parameters $\dot{M}_{\text {inj }}(\tilde{\tau})$ and $\tilde{R}_{\text {inj }}$. We will, therefore, for the remainder of this work, use the asymptotic surface density function $\Sigma_{0}(\tilde{\tau})$ as a measure of the mass and angular momentum injection into our disk models. 

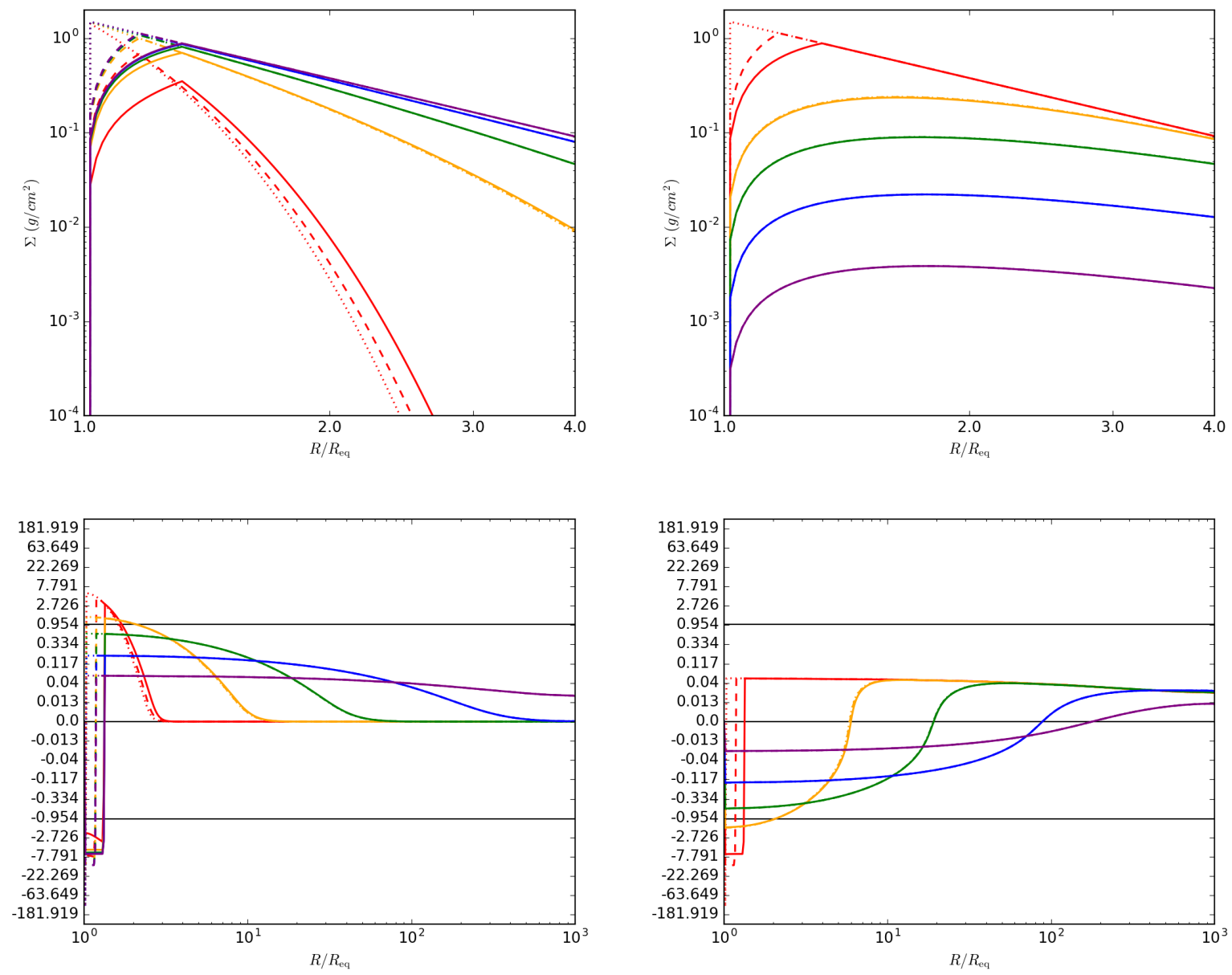

Figure 3.2: Simulation of a constant build up phase $\left(\Sigma_{0}=1.549 \mathrm{~g} \mathrm{~cm}^{-2}\right)$, during $\tilde{\tau}_{\mathrm{bu}}=30$, followed by a complete dissipation $\left(\Sigma_{0}=0\right)$. Three injection radii were used: $\tilde{R}_{\text {inj }}=1.017$ (dotted), 1.169 (dashed), 1.319 (solid). The different colors corresponds to different times after the beginning (left) or the end of injection (right): $\tilde{\tau} \sim 0$ (red), $\tilde{\tau}=0.2$ (orange), $\tilde{\tau}=1$ (green), $\tilde{\tau}=6$ (blue), $\tilde{\tau}=25$ (purple). Top: Surface density in the first 4 stellar radii, where most of the visual continuum observables are generated. Bottom: Mass flux, as a function of the typical decretion rate (Eq. 3.2.15), in the whole simulated disk.

The time-dependent solutions of Eq. (3.1.12) generally show that, for Be stars dynamically feeding the disk, but still far from steady-state, the mass flux close to $\tilde{R}_{\text {inj }}$ has absolute values of the order of $(-\partial M / \partial t)_{\text {typ }}$, defined by Eq. (3.2.15). Therefore, we refer to this quantity as the typical decretion rate, which depends on parameters that are relatively easy to estimate from SEDs of Be stars. As an illustration of how the values of the mass flux in the disk compares with the typical decretion rate, we show, in the two lower panels of Fig. 3.2, the mass fluxes, divided by the typical decretion rate, of the simulated dynamical models. ${ }^{1}$ Two horizontal straight lines mark the position of the

\footnotetext{
${ }^{1}$ The vertical axis of the plates are in arcsinh scale, to better show the smaller values, positive or
} 
positive and negative typical decretion rates. The lower-left and lower-right panels show the mass fluxes at the 5 selected times during the build-up phase and the dissipation phase, respectively.

From the lower-left panel, we see that, in the build-up phase, the mass flux outwards begins quite high, in the vicinity of the radius of mass injection. This flux diffuses outwards, progressively diminishig its value near the mass injection radius. We see, however, that, at least during the interval $\Delta \tilde{\tau}=1$ (comprised by the red, orange and green curves), the mass flux near the radius of mass injection is very close to the typical decretion rate. As time passes, the mass flux approaches its steady state value, given by the constant value of $\dot{M}_{\text {inj }}(1-\Xi)$ through the whole domain $\tilde{R}_{\text {inj }} \leq \tilde{R} \leq \tilde{R}_{\text {out }}$. The purple curve (for $\tilde{\tau}=25$ ), in particular, shows that at this time, there is a significant mass loss through the outer boundary, which in our models was set to $\tilde{R}_{\text {out }}=1000$.

The lower-right panel shows that, in the dissipation phase, which started at $\tilde{\tau}_{\mathrm{bu}}=30$, the inner disk rapidly becomes an accretion disk. Again, at least during the first interval $\Delta \tilde{\tau}=1$ (comprised by the red, orange and green curves), the mass flux near the radius of mass injection is very close to the negative typical decretion rate. The radius where the mass flux is zero is the "stagnation point" (Haubois et al., 2012). At $\tilde{\tau}=31$, the stagnation point is already beyond $\tilde{R}=10$. At $\tilde{\tau}=55$, it is already beyond $\tilde{R}=100$. Further away, however, the disk is still a decretion disk (that is: a disk with a positive mass flux). As $\tilde{\tau} \rightarrow \infty$, the mass flux tends to zero in the whole disk.

In the simulations done in this work, since the values of $\dot{M}_{\text {inj }}$ and $\tilde{R}_{\text {inj }}$ are of no interest and the value of $\tilde{R}_{\text {out }}$ is quite uncertain, we arbitrarily chose $\tilde{R}_{\text {inj }}=1.017$ and $\tilde{R}_{\text {out }}=1000$. Eq. (3.2.15), therefore, shows that the typical decretion rate is much smaller than the mass injection rate $\dot{M}_{\text {inj }}(\tilde{\tau})$. In our case, the typical decretion rate is only $8.46 \times 10^{-3} \dot{M}_{\text {inj }}$. This means that, as seen above, the majority of the injected mass flows inwards and is absorbed by the inner boundary at the stellar equator, and only a small remaining fraction of the injected mass is responsible for the growth of the disk. These results were first obtained from SPH simulations of Be disks by Okazaki et al. (2002), who found that only about $0.1 \%$ of the injected material flows outward, as a direct result of their choice for $R_{\text {inj }}$.

In steady-state, only the fraction of the injected mass given by $\dot{M}_{\text {inj }}(1-\Xi)$ is flowing outwards through the disk and crossing the outer radius $R_{\text {out }}$, thus leaving the system (in our case, $\left.1-\Xi=2.84 \times 10^{-4}\right)$. Since the mass of the disk is not varying in steady-state, the mass flux given by $\dot{M}_{\text {inj }}(1-\Xi)$ is actually the rate of mass being lost by the star, which we indicate by $(-\partial M / \partial t)_{\text {steady }}$. It is easily seen that it is related to the typical decretion rate by the following equation

$$
\left(-\frac{\partial M}{\partial t}\right)_{\text {steady }}=\frac{\Lambda}{\tilde{R}_{\text {out }}^{\frac{1}{2}}}\left(-\frac{\partial M}{\partial t}\right)_{\text {typ }},
$$

negative, and compress the regions of high absolute value in a similar way as the log scale does. 

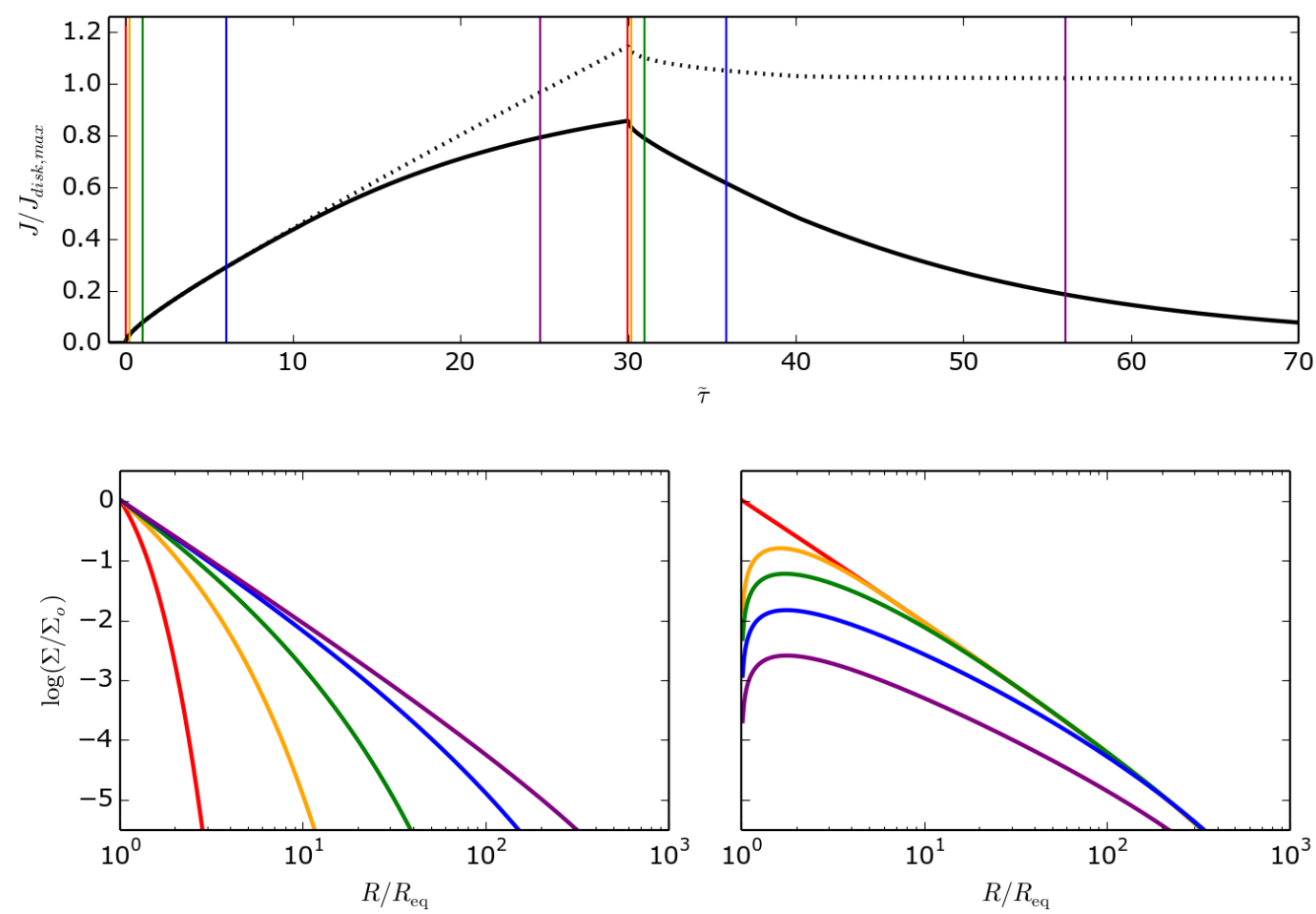

Figure 3.3: Dynamical bump model with $\tilde{\tau}_{\mathrm{bu}}=30$. Above: the amount of angular momentum in the disk (solid black curve) and the amount of angular momentum that is lost by the star (dotted black curve). Colored vertical straight lines mark the 5 instants $\tilde{\tau}=0,0.2,1,6,25$ (during the build-up phase), and the 5 instants $\tilde{\tau}=30,30.2,31,36,55$ (during the dissipation phase). Surface density profiles are shown at the first 5 instants (lower-left) and at the last 5 instants (lower-right).

where $\Lambda=1 /\left(1-\tilde{R}_{\text {out }}^{-\frac{1}{2}}\right)$ is a number very close to 1 for any Be disk in general. In our case, $\Lambda-1=3.27 \times 10^{-2}$.

In steady-state, the angular momentum escaping the system at the outer boundary (and also ultimately being lost by the star) is $\left(G M R_{\text {out }}\right)^{\frac{1}{2}} \dot{M}_{\text {inj }}(1-\Xi)$, being given by

$$
\left(-\frac{\partial J}{\partial t}\right)_{\text {steady }}=\Lambda\left(G M R_{\mathrm{eq}}\right)^{\frac{1}{2}}\left(-\frac{\partial M}{\partial t}\right)_{\mathrm{typ}} .
$$

Therefore, from Eq. (3.2.17) we see that knowing $\tilde{R}_{\text {out }}$ is essential for estimating the rate of mass being lost by the star. This, however, is not the case for the rate of angular momentum being lost by the star, given by Eq. (3.2.18). If follows that the latter quantity can be much more reliably obtained from light curve modellig than the former.

\subsubsection{On the importance of the angular momentum loss rate}

The same model used above for Fig. 3.2 will be used again to explore some other important properties of the dynamical models.

The upper panel of Fig. 3.3 shows the amount of angular momentum in the disk, given 
by

$$
J_{\text {disk }}=\int_{R_{\mathrm{eq}}}^{R_{\mathrm{out}}}(G M R)^{\frac{1}{2}} \Sigma 2 \pi R \mathrm{~d} R,
$$

and the amount of angular momentum that is lost by the star, given by the angular momentum that is injected at the radius of mass injection minus the angular momentum that falls back to the stellar equator:

$$
-\Delta J_{*}=\int_{t_{\text {init }}}^{t}\left[\left(G M R_{\mathrm{inj}}\right)^{\frac{1}{2}} \dot{M}_{\mathrm{inj}}+\left(G M R_{\mathrm{eq}}\right)^{\frac{1}{2}} \dot{M}_{\mathrm{eq}}\right] \mathrm{d} t^{\prime} .
$$

By the conservation of angular momentum, the difference between the dotted and the solid curves is the angular momentum that escapes the system through the outer boundary at $R_{\text {out }}$. All values were scaled by the maximum angular momentum supported by the disk, which is given by

$$
J_{\text {disk,max }}=\int_{R_{\text {eq }}}^{R_{\text {out }}}(G M R)^{\frac{1}{2}} \Sigma_{\text {steady }} 2 \pi R \mathrm{~d} R .
$$

The plot shows that, as the build-up process occurs, the disk's angular momentum and mass increase continuously. Eventually (in our example, roughly after $\tilde{\tau}=10$ ), we see that a non-negligible amount of angular momentum starts to reach the outer radius $\tilde{R}_{\text {out }}=1000$, thus leaving the system through the outer boundary. After the end of the build-up phase (which, in our example, happens at $\tilde{\tau}=30$ ), the disk starts to dissipate: the black curve shows that the disk loses angular momentum until it reaches zero. However, as the dotted curve shows, only a fraction of the angular momentum of the disk returns to the star by re-accretion. The dotted curve tends to a non-zero value, which is the angular momentum that was lost by the star in the whole process.

This non-zero total angular momentum lost by the star was verified in our simulations, for the case of a constant $\alpha$, to be given exactly by

$$
-\Delta J_{*}=\left(-\frac{\partial J}{\partial t}\right)_{\text {steady }} \Delta t
$$

where $\Delta t$ is the duration of the build-up time.

The two lower panels of Fig. 3.3 show surface density radial profiles in the whole disk range (the $\Sigma$ profiles of Fig. 3.2 were limited only to the first four radii) at the specified times marked in the above panel. During build-up, the disk grows from the inside outward, with the inner regions reaching a near steady-state earlier than the outer parts. During dissipation, however, the disk becomes less and less dense as a whole, more or less in a self-similar way, because the entire disk is coupled by viscous forces.

It is implicit in our assumptions for the mass source (Eq. 3.1.5) that matter is injected with Keplerian velocity at $R_{\text {inj }}$ and its removed with Keplerian velocity at $R_{\text {eq }}$ and at $R_{\text {out }}$. Therefore, at the radius of mass injection, there is an angular momentum injection 
rate given by $\left(G M R_{\text {inj }}\right)^{\frac{1}{2}} \dot{M}_{\text {inj }}$, and at the stellar equator, there is an angular momentum removal rate given by $\left(G M R_{\text {eq }}\right)^{\frac{1}{2}} \dot{M}_{\text {eq }}$. Be stars, however, do not rotate at critical velocities. Their mean rotational parameter is $W=0.81 \pm 0.12$ (Rivinius et al., 2006). We may therefore assume that the inner boundary condition is located at just the place where the Keplerian velocity assumption starts to break down. There is strong evidence, however, at least for the Be star Achernar (Rivinius et al., 2013), the closest Be star, that the equatorial velocity may be near-Keplerian during the whole period of Be activity (build-up and dissipation). Rivinius et al. (2013) noticed a broadening in the wings of pure photospheric lines during an activity period of Achernar. I believe that it indeed makes sense that during activity the connection between the matter being injected and absorbed in the build-up phase (or being absorbed only) in the dissipation phase, might put at least the stellar equator of the star, which is a region not well separated from the disk, in an near orbital velocity. If that is the case, our adopted inner boundary condition in which matter is absorbed with azimutal Keplerian velocity should be closer to reality.

In any case, the rate of angular momentum injected minus the rate of angular momentum absorbed is the rate of variation of the angular momentum of the star. Therefore,

$$
\begin{aligned}
-\frac{\partial J}{\partial t}=\dot{J}_{\mathrm{disk}}-\left(G M R_{\mathrm{out}}\right)^{\frac{1}{2}} \dot{M}_{\mathrm{out}} & =\left(G M R_{\mathrm{inj}}\right)^{\frac{1}{2}} \dot{M}_{\mathrm{inj}}+\left(G M R_{\mathrm{eq}}\right)^{\frac{1}{2}} \dot{M}_{\mathrm{eq}} \\
& =\left(G M R_{\mathrm{eq}}\right)^{\frac{1}{2}}\left(\tilde{R}_{\mathrm{inj}}^{\frac{1}{2}} \dot{M}_{\mathrm{inj}}+\dot{M}_{\mathrm{eq}}\right) \\
& =\left(G M R_{\mathrm{eq}}\right)^{\frac{1}{2}} \dot{M}_{\mathrm{inj}}\left(\tilde{R}_{\mathrm{inj}}^{\frac{1}{2}}-\frac{-\dot{M}_{\mathrm{eq}}}{\dot{M}_{\mathrm{inj}}}\right) .
\end{aligned}
$$

In steady-state, we have that $\dot{M}_{\text {eq }}=-\dot{M}_{\text {inj }} \Xi \gtrsim-\dot{M}_{\text {inj }}$, where the last approximation comes from the fact that almost all matter injected comes back to the star. Therefore, we have that the angular momentum loss rate of the star is given by

$$
-\frac{\partial J}{\partial t}=\dot{J}_{\text {disk }}-\left(G M R_{\text {out }}\right)^{\frac{1}{2}} \dot{M}_{\text {out }} \approx\left(G M R_{\text {eq }}\right)^{\frac{1}{2}} \dot{M}_{\text {inj }}\left(\tilde{R}_{\text {inj }}^{\frac{1}{2}}-1\right) .
$$

Eq. (3.2.15), however, says that $\alpha \Sigma_{0} \propto \dot{M}_{\text {inj }}\left(\tilde{R}_{\text {inj }}^{\frac{1}{2}}-1\right)$. Therefore, we conclude that $\Sigma_{0}$, apart from a determination of $\alpha$, is closely related to the net angular momentum that is injected into the disk. The product $\alpha \Sigma_{0}$ is just another way of specifying that physical quantity.

Also, noticing that $\dot{M}_{\text {inj }}\left(\tilde{R}_{\text {inj }}^{\frac{1}{2}}-1\right)$ is the typical decretion rate, we may compare Eq. (3.2.23) with Eq. (3.2.18), from where we conclude that

$$
-\frac{\partial J}{\partial t} \approx\left(-\frac{\partial J}{\partial t}\right)_{\text {steady }}
$$

which means that the angular momentum loss rate of the star is roughly given by the steady-state angular momentum loss rate. 
I conclude this section reflecting on the fact that, although we commonly associate Be stars with "mass loss", probably as a result of Be stars being traditionally linked with stellar winds, the fact is that these stars have outflowing rotating disks, and our results suggest that, in this context, it might be better to talk about "angular momentum loss". Any future theory explaining the Be mechanism will probably be linked with the angular momentum as the fundamental physical quantity to be explained.

\subsection{The mass reservoir effect}

It is important to stress that the solution $\Sigma(\tilde{R}, \tilde{\tau})$ is shaped not just by the mass injection rate $\Sigma_{0}(\tilde{\tau})$ at the specific instant $\tilde{\tau}$, but by the whole mass injection history before the instant $\tilde{\tau}$. Therefore, the advantage of studying the relatively isolated bumps like the ones exemplified in Fig. 3.1, which started after a clear inactive phase, is that there is no disk present when the bump starts developing; thus, no previous history of mass injection has to be taken into account in the beginning of their modeling.

The light curves of several Be stars show that the duration of the build-up phase, which we refer to as the build-up time, is variable between Be stars and even between different bumps from the same star, ranging from a few days to years. The dissipation phase that follows, however, contrary to the build-up phase, depends on the previous history of mass injection. For this reason, the modeling of the dissipation phase must not be disconnected from the modeling of the build-up phase that happened before it. This was one of the main realizations of this thesis. One of the main consequences of this fact is what we call the "mass reservoir effect" (see also Ghoreyshi et al., 2016).

It is possible that two Be star disks with roughly the same visual magnitude have two very different total masses. This is because the visual continuum emission of a Be disk comes almost entirelly from the inner parts of the disk, roughly within the very first few stellar radii (see Fig. 1.11). Depending on the magnitude of the viscosity, a disk under a constant mass injection rate from the star may effectively reach a near steady density distribution in the region of the first few stellar radii within a few months. The outer parts, however, would not have reached a near steady-state yet, and would continue to grow in density, had the mass injection continued, increasing the total mass of the disk in the process. It could take several years for these outer regions to reach a near-steady state. But despite that, the effect on the visual continuum of this growth would be barely noticeable, since these regions contribute negligibly to the visible continuum emission. This is illustrated in Fig. 3.4.

The total mass of the disk is shown in the upper panel of Fig. 3.4. We notice that, although the mass injection rate $\dot{M}_{\text {inj }}$ is constant in the disk, during the build-up the total 

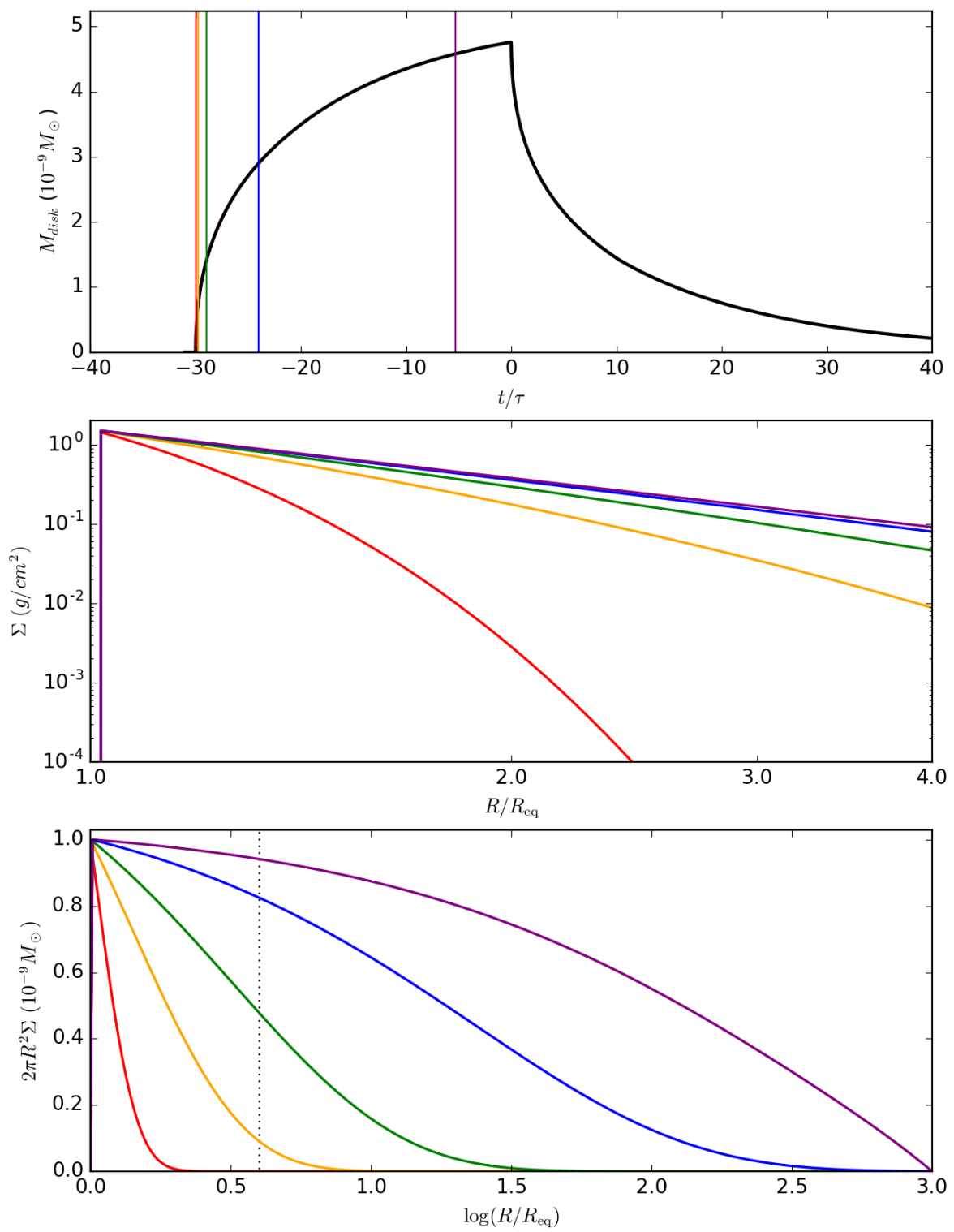

Figure 3.4: Simulation of a constant build up phase $\left(\Sigma_{0}=1.549 \mathrm{~g} \mathrm{~cm}^{-2}\right)$, during $\tilde{\tau}_{\mathrm{bu}}=30$, followed by a complete dissipation $\left(\Sigma_{0}=0\right)$. The different colors corresponds to different instants after beginning of the build-up phase: $\tilde{\tau}+30 \sim 0$ (red), $\tilde{\tau}+30=0.2$ (orange), $\tilde{\tau}+30=1$ (green), $\tilde{\tau}+30=6$ (blue), $\tilde{\tau}+30=25$ (purple). Above: Total mass of the disk. Middle: Surface density profile in the first four radii of the disk. Below: Mass distribution $\left(\partial M_{\text {disk }} / \partial \ln R\right)$ of the disk.

mass of the disk continually increases but at a slower rate. This is due to the fact that the stellar equator absorbs an increasing fraction of the mass being injected, owing to the increasing angular momentum loss rate as the outer radius grows. In the middle panel 

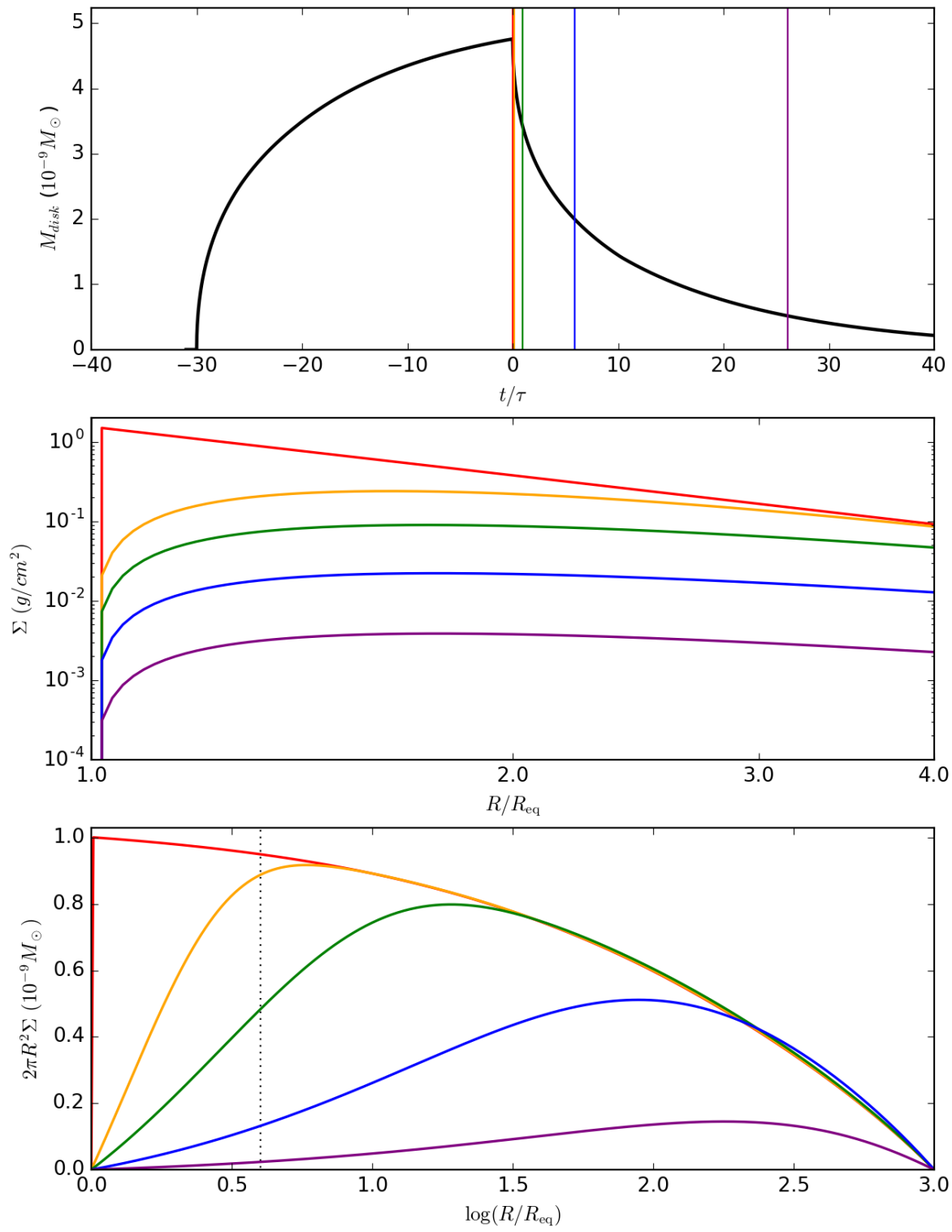

Figure 3.5: Simulation of two constant build-up phases $\left(\Sigma_{0}=1.549 \mathrm{~g} \mathrm{~cm}^{-2}\right)$, one during $\tilde{\tau}_{\mathrm{bu}}=30$ (solid line), and another during $\tilde{\tau}_{\mathrm{bu}}=30$ (dashed line). Both were followed by a complete dissipation $\left(\Sigma_{0}=0\right)$. The different colors corresponds to different instants after beginning of the build-up phase: $\tilde{\tau} \sim 0$ (red), $\tilde{\tau}=0.2$ (orange), $\tilde{\tau}=1$ (green), $\tilde{\tau}=6$ (blue), $\tilde{\tau}=25$ (purple). The three panels are the same as the ones of Fig. 3.4.

of Fig. 3.4 we show the surface density of the disk, at $1 \leq \tilde{R} \leq 4$ (from where the most of the visible continuum is generated) at the five times defined in the upper panel. From this panel, we can see that, at $\tilde{\tau}+30=1$ (see green curve), the inner disk has reached 
a state very close to the steady-state. Therefore, the photometric visual bump (or dip) must already be reaching a plateau by this time. The upper panel shows, however, that the disk's mass is still increasing and will still increase much more, as time passes. This is further illustrated in the lower left panel of Fig.3.4, where we plot $2 \pi R^{2} \Sigma$ versus $\log \tilde{R}$. A dotted vertical line marks the radius $\tilde{R}=4$. The mass of the disk is given by the integral $\int\left(2 \pi R^{2} \Sigma\right) \mathrm{d} \ln R$. Therefore, in the lower panel, any area below each curve and between two radii gives the amount of mass between these radii.

Now, when the mass injection rate from the star to the disk eventually stops, the viscous forces that couple all the matter in the disk lead to the reacretion of most of the disk mass (see the lower-right panel of Fig. 3.2). In that case, we may expect that disks that are very similar in their inner regions (having, therefore, similar visual excesses) but are very different in their outer regions will have a different observed photometric dissipation, due to the fact that the inner region will be viscously coupled to the outer regions. A more massive outer disk represents a bigger reservoir of mass for the inner disk in the dissipation process.

In Fig. 3.5, it is shown that a more massive disk can supply the inner regions with mass for a longer time than a less massive outer disk, eventually making the dissipation of the more massive disk appear slower than the dissipaton of the less massive one.

In the upper panel of Fig. 3.5, we compare two disk models that have been fed with the same $\Sigma_{0}$ but by two very different durations $\left(\tilde{\tau}_{\mathrm{bu}}=30\right.$ and $\left.\tilde{\tau}_{\mathrm{bu}}=3\right)$. In that case, the inner regions of both disks have reached a near-steady surface density by the time of the end of the smallest build-up time $(\tilde{\tau}=3)$. The solid and dashed red lines on the middle panel show that the inner disk of these two disks are very similar, and, so, their visual photometric emission should be very similar. The upper panel, on the other hand, shows that, just before $\tilde{\tau}=0$, the disk that had a build-up time ten times longer ended having more than twice the mass of the other.

As the dissipation phase takes place $(\tilde{\tau}>0)$, the middle panel shows that the surface density of the more massive disk diminishes at a slower rate. Therefore, the initially more massive disk should reduce its visible brightness at a slower rate. Since the viscosity parameter $\alpha$ influences the timescale parameter $\tau$ (see Eq. 3.1.13), we see from the middle panel that a more massive disk, when dissipating, could mimic the dissipation of a less massive disk with a smaller $\alpha$. This is the effect of the mass reservoir on the determination of the $\alpha$ viscosity parameter of VDDs.

The contribution of the outer mass reservoir is further illustrated by the lower panel. We see that the initial distribution of mass of the two disks before the dissipation (given by the solid and dashed red curves) was very different. Since the mass flux is given by $2 \pi R \Sigma\left\langle v_{R}\right\rangle=2 /(G M R)^{\frac{1}{2}}\left(\partial\left(\alpha c_{s}^{2} 2 \pi R^{2} \Sigma\right) / \partial \ln R\right)$, it follows that the slope of the curves of the lower panel is a measure of the mass flux: if the distribution of mass is either locally increasing or decreasing, it means, respectivelly, that mass is flowing inwards or 
outwards. The points of maxima of each curve are the "stagnation points" (the points where $2 \pi R \Sigma\left\langle v_{R}\right\rangle=0$ ). The lower panel, therefore, shows that the more massive disk always accretes mass back to the star at a higher rate, since the slope of the surface density at $\log \tilde{R}=0$ is always steeper for the more massive disk. Despite that, the more massive disk still takes longer to decrease because the accretion region of this disk (the region of positive inclination in its curve) rapidly becomes increasingly bigger than the one of the less massive disk and the reservoir of mass available of the former is bigger than that of the latter.

The importance of the mass reservoir effect can be assessed by the reevaluation of the $\alpha$ parameter in $28 \mathrm{CMa}$ by Ghoreyshi et al. (2016). Carciofi et al. (2012) modeled the 2003 dissipation phase of $28 \mathrm{CMa}$ by considering a very long previous build-up time, and found that a high value of $\alpha$ was necessary to match the observed dissipation rate. Ghoreyshi et al. (2016) have shown that when the prior mass injection history is properly accounted for in the modeling, the value of $\alpha$ required to match the dissipation rate is much smaller: $\alpha=0.21 \pm 0.05$ (M. Ghoreyshi, in preparation).

In Fig. 3.6, we show M. Ghoreyshi et al. ${ }^{2}$ 's current best results for the photometric modeling of the light curve of $28 \mathrm{CMa}$, covering more than 33 years. From the first panel, we see that there are 4 major bumps. These bumps, however, contain smaller bumps in it, suggesting a more erratic mass injection rate superimposed to a more near periodic one. Their values of $\dot{M}_{\text {inj }}$ are shown in the third panel (where they have set $\tilde{R}_{\text {inj }}=1.017$, like we did in our models). In the first panel, we see that the dissipation of the third major bump is the dissipation previously modelled by Carciofi et al. (2012). In their modelling, Carciofi et al. (2012), who were unaware of the mass reservoir effect, used a very long build-up phase before turning the mass injection off to model the dissipation with the dynamical solutions coupled with the radiative transfer code HDUST (A. Carciofi, priv. comm.). Therefore, they required a very large value of $\alpha=1$ in order to model the observed dissipation. In contrast, by modeling the whole available light curve, $\mathrm{M}$. Ghoreyshi et al. obtained the values of $\alpha$ for several build-up and dissipations, properly taking the disk's history into account.

An important fact seen in the first panel of Fig. 3.6 is that one single value of $\alpha$ is not able to fit all the observed bumps in the light curve. M. Ghoreyshi et al.'s results indicate that the $\alpha$ parameter of a Be star can be variable, at least in time. Another striking fact, related to the first, is that we see that the values of $\alpha$ during the build-up phase are often greater than the values of $\alpha$ during the dissipations. The only exception to that is the third major bump. We still don't have an explanation for this fact and will come back to it when we discuss our own results.

The second panel of Fig. 3.6 shows the mass flux at three different radii as a function of time. This panel is a complementary version of the two lower panels of Fig. 3.2. We

\footnotetext{
${ }^{2}$ His $\mathrm{PhD}$ thesis is on going, with a paper in the last steps of preparation.
} 


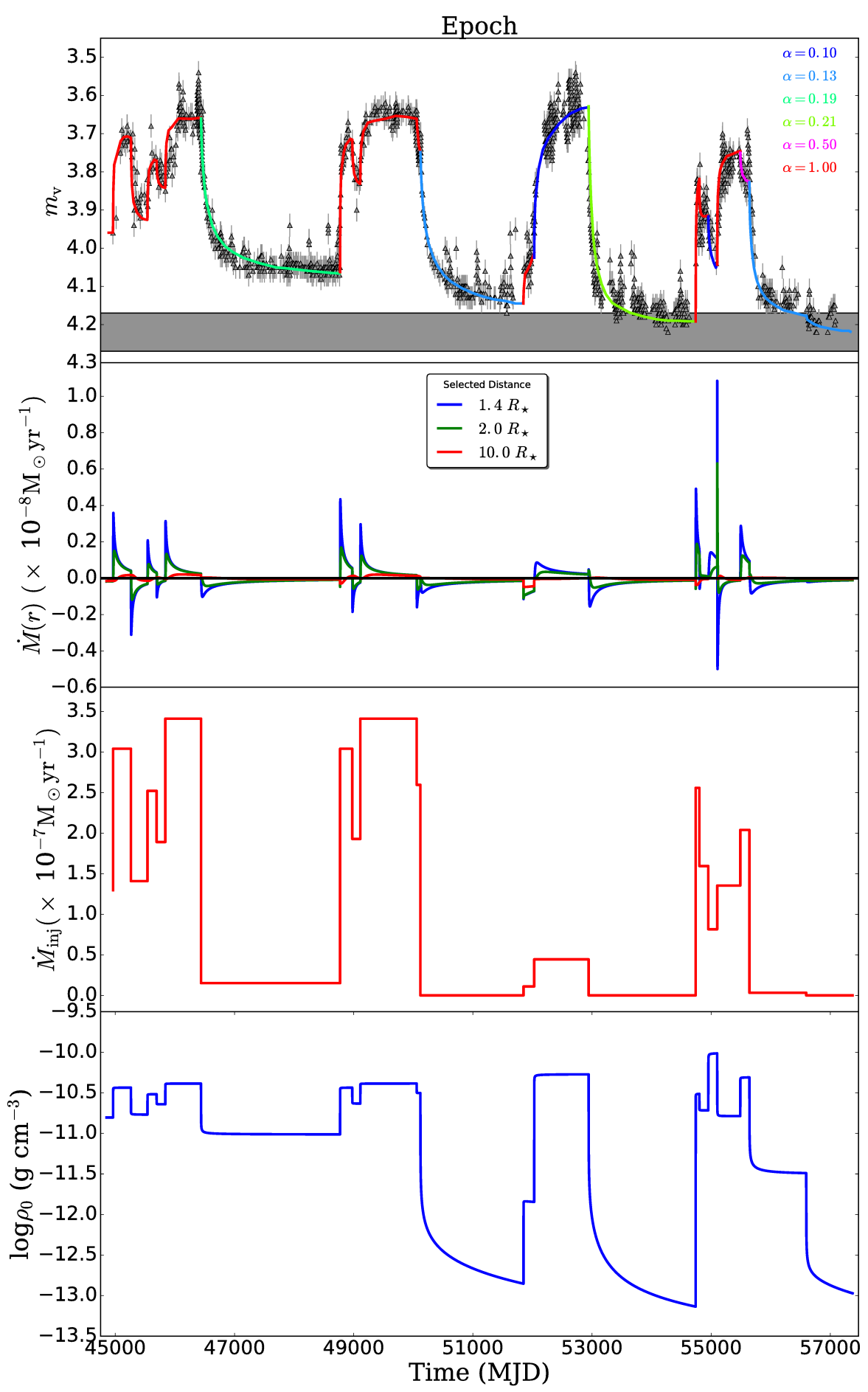

Figure 3.6: M. Ghoreyshi et al.'s current best results for the photometric modeling of the light curve of 28 CMa. First: A more than 33 years-long light curve of the $28 \mathrm{CMa}$ with the best fitted synthetic light curve generated by a dynamical model computed with SINGLEBE feeding the radiative transfer code HDUST. The different colors correspond to different values of $\alpha$ used in separated portions of the curve. Second: Mass flux at $\tilde{R}=1.4$ (blue), $\tilde{R}=2.0$ (green), $\tilde{R}=10.0$ (red), as a function of time. Third: Mass injection rate $\left(\dot{M}_{\mathrm{inj}}\right.$, with $\left.\tilde{R}_{\mathrm{inj}}=1017\right)$ into the disk as a function of time. Forth: mass density at the radius of mass injection, $\rho_{\text {inj }}=\Sigma_{\text {inj }} /(2 \pi)^{\frac{1}{2}} H$. 
see that, after the abrupt changes in $\dot{M}_{\text {inj }}$ (or $\Sigma_{0}$, in our work), the mass flux in the inner disk abruptly changes. The absolute value of the mass flux at radii nearer to the radius of mass injection always show bigger amplitudes in the variations after the changes of $\dot{M}_{\text {inj. }}$. At larger radii, the variations in the mass flux are smaller, meaning that the inner disk retains part of the mass flux, vary more intenselly and leave less mass to the outer regions. 
Chapter 4

\section{A model grid of disk formation and dissipation events}

God used beautiful mathematics in creating the world.

Paul Dirac, The Cosmic Code:

Quantum Physics As The Language

Of Nature (1982)

\subsection{The dynamical model grid}

For building a comprehensive grid of dynamical models that are solutions of Eq. 3.1.12, we make usage of the definitions of Chapter 3 that allow us to write the solution $\Sigma(\tilde{R}, \tilde{\tau})$ in terms of $\Sigma_{0}(\tilde{\tau})$ (see source term in Eq. 3.2.16) and the dimensionless time parameter $\tilde{\tau}$ (see Eq. 3.1.14).

As discussed in Sect. 3.3, the advantage of studying relatively isolated bumps like the ones exemplified in Fig. 3.1, which started after a clear inactive phase, is that there is no previous history of mass injection to be taken into account for the modeling, so that during build-up, the shape of the curve is controlled solely by $\Sigma_{0}(\tilde{\tau})$ and $\tilde{\tau}$, while at dissipation, an additional parameter, the previous disk build-up time, must be considered (see Sect 3.3). By using the time parameter (Eq. 3.1.14) instead of the physical time, our dynamical models are independent of the specific physical parameters $M, T_{\text {eff }}, R_{\text {eq }}$ and $\alpha(t)$ of the Be star under consideration (recall that these parameters - see Eq. 3.1.13 - define the timescale parameter $\tau(t)$, which in turn makes the connection between the physical time $t$ and the time parameter $\tilde{\tau}$.) Also, from the linearity of Eq. 3.1.12, it follows that multiplying $\Sigma_{0}(\tilde{\tau})$ by some constant results in the solution $\Sigma(\tilde{R}, \tilde{\tau})$ multiplied by the same constant. Consequently, the dynamical models need to be run for just one arbitrary value of $\Sigma_{0}$.

For our grid of dynamical models of bumps and dips of Be stars, we assume that the stars start diskless. Then, at time $\tilde{\tau}=0$, mass injection into the disk begins, with some asymptotic surface density $\left(\Sigma_{0}>0\right)$, which lasts until $\tilde{\tau}=\tilde{\tau}_{\text {bu }}$. This last quantity is the 
scaled build-up time, related to the above mentioned build-up time, but scaled by the timescale parameter. After that $\left(\tilde{\tau}>\tilde{\tau}_{\text {bu }}\right)$, mass injection no longer occurs $\left(\Sigma_{0}=0\right)$ and the disk dissipates.

We generated 11 dynamical models following the description given in the last paragraph, for 11 different values of $\tilde{\tau}_{\mathrm{bu}}$, listed as the numerical values in the forth column of Table 4.1. In addition to these dynamical models, we also generated 1 dynamical model composed only of a very long build-up phase. This model that is not of a bump (or dip) is represented in the forth column of Table 4.1 as the $\tilde{\tau}_{\mathrm{bu}}=$ bu model. Finally, we also generated 1 dynamical model that did not start from a diskless state, but rather from the steady-state (see Eq. 3.2.13) configuration for the given $\Sigma_{0}$. This model corresponds to a bump that dissipates after an infinitely long build-up phase. In the forth column of Table 4.1, this model is the $\tilde{\tau}_{\mathrm{bu}}=\infty$ model.

For the models with $\tilde{\tau}_{\mathrm{bu}} \neq \mathrm{bu}$, it is important to remember that their build-up phases are all indistinguishable from sections of the very long build-up model described by $\tilde{\tau}_{\mathrm{bu}}=$ bu. Therefore, we only need to model specific instants of the dynamical models with $\tilde{\tau}_{\mathrm{bu}}=\mathrm{bu}$ to have the photometric description of the build-up phases of all the other models. We hence, only have to model the dissipation phases of the other models. These dissipation phases differ from one another because of the mass reservoir effect, outlined in Sect. 3.3.

Since the timescale parameter (Eq. 3.1.13) is roughly given by $\sim(100-200) / \alpha$ days for early Be stars with $\alpha \lesssim 1$, our chosen values of $\tilde{\tau}_{\mathrm{bu}}$ correspond to real build-up times of at least 15 days, which brackets the observed build-up times of the sample described below (Sect. 5.2, Table 5.1).

\subsection{Radiative transfer models}

Having selected a set of suitable hydrodynamic bump models, the next step is to produce photometric light curves of these models.

In this work, at selected time parameters $\tilde{\tau}$, a particular dynamical model, which is a function $\Sigma(\tilde{R}, \tilde{\tau})$ is input to the Monte Carlo 3-D radiative transfer code HDUST (Carciofi et al., 2004; Carciofi and Bjorkman, 2006, 2008) which calculates the emergent spectrum of the star+disk system.

To convert between surface density and mass density, it is assumed that the disk is vertically supported by hydrostatic pressure (see Eq. 2.2.14), in which case the vertical density profile is approximately a Gaussian, and

$$
\rho(\tilde{R}, z, \tilde{\tau})=\frac{\Sigma(\tilde{R}, \tilde{\tau})}{\sqrt{2 \pi} H} e^{-\frac{z^{2}}{2 H^{2}}}
$$

where

$$
\frac{H}{R_{\text {eq }}}=\left(\frac{c_{s}}{v_{\text {orb }}}\right) \tilde{R}^{\frac{3}{2}}
$$


is the scale height.

Before making further specifications in our models of bumps (and dips), let us briefly introduce the radiative transfer code HDUST, used in this work to generate the photometric observables associated with our dynamical models of bumps.

\subsubsection{The Monte Carlo Radiative Transfer code HDUST}

C.P. Dullemond, in his lecture notes ${ }^{1}$, states: "When you hear that radiative transfer is a very challenging topic, the reason is that in many cases we do not know the values of $j_{\nu}$ and/or $k_{\nu}$ in advance. The radiation field $I_{\nu}$ that we wish to compute can affect the medium in such a way as to modify $j_{\nu}$ and $k_{\nu}$. We are then faced with a "chicken or egg" effect: to compute $I_{\nu}$ we need to know $j_{\nu}$ and $k_{\nu}$, and to compute $j_{\nu}$ and $k_{\nu}$ we need to know $I_{\nu} . "$

HDUST is a Monte Carlo code that solves simultaneously the radiative transfer, radiative equilibrium and statistical equilibrium equations for a physical system composed of sources of radiation and an arbitrary distribution of gas (hydrogen) and dust (Carciofi et al., 2004; Carciofi and Bjorkman, 2006, 2008). The gas and dust compose the "interaction region". This interaction region, which is the circumstellar envelope of a Be star for our purposes, is divided into several small cells where the physical properties are assumed to be constant (number of particles, kinetic temperature, velocity, velocity gradient and level populations). Consequently, the emissivity $j_{\nu}$ and the opacity $k_{\nu}$ are also constants within each cell.

The radiative transfer and radiative equilibrium problems and the statistical equilibrium problem are solved in an iterative alternating fashion. The code alternates from "global" to "local" at each iteration. The "global problem" is the Monte Carlo solution of the radiative transfer problem, where the radiation field is simulated. The "local problem" is the updating of the states of each cell to new states that are more in equilibrium with the radiation field just simulated.

For each iteration, in the radiative transfer part of the problem, the total luminosity of the physical system is divided into $N$ "photon packets" $(\mathrm{PP})^{2}$. These $N$ PPs will be emitted from points from the stellar surface and from the cells, and they will be propagated until they escape the envelope. During the propagation of the PPs, when one crosses the surface of a cell and is absorbed within that cell, the interaction with the gas is tracked by sampling (counting) the number of radiative transitions (e.g., bound-bound, bound-free) caused by the absorption. Then, in the rest frame of the fluid, a PP is reemitted at the same location and with the same total energy (but with a different frequency), as that of the incoming PP, in order to ensure radiative equilibrium $\left(\nabla \cdot \boldsymbol{F}_{\nu}=0\right)$ in the rest frame of the fluid.

\footnotetext{
${ }^{1}$ http://www.ita.uni-heidelberg.de/ dullemond/lectures/radtrans_2012/

${ }^{2}$ Actually, they are "luminosity packets".
} 


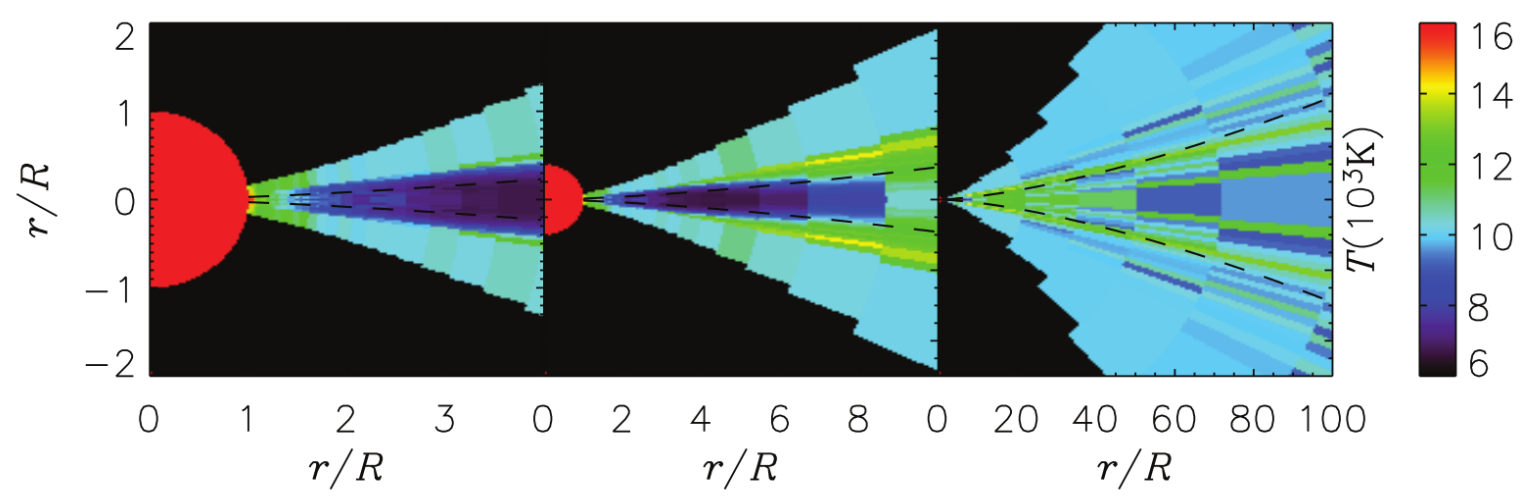

Figure 4.1: Temperatures in the cells and on the stellar surface in a simulation of near-Keplerian disk of a Be star made by HDUST. (Credits: Carciofi and Bjorkman, 2006)

At the end of the radiative transfer part, the code has sampled all relevant radiative rates for all cells. Then, for each cell, a linearized scheme produces a new updated state to be used in the next iteration.

After a sufficient number of iterations, both the global and local problems converge to a self-consistent system in which the microphysics within each cell is in equilibrium with the radiation field of the whole system. In the last iteration, in particular, when the PPs escape the envelope, their luminosities are binned in into solid angle bins (corresponding to the observer's viewing angles) and frequency bins (which will compose the synthetic SEDs).

Fig. 4.1, from Carciofi and Bjorkman (2006), shows the temperatures for several HDUST cells, defined in spherical coordinates, in the simulation of a Be star with its near-Keplerian circumstellar disk. The stellar surface assumed in this figure, in particular, was a spherical one.

\subsubsection{Radiative transfer models}

The radiative transfer part of the problem requires a stellar model, which will be the main source of radiation. The stellar model defines the physical parameters $M, R_{\text {eq }}$ and $T_{\text {eff }}$, which were left unspecified in the dynamical model grid. In addition, another parameter must be specified: the inclination, $i$ (where $i=0$ means pole-on orientation).

As we know, one important feature of the central stars of Be stars is that they are fast rotators. Fast rotation causes the star to be oblate, with hotter poles and colder equatorial regions. The rotation rate is specified by the ratio of the rotation velocity at the equator to the Keplerian velocity at the equator, $W=v_{\text {eq }} / v_{\text {orb }}$. The ratio between the equatorial radius to the polar radius is given by $R_{\mathrm{eq}} / R_{\text {pole }}=1+W^{2} / 2$ for a Roche-shaped star. All these parameters evolve in time as a consequence of stellar evolution, and Be stars can be found in luminosity classes from V to III (Rivinius et al., 2013). 


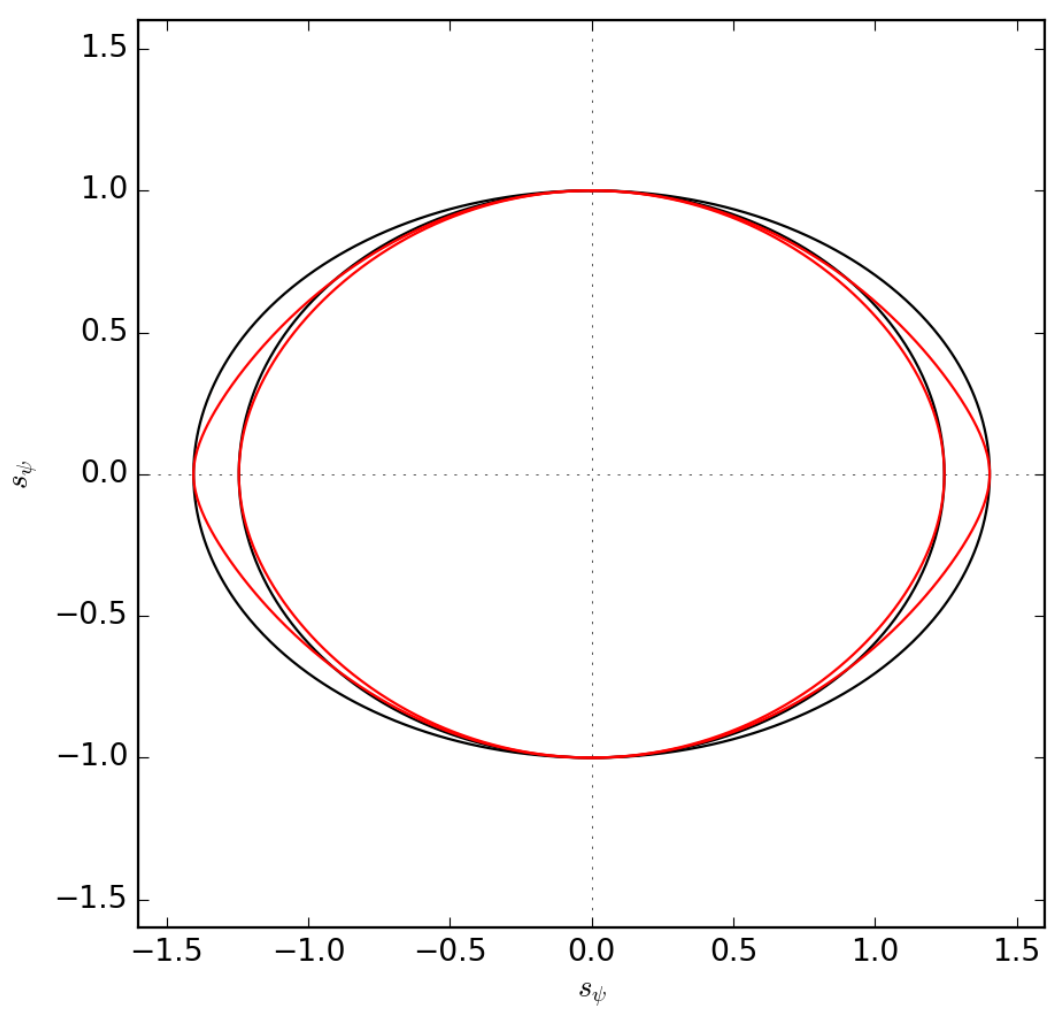

Figure 4.2: HDUST's current ellipsoid stars (black) versus Roche-shaped stars with $W=0.7$ and $W=0.9$ (red).

As the sources of the physical parameters of our rotating stars, we used the evolutionary tracks generated by the Geneva stellar evolution code (Georgy et al., 2013) . Therefore, the physical parameters of the central stars that enter as input into HDUST (e.g., $R_{\text {eq }}$ and $\left.T_{\text {eff }}\right)$ are obtained by interpolating the tracks, in metallicity $Z$, stellar mass $M$, rotation parameter $W$ and the age during the main sequence $t / t_{\mathrm{MS}}$, where $t_{\mathrm{MS}}$ is the timespan of the main sequence for each stellar model.

The current version of HDUST allows for a spheroidal oblate star, with the latitudedependent surface temperature being given by (Carciofi et al., 2008)

$$
T_{\text {surf }} \propto g_{\text {eff }}^{\beta} .
$$

In Fig. 4.2, we show the ellipsoidal shape compared to the shape given by Roche equipotentials for $W=0.7$ and $W=0.9$, roughly corresponding to the $\pm 1 \sigma$ limits of the distribution of values of $W$ for Galactic Be stars (Rivinius et al., 2006).

The coefficient $\beta(W)$ in Eq. (4.2.3) is calculated by fitting a straight line to the curve given by $\ln T_{\text {surf }}$ versus $\ln g_{\text {eff }}$ given by the flux theory of Espinosa Lara and Rieutord (2011) (see the lower-right panel of Fig. 2.2). For the disk scale height (Eq. 4.2.2), we assume

\footnotetext{
${ }^{3}$ Their tracks are available online at https://obswww. unige.ch/Recherche/evol/-Database-
} 


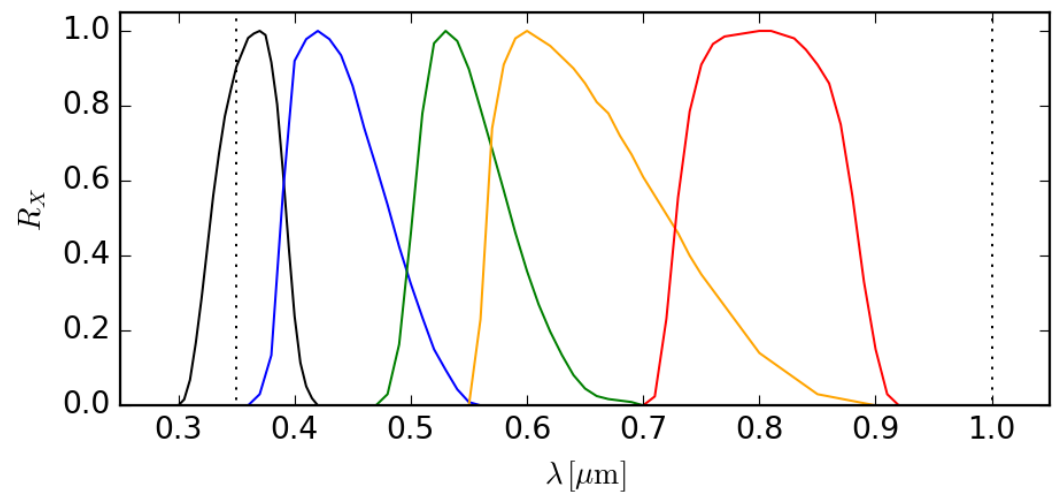

Figure 4.3: UBVRI Johnson-Cousins passbands from Bessell (1990), in black, blue, green, orange and red, respectively. The vertical dotted lines mark the limits in frequency of the SEDs generated by HDUST in this work.

an isothermal disk with $T_{\text {disk }}=0.6 T_{\text {eff }}$, where $T_{\text {eff }}$ is the mean effective temperature of the star, defined by $T_{\text {eff }}=\left(L_{*} / \sigma S_{*}\right)^{1 / 4}$, with $S_{*}$ being the surface area of the star (here calculated as the surface of the Roche equipotential).

For CCD observations (photon counting devices), a photometric observable is proportional to the number of photons arriving per units of time and area, given by

$$
\int_{0}^{\infty}(\lambda / h c) F_{\lambda}(\lambda) R_{X}(\lambda) \mathrm{d} \lambda
$$

where $F_{\lambda}(\lambda)$ is the flux density and $R_{X}(\lambda)$ is the passband of a given standard filter $X$. The function $F_{\lambda}$ is one of the main outputs of the code HDUST.

The Johnson Magnitude System is a special case of a "Vega magnitude system". Therefore, measurements in any filter $X$ are compared to measurements of Vega in that same filter, in the following way

$$
M_{X}=-\frac{5}{2} \log \left(\frac{\left\langle\lambda F_{\lambda}\right\rangle_{X}}{\left\langle\lambda F_{\lambda}^{\mathrm{Vega}}\right\rangle_{X}}\right)+M_{X}^{\mathrm{Vega}}
$$

where the magnitude of Vega is defined to be $M_{X}^{\mathrm{Vega}}=0.03$ in every filter and

$$
\left\langle\lambda F_{\lambda}\right\rangle_{X}=\frac{\int_{0}^{\infty}\left(\lambda F_{\lambda}\right) R_{X}(\lambda) \mathrm{d} \lambda}{\int_{0}^{\infty} R_{X}(\lambda) \mathrm{d} \lambda} .
$$

In order to generate synthetic magnitudes, we used the standard BVRI JohnsonCousins passbands from Bessell (1990). They are sketched as the solid curves in Fig. 4.2. To calibrate our system to cgs units, we used the Vega flux from ATLAS9 model of Vega described in Castelli and Kurucz (1994), and we normalized HDUST's flux as if the objects were at $10 p c$ of distance. 
Table 4.1 - Parameters of the grid of photometric models of bumps

\begin{tabular}{rrrr}
\hline \hline Star & $i[\mathrm{deg}]$ & $\Sigma_{0}\left[\mathrm{~g} \mathrm{~cm}^{-2}\right]$ & $\tilde{\tau}_{\text {bu }}$ \\
\hline Star 1 & 00.0 & 0.30 & $\mathrm{bu}$ \\
Star 2 & 21.8 & 0.41 & 00.15 \\
Star 3 & 31.0 & 0.56 & 00.45 \\
& 38.2 & 0.75 & 00.75 \\
& 44.4 & 1.01 & 01.50 \\
& 50.0 & 1.37 & 02.25 \\
& 55.2 & 1.85 & 03.00 \\
& 60.0 & 2.50 & 04.50 \\
& 64.6 & & 06.00 \\
& 69.1 & & 09.00 \\
& 73.4 & & 15.00 \\
& 77.6 & & 30.00 \\
& 81.8 & & $\infty$ \\
& 85.9 & & \\
& 90.0 & & \\
\hline
\end{tabular}

Table 4.2 - Parameters of the stellar models of Table 4.1

\begin{tabular}{r|rrrr|r}
\hline \hline Star & $Z$ & $M / M_{\odot}$ & $W$ & $t / t_{\mathrm{MS}}$ & $\alpha \tau[\mathrm{d}]$ \\
\hline Star 1 & 0.002 & 7 & 0.81 & 0.5 & 125.3 \\
Star 2 & 0.002 & 11 & 0.81 & 0.5 & 138.8 \\
Star 3 & 0.002 & 15 & 0.81 & 0.5 & 160.4 \\
\hline
\end{tabular}

The SEDs generated by HDUST were chosen, in this work, to be given in the (visual) frequency domain $0.35-1 \mu \mathrm{m}$. This limit is shown in Fig. 4.3, covering the limits of the four $B V R I$ passbands.

A grid of model light curves was computed using the $13 \times 8$ dynamical models described in Sect. 4.1 (third and forth columns of Table 4.1). For each of these disk models, 17 radiative transfer models were calculated with HDUST at different instants of time parameters (not shown in the table) $)^{4}$ and 15 equaly-spaced values of $\cos i$ (second column). This whole process was done for 3 different stellar models ("Star 1", "Star 2" and "Star 3", first column of Table 4.1). Details of the stellar models are given in Table 4.2. They

\footnotetext{
${ }^{4}$ The time parameters were: $0,0.02,0.05,0.09,0.15,0.21,0.3,0.6,0.9,1.5,2.1,3,6,9,15,30, \infty$. For the long build-up light curves $\left(\tilde{\tau}_{\mathrm{bu}}=\mathrm{bu}\right)$, this list of values are for $\tilde{\tau}$. For the other cases, the list of values is for $\tilde{\tau}-\tilde{\tau}_{\mathrm{bu}}$.
} 
Table 4.3 - Parameters of the grid of photometric models of diskless stars

\begin{tabular}{rrrr|r}
\hline \hline$Z$ & $M / M_{\odot}$ & $W$ & $t / t_{\mathrm{MS}}$ & $i[\mathrm{deg}]$ \\
\hline 0.002 & 2.50 & 0.600 & 0.00 & 00.0 \\
& 3.68 & 0.699 & 0.25 & 27.3 \\
& 4.85 & 0.770 & 0.50 & 38.9 \\
& 6.00 & 0.833 & 0.75 & 48.2 \\
& 7.15 & 0.901 & 1.00 & 56.3 \\
& 8.29 & 0.990 & & 63.6 \\
9.42 & & & 70.5 \\
10.54 & & & 77.2 \\
11.66 & & & 83.6 \\
12.78 & & & 90.0 \\
13.89 & & & \\
15.00 & & & \\
\hline
\end{tabular}

were chosen to represent early B-type stars from the $\operatorname{SMC}(Z=0.002)$, in the middle of their life in the main sequence, with the rotation parameter given by the mean value obtained for Be stars ( $W=0.81$, Rivinius et al., 2006). In the sixth column of Table 4.2, we present the values of $\alpha \tau$ (Eq. 3.1.13) for the disks of these stars (with the assumption that $T_{\text {disk }}=0.6 T_{\text {eff }}$.

In short, a single light curve is specified by taking one element of each column of Table 4.1. The end result was a grid of $3 \times 14 \times 8 \times 13=4368$ light curves, for each of the $B V R I$ bands. A study of these light curves will be presented in Sect. 4.3 below.

We also generated a grid of inactive (diskless) stellar models. Because these models can be computed much faster than the bump models, we were able to cover a much finer grid of stellar parameters (Table 4.3). The grid is composed by models for 12 different masses (second column), 6 different rotation rates (third column), 5 equally spaced values for the age in the main sequence (forth column) and 10 equally spaced values of $\cos i$ (fifth column), resulting in a total of $12 \times 6 \times 5 \times 10=3600$ photometric models for each of the $B V R I$ bands.

In the left panel of Fig. 4.4, we show a color-magnitude diagram (CMD) in the $V$ and $I$ bands of the stellar models of Table 4.3. This figure will be used in this work to represent all the possible main sequence central stars of the Be star system. The mass of the star increases with the size of its marker. The 5 different stellar ages are seen as the groups of points move in the upper-right direction. This is better seen in the lower, less crowded, portion of the diagram. For each star, the effect changing $i$ from 90 deg to $0 \mathrm{deg}$ is to move in the upper-left direction. From this diagram, therefore, we see that 

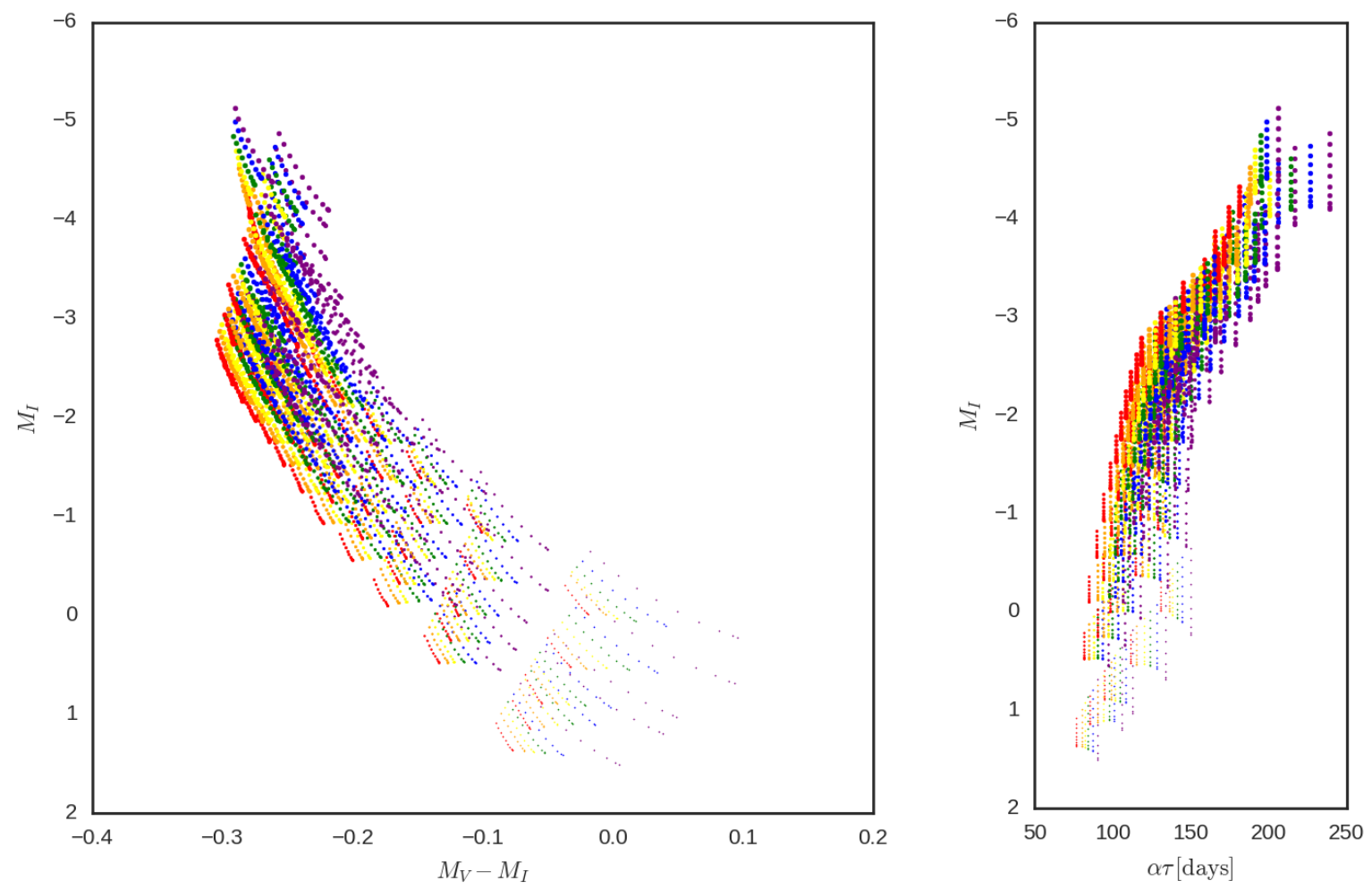

Figure 4.4: Left: CMD in bands $V$ and $I$ of the diskless stars of Table 4.3. The colors red, orange, yellow, green, blue and purple correspond to the 6 values of $W$ in increasing order. The mass of the star increases with the size of its marker. The 5 different stellar ages are seen as the groups of points move in the upper-right direction. For each star, the effect of going from inclination angle $90 \mathrm{deg}$ to $0 \mathrm{deg}$ is to move in the upper-left direction. Right: diagram showing $M_{I}$ (the same vertical axis of the upper-left one) versus $\alpha \tau$ (in days) for the diskless stars.

for stars seen pole-on, the effect of increasing $W$ is to increase its apparent magnitude (owing to the polar brightening in the "von Zeipel effect", the flux is enhanced in the poles - see Sect. 2.3.14) and to make it a little redder (since the temperature decreases in the equatorial regions). For stars seen edge-on, there is little variation in the apparent magnitude, but the star becomes much redder, because of the decrease in the temperature in the equatorial region.

In the right panel of Fig. 4.4, we show the same models in a diagram of $M_{I}$ (the same vertical axis of the upper-left one) versus $\alpha \tau$ (in days). From this diagram, we see that the values of the disk parameter $\alpha \tau$ (Eq. 3.1.13, with the assumption that $T_{\text {disk }}=0.6 T_{\text {eff }}$ ) are roughly between 100 and 200 days for early Be stars.

\subsection{Empirical law}

In order to facilitate the comparison of the synthetic light curves (Sect. 4.2.2) with the observed ones (Sect. 5.2), we developed two empirical laws that match quite closely 
the synthetic light curves for build-up and dissipation. The usefulness of these formulae will become clear in the next section.

In our discussion of the features of the light curves, it is useful to separate them in three groups: pole-on light curves, of stars seen at small inclination angles ( $0 \leq i \lesssim 70 \mathrm{deg}$ ), which should statistically correspond to the majority of the observed light curves; edge-on light curves, of shell stars $(i \approx 90 \mathrm{deg})$; and, intermediate light curves, of stars seen at intermediate angles $(70 \lesssim i \lesssim 85 \mathrm{deg}$ - the extension of this intermediate region varies depending the photometric band under consideration and will be defined below). Pole-on light curves show an increase in apparent brightness, due to the additional flux coming from the disk. Conversely, edge-on light curves show a decrease in apparent brightness, due to obscuration of the star by the disk. The intermediate case shows the smallest variations in apparent brightness and frequently the light curve has a more complicated shape, as it is influenced by variable amounts of disk emission/absorption.

A computed light curve is a sequence of absolute magnitudes for a set of time parameters, in a given photometric band $X$, given by

$$
M_{X}(\tilde{\tau})=M_{X *}+\Delta X(\tilde{\tau})
$$

where $M_{X *}$ is the absolute magnitude of the inactive Be star at band $X$, and $\Delta X(\tilde{\tau})$ is the magnitude difference caused by the disk $(\Delta X(\tilde{\tau})$ can be either positive or negative). $M_{X *}$ can be estimated from the flux during the inactive phase (e.g., the purple points of Fig. 3.1).

A build-up light curve (for $\tilde{\tau}-\tilde{\tau}_{\mathrm{bu}}<0$ ) is denoted by $\Delta X_{\mathrm{bu}}(\tilde{\tau})$. Its limiting value, if the build-up phase were to have an infinite duration, is denoted by $\Delta X_{\mathrm{bu}}^{\infty}$ - the photometric excess of the disk when in steady-state. A dissipation light curve (for $\tilde{\tau}-\tilde{\tau}_{\mathrm{bu}} \geq 0$ ) is denoted by $\Delta X_{\mathrm{d}}(\tilde{\tau})$. Its value at the beginning of the dissipation $\left(\tilde{\tau}=\tilde{\tau}_{\mathrm{bu}}\right)$ is given by $\Delta X_{\mathrm{d}}^{0}$.

Since every dynamical model starts from a diskless state and asymptotically ends at a diskless state, it follows that, regardless of the viewing angle, for every build-up light curve the quantity $\Delta X_{\mathrm{bu}}(\tilde{\tau}) / \Delta X_{\mathrm{bu}}^{\infty}$ is a function that goes from 0 to 1 as $\tilde{\tau}$ goes from 0 to $\infty$, and for every dissipation light curve, the quantity $\Delta X_{\mathrm{d}}(\tilde{\tau}) / \Delta X_{\mathrm{d}}^{0}$ is a function that goes from 1 to 0 as $\tilde{\tau}-\tilde{\tau}_{\text {bu }}$ goes from 0 to $\infty$.

Figure 4.5 shows examples of $I$-band light curves from the grid (see Table 4.1). The dashed black curves correspond to $\Delta I_{\text {bu }} / \Delta I_{\text {bu }}^{\infty}$ versus $\tilde{\tau}$. The solid curves correspond to $\Delta I_{\mathrm{d}} / \Delta I_{\mathrm{d}}^{0}$ versus $\tilde{\tau}-\tilde{\tau}_{\mathrm{bu}}$ for four dissipating light curves with increasing scaled build-up times. Vertical colored straight lines (with the same color code as the one of Fig. 3.3) mark the times $\tilde{\tau}$ or $\tilde{\tau}-\tilde{\tau}_{\mathrm{bu}}=0,0.2,1$.

The light curves show that, at $\tilde{\tau} \approx 1$, the simulated bump has reached a significant fraction of its limiting value, and, at $\tilde{\tau}-\tilde{\tau}_{\text {bu }} \approx 1$, the bump has already fallen considerably from its previous magnitude before the beginning of the dissipation. 

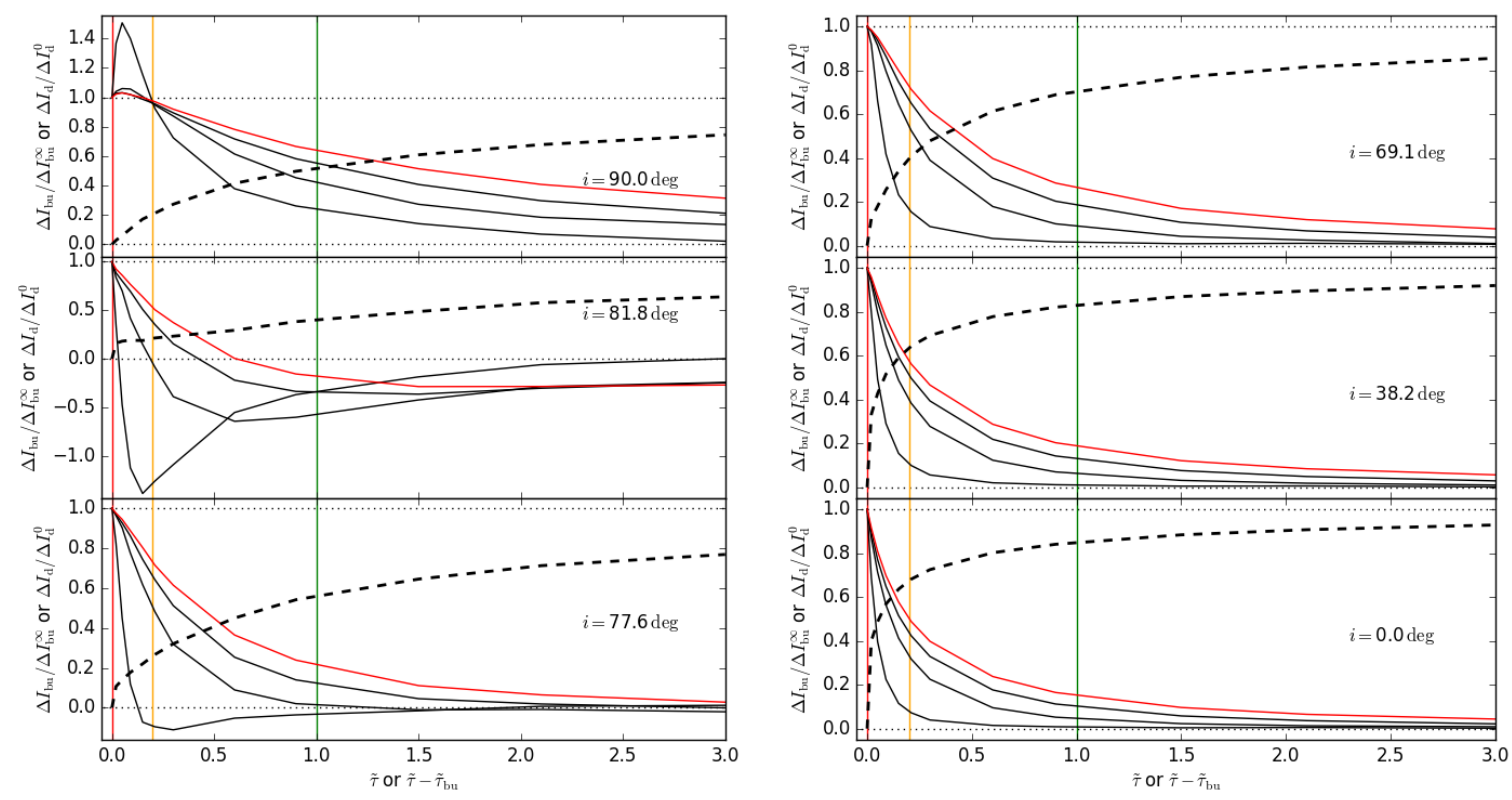

Figure 4.5: Examples of model $I$-band light curves, for several inclination angles, as indicated. The dashed black curves correspond to $\Delta I_{\mathrm{bu}} / \Delta I_{\mathrm{bu}}^{\infty}$ versus $\tilde{\tau}$, and the solid curves correspond to $\Delta I_{\mathrm{d}} / \Delta I_{\mathrm{d}}^{0}$ versus $\tilde{\tau}-\tilde{\tau}_{\text {bu }}$, for four dissipating light curves with scaled build-up times given by $\tilde{\tau}_{\text {bu }}=0.15,1.5,6$ (in black) and $\tilde{\tau}_{\mathrm{bu}}=30$ (in red). Vertical colored straight lines mark the instants $\tilde{\tau}$ or $\tilde{\tau}-\tilde{\tau}_{\mathrm{bu}}=0,0.2,1$. All light curves are specifically for Star 2 and disks with $\Sigma_{0}=1.37 \mathrm{~g} \mathrm{~cm}^{-2}$.

It can be seen in the panels that the dissipating curves with bigger scaled build-up times dissipate at slower rates. This is a consequence of the mass reservoir effect.

The light curves at near pole-on angles (the three panels from the right) show that, during the build-up phase, there is brightening due to the construction of the disk (and a usually negligible obscuration of the star by the disk), and during the dissipation phase, there is a decrease in brightening due to the disappearance of the disk. These light curves also show that, when viewed at angles closer to pole-on ( $\cos i$ closer to 1$)$, they build-up and dissipate faster. We will return to this effect below.

In the light curves at intermediate angles (middle-left and lower-left panels), both the effects of brightening from the disk and dimming from the obscuration of the star take place. The dissipations in the middle-left panel, for instance, show that, first, the gradual disappearance of the disk results in decrease in brightness. However, in time, the star becomes also less obscured by the disk and a small brightening happens.

For light curves at near edge-on angles (upper-left panel), the build-up light curves are dimmings due to obscuration of the star by the disk and the dissipation light curves are brightenings due to the disappearance of the disk and the disobscuration of the star.

The values of $\Delta I_{\mathrm{bu}}^{\infty}$ and $\Delta V_{\mathrm{bu}}^{\infty}$ for our grid are shown on the left and right panels of Fig. 4.6, respectively, plotted against $\cos i$. The values for the $B$ and $R$ bands show qualitatively similar patterns to the ones presented in this figure. Each panel shows the 

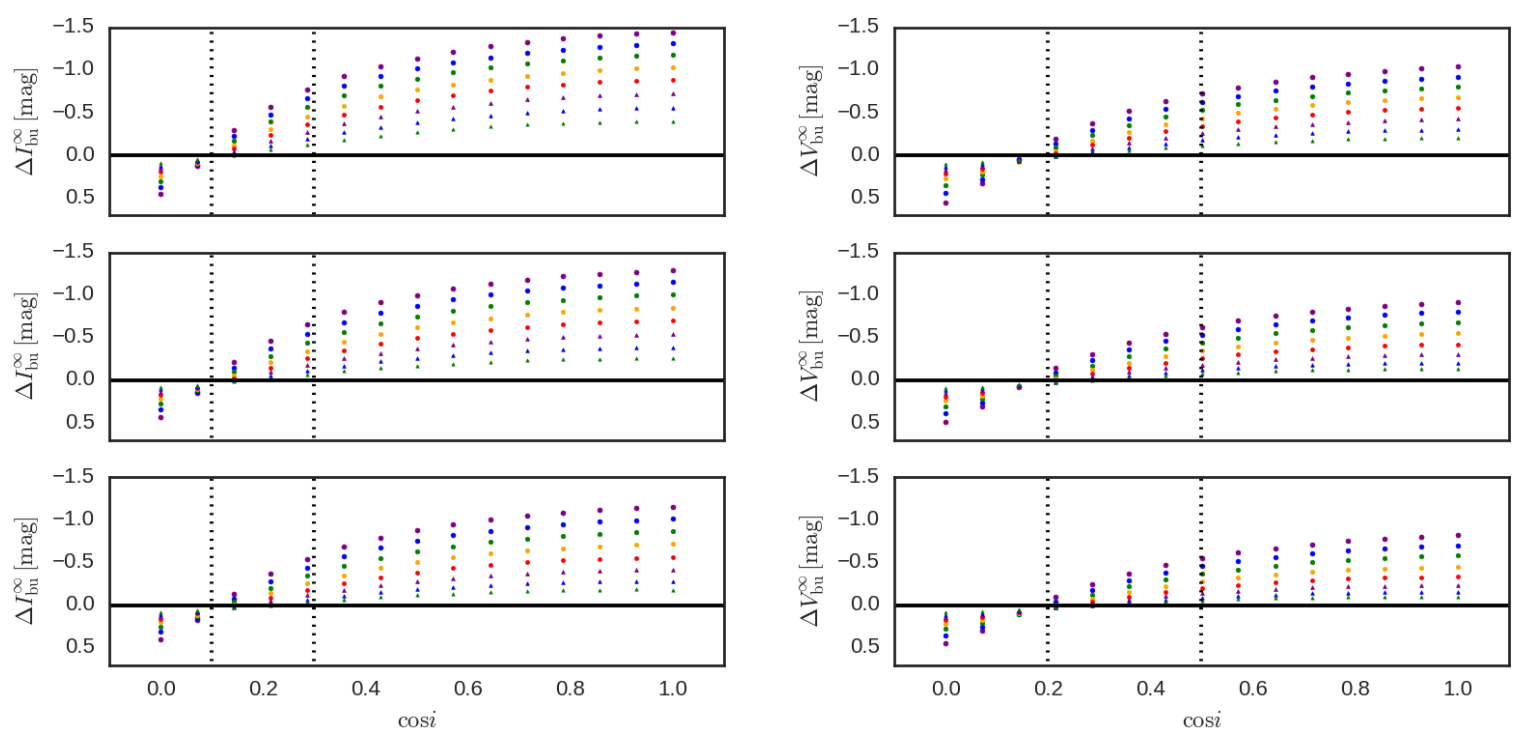

Figure 4.6: The values of $\Delta I_{\mathrm{bu}}^{\infty}$ versus $\cos i$ (left) and $\Delta V_{\mathrm{bu}}^{\infty}$ versus $\cos i$ (right) for our grid. In both panels, from top to bottom, the results are for Star 1, Star 2 and Star 3, respectively. Purple, blue, green, orange and red circles correspond to $\Sigma_{0}=2.50,1.85,1.37,1.01,0.75 \mathrm{~g} \mathrm{~cm}^{-2}$. Purple, blue and green diamonds correspond to $\Sigma_{0}=0.56,0.41,0.30 \mathrm{~g} \mathrm{~cm}^{-2}$. Vertical dotted lines define the region of intermediate angles for the $I$-band (left, $73 \lesssim i \lesssim 84 \mathrm{deg}$ ) and for the $V$-band (right, $60 \lesssim i \lesssim 78 \mathrm{deg}$ ).

results for a different star, and all 8 values of $\Sigma_{0}$ (Table 4.1) are represented in the figure by different colors and symbols. The curves monotonically increase with $\cos i$, starting with negative values at edge-on orientation and reaching a maximum for pole-on viewing. The angle for which $\Delta X_{\mathrm{bu}}^{\infty}=0$, where the disk excess emission is exactly matched by the absorption of photospheric light by the disk, depends both on the density scale (as shown in the figure) and (most importantly) on the band pass.

An analysis of our model grid allowed us to determine the ranges in inclination angle for which the light curve displays the intermediate behavior described above. They were determined by visual inspection of our model grid, as the angles for which the light curves present more complex shapes (see, e.g., the $I$-band light curves seen at $i=81.8 \mathrm{deg}$ and $i=77.6 \mathrm{deg}$ in Fig. 4.5). Their adopted values are 53-78 deg, 60-78 deg, 66-84 deg and 73-84 deg for the BVRI bands, respectively.

For pole-on orientations, the observed excess is given by $\Delta X \approx-2.5 \log \left(1+F_{\text {disk }} / F_{*}\right) \approx$ $-F_{\text {disk }} / F_{*}$. Vieira et al. (2015) studied the continuum emission from gaseous disks, and showed that it can be approximated by the sum of the flux coming from an optically thick inner part (the so-called pseudophotosphere) with the contribution from an optically thin outer part, i.e.,

$$
F_{\text {disk }} \propto \cos i F_{\text {thick }}+F_{\text {thin }} .
$$

If the contribution of the optically thin part of the disk were negligible and the stellar flux, $F_{*}$, did not depend on $\cos i$ (see Fig. 4.4), $\Delta X$ would be a linear function of $\cos i$. Clearly 

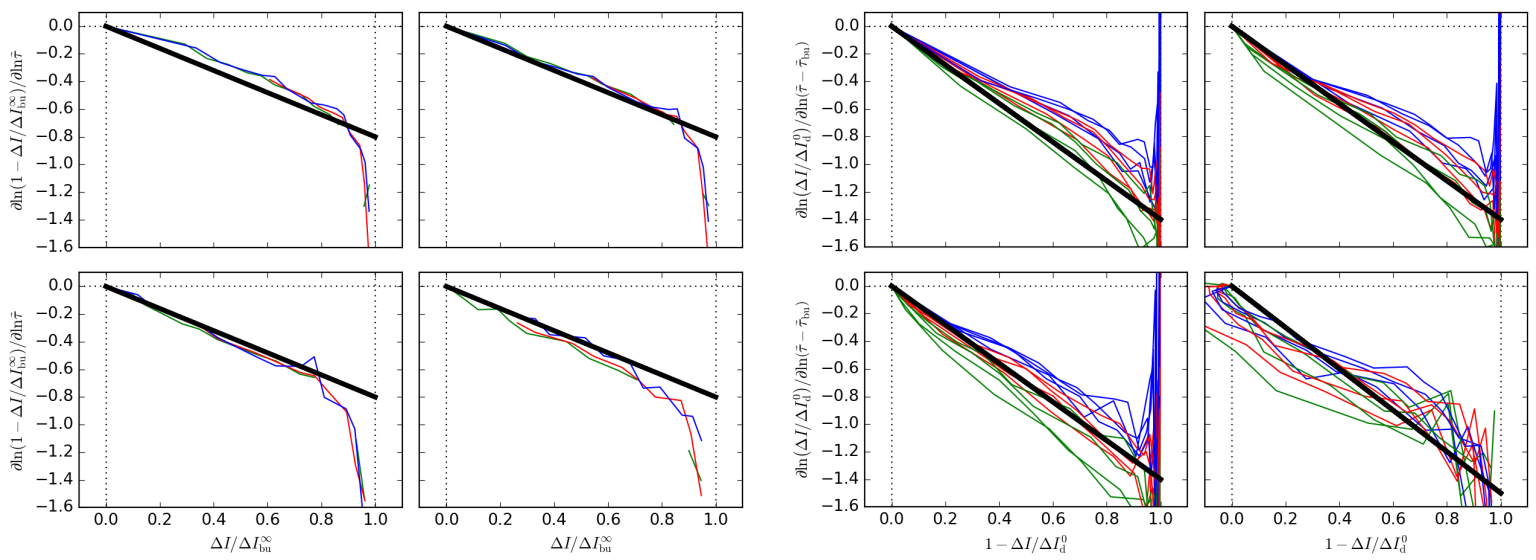

Figure 4.7: Examples of the $\log -\log$ derivative of $1-\Delta I_{\mathrm{bu}}(\tilde{\tau}) / \Delta I_{\mathrm{bu}}^{\infty}$ vs. $\quad \Delta I_{\mathrm{bu}}(\tilde{\tau}) / \Delta I_{\mathrm{bu}}^{\infty}$ (top four panels) and the $\log -\log$ derivative of $\Delta I_{\mathrm{d}} / \Delta I_{\mathrm{d}}^{0}$ vs. $1-\Delta I_{\mathrm{d}} / \Delta I_{\mathrm{d}}^{0}$, for values of $\tilde{\tau}_{\mathrm{bu}}$ equal to $0.45,1.5,6$ and 30 (bottom four panels). They correspond to light curves of Star 2, seen at $i=0 \operatorname{deg}$ (upper left), $i=44.42 \mathrm{deg}$ (upper right), $i=69.98 \mathrm{deg}$ (lower left), and $i=90 \mathrm{deg}$ (lower right). The green, red and blue curves correspond to $\Sigma_{0}$ equal to $1.37,0.75$ and $0.41 \mathrm{~g} \mathrm{~cm}^{-2}$. Black straight lines are the derived empirical laws.

this is not the case, and both the optically thin and thick parts of the disk contribute to observed behavior of $\Delta X$ vs. cos $i$. This pseudo-photosphere concept will be important to understand the growth and decay rates of the light curves, discussed below. Figure 4.6 also shows that the excesses increase a little when moving from a low- to a high-mass star, for disks with the same other features. This is a consequence of the fact that the stellar flux relative to the disk flux increases with the luminosity of the star. Finally, by comparing the left and right panels of Figure 4.6, we see that, for pole-on angles, the excesses increase with the wavelength, as we notice that the excesses on the left panel are bigger than the ones on the right panel. This doesn't happen, however, for near-edge-on angles, where both $\Delta I_{\mathrm{bu}}^{\infty}$ and $\Delta V_{\mathrm{bu}}^{\infty}$ have similar values.

Both the pole-on and edge-on light curves have functions $\Delta X_{\mathrm{bu}}(\tilde{\tau}) / \Delta X_{\mathrm{bu}}^{\infty}$ and $\Delta X_{\mathrm{d}}(\tilde{\tau}) / \Delta X_{\mathrm{d}}^{0}$ that are qualitatively similar to each other, as examplified by the upperleft and right panels of Fig. 4.5, suggesting that they could be approximated by simple and general formulas of $\tilde{\tau}$. This is illustrated in Fig. 4.7, where we compare the values of $\Delta I_{\mathrm{bu}}(\tilde{\tau}) / \Delta I_{\mathrm{bu}}^{\infty}$ and $\Delta I_{\mathrm{d}}(\tilde{\tau}) / \Delta I_{\mathrm{d}}^{0}$ (in the horizontal axis) with their log - log derivatives (in the vertical axis). As the panels exemplify, the curves are similar to each other in a wide range parameters $\left(\cos i, \Sigma_{0}\right.$ and $\left.\tilde{\tau}_{\text {bu }}\right)$, and they can be roughly approximated by straight lines (shown in black). Therefore, the build-up and dissipation light curves (for inclinations not in the intermediate region) should roughly obey the following differential equations:

$$
\frac{\partial \ln }{\partial \ln \tilde{\tau}}\left(1-\frac{\Delta X_{\mathrm{bu}}}{\Delta X_{\mathrm{bu}}^{\infty}}\right) \approx-\eta_{\mathrm{bu}} \frac{\Delta X_{\mathrm{bu}}}{\Delta X_{\mathrm{bu}}^{\infty}},
$$


and

$$
\frac{\partial \ln }{\partial \ln \left(\tilde{\tau}-\tilde{\tau}_{\mathrm{bu}}\right)} \frac{\Delta X_{\mathrm{d}}}{\Delta X_{\mathrm{d}}^{0}} \approx-\eta_{\mathrm{d}}\left(1-\frac{\Delta X_{\mathrm{d}}}{\Delta X_{\mathrm{d}}^{0}}\right) .
$$

Eq. (4.3.2) is an ordinary differential equation (ODE) with the form given by $\mathrm{d} \ln (1-$ $v) / \mathrm{d} \ln x=-\eta v$. By defining the variable $y=1-v$, the ODE becomes $\mathrm{d} \ln y / \mathrm{d} \ln x=$ $-\eta(1-y)$, which is the exactly the form of Eq. (4.3.3). Therefore, we just have to solve the ODE

$$
\frac{\mathrm{d} \ln y}{\mathrm{~d} \ln x}=-\eta(1-y),
$$

which is a special case a class of non-linear ODEs called "Bernoulli equations" 5 . The secret for solving Eq. (4.3.4) is defining $w=y^{-1}$. In that case, Eq. (4.3.4) becomes

$$
\frac{\mathrm{d} w}{w-1}=\eta \mathrm{d} \ln x
$$

which now is a linear ODE. Its solution is

$$
w-1=(\xi x)^{\eta},
$$

where $\xi$ is a constant of integration.

Now, returning to $y=w^{-1}$, we have the solution of Eq. (4.3.4), which corresponds to Eq. (4.3.3), and further making $v=y-1$, we have the solution to the ODE that corresponds to Eq. (4.3.2). Therefore, the solutions of Eqs. (4.3.2) and (4.3.3) are, respectively,

$$
\Delta X_{\mathrm{bu}}=\Delta X_{\mathrm{bu}}^{\infty}\left[1-\frac{1}{1+\left(\xi_{\mathrm{bu}} \tilde{\tau}\right)^{\eta_{\mathrm{bu}}}}\right]
$$

and

$$
\Delta X_{\mathrm{d}}=\Delta X_{\mathrm{d}}^{0}\left[\frac{1}{1+\left(\xi_{\mathrm{d}}\left(\tilde{\tau}-\tilde{\tau}_{\mathrm{bu}}\right)\right)^{\eta_{\mathrm{d}}}}\right] .
$$

The continuity condition given by the fact that a bump or dip has a build-up phase followed by a dissipation phase requires that

$$
\Delta X_{\mathrm{d}}^{0}=\Delta X_{\mathrm{bu}}^{\infty}\left[1-\frac{1}{1+\left(\xi_{\mathrm{bu}} \tilde{\tau}_{\mathrm{bu}}\right)^{\eta_{\mathrm{bu}}}}\right] .
$$

The parameters $\xi_{\text {bu }}$ and $\xi_{\mathrm{d}}$ are constants of integration whose values were determined by fitting Eqs. (4.3.7) and (4.3.8) to the computed light curves. The tables of the parameters $\Delta X_{\mathrm{bu}}^{\infty}$ and the fitted parameters $\xi_{\mathrm{bu}}$ and $\xi_{\mathrm{d}}$ are given in Appendix A, in Tables A.1, A.2, A.3 and A.4 for the BVRI bands, respectively. Examples of plots of the light curves given by Eqs. (4.3.7) and (4.3.8) compared with the synthetic light curves generated by our grid are shown in Appendix B. As briefly explained in Appendix A, the values of the exponents $\eta_{\mathrm{bu}}$ and $\eta_{\mathrm{d}}$ were empirically determined to best match the model light curves. Their values are dependent on the photometric band, and are listed in Table 4.4.

\footnotetext{
${ }^{5}$ http://eqworld.ipmnet.ru/en/solutions/ode/ode0104.pdf
} 
Table 4.4 - Values of $\eta$ 's.

\begin{tabular}{rr|rrrr}
\hline \hline & & $B$ & $V$ & $R$ & $I$ \\
\hline$\eta_{\text {bu }}$ & $($ edge-on) & 0.8 & 0.8 & 0.8 & 0.8 \\
& $($ pole-on) & 0.8 & 0.8 & 0.8 & 0.8 \\
\hline$\eta_{\mathrm{d}}$ & $($ edge-on) & 1.5 & 1.5 & 1.5 & 1.5 \\
& $($ pole-on) & 1.1 & 1.2 & 1.3 & 1.4 \\
\hline
\end{tabular}
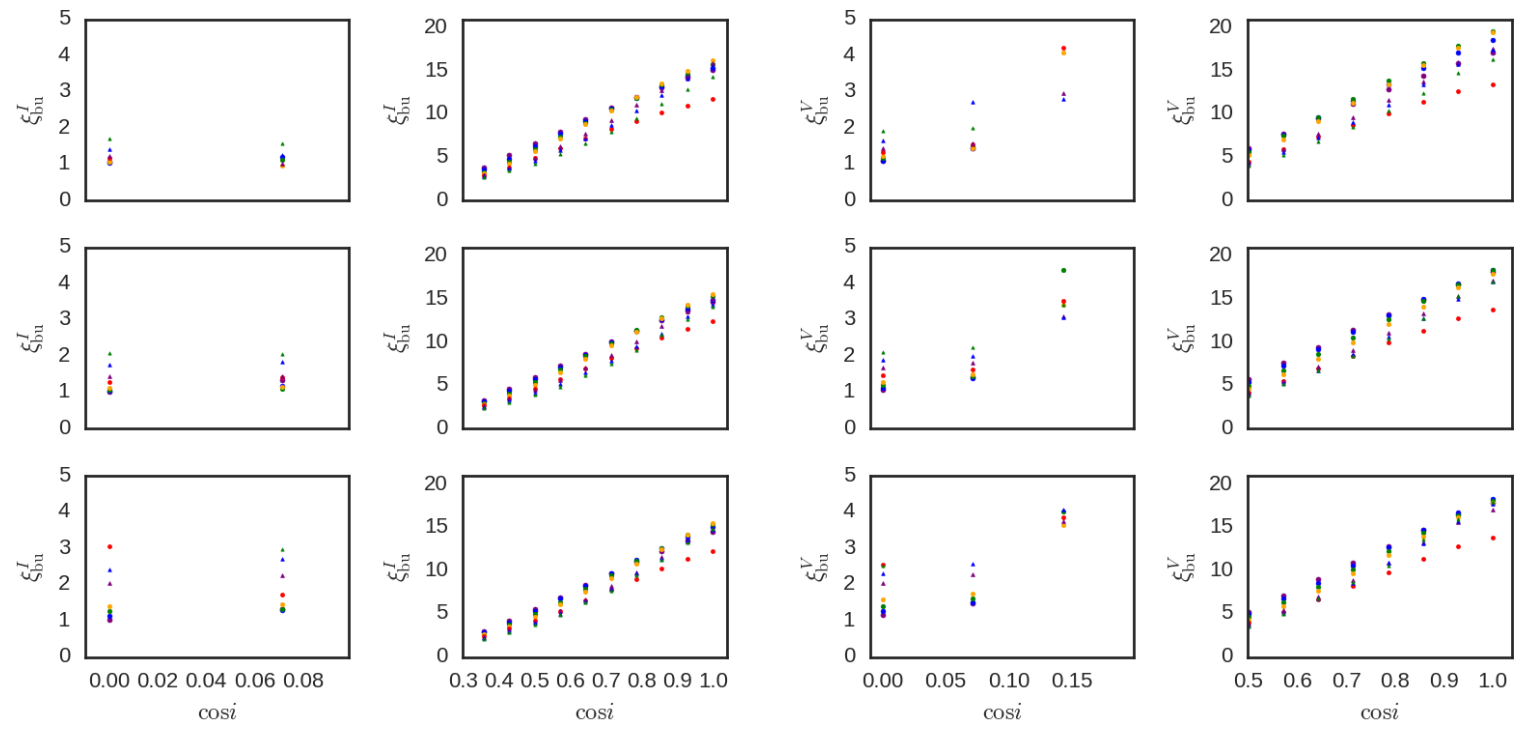

Figure 4.8: The $I$ - and $V$-band values of $\xi_{\text {bu }}$ for our grid vs. cos $i$. First column: Edge-on models for the I-band. Second column: Pole-on models for the I-band. Third column: Edge-on models for the $V$-band. Forth column: Pole-on models for the $V$-band. From top to bottom, the results for Star 1, 2, and 3, respectively. The markers are the same as in Fig. 4.6.

Representative $I$ - and $V$-band values of $\xi_{\text {bu }}$ and $\xi_{\mathrm{d}}$ for our grid are shown in Figs. 4.8, 4.9 and 4.10 (the values of $\xi_{\mathrm{bu}}$ and $\xi_{\mathrm{d}}$ for the $B R$ bands show qualitatively similar patterns to the ones presented in these figures). Each row shows the results for a different star. The panels of the first and third columns are for edge-on models in the $I$ and $V$ bands, respectively. The panels of the second and forth columns are for pole-on models in the $I$ and $V$ bands, respectively.

The values of $\xi$ are directly related to the rate of photometric variations: the smaller the $\xi$ the slower the variation (see Eqs. 4.3.7 and 4.3.8). Figure 4.8 exemplify the strong variation of $\xi_{\text {bu }}$ with $i$, for the pole-on case (see second and forth columns). This is probably due to the fact that, in the build-up process, the density grows inside-out (see lower panels of Fig. 3.3), which means that the optically thick part of the disk, which is an expanding pseudo-photosphere, forms first, and the optically thin part of the disk takes longer to be built. Since the optical excess of the disk is given by $\Delta X \propto \cos i F_{\text {thick }}+$ 

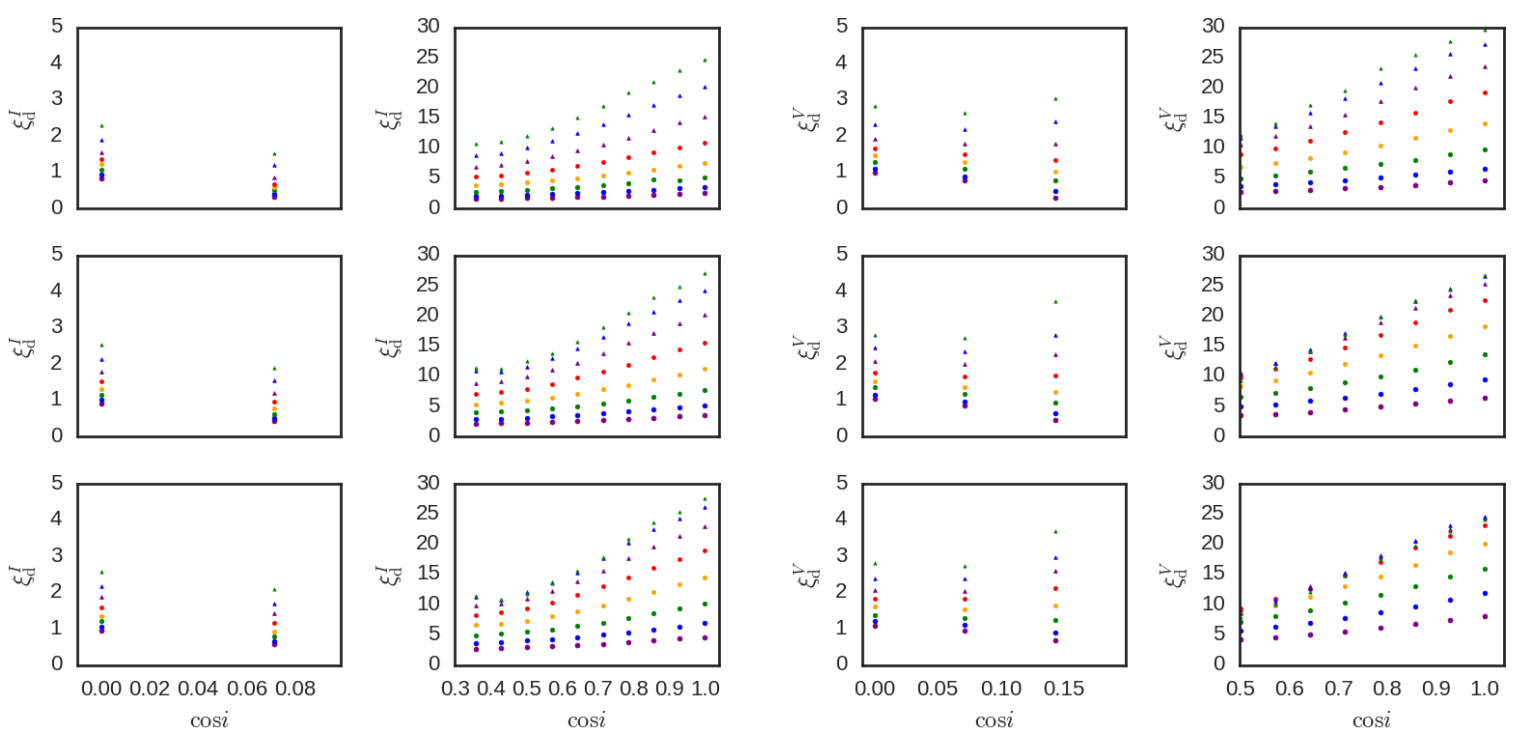

Figure 4.9: Selected $I$ and $V$-band values of $\xi_{\mathrm{d}}$ for our grid vs. $\cos i$. The scaled build-up time was fixed to $\tilde{\tau}_{\mathrm{bu}}=2.25$. First column: Edge-on models for the I-band. Second column: Pole-on models for the $I$-band. Third column: Edge-on models for the $V$-band. Forth column: Pole-on models for the $V$-band. From top to bottom, the results for Star 1, 2, and 3, respectively. The markers are the same as in Fig. 4.6.
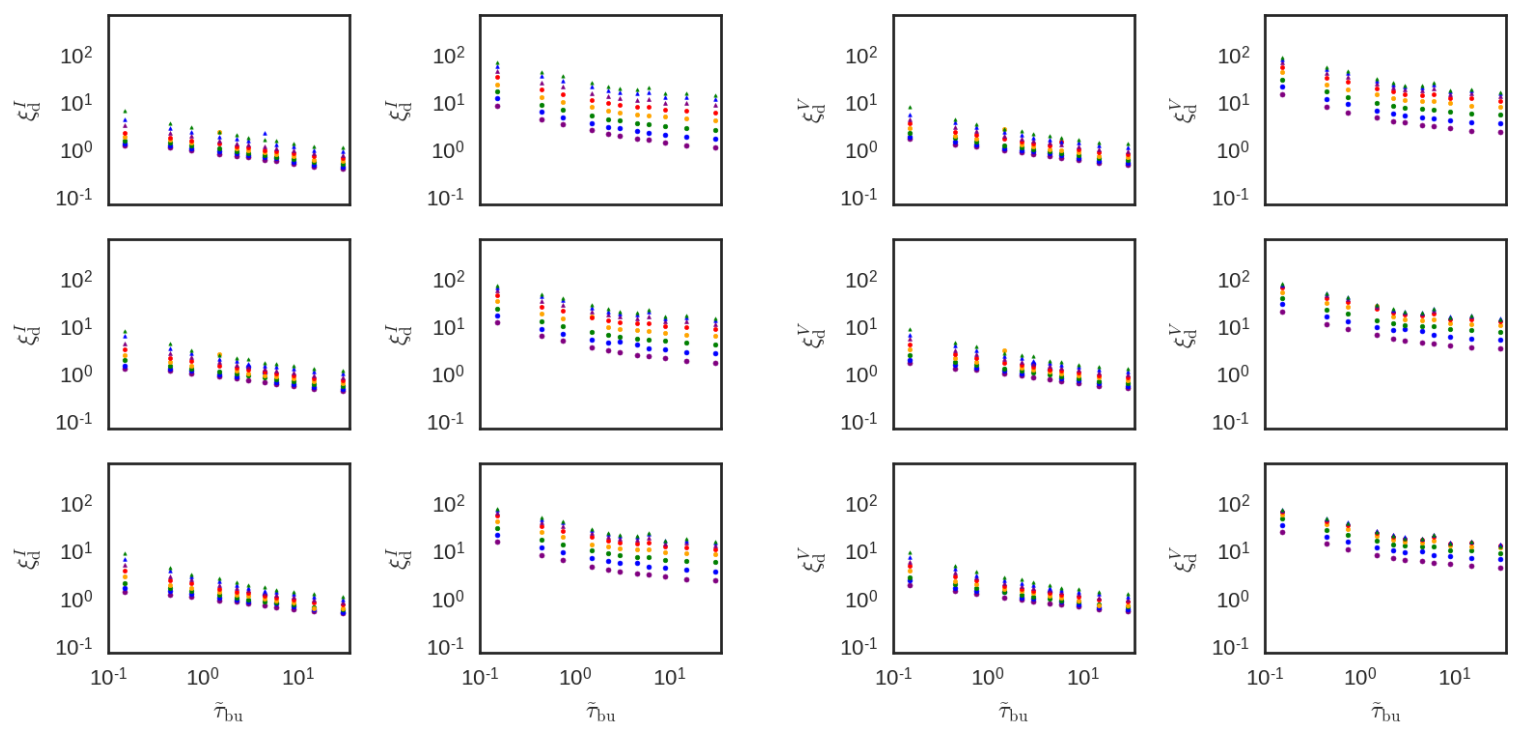

Figure 4.10: Selected $I$ and $V$-band values of $\xi_{\mathrm{d}}$ for our grid vs. $\tilde{\tau}_{\mathrm{bu}}$. First column: Edge-on models for the $I$-band with $i=90 \mathrm{deg}$. Second column: Pole-on models for the $I$-band with $i=0 \mathrm{deg}$. Third column: Edge-on models for the $V$-band with $i=90 \mathrm{deg}$. Forth column: Pole-on models for the $V$-band with $i=0 \mathrm{deg}$. From top to bottom, the results for Star 1, 2, and 3, respectively. The markers are the same as in Fig. 4.6.

$F_{\text {thin }}$, it follows that, as we move from pole-on to edge-on angles, only the optically thick contribution (proportional to $\cos i$ ) varies. As a consequence, the observed rate of increase in flux moves from being more to less optically thick dominated. 
In the dissipation process, the density rapidly adjusts to a self-similar dissipation pattern in the inner disk (see lower panels of Fig. 3.3). Therefore, the flux from the disk is the result of the decrease and disappearance of the optically thick region - transformed into an optically thin region - and the diminishing of the whole optically thin region. The pole-on values of $\xi_{\mathrm{d}}$ (second and forth panels of Fig. 4.9) are affected by $\cos i$ to a lesser extent, when compared to the values of $\xi_{\mathrm{bu}}$. In the dissipation process, by the same reasoning applied to the build-up process, since the optically thick emission is attenuated by the effect of $\cos i$, its disappearance has a reduced effect for more inclined disks and, therefore, the disk should apparently disappear at a slower rate.

The values of $\xi_{\mathrm{d}}$ also show great variation with the asymptotic surface density (Figs. 4.9 and 4.10). More specifically, increasing $\Sigma_{0}$ results in a light curve with a slower decay rate. This is probably due to different levels of saturation in the optically thick region. The denser the optically thick region, the bigger its optical depth and the greater the amount of time for it to turn into an optically thin region.

In addition, Fig. 4.10 shows that $\xi_{\mathrm{d}}$ strongly depends on the scaled build-up time. As expected from the mass reservoir effect (Sect. 3.3), increasing $\tilde{\tau}_{\mathrm{bu}}$ results in smaller values of $\xi_{\mathrm{d}}$, which implies slower decay rates.

From the above an important conclusion can be drawn: viscosity is not the only parameter affecting the rate of photometric variations in a Be light curve. The stellar parameters, the asymptotic surface density, as well as the inclination angle, all affect the observed shape of the light curve. Thus, extracting $\alpha$ from light curves, one of the main goals of this work, cannot be done without some knowledge about these parameters.

\subsection{Fitting pipeline}

So far, our model light curves were given in terms of the dimensionless time parameter, $\tilde{\tau}$. Thus, an equation is necessary to transform from the physical time $t$ to $\tilde{\tau}$, in order to connect the real light curves to our simulated ones.

A variation in the time parameter, $\mathrm{d} \tilde{\tau}$, is related to a variation in physical time by $\mathrm{d} \tilde{\tau}=\mathrm{d} t / \tau(t)$, where $\tau(t)$ depends on 3 stellar parameters $\left(M, R_{\text {eq }}, T_{\text {eff }}\right)$ and the viscous parameter $\alpha(t)$ (Eq. 3.1.13). For a given Be star, the build-up phase starts at $t_{1}$, and ends at $t_{2}$, when dissipation begins. Thus, the build-up time is given by $t_{2}-t_{1}$. In this work, following the results of Ghoreyshi et al. (2016), we explore the possibility that the viscosity parameter may be different at build-up $\left(\alpha(t)=\alpha_{\mathrm{bu}}\right.$, for $\left.t_{1} \leq t<t_{2}\right)$ and dissipation $\left(\alpha(t)=\alpha_{\mathrm{d}}\right.$, for $\left.t \geq t_{2}\right)$. Therefore, the transformation equation from $t$ to $\tilde{\tau}$ is

$$
\tilde{\tau}=\left\{\begin{array}{ll}
\alpha_{\mathrm{bu}} \frac{t-t_{1}}{\alpha \tau}, & t_{1} \leq t<t_{2} \\
\alpha_{\mathrm{bu}} \frac{t_{2}-t_{1}}{\alpha \tau}+\alpha_{\mathrm{d}} \frac{t-t_{2}}{\alpha \tau}, & t \geq t_{2}
\end{array},\right.
$$


which is such that, as $t$ goes from $t_{1}$ to $t_{2}, \tilde{\tau}$ goes from 0 to $\tilde{\tau}_{\mathrm{bu}}$, given by

$$
\tilde{\tau}_{\mathrm{bu}}=\alpha_{\mathrm{bu}} \frac{t_{2}-t_{1}}{\alpha \tau}
$$

and for $t$ larger than $t_{2}$, we see that $\tilde{\tau}>\tilde{\tau}_{\text {bu }}$. Recall that $\alpha \tau$, defined in Eq. 3.1.13, is a quantity dependent only on the stellar parameters and the disk temperature (see the upper-right panel of Fig. 4.4 for the values of $\alpha \tau$ for the stellar models of Table 4.3).

Substitution of Eq. (4.4.1) into Eqs. (4.3.7), (4.3.8) and (4.3.9), gives the following equation for fitting an observed bump

$$
\Delta X(t)= \begin{cases}\Delta X_{\mathrm{bu}}^{\infty}\left(1-\frac{1}{1+\left[C_{\mathrm{bu}}\left(t-t_{1}\right)\right]^{\eta_{\mathrm{bu}}}}\right), & t_{1} \leq t<t_{2} \\ \Delta X_{\mathrm{bu}}^{\infty}\left(1-\frac{1}{1+\left[C_{\mathrm{bu}}\left(t_{2}-t_{1}\right)\right]^{\eta_{\mathrm{bu}}}}\right) \frac{1}{1+\left[C_{\mathrm{d}}\left(t-t_{2}\right)\right]^{\eta_{\mathrm{d}}}}, & t \geq t_{2}\end{cases}
$$

where

$$
C_{\mathrm{bu}}=\alpha_{\mathrm{bu}} \frac{\xi_{\mathrm{bu}}}{\alpha \tau}
$$

and

$$
C_{\mathrm{d}}=\alpha_{\mathrm{d}} \frac{\xi_{\mathrm{d}}}{\alpha \tau}
$$

are coefficients related to the rate of photometric variations. The values of the parameters $\Delta X_{\mathrm{bu}}^{\infty}, \xi_{\mathrm{bu}}$ and $\xi_{\mathrm{d}}$ were tabulated in Appendix A, by fitting the respective empirical laws to the model grid.

Our goal is to fit an observed light curve with Eq. 4.4.3, in order to obtain, in a self-consistent way, all the stellar and disk parameters of interest. For that, the following chain of procedures is adopted:

1. Find a light curve of a Be star that contains at least one clear inactive phase and one complete photometric bump.

2. Obtain the magnitudes $X_{*}$ at the inactive phase. Subtract these magnitudes from the light curve and obtain the excesses $\Delta X(t)$.

Without a clear inactive phase, it is not possible to obtain the pure photospheric brightness (e.g., the horizontal purple straight lines in Fig. 3.1) and, consequently, it is not possible to know how much of the observed bumps represent the disk contribution to the total flux. In addition, the photometric bump must contain a completely identified buildup phase (from which the instants $t_{1}$ and $t_{2}$ can be extracted) along with a considerable fraction of the dissipation phase.

3. Fit Eq. (4.4.3) to the selected bumps, obtaining the coefficients $\Delta X_{\mathrm{bu}}^{\infty}, C_{\mathrm{bu}}$ and $C_{\mathrm{d}}$, as well as the times $t_{1}$ and $t_{2}$ for the onsets of build-up and dissipation. 
4. Transform the magnitudes at the inactive phase, $X_{*}$, to absolute magnitudes, $M_{X *}$, by correcting for the distance to the star and reddening at each observed band.

Given the theoretical dependence of the coefficients in Eq. 4.4.3 on the stellar parameters, the absolute magnitudes are required to estimate the stellar parameters $(M, W$ and $t / t_{\mathrm{MS}}$ ). From them, the parameter $\alpha \tau$ (see Eq. 3.1.13) can be estimated. Clearly, if the stellar parameters are known from some other way (e.g., by spectroscopic analysis), this requirement is no longer necessary. Unfortunately, this is not the case for our sample (see Chapter 5 below).

5. Estimate the stellar parameters, the geometric parameter $(\cos i)$ and the bump parameters $\left(\Sigma_{0}, \alpha_{\mathrm{bu}}\right.$ and $\alpha_{\mathrm{d}}$, for each bump) that best reproduce the fitted stellar $\left(M_{X *}\right)$ and bump $\left(\Delta X_{\mathrm{bu}}^{\infty}, C_{\mathrm{bu}}\right.$ and $\left.C_{\mathrm{d}}\right)$ parameters (see Eqs. 4.4.4 and 4.4.5 and the parameters $\Delta X_{\mathrm{bu}}^{\infty}, \xi_{\mathrm{bu}}$ and $\xi_{\mathrm{d}}$ from Sect. 4.3).

In practice, the above process involves several complications (e.g., estimating the goodnees of the fit) that are described in the next section.

\subsection{Fitting using $M C M C$ sampling}

Never tell me the odds!

Han Solo to C-3PO, in Star Wars:

The Empire Strikes Back

The task of fitting the measured stellar $\left(M_{X *}\right)$ and bump parameters $\left(\Delta X_{\mathrm{bu}}^{\infty}, C_{\mathrm{bu}}\right.$ and $C_{\mathrm{d}}$ ) and estimating the model parameters - step 5, above - was done using the Markov-Chain Monte Carlo (MCMC) sampling technique. In this section, we describe this technique and how it was used to estimate the parameters of our models based on light curves described in Chapter 6.

\subsubsection{Statistical considerations}

A certain physical system will be defined by $N_{m}$ model parameters. Together, they compose the $N_{m}$-dimensional vector $\boldsymbol{m}$, which represents the state of the physical system. The physical system will also be associated to $N_{D}$ observed data quantities, which, together, compose the $N_{D}$-dimensional vector $\boldsymbol{D}$. When fitting models to observations, we are interested in obtaining a probabilistic distribution of states $\boldsymbol{m}$ given the observed data $\boldsymbol{D}$. With such a distribution, we can calculate mean values, standard deviations, medians and percentiles, etc.

In the whole space of possibilities of our different physical systems of Be stars, there is a small subset of those systems, which we refer to as $\mathrm{d} \mathcal{M}$, which contains a small list of 
possible states, and there is also a small subset of those systems, which we refer to as $\mathrm{d} \mathcal{D}$, which contains a small list of possible observations. The probability of getting physical systems of Be stars with states in the small set $\mathrm{d} \mathcal{M}$ and with observed data in the small set $\mathrm{d} \mathcal{D}$ is

$$
P(\mathrm{~d} \mathcal{M} \cap \mathrm{d} \mathcal{D})=P(\mathrm{~d} \mathcal{M} \mid \mathrm{d} \mathcal{D}) P(\mathrm{~d} \mathcal{D})=P(\mathrm{~d} \mathcal{D} \mid \mathrm{d} \mathcal{M}) P(\mathrm{~d} \mathcal{M})
$$

where we have applied the definition of conditional probabilities that say that the "probability of A and B" equals the "probability of B" times the "probability of A, given B".

Now, these small probabilities can be rewritten in terms of probability density functions, whose interpretations are as follows:

- $P(\mathrm{~d} \mathcal{M}) \rightarrow \pi(\boldsymbol{m}) \mathrm{d}^{N_{m}} m$, where $\pi(\boldsymbol{m})$ is the probability density of a Be star with the state $\boldsymbol{m}$, independent of the observations. It represents, therefore, our prior knowledge of the distribution of Be stars. We refer to $\pi(\boldsymbol{m})$ as the "prior distribution".

- $P(\mathrm{~d} \mathcal{D} \mid \mathrm{d} \mathcal{M}) \rightarrow L(\boldsymbol{D} \mid \boldsymbol{m}) \mathrm{d}^{N_{D}} D$, where $L(\boldsymbol{D} \mid \boldsymbol{m})$ is the probability density referred to as the "likelihood of a Be star with the observed data $\boldsymbol{D}$, given the state $\boldsymbol{m}$ ". This distribution depends on the uncertainties of the observed data. The smaller those are, the sharper the likelihood of the data $\boldsymbol{D}$ around the state $\boldsymbol{m}$.

- $P(\mathrm{~d} \mathcal{M} \mid \mathrm{d} \mathcal{D}) \rightarrow p(\boldsymbol{m} \mid \boldsymbol{D}) \mathrm{d}^{N_{m}} m$, where $p(\boldsymbol{m} \mid \boldsymbol{D})$ is our desired distribution: the probability density of a Be star with state $\boldsymbol{m}$, given the observed data $\boldsymbol{D}$. It is called the "posterior probability": the probability obtained by multiplying our prior knowledge with the likelihood of the model after observations have been made.

- $P(\mathrm{~d} \mathcal{D}) \rightarrow g(\boldsymbol{D}) \mathrm{d}^{N_{D}} D$, where $g(\boldsymbol{D})$ is just a normalizing constant for the distribution $p(\boldsymbol{m} \mid \boldsymbol{D})$.

Substitution of these probability density functions into Eq. (4.5.1) results in the following equation for the probability densities

$$
p(\boldsymbol{m} \mid \boldsymbol{D})=\frac{L(\boldsymbol{D} \mid \boldsymbol{m}) \pi(\boldsymbol{m})}{g(\boldsymbol{D})}
$$

where $g(\boldsymbol{D})=\int L(\boldsymbol{D} \mid \boldsymbol{m}) \pi(\boldsymbol{m}) \mathrm{d}^{N_{m}} m$. 


\subsubsection{MCMC sampling with emcee}

Spock: "Random chance seems to have operated in our favor" McCoy: "In plain, non-Vulcan English, we've been lucky" Spock: "I believe I said that, Doctor."

Spock to McCoy in Star Trek: The Original Series, episode "The Doomsday Machine"

In statistics, the quantities of interest are usually integrals of functions $\phi$, weighed by the probability density $p$, over a certain domain: $\int p(\boldsymbol{m} \mid \boldsymbol{D}) \phi(\boldsymbol{m}) \mathrm{d}^{N_{m}} m$. If, however, the dimension of the parameter space $N_{m}$ is too big and/or the probability density $p$ is too "complicated", the problem of calculating such integral easily becomes untractable by ordinary means.

A Markov Chain (MC) is a mathematical system that undergoes random transitions from one state to another on a state space. The next state depends only on the current state and not on the sequence of events that preceded it. A Markov Chain, therefore, is a memoriless process.

Some MCs have stationary distributions. For these MCs, the stationary distributions will be asymptotically reached by the states after a sufficiently large number of iterations, regardless of the initial state of the chain. One example of such a Markov Chain is the one that is constructed by the famous "Metropolis algorithm". In that algorithm, a certain probability density function is given as input and a Markov Chain is generated in which the states converge to a sample distributed according to the probability density that was given $^{6}$.

What the Metropolis algorithm does is exactly the purpose of any Markov Chain Monte Carlo (MCMC) algorithm. MCMC methods are, therefore, a class of algorithms, which utilize random numbers, for generating samples of states $\left\{\boldsymbol{m}_{r}\right\}_{r=1}^{R}$ from a probability distribution $p(\boldsymbol{m} \mid \boldsymbol{D})$ which is given as input. They construct a Markov chain that has the distribution $p(\boldsymbol{m} \mid \boldsymbol{D})$ as its stationary distribution (Mackay, 2003). The output of the MCMC algorithm is the sample of states $\left\{\boldsymbol{m}_{r}\right\}_{r=1}^{R}$.

In the stationary distribution, the states are more densely distributed where the probability density is higher. In fact the stationary sample is such that $p\left(\boldsymbol{m}_{r} \mid \boldsymbol{D}\right) \mathrm{d}^{N_{m}} m_{r} \rightarrow \frac{1}{R}$, where $\mathrm{d}^{N_{m}} m_{r}$ is a measure of the hypervolume occupied by a single state. Therefore, one of the most important consequences of this fact is the ease of calculating the aforementi-

\footnotetext{
6 The Metropolis algorithm was presented in 1953, in a work whose main purpose was the numerical calculation of the equation of state for a system of rigid spheres in two dimensions.
} 
oned integrals required for statistical analysis, namely

$$
\int p(\boldsymbol{m} \mid \boldsymbol{D}) \phi(\boldsymbol{m}) \mathrm{d}^{N_{m}} m \rightarrow \frac{1}{R} \sum_{r=1}^{R} \phi\left(\boldsymbol{m}_{r}\right) .
$$

One final and crucial feature of MCMC algorithms (such as the Metropolis algorithm) is that the probability density $p(\boldsymbol{m} \mid \boldsymbol{D})$, which is given as input, does not need to be normalized, because the algorithms deal only with ratios of these distributions. Therefore, we don't need to calculate the integral $g(\boldsymbol{D})$ in Eq. 4.5.2. If we had to perform such a calculation, there would be no advantage in the MCMC technique.

In this work, we use emcee (Foreman-Mackey et al., 2013), which is Python implementation of the Affine Invariant MCMC Ensemble sampler of Goodman and Weare (2010). Other samplers, however, are also available in emcee, like the classic Metropolis Algorithm, for example. In the context of emcee, the "states" are referred to as "walkers". According to the emcee website": "Walkers are the members of the ensemble. They are almost like separate Metropolis-Hastings chains but, of course, the proposal distribution for a given walker depends on the positions of all the other walkers in the ensemble."

When emcee is used for approximating a multi-dimensional posterior probability density, the ensemble of "walkers" starts to move around the $N_{m}$-dimensional space, according to the MCMC algorithm. There will be a certain number of $\mathrm{MC}$ iterations required before a near stationary state, according to the probability density $p(\boldsymbol{m} \mid \boldsymbol{D})$, is reached by the walkers. This first phase where the walkers move towards the regions of high probability is called the "burn-in" phase, and its resulting sampling is discarded because it is not the desired stationary sampling. It is usually a trial-and-error problem to determine the minimum number of steps that are needed to converge to the stationary distribution within an acceptable error. After the burn-in, the walkers are well localized near the most likely regions after which their subsequent positions are then recorded. This is the sampling phase of the Markov Chain, which never ends. This chain of states is used as the sample of the desired distribution $p(\boldsymbol{m} \mid \boldsymbol{D})$.

\subsubsection{The likelihood and prior distributions of our problem}

In our fitting procedure for each light curve containing $N_{\text {bumps }}$ identified bumps, there are $4+5 N_{\text {bumps }}$ model parameters. These are the 3 stellar parameters $\left(M, t / t_{\mathrm{MS}}, W\right)$, the geometric parameter $(\cos i)$, and, for each bump in the light curve, there are 5 additional parameters: the initial times of the build-up and dissipation phases $\left(t_{1}\right.$ and $\left.t_{2}\right)$, the asymptotic surface density $\left(\Sigma_{0}\right)$, and the viscosity parameters during the build-up and dissipation phases $\left(\alpha_{\mathrm{bu}}\right.$ and $\left.\alpha_{\mathrm{d}}\right)$. The state space of our system is thus a $\left(4+5 N_{\text {bumps }}\right)$ dimensional space, and the problem of estimating these $4+5 N_{\text {bumps }}$ model parameters,

\footnotetext{
${ }^{7}$ http://dan.iel.fm/emcee/current/
} 
which can be represented by the $\left(4+5 N_{\text {bumps }}\right)$-dimensional vector $\boldsymbol{m}$, can be adressed by MCMC sampling of the probability distribution of these models, given the data $\boldsymbol{D}$ from the light curves.

We assume that the errors of each of the observations in the light curves follow a Gaussian distribution and, therefore, the likelihood of a Be star with the observed data, given some model parameters, is

$$
L(\boldsymbol{D} \mid \boldsymbol{m}) \propto e^{-\frac{1}{2} \chi^{2}}
$$

where

$$
\chi^{2}=\chi_{\text {diskless }}^{2}+\chi_{\text {bump }}^{2}
$$

and

$$
\chi_{\text {diskless }}^{2}=\sum_{\text {bands }} \frac{\left(M_{X *}^{\text {model }}-M_{X *}^{\text {obs }}\right)^{2}}{\sigma^{2}\left(M_{X *}^{\text {obs }}\right)},
$$

and

$$
\chi_{\text {bump }}^{2}=\sum_{\text {bands bumps }} \sum_{\frac{1}{N_{t}}} \sum_{i=1}^{N_{t}} \frac{\left(\Delta X_{i}^{\text {model }}-\Delta X_{i}^{\text {obs }}\right)^{2}}{\sigma^{2}\left(\Delta X_{i}^{\text {obs }}\right)},
$$

where $N_{t}$ is the number of datapoints for a given bump at a given photometric band.

From Eq. (4.5.7), we see that the goodness of the fit for each bump is given by the reduced chi-square of a model light curve with respect to the data points. The sums in Eqs. (4.5.6) and (4.5.7) then show that each magnitude during inactivity and each bump in each band are treated with equal weight in the final $\chi^{2}$ used in the likelihood function (Eq. 4.5.5).

The prior distribution $\pi$ represents our prior knowledge of the distribution of Be stars. We assume it to be

$$
\pi(\boldsymbol{m}) \propto M^{-2.3} f_{\mathrm{Be}}(M) e^{-\frac{(W-\langle W\rangle)^{2}}{2 \sigma_{W}^{2}}},
$$

where the factor $M^{-2.3}$ is the initial mass function (IMF) of Kroupa (2001), and $f_{\mathrm{Be}}(M)$ represents the fraction of Be stars relative to the number of B stars, estimated by Martayan et al. (2007, their Fig. 6). Finally, the Gaussian factor comes from the distribution of rotational velocities in the sample of Be stars, here estimated from Rivinius et al. (2006), assuming $\langle W\rangle=0.81$ and $\sigma_{W}=0.12$.

For sampling of the model parameters, we have chosen $50 \times\left(4+5 N_{\text {bumps }}\right)$ "walkers", for each light curve. For a randomly chosen set of parameters sampled by emcee in the course of the simulation, the corresponding stellar and bump observables are calculated by a multidimensional linear interpolation of the model grid (see the tables in Appendix A and the diskless models of Table 4.3). During the raffle of parameters the prior probability is set to zero if one of the sampled values lies outside of this allowed range (Tables 4.1 and 4.3). We found that 1000 iterations in the burn-in phase is sufficient for the convergence of all our models. 
In Chapter 5 we present a selection of light curves of Be stars from the SMC, and measure their stellar and bump quantities (steps 1 to 4 of the pipeline). Later, in Chapter 6, we apply step 5 of the pipeline, as described in Sect 4.4, in order to estimate the relevant parameters of the selected Be stars. 


\section{Chapter 5 \\ The remarkable light curves of Be stars from the Magellanic Clouds}

Look how they shine for you

Guy Berryman, in the song "Yellow",

from Coldplay

From the previous chapters, we conclude that the most reliable and direct way of estimating the $\alpha$ viscosity parameter is to consider time-dependent disk behavior, where the diffusive effect of viscosity will have clear observational counterparts. Therefore, light curves of long temporal coverage, such as the ones given by microlensing or planetary transit surveys are excellent instruments to study the dynamical processes acting on the disk, as it builds-up and dissipates. Also, our studies have shown that other important byproducts of studying the light curves of Be stars are the estimations of the mass and angular momentum loss rates of the star. These physical quantities are important estimates for stellar evolutionary models and may give us clues about the "Be mechanism".

In 1986, astronomer Bohdan Paczyński proposed to use of gravitational microlensing to look for dark matter in the form of massive compact halo objects (MACHOs). By observing fields crowded with background stars, a MACHO would be detected when passing nearly in front of a background star and causing the star to appear brighter in a very distinct way (Aubourg et al., 1993). Since then, microlensing surveys like OGLE (Udalski et al., 1992), MACHO (Alcock et al., 1997) and EROS (Aubourg et al., 1993) resulted in light curves of millions of stars. It suffices to say that MACHOs were indeed found soon after the campaings started. But, what is important for our work is that many thousands of light curves of variable Be stars, with years of coverage, were recorded in these campaigns.

In the recent decades, astronomers also started to detect exoplanets (planets far outside our Solar System). One of the methods of detecting such planets is the "transit method", in which a planet in an edge-on orbit around the star passes in front of the star, causing a minute dimming. Periodic dimmings are the signature of planets orbiting other 
stars. OGLE-TR-56b was the first exoplanet discovered by the transit method alone, in November $2002^{1}$, and in the last ten to fifteen years, there has been an enormous increase in the number of high precision photometric surveys, looking for transits of exoplanets. Some of these surveys are ground based, like KELT (Pepper et al., 2007) and SuperWASP (Pollacco et al., 2006). Some are space missions, like CoRoT ${ }^{2}$ and $\mathrm{Kepler}^{3}$. In order to find transiting planets at the moment of transit, and, later, to estabilish that they are periodic, the photometric surveys must continuously cover portions of sky containing many stars for long periods of time. Consequently, light curves of many Be stars with years of coverage were also recorded by these surveys.

This chapter is devoted to a description of the features of light curves of Be star candidates from the Magellanic Clouds and to a selection of some of these light curves. We obtained hundreds of light curves from the OGLE-II (Udalski et al., 1997) and OGLE-III (Udalski, 2003; Udalski et al., 2008) photometric surveys, for stars selected from catalogs of Be star candidates in the SMC (Mennickent et al., 2002) and the LMC (Sabogal et al., 2005). We ultimately, selected light curves for 54 SMC Be star candidates containg clear inactive phases and 81 bumps and dips.

\subsection{Selections of Be stars from the SMC and $L M C$}

Keller et al. (2002) selected 1279 light curves from the MACHO photometric survey from the Large Magellanic Cloud (LMC) galaxy which were believed to be light curves of variable Be stars. They did so by: 1) selecting light curves with colors and magnitudes in regions that correspond to the locations of Be stars in color-magnitude diagrams (CMD); 2) selecting (automatically) from these light curves the ones that satisfied a certain criterium of variability; and 3) excluding the "contaminants" (e.g., eclipsing binaries) by visual inspection. The remaining 1279 light curves showed the following morphologies, illustrated in Fig. 5.1:

- mode 1 - "bumper events"

- mode 2 - "flicker events"

- mode 3 - "step events"

- mode 4 - "baseline variations"

- mode 5 - "fading events"

\footnotetext{
${ }^{1}$ The exoplanet HD 209458 b (sometimes unofficially called "Osiris"), however, discovered in November 1999 by the "radial velocity method", was also found to be transiting before the discovery of OGLE-TR$56 \mathrm{~b}$

2 https://corot.cnes.fr/en/COROT/index.htm

3 https://www.nasa.gov/mission_pages/kepler/main/index.html
} 


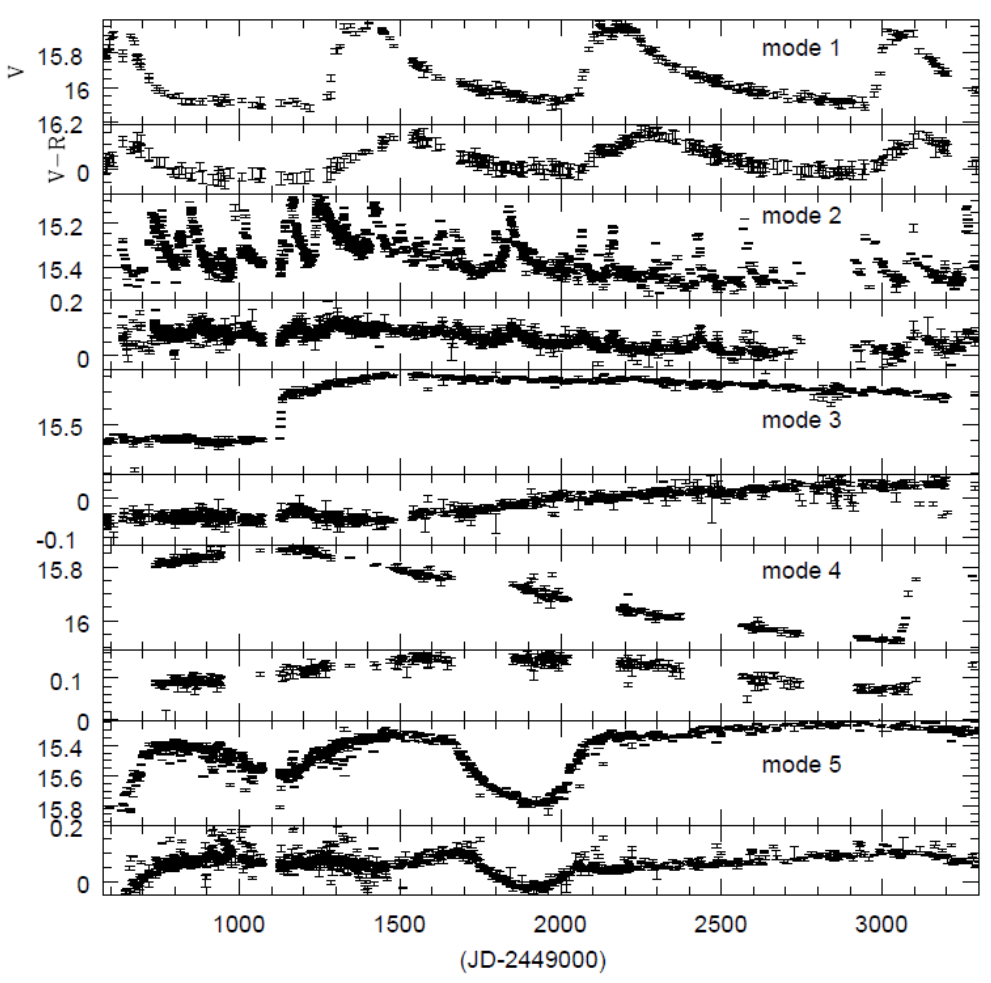

Figure 5.1: $V$ light curves and $V-R$ color curves for examples of the five modes of variability of Keller et al. (2002).

In fact, several different modes frequently appear in the light curves for a single star.

A subset of 102 of those stars also were examined spectroscopically in a limited number of instances. The majority (91\%) of the spectroscopic sample was found to exhibit Balmer emission lines in at least one epoch. Therefore, it was confirmed that at least $\sim 90 \%$ of the light curves obtained by their photometric seletion method were Be stars.

Mennickent et al. (2002) selected 1056 OGLE-II light curves of Be star candidates from the Small Magellanic Cloud (SMC) galaxy, with a method similar to the one of Keller et al. (2002). They also: 1) selected light curves inside regions in CMDs; and 2) visually inspected the light curves, removing spurious variables, Cepheids and eclipsing binaries. They classified the morphologies of their light curves in a little different way, shown in Figs. 5.2 and 5.3:

- Type-1 events (showing sharp outbursts or hump-like outbursts, roughly corresponding to mode 1 and mode 2 events of Keller et al. (2002))

- Type-2 events (showing "high" and "low" states, roughly corresponding to the mode 3 events of Keller et al. (2002))

- Type-3 events (showing periodic or near-periodic events)

- Type-4 events (showing non-periodic outbursts) 

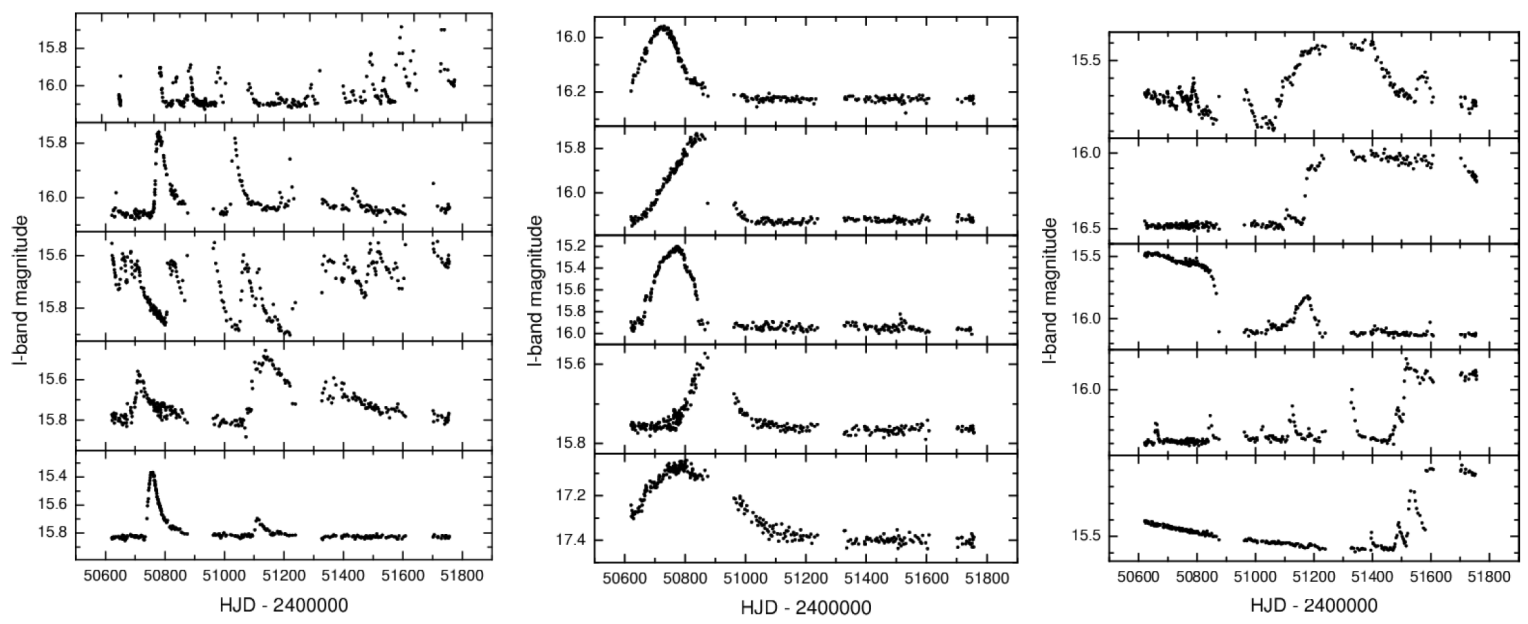

Figure 5.2: Examples of OGLE II light curves selected as Be star candidates. Left: Type-1 light curves showing sharp outbursts. Middle: Type-1 light curves showing hump-like outbursts. Right: Type1/Type-2 light curves showing outbursts and "high" and "low" states. (Credits: Mennickent et al., 2002)

The authors found that the majority of the light curves fall into the type- 4 category. In fact, the relative fraction of these light curves was: $64.9 \%$ objects showing type- 4 light curves, $13.2 \%$ objects showing type- 1 light curves, $14.6 \%$ showing type-2 light curves, and $7.4 \%$ stars showing type-3 light curves. Figure 5.4 (left) shows the distributions of these objects in a color-magnitude diagram, together with a main sequence track.

In a continuation of the previous work, Sabogal et al. (2005) selected 2446 OGLE-II light curves of Be star candidates of from the LMC galaxy. They found $60.0 \%$ type- 4 , $23.1 \%$ type-1, 6.1\% type-2, $6.1 \%$ type-3 and $4.0 \%$ type-1/type-2 light curves. Figure 5.4 (right) shows the distributions of their objects also in a color-magnitude diagram, together with a main sequence track. As for the case of the selection of Mennickent et al. (2002), we see that their objects occur mostly for very late O-type stars and early B-type stars.

The type-4 light curves should correspond to Be stars showing episodes of mass injection more complicated than the simple build-up followed by dissipation scenario described in Sect. 4.1.

Most interesting for us is the type-1 group ( $\sim 13 \%$ of the sample), composed by light curves that show single sharp or hump-like bumps, like the bumps of the light curve of SMC_SC1 75701 (Fig. 3.1). These bumps should be the result of single nearly continuous episodes of mass injection followed by dissipation of the disk, like the theoretical scenario explored in Sect. 4.1. The "bumper events" and "flicker events" of Mennickent et al. (2002) more or less correspond to the bumps of type-1 light curves, but also to features of the more irregular type-4 light curves. The bumpers have durations of a few hundred days, while the flicker events are faster, with durations of a few dozens of days. Dips like the one exemplified by the light curve of SMC_SC6 128831 (Fig. 3.1) were called "fading events" by Mennickent et al. (2002). The frequency of these events was much smaller than that of the bumpers (Mennickent et al., 2002), which is in accordance with the picture 

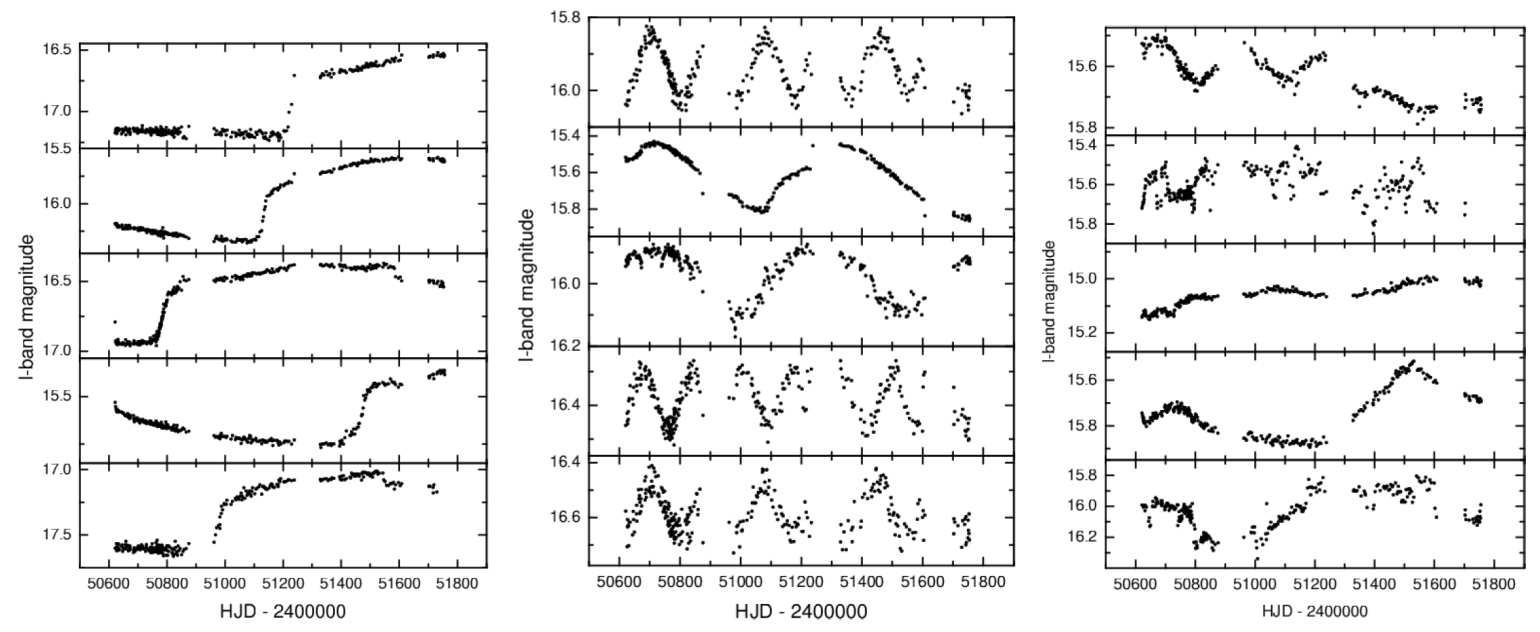

Figure 5.3: Examples of OGLE II light curves selected as Be star candidates. Left: Type-2 light curves showing "high" and "low" states. Middle: Type-3 light curves showing periodic oscillations. Right: Type-4 light curves showing random variability. (Credits: Mennickent et al., 2002)
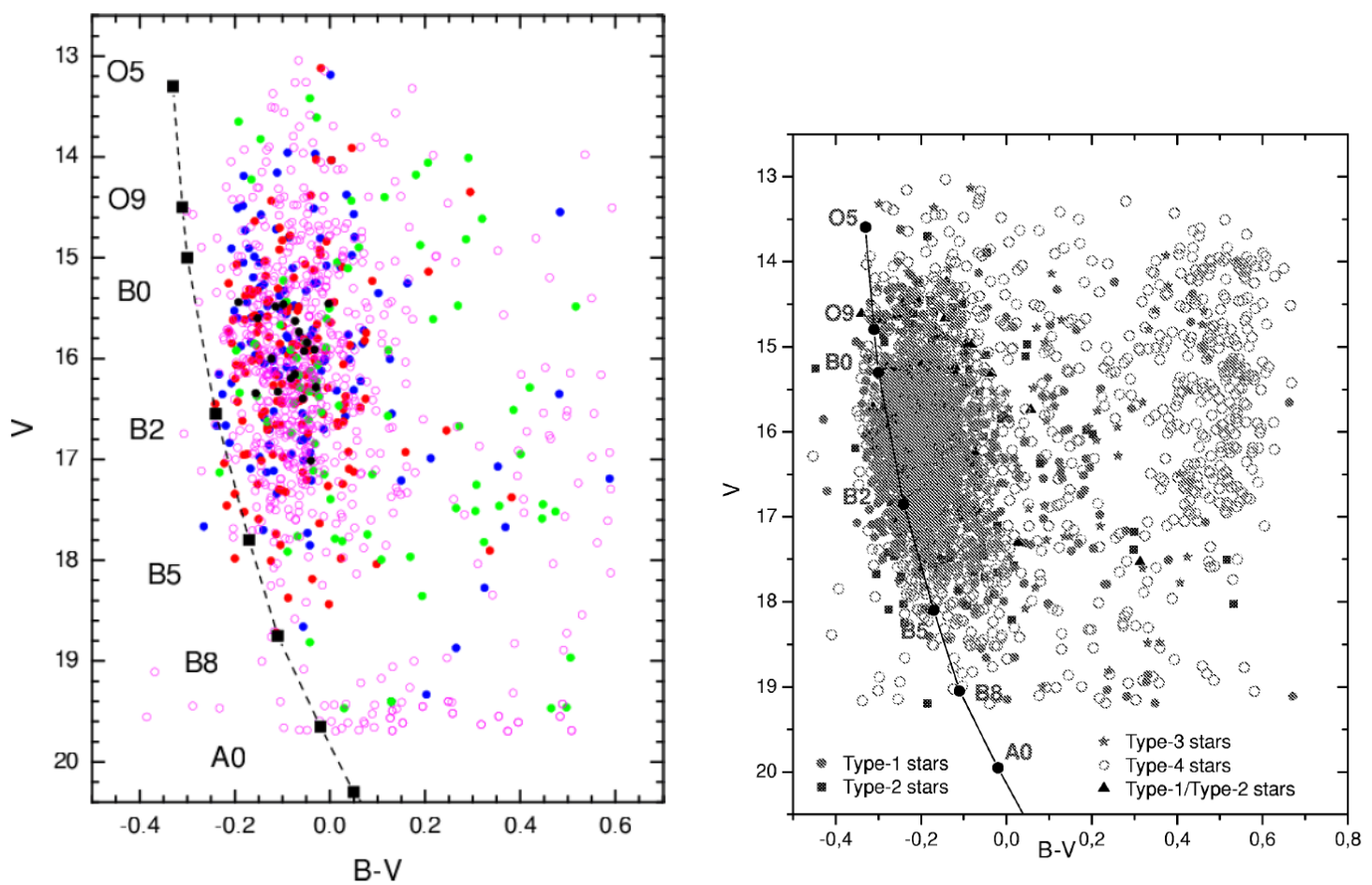

Figure 5.4: Left: CMD diagram for the selected stars from the SMC. Blue dots correspond to type1 stars, red dots to type- 2 stars, green dots to type- 3 stars, circles to type- 4 stars and black dots to type-1/type-2 stars. A main sequence track is shown for reference. (Credits: Mennickent et al., 2002) Right: CMD diagram for the selected stars from the LMC. A main sequence track is shown for reference. (Credits: Sabogal et al., 2005)

that fading events are associated with the less numerous shell stars.

The type-2 group ( 14\% of the sample of Mennickent et al. (2002)), containing light curves showing high and low plateaus, also has some interesting cases for our purposes. 
High plateaus are usually the photometric result of a longer build-up process in which a near steady-state has been reached in the inner disk. The low plateaus are frequently the portions of the light curve during inactive phases.

Paul et al. (2012) studied the spectral properties of stars from the catalogues of Mennickent et al. (2002) and Sabogal et al. (2005). For the candidates from the SMC, they found that the majority of type- 1 and type-2 light curves belong to early B type stars with emission features characteristic of circumstellar material (Paul et al., 2012). Therefore, these light curves are very likely from Be stars. For the candidates of the LMC, however, there wasn't a clear emission feature for a fraction of the type-1 light curves.

\subsection{OGLE light curves of Be star candidates}

In this work, we used both the catalogs of Be star candidates of Mennickent et al. (2002) and Sabogal et al. (2005), and, later, we focused just on the former.

In 2012, our colaborators Thomas Rivinius and Xavier Haubois downloaded public I-band light curves of the Be star candidates of these two catalogs, from the OGLE-II survey $^{4}$. Later, they contacted the OGLE team, in order to obtain the data in the other bands and the OGLE-III data for the stars in the catalogs. They were kindly sent by Igor Soszyński from the OGLE team, who provided the $V$ - and $B$-band OGLE-II data of the stars and the corresponding OGLE-III $V$ - and $I$-band data for a fraction of the stars, obtained by cross-identifications. The OGLE-II portions of the light curves range roughly from January 1997 to December 2000. The OGLE-III portions of the light curves range roughly from June 2001 to May 2009. Our resulting light curves, therefore, covered roughly 12 years.

\subsubsection{Our selection of bumps from the SMC}

The light curves were visually inspected according to the criteria of item 1, Sect. 4.4; i.e., light curves with at least one clear inactive phase and one bump. In this initial work we focussed on well-behaved light curves with clear bumps. We also avoided the short events (flickers, with build-up times $\lesssim 15$ days), due to the fact that most of them are poorly sampled.

We selected light curves from the catalogue of Be star candidates from the SMC of Mennickent et al. (2002). Due to a callibration issue between the OGLE-II and OGLE-III portions of the light curves, namely a shift in the zero points present in some of the light curves, it was necessary to find separate intervals of inactivity in both the OGLE-II and OGLE-III portions of several light curves to correct the problem. Fig. 5.5 shows examples of light curves with such zero point shifts.

\footnotetext{
4 http://ogledb.astrouw.edu.pl/ ogle/photdb/
} 

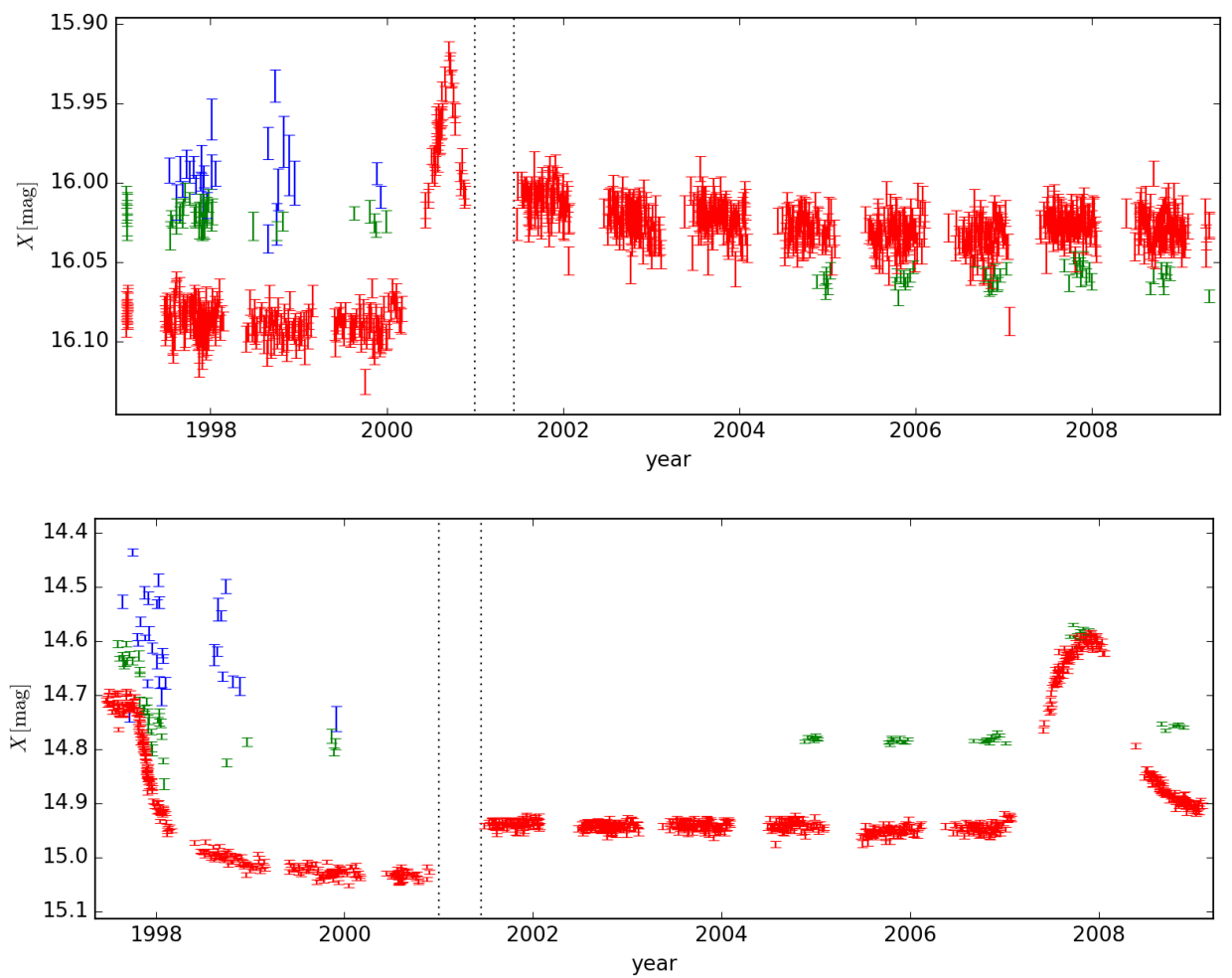

Figure 5.5: Examples of SMC light curves showing clear zero point shifts between the OGLE-II and OGLE-III components. The blue, green and red points correspond to the $B, V$ and $I$ bands, respectively. Above: SMC_SC5 21134. Below: SMC_SC8 183240. Both light curves in this figure were used in this work (see Table 5.1).

Several other light curves failed to meet the criteria of item 1 of Sect. 4.4. In Fig. 5.6, we show an example of a light curve without a clear inactive phase (above) and a light curve (below), probably belonging to the type-4 category, without a clear dissipation phase and with variability that suggests a more complicated scenario of mass injection than the one described in the Chapter 3.

The end result was a sample of 54 stars, containing 81 selected bumps, shown in Table 5.1. In the table, horizontal lines separate the data for each of the 54 stars. Each row in the table contain the data for each of the 81 selected bumps. The fifth and sixth columns in the table contain the beginning and ending of the selected inactive interval for the light curve. The seventh, eighth and ninth columns contain the $B_{*}, V_{*}$ and $I_{*}$ magnitudes obtained at the inactive phase for the light curve. Due to the nature of the OGLE survey, the $B_{*}$ and $V_{*}$ magnitudes are not available for all sources. The eleventh column contains the bands that were considered in the fitting process of the specific bumps, depending on the availability of measurements in each band. The last two columns are initial visual estimates of $t_{1}$ and $t_{2}$, which were used as input for emcee.

As explained in Sect. 4.4, the magnitudes at the inactive phase are necessary to set the baseline level of the light curves, which will be used to get an estimate of the stellar 

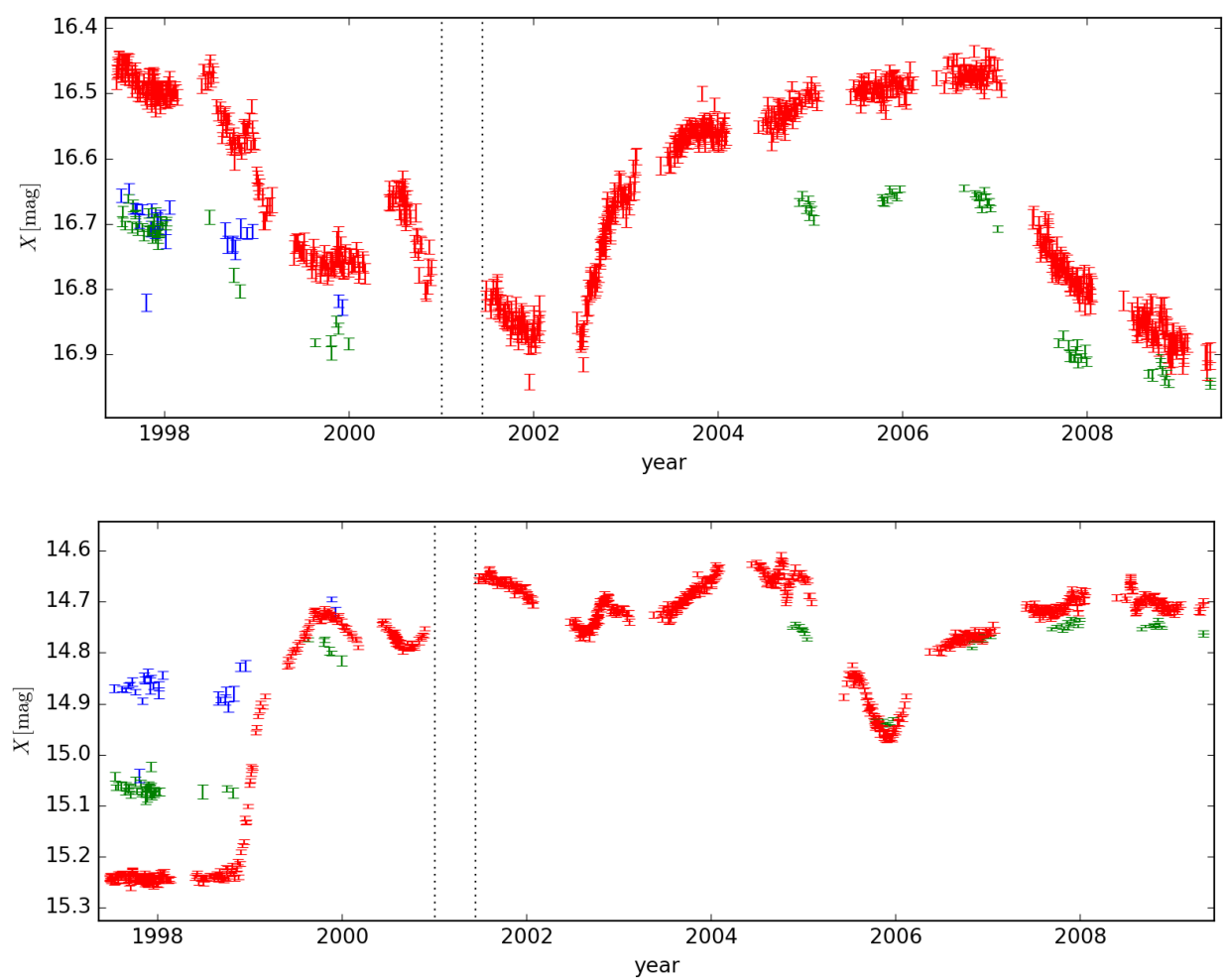

Figure 5.6: Examples of SMC light curves found to have no clear inactive phase (e.g., above: SMC_SC4 $60186)$ or to clearly be associated with a more complicated scenario of mass injection than the one described in the Chapter 3 (e.g., below: SMC_SC4 116957). The blue, green and red points correspond to the $B, V$ and $I$ bands, respectively.

parameters. In order to do the latter, these apparent magnitudes must be color-corrected and converted to absolute magnitudes by the standard formula $M_{X *}=X_{*}-(5 \log d-5)-$ $A_{X}$. We adopted as the distance to the SMC $d=60.3 \pm 3.8 \mathrm{kpc}$ from Hilditch et al. (2005). The mean $V$-band reddening of the bright stars over the whole SMC is $A_{V}=0.470 \pm 0.326$ (Zaritsky et al., 2004). The reddening in the other bands were obtained by the relations $A_{X} / A_{V}$ given by Gordon et al. (2003).

The color-magnitude diagram (CMD) of Fig. 5.7 compares the grid of diskless models (Table 4.3) to our sample of Be stars. Most of the stars lie in the upper right corner of the CMD, which means that the majority of our sample is comprised of early-type Be stars, as further discussed in Chapter 6. 


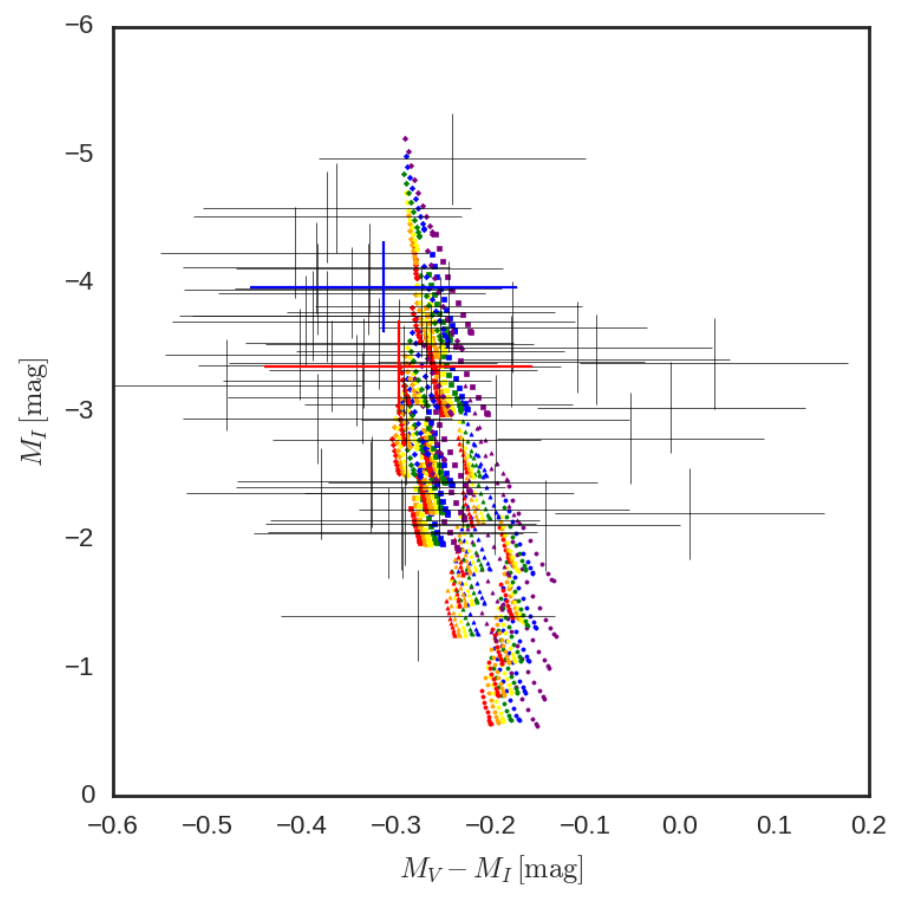

Figure 5.7: Color-magnitude diagram of simulated diskless stars (Table 4.3). Circles, triangles, squares and diamonds correspond to stellar models with $M=6.0,8.3,11.7,15.0 M_{\odot}$, respectively. The colors red, orange, yellow, green, blue and purple correspond, respectively, to the 6 values of $W$ in increasing order. The 5 different stellar ages are seen as the groups of points move in the upper-right direction. For each star, the effect of going from an inclination angle of $90 \mathrm{deg}$ to $0 \mathrm{deg}$ is to move in the upperleft direction. Also shown, with errorbars, are the positions of our selected stars (see eighth and ninth columns of Table 5.1). The blue and red errorbars mark the positions of SMC_SC1 75701 and SMC_SC6 128831 , respectively. 
Table 5.1 - List of Be stars and their respective bumps selected for this study

\begin{tabular}{|c|c|c|c|c|c|c|c|c|c|c|c|c|}
\hline \multicolumn{2}{|c|}{ OGLE-II } & \multicolumn{2}{|c|}{ OGLE-III } & \multirow{2}{*}{\multicolumn{2}{|c|}{$\begin{array}{r}\text { diskless interval } \\
(\mathrm{JD}-2450000)\end{array}$}} & \multirow[t]{2}{*}{$B_{*}$} & \multirow[t]{2}{*}{$V_{*}$} & \multirow[t]{2}{*}{$I_{*}$} & \multirow{2}{*}{$\begin{array}{r}\text { Bump } \\
\text { ID }\end{array}$} & \multirow[t]{2}{*}{ Bands } & \multirow{2}{*}{\multicolumn{2}{|c|}{$\begin{array}{r}\text { Bump interval } \\
\text { (JD-2450000) }\end{array}$}} \\
\hline Field & ID & Field & ID & & & & & & & & & \\
\hline \multirow[t]{2}{*}{ smc_sc1 } & 7612 & smc133.4 & 8877 & 600 & 1300 & $15.926 \pm 0.01$ & $16.164 \pm 0.009$ & $16.443 \pm 0.009$ & 01 & $I$ & 1420 & 2000 \\
\hline & & & & & & & & & 02 & $I$ & 3250 & 3800 \\
\hline smc_sc1 & 60553 & smc128.6 & 57 & 3000 & 3500 & - & $15.418 \pm 0.003$ & $15.601 \pm 0.007$ & 01 & $V I$ & 3500 & 5000 \\
\hline smc_sc1 & 75701 & smc125.7 & 20383 & 3000 & 3500 & - & $15.397 \pm 0.003$ & $15.51 \pm 0.006$ & 01 & $V I$ & 3650 & 5000 \\
\hline \multirow[t]{2}{*}{ smc_sc1 } & 92262 & smc128.6 & 147 & 3500 & 3800 & - & $15.623 \pm 0.003$ & $15.811 \pm 0.007$ & 01 & $I$ & 2600 & 3100 \\
\hline & & & & & & & & & 02 & $V I$ & 3900 & 5000 \\
\hline smc_sc2 & 94939 & smc125.3 & 52 & 1000 & 1100 & $15.832 \pm 0.008$ & $15.991 \pm 0.013$ & $16.126 \pm 0.007$ & 01 & $I$ & 1100 & 2000 \\
\hline smc_sc3 & 5719 & smc125.1 & 20231 & 2980 & 3020 & - & - & $16.233 \pm 0.01$ & 01 & $I$ & 1200 & 4500 \\
\hline \multirow[t]{3}{*}{ smc_sc3 } & 15970 & smc125.2 & 28056 & 700 & 750 & $15.282 \pm 0.01$ & $15.412 \pm 0.006$ & $15.541 \pm 0.006$ & 01 & $B V I$ & 750 & 2000 \\
\hline & & & & & & & & & 02 & & 2200 & 3500 \\
\hline & & & & & & & & & 03 & $V I$ & 4000 & 5000 \\
\hline \multirow[t]{2}{*}{ smc_sc3 } & 71445 & smc125.2 & 34818 & 700 & 800 & $16.227 \pm 0.01$ & $16.425 \pm 0.009$ & $16.608 \pm 0.012$ & 01 & $I$ & 1450 & 2400 \\
\hline & & & & & & & & & 02 & $I$ & 4400 & 4700 \\
\hline smc_sc3 & 125899 & smc125.2 & 6200 & 3500 & 3750 & - & $15.837 \pm 0.004$ & $15.956 \pm 0.008$ & 01 & $I$ & 2320 & 3400 \\
\hline smc_sc3 & 197941 & smc125.3 & 25034 & 4000 & 4060 & - & $15.671 \pm 0.003$ & $15.724 \pm 0.006$ & 01 & $V I$ & 2350 & 4000 \\
\hline smc_sc4 & 22859 & smc125.4 & 22723 & 700 & 1200 & $17.068 \pm 0.013$ & $17.133 \pm 0.012$ & $17.129 \pm 0.015$ & 01 & $V I$ & 1200 & 3500 \\
\hline \multirow[t]{2}{*}{ smc_sc4 } & 71499 & smc100.7 & 34896 & 1000 & 1450 & $15.414 \pm 0.011$ & $15.553 \pm 0.007$ & $15.597 \pm 0.006$ & 01 & $V I$ & 1430 & 2400 \\
\hline & & & & & & & & & 02 & $V I$ & 2900 & 4400 \\
\hline smc_sc4 & 120783 & smc100.6 & 7129 & 4250 & 4500 & - & $14.401 \pm 0.003$ & $14.442 \pm 0.005$ & 01 & $V I$ & 2750 & 4500 \\
\hline smc_sc4 & 127840 & smc100.6 & 38372 & 3500 & 4500 & - & $14.853 \pm 0.003$ & $15.026 \pm 0.006$ & 01 & $I$ & 2800 & 3500 \\
\hline smc_sc4 & 156248 & smc100.8 & 14683 & 1000 & 1200 & $15.91 \pm 0.01$ & $15.964 \pm 0.007$ & $15.852 \pm 0.006$ & 01 & $I$ & 1320 & 2300 \\
\hline \multirow[t]{3}{*}{ smc_sc4 } & 156251 & smc100.8 & 14642 & 600 & 680 & $14.881 \pm 0.005$ & $15.13 \pm 0.007$ & $15.338 \pm 0.005$ & 01 & $B V I$ & 650 & 1200 \\
\hline & & & & & & & & & 02 & $V I$ & 3170 & 3600 \\
\hline & & & & & & & & & 03 & $V I$ & 3770 & 4600 \\
\hline \multirow[t]{2}{*}{ smc_sc4 } & 159829 & smc100.8 & 37214 & 4450 & 4500 & - & $15.895 \pm 0.005$ & $15.959 \pm 0.009$ & 01 & $I$ & 2600 & 3000 \\
\hline & & & & & & & & & 02 & $V I$ & 3430 & 4500 \\
\hline smc_sc4 & 159857 & smc100.8 & 45127 & 3000 & 3500 & - & $15.626 \pm 0.004$ & $15.8 \pm 0.008$ & 01 & $I$ & 780 & 1400 \\
\hline
\end{tabular}


Table 5.1 - continued.

\begin{tabular}{|c|c|c|c|c|c|c|c|c|c|c|c|c|}
\hline \multicolumn{2}{|c|}{ OOGLE-II } & \multicolumn{2}{|c|}{ OGLE-III } & \multirow{2}{*}{\multicolumn{2}{|c|}{$\begin{array}{r}\text { diskless interval } \\
(\mathrm{JD}-2450000) \\
\end{array}$}} & \multirow{3}{*}{$B_{*}$} & \multirow[t]{2}{*}{$V_{*}$} & \multirow[t]{3}{*}{$I_{*}$} & \multirow{3}{*}{$\begin{array}{r}\text { Bump } \\
\text { ID } \\
02\end{array}$} & \multirow{3}{*}{$\begin{array}{r}\text { Bands } \\
V I\end{array}$} & \multirow{2}{*}{\multicolumn{2}{|c|}{$\begin{array}{r}\text { Bump interval } \\
\text { (JD-2450000) }\end{array}$}} \\
\hline \multirow[t]{2}{*}{ Field } & \multirow[t]{2}{*}{ ID } & \multirow{2}{*}{ Field } & \multirow[t]{2}{*}{ ID } & & & & & & & & & \\
\hline & & & & & & & & & & & 3600 & 4300 \\
\hline smc_sc4 & 163828 & smc100.7 & 8813 & 600 & 700 & $17.171 \pm 0.009$ & $17.164 \pm 0.009$ & $16.953 \pm 0.013$ & 01 & $B V I$ & 700 & 2400 \\
\hline \multirow[t]{2}{*}{ smc_sc4 } & \multirow[t]{2}{*}{167554} & \multirow[t]{2}{*}{ smc100.7 } & \multirow[t]{2}{*}{51098} & \multirow[t]{2}{*}{3000} & \multirow[t]{2}{*}{4500} & \multirow[t]{2}{*}{-} & \multirow{2}{*}{$17.258 \pm 0.009$} & \multirow[t]{2}{*}{$17.2 \pm 0.016$} & 01 & $I$ & 1620 & 1900 \\
\hline & & & & & & & & & 02 & $V I$ & 4700 & 5000 \\
\hline \multirow[t]{2}{*}{ smc_sc4 } & \multirow[t]{2}{*}{171253} & \multirow[t]{2}{*}{ smc100.7 } & \multirow[t]{2}{*}{42620} & \multirow[t]{2}{*}{700} & \multirow[t]{2}{*}{1200} & $15.674 \pm 0.01$ & $15.714 \pm 0.009$ & $15.691 \pm 0.006$ & 01 & $I$ & 1410 & 1900 \\
\hline & & & & & & & & & 02 & $V I$ & 3245 & 4000 \\
\hline smc_sc4 & 175272 & smc100.6 & 7362 & 3950 & 4000 & - & $16.579 \pm 0.005$ & $16.431 \pm 0.009$ & 01 & $I$ & 2600 & 3800 \\
\hline smc_sc4 & 179053 & smc100.6 & 38443 & 0 & 1000 & $16.304 \pm 0.009$ & $16.339 \pm 0.009$ & $16.148 \pm 0.008$ & 01 & $I$ & 1300 & 2400 \\
\hline smc_sc5 & 11453 & smc100.8 & 14734 & 3300 & 3400 & - & $15.871 \pm 0.004$ & $15.779 \pm 0.008$ & 01 & $I$ & 3400 & 5000 \\
\hline smc_sc5 & 21117 & smc100.8 & 52883 & 3650 & 3750 & - & $16.051 \pm 0.004$ & $16.143 \pm 0.009$ & 01 & $V I$ & 970 & 2000 \\
\hline & & & & & & & & & 02 & $I$ & 2200 & 2800 \\
\hline & & & & & & & & & 03 & $I$ & 2900 & 3400 \\
\hline & & & & & & & & & 04 & $V I$ & 3850 & 5000 \\
\hline smc_sc5 & 21134 & smc100.8 & 45175 & 1000 & 1500 & $15.994 \pm 0.013$ & $16.023 \pm 0.006$ & $16.091 \pm 0.007$ & 01 & $I$ & 1600 & 2000 \\
\hline smc_sc5 & 32377 & smc100.7 & 50838 & 4050 & 4100 & - & $15.844 \pm 0.003$ & $15.941 \pm 0.008$ & 01 & $I$ & 3030 & 3400 \\
\hline & & & & & & & & & 02 & $I$ & 4350 & 5000 \\
\hline smc_sc5 & 43650 & smc100.6 & 15248 & 1400 & 2000 & $17.177 \pm 0.013$ & $17.214 \pm 0.01$ & $17.305 \pm 0.017$ & 01 & $V I$ & 750 & 2000 \\
\hline & & & & & & & & & 02 & $V I$ & 2550 & 5000 \\
\hline smc_sc5 & 54851 & smc100.5 & 14725 & 600 & 850 & $16.264 \pm 0.01$ & $16.311 \pm 0.009$ & $16.366 \pm 0.008$ & 01 & $B V I$ & 850 & 2000 \\
\hline & & & & & & & & & 02 & & 2120 & 3600 \\
\hline & & & & & & & & & 03 & $V I$ & 3650 & 5000 \\
\hline smc_sc5 & 65500 & smc101.8 & 21127 & 1000 & 1500 & $16.034 \pm 0.011$ & $15.981 \pm 0.007$ & $15.959 \pm 0.006$ & 01 & $B V I$ & 600 & 1000 \\
\hline smc_sc5 & 129535 & smc100.6 & 53957 & 4700 & 4800 & - & $16.923 \pm 0.008$ & $16.953 \pm 0.013$ & 01 & $V I$ & 3300 & 5000 \\
\hline smc_sc5 & 145724 & smc101.8 & 21370 & 3000 & 3200 & - & - & $17.116 \pm 0.019$ & 01 & $I$ & 3230 & 5000 \\
\hline smc_sc5 & 180034 & smc100.1 & 27826 & 3900 & 4100 & - & $16.436 \pm 0.005$ & $16.431 \pm 0.009$ & 01 & $V I$ & 4120 & 5000 \\
\hline smc_sc5 & 260841 & smc100.1 & 36050 & 800 & 900 & $15.858 \pm 0.011$ & $16.013 \pm 0.008$ & $16.182 \pm 0.008$ & 01 & $I$ & 1500 & 2200 \\
\hline & & & & & & & & & 02 & $V I$ & 3800 & 4800 \\
\hline
\end{tabular}


Table 5.1 - continued.

\begin{tabular}{|c|c|c|c|c|c|c|c|c|c|c|c|c|}
\hline \multirow{3}{*}{$\begin{array}{r}\text { Field } \\
\text { smc_sc5 }\end{array}$} & OGLE-II & \multicolumn{2}{|c|}{ OGLE-III } & \multirow{2}{*}{\multicolumn{2}{|c|}{$\begin{array}{r}\text { diskless interval } \\
(\mathrm{JD}-2450000)\end{array}$}} & \multirow[t]{2}{*}{$B_{*}$} & \multirow[t]{2}{*}{$V_{*}$} & \multirow[t]{2}{*}{$I_{*}$} & \multirow{2}{*}{$\begin{array}{r}\text { Bump } \\
\text { ID }\end{array}$} & \multirow[t]{2}{*}{ Bands } & \multirow{2}{*}{\multicolumn{2}{|c|}{$\begin{array}{r}\text { Bump interval } \\
\text { (JD-2450000) }\end{array}$}} \\
\hline & ID & Field & ID & & & & & & & & & \\
\hline & 260957 & smc100.1 & 36101 & 1200 & 1700 & $16.747 \pm 0.009$ & $16.917 \pm 0.009$ & $17.043 \pm 0.015$ & 01 & $V I$ & 3620 & 5000 \\
\hline smc_sc5 & 266088 & smc100.2 & 9240 & 700 & 750 & $17.227 \pm 0.012$ & $17.315 \pm 0.01$ & $17.408 \pm 0.016$ & 01 & $B V I$ & 750 & 2000 \\
\hline smc_sc5 & 276982 & smc100.3 & 9403 & 4400 & 4500 & - & $15.993 \pm 0.005$ & $15.757 \pm 0.008$ & 01 & $V I$ & 3030 & 4500 \\
\hline smc_sc5 & 282963 & smc100.3 & 9408 & 1000 & 1500 & $15.431 \pm 0.009$ & $15.591 \pm 0.006$ & $15.665 \pm 0.005$ & 01 & $I$ & 1600 & 4300 \\
\hline \multirow[t]{2}{*}{ smc_sc6 } & 11085 & smc100.1 & 36096 & 700 & 1100 & $15.52 \pm 0.01$ & $15.667 \pm 0.007$ & $15.863 \pm 0.006$ & 01 & $V I$ & 1400 & 2000 \\
\hline & & & & & & & & & 02 & $V I$ & 3900 & 5000 \\
\hline smc_sc6 & 17538 & smc100.2 & 9240 & 0 & 750 & $17.213 \pm 0.012$ & $17.315 \pm 0.013$ & $17.424 \pm 0.015$ & 01 & $V I$ & 800 & 3000 \\
\hline smc_sc6 & 42440 & smc100.3 & 56046 & 700 & 1100 & $16.867 \pm 0.012$ & $17.01 \pm 0.011$ & $17.19 \pm 0.017$ & 01 & $I$ & 1400 & 3500 \\
\hline \multirow[t]{4}{*}{ smc_sc6 } & 99991 & smc100.1 & 43700 & 1300 & 1800 & $15.755 \pm 0.007$ & $15.923 \pm 0.007$ & $16.126 \pm 0.009$ & 01 & $I$ & 650 & 1400 \\
\hline & & & & & & & & & 02 & $I$ & 1800 & 2500 \\
\hline & & & & & & & & & 03 & $I$ & 3150 & 4000 \\
\hline & & & & & & & & & 04 & $I$ & 4200 & 5000 \\
\hline \multirow[t]{2}{*}{ smc_sc6 } & 105368 & smc100.2 & 17645 & 600 & 1200 & $16.462 \pm 0.011$ & $16.592 \pm 0.008$ & $16.681 \pm 0.011$ & 01 & $V I$ & 1150 & 2000 \\
\hline & & & & & & & & & 02 & $I$ & 2700 & 4000 \\
\hline smc_sc6 & 116294 & smc100.2 & 49901 & 0 & 900 & $16.775 \pm 0.012$ & $16.958 \pm 0.01$ & $17.085 \pm 0.013$ & 01 & $I$ & 985 & 2000 \\
\hline smc_sc6 & 128831 & smc100.3 & 55954 & 600 & 1200 & $15.849 \pm 0.009$ & $16.018 \pm 0.007$ & $16.116 \pm 0.007$ & 01 & $I$ & 1445 & 2600 \\
\hline smc_sc6 & 199611 & smc100.3 & 29080 & 600 & 1300 & $15.265 \pm 0.011$ & $15.447 \pm 0.008$ & $15.594 \pm 0.006$ & 01 & $I$ & 1500 & 2000 \\
\hline smc_sc6 & 272665 & smc106.6 & 26640 & 1000 & 1500 & $17.784 \pm 0.016$ & $17.962 \pm 0.016$ & $18.04 \pm 0.029$ & 01 & $I$ & 1620 & 4500 \\
\hline smc_sc7 & 57131 & smc105.6 & 33029 & 1200 & 2000 & $16.037 \pm 0.015$ & $16.127 \pm 0.008$ & $16.269 \pm 0.008$ & 01 & $I$ & 2780 & 3500 \\
\hline smc_sc8 & 183240 & smc105.2 & 32029 & 3000 & 4000 & - & $14.783 \pm 0.003$ & $14.946 \pm 0.005$ & 01 & $V I$ & 4150 & 4800 \\
\hline \multirow[t]{2}{*}{ smc_sc9 } & 105383 & smc110.6 & 114 & 1000 & 1300 & $16.115 \pm 0.01$ & $16.264 \pm 0.007$ & $16.4 \pm 0.009$ & 01 & $V I$ & 1240 & 3500 \\
\hline & & & & & & & & & 02 & $V I$ & 3780 & 4800 \\
\hline smc_sc9 & 168422 & smc113.7 & 6330 & 4700 & 4850 & - & $17.002 \pm 0.009$ & $17.057 \pm 0.014$ & 01 & $V I$ & 2700 & 4850 \\
\hline \multirow[t]{2}{*}{ smc_sc10 } & 8906 & smc110.6 & 22338 & 4500 & 5000 & - & $15.253 \pm 0.003$ & $15.382 \pm 0.006$ & 01 & $I$ & 2935 & 3300 \\
\hline & & & & & & & & & 02 & $V I$ & 3650 & 3710 \\
\hline smc_sc11 & 28090 & smc113.2 & 4458 & 4500 & 5000 & - & $15.248 \pm 0.004$ & $15.433 \pm 0.006$ & 01 & $V I$ & 2300 & 4500 \\
\hline smc_sc11 & 46587 & smc110.3 & 16096 & 600 & 1000 & $17.087 \pm 0.013$ & $17.248 \pm 0.01$ & $17.343 \pm 0.021$ & 01 & $I$ & 1110 & 4500 \\
\hline
\end{tabular}


Chapter 6

\section{Results}

I hate being "allowed for", as if I

were some incalculable quantity in an astronomical equation.

Dorothy L. Sayers and Robert

Eustace, The Documents in the Case

In this section, the results obtained by applying the pipeline (Sects. 4.4 and 4.5) to the stars and bumps of our sample are described. Initially, the results for the two objects of Fig. 3.1 are examined in detail (Sect. 6.1), followed by an analysis of the results obtained for the entire sample (Sect. 6.2).

\subsection{SMC_SC1 75701 and SMC_SC6 128831}

The results for SMC_SC1 75701 and SMC_SC6 128831 are shown in Figs. 6.1 and 6.2, respectively. For SMC_SC1 75701 there was enough data for both the $I$ and $V$ bands to allow these two light curves to be fitted simultaneously. For SMC_SC6 128831, however, only $I$-band data was available. The times for the beginning of the build-up $\left(t_{1}\right)$ and dissipation $\left(t_{2}\right)$ are fitted quantities in the pipeline, but an inicial estimate for them is provided to emcee by graphically analyzing each light curve. These estimates are shown as the purple and orange segments in the horizontal straight lines in the figure. As explained in Sect. 4.5.1.1, in the MCMC sampling, after a sufficient number of iterations, a stationary sample is obtained, for which the model parameters are more concentrated in the regions of higher posterior probability. In the plots we show 100 sets of randomly selected model curves obtained after the stationary sample was reached. The dispersion of the curves gives a visual measure of the goodness of the fits.

The goodness of the fit can be quantitatively assessed from the distribution of the posterior probabilities of each fitted parameter shown in Figs. 6.1 and 6.2. The main diagonal of the triangular diagram plots the distributions of the stellar $\left(M, t / t_{\mathrm{MS}}\right.$ and $W)$, geometrical $(\cos i)$, and bump $\left(\Sigma_{0}, \alpha_{\mathrm{bu}}\right.$ and $\left.\alpha_{\mathrm{d}}\right)$ parameters, and they can be used to 


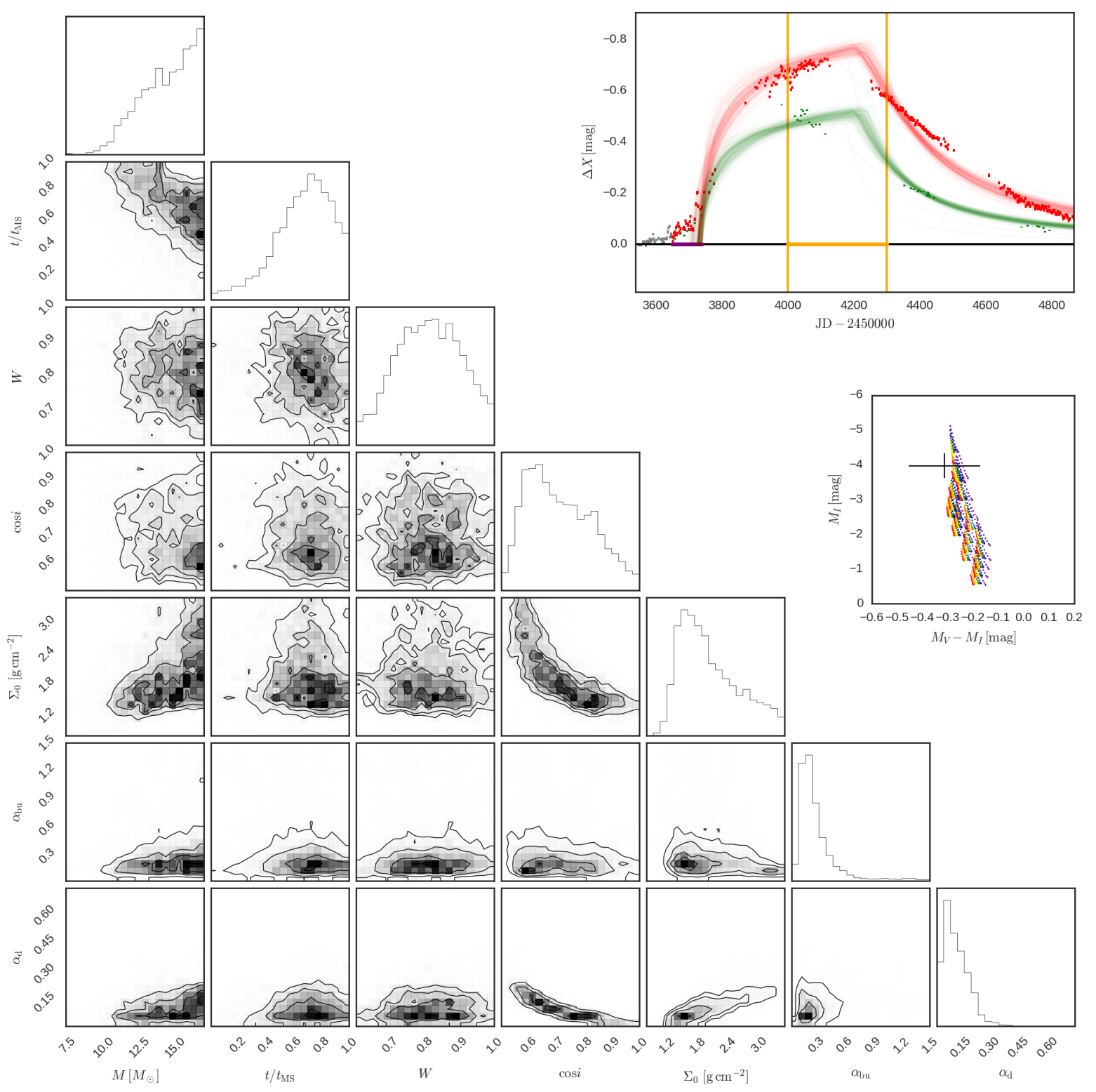

Figure 6.1: Upper-right: Light curve of SMC_SC1 75701. Thin lines: 100 model curves randomly selected from the stationary sample of the emcee code. The red (green) color indicates the $I(V)$ band. The purple and orange time intervals marked in the horizontal straight lines are the allowed intervals for the model parameters $t_{1}$ and $t_{2}$, respectively. Middle-right: CMD displaying the model grid of inactive Be stars and the position of SMC_SC1 75701 (see Fig. 5.7 for details). Below: Results of the emcee run for SMC_SC1 75701. Histogram distributions of the posterior probabilities (top panels) and two-by-two correlations of the stellar $\left(M, t / t_{\mathrm{MS}}\right.$ and $\left.W\right)$, geometrical $(\cos i)$, and bump $\left(\Sigma_{0}, \alpha_{\mathrm{bu}}\right.$ and $\left.\alpha_{\mathrm{d}}\right)$ parameters (off-diagonal panels). The parameters $t_{1}$ and $t_{2}$ were not shown for convenience. The normalized density contour levels shown in the off-diagonal panels are $12 \%, 39 \%, 68 \%, 87 \%$ of the peak probability.

assess how well-constrained each parameter is. The images below the diagonal show how the parameters correlate with each other.

The stellar parameters $\left(M, t / t_{\mathrm{MS}}\right.$ and $\left.W\right)$ are mainly constrained by the magnitudes 


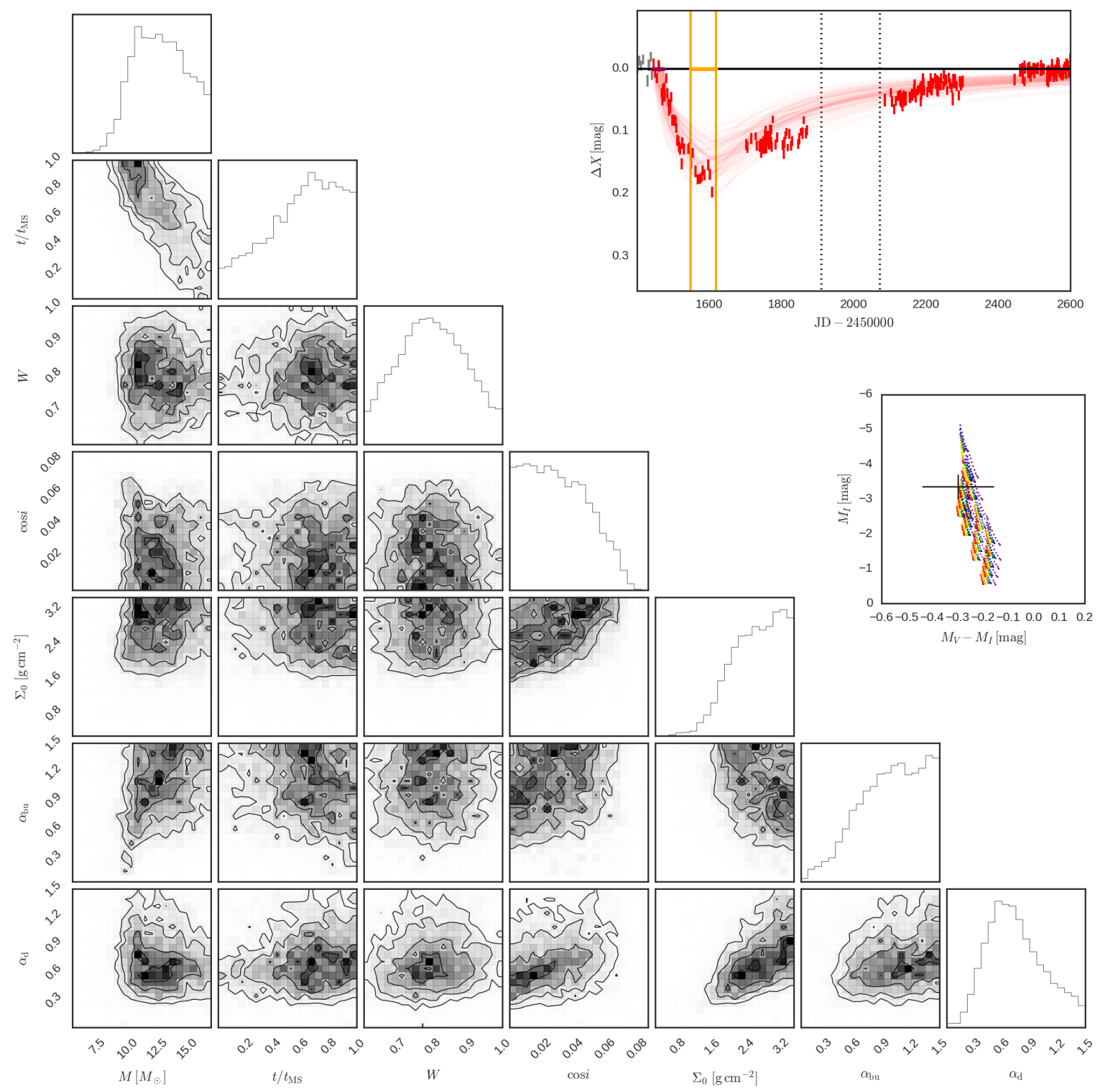

Figure 6.2: Same as Fig. 6.1 for SMC_SC6 128831.

at the inactive phase. In Fig. 6.1, the three leftmost histograms along the diagonal have broad distributions, which means that these parameters are not well constrained. The first histogram shows that SMC_SC1 75701 is an early Be star, probably even more massive than the available stellar models (Table 4.3). This agrees with the position of the star in the CMD (Fig. 5.7). The mass is anti-correlated with the main sequence age (see $t / t_{\mathrm{MS}} \times M$ plane), as expected from the fact that a less massive but more evolved star can have a similar absolute magnitude to that of a younger, more massive star.

The bump parameters $\left(\Sigma_{0}, \alpha_{\mathrm{bu}}\right.$ and $\left.\alpha_{\mathrm{d}}\right)$ are mainly constrained by the shape of the observed bump. Roughly, the amplitude of the bump depends mostly on $\Sigma_{0}$ (and $\cos i$, 
see Fig. 4.6), while the value of viscosity parameter in each phase controls the rate of brightness variation. For SMC_SC1 75701, $\Sigma_{0}$ has a broad distribution peaking around $\sim 1.5 \mathrm{~g} \mathrm{~cm}^{-2}$, indicating a quite dense disk, close to the densest cases in the sample of Vieira et al. (2017) for the same spectral type. This fact can also be inferred from Fig. 4.6, given the large observed $\Delta I_{\mathrm{bu}}^{\infty}$. The best-fit viscosity parameters are $\alpha_{\mathrm{bu}}=0.24_{-0.08}^{+0.18}$ and $\alpha_{\mathrm{d}}=0.11_{-0.05}^{+0.08}$.

Of the three bump parameters derived for SMC_SC1 75701, $\Sigma_{0}$ and $\alpha_{\mathrm{d}}$ clearly anticorrelate with $\cos i$, while $\alpha_{\mathrm{bu}}$ shows no strong sign of correlation. In fact, an anticorrelation of these three parameters with $\cos i$ is expected, as a consequence of the dependency of $\Delta X_{\mathrm{bu}}^{\infty}, \xi_{\mathrm{bu}}$ and $\xi_{\mathrm{d}}$, defined in Sect. 4.3, on $\cos i$. Fig. 4.6 shows that, if the star is seen more pole-on (higher values of $\cos i$ ), smaller values of $\Sigma_{0}$ are required in order to obtain the fitted $\Delta X_{\mathrm{bu}}^{\infty}$, hence the strong anti-correlation seen in the $\Sigma_{0} \times \cos i$ plane. Eq. (4.4.4) shows that the fitted coefficient $C_{\mathrm{bu}}$ is proportional to the product of $\alpha_{\mathrm{bu}}$ and $\xi_{\text {bu }}$, and it was shown (second and fourth columns of panels of Fig. 4.8) that disks seen more pole-on (higher values of $\cos i$ ) appear to build-up faster (having higher values of $\left.\xi_{\mathrm{bu}}\right)$. Therefore, for higher values of $\cos i$, smaller values of $\alpha_{\mathrm{bu}}$ are required to obtain the fitted $C_{\mathrm{bu}}$, which would produce an anti-correlation in the $\alpha_{\mathrm{bu}} \times \cos i$ plane. Even though this last anti-correlation was not observed for SMC_SC1 75701, it is clearly seen for many objects of our sample, for instance, SMC_SC3 71445 and SMC_SC3 125899 (see Figs. C11 and $\mathrm{C} 13$ from the paper in Appendix C). Eq. (4.4.5) shows that $C_{\mathrm{d}}$ is proportional to the product of $\alpha_{\mathrm{d}}$ and $\xi_{\mathrm{d}}$, and it was shown in the second and fourth columns of panels of Fig. 4.9 that the more pole-on and the less dense the disk, the faster the rate of brightness variation in the dissipation, thus the anti-correlation seen in the $\alpha_{\mathrm{d}} \times \cos i$ plane. Finally, the plane $\alpha_{\mathrm{d}} \times \Sigma_{0}$ shows a correlation which is a consequence of the above mentioned relationship between $\xi_{\mathrm{d}}$ with $\Sigma_{0}$.

The results for SMC_SC6 128831 (Fig. 6.2) indicate a less massive $\operatorname{star}\left(M=12.2_{-2.2}^{+2.7} M_{\odot}\right)$ surrounded by a much more massive disk $\left(\Sigma_{0}=2.6_{-0.6}^{+0.5} \mathrm{~g} \mathrm{~cm}^{-2}\right)$. SMC_SC6 128831 is an example of a dip, which means that this Be star is seen at a near edge-on angles, with $\cos i$ being well constrained. The very steep build-up phase of SMC_SC6 128831 hints to large mass injection rate and viscosity during build-up, as confirmed by the fifth and sixth histograms along the diagonal of Fig. 6.2. The viscosity parameter during dissipation was found to be $\alpha_{\mathrm{d}}=0.69_{-0.27}^{+0.34}$. The plane $\alpha_{\mathrm{d}} \times \Sigma_{0}$ shows a correlation, just as for the case of SMC_SC1 75701, which is a consequence of the relationship between $\xi_{\mathrm{d}}$ with $\Sigma_{0}$, also expected for near-edge-on inclinations (see Fig. 4.9).

For SMC_SC6 128831 a positive correlation between $\Sigma_{0}, \alpha_{\mathrm{bu}}$ and $\alpha_{\mathrm{d}}$ with $\cos i$ was observed. Fig. 4.6 shows that, if the star moves away from the edge-on case $(\cos i=0)$, bigger values of $\Sigma_{0}$ are required in order to obtain the fitted $\Delta X_{\mathrm{bu}}^{\infty}$, hence the correlation seen in the $\Sigma_{0} \times \cos i$ plane. The first column of panels of Fig. 4.8 (for the $I$-band) show that, for the edge-on case there is no strong variation of $\xi_{\mathrm{bu}}$ with $\cos i$. The third column 
of panels (for the $V$-band), however, show an increase of $\xi_{\text {bu }}$ with $\cos i$, which does not affect the estimates for this dip, since there are no measurements in the $V$-band for this dip. In any case, however, in both the first and third columns of panels, there is the trend that a more tenuous disk appears to build faster, especially when the star is hotter. Therefore, since Eq. 4.4.4 shows that $C_{\mathrm{bu}} \propto \alpha_{\mathrm{bu}} \xi_{\mathrm{bu}}$, it follows that, with the increase of $\Sigma_{0}$ with $\cos i$, the function $\xi_{\text {bu }}$ decreases and, hence, $\alpha_{\text {bu }}$ increases. Finally, Eq. 4.4.5 shows that $C_{\mathrm{d}} \propto \alpha_{\mathrm{d}} \xi_{\mathrm{d}}$ and Fig. 4.10 shows that $\xi_{\mathrm{d}}$ decreases with $\cos i$ and $\Sigma_{0}$. Therefore, as $\Sigma_{0}$ increases with $\cos i$, it follows that $\xi_{\mathrm{d}}$ decreases and, hence, $\alpha_{\mathrm{d}}$ must increase. Similar trends were found for the other two edge-on stars in our sample (SMC_SC1 92262, Figs. C4 and C5, and SMC_SC4 179053, Fig. C34 from the paper in Appendix C).

In general, the histograms of Fig. 6.2 are broader than the ones in Fig. 6.1, indicating that parameters are more poorly constrained than for SMC_SC1 75701. There are two main reasons for this. First, only I-band data was available for this star, which has a negative impact on the pipeline's ability to constrain the stellar parameters. The MCMC method ensures that the uncertainties in the stellar parameters are properly propagated into the other model parameters. Second, the fact that the dips have smaller amplitudes than the bumps of pole-on stars, even for higher values of $\Sigma_{0}$, is a great disadvantage, because the bump amplitude is much closer to the noise level of the measurements.

The results of the pipeline for all stars in Table 5.1 are shown in Figs. C1 to C79, in the paper in Appendix C. The best-fit model parameters for all stars and bumps are listed in Table 6.1.

\subsection{Results for the whole sample}

Geordi: "Suddenly it's like the laws of physics went right out the window." Q: "And why shouldn't they? They're so inconvenient!"

Georgi to Q, in Star Trek: The Next Generation, episode "True Q"

As seen previously, the scarcity of information about the central star (one, two, or at most 3 photometric bands only) causes a poor determination of its fundamental stellar parameters, which, owing to the nature of the MCMC method, propagates onto the disk parameters. The main result of this work, therefore, does not lie on the individual determination of the bump parameters, but on the statistical properties of the sample as a whole. 


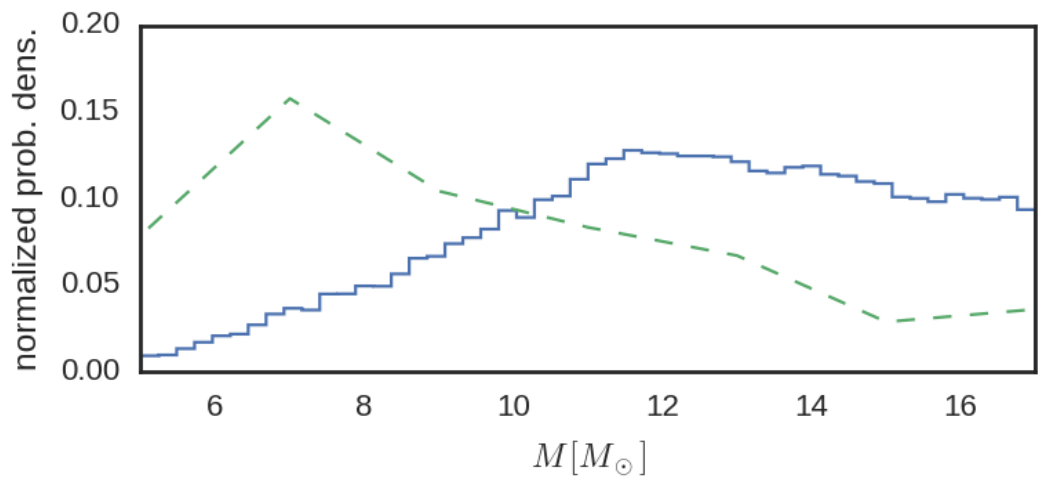

Figure 6.3: Solid line: Histogram of the sum of the posterior probabilities of parameter $M$ for all stars in our sample. Dashed line: IMF of Kroupa (2001) weighed by the fraction of Be stars over B stars of Martayan et al. (2007), given by the factor $M^{-2.3} f_{\mathrm{Be}}(M)$ in Eq. (4.5.8).

\subsubsection{Mass distribution}

Let us initially discuss the properties of our sample, in order to determine whether it represents a typical population of Be stars in SMC, or whether one or more selection biases were introduced.

Our selection of stars and bumps comes from the catalogue of visual photometric Be star candidates of Mennickent et al. (2002), where the candidate stars were selected according to the expected location of Be stars in color-magnitude diagrams and according to the observed variability in the light curves. In Fig. 6.3, we show the sum of the posterior probabilities of parameter $M$ for all our sample of stars (solid line). Clearly, most of our stars are early-type Be stars, in agreement to the position of our sample in the CMD (Fig. 5.7). We also show the factor $\propto M^{-2.3} f_{\mathrm{Be}}(M)$ of Eq. 4.5.8 (dashed line). We recall that this factor was assumed as a prior in the MCMC fitting, and it represents our current knowledge about the populations of Be stars in the SMC. The green curve shows that, although the fraction of Be stars over B stars $\left(f_{\mathrm{Be}}(M)\right.$, estimated by Martayan et al. (2007) from a cluster of the SMC) generally increases with $M$, the higher probability of the formation of less massive stars expressed in the IMF of Kroupa (2001) results in a bigger incidence of late type over early type Be stars. Our sample, therefore, is biased towards more massive stars.

This bias likely has several reasons:

1. The typical apparent $I$-band magnitudes of a B0 and a B9 star in the SMC are $\sim 15.5$ and $\sim 19.5$, with rms uncertainties given by $\gtrsim 0.005$ and $\gtrsim 0.15$, respectively (Wyrzykowski et al., 2009, and see also Fig. 6.4). Consequently, it is more difficult to detect small amplitude bumps for late-type Be stars.

2. Late-type Be stars tend to have more tenuous disks than early-type ones (Vieira 


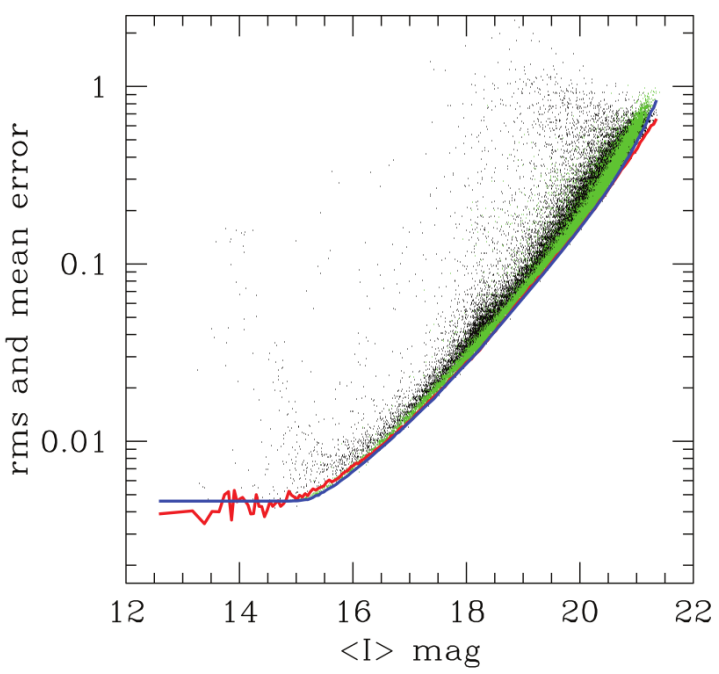

Figure 6.4: Mean error (green) and rms (black) for a sample of stars from one of the fields of the OGLE-II survey. (Credits: Wyrzykowski et al., 2009)

et al., 2017, see also next subsections), and therefore should develop bumps with much smaller amplitudes.

3. Late-type Be stars tend to show less variability (Rivinius et al., 2013, see also next subsections), which would make it less probable to identify bumps in their light curves.

\subsubsection{Asymptotic surface density}

In the upper panel of Fig. 6.5 we show how $\Sigma_{0}$ varies with stellar mass in our sample, demonstrating a clear tendency toward denser disks around the more massive stars. Vieira et al. (2017) have shown that, for the Be stars in the MW, the incidence of denser disks increases with the mass of the stars. Comparison of our results with the ones of Vieira et al. are shown in Fig. 6.6. While our sample is biased towards large masses, their sample is more evenly distributed in mass. Another difference is that our results are all concentrated in a region of high disk density, while theirs cover a much wider range of densities for all spectral types. The reason for this lies in the fact that for this initial study we selected light curves with large and well-defined bumps, disregarding low-amplitude and short-duration ones. In fact, the detection of tenuous disks by Vieira et al. was only possible because they studied the SED in the IR (typically between 9 and $60 \mu \mathrm{m}$ ), where the disk emission is much stronger than in the visible range. Therefore, all but the most dense of their disks would be too tenuous to generate appreciable photometric excesses in the visual photometric bands, suitable for our fitting procedure.

We conclude that our sample of visual bumps should represent the upper limit for the densities found in the disks of SMC Be stars. In the Milky Way (MW), these large 

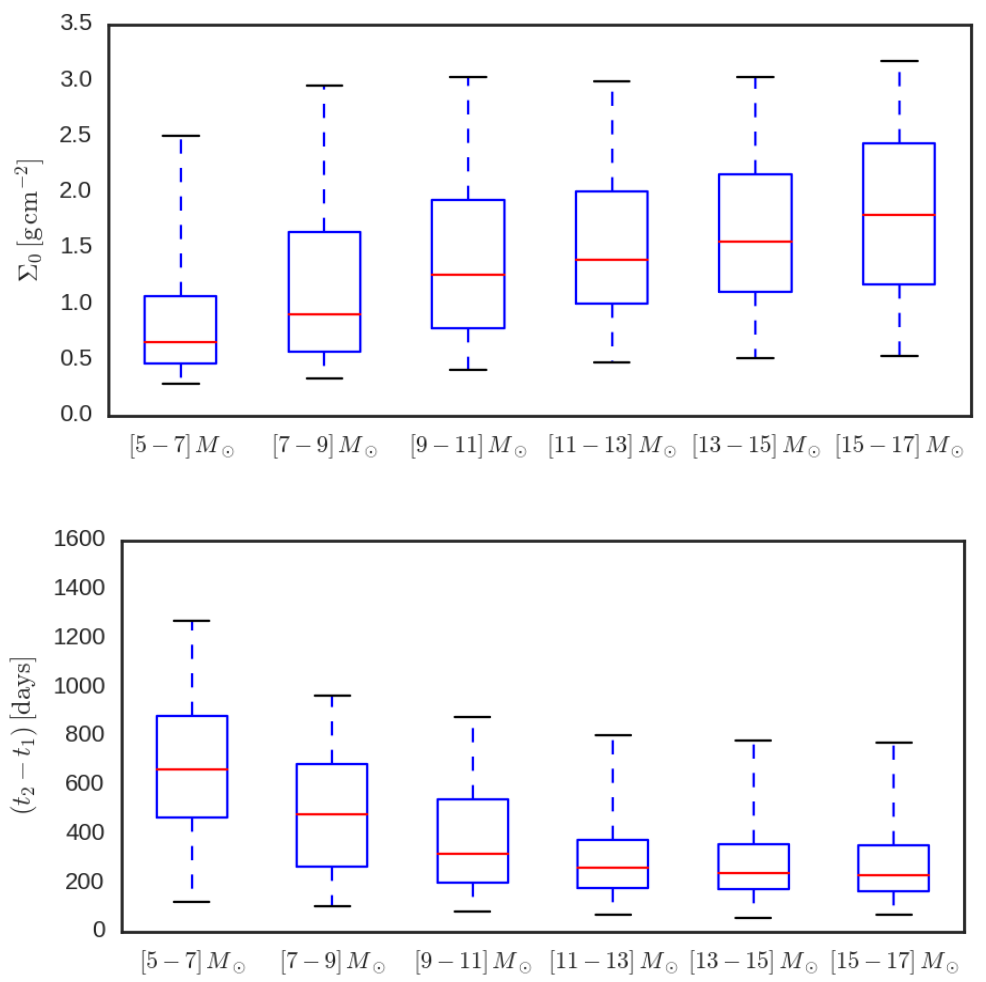

Figure 6.5: Boxplots of $\Sigma_{0}$ (above) and $t_{2}-t_{1}$ (below) for the summed posterior probabilities of our sample of bumps, separated in six equal intervals of mass, ranging from 5 to 17 solar masses. The middle line of the boxes mark the median (50\%) of the samples. The lower and upper ends of the boxes mark $25 \%$ and $75 \%$ of the samples. The lower and upper whiskers mark $5 \%$ and $95 \%$ of the samples.

densities are only found in early type Be stars. The median of the $\Sigma_{0}$ for our sample is $\left\langle\Sigma_{0}\right\rangle=1.44_{-0.75}^{+1.01} \mathrm{~g} \mathrm{~cm}^{-2}$. Furthermore, there may be some indication that the Be stars in the SMC may have more massive disks, on average, than their MW counterparts, in line with results from the literature that report higher $\mathrm{H} \alpha$ equivalent widths in the $\mathrm{SMC} \mathrm{Be}$ stars than in the MW (Martayan et al., 2007). This last point, however, should be viewed with some caution given the large biases present in our sample.

\subsubsection{Disk life cycles}

In the lower panel of Fig. 6.5, we plot the distribution of the build-up time, $t_{2}-t_{1}$, versus the stellar mass. We see that the duration of the bump is much shorter for massive stars, which indicates that these stars are much more variable than their late type siblings. Similar findings were reported in the MW (e.g. Rivinius et al., 2013). For a complete characterization of the disk life cycles we would require a full census of the number of bumps present during the timespan of OGLE-II and OGLE-III observations (roughly 12 years). Unfortunately, this cannot be done for our sample because in this study we selected only the well-defined bumps. The median of the build-up time for our sample is $\left\langle t_{2}-t_{1}\right\rangle=305_{-171}^{+351}$ days. 


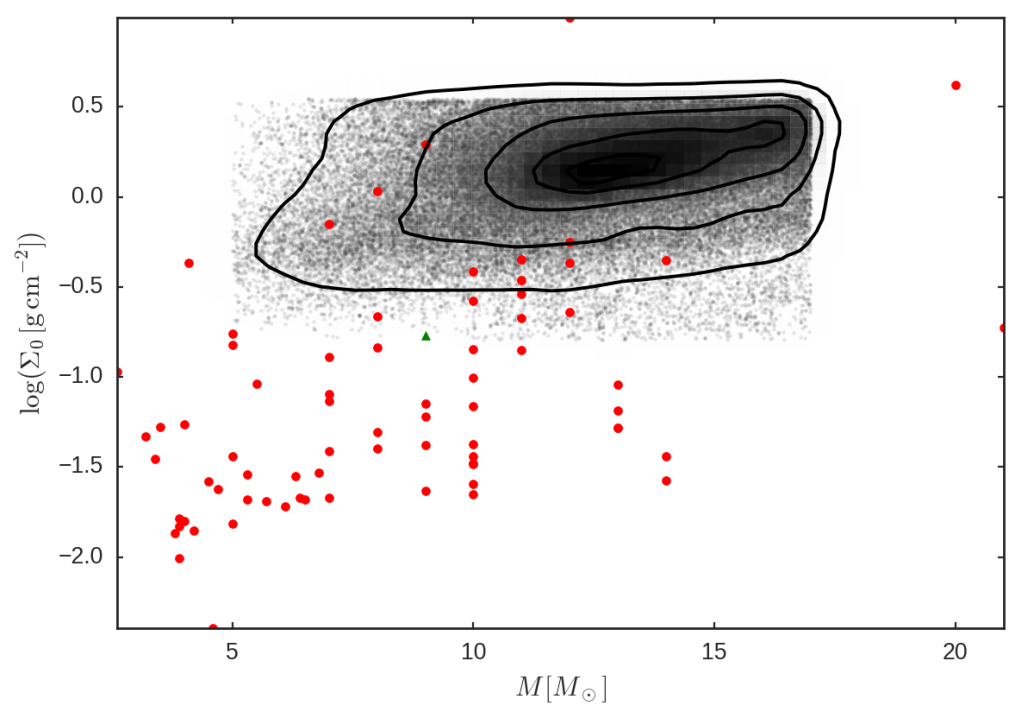

Figure 6.6: Distribution of the parameters $M$ and $\Sigma_{0}$ for our sample. The contour levels are the same as in Fig. 6.1. The red dots correspond to the surface densities at the base of the disk of MW Be stars, measured by Vieira et al. (2017). The green triangle corresponds to the initial state of the ablating disk model of Kee et al. (2016) for a B2e star.
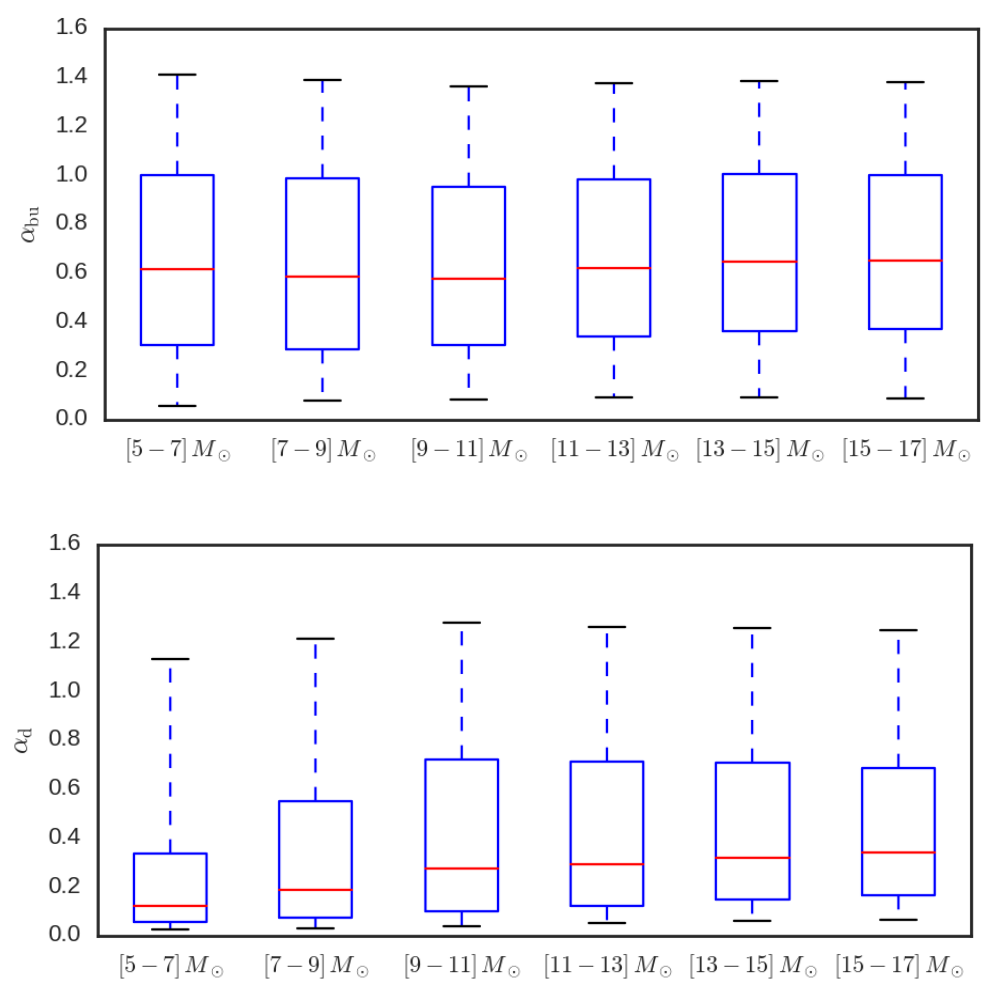

Figure 6.7: Same as Fig. 6.5 for $\alpha_{\text {bu }}$ (above) and $\alpha_{\mathrm{d}}$ (below). 


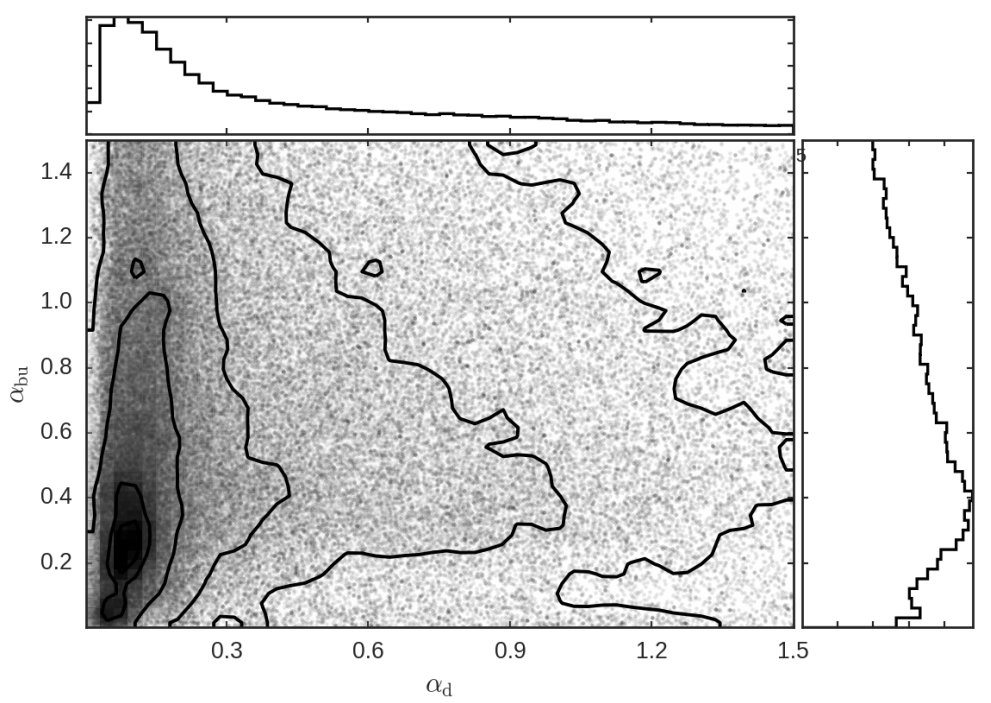

Figure 6.8: Distribution of the two viscosity parameters $\alpha_{\mathrm{bu}}$ and $\alpha_{\mathrm{d}}$. The histograms above and on the right are the projections for $\alpha_{\mathrm{d}}$ and $\alpha_{\mathrm{bu}}$, respectively. The contour levels are the same as in Fig. 6.1.

\subsubsection{Viscosity parameter}

The majority of our determinations of the viscosity parameter had broad uncertainty distributions, and it was found that the errors in the determinations of $\alpha_{\text {bu }}$ were generally greater than those of $\alpha_{\mathrm{d}}$. Considering the sample as a whole, we find that there is no variation of $\alpha_{\mathrm{bu}}$ with the stellar mass (Fig. 6.7, top), but there is a slight hint that $\alpha_{\mathrm{d}}$ may grow with the stellar mass (Fig. 6.7, bottom). Furthermore, we find that on average the viscosity parameter is roughly two times larger at build-up than at dissipation $\left(\left\langle\alpha_{\text {bu }}\right\rangle=0.63_{-0.39}^{+0.52}\right.$ vs. $\left.\left\langle\alpha_{\mathrm{d}}\right\rangle=0.29_{-0.20}^{+0.61}\right)$.

The estimated values of $\alpha$ in the range of a few tenths to one are in agreement with the usual values obtained for the hot and variable disks of dwarf novae (King et al., 2007; Kotko and Lasota, 2012). They are, however, an order of magnitude or more above the usual values obtained in magnetohydrodynamic (MHD) simulations, where the magnetorotational instability (MRI, Balbus and Hawley, 1991) is the main theoretical assumption for the mechanism that generates the necessary viscosity (King et al., 2007).

In Fig. 6.8, we show the distributions of $\alpha_{\mathrm{bu}}$ (right) and $\alpha_{\mathrm{d}}$ (above) and the distribution in the $\alpha_{\mathrm{bu}} \times \alpha_{\mathrm{d}}$ plane. We found that, for most of the bumps, there was a correlation between $\alpha_{\mathrm{bu}}$ and $\alpha_{\mathrm{d}}$, with values of $\alpha_{\mathrm{bu}}$ greater than values of $\alpha_{\mathrm{d}}$ being more likely. This trend can be seen in the darker areas of the $\alpha_{\mathrm{bu}} \times \alpha_{\mathrm{d}}$ plane. Ghoreyshi and Carciofi (2017) modeled several build-up and dissipation phases of the Be star $28 \mathrm{CMa}$ and found the same trend for that star.

It is unclear wether the the higher likelihood of $\alpha_{\mathrm{bu}}>\alpha_{\mathrm{d}}$ is real a phenomenon or a result of the approximations employed in this work. One key approximation made in our model is that the hydrodynamical equations are solved assuming that the entire disk 


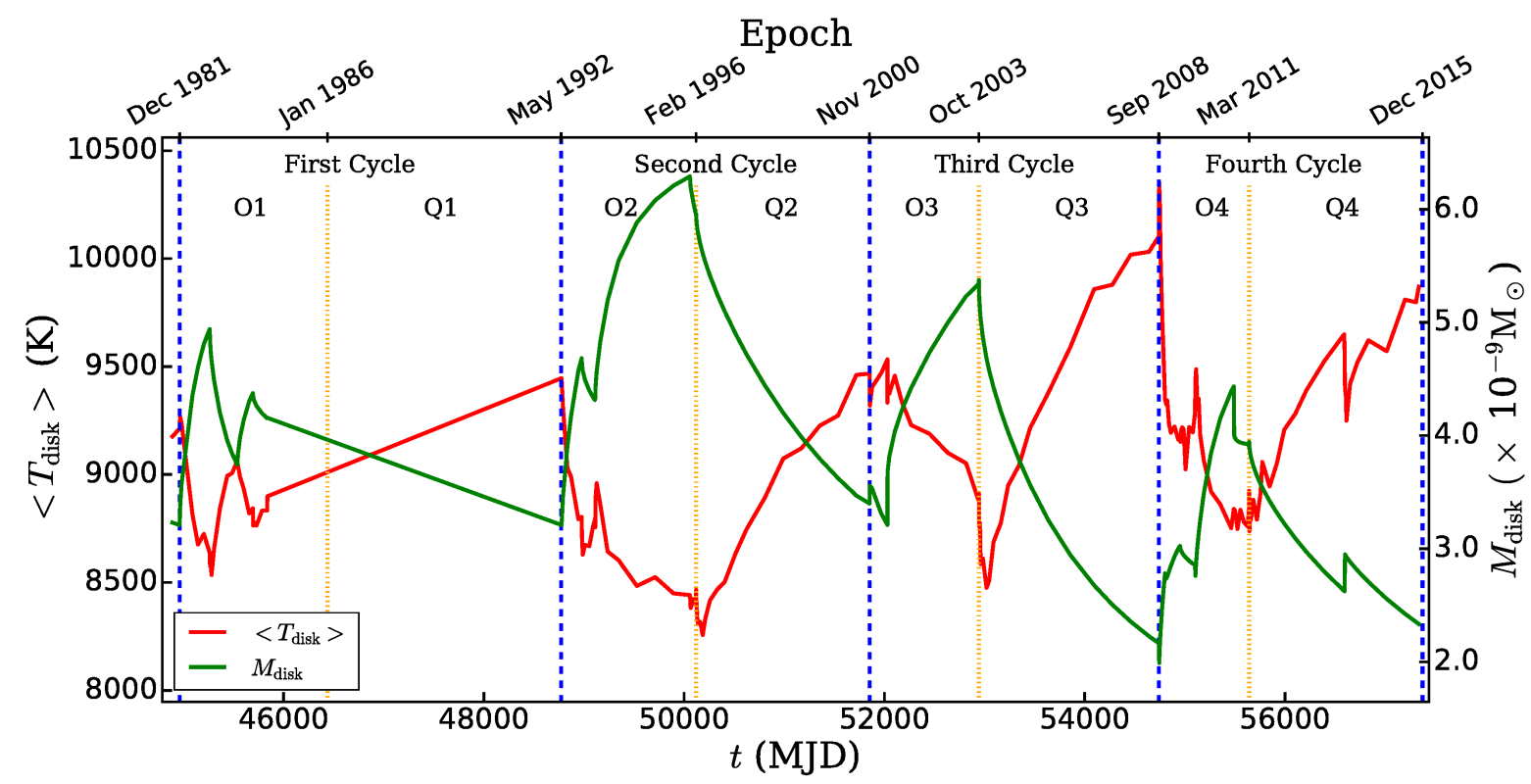

Figure 6.9: Evolution of the disk mass and mean disk temperature of the Be star 28 CMa, complementing the panels of Fig. 3.6. The green curve shows the total mass of the disk. The red curve shows the mean temperature of the disk, calculated by Eq. (6.2.1). (Credits: M. Ghoreyshi, priv. comm.)

is isothermal. Earlier studies, however, (e.g., Jones et al., 2004; Carciofi and Bjorkman, 2006) have shown the disk to be highly non-isothermal, which means that $c_{s}$ in Eq. 3.1.2 is a complicated function both of $R$ and time. There is not any strong correlation between the viscosity parameters and the temperatures of the disks, because in Fig. 6.7 we do not see strong correlations between the viscosity parameters and the mass of the stars. However, the fitted parameters $C_{\mathrm{bu}}$ and $C_{\mathrm{d}}$ are proportional to $\alpha_{\mathrm{bu}} c_{s}^{2}$ and $\alpha_{\mathrm{d}} c_{s}^{2}$, respectively (see Eqs. 3.1.13, 4.4.4, and 4.4.5). Therefore, if we erroneously understimated the temperature during build-up phase, characterized by a surface density that starts from zero and then increases, and overestimated the temperature during dissipation, where the density starts higher and begins to decrease, that might contribute to our determination of $\alpha_{\mathrm{bu}}>\alpha_{\mathrm{d}}$ being more likely.

The Fig. 6.9 was very recently generated by M. Ghoreyshi (priv. comm.). It must be compared with the modeling of the light curve of $28 \mathrm{CMa}$ in Fig. 3.6. The green curve shows the total mass of the disk of his dynamical model of the star 28 CMa. During the build-up phases, the mass of the disk increases and then decreases during the dissipation phases. The red curve shows the average temperature of the disk, given by

$$
\left\langle T_{\text {disk }}\right\rangle=\frac{\sum_{\text {cells }} \rho_{i} T_{i, \text { disk }} \delta V_{i}}{\sum_{\text {cells }} \rho_{i} \delta V_{i}},
$$

where the average was made over all the cells of the interaction region of HDUST. The volume of each cell is given by $\delta V_{i}$. 
The red curve, therefore, shows that, at the beginning of the build-up phases, the mean temperature of the disk is usually higher, and it decreases as time passes and the disk becomes denser, especially in the equatorial plane. Converselly, at the beginning of the dissipations, the mean temperature is usually lower, and it increases as time passes and the disk becomes tenuous. These observed variations in temperature, around $10-20 \%$, may help explaining physically why $\alpha_{\mathrm{bu}}>\alpha_{\mathrm{d}}$ was found to be more likely. It does not invalidate the conclusion that indeed $\alpha_{\mathrm{bu}}>\alpha_{\mathrm{d}}$, however.

Another approximation is that the possible effects of line forces were neglected. Recently, Kee et al. (2016) simulated the effects of a line-driving on the disks of early B-type stars. They showed that the line-driving was able to ablate and destroy the disk in timescales compatible with the observed large-amplitude photometric variations of Be stars. They argued that the presence of the line-driven ablation might be the cause of the apparent abnormal value estimated for $\alpha$ by Carciofi et al. (2012); Ghoreyshi and Carciofi (2017), and this work. Future work must explore if the viscous force and the line force working together can reproduce the variability of Be stars with smaller values of $\alpha$.

The line force acting on an outwardly diffusing near-Keplerian optically thin disk under irradiation by the hot star will have a positive component in the azimuthal direction, giving angular momentum to the gas (Gayley et al., 2001). It is possible that this additional source of angular momentum may help viscosity in pushing material outwards during the build-up phase and act to oppose to the reaccretion of the inner disk during dissipation, thus contributing to the observed trend of $\alpha_{\mathrm{bu}}>\alpha_{\mathrm{d}}$. We expect, however, that for our low-metallicity SMC Be stars, the possible effect of the line force will be greatly diminished. Furthermore, so far all studies of the effects of line forces in gaseous Keplerian disks assumed that the gas is optically thin, which is not the case for the inner parts of the disk, near the equatorial plane. The green triangle in Fig. 6.6 marks the mass and density at the stellar equator of the initial state of the ablating disk model of Kee et al. (2016) for a B2e star. Our calculations show that their initial state would generate only a modest excess $\Delta I=-0.1 \mathrm{mag}$, if seen pole-on.

On the other hand, it is possible that an opposite scenario might happen. The line force might operate to ablate the tenuous material above the disk plane. These regions would receive radiation from the stellar surface and radiation reprocessed by the optically thick disk, behaving as a sink of mass and angular momentum from the disk (e.g. Krtička et al., 2011). In that case, the line-driven wind would actually slow down the build-up phase, because it would take a longer time for the disk to reach a near steady-state, and would speed up the dissipation phase. If the above were true, that would result in $\alpha_{\mathrm{bu}}<\alpha_{\mathrm{d}}$, contrary to the results of Ghoreyshi and Carciofi (2017) and this work. 

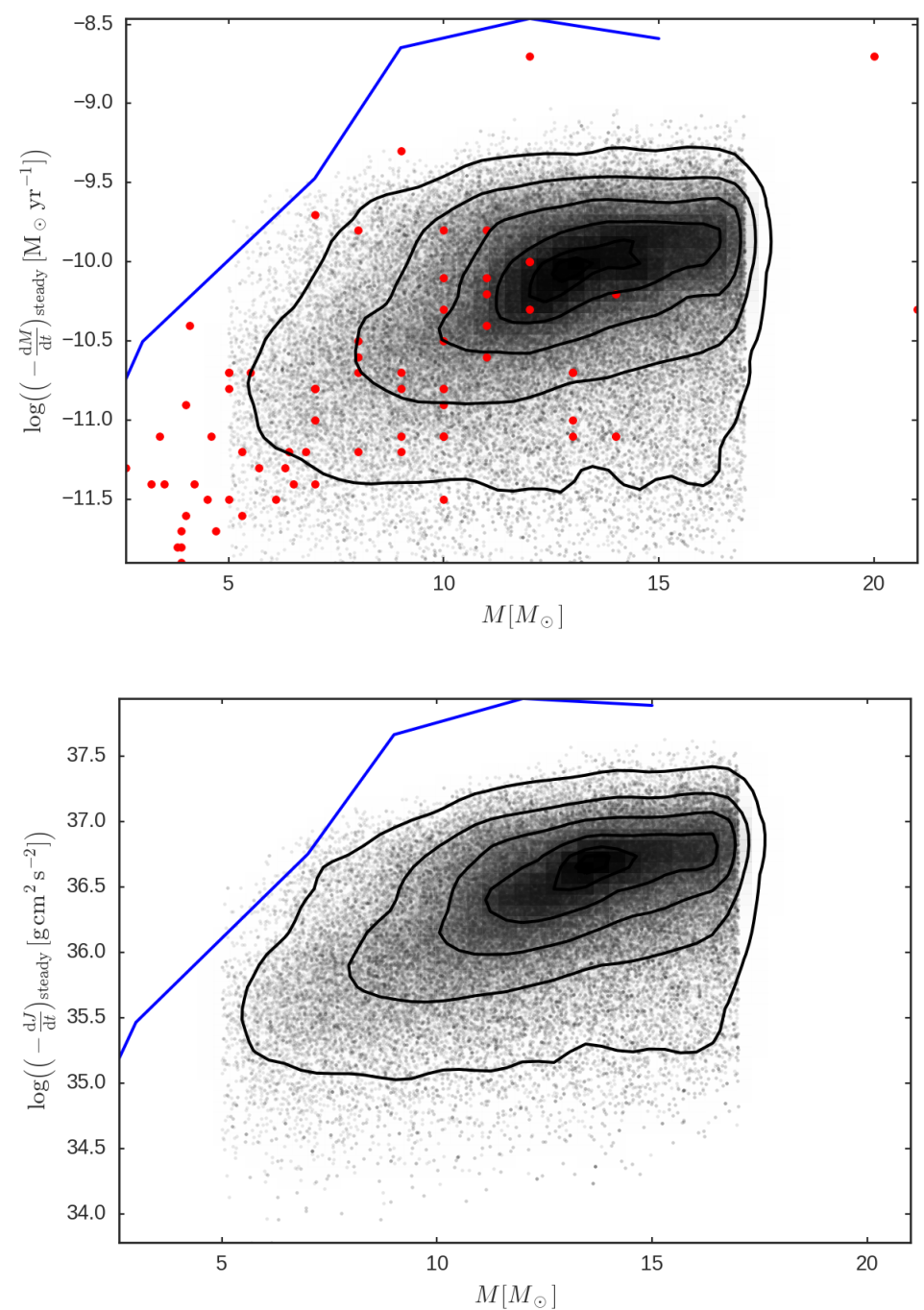

Figure 6.10: Distributions of the steady-state mass (above) and angular momentum (below) loss rates for our sample. The contour levels are the same as in Fig. 6.1. The red dots (upper panel) are the values of $(-\partial M / \partial t)_{\text {steady }}$, calculated from the results of Vieira et al. (2017) for MW Be stars. The blue curves are the estimations made by Granada et al. (2013) of the steady-state mass and angular momentum loss of their $Z=0.002$ stars during their episodes of disk formation.

\subsubsection{Mass and angular momentum loss}

Although the disks of Be stars in our sample are generally not in steady-state, their steady-state mass and angular momentum loss rates (Eqs. 3.2.17 and 3.2.18) are useful estimates of the actual quantities that are lost by the star after the bump event ends (see Sect 3.2). The panels of Fig. 6.10 show our distributions of the steady-state mass (above) and angular momentum (below) loss rates. For the calculation of $(-\partial M / \partial t)_{\text {steady }}$, we considered the radius of the outer boundary to be given by the radius at which the disk outflow becomes angular momentum conserving (which can be seen as an outer radius of the viscous disk), estimated as $\tilde{R}_{\text {out }}=0.3\left(v_{\text {orb }} / c_{s}\right)^{2}$ by Krtička et al. (2011). The red dots 
(upper panel) are the estimates of $(-\partial M / \partial t)_{\text {steady }}$ made from the results of Vieira et al. (2017) for MW Be stars, assuming that $\alpha=1$.

The steady-state mass and angular momentum loss rates for our densest bumps are of the order of $\sim 10^{-10} M_{\odot} \mathrm{yr}^{-1}$ and $\sim 5 \times 10^{36} \mathrm{~g} \mathrm{~cm}^{2} \mathrm{~s}^{-2}$, respectively. The typical decretion rate, which estimates the flux of mass in the disk near the stellar equator, is an order of magnitude higher than $(-\partial M / \partial t)_{\text {steady }}$, being of the order of $\sim 10^{-9} M_{\odot} \mathrm{yr}^{-1}$, which also corresponds to the upper limit of the observed wind mass loss rate of B stars (Snow, 1981; Puls et al., 2008). Values of the typical decretion rate and steady-state angular momentum loss rate are given in the eleventh and twelfth columns of Table 6.1, respectively. The total angular momentum lost by the star as a consequence of the bump, $-\Delta J_{*}$, is given by $(-\partial J / \partial t)_{\text {steady }}$ times the build-up time (Eq. 3.2.22), and the total mass lost is simply $-\Delta M_{*}=-\Delta J_{*} /\left(G M R_{\text {out }}\right)^{\frac{1}{2}}$, if we still approximate the VDD as a Keplerian disk at $R_{\text {out }}$.

If we consider that a typical bump has a build-up time of roughly one year, then the mass and angular momentum lost by the star as a consequence of one dense complete bump are of the order of $\sim 10^{-10} M_{\odot}$ and $\sim 10^{44} \mathrm{~g} \mathrm{~cm}^{2} \mathrm{~s}^{-1}$ (or $\sim 0.01$ Moon masses and $\sim 10^{-3}$ times Earth's orbital angular momentum around the Sun, respectively). The VDD is, thus, a physical mechanism capable of extracting a large quantity of angular momentum from the outer layers of the star, without requiring the loss of too much mass. It is, therefore, a rotational breaking mechanism for the outer layers. The angular momentum lost by the star for each individual bump is given in the thirteenth column of Table 6.1.

It has been proposed (e.g., Krtička et al., 2011) that, during the evolution of the star, the formation of the VDD might be a natural mechanism to extract angular momentum from the outer layers of the star, preventing them from exceding the break up velocity. Granada et al. (2013) assumed the appearance of a steady-state VDD in the Geneva stellar evolution code every time the outer layers of the star reached $W>0.88(\omega>0.99$, in their notation, where $\left.\omega=\Omega /\left(8 G M / 27 R_{\text {pole }}^{3}\right)^{\frac{1}{2}}\right)$. The blue curves in Fig. 6.10 are the estimates made by Granada et al. (2013) of the steady-state mass and angular momentum loss of their $Z=0.002$ stars during their episodes of disk formation. It is remarkable that our estimates of $(-\partial M / \partial t)_{\text {steady }}$ and $(-\partial J / \partial t)_{\text {steady }}$ made using our dynamical VDD models of bumps in the light curves of Be stars and the estimations of $(-\partial M / \partial t)_{\text {steady }}$ made using near-IR SED fitting by Vieira et al. (2017) are both similar to the estimates of these physical quantities made by Granada et al. (2013), by assuming that the disks are formed when a certain criterion of maximum surface angular velocity in the evolving stars is reached, though our results are still below (by an order of magnitude for $M \gtrsim 12 M_{\odot}$ ) their results. That means that, even if $\alpha=1$ in their disks, they will still have to be denser than ours in order for them to extract the required angular momentum from the star. Also, the red dots from are for the assumption of $\alpha=1$ in their VDDs, which means that they must be roughly the upper limits of their determinations of $(-\partial M / \partial t)_{\text {steady }}$. 
It is therefore an important fact that the compatibility of our three works require that $\alpha_{\mathrm{bu}} \sim 1$, which was obtained in our results.

The fact that the curves of Granada et al. (2013) lie up to one order of magnitude above our results and the ones of Vieira et al. suggests that their assumed disks were made much too dense to be able to remove the needed angular momentum. The evolutionary models of Granada et al. (2013) with the disk prescription only generated decretion disks if their initial rotation rate was $W_{\mathrm{ZAMS}}>0.66$ (or $\omega_{\mathrm{ZAMS}}>0.9$ ) and if $W>0.88$ was reached. That happened during $\lesssim 0.1 \%$ of the stars' life in the main sequence. The authors showed however that by lowering the maximum velocity criterion of one of their models to a maximum velocity that is satisfied by most Be stars, they could make the appearance of disks with a frequency more compatible with the fraction of Be stars found in clusters. The obtained mass and angular momentum loss rates didn't change appreciably from the obtained with previous models, however. This may lead us to believe that the lowering of the maximum velocity criterion may not explain why their required disks are denser than the observed ones.

The authors pointed that the inclusion of other mechanisms taking place inside the star (e.g., magnetic coupling between the core and the envelope) would lead to faster surface rotation, enhancing the integrated time of appearance of decretion disks. Our results, however, seem to point in the other direction. Maybe, the internal coupling assumed in their evolutionary models is too strong, resulting in an excessive transport of angular momentum from the contracting core to the outer layers, resulting in the necessity of creating a too dense VDD in order to extract angular momentum from the surface of the star and prevent it from breaking-up.

We conclude that, although the Be phenomenon is probably a powerful velocity breaking mechanism for the outer layers of the star, its effect on the star as a whole is expected to be modest. Our determinations of angular momentum loss show that, even if the Be phenomenon happened during $\sim 30 \%$ of the stellar main sequence lifetime, and the integrated time of all build-up phases were $\sim 30 \%$ of that time, that would lead to the removal of $\sim 1 \%$ of the initial angular momentum of the star, which, for fast rotating stars of masses from $7 M_{\odot}$ to $15 M_{\odot}$ is of $8-30 \times 10^{51} \mathrm{~g} \mathrm{~cm}^{2} \mathrm{~s}^{-1}$ (Granada et al., 2013). 
Table 6.1 - Results of the pipeline for each star and bump of the sample

\begin{tabular}{|c|c|c|c|c|c|c|c|c|c|c|c|c|}
\hline OGLE-II ID & $\begin{array}{r}\text { ump } \\
\text { ID }\end{array}$ & $M / M_{\odot}$ & $t / t_{\mathrm{MS}}$ & $W$ & $\cos i$ & $\begin{array}{r}\Sigma_{0} \\
{\left[\mathrm{~g} \mathrm{~cm}^{-2}\right]}\end{array}$ & $\alpha$ & $\alpha_{\mathrm{d}}$ & $\tilde{\tau}_{\mathrm{bu}}$ & $\begin{array}{r}\left(-\frac{\partial M}{\partial t}\right)_{\text {typ }} \\
{\left[10^{-9} \times\right.} \\
\left.M_{\odot} \mathrm{yr}^{-1}\right]\end{array}$ & $\begin{array}{r}\left(-\frac{\partial J}{\partial t}\right)_{\mathrm{std}} \\
{\left[10^{36} \times\right.} \\
\left.\mathrm{g} \mathrm{cm}^{2} \mathrm{~s}^{-2}\right]\end{array}$ & $\begin{array}{r}-\Delta J_{*} \\
{\left[10^{44} \times\right.} \\
\left.\mathrm{g} \mathrm{cm}^{2} \mathrm{~s}^{-1}\right]\end{array}$ \\
\hline \multirow[t]{2}{*}{ smc_sc1 7612} & 01 & $12.8_{-2.6}^{+2.6}$ & $0.5_{-0.3}^{+0.3}$ & $0.81_{-0.1}^{+0.11}$ & $0.36_{-0.04}^{+0.11}$ & $1.1_{-0.6}^{+1.3}$ & $0.33_{-0.24}^{+0.68}$ & $0.65_{-0.41}^{+0.54}$ & $0.15_{-0.11}^{+0.32}$ & $0.62_{-0.38}^{+0.65}$ & $1.09_{-0.68}^{+1.35}$ & $0.07_{-0.04}^{+0.08}$ \\
\hline & 02 & & & & & $1.3_{-0.7}^{+1.2}$ & $0.38_{-0.27}^{+0.64}$ & $0.62_{-0.4}^{+0.53}$ & $0.15_{-0.1}^{+0.28}$ & $0.77_{-0.45}^{+0.8}$ & $1.35_{-0.81}^{+1.66}$ & $0.07_{-0.04}^{+0.08}$ \\
\hline smc_sc1 60553 & 01 & $12.7_{-1.9}^{+2.8}$ & $0.7_{-0.3}^{+0.2}$ & $0.81_{-0.11}^{+0.1}$ & $0.66_{-0.1}^{+0.12}$ & $1.6_{-0.4}^{+0.7}$ & $1.25_{-0.25}^{+0.18}$ & $0.09_{-0.03}^{+0.04}$ & $2.76_{-0.57}^{+0.5}$ & $4.32_{-1.54}^{+2.2}$ & $8.24_{-3.31}^{+5.62}$ & $2.63_{-1.05}^{+1.73}$ \\
\hline smc_sc1 75701 & 01 & $14.7_{-2.3}^{+1.7}$ & $0.7_{-0.2}^{+0.2}$ & $0.81_{-0.1}^{+0.1}$ & $0.7_{-0.1}^{+0.15}$ & $1.9_{-0.4}^{+0.8}$ & $0.24_{-0.08}^{+0.18}$ & $0.11_{-0.05}^{+0.08}$ & $0.66_{-0.22}^{+0.48}$ & $1.15_{-0.52}^{+1.06}$ & $2.47_{-1.24}^{+2.62}$ & $1.03_{-0.52}^{+1.08}$ \\
\hline \multirow[t]{2}{*}{ smc_sc1 92262} & 01 & $12.7_{-1.8}^{+2.5}$ & $0.6_{-0.3}^{+0.2}$ & $0.8_{-0.09}^{+0.11}$ & $0.03_{-0.02}^{+0.03}$ & $1.5_{-0.9}^{+1.0}$ & $0.47_{-0.29}^{+0.6}$ & $0.84_{-0.38}^{+0.4}$ & $0.53_{-0.34}^{+0.63}$ & $1.07_{-0.56}^{+1.62}$ & $2.01_{-1.06}^{+2.83}$ & $0.31_{-0.16}^{+0.4}$ \\
\hline & 02 & & & & & $2.4_{-0.5}^{+0.4}$ & $0.99_{-0.23}^{+0.26}$ & $0.94_{-0.3}^{+0.35}$ & $1.2_{-0.37}^{+0.58}$ & $4.42_{-1.42}^{+2.72}$ & $8.36_{-3.24}^{+6.38}$ & $1.51_{-0.62}^{+1.01}$ \\
\hline smc_sc2 94939 & 01 & $12.2_{-2.3}^{+2.7}$ & $0.6_{-0.3}^{+0.2}$ & $0.81_{-0.11}^{+0.1}$ & $0.45_{-0.07}^{+0.13}$ & $2.4_{-0.8}^{+0.7}$ & $0.88_{-0.45}^{+0.42}$ & $0.69_{-0.33}^{+0.43}$ & $1.15_{-0.56}^{+0.53}$ & $3.44_{-1.75}^{+2.61}$ & $6.05_{-3.17}^{+5.42}$ & $1.06_{-0.56}^{+0.92}$ \\
\hline smc_sc3 5719 & 01 & $12.3_{-2.3}^{+2.7}$ & $0.6_{-0.3}^{+0.3}$ & $0.81_{-0.11}^{+0.1}$ & $0.38_{-0.05}^{+0.11}$ & $2.6_{-0.8}^{+0.5}$ & $0.72_{-0.33}^{+0.42}$ & $0.47_{-0.22}^{+0.36}$ & $3.83_{-1.72}^{+2.41}$ & $3.25_{-1.77}^{+2.3}$ & $5.73_{-3.18}^{+4.5}$ & $4.09_{-2.27}^{+3.25}$ \\
\hline \multirow[t]{3}{*}{ smc_sc3 15970} & 01 & $13.5_{-1.3}^{+1.5}$ & $0.8_{-0.2}^{+0.1}$ & $0.87_{-0.09}^{+0.07}$ & $0.63_{-0.03}^{+0.05}$ & $1.1_{-0.1}^{+0.2}$ & $0.89_{-0.45}^{+0.31}$ & $0.13_{-0.04}^{+0.05}$ & $1.64_{-0.79}^{+0.67}$ & $2.52_{-1.14}^{+1.15}$ & $5.31_{-2.54}^{+3.1}$ & $1.55_{-0.74}^{+0.95}$ \\
\hline & 02 & & & & & $1.4_{-0.2}^{+0.2}$ & $0.85_{-0.31}^{+0.29}$ & $0.17_{-c}^{+c}$ & $1.92_{-0.7}^{+0.77}$ & $2.89_{-1.03}^{+1.38}$ & $6.17_{-2.52}^{+3.61}$ & $2.24_{-0.92}^{+1.22}$ \\
\hline & 03 & & & & & $1.2_{-0.1}^{+0.2}$ & $1.1_{-0.3}^{+0.25}$ & $0.12_{-0.05}^{+0.05}$ & $2.62_{-0.9}^{+0.83}$ & $3.27_{-1.12}^{+1.37}$ & $6.97_{-2.73}^{+3.75}$ & $2.62_{-1.18}^{+1.66}$ \\
\hline \multirow[t]{2}{*}{ smc_sc3 71445} & 01 & $11.2_{-2.3}^{+2.7}$ & $0.5_{-0.3}^{+0.3}$ & $0.81_{-0.1}^{+0.1}$ & $0.4 e_{-0.06}$ & $2.5_{-0.6}^{+0.6}$ & $0.57_{-0.25}^{+0.41}$ & $0.75_{-0.31}^{+0.37}$ & $1.11_{-0.48}^{+0.8}$ & $2.14_{-1.0}^{+1.74}$ & $3.42_{-1.7}^{+3.2}$ & $0.83_{-0.41}^{+0.77}$ \\
\hline & 02 & & & & & $1.4_{-0.5}^{+0.7}$ & $0.65_{-0.34}^{+0.53}$ & $1.05_{-0.38}^{+0.31}$ & $0.47_{-0.24}^{+0.41}$ & $1.38_{-0.61}^{+0.91}$ & $2.19_{-1.02}^{+1.74}$ & $0.2_{-0.09}^{+0.17}$ \\
\hline smc_sc3 125899 & 01 & $13.4_{-2.4}^{+2.3}$ & $0.6_{-0.3}^{+0.2}$ & $0.82_{-0.11}^{+0.1}$ & $0.37_{-0}^{+0}$ & $1.7_{-0.7}^{+0.9}$ & $0.7_{-0.44}^{+0.54}$ & $0.41_{-0.22}^{+0.33}$ & $0.89_{-0.53}^{+0.7}$ & $2.14_{-1.32}^{+2.11}$ & $4.04_{-2.55}^{+4.74}$ & $0.73_{-0.46}^{+0.88}$ \\
\hline smc_sc3 197941 & 01 & $12.0_{-1.8}^{+2.5}$ & $0.8_{-0.2}^{+0.2}$ & $0.82_{-0.11}^{+0.1}$ & $0.57_{-0.05}^{+0.15}$ & $0.8_{-0.2}^{+0.3}$ & $0.4_{-0.23}^{+0.52}$ & $0.1_{-0}^{+0}$ & $1.43_{-0.81}^{+1.79}$ & $0.69_{-0.38}^{+0.82}$ & $1.3_{-0.75}^{+1.69}$ & $0.66_{-0.38}^{+0.85}$ \\
\hline smc_sc4 22859 & 01 & $9.5_{-2.1}^{+2.5}$ & $0.4_{-0.3}^{+0.3}$ & $0.81_{-0.11}^{+0.1}$ & $0.64_{-0.1}^{+0.19}$ & $0.4_{-0.1}^{+0.2}$ & $0.66_{-0.51}^{+0.57}$ & $0.04_{-0}^{+0}$ & $3.5_{-2.71}^{+3.15}$ & $0.27_{-0.19}^{+0.26}$ & $0.35_{-0.25}^{+0.42}$ & $0.21_{-0.15}^{+0.25}$ \\
\hline \multirow[t]{2}{*}{ smc_sc4 71499} & 01 & $14.5_{-2.2}^{+1.8}$ & $0.7_{-0.2}^{+0.2}$ & $0.82_{-0.09}^{+0.1}$ & $0.54_{-0.03}^{+0.07}$ & $1.3_{-0.3}^{+0.5}$ & $0.7_{-0.35}^{+0.46}$ & $0.25_{-0.08}^{+0.11}$ & $0.96_{-0.47}^{+0.66}$ & $2.15_{-0.94}^{+1.3}$ & $4.6_{-2.26}^{+3.3}$ & $0.94_{-0.46}^{+0.67}$ \\
\hline & 02 & & & & & $1.5_{-0.3}^{+0.5}$ & $0.64_{-0.34}^{+0.47}$ & $0.19_{-0.06}^{+0.06}$ & $1.16_{-0.63}^{+0.83}$ & $2.18_{-1.0}^{+1.77}$ & $4.59_{-2.23}^{+4.38}$ & $1.26_{-0.61}^{+1.19}$ \\
\hline smc_sc4 120783 & 01 & $15.2_{-1.8}^{+1.2}$ & $0.9_{-0.1}^{+0.1}$ & $0.84_{-0.1}^{+0.09}$ & $0.64_{-0.11}^{+0.2}$ & $\begin{array}{l}0.7_{-0.2}^{+0.3} \\
\end{array}$ & $0.63_{-0.43}^{+0.54}$ & $0.11_{-0.05}^{+0.06}$ & $1.56_{-1.06}^{+1.33}$ & $1.47_{-0.93}^{+1.37}$ & $3.67_{-2.41}^{+3.63}$ & $1.53_{-1.01}^{+1.46}$ \\
\hline 4127840 & 01 & $14.3_{-1.8}^{+1.7}$ & $0.9_{-0.2}^{+0.1}$ & $0.83_{-0.11}^{+0.09}$ & $0.6_{-0.09}^{+0.15}$ & $2.6_{-0.6}^{+0.5}$ & $0.4_{-0.15}^{+0.23}$ & $0.85_{-0.41}^{+0.4}$ & $0.54_{-0.19}^{+0.3}$ & $3.19_{-1.53}^{+2.4}$ & $7.35_{-3.72}^{+6.28}$ & $1.6_{-0.8}^{+1.3}$ \\
\hline smc_sc4 156248 & 01 & $12.3_{-1.9}^{+2.8}$ & $0.7_{-0.3}^{+0.2}$ & $0.81_{-0.1}^{+0.09}$ & $0.07_{-0}^{+0 .}$ & $1.3_{-0.9}^{+1.4}$ & $0.5_{-0.34}^{+0.73}$ & $0.78_{-0.41}^{+0.43}$ & $1.44_{-1.0}^{+1.98}$ & $0.83_{-0.56}^{+3.31}$ & $1.6_{-1.07}^{+6.2}$ & $0.63_{-0.43}^{+2.39}$ \\
\hline \multirow[t]{3}{*}{ smc_sc4 156251} & 01 & $15.3_{-1.7}^{+1.2}$ & $0.7_{-0.2}^{+0.1}$ & $0.83_{-0.1}^{+0.1}$ & $0.76_{-0.07}^{+0.08}$ & $2.2_{-0.4}^{+0.4}$ & $0.4_{-0.11}^{+0.16}$ & 0.78 & $0.37_{-0.1}^{+0.14}$ & $2.52_{-0.91}^{+1.17}$ & $5.83_{-2.42}^{+3.14}$ & $0.86_{-0.37}^{+0.48}$ \\
\hline & 02 & & & & & $1.5_{-0.2}^{+0.2}$ & $0.65_{-0.18}^{+0.29}$ & 0.61 & $0.62_{-0.16}^{+0.25}$ & $2.7_{-0.98}^{+1.46}$ & $6.27_{-2.6}^{+3.88}$ & $0.94_{-0.39}^{+0.57}$ \\
\hline & 03 & & & & & $2.5_{-0.4}^{+0.4}$ & $0.8_{-0.33}^{+0.18}$ & $0.49_{-0.13}^{+0.18}$ & $0.96_{-0.36}^{+0.47}$ & $5.27_{-2.16}^{+2.84}$ & $11.98_{-5.13}^{+7.6}$ & $2.31_{-0.92}^{+1.45}$ \\
\hline \multirow[t]{2}{*}{ smc_sc4 159829} & 01 & $13.1_{-2.3}^{+2.6}$ & $0.6_{-0.3}^{+0.3}$ & $0.8_{-0.1}^{+0.11}$ & $0.61_{-0.07}^{+0.1}$ & $2.0_{-0.5}^{+0.7}$ & $1.07_{-0.34}^{+0.28}$ & $0.25_{-0.08}^{+0.1}$ & $1.47_{-0.48}^{+0.48}$ & $3.91_{-1.44}^{+1.91}$ & $7.21_{-2.97}^{+4.56}$ & $1.37_{-0.56}^{+0.84}$ \\
\hline & 02 & & & & & & $0.96_{-0.36}^{+0.36}$ & & $2.26_{-0.77}^{+0.84}$ & $3.64_{-1.56}^{+1.99}$ & $6.74_{-3.15}^{+4.54}$ & $2.18_{-1.0}^{+1.49}$ \\
\hline
\end{tabular}


Table 6.1 - continued.

\begin{tabular}{|c|c|c|c|c|c|c|c|c|c|c|c|c|}
\hline OGLE-II ID & $\begin{array}{r}\mathrm{ump} \\
\text { ID }\end{array}$ & $M / M_{\odot}$ & $t / t_{\mathrm{MS}}$ & $W$ & $\cos i$ & $\begin{array}{r}\Sigma_{0} \\
{\left[\mathrm{~g} \mathrm{~cm}^{-2}\right]}\end{array}$ & $\alpha_{\mathrm{bu}}$ & $\alpha_{\mathrm{d}}$ & $\tilde{\tau}_{\mathrm{bu}}$ & $\begin{array}{r}\left(-\frac{\partial M}{\partial t}\right)_{\text {typ }} \\
{\left[10^{-9} \times\right.} \\
\left.M_{\odot} \mathrm{yr}^{-1}\right]\end{array}$ & $\begin{array}{r}\left(-\frac{\partial J}{\partial t}\right)_{\text {std }} \\
{\left[10^{36} \times\right.} \\
\left.\mathrm{g} \mathrm{cm}^{2} \mathrm{~s}^{-2}\right]\end{array}$ & $\begin{array}{r}-\Delta J_{*} \\
{\left[10^{44} \times\right.} \\
\left.\mathrm{g} \mathrm{cm}^{2} \mathrm{~s}^{-1}\right] \\
\end{array}$ \\
\hline \multirow[t]{2}{*}{ smc_sc4 159857} & 01 & $13.7_{-2.3}^{+1.9}$ & $0.7_{-0}^{+0}$ & $0.81_{-0.1}^{+0.1}$ & $0.55_{-0.03}^{+0.08}$ & $0.6_{-0.2}^{+1.0}$ & $0.42_{-0.32}^{+0.64}$ & $0.66_{-0.35}^{+0.46}$ & $0.28_{-0.22}^{+0.45}$ & $0.62_{-0.36}^{+0.5}$ & $1.22_{-0.75}^{+1.15}$ & $0.12_{-0.07}^{+0.11}$ \\
\hline & 02 & & & & & $1.0_{-0.2}^{+0.6}$ & $0.7_{-0.43}^{+0.48}$ & $0.34_{-0.16}^{+0.56}$ & $0.42_{-0.27}^{+0.28}$ & $1.38_{-0.65}^{+0.94}$ & $2.7_{-1.36}^{+2.24}$ & $0.22_{-0.11}^{+0.19}$ \\
\hline smc_sc4 163828 & 01 & $9.7_{-2.0}^{+2.4}$ & $0.4_{-0.3}^{+0.4}$ & $0.79_{-0.1}^{+0.11}$ & $0.71_{-0.08}^{+0.14}$ & $0.7_{-0.1}^{+0.2}$ & $0.53_{-0.33}^{+0.58}$ & $0.06_{-0.02}^{+0.05}$ & $3.42_{-2.13}^{+3.83}$ & $0.43_{-0.25}^{+0.5}$ & $0.59_{-0.36}^{+0.78}$ & $0.44_{-0.26}^{+0.58}$ \\
\hline \multirow[t]{2}{*}{ smc_sc4 167554} & 01 & $9.9_{-2.1}^{+2.4}$ & $0.4_{-0.2}^{+0.3}$ & $0.82_{-0.1}^{+0.1}$ & $0.63_{-0.09}^{+0.12}$ & $0.9_{-0.3}^{+0.7}$ & $0.54_{-0.36}^{+0.51}$ & $0.6_{-0.29}^{+0.45}$ & $0.45_{-0.3}^{+0.42}$ & $0.59_{-0.31}^{+0.46}$ & $0.82_{-0.46}^{+0.76}$ & $0.08_{-0.04}^{+0.08}$ \\
\hline & 02 & & & & & $1.6_{-0.5}^{+0.7}$ & $0.42_{-0}^{+0}$ & $0.86_{-0}^{+0}$ & $0.42_{-0.2}^{+0.5}$ & $0.86_{-0.41}^{+0.82}$ & $1.19_{-0.61}^{+1.44}$ & $0.14_{-0.07}^{+0.15}$ \\
\hline \multirow[t]{2}{*}{ smc_sc4 171253} & 01 & $14.3_{-2.5}^{+1.9}$ & $0.6_{-0.3}^{+0.2}$ & $0.81_{-0.11}^{+0.11}$ & $0.58_{-0 .}^{+0.1}$ & $0.3_{-0.1}^{+1.2}$ & $0.29_{-0}^{+0}$ & $0.81_{-0}^{+0}$ & $0.24_{-0.23}^{+0.6}$ & $0.16_{-0.12}^{+0.3}$ & $0.32_{-0.23}^{+0.65}$ & $0.04_{-0.03}^{+0.07}$ \\
\hline & 02 & & & & & $0.3_{-0.1}^{+0.8}$ & $0.48_{-0}^{+0}$ & $0.64_{-0.42}^{+0.52}$ & $0.31_{-0.29}^{+0.45}$ & $0.29_{-0.2}^{+0.32}$ & $0.58_{-0.4}^{+0.7}$ & $0.05_{-0.04}^{+0.06}$ \\
\hline smc_sc4 175272 & 01 & $12.1_{-2.4}^{+2.8}$ & $0.5_{-0}^{+0}$ & 0.81 & $\overline{0}$ & $1.8_{-0.7}^{+0.8}$ & $0.75_{-0}^{+0}$ & 0.48 & $2.31_{-1.21}^{+1.52}$ & $2.07_{-1.16}^{+1.73}$ & $3.52_{-2.05}^{+3.33}$ & $1.37_{-0.8}^{+1.31}$ \\
\hline smc_sc4 179053 & 01 & $12.6_{-2.3}^{+2.6}$ & $0.6_{-0.4}^{+0.3}$ & $0.8_{-0.11}^{+0.11}$ & $0.02_{-0}^{+0}$ & $2.1_{-0.6}^{+0.7}$ & $0.96_{-0.52}^{+0.37}$ & $1.1_{-0.32}^{+0.27}$ & $2.14_{-1.16}^{+1.11}$ & $3.1_{-1.32}^{+1.84}$ & $5.5_{-2.58}^{+4.29}$ & $1.74_{-0.86}^{+1.19}$ \\
\hline smc_sc5 11453 & 01 & $13.6_{-2.3}^{+2.2}$ & $0.7_{-0.3}^{+0.2}$ & $0.81_{-0.11}^{+0.11}$ & $0.38_{-0}^{+0}$ & $1.8_{-0.7}^{+0.7}$ & $0.9_{-0.4}^{+0.38}$ & $0.26_{-0.11}^{+0.15}$ & $4.17_{-1.74}^{+1.82}$ & $3.3_{-1.81}^{+2.78}$ & $6.49_{-3.7}^{+6.33}$ & $4.41_{-2.51}^{+4.36}$ \\
\hline \multirow[t]{4}{*}{ smc_sc5 21117} & 01 & $13.5_{-1.7}^{+1.9}$ & $0.7_{-0.2}^{+0.1}$ & $0.84_{-0.1}^{+0.09}$ & $0.52_{-0.02}^{+0.04}$ & $1.3_{-0.2}^{+0.3}$ & $0.5_{-0.17}^{+0.32}$ & $0.61_{-0.32}^{+0.48}$ & $0.64_{-0.23}^{+0.39}$ & $1.46_{-0.57}^{+1.04}$ & $2.97_{-1.34}^{+2.63}$ & $0.56_{-0.26}^{+0.47}$ \\
\hline & 02 & & & & & $1.8_{-0.4}^{+0.5}$ & $0.58_{-0.26}^{+0.4}$ & $0.17_{-0.05}^{+0.08}$ & $0.85_{-0.38}^{+0.55}$ & $2.44_{-1.04}^{+1.63}$ & $4.99_{-2.39}^{+3.88}$ & $1.08_{-0.51}^{+0.78}$ \\
\hline & 03 & & & & & $1.9_{-0.5}^{+0.6}$ & $0.57_{-0.19}^{+0.28}$ & $0.35_{-0.13}^{+0.23}$ & $0.57_{-0.18}^{+0.28}$ & $2.53_{-1.01}^{+1.34}$ & $5.13_{-2.37}^{+3.52}$ & $0.76_{-0.35}^{+0.54}$ \\
\hline & 04 & & & & & $1.3_{-0.2}^{+0.2}$ & $0.87_{-0.3}^{+0.36}$ & $0.16_{-0.05}^{+0.07}$ & $3.11_{-1.03}^{+1.26}$ & $2.65_{-1.09}^{+1.36}$ & $5.36_{-2.46}^{+3.51}$ & $2.83_{-1.29}^{+1.95}$ \\
\hline smc_sc5 21134 & 01 & $12.7_{-2.4}^{+2.5}$ & $0.6_{-0.3}^{+0.2}$ & $0.8_{-0.09}^{+0.11}$ & $0.38_{-0 .}^{+0 .}$ & $0.8_{-0.4}^{+1.4}$ & $0.4_{-0.33}^{+0.68}$ & $0.18_{-0.11}^{+0.26}$ & $0.34_{-0.28}^{+0.59}$ & $0.54_{-0.36}^{+0.58}$ & $0.97_{-0.66}^{+1.22}$ & $0.12_{-0.08}^{+0.15}$ \\
\hline \multirow[t]{2}{*}{ smc_sc5 32377} & 01 & $13.3_{-2.2}^{+2.3}$ & $0.7_{-0.3}^{+0.2}$ & $0.81_{-0.11}^{+0.1}$ & $0.34_{-0.03}^{+0.06}$ & $1.9_{-0.7}^{+0.9}$ & $0.71_{-0.4}^{+0.45}$ & $0.66_{-0.33}^{+0.48}$ & $0.3_{-0.17}^{+0.28}$ & $2.53_{-1.38}^{+2.18}$ & $4.93_{-2.77}^{+4.81}$ & $0.31_{-0.17}^{+0.3}$ \\
\hline & 02 & & & & & $2.2_{-0.6}^{+0.8}$ & $0.51_{-0.24}^{+0.41}$ & $0.32_{-0.12}^{+0.15}$ & $0.84_{-0.38}^{+0.69}$ & $2.31_{-1.12}^{+1.89}$ & $4.49_{-2.34}^{+4.36}$ & $1.08_{-0.56}^{+1.03}$ \\
\hline \multirow[t]{2}{*}{ smc_sc5 43650} & 01 & $10.7_{-2.6}^{+3.0}$ & $0.4_{-0.3}^{+0.3}$ & $0.81_{-0.11}^{+0.1}$ & $0.55_{-0.04}^{+0.09}$ & $0.5_{-0.2}^{+1.2}$ & $0.35_{-0.3}^{+0.75}$ & $0.39_{-0.3}^{+0.71}$ & $0.61_{-0.53}^{+1.32}$ & $0.24_{-0.16}^{+0.33}$ & $0.35_{-0.23}^{+0.59}$ & $0.07_{-0.05}^{+0.12}$ \\
\hline & 02 & & & & & $0.6_{-0.2}^{+0.2}$ & $0.72_{-0.53}^{+0.53}$ & $0.53_{-0.42}^{+0.67}$ & $6.35_{-4.65}^{+4.94}$ & $0.5_{-0.33}^{+0.45}$ & $0.71_{-0.46}^{+0.83}$ & $0.74_{-0.47}^{+0.92}$ \\
\hline \multirow[t]{3}{*}{ smc_sc5 54851} & 01 & $11.7_{-1.5}^{+1.6}$ & $0.7_{-0.1}^{+0.1}$ & $0.78_{-0.08}^{+0.1}$ & $0.62_{-0.01}^{+0.04}$ & $1.3_{-0.2}^{+0.3}$ & $0.38_{-0.14}^{+0.35}$ & $0.11_{-0.03}^{+0.04}$ & $0.82_{-0.3}^{+0.73}$ & $1.06_{-0.42}^{+0.08}$ & $1.95_{-0.9}^{+1.69}$ & $0.59_{-0.28}^{+0.5}$ \\
\hline & 02 & & & & & & $0.48_{-c}^{+c}$ & $0.12_{-0}^{+0}$ & $\begin{array}{l}-0.3 \\
1.19_{-0.54}^{+0.99}\end{array}$ & $1.28_{-0.58}^{+1.07}$ & $\begin{array}{c}-0.9 \\
2.33_{-1.18}^{+2.28}\end{array}$ & $\begin{array}{r}-0.28 \\
0.8_{-0.41}^{+0.79}\end{array}$ \\
\hline & 03 & & & & & $\begin{array}{l}-0.2 \\
-0.1\end{array}$ & ${ }_{-0.2}^{+0.25}$ & $\begin{array}{l}+0.01 \\
+0.01\end{array}$ & $\begin{array}{l}+0.99 \\
+0.8\end{array}$ & $1.12_{-0.49}^{+0.07}$ & $2.04_{-0.97}^{+1.52}$ & $1.19_{-0.57}^{+0.88}$ \\
\hline smc_sc5 65500 & 01 & $12.1_{-2.4}^{+2.9}$ & $0.6_{-0.3}^{+0.2}$ & $0.81_{-0.1}^{+0.1}$ & 0.71 & $1.7_{-0.4}^{+0.6}$ & $0.7_{-0.34}^{+0.38}$ & $0.26_{-0.1}^{+0.13}$ & $0.44_{-0.19}^{+0.24}$ & $2.07_{-1.02}^{+1.66}$ & $3.7_{-2.05}^{+3.62}$ & $0.32_{-0.17}^{+0.31}$ \\
\hline smc_sc5 129535 & 01 & $\begin{array}{r}-2.4 \\
9.4_{-2.0}^{+2.2}\end{array}$ & $\begin{array}{l}-0.3 \\
0.5_{-0.3}^{+0.3}\end{array}$ & $0.81_{-0.11}^{+0.1}$ & $0.58_{-0}^{+0}$ & $0.7_{-0.2}^{+0.2}$ & $\begin{array}{r}-0.04 \\
0.65_{-0.37}^{+0.52}\end{array}$ & $0.25_{-0.13}^{+0.36}$ & $\begin{array}{r}-0.19 \\
3.88_{-2.29}^{+3.02}\end{array}$ & $0.58_{-0.32}^{+0.54}$ & $0.8_{-0.46}^{+0.86}$ & $0.56_{-0.33}^{+0.6}$ \\
\hline smc_sc5 145724 & 01 & $9.2_{-1.8}^{+2.1}$ & $0.5_{-0.3}^{+0.3}$ & $0.81_{-0.1}^{+0.1}$ & $0.4_{-0}^{+0}$ & $2.6_{-1.0}^{+0.7}$ & $0.88_{-0.46}^{+0.4}$ & $0.16_{-0.08}^{+0.19}$ & $3.87_{-1.98}^{+1.89}$ & $\begin{array}{r}-0.02 \\
2.6_{-1.55}^{+2.03}\end{array}$ & $\begin{array}{r}-0.40 \\
3.46_{-2.12}^{+3.23}\end{array}$ & $\begin{array}{c}-0.33 \\
1.79_{-1.11}^{+1.7}\end{array}$ \\
\hline smc_sc5 180034 & 01 & $12.6_{-2.6}^{+2.6}$ & $\begin{array}{l}-0.3 \\
0.4_{-0.3}^{+0.3}\end{array}$ & $\begin{array}{r}-0.1 \\
0.8_{-0.1}^{+0.1}\end{array}$ & $\begin{array}{r}-0.00 \\
0.62_{-0.09}^{+0.17}\end{array}$ & $1.0_{-0.3}^{+0.5}$ & $\begin{array}{r}-0.40 \\
0.4_{-0.25}^{+0.65}\end{array}$ & $\begin{array}{r}-0.00 \\
0.22_{-0.09}^{+0.17}\end{array}$ & $0.97_{-0.57}^{+1.54}$ & $0.68_{-0.4}^{+0.81}$ & $1.17_{-0.71}^{+1.57}$ & $0.36_{-0.22}^{+0.47}$ \\
\hline
\end{tabular}


Table 6.1 - continued.

\begin{tabular}{|c|c|c|c|c|c|c|c|c|c|c|c|c|}
\hline OGLE-II ID & $\begin{array}{r}\mathrm{ump} \\
\text { ID }\end{array}$ & $M / M_{\odot}$ & $t / t_{\mathrm{N}}$ & $W$ & $\cos i$ & $\begin{array}{r}\Sigma_{0} \\
{\left[\mathrm{~g} \mathrm{~cm}^{-2}\right]}\end{array}$ & $\alpha_{\mathrm{bu}}$ & $\alpha_{\mathrm{d}}$ & $\tilde{\tau}_{\mathrm{bu}}$ & $\begin{array}{r}\left(-\frac{\partial M}{\partial t}\right)_{\text {typ }} \\
{\left[10^{-9} \times\right.} \\
\left.M_{\odot} \mathrm{yr}^{-1}\right]\end{array}$ & $\begin{array}{r}\left(-\frac{\partial J}{\partial t}\right)_{\mathrm{std}} \\
{\left[10^{36} \times\right.} \\
\left.\mathrm{g} \mathrm{cm}^{2} \mathrm{~s}^{-2}\right]\end{array}$ & $\begin{array}{r}-\Delta J_{*} \\
{\left[10^{44} \times\right.} \\
\left.\mathrm{g} \mathrm{cm}^{2} \mathrm{~s}^{-1}\right]\end{array}$ \\
\hline \multirow[t]{2}{*}{ smc_sc5 260841} & 01 & $12.7_{-2.0}^{+2.4}$ & $0.6_{-0.3}^{+0.2}$ & $0.83_{-0.1}^{+0.09}$ & $0.54_{-0}^{+0}$ & & & & $1.42_{-}^{+}$ & $0.86_{-0.45}^{+0.71}$ & $1.58_{-0.88}^{+1.61}$ & $0.38_{-0.22}^{+0.38}$ \\
\hline & 02 & & & & & $0.5_{-0.1}^{+0.2}$ & $0.78_{-0.41}^{+0.4}$ & $0.08_{-0}^{+0}$ & $2.49_{-1}^{+1}$ & $0.78_{-0.41}^{+0.6}$ & $1.44_{-0.82}^{+1.39}$ & $0.64_{-0.36}^{+0.64}$ \\
\hline smc_sc5 260957 & 01 & $9.4_{-1.9}^{+2.5}$ & $0.5_{-0.3}^{+0.3}$ & $82_{-0.11}^{+0.1}$ & $0.58_{-0.06}^{+0.13}$ & $1.3_{-0.3}^{+0.4}$ & $0.64_{-0.3}^{+0.46}$ & $0.05_{-0.02}^{+0.02}$ & $2.41_{-1.1}^{+1.92}$ & $1.07_{-0.57}^{+0.89}$ & $1.48_{-0.85}^{+1.49}$ & $0.66_{-0.38}^{+0.68}$ \\
\hline smc_sc5 266088 & 01 & $8.8_{-2.1}^{+2.4}$ & $0.4_{-0.3}^{+0.4}$ & $0.8_{-0.1}^{+0.1}$ & $0.71_{-0.08}^{+0.14}$ & $0.7_{-0.2}^{+0.4}$ & $0.29_{-0.22}^{+0.73}$ & $0.07_{-0.03}^{+0.07}$ & $1.53_{-1.17}^{+3.83}$ & $0.23_{-0.15}^{+0.46}$ & $0.31_{-0.21}^{+0.58}$ & $0.18_{-0.12}^{+0.34}$ \\
\hline smc_sc5 276982 & 01 & $15.2_{-2.0}^{+1.3}$ & $0.5_{-0.2}^{+0.2}$ & $0.81_{-0.11}^{+0.1}$ & $0.78_{-0.11}^{+0.11}$ & $1.0_{-0.3}^{+0.4}$ & $0.05_{-0.03}^{+0.18}$ & $0.06_{-0.02}^{+0.05}$ & $0.25_{-0.13}^{+0.8}$ & $0.13_{-0.06}^{+0.26}$ & $0.26_{-0.14}^{+0.57}$ & $0.17_{-0.09}^{+0.34}$ \\
\hline smc_sc5 282963 & 01 & $13.8_{-2.4}^{+2.1}$ & $0.7_{-0.3}^{+0.2}$ & $0.82_{-0.11}^{+0.1}$ & $0.37_{-0.05}^{+0.11}$ & $1.5_{-0.5}^{+0.7}$ & $0.69_{-0.35}^{+0.47}$ & $0.44_{-0.19}^{+0.29}$ & $5.52_{-2}^{+3}$ & $2.46_{-1.47}^{+1.98}$ & $5.05_{-3.2}^{+4.7}$ & $6.01_{-3.82}^{+5.53}$ \\
\hline \multirow[t]{2}{*}{ smc_sc6 11085} & 01 & $15.7_{-2.0}^{+1.0}$ & $0.6_{-0.3}^{+0.2}$ & $0.82_{-0.1}^{+0.09}$ & $0.69_{-}^{+}$ & $2.3_{-0.4}^{+0.6}$ & $0.63_{-0}^{+0}$ & $\begin{array}{l}9 \\
9 \\
5\end{array}$ & $\begin{array}{l}3 \\
3 \\
6\end{array}$ & $3.17_{-1.35}^{+2.3}$ & $\begin{array}{r}-3.2 \\
6.76_{-3.13}^{+5.89}\end{array}$ & $1.39_{-0.65}^{+1.2}$ \\
\hline & 02 & & & & & $2.5_{-0.6}^{+0.6}$ & $0.65_{-}^{+}$ & $0.34_{-0.1}^{+0.2}$ & $1.04_{-0}^{+0}$ & $3.66_{-1.49}^{+2.39}$ & $7.79_{-3.49}^{+6.18}$ & $1.82_{-0.8}^{+1.43}$ \\
\hline smc_sc6 17538 & 01 & $8.8_{-2.1}^{+2.5}$ & $0.3_{-0.2}$ & $0.81_{-0.1}^{+0.1}$ & $0.02_{-0}$ & $0.8_{-0.2}^{+0.4}$ & $0.63_{-}^{+}$ & $0.1_{2}$ & $3.15_{-2.3}^{+2.77}$ & $\begin{array}{r}-1.49 \\
0.5_{-0.29}^{+0.49}\end{array}$ & $\begin{array}{r}-3.49 \\
0.61_{-0.36}^{+0.74}\end{array}$ & $0.33_{-0.2}^{+0.8}$ \\
\hline smc_sc6 42440 & 01 & $9.4_{-2.1}^{+2.6}$ & $0.4_{-0.3}^{+0.4}$ & $0.8_{-0.11}^{+0.11}$ & $0.37_{-c}^{+c}$ & $1.8_{-0.8}^{+0.8}$ & $0.76_{-0}^{+0}$ & $0.2_{-0}^{+0}$ & $2.4_{-1.29}^{+1.65}$ & $1.5_{-0.83}^{+1.6}$ & $2.01_{-1.15}^{+2.62}$ & $0.75_{-0.44}^{+0.97}$ \\
\hline \multirow[t]{4}{*}{ smc_sc6 99991} & 01 & $13.0_{-2.2}^{+2.2}$ & $0.7_{-0.2}^{+0.2}$ & $0.82_{-0.1}^{+0.1}$ & $0.34_{-0.03}^{+0.04}$ & $\begin{array}{l}-0.8 \\
1.6_{-0.5}^{+0.6}\end{array}$ & $\begin{array}{l}-0.41 \\
0.53_{-0.23}^{+0.41}\end{array}$ & $\begin{array}{l}-0.11 \\
0.94_{-0.28}^{+0.33}\end{array}$ & $\begin{array}{l}-1.29 \\
0.64_{-0.27}^{+0.51}\end{array}$ & $\begin{array}{r}-0.83 \\
1.82_{-0.72}^{+1.03}\end{array}$ & $3.54_{-1.6}^{+2.38}$ & $\begin{array}{l}-0.44 \\
0.61_{-0.27}^{+0.41}\end{array}$ \\
\hline & 0 & & & & & $1.8_{-0.4}^{+0.7}$ & $0.67_{-0}^{+0}$ & $0.75_{-0.26}^{+0.31}$ & $1.07_{-0.49}^{+0.73}$ & $2.42_{-1.06}^{+1.7}$ & $4.68_{-2.24}^{+3.84}$ & $1.09_{-0.52}^{+0.85}$ \\
\hline & 03 & & & & & $1.8_{-0.4}^{+0.5}$ & $0.51_{-0}^{+0}$ & $0.82_{-0}^{+0}$ & $0.81_{-0.34}^{+0.63}$ & $1.89_{-0.77}^{+1.26}$ & $3.64_{-1.66}^{+2.95}$ & $0.82_{-0.36}^{+0.65}$ \\
\hline & \pm & & & & & $1.7_{-0.5}^{+0.6}$ & $0.42_{-0}^{+0}$ & $0.94_{-0}^{+0}$ & $0.78_{-0.36}^{+1.0}$ & $1.56_{-0.66}^{+1.05}$ & $3.04_{-1.44}^{+2.29}$ & $0.8_{-0.37}^{+0.61}$ \\
\hline \multirow[t]{2}{*}{ smc_sc6 105368} & 01 & $12.4_{-2.5}^{+2.7}$ & $0.5_{-0.3}^{+0.2}$ & $0.82_{-0.1}^{+0.11}$ & $0.53_{-0.03}^{+0.05}$ & $1.0_{-0.2}^{+0.4}$ & $0.56_{-0.32}^{+0.5}$ & $0.2_{-0.07}^{+0.12}$ & $1.03_{-0.59}^{+0.9}$ & $0.89_{-0.45}^{+0.79}$ & $1.52_{-0.81}^{+1.66}$ & $0.36_{-0.19}^{+0.39}$ \\
\hline & 02 & & & & & $1.5_{-0.5}^{+0.7}$ & $0.24_{-0.12}^{+0.45}$ & $0.22_{-0.09}^{+0.2}$ & $0.56_{-0.27}^{+1.02}$ & $0.65_{-0.31}^{+0.76}$ & $1.17_{-0.63}^{+1.53}$ & $0.34_{-0.18}^{+0.43}$ \\
\hline smc_sc6 116294 & 01 & $9.2_{-1.8}^{+2.3}$ & $0.5_{-0.3}^{+0.3}$ & $0.81_{-0.11}^{+0.1}$ & $0.4_{-0.06}^{+0.14}$ & $2.6_{-1.0}^{+0.7}$ & $0.89_{-0.38}^{+0.38}$ & $0.18_{-0.09}^{+0.19}$ & $2.39_{-1.09}^{+1.03}$ & $2.67_{-1.5}^{+2.3}$ & $3.63_{-2.09}^{+3.66}$ & $1.13_{-0.65}^{+1.13}$ \\
\hline smc_sc6 128831 & 01 & $12.2_{-2.2}^{+2.7}$ & $0.6_{-0.3}^{+0.2}$ & $0.8_{-0.09}^{+0.11}$ & $0.03_{-0.02}^{+0.02}$ & $2.6_{-0.6}^{+0.5}$ & $0.98_{-0.4}^{+0.35}$ & $0.69_{-0}^{+0}$ & $0.82_{-0.36}^{+0.32}$ & $4.18_{-1.72}^{+2.2}$ & $7.47_{-3.44}^{+4.69}$ & $0.84_{-0.4}^{+0.61}$ \\
\hline smc_sc6 199611 & 01 & $13.8_{-2.0}^{+2.1}$ & $0.8_{-0.3}^{+0.2}$ & $0.81_{-0.1}^{+0.1}$ & $0.37_{-c}^{+0}$ & $1.4_{-0.7}^{+1.4}$ & $0.47_{-0.33}^{+0.62}$ & $0.34_{-0.18}^{+0.34}$ & $0.35_{-0.25}^{+0.48}$ & $1.48_{-0.91}^{+1.51}$ & $3.03_{-1.93}^{+3.73}$ & $0.34_{-0.21}^{+0.42}$ \\
\hline smc_sc6 272665 & 01 & $\begin{array}{r}-2.0 \\
7.0_{-1.2}^{+1.8}\end{array}$ & $0.4_{-0.3}^{+0.3}$ & $0.8_{-0.1}^{+0.11}$ & $0.37_{-0}^{+0}$ & $0.7_{-0.2}^{+0.4}$ & $0.68_{-0.46}^{+0.55}$ & $\begin{array}{l}-0.10 \\
0.08_{-0.05}^{+0.25}\end{array}$ & $\begin{array}{r}-0.00 \\
5.97_{-4.09}^{+5.43}\end{array}$ & $0.37_{-0.22}^{+0.37}$ & $0.4_{-0.24}^{+0.46}$ & $0.37_{-0.24}^{+0.42}$ \\
\hline smc_sc7 57131 & 01 & $\begin{array}{l}-1.2 \\
12.1_{-2.2}^{+2.6}\end{array}$ & $0.6^{+}$ & .1 & 0.09 & 2.0 & 0.7 & 0 . & 1.0 & $\begin{array}{l}-0.22 \\
2.48_{-1.36}^{+1.97}\end{array}$ & $\begin{array}{c}-0.24 \\
4.32_{-2.46}^{+3.9}\end{array}$ & $1.26_{-0.73}^{+1.24}$ \\
\hline smc_sc8 183240 & 01 & $14.7_{-1.8}^{+1.6}$ & $0.8_{-0.2}^{+0.1}$ & $0.82_{-0.11}^{+0.1}$ & $0.64_{-0.11}^{+0.18}$ & $0.7_{-0.2}^{+0.3}$ & $0.63_{-0.38}^{+0.51}$ & $0.25_{-0.12}^{+0.21}$ & $1.1_{-0.63}^{+0.86}$ & $1.37_{-0.8}^{+1.24}$ & $3.2_{-1.94}^{+3.22}$ & $0.92_{-0.54}^{+0.89}$ \\
\hline \multirow[t]{2}{*}{ smc_sc9 105383} & 01 & $13.2_{-2.5}^{+2.6}$ & $0.5_{-0.3}^{+0.2}$ & $0.83_{-0.1}^{+0.09}$ & $0.56_{-0.05}^{+0.08}$ & $1.9_{-0.5}^{+0.8}$ & $0.52_{-0.25}^{+0.5}$ & $0.1_{-0.03}^{+0.04}$ & $1.17_{-0.58}^{+1.2}$ & $1.82_{-0.86}^{+1.58}$ & $3.36_{-1.76}^{+3.43}$ & $1.01_{-0.51}^{+1.07}$ \\
\hline & 02 & & & & & & $0.86_{-0.39}^{+0.38}$ & $0.1_{-0.03}^{+0.05}$ & $1.76_{-0.75}^{+0.73}$ & $2.58_{-1.15}^{+1.8}$ & $4.75_{-2.38}^{+4.14}$ & $1.3_{-0.62}^{+1.12}$ \\
\hline smc_sc9 168422 & 01 & $9.4_{-2.0}^{+2.3}$ & $0.4_{-0.3}^{+0.3}$ & $0.82_{-0.11}^{+0.1}$ & $0.61_{-0.08}^{+0.10}$ & $0.5_{-0.2}^{+0.4}$ & $0.56_{-0.45}^{+0.62}$ & $0.08_{-0.04}^{+0.12}$ & $2.15_{-1.71}^{+2.35}$ & $0.33_{-0.22}^{+0.41}$ & $0.44_{-0.3}^{+0.63}$ & $0.2_{-0.13}^{+0.29}$ \\
\hline smc_sc10 8906 & 01 & $13.9_{-2.1}^{+2.0}$ & $0.8_{-0.2}^{+0.1}$ & $0.81_{-0.1}^{+0.11}$ & $0.58_{-0.05}^{+0.1}$ & $2.0_{-0.7}^{+1.0}$ & $0.79_{-0.34}^{+0.44}$ & $0.47_{-0.18}^{+0.26}$ & $0.16_{-0.07}^{+0.1}$ & $3.88_{-1.57}^{+2.17}$ & $8.2_{-3.7}^{+5.63}$ & $0.26_{-0.12}^{+0.19}$ \\
\hline
\end{tabular}


Table 6.1 - continued.

\begin{tabular}{|c|c|c|c|c|c|c|c|c|c|c|c|c|}
\hline OOGLE-II ID & $\begin{array}{r}\text { ump } \\
\text { ID }\end{array}$ & $\overline{\bar{M} M / M_{\odot}}$ & $t / t_{\mathrm{MS}}$ & $W$ & $\cos i$ & $\begin{array}{r}\Sigma_{0} \\
{\left[\mathrm{~g} \mathrm{~cm}^{-2}\right]}\end{array}$ & $\alpha_{\mathrm{bu}}$ & $\alpha_{\mathrm{d}}$ & $\tilde{\tau}_{\mathrm{bu}}$ & $\begin{array}{r}\left(-\frac{\partial M}{\partial t}\right)_{\text {typ }} \\
{\left[10^{-9} \times\right.} \\
\left.M_{\odot} \mathrm{yr}^{-1}\right]\end{array}$ & $\begin{array}{r}\left(-\frac{\partial J}{\partial t}\right)_{\mathrm{std}} \\
{\left[10^{36} \times\right.} \\
\left.\mathrm{g} \mathrm{cm}^{2} \mathrm{~s}^{-2}\right]\end{array}$ & $\begin{array}{r}-\Delta J_{*} \\
{\left[10^{44} \times\right.} \\
\left.\mathrm{g} \mathrm{cm}^{2} \mathrm{~s}^{-1}\right]\end{array}$ \\
\hline & 02 & & & & & 1.8 & $0.81_{-}^{+}$ & & 0.06 & $3.39_{-1.5}^{+2.05}$ & $7.26_{-3.55}^{+5.01}$ & $0.09_{-0.04}^{+0.06}$ \\
\hline smc_sc11 28090 & 01 & $13.7_{-2.1}^{+2.1}$ & $0.8_{-0}^{+0}$ & $.83_{-0.11}^{+0.09}$ & $56_{-0}^{+0 .}$ & $1.7_{-}^{+}$ & $0.1_{-0.05}^{+0.1}$ & $0.12_{-0}^{+0}$ & $0.41_{-0.18}^{+0.37}$ & $0.48_{-}^{+}$ & $1.02_{-0.54}^{+1.01}$ & $0.64_{-0.34}^{+0.64}$ \\
\hline smc_sc11 46587 & 01 & $9.0_{-2.1}^{+2.5}$ & $0.4_{-0.3}^{+0.4}$ & $0.81_{-0.11}^{+0.1}$ & $0.39_{-0.07}^{+0.19}$ & $0.9_{-0.4}^{+0.7}$ & $0.6_{-0.43}^{+0.57}$ & $0.36_{-0.22}^{+0.51}$ & $\begin{array}{l}4.02_{-2.89}^{+3.88} \\
\end{array}$ & $0.55_{-0.34}^{+0.58}$ & $0.72_{-0.47}^{+0.91}$ & $0.55_{-0.36}^{+0.7}$ \\
\hline
\end{tabular}


Chapter 7

\section{Conclusions}

Though my soul may set in darkness, it will rise in perfect light;

I have loved the stars too fondly to be fearful of the night.

Sarah Williams, last lines of the fourth paragraph of the poem The Old Astronomer to His Pupil.

Be stars can be selected from the bulk of light curves from photometric surveys by their characteristic magnitudes and colors and by their quite irregular variability of a few tenths of a magnitude at optical wavelenths (e.g., Mennickent et al., 2002; Paul et al., 2012). The variability is understood to be due to variable rates of disk feeding by the central fast rotating star.

We presented a new method to model the light curves of Be stars with the goal of extracting quantitative information about the fundamental parameters of their disks, such as the viscosity parameter, $\alpha$, and the asymptotic surface density $\left(\Sigma_{0}\right)$. We demonstrated that the new parameter $\Sigma_{0}$, the asymptotic surface density, which can be thought to correspond to the steady-state surface density of the disk at the stellar equator, is a much more useful way of expressing the mass injection rate and the net angular momentum injection rate of the star into the disk than the pair given by the mass injection rate and the radius of mass injection. Also, we took advantage of the scaling relations of the equation that governs the diffusion of mass through the disk, in order to generate a grid of dynamical models that is independent of the viscosity parameter $\alpha$, which in this work, was assumed to be time-dependent.

The method we developed uses a large pre-computed grid of synthetic Be light curves, calculated using detailed hydrodynamic calculations coupled with three-dimensional NLTE radiative transfer calculations. The comparison between the model grid and the observed light curves was made possible by the identification of two empirical laws that consists of simple formulas that closely match the photometric behaviour during disk build-up and dissipation. 
An initial analysis of our model grid allowed us to draw important conclusions about the properties of viscous decretion discs (VDD) around Be stars, and their effect on the stellar SED as they evolve in time:

- The viscosity parameter $\alpha$ is the most important parameter controlling the observed rate of photometric variations in Be light curves, but it is not the only one. Stellar parameters (mass, radius and effective temperature), as well as the disk viewing angle and density level, all contribute to change the rate of brightness variations in complicated ways, which means that if meaningful information about $\alpha$ is to be extracted from the data, these parameters must be estimated somehow;

- We identified a previously unknown effect, dubbed the mass-reservoir effect, which also controls the rate of photometric variations during phases of disk dissipation. This effect is a consequence of the fact that VDDs build a mass reservoir in their outer regions, which is unnoticed at short wavelengths (e.g., visible). The longer the build-up phase of a disk, the bigger its mass reservoir. When mass injection from the star stops and reaccretion occurs, the reservoir feeds the inner disk with mass. Clearly, the larger the reservoir, the longer it will be able to supply mass to the inner disk, and the slower the photometric dissipation will appear.

We applied our fitting pipeline to a sample of light curves of 54 Be star candidates from the SMC (Mennickent et al., 2002) containing 81 clearly identified events of disk formation/dissipation (here referred to as bumps). The light curves come from OGLE-II and OGLE-III data, covering roughly 12 years. A Markov Chain Monte Carlo (MCMC) technique was used to properly estimate the posterior probabilities of each fitted parameter.

It was found that our sample is biased towards early type Be stars, likely because these stars are more variable and their disks are denser, resulting in clearer bumps. Also, photometric uncertainties increase for late type Be stars. Since our sample was selected based on the appearance of their bumps, we conclude that our Be disks must be among the densest found for Be stars in the SMC. We verified an increase of $\Sigma_{0}$ with the stellar mass and the median for our whole sample is $\left\langle\Sigma_{0}\right\rangle=1.44_{-0.75}^{+1.01} \mathrm{~g} \mathrm{~cm}^{-2}$. Our results may suggest that Be disks in the SMC are denser than their siblings in the Galaxy, in line with $\mathrm{H} \alpha$ surveys that found stronger line emission in the SMC than in our Galaxy.

The durations of the build-up phases become shorter for more massive stars, which indicates that, as was already observed in the Galaxy, late-type Be stars are less variable than early-type ones. The median of the build-up time for our sample is $\left\langle t_{2}-t_{1}\right\rangle=$ $305_{-171}^{+351}$ days.

We obtained, for the first time, estimates of $\alpha$ for a statistically significant sample of Be stars. In our work, we explored the possibility that the viscosity parameter might be different at build-up $\left(\alpha_{\mathrm{bu}}\right)$ and dissipation $\left(\alpha_{\mathrm{d}}\right)$. We found no significant variation of $\alpha_{\mathrm{bu}}$ 
with the stellar mass (Fig. 6.7, top), but some evidence points to a correlation between $\alpha_{\mathrm{d}}$ with $M$ (Fig. 6.7, bottom). Furthermore, we find that on average the viscosity parameter is larger at build-up than at dissipation. The medians of the two viscosity parameters are $\left\langle\alpha_{\mathrm{bu}}\right\rangle=0.63_{-0.39}^{+0.52}$ and $\left\langle\alpha_{\mathrm{d}}\right\rangle=0.29_{-0.20}^{+0.61}$. These values are in agreement in magnitude with the determinations of Carciofi et al. (2012) and Ghoreyshi and Carciofi (2017) for the Galactic Be star $28 \mathrm{CMa}$. They are also similar to the values of $\alpha$ usually found in cataclysmic variables (King et al., 2007; Kotko and Lasota, 2012).

The trend that $\alpha_{\mathrm{bu}}>\alpha_{\mathrm{d}}$ was also seen by Ghoreyshi and Carciofi (2017) in the different cycles of activity of the Be star 28 CMa. Further work is necessary to establish whether this trend is real or simply a result of our model assumptions. In particular, two important physical effects were ignored in this work, namely the fact that Be disks are nonisothermal and the line forces known to act on the disk material. This last point, however, is likely of little importance for Be stars in the SMC, given their low metallicity. The $\alpha$ determinations made in this work should help in investigating the physical mechanisms producing the anomalous viscosity in circumstellar disks environments.

It must be further emphasised that in this work what is really measured are the timescales for disk build-up and dissipation. Under the assumption that viscosity is the only driving mechanism operating on the disk, these timescales can in turn be converted to estimates of the viscosity parameter. The presence of other driving mechanisms (such as the aforementioned ablation) might affect the determination of $\alpha$ in unpredictable ways.

The steady-state mass loss rate for the bumps is of the order of $\sim 10^{-10} M_{\odot} \mathrm{yr}^{-1}$. The typical decretion rate is of the order of $\sim 10^{-9} M_{\odot} \mathrm{yr}^{-1}$. These values are in agreement with the upper limit of the observed wind mass loss rate of B stars (Snow, 1981; Puls et al., 2008). In addition, these values roughly agree in magnitude with the work of Vieira et al. (2017), who studied a sample of 80 Galactic Be stars.

The steady-state angular momentum loss rate for the bumps, however, was found to be of the order of $\sim 5 \times 10^{36} \mathrm{~g} \mathrm{~cm}^{2} \mathrm{~s}^{-2}$, which is roughly one order of magnitude below the angular momentum loss rates required by the Geneva evolutionary models (see Fig. 6.10), so the evolving star does not reach its break-up velocity. Therefore, our results, for the first time, put constraints on the internal mechanisms of angular momentum transport of massive fast rotating stars. 


\section{Chapter 8}

\section{Prospectives}

That is the exploration that awaits you! Not mapping stars and studying nebula, but charting the unknown possibilities of existence.

Q to Picard, in Star Trek: The Next Generation, episode "All Good Things..."

It's not a silly question if you can't answer it.

Jostein Gaarder, Sophie's World

There are observational and theoretical future prospectives for this work.

On the observational side, the first obvious continuation is the modelling of the light curves containing bumps and inactive phases of the LMC, using the catalog of Be star candidates of Sabogal et al. (2005). We are already working on this project.

Our work, however, has shown that it is important to make an effort to remove the biases of our samples, by trying to include late-type Be stars and smaller-amplitude bumps. It is possible to find smaller amplitude bumps in the light curves of the catalogs of Mennickent et al. (2002) and Sabogal et al. (2005), but, for such bumps, the uncertainties will be more comparable in size with the amplitude of the bump, making the determinations of all the disk parameters more uncertain. We can, however, extend our light curves by adding OGLE-IV data, which is a continuation of the OGLE project (Udalski et al., 2015), for the stars of the catalogs of Mennickent et al. (2002) and Sabogal et al. (2005) and combine them with the near-infrared light curves of the VISTA Magellanic Survey (VMC) (Cioni et al., 2011). Since the flux excess in Be stars increases for longer wavelengths, with VMC data, we should be able to obtain bumps in the infrared that are associated with less dense disks, which would have much smaller amplitudes in the visual 
light curves of OGLE-IV. The OGLE-IV and VMC surveys started in March 2010 and November 2009, respectively. In both surveys, the data is collected mostly from August to January, because of the position of the Magellanic Clouds in the sky. VMC, however, only guarantees at least 12 measurements in the $K_{s}$ band $(\sim 2-2.4 \mu \mathrm{m})$ in this period, which hopefully means a measurement once every $\sim 15$ days.

The metallicity of the stars in the Magellanic Clouds is smaller than the galactic ones. The effects of the line-driven force in these regions, must, therefore, be less pronounced, and, hence, the disks of Be stars in these regions are essentially driven by viscosity. This is a great important justification for studying Be stars in the SMC and LMC. Despite that, we should however, extend our studies to the Galaxy, where there are many photometric surveys being carried out today, usually with smaller telescopes, limited to $V \sim 13-$ 14 mag. With the releases of parallaxes (distances) of the Gaia spacecraft ${ }^{1}$, we can have the distances of each individual star whose light curve came from these Galactic stellar variability surveys. Such surveys are general variability surveys like ASAS (Pojmanski, 1997) or HATNet (Bakos et al., 2002) and surveys primarily focusing on planetary transits, like KELT (Pepper et al., 2007) and SuperWASP (Pollacco et al., 2006).

Finally, it is also important to obtain a better estimate of the central star properties, e.g., via spectroscopic modelling or using stars belonging to clusters with known ages. Our current analysis was limited by the amount of information available on the central stars. This can be done for Galactic stars and for stars in the LMC and SMC, though, in the latter cases, bigger telescopes are required. Spectra and multicolor polarimetry of Galactic southern stars can be made by our group with the Brazilian telescopes at Observatório Pico dos Dias (OPD). In the near future, optical broad-band polarimetry will also be available from the SOUTH-POL survey (Magalhães et al., 2012), which will image the entire southern sky (from the Cerro Tololo Inter-American Observatory - CTIO). Polarimetry gives us information about the density of the disk, and, specially, constraints on its inclination angle.

From the theoretical side, one primary future prospective is the non-isothermal treatment of the disk evolution. Recent results show that the non-isothermality of the disk can affect all our determinations, as shown in Fig. 6.9. The non-isothermal treatment of the disk shouldn't be difficult to implement. It could be done with SINGLEBE and HDUST working in a "predictor-corrector" way involving a few iterations.

We must, however, investigate the importance of line-driving in our viscous decretion disk formalism, especially when dealing with stars from the Galaxy. In particular, how will ablation, as suggested by the work of Kee et al. (2016) or by the work of Krtička et al. (2011) affect the evolution of viscous disks of early Be stars?

In this work, we have shown that the angular momentum lost by early Be stars, even in the case of the most massive disks, are still below the angular momentum loss that the

\footnotetext{
${ }^{1}$ http://sci.esa.int/gaia/
} 
Geneva stellar evolutionary models (Granada et al., 2013) require so that rotating stars do not exceed their break-up velocities during the main sequence evolution. Could this be an observational constraint to their internal angular momentum transport, implying their transport is too efficient? With our increasing sample of Be stars with angular momentum loss estimates, we hope to collaborate with the Geneva team in testing their evolutionary models in the future.

Finally, with our estimates of the $\alpha$ parameter, we hope to attract the attention of turbulence theoreticians. Is it possible for the MRI to generate the observed viscosities? Or, since this and other work on Be stars have shown that late-type Be stars have more stable disks, could the turbulence be induced instead by the stellar radiation? 


\section{Bibliography}

Alcock C., Allsman R. A., Alves D., Axelrod T. S., Becker A. C., Bennett D. P., Cook K. H., Freeman K. C., Griest K., Guern J., Lehner M. J., Marshall S. L., Peterson B. A., Pratt M. R., Quinn P. J., Rodgers A. W., Stubbs C. W., Sutherland W., Welch D. L., The MACHO Project Large Magellanic Cloud Microlensing Results from the First Two Years and the Nature of the Galactic Dark Halo, ApJ, 1997, vol. 486, p. 697

Aubourg E., Bareyre P., Bréhin e. a., Evidence for gravitational microlensing by dark objects in the Galactic halo, Nature, 1993, vol. 365, p. 623

Baade D., Rivinius T., Pigulski A., Carciofi A. C., Martayan C., Moffat A. F. J., Wade G. A., Weiss W. W., Grunhut J., Handler G., Kuschnig R., Mehner A., Pablo H., Popowicz A., Rucinski S., Whittaker G., Short-term variability and mass loss in Be stars. I. BRITE satellite photometry of $\eta$ and $\mu$ Centauri, A\&A, 2016, vol. 588, p. A56

Bakos G. Á., Lázár J., Papp I., Sári P., Green E. M., System Description and First Light Curves of the Hungarian Automated Telescope, an Autonomous Observatory for Variability Search, PASP, 2002, vol. 114, p. 974

Balbus S. A., Hawley J. F., A powerful local shear instability in weakly magnetized disks. I - Linear analysis. II - Nonlinear evolution, ApJ, 1991, vol. 376, p. 214

Bessell M. S., UBVRI passbands, PASP, 1990, vol. 102, p. 1181

Bjorkman J. E., Circumstellar Disks. In Stellar Atmospheres: Theory and Observations , vol. 497 of Lecture Notes in Physics, Berlin Springer Verlag, 1997, p. 239

Bjorkman J. E., Carciofi A. C., Modeling the Structure of Hot Star Disks. In The Nature and Evolution of Disks Around Hot Stars, vol. 337 of Astronomical Society of the Pacific Conference Series, 2005, p. 75

Bjorkman J. E., Cassinelli J. P., Equatorial disk formation around rotating stars due to Ram pressure confinement by the stellar wind, ApJ, 1993, vol. 409, p. 429 
Carciofi A. C., The circumstellar discs of Be stars. In Active OB Stars: Structure, Evolution, Mass Loss, and Critical Limits , vol. 272 of IAU Symposium, 2011, p. 325

Carciofi A. C., Bjorkman J. E., Non-LTE Monte Carlo Radiative Transfer. I. The Thermal Properties of Keplerian Disks around Classical Be Stars, ApJ, 2006, vol. 639, p. 1081

Carciofi A. C., Bjorkman J. E., Non-LTE Monte Carlo Radiative Transfer. II. Nonisothermal Solutions for Viscous Keplerian Disks, ApJ, 2008, vol. 684, p. 1374

Carciofi A. C., Bjorkman J. E., Magalhães A. M., Effects of Grain Size on the Spectral Energy Distribution of Dusty Circumstellar Envelopes, ApJ, 2004, vol. 604, p. 238

Carciofi A. C., Bjorkman J. E., Otero S. A., Okazaki A. T., Štefl S., Rivinius T., Baade D., Haubois X., The First Determination of the Viscosity Parameter in the Circumstellar Disk of a Be Star, ApJ, 2012, vol. 744, p. L15

Carciofi A. C., Domiciano de Souza A., Magalhães A. M., Bjorkman J. E., Vakili F., On the Determination of the Rotational Oblateness of Achernar, ApJ, 2008, vol. 676, p. L41

Carciofi A. C., Magalhães A. M., Leister N. V., Bjorkman J. E., Levenhagen R. S., Achernar: Rapid Polarization Variability as Evidence of Photospheric and Circumstellar Activity, ApJ, 2007, vol. 671, p. L49

Carciofi A. C., Miroshnichenko A. S., Kusakin A. V., Bjorkman J. E., Bjorkman K. S., Marang F., Kuratov K. S., García-Lario P., Calderón J. V. P., Fabregat J., Magalhães A. M., Properties of the $\delta$ Scorpii Circumstellar Disk from Continuum Modeling, ApJ, 2006, vol. 652, p. 1617

Carciofi A. C., Okazaki A. T., Le Bouquin J.-B., Štefl S., Rivinius T., Baade D., Bjorkman J. E., Hummel C. A., Cyclic variability of the circumstellar disk of the Be star $\zeta$ Tauri. II. Testing the 2D global disk oscillation model, A\&A, 2009, vol. 504, p. 915

Cassinelli J. P., Brown J. C., Maheswaran M., Miller N. A., Telfer D. C., A Magnetically Torqued Disk Model for Be Stars, ApJ, 2002, vol. 578, p. 951

Castelli F., Kurucz R. L., Model atmospheres for VEGA, A\&A, 1994, vol. 281, p. 817

Cioni M.-R. L., Clementini G., Girardi L. e. a., The VMC survey. I. Strategy and first data, A\&A, 2011, vol. 527, p. A116

Collins II G. W., The use of terms and definitions in the study of Be stars. In IAU Colloq. 92: Physics of Be Stars , 1987, p. 3 
Cranmer S. R., Owocki S. P., The effect of oblateness and gravity darkening on the radiation driving in winds from rapidly rotating B stars, ApJ, 1995, vol. 440, p. 308

Ekström S., Meynet G., Maeder A., Barblan F., Evolution towards the critical limit and the origin of Be stars, A\&A, 2008, vol. 478, p. 467

Espinosa Lara F., Theory of stellar distortion by fast rotators. In EAS Publications Series , vol. 69 of EAS Publications Series, 2014, p. 297

Espinosa Lara F., Rieutord M., Gravity darkening in rotating stars, A\&A, 2011, vol. 533, p. A43

Foreman-Mackey D., Hogg D. W., Lang D., Goodman J., emcee: The MCMC Hammer, PASP, 2013, vol. 125, p. 306

Gayley K. G., Ignace R., Owocki S. P., Line Forces in Keplerian Circumstellar Disks and Precession of Nearly Circular Orbits, ApJ, 2001, vol. 558, p. 802

Gehrz R. D., Hackwell J. A., Jones T. W., Infrared observations of Be stars from 2.3 to 19.5 microns., ApJ, 1974, vol. 191, p. 675

Georgy C., Ekström S., Granada A., Meynet G., Mowlavi N., Eggenberger P., Maeder A., Populations of rotating stars. I. Models from 1.7 to $15 \mathrm{M}_{\odot}$ at $\mathrm{Z}=0.014,0.006$, and 0.002 with $\Omega / \Omega_{\text {crit }}$ between 0 and 1 , A\&A, 2013, vol. 553, p. A24

Ghoreyshi M. R., Carciofi A. C., Analysis of the V-Band Light Curve of the Be Star $\omega$ CMa with the Viscous Decretion Disk Model. In Astronomical Society of the Pacific Conference Series, vol. 508 of Astronomical Society of the Pacific Conference Series, 2017, p. 323

Ghoreyshi M. R., Carciofi A. C., Bjorkman J., Rivinius T., Baade D., Štefl S., Okazaki A., Modeling the Complete Lightcurve of $\omega$ CMa. In Bright Emissaries: Be Stars as Messengers of Star-Disk Physics, vol. 506 of Astronomical Society of the Pacific Conference Series, 2016, p. 315

Goodman J., Weare J., Ensemble samplers with affine invariance, Communications in applied mathematics and computational science, 2010, vol. 5, p. 65

Gordon K. D., Clayton G. C., Misselt K. A., Landolt A. U., Wolff M. J., A Quantitative Comparison of the Small Magellanic Cloud, Large Magellanic Cloud, and Milky Way Ultraviolet to Near-Infrared Extinction Curves, ApJ, 2003, vol. 594, p. 279

Granada A., Ekström S., Georgy C., Krtička J., Owocki S., Meynet G., Maeder A., Populations of rotating stars. II. Rapid rotators and their link to Be-type stars, A\&A, 2013, vol. 553, p. A25 
Haubois X., Carciofi A. C., Rivinius T., Okazaki A. T., Bjorkman J. E., Dynamical Evolution of Viscous Disks around Be Stars. I. Photometry, ApJ, 2012, vol. 756, p. 156

Haubois X., Mota B. C., Carciofi A. C., Draper Z. H., Wisniewski J. P., Bednarski D., Rivinius T., Dynamical Evolution of Viscous Disks around Be Stars. II. Polarimetry, ApJ, 2014, vol. 785, p. 12

Heinbockel J., Introduction to Tensor Calculus and Continuum Mechanics. Trafford, 2001

Hilditch R. W., Howarth I. D., Harries T. J., Forty eclipsing binaries in the Small Magellanic Cloud: fundamental parameters and Cloud distance, MNRAS, 2005, vol. 357, p. 304

Jaschek M., Slettebak A., Jaschek C., , 1981 Be star terminology. Be Star Newsletter

Jones C. E., Sigut T. A. A., Marlborough J. M., Iron line cooling of Be star circumstellar discs, MNRAS, 2004, vol. 352, p. 841

Jones C. E., Sigut T. A. A., Porter J. M., The circumstellar envelopes of Be stars: viscous disc dynamics, MNRAS, 2008, vol. 386, p. 1922

Jones C. E., Tycner C., Sigut T. A. A., Benson J. A., Hutter D. J., A Parameter Study of Classical Be Star Disk Models Constrained by Optical Interferometry, ApJ, 2008, vol. 687 , p. 598

Kee N. D., Owocki S., Sundqvist J. O., Line-driven ablation of circumstellar discs - I. Optically thin decretion discs of classical Oe/Be stars, MNRAS, 2016, vol. 458, p. 2323

Keller S. C., Bessell M. S., Cook K. H., Geha M., Syphers D., Blue Variable Stars from the MACHO Database. I. Photometry and Spectroscopy of the Large Magellanic Cloud Sample, AJ, 2002, vol. 124, p. 2039

King A. R., Pringle J. E., Livio M., Accretion disc viscosity: how big is alpha?, MNRAS, 2007, vol. 376, p. 1740

Kippenhahn R., Weigert A., Weiss A., Stellar Structure and Evolution, 2012

Klement R., Carciofi A. C., Rivinius T., Panoglou D., Vieira R. G., Bjorkman J. E., Štefl S., Tycner C., Faes D. M., Korčáková D., Müller A., Zavala R. T., Curé M., Multitechnique testing of the viscous decretion disk model. I. The stable and tenuous disk of the late-type Be star $\beta$ CMi, A\&A, 2015, vol. 584, p. A85

Kotko I., Lasota J.-P., The viscosity parameter $\alpha$ and the properties of accretion disc outbursts in close binaries, A\&A, 2012, vol. 545, p. A115 
Kroupa P., On the variation of the initial mass function, MNRAS, 2001, vol. 322, p. 231

Krtička J., Owocki S. P., Meynet G., Mass and angular momentum loss via decretion disks, A\&A, 2011, vol. 527, p. A84

Landau L., Lifshitz E., Fluid Mechanics. No. v. 6, Elsevier Science, 2013

Ledrew G., The Real Starry Sky, JRASC, 2001, vol. 95, p. 32

Lee U., Osaki Y., Saio H., Viscous excretion discs around Be stars, MNRAS, 1991, vol. 250, p. 432

MacKay D., Information Theory, Inference and Learning Algorithms. Cambridge University Press, 2003

Magalhães A. M., de Oliveira C. M., Carciofi A., Costa R., Dal Pino E. M. G., Diaz M., Ferrari T., Fernandez C., Gomes A. L., Marrara L., Pereyrac A., Ribeiro N. L., Rodrigues C. V., Rubinho M. S., Seriacopi D. B., Taylor K., South Pol: Revealing the polarized southern sky. In American Institute of Physics Conference Series , vol. 1429 of American Institute of Physics Conference Series, 2012, p. 244

Martayan C., Floquet M., Hubert A. M., Gutiérrez-Soto J., Fabregat J., Neiner C., Mekkas M., Be stars and binaries in the field of the SMC open cluster NGC 330 with VLT-FLAMES, A\&A, 2007, vol. 472, p. 577

Martayan C., Frémat Y., Hubert A.-M., Floquet M., Zorec J., Neiner C., Effects of metallicity, star-formation conditions, and evolution in B and Be stars. II. Small Magellanic Cloud, field of NGC 330, A\&A, 2007, vol. 462, p. 683

Martayan C., Rivinius T., Baade D., Hubert A.-M., Zorec J., Populations of Be stars: stellar evolution of extreme stars. In Active OB Stars: Structure, Evolution, Mass Loss, and Critical Limits, vol. 272 of IAU Symposium, 2011, p. 242

Mennickent R. E., Pietrzyński G., Gieren W., Szewczyk O., On Be star candidates and possible blue pre-main sequence objects in the Small Magellanic Cloud, A\&A, 2002, vol. 393 , p. 887

Okazaki A. T., On the confinement of one-armed oscillations in discs of Be stars., A\&A, 1997, vol. 318 , p. 548

Okazaki A. T., Viscous Transonic Decretion in Disks of Be Stars, PASJ, 2001, vol. 53, p. 119

Okazaki A. T., Theory vs. Observation of Circumstellar Disks and Their Formation. In Active OB-Stars: Laboratories for Stellare and Circumstellar Physics, vol. 361 of Astronomical Society of the Pacific Conference Series, 2007, p. 230 
Okazaki A. T., Bate M. R., Ogilvie G. I., Pringle J. E., Viscous effects on the interaction between the coplanar decretion disc and the neutron star in Be/X-ray binaries, MNRAS, 2002, vol. 337, p. 967

Owocki S., Ud-Doula A., Magnetic Spin-Up of Line-Driven Stellar Winds. In Magnetic Fields in O, B and A Stars: Origin and Connection to Pulsation, Rotation and Mass Loss , vol. 305 of Astronomical Society of the Pacific Conference Series, 2003, p. 350

Owocki S. P., Cranmer S. R., Gayley K. G., Inhibition FO Wind Compressed Disk Formation by Nonradial Line-Forces in Rotating Hot-Star Winds, ApJ, 1996, vol. 472, p. L115

Paul K. T., Subramaniam A., Mathew B., Mennickent R. E., Sabogal B., Study of candidate Be stars in the Magellanic Clouds using near-infrared photometry and optical spectroscopy, MNRAS, 2012, vol. 421, p. 3622

Pepper J., Pogge R. W., DePoy D. L., Marshall J. L., Stanek K. Z., Stutz A. M., Poindexter S., Siverd R., O'Brien T. P., Trueblood M., Trueblood P., The Kilodegree Extremely Little Telescope (KELT): A Small Robotic Telescope for Large-Area Synoptic Surveys, PASP, 2007, vol. 119, p. 923

Pletcher R., Tannehill J., Anderson D., Computational Fluid Mechanics and Heat Transfer, Second Edition. Series in Computational and Physical Processes in Mechanics and Thermal Sciences, Taylor \& Francis, 1997

Pojmanski G., The All Sky Automated Survey, Acta Astron., 1997, vol. 47, p. 467

Pollacco D. L., Skillen I., Collier Cameron e. a., The WASP Project and the SuperWASP Cameras, PASP, 2006, vol. 118, p. 1407

Porter J. M., On outflowing viscous disc models for Be stars, A\&A, 1999, vol. 348, p. 512

Porter J. M., Rivinius T., Classical Be Stars, PASP, 2003, vol. 115, p. 1153

Puls J., Vink J. S., Najarro F., Mass loss from hot massive stars, A\&A Rev., 2008, vol. 16, p. 209

Quirrenbach A., Buscher D. F., Mozurkewich D., Hummel C. A., Armstrong J. T., Maximum-entropy maps of the Be shell star zeta Tauri from optical long-baseline interferometry, A\&A, 1994, vol. 283, p. L13

Rivinius T., Baade D., Carciofi A. C., Short-term variability and mass loss in Be stars. II. Physical taxonomy of photometric variability observed by the Kepler spacecraft, A\&A, 2016, vol. 593, p. A106 
Rivinius T., Baade D., Stefl S., Stahl O., Wolf B., Kaufer A., Stellar and circumstellar activity of the Be star MU Centauri. I. Line emission outbursts, A\&A, 1998, vol. 333, p. 125

Rivinius T., Baade D., Townsend R. H. D., Carciofi A. C., Štefl S., Variable rotational line broadening in the Be star Achernar, A\&A, 2013, vol. 559, p. L4

Rivinius T., Carciofi A. C., Martayan C., Classical Be stars. Rapidly rotating B stars with viscous Keplerian decretion disks, A\&A Rev., 2013, vol. 21, p. 69

Rivinius T., Štefl S., Baade D., Bright Be-shell stars, A\&A, 2006, vol. 459, p. 137

Sabogal B. E., Mennickent R. E., Pietrzyński G., Gieren W., Be star candidates in the Large Magellanic Cloud: the catalogue and comparison with the Small Magellanic Cloud sample, MNRAS, 2005, vol. 361, p. 1055

Sana H., de Mink S. E., de Koter A., Langer N., Evans C. J., Gieles M., Gosset E., Izzard R. G., Le Bouquin J.-B., Schneider F. R. N., Binary Interaction Dominates the Evolution of Massive Stars, Science, 2012, vol. 337, p. 444

Secchi A., Schreiben des Herrn Prof. Secchi, Directors der Sternwarte des Collegio Romano, an den Herausgeber, Astronomische Nachrichten, 1866, vol. 68, p. 63

Shakura N. I., Sunyaev R. A., Black holes in binary systems. Observational appearance., A\&A, 1973, vol. 24, p. 337

Silaj J., Jones C. E., Tycner C., Sigut T. A. A., Smith A. D., A Systematic Study of H $\alpha$ Profiles of Be Stars, ApJS, 2010, vol. 187, p. 228

Snow Jr. T. P., Stellar winds and mass-loss rates from Be stars, ApJ, 1981, vol. 251, p. 139

Sparke L. S., Gallagher III J. S., Galaxies in the Universe: An Introduction. Cambridge University Press, 2007

Strom S. E., Edwards S., Skrutskie M. F., Evolutionary time scales for circumstellar disks associated with intermediate- and solar-type stars. In Protostars and Planets III , 1993, p. 837

Touhami Y., Gies D. R., Schaefer G. H., The Infrared Continuum Sizes of Be Star Disks, ApJ, 2011, vol. 729, p. 17

Udalski A., The Optical Gravitational Lensing Experiment. Real Time Data Analysis Systems in the OGLE-III Survey, Acta Astron., 2003, vol. 53, p. 291 
Udalski A., Kubiak M., Szymanski M., Optical Gravitational Lensing Experiment. OGLE2 - the Second Phase of the OGLE Project, Acta Astron., 1997, vol. 47, p. 319

Udalski A., Szymanski M., Kaluzny J., Kubiak M., Mateo M., The Optical Gravitational Lensing Experiment, Acta Astron., 1992, vol. 42, p. 253

Udalski A., Szymanski M. K., Soszynski I., Poleski R., The Optical Gravitational Lensing Experiment. Final Reductions of the OGLE-III Data, Acta Astron., 2008, vol. 58, p. 69

Udalski A., Szymański M. K., Szymański G., OGLE-IV: Fourth Phase of the Optical Gravitational Lensing Experiment, Acta Astron., 2015, vol. 65, p. 1

Štefl S., Rivinius T., Carciofi A. C., Le Bouquin J.-B., Baade D., Bjorkman K. S., Hesselbach E., Hummel C. A., Okazaki A. T., Pollmann E., Rantakyrö F., Wisniewski J. P., Cyclic variability of the circumstellar disk of the Be star $\zeta$ Tauri. I. Long-term monitoring observations, A\&A, 2009, vol. 504, p. 929

Vieira R. G., Carciofi A. C., Bjorkman J. E., The pseudo-photosphere model for the continuum emission of gaseous discs, MNRAS, 2015, vol. 454, p. 2107

Vieira R. G., Carciofi A. C., Bjorkman J. E., Rivinius T., Baade D., Rímulo L. R., The life cycles of Be viscous decretion discs: time-dependent modelling of infrared continuum observations, MNRAS, 2017, vol. 464, p. 3071

von Zeipel H., The radiative equilibrium of a rotating system of gaseous masses, MNRAS, 1924, vol. 84, p. 665

Wade G. A., Grunhut J. H., MiMeS Collaboration The MiMeS Survey of Magnetism in Massive Stars. In Circumstellar Dynamics at High Resolution, vol. 464 of Astronomical Society of the Pacific Conference Series, 2012, p. 405

Waters L. B. F. M., Cote J., Lamers H. J. G. L. M., IRAS observations of Be stars. II Far-IR characteristics and mass loss rates, A\&A, 1987, vol. 185, p. 206

Wood K., Bjorkman K. S., Bjorkman J. E., Deriving the Geometry of Be Star Circumstellar Envelopes from Continuum Spectropolarimetry. I. The Case of $\zeta$ Tauri, ApJ, 1997, vol. 477 , p. 926

Wyrzykowski Ł., Kozłowski S., Skowron J., Belokurov V., Smith M. C., Udalski A., Szymański M. K., Kubiak M., Pietrzyński G., Soszyński I., Szewczyk O., Żebruń K., The OGLE view of microlensing towards the Magellanic Clouds - I. A trickle of events in the OGLE-II LMC data, MNRAS, 2009, vol. 397, p. 1228 
Zaritsky D., Harris J., Thompson I. B., Grebel E. K., The Magellanic Clouds Photometric Survey: The Large Magellanic Cloud Stellar Catalog and Extinction Map, AJ, 2004, vol. 128 , p. 1606 
Appendix 

Appendix A

\section{Long tables}

Here we present the parameters $\Delta X_{\mathrm{bu}}^{\infty}, \xi_{\mathrm{bu}}$ and $\xi_{\mathrm{d}}$ of the empirical laws given by Eqs. (4.3.7) and (4.3.8), and with $\eta$ 's given by Table 4.4, for the BVRI bands in Tables A.1, A.2, A.3 and A.4, respectively.

The fits were performed with the MCMC emcee code (Sect. 4.5). The values in the tables are for the fits with the highest probability. The standard deviations from the fits were small for edge-on and pole-on angles (see forth column of the tables). The standard deviations from the fits of light curves of intermediate angles were high (and the residuals also) and they were the best measure in the definition of the region of intermediate angles for each band (see the extensions of the regions of intermediate angles for each band in the text of Sect. 4.3).

For the intermediate angles, in particular, the $\eta$ used was the one for "edge-on" in Table 4.4 if $\Delta X_{\mathrm{bu}}^{\infty}$ was positive and the one from "pole-on" in that table if $\Delta X_{\mathrm{bu}}^{\infty}$ was negative.

The values given in Table 4.4 were the values that gave the best fittings (like the ones exemplified in Appendix B) for the greatest number of light curves. 
Table A.1 - Empirical law parameters for band $B$.

\begin{tabular}{|c|c|c|c|c|c|c|c|c|c|c|c|c|c|c|c|c|c|}
\hline & & & $\tilde{\tau}_{\mathrm{bu}}$ & & $\mathrm{bu}$ & 0.15 & 0.45 & 0.75 & 1.50 & 2.25 & 3.00 & 4.50 & 6.00 & 9.00 & 15.00 & 30.00 & $\infty$ \\
\hline Star & $\Sigma_{0}\left[\mathrm{~g} \mathrm{~cm}^{-2}\right]$ & $\cos i$ & ang. type & $\Delta B_{\mathrm{bu}}^{\infty}$ & $\xi_{\mathrm{bu}}^{B}$ & $\xi_{d}^{B}$ & & & & & & & & & & & \\
\hline S1 & 2.5 & 0.0 & edge-on & 0.511 & 1.232 & 2.02 & 1.56 & 1.375 & 1.15 & 1.024 & 0.952 & 0.846 & 0.785 & 0.707 & 0.611 & 0.555 & 0.535 \\
\hline S1 & 2.5 & 0.071 & edge-on & 0.363 & 1.603 & 2.03 & 1.485 & 1.287 & 1.047 & 0.929 & 0.853 & 0.759 & 0.697 & 0.631 & 0.542 & 0.491 & 0.466 \\
\hline $\mathrm{S} 1$ & 2.5 & 0.143 & edge-on & 0.156 & 7.166 & 2.686 & 1.758 & 1.398 & 1.003 & 0.822 & 0.76 & 0.662 & 0.584 & 0.506 & 0.413 & 0.374 & 0.34 \\
\hline S1 & 2.5 & 0.215 & interm. & -0.025 & 4.25 & 3.513 & 1.801 & 1.183 & 2.419 & 71.635 & 75.725 & 153.281 & 272.649 & 255.478 & 84.525 & 45.069 & 35.747 \\
\hline $\mathrm{S} 1$ & 2.5 & 0.286 & interm. & -0.185 & 0.769 & 155.721 & 18.299 & 11.439 & 7.662 & 5.885 & 5.178 & 4.045 & 3.732 & 3.617 & 3.184 & 2.792 & 2.994 \\
\hline $\mathrm{S} 1$ & 2.5 & 0.357 & interm. & -0.312 & 1.931 & 10.595 & 7.183 & 5.953 & 4.712 & 4.03 & 3.587 & 3.007 & 2.833 & 2.676 & 2.381 & 2.138 & 2.226 \\
\hline S1 & 2.5 & 0.429 & interm. & -0.416 & 3.477 & 10.616 & 6.947 & 5.559 & 4.415 & 3.837 & 3.464 & 2.933 & 2.773 & 2.602 & 2.338 & 2.11 & 2.171 \\
\hline S1 & 2.5 & 0.5 & interm. & -0.498 & 5.407 & 11.771 & 7.266 & 5.75 & 4.549 & 3.98 & 3.594 & 3.122 & 2.932 & 2.758 & 2.464 & 2.243 & 2.291 \\
\hline $\mathrm{S} 1$ & 2.5 & 0.571 & interm. & -0.566 & 7.522 & 13.058 & 7.813 & 6.198 & 4.877 & 4.256 & 3.875 & 3.368 & 3.173 & 2.989 & 2.672 & 2.463 & 2.495 \\
\hline S1 & 2.5 & 0.643 & pole-on & -0.625 & 9.756 & 14.636 & 8.597 & 6.779 & 5.32 & 4.663 & 4.254 & 3.725 & 3.52 & 3.284 & 2.973 & 2.748 & 2.788 \\
\hline S1 & 2.5 & 0.714 & pole-on & -0.673 & 12.049 & 16.554 & 9.566 & 7.512 & 5.891 & 5.163 & 4.714 & 4.168 & 3.929 & 3.672 & 3.322 & 3.068 & 3.097 \\
\hline $\mathrm{S} 1$ & 2.5 & 0.786 & pole-on & -0.712 & 14.292 & 18.555 & 10.687 & 8.414 & 6.577 & 5.765 & 5.251 & 4.666 & 4.422 & 4.124 & 3.743 & 3.455 & 3.477 \\
\hline S1 & 2.5 & 0.857 & pole-on & -0.746 & 16.451 & 21.096 & 12.034 & 9.453 & 7.378 & 6.466 & 5.909 & 5.267 & 5.007 & 4.63 & 4.195 & 3.894 & 3.914 \\
\hline S1 & 2.5 & 0.928 & pole-on & -0.773 & 18.691 & 24.098 & 13.651 & 10.698 & 8.285 & 7.227 & 6.629 & 5.932 & 5.647 & 5.18 & 4.691 & 4.374 & 4.433 \\
\hline $\mathrm{S} 1$ & 2.5 & 1.0 & pole-on & -0.794 & 20.472 & 27.2 & 15.255 & 11.939 & 9.202 & 7.988 & 7.309 & 6.589 & 6.24 & 5.72 & 5.225 & 4.878 & 5.016 \\
\hline S1 & 1.85 & 0.0 & edge-on & 0.413 & 1.251 & 2.424 & 1.838 & 1.607 & 1.318 & 1.154 & 1.082 & 0.999 & 0.911 & 0.808 & 0.703 & 0.638 & 0.619 \\
\hline S1 & 1.85 & 0.071 & edge-on & 0.306 & 1.613 & 2.489 & 1.83 & 1.535 & 1.248 & 1.074 & 1.013 & 0.896 & 0.823 & 0.743 & 0.651 & 0.584 & 0.571 \\
\hline $\mathrm{S} 1$ & 1.85 & 0.143 & edge-on & 0.157 & 4.792 & 3.161 & 2.285 & 1.806 & 1.357 & 1.149 & 1.029 & 0.811 & 0.776 & 0.697 & 0.616 & 0.534 & 0.528 \\
\hline S1 & 1.85 & 0.215 & interm. & 0.015 & 469.674 & 4.309 & 2.719 & 1.995 & 1.213 & 0.938 & 3.7 & 0.382 & 5.935 & 0.361 & 16.244 & 36.156 & 8.417 \\
\hline $\mathrm{S} 1$ & 1.85 & 0.286 & interm. & -0.124 & 0.59 & 43.077 & 58.661 & 19.877 & 10.796 & 7.894 & 6.67 & 6.381 & 5.694 & 4.771 & 3.963 & 3.581 & 3.412 \\
\hline S1 & 1.85 & 0.357 & interm. & -0.237 & 1.794 & 15.156 & 8.289 & 6.988 & 5.409 & 4.626 & 4.143 & 3.996 & 3.691 & 3.269 & 2.824 & 2.565 & 2.531 \\
\hline S1 & 1.85 & 0.429 & interm. & \begin{tabular}{|l}
-0.332 \\
\end{tabular} & 3.442 & 14.041 & 8.325 & 6.879 & 5.383 & 4.656 & 4.142 & 3.988 & 3.679 & 3.314 & 2.892 & 2.682 & 2.66 \\
\hline $\mathrm{S} 1$ & 1.85 & 0.5 & interm. & -0.408 & 5.51 & 15.669 & 9.051 & 7.438 & 5.798 & 5.039 & 4.487 & 4.277 & 3.998 & 3.614 & 3.179 & 2.946 & 2.914 \\
\hline S1 & 1.85 & 0.571 & interm. & -0.473 & 7.837 & 17.521 & 10.028 & 8.206 & 6.405 & 5.556 & 4.99 & 4.734 & 4.432 & 4.029 & 3.566 & 3.321 & 3.276 \\
\hline S1 & 1.85 & 0.643 & pole-on & -0.528 & 10.319 & 19.698 & 11.333 & 9.201 & 7.127 & 6.251 & 5.612 & 5.304 & 4.987 & 4.541 & 4.07 & 3.779 & 3.739 \\
\hline $\mathrm{S} 1$ & 1.85 & 0.714 & pole-on & -0.573 & 12.938 & 22.365 & 12.755 & 10.407 & 8.074 & 7.044 & 6.389 & 5.996 & 5.624 & 5.145 & 4.615 & 4.322 & 4.301 \\
\hline S1 & 1.85 & 0.786 & pole-on & -0.611 & 15.658 & 25.441 & 14.548 & 11.794 & 9.165 & 7.958 & 7.203 & 6.758 & 6.326 & 5.826 & 5.276 & 4.951 & 4.994 \\
\hline S1 & 1.85 & 0.857 & pole-on & \begin{tabular}{|l}
-0.642 \\
\end{tabular} & 18.312 & 29.255 & 16.661 & 13.435 & 10.334 & 8.978 & 8.124 & 7.633 & 7.145 & 6.576 & 6.029 & 5.691 & 5.837 \\
\hline $\mathrm{S} 1$ & 1.85 & 0.928 & pole-on & -0.667 & 20.872 & 33.733 & 19.016 & 15.265 & 11.64 & 10.092 & 9.213 & 8.664 & 8.086 & 7.475 & 6.941 & 6.542 & 6.817 \\
\hline S1 & 1.85 & 1.0 & pole-on & -0.686 & 22.881 & 38.355 & 21.483 & 17.226 & 13.051 & 11.276 & 10.114 & 9.767 & 9.079 & 8.372 & 7.871 & 7.397 & 7.781 \\
\hline S1 & 1.37 & 0.0 & edge-on & 0.329 & 1.304 & 2.926 & 2.109 & 1.822 & 1.495 & 1.328 & 1.222 & 1.105 & 1.017 & 0.921 & 0.805 & 0.731 & 0.714 \\
\hline S1 & 1.37 & 0.071 & edge-on & 0.254 & 1.636 & 3.011 & 2.133 & 1.833 & 1.471 & 1.321 & 1.193 & 1.072 & 0.985 & 0.893 & 0.775 & 0.714 & 0.696 \\
\hline S1 & 1.37 & 0.143 & edge-on & 0.146 & 3.827 & 3.818 & 2.702 & 2.271 & 1.735 & 1.531 & 1.346 & 1.2 & 1.12 & 0.977 & 0.851 & 0.777 & 0.761 \\
\hline $\mathrm{S} 1$ & 1.37 & 0.215 & interm. & 0.036 & 90.464 & 5.094 & 3.486 & 2.808 & 2.008 & 1.822 & 1.412 & 1.395 & 1.289 & 0.917 & 0.896 & 0.866 & 0.815 \\
\hline $\mathrm{S} 1$ & 1.37 & 0.286 & interm. & -0.08 & 0.482 & 14.374 & 328.28 & 199.606 & 20.143 & 11.852 & 10.675 & 6.22 & 5.928 & 6.413 & 4.721 & 3.953 & 3.614 \\
\hline
\end{tabular}


Table A.1 - continued.

\begin{tabular}{|c|c|c|c|c|c|c|c|c|c|c|c|c|c|c|c|c|c|}
\hline & & & $\tilde{\tau}_{\mathrm{bu}}$ & & $\mathrm{bu}$ & 0.15 & 0.45 & 0.75 & 1.50 & 2.25 & 3.00 & 4.50 & 6.00 & 9.00 & 15.00 & 30.00 & $\infty$ \\
\hline Star & $\Sigma_{0}\left[\mathrm{~g} \mathrm{~cm}^{-2}\right]$ & $\cos i$ & ang. type & $\Delta B_{\mathrm{bu}}^{\infty}$ & $\xi_{\mathrm{bu}}^{B}$ & $\xi_{\mathrm{d}}^{B}$ & & & & & & & & & & & \\
\hline S1 & 1.37 & 0.357 & interm. & -0.177 & 1.787 & 24.461 & 10.48 & 8.302 & 5.968 & 5.253 & 4.785 & 4.129 & 3.892 & 3.808 & 3.23 & 2.931 & 2.844 \\
\hline S1 & 1.37 & 0.429 & interm. & -0.262 & 3.468 & 17.74 & 10.537 & 8.522 & 6.399 & 5.547 & 5.149 & 4.505 & 4.264 & 4.062 & 3.545 & 3.286 & 3.198 \\
\hline S1 & 1.37 & 0.5 & interm. & -0.332 & 5.483 & 19.517 & 11.696 & 9.427 & 7.185 & 6.274 & 5.833 & 5.141 & 4.864 & 4.65 & 4.046 & 3.755 & 3.778 \\
\hline S1 & 1.37 & 0.571 & interm. & -0.391 & 7.876 & 22.373 & 13.264 & 10.682 & 8.164 & 7.214 & 6.601 & 5.902 & 5.594 & 5.294 & 4.718 & 4.373 & 4.445 \\
\hline S1 & 1.37 & 0.643 & pole-on & -0.44 & 10.508 & 25.776 & 15.174 & 12.253 & 9.347 & 8.25 & 7.624 & 6.816 & 6.507 & 6.144 & 5.492 & 5.111 & 5.239 \\
\hline S1 & 1.37 & 0.714 & pole-on & -0.482 & 13.285 & 29.779 & 17.297 & 14.11 & 10.658 & 9.45 & 8.708 & 7.862 & 7.554 & 7.082 & 6.349 & 5.931 & 6.185 \\
\hline S1 & 1.37 & 0.786 & pole-on & -0.515 & 16.154 & 34.411 & 19.994 & 16.024 & 12.336 & 10.809 & 10.042 & 9.083 & 8.798 & 8.193 & 7.41 & 6.96 & 7.327 \\
\hline S1 & 1.37 & 0.857 & pole-on & -0.543 & 19.074 & 40.04 & 23.065 & 18.571 & 14.173 & 12.435 & 11.461 & 10.434 & 10.242 & 9.363 & 8.618 & 8.012 & 8.72 \\
\hline S1 & 1.37 & 0.928 & pole-on & -0.565 & 22.011 & 46.584 & 26.792 & 21.498 & 16.251 & 14.186 & 12.99 & 12.03 & 12.019 & 10.647 & 10.013 & 9.223 & 10.334 \\
\hline $\mathrm{S} 1$ & 1.37 & 1.0 & pole-on & -0.58 & 24.289 & 53.4 & 30.568 & 24.635 & 18.443 & 15.867 & 14.514 & 13.669 & 13.804 & 11.928 & 11.377 & 10.423 & 12.057 \\
\hline S1 & 1.01 & 0.0 & edge-on & 0.26 & 1.364 & 3.437 & 2.417 & 2.088 & 3.123 & 1.516 & 1.4 & 1.232 & 1.149 & 1.043 & 0.841 & 0.839 & 0.842 \\
\hline $\mathrm{S} 1$ & 1.01 & 0.071 & edge-on & 0.208 & 1.594 & 3.547 & 2.51 & 2.142 & 2.87 & 1.551 & 1.423 & 1.252 & 1.161 & 1.056 & 0.84 & 0.844 & 0.848 \\
\hline $\mathrm{S} 1$ & 1.01 & 0.143 & edge-on & 0.132 & 3.075 & 4.495 & 3.249 & 2.769 & 2.776 & 1.928 & 1.758 & 1.54 & 1.45 & 1.304 & 1.054 & 1.042 & 1.056 \\
\hline $\mathrm{S} 1$ & 1.01 & 0.215 & interm. & 0.048 & 16.107 & 6.121 & 4.479 & 3.745 & 56.935 & 2.609 & 2.429 & 2.079 & 2.133 & 1.888 & 1.552 & 1.619 & 1.727 \\
\hline S1 & 1.01 & 0.286 & interm. & -0.046 & 0.384 & 26.954 & 110.929 & 381.087 & 286.612 & 79.824 & 19.037 & 11.967 & 7.118 & 6.707 & 6.712 & 3.021 & 2.581 \\
\hline S1 & 1.01 & 0.357 & interm. & -0.128 & 1.652 & 82.201 & 11.828 & 9.155 & 9.959 & 5.699 & 5.035 & 4.7 & 4.275 & 3.837 & 3.943 & 3.112 & 2.889 \\
\hline $\mathrm{S} 1$ & 1.01 & 0.429 & interm. & -0.202 & 3.266 & 21.087 & 11.998 & 9.509 & 9.861 & 6.53 & 5.912 & 5.428 & 5.043 & 4.573 & 4.522 & 3.827 & 3.648 \\
\hline $\mathrm{S} 1$ & 1.01 & 0.5 & interm. & -0.262 & 5.086 & 23.464 & 13.865 & 11.24 & 10.559 & 7.608 & 7.059 & 6.313 & 6.052 & 5.493 & 5.338 & 4.682 & 4.611 \\
\hline $\mathrm{S} 1$ & 1.01 & 0.571 & interm. & -0.314 & 7.212 & 27.125 & 16.133 & 13.094 & 11.883 & 8.888 & 8.214 & 7.459 & 7.147 & 6.644 & 6.309 & 5.612 & 5.705 \\
\hline $\mathrm{S} 1$ & 1.01 & 0.643 & pole-on & -0.357 & 9.637 & 31.688 & 18.734 & 15.38 & 13.408 & 10.399 & 9.666 & 8.773 & 8.51 & 7.872 & 7.377 & 6.669 & 7.163 \\
\hline $\mathrm{S} 1$ & 1.01 & 0.714 & pole-on & -0.393 & 12.152 & 37.111 & 21.927 & 18.078 & 15.006 & 12.196 & 11.262 & 10.267 & 10.196 & 9.377 & 8.56 & 7.88 & 9.047 \\
\hline $\mathrm{S} 1$ & 1.01 & 0.786 & pole-on & -0.422 & 15.018 & 44.002 & 25.979 & 21.177 & 17.023 & 14.226 & 13.112 & 11.95 & 11.956 & 10.921 & 9.878 & 9.235 & 11.175 \\
\hline $\mathrm{S} 1$ & 1.01 & 0.857 & pole-on & -0.445 & 17.938 & 51.401 & 30.354 & 24.916 & 19.173 & 16.424 & 15.152 & 13.767 & 14.091 & 12.639 & 11.385 & 10.65 & 13.754 \\
\hline $\mathrm{S} 1$ & 1.01 & 0.928 & pole-on & -0.463 & 20.658 & 60.802 & 35.672 & 29.305 & 21.723 & 18.636 & 17.092 & 15.91 & 16.612 & 14.485 & 13.099 & 12.202 & 16.826 \\
\hline $\mathrm{S} 1$ & 1.01 & 1.0 & pole-on & -0.473 & 22.891 & 70.496 & 41.114 & 33.665 & 24.425 & 20.773 & 18.827 & 18.111 & 19.127 & 16.094 & 14.811 & 13.705 & 19.92 \\
\hline $\mathrm{S} 1$ & 0.75 & 0.0 & edge-on & 0.204 & 1.468 & 4.324 & 2.839 & 2.404 & 1.939 & 1.695 & 1.567 & 1.397 & 1.292 & 1.183 & 1.042 & 0.948 & 0.931 \\
\hline $\mathrm{S} 1$ & 0.75 & 0.071 & edge-on & 0.167 & 1.765 & 4.425 & 2.967 & 2.495 & 2.019 & 1.772 & 1.638 & 1.465 & 1.345 & 1.242 & 1.091 & 0.981 & 0.968 \\
\hline $\mathrm{S} 1$ & 0.75 & 0.143 & edge-on & 0.112 & 3.131 & 5.397 & 3.776 & 3.202 & 2.604 & 2.265 & 2.097 & 1.912 & 1.759 & 1.617 & 1.468 & 1.344 & 1.318 \\
\hline $\mathrm{S} 1$ & 0.75 & 0.215 & interm. & 0.046 & 80.87 & 7.398 & 5.169 & 4.539 & 3.67 & 3.371 & 3.361 & 3.087 & 2.947 & 3.476 & 2.616 & 2.537 & 2.577 \\
\hline $\mathrm{S} 1$ & 0.75 & 0.286 & interm. & -0.022 & 39.937 & 21.617 & 60.179 & 180.416 & 363.804 & 327.329 & 366.691 & 247.691 & 99.498 & 37.139 & 20.082 & 12.447 & 3.774 \\
\hline $\mathrm{S} 1$ & 0.75 & 0.357 & interm. & -0.09 & 1.659 & 234.545 & 16.004 & 9.981 & 6.795 & 5.873 & 4.704 & 4.506 & 4.203 & 3.71 & 3.158 & 2.798 & 2.914 \\
\hline S1 & 0.75 & 0.429 & interm. & $\mid-0.149$ & 3.018 & 26.246 & 13.634 & 10.765 & 8.098 & 7.148 & 6.355 & 5.776 & 5.449 & 4.93 & 4.639 & 4.039 & 4.213 \\
\hline $\mathrm{S} 1$ & 0.75 & 0.5 & interm. & -0.2 & 4.531 & 26.875 & 15.551 & 12.762 & 9.719 & 8.58 & 7.823 & 7.114 & 6.883 & 6.193 & 5.692 & 5.265 & 5.582 \\
\hline $\mathrm{S} 1$ & 0.75 & 0.571 & interm. & -0.242 & 6.105 & 30.787 & 18.843 & 15.26 & 11.784 & 10.3 & 9.42 & 8.588 & 8.492 & 7.613 & 6.878 & 6.519 & 6.961 \\
\hline $\mathrm{S} 1$ & 0.75 & 0.643 & pole-on & -0.278 & 7.927 & 37.009 & 22.443 & 18.222 & 13.976 & 12.388 & 11.315 & 10.223 & 10.267 & 9.102 & 8.388 & 7.888 & 8.832 \\
\hline
\end{tabular}


Table A.1 - continued.

\begin{tabular}{|c|c|c|c|c|c|c|c|c|c|c|c|c|c|c|c|c|c|}
\hline & & & $\tilde{\tau}_{\mathrm{bu}}$ & & $\mathrm{bu}$ & 0.15 & 0.45 & 0.75 & 1.50 & 2.25 & 3.00 & 4.50 & 6.00 & 9.00 & 15.00 & 30.00 & $\infty$ \\
\hline Star & $\Sigma_{0}\left[\mathrm{~g} \mathrm{~cm}^{-2}\right]$ & $\cos i$ & ang. type & $\Delta B_{\mathrm{bu}}^{\infty}$ & $\xi_{\mathrm{bu}}^{B}$ & $\xi_{\mathrm{d}}^{B}$ & & & & & & & & & & & \\
\hline S1 & 0.75 & 0.714 & pole-on & -0.307 & 9.676 & 43.557 & 26.679 & 21.866 & 16.711 & 14.65 & 13.376 & 12.234 & 12.351 & 10.716 & 10.042 & 9.394 & 10.905 \\
\hline S1 & 0.75 & 0.786 & pole-on & -0.33 & 11.4 & 51.798 & 31.817 & 25.889 & 19.893 & 17.469 & 15.794 & 14.251 & 14.919 & 12.51 & 11.989 & 11.013 & 13.405 \\
\hline $\mathrm{S} 1$ & 0.75 & 0.857 & pole-on & -0.347 & 13.112 & 61.387 & 37.811 & 30.967 & 23.249 & 19.912 & 18.031 & 16.609 & 17.544 & 14.39 & 13.949 & 12.648 & 16.391 \\
\hline S1 & 0.75 & 0.928 & pole-on & -0.36 & 14.656 & 72.95 & 44.345 & 36.76 & 26.421 & 22.601 & 20.335 & 19.261 & 21.001 & 16.179 & 16.398 & 14.444 & 19.954 \\
\hline S1 & 0.75 & 1.0 & pole-on & -0.366 & 15.691 & 83.592 & 51.718 & 42.315 & 29.767 & 25.099 & 22.42 & 21.575 & 23.96 & 17.699 & 18.538 & 16.006 & 23.423 \\
\hline $\mathrm{S} 1$ & 0.56 & 0.0 & edge-on & 0.16 & 1.543 & 5.083 & 3.389 & 2.8 & 2.186 & 1.933 & 1.784 & 1.575 & 1.45 & 1.337 & 1.152 & 1.068 & 1.033 \\
\hline S1 & 0.56 & 0.071 & edge-on & 0.134 & 1.745 & 4.924 & 3.558 & 2.928 & 2.3 & 2.042 & 1.893 & 1.69 & 1.538 & 1.415 & 1.235 & 1.132 & 1.093 \\
\hline S1 & 0.56 & 0.143 & edge-on & 0.093 & 2.75 & 6.245 & 4.489 & 3.776 & 3.066 & 2.717 & 2.519 & 2.284 & 2.125 & 1.979 & 1.743 & 1.636 & 1.578 \\
\hline $\mathrm{S} 1$ & 0.56 & 0.215 & interm. & 0.044 & 6.942 & 8.044 & 6.403 & 5.75 & 4.693 & 4.443 & 4.154 & 4.159 & 3.76 & 3.633 & 3.298 & 3.334 & 3.238 \\
\hline $\mathrm{S} 1$ & 0.56 & 0.286 & interm. & -0.007 & 67.764 & 81.412 & 40.885 & 117.976 & 231.031 & 258.856 & 351.47 & 297.635 & 328.064 & 293.562 & 266.423 & 284.651 & 258.177 \\
\hline S1 & 0.56 & 0.357 & interm. & -0.062 & 1.556 & 299.725 & 23.977 & 10.324 & 6.464 & 4.99 & 4.293 & 3.571 & 3.549 & 2.963 & 2.861 & 2.459 & 2.722 \\
\hline $\mathrm{S} 1$ & 0.56 & 0.429 & interm. & -0.107 & 3.151 & 35.694 & 14.542 & 11.33 & 7.978 & 6.88 & 6.225 & 5.507 & 5.491 & 4.968 & 4.62 & 4.03 & 4.459 \\
\hline S1 & 0.56 & 0.5 & interm. & -0.146 & 4.659 & 31.372 & 17.101 & 13.491 & 10.224 & 8.905 & 7.98 & 7.183 & 7.287 & 6.482 & 5.982 & 5.47 & 6.088 \\
\hline S1 & 0.56 & 0.571 & interm. & -0.179 & 6.464 & 34.22 & 19.973 & 16.41 & 12.705 & 10.942 & 10.234 & 8.909 & 9.149 & 8.034 & 7.36 & 7.043 & 7.886 \\
\hline $\mathrm{S} 1$ & 0.56 & 0.643 & pole-on & -0.206 & 8.467 & 40.658 & 24.707 & 20.285 & 15.267 & 13.284 & 12.394 & 10.977 & 11.314 & 9.876 & 9.221 & 8.562 & 10.187 \\
\hline S1 & 0.56 & 0.714 & pole-on & -0.228 & 10.856 & 49.07 & 29.892 & 24.37 & 18.679 & 16.15 & 15.012 & 13.269 & 13.85 & 11.802 & 11.029 & 10.294 & 12.736 \\
\hline $\mathrm{S} 1$ & 0.56 & 0.786 & pole-on & -0.245 & 13.35 & 58.049 & 35.679 & 29.818 & 22.331 & 19.487 & 17.627 & 15.539 & 16.912 & 13.933 & 13.233 & 12.233 & 15.958 \\
\hline S1 & 0.56 & 0.857 & pole-on & -0.257 & 16.109 & 69.184 & 42.401 & 35.768 & 26.147 & 22.415 & 20.316 & 18.251 & 20.275 & 15.84 & 15.627 & 13.811 & 19.785 \\
\hline S1 & 0.56 & 0.928 & pole-on & -0.265 & 18.969 & 82.209 & 51.033 & 43.337 & 30.001 & 25.218 & 22.586 & 21.285 & 23.922 & 17.756 & 18.331 & 16.087 & 24.183 \\
\hline S1 & 0.56 & 1.0 & pole-on & -0.267 & 20.724 & 93.501 & 59.484 & 49.343 & 33.391 & 27.182 & 24.706 & 23.785 & 28.089 & 19.59 & 20.667 & 17.62 & 28.36 \\
\hline S1 & 0.41 & 0.0 & edge-on & 0.124 & 1.734 & 6.102 & 3.922 & 3.296 & 2.54 & 2.229 & 2.021 & 1.811 & 1.624 & 1.502 & 1.291 & 1.205 & 1.154 \\
\hline S1 & 0.41 & 0.071 & edge-on & 0.106 & 1.85 & 6.036 & 4.097 & 3.433 & 2.756 & 2.356 & 2.162 & 1.944 & 1.705 & 1.612 & 1.397 & 1.297 & 1.269 \\
\hline S1 & 0.41 & 0.143 & edge-on & 0.075 & 2.682 & 7.005 & 5.217 & 4.365 & 3.528 & 3.278 & 2.976 & 2.763 & 2.507 & 2.442 & 2.126 & 1.949 & 1.945 \\
\hline $\mathrm{S} 1$ & 0.41 & 0.215 & interm. & 0.038 & 6.274 & 16.04 & 7.643 & 6.67 & 5.841 & 5.475 & 5.241 & 4.9 & 4.844 & 4.75 & 4.288 & 4.308 & 4.147 \\
\hline S1 & 0.41 & 0.286 & interm. & 0.001 & 298.321 & 115.416 & 57.898 & 145.617 & 126.343 & 223.322 & 282.342 & 264.2 & 302.723 & 303.543 & 305.299 & 310.457 & 323.879 \\
\hline S1 & 0.41 & 0.357 & interm. & -0.04 & 1.76 & 302.226 & 174.255 & 21.389 & 6.557 & 4.004 & 3.518 & 3.264 & 3.293 & 2.227 & 2.185 & 1.689 & 2.079 \\
\hline $\mathrm{S} 1$ & 0.41 & 0.429 & interm. & -0.073 & 3.405 & 102.958 & 15.902 & 12.009 & 7.445 & 6.486 & 5.661 & 5.162 & 5.26 & 4.133 & 4.002 & 3.574 & 4.219 \\
\hline S1 & 0.41 & 0.5 & interm. & \begin{tabular}{|l}
-0.102 \\
\end{tabular} & 4.598 & 33.656 & 17.297 & 13.691 & 10.436 & 8.561 & 7.613 & 7.165 & 7.195 & 5.92 & 5.819 & 5.088 & 5.935 \\
\hline S1 & 0.41 & 0.571 & interm. & -0.125 & 6.359 & 37.578 & 21.466 & 17.126 & 12.585 & 11.062 & 10.058 & 9.147 & 9.375 & 7.817 & 7.518 & 6.942 & 8.133 \\
\hline S1 & 0.41 & 0.643 & pole-on & -0.145 & 8.444 & 42.85 & 25.447 & 21.023 & 15.901 & 13.536 & 12.399 & 11.171 & 11.661 & 9.775 & 9.323 & 8.548 & 10.586 \\
\hline S1 & 0.41 & 0.714 & pole-on & -0.16 & 10.609 & 51.83 & 31.001 & 25.966 & 19.521 & 16.645 & 15.16 & 13.755 & 14.321 & 11.719 & 11.604 & 10.596 & 13.468 \\
\hline S1 & 0.41 & 0.786 & pole-on & -0.171 & 13.262 & 61.077 & 38.581 & 31.76 & 23.543 & 20.293 & 18.124 & 16.462 & 17.674 & 13.981 & 13.702 & 12.352 & 17.41 \\
\hline S1 & 0.41 & 0.857 & pole-on & -0.179 & 16.006 & 73.851 & 45.109 & 37.971 & 27.697 & 23.618 & 20.737 & 19.086 & 21.435 & 16.205 & 16.295 & 14.441 & 21.814 \\
\hline S1 & 0.41 & 0.928 & pole-on & $\mid-0.183$ & 18.986 & 88.284 & 55.612 & 44.724 & 31.551 & 26.597 & 23.706 & 21.985 & 25.667 & 17.919 & 19.415 & 16.318 & 26.992 \\
\hline $\mathrm{S} 1$ & 0.41 & 1.0 & pole-on & -0.182 & 21.209 & 99.68 & 63.632 & 53.344 & 35.447 & 29.091 & 25.337 & 25.031 & 29.871 & 19.711 & 21.596 & 18.456 & 31.369 \\
\hline
\end{tabular}


Table A.1 - continued.

\begin{tabular}{|c|c|c|c|c|c|c|c|c|c|c|c|c|c|c|c|c|c|}
\hline & & & $\tilde{\tau}_{\text {bu }}$ & & $\mathrm{bu}$ & 0.15 & 0.45 & 0.75 & 1.50 & 2.25 & 3.00 & 4.50 & 6.00 & 9.00 & 15.00 & 30.00 & $\infty$ \\
\hline Star & $\Sigma_{0}\left[\mathrm{~g} \mathrm{~cm}^{-2}\right]$ & $\cos i$ & ang. type & $\Delta B_{\mathrm{bu}}^{\infty}$ & $\xi_{\mathrm{bu}}^{B}$ & $\xi_{\mathrm{d}}^{B}$ & & & & & & & & & & & \\
\hline S1 & 0.3 & 0.0 & edge-on & 0.096 & 6.629 & 7.678 & 4.542 & 3.661 & 2.884 & 2.474 & 2.284 & 1.97 & 1.809 & 1.699 & 1.474 & 1.322 & 1.251 \\
\hline S1 & 0.3 & 0.071 & edge-on & 0.082 & 1.908 & 7.744 & 4.674 & 3.865 & 3.059 & 2.635 & 2.411 & 2.15 & 1.955 & 1.84 & 1.564 & 1.459 & 1.395 \\
\hline S1 & 0.3 & 0.143 & edge-on & 0.058 & 2.771 & 12.337 & 5.854 & 4.887 & 4.111 & 3.79 & 3.456 & 3.04 & 2.86 & 2.804 & 2.441 & 2.31 & 2.211 \\
\hline S1 & 0.3 & 0.215 & interm. & 0.03 & 36.55 & 32.934 & 9.423 & 7.624 & 6.956 & 6.679 & 6.504 & 6.155 & 5.74 & 5.888 & 5.256 & 5.308 & 5.2 \\
\hline S1 & 0.3 & 0.286 & interm. & 0.003 & 282.936 & 228.636 & 150.817 & 157.266 & 138.707 & 227.599 & 198.369 & 260.657 & 281.333 & 295.088 & 309.106 & 257.1 & 259.74 \\
\hline S1 & 0.3 & 0.357 & interm. & -0.025 & 20.874 & 319.978 & 267.356 & 166.275 & 41.016 & 7.152 & 11.906 & 5.109 & 4.425 & 4.918 & 2.171 & 1.793 & 1.99 \\
\hline S1 & 0.3 & 0.429 & interm. & -0.05 & 3.491 & 180.182 & 20.781 & 11.975 & 6.718 & 6.148 & 4.69 & 5.022 & 4.799 & 3.69 & 3.538 & 3.494 & 3.701 \\
\hline S1 & 0.3 & 0.5 & interm. & -0.069 & 4.77 & 46.535 & 17.842 & 13.784 & 9.149 & 8.463 & 7.194 & 6.472 & 6.893 & 5.532 & 5.253 & 4.418 & 5.418 \\
\hline S1 & 0.3 & 0.571 & interm. & -0.086 & 6.126 & 39.222 & 22.698 & 17.548 & 12.305 & 10.786 & 9.251 & 8.511 & 9.033 & 7.278 & 6.994 & 6.311 & 7.719 \\
\hline S1 & 0.3 & 0.643 & pole-on & \begin{tabular}{|l|}
-0.099 \\
\end{tabular} & 8.16 & 46.058 & 25.705 & 21.561 & 15.212 & 13.641 & 11.73 & 10.621 & 11.186 & 9.461 & 8.896 & 8.081 & 10.783 \\
\hline S1 & 0.3 & 0.714 & pole-on & -0.109 & 10.172 & 54.44 & 31.811 & 26.222 & 18.999 & 16.963 & 14.692 & 13.042 & 14.276 & 11.471 & 11.129 & 10.063 & 13.474 \\
\hline S1 & 0.3 & 0.786 & pole-on & $\mid-0.116$ & 12.715 & 62.874 & 40.385 & 31.587 & 23.429 & 20.542 & 17.778 & 15.928 & 17.711 & 13.653 & 13.155 & 11.843 & 17.061 \\
\hline S1 & 0.3 & 0.857 & pole-on & -0.12 & 15.634 & 76.752 & 47.51 & 39.025 & 27.428 & 23.989 & 20.049 & 19.089 & 21.911 & 15.561 & 16.023 & 13.834 & 22.137 \\
\hline S1 & 0.3 & 0.928 & pole-on & \begin{tabular}{|l}
-0.122 \\
\end{tabular} & 18.332 & 90.184 & 58.474 & 46.453 & 32.451 & 26.737 & 23.067 & 21.902 & 26.817 & 17.337 & 18.328 & 16.042 & 27.296 \\
\hline S1 & 0.3 & 1.0 & pole-on & -0.12 & 20.383 & 113.511 & 64.429 & 52.943 & 34.938 & 29.936 & 26.434 & 24.125 & 30.351 & 20.279 & 20.827 & 17.738 & 32.619 \\
\hline $\mathrm{S} 2$ & 2.5 & 0.0 & edge-on & 0.474 & 1.176 & 2.092 & 1.629 & 1.478 & 1.2 & 1.086 & 0.996 & 0.904 & 0.833 & 0.757 & 0.658 & 0.595 & 0.58 \\
\hline $\mathrm{S} 2$ & 2.5 & 0.071 & edge-on & 0.346 & 1.525 & 2.052 & 1.586 & 1.403 & 1.142 & 1.022 & 0.936 & 0.84 & 0.788 & 0.702 & 0.606 & 0.543 & 0.531 \\
\hline S2 & 2.5 & 0.143 & edge-on & 0.166 & 4.842 & 2.573 & 1.919 & 1.638 & 1.226 & 1.049 & 0.917 & 0.795 & 0.752 & 0.638 & 0.562 & 0.467 & 0.458 \\
\hline $\mathrm{S} 2$ & 2.5 & 0.215 & interm. & 0.01 & 439.245 & 3.327 & 2.168 & 1.674 & 1.016 & 0.809 & 0.598 & 0.802 & 0.478 & 4.605 & 0.291 & 47.653 & 31.689 \\
\hline $\mathrm{S} 2$ & 2.5 & 0.286 & interm. & -0.134 & 0.687 & 122.926 & 40.367 & 16.438 & 8.664 & 6.749 & 5.816 & 4.758 & 4.183 & 3.93 & 3.241 & 3.01 & 2.772 \\
\hline $\mathrm{S} 2$ & 2.5 & 0.357 & interm. & -0.25 & 2.01 & 16.376 & 8.374 & 6.546 & 4.942 & 4.226 & 3.878 & 3.37 & 3.042 & 2.803 & 2.436 & 2.267 & 2.149 \\
\hline $\mathrm{S} 2$ & 2.5 & 0.429 & interm. & -0.346 & 3.736 & 13.696 & 8.034 & 6.434 & 4.92 & 4.225 & 3.887 & 3.428 & 3.142 & 2.925 & 2.523 & 2.331 & 2.251 \\
\hline $\mathrm{S} 2$ & 2.5 & 0.5 & interm. & -0.423 & 5.811 & 14.751 & 8.587 & 6.842 & 5.281 & 4.557 & 4.189 & 3.699 & 3.468 & 3.172 & 2.789 & 2.593 & 2.507 \\
\hline S2 & 2.5 & 0.571 & interm. & -0.487 & 8.099 & 16.529 & 9.427 & 7.57 & 5.796 & 5.038 & 4.673 & 4.136 & 3.879 & 3.573 & 3.152 & 2.915 & 2.848 \\
\hline $\mathrm{S} 2$ & 2.5 & 0.643 & pole-on & -0.542 & 10.579 & 18.593 & 10.598 & 8.493 & 6.481 & 5.668 & 5.241 & 4.683 & 4.381 & 4.034 & 3.57 & 3.335 & 3.284 \\
\hline $\mathrm{S} 2$ & 2.5 & 0.714 & pole-on & -0.587 & 13.121 & 20.974 & 11.9 & 9.547 & 7.322 & 6.374 & 5.912 & 5.306 & 4.965 & 4.571 & 4.089 & 3.82 & 3.8 \\
\hline $\mathrm{S} 2$ & 2.5 & 0.786 & pole-on & -0.623 & 15.636 & 23.851 & 13.445 & 10.804 & 8.273 & 7.189 & 6.638 & 5.977 & 5.645 & 5.18 & 4.673 & 4.392 & 4.432 \\
\hline $\mathrm{S} 2$ & 2.5 & 0.857 & pole-on & -0.653 & 18.094 & 27.384 & 15.338 & 12.273 & 9.327 & 8.159 & 7.448 & 6.787 & 6.448 & 5.899 & 5.374 & 5.063 & 5.179 \\
\hline $\mathrm{S} 2$ & 2.5 & 0.928 & pole-on & -0.677 & 20.495 & 31.508 & 17.44 & 13.917 & 10.557 & 9.147 & 8.449 & 7.704 & 7.407 & 6.735 & 6.197 & 5.813 & 6.016 \\
\hline $\mathrm{S} 2$ & 2.5 & 1.0 & pole-on & -0.695 & 22.348 & 35.837 & 19.778 & 15.671 & 11.812 & 10.193 & 9.446 & 8.693 & 8.424 & 7.535 & 7.036 & 6.584 & 6.95 \\
\hline $\mathrm{S} 2$ & 1.85 & 0.0 & edge-on & 0.375 & 1.242 & 2.563 & 1.88 & 1.66 & 1.358 & 1.189 & 1.866 & 1.427 & 0.952 & 0.84 & 0.736 & 0.677 & 0.653 \\
\hline $\mathrm{S} 2$ & 1.85 & 0.071 & edge-on & 0.286 & 1.56 & 2.532 & 1.865 & 1.636 & 1.319 & 1.15 & 1.513 & 1.182 & 0.917 & 0.821 & 0.712 & 0.647 & 0.621 \\
\hline $\mathrm{S} 2$ & 1.85 & 0.143 & edge-on & 0.156 & 3.891 & 3.198 & 2.281 & 1.961 & 1.497 & 1.275 & 1.262 & 1.098 & 0.955 & 0.868 & 0.743 & 0.663 & 0.641 \\
\hline $\mathrm{S} 2$ & 1.85 & 0.215 & interm. & 0.031 & 265.893 & 4.293 & 2.789 & 2.331 & 1.594 & 1.305 & 0.725 & 0.801 & 0.895 & 0.781 & 0.648 & 0.55 & 0.539 \\
\hline S2 & 1.85 & 0.286 & interm. & -0.093 & 0.876 & 59.812 & 348.518 & 77.876 & 16.625 & 11.526 & 17.326 & 11.39 & 5.987 & 4.897 & 4.187 & 3.692 & 3.429 \\
\hline
\end{tabular}


Table A.1 - continued.

\begin{tabular}{|c|c|c|c|c|c|c|c|c|c|c|c|c|c|c|c|c|c|}
\hline & & & $\tilde{\tau}_{\mathrm{bu}}$ & & $\mathrm{bu}$ & 0.15 & 0.45 & 0.75 & 1.50 & 2.25 & 3.00 & 4.50 & 6.00 & 9.00 & 15.00 & 30.00 & $\infty$ \\
\hline Star & $\Sigma_{0}\left[\mathrm{~g} \mathrm{~cm}^{-2}\right]$ & $\cos i$ & ang. type & $\Delta B_{\mathrm{bu}}^{\infty}$ & $\xi_{\mathrm{bu}}^{B}$ & $\xi_{\mathrm{d}}^{B}$ & & & & & & & & & & & \\
\hline S2 & 1.85 & 0.357 & interm. & -0.195 & 1.804 & 23.258 & 10.965 & 7.849 & 5.927 & 5.229 & 6.512 & 5.374 & 3.805 & 3.349 & 2.961 & 2.733 & 2.658 \\
\hline S2 & 1.85 & 0.429 & interm. & -0.282 & 3.366 & 16.386 & 9.948 & 7.79 & 6.116 & 5.299 & 6.13 & 5.197 & 4.017 & 3.63 & 3.21 & 2.991 & 2.938 \\
\hline $\mathrm{S} 2$ & 1.85 & 0.5 & interm. & -0.352 & 5.33 & 18.053 & 10.964 & 8.708 & 6.699 & 5.914 & 6.495 & 5.648 & 4.547 & 4.135 & 3.665 & 3.416 & 3.407 \\
\hline S2 & 1.85 & 0.571 & interm. & -0.41 & 7.558 & 20.234 & 12.345 & 9.763 & 7.567 & 6.654 & 7.137 & 6.334 & 5.137 & 4.719 & 4.225 & 3.981 & 3.997 \\
\hline S2 & 1.85 & 0.643 & pole-on & -0.459 & 9.941 & 23.452 & 14.034 & 11.099 & 8.552 & 7.597 & 8.066 & 7.176 & 5.922 & 5.43 & 4.925 & 4.612 & 4.727 \\
\hline S2 & 1.85 & 0.714 & pole-on & -0.5 & 12.498 & 26.851 & 15.934 & 12.744 & 9.79 & 8.644 & 9.123 & 8.222 & 6.786 & 6.299 & 5.742 & 5.395 & 5.589 \\
\hline S2 & 1.85 & 0.786 & pole-on & -0.532 & 15.154 & 31.201 & 18.357 & 14.509 & 11.263 & 9.899 & 10.435 & 9.524 & 7.865 & 7.254 & 6.664 & 6.25 & 6.63 \\
\hline $\mathrm{S} 2$ & 1.85 & 0.857 & pole-on & -0.559 & 17.796 & 36.203 & 21.096 & 16.789 & 12.896 & 11.295 & 11.971 & 10.985 & 9.165 & 8.317 & 7.799 & 7.242 & 7.832 \\
\hline $\mathrm{S} 2$ & 1.85 & 0.928 & pole-on & -0.58 & 20.28 & 42.311 & 24.416 & 19.502 & 14.882 & 12.931 & 13.836 & 12.845 & 10.719 & 9.512 & 9.008 & 8.307 & 9.288 \\
\hline $\mathrm{S} 2$ & 1.85 & 1.0 & pole-on & -0.594 & 22.485 & 48.582 & 27.978 & 22.24 & 16.778 & 14.456 & 15.838 & 14.756 & 12.397 & 10.654 & 10.304 & 9.421 & 10.738 \\
\hline $\mathrm{S} 2$ & 1.37 & 0.0 & edge-on & 0.296 & 1.346 & 3.236 & 2.204 & 1.909 & 1.524 & 1.371 & 1.262 & 1.13 & 1.044 & 0.949 & 0.834 & 0.754 & 0.732 \\
\hline $\mathrm{S} 2$ & 1.37 & 0.071 & edge-on & 0.235 & 1.602 & 3.21 & 2.238 & 1.915 & 1.54 & 1.387 & 1.279 & 1.129 & 1.046 & 0.962 & 0.841 & 0.757 & 0.735 \\
\hline $\mathrm{S} 2$ & 1.37 & 0.143 & edge-on & 0.14 & 3.383 & 3.88 & 2.811 & 2.374 & 1.873 & 1.624 & 1.503 & 1.328 & 1.231 & 1.125 & 0.981 & 0.891 & 0.861 \\
\hline $\mathrm{S} 2$ & 1.37 & 0.215 & interm. & 0.045 & 27.624 & 5.394 & 3.731 & 3.122 & 2.35 & 2.007 & 1.875 & 1.758 & 1.569 & 1.394 & 1.298 & 1.199 & 1.175 \\
\hline $\mathrm{S} 2$ & 1.37 & 0.286 & interm. & -0.058 & 0.37 & 17.181 & 184.088 & 369.423 & 117.264 & 30.251 & 17.269 & 10.318 & 8.286 & 6.203 & 4.262 & 3.82 & 3.907 \\
\hline $\mathrm{S} 2$ & 1.37 & 0.357 & interm. & -0.144 & 1.624 & 54.387 & 12.599 & 8.742 & 6.796 & 5.734 & 4.922 & 4.34 & 4.151 & 3.689 & 3.243 & 2.932 & 2.957 \\
\hline $\mathrm{S} 2$ & 1.37 & 0.429 & interm. & -0.219 & 3.124 & 19.171 & 11.227 & 8.884 & 6.923 & 6.104 & 5.496 & 4.913 & 4.662 & 4.288 & 3.792 & 3.507 & 3.571 \\
\hline S2 & 1.37 & 0.5 & interm. & -0.28 & 4.96 & 20.904 & 12.723 & 10.22 & 7.913 & 6.967 & 6.308 & 5.74 & 5.466 & 5.016 & 4.52 & 4.199 & 4.358 \\
\hline $\mathrm{S} 2$ & 1.37 & 0.571 & interm. & -0.331 & 7.008 & 24.248 & 14.736 & 11.828 & 9.163 & 8.116 & 7.384 & 6.683 & 6.46 & 5.851 & 5.347 & 5.03 & 5.249 \\
\hline $\mathrm{S} 2$ & 1.37 & 0.643 & pole-on & -0.374 & 9.34 & 28.247 & 17.084 & 13.777 & 10.758 & 9.421 & 8.637 & 7.885 & 7.617 & 6.951 & 6.334 & 5.921 & 6.337 \\
\hline $\mathrm{S} 2$ & 1.37 & 0.714 & pole-on & \begin{tabular}{|l}
-0.409 \\
\end{tabular} & 11.918 & 33.003 & 19.981 & 16.073 & 12.478 & 10.986 & 10.088 & 9.139 & 9.05 & 8.105 & 7.508 & 6.976 & 7.646 \\
\hline $\mathrm{S} 2$ & 1.37 & 0.786 & pole-on & -0.436 & 14.567 & 38.642 & 23.394 & 18.931 & 14.662 & 12.793 & 11.726 & 10.658 & 10.692 & 9.364 & 8.817 & 8.149 & 9.204 \\
\hline $\mathrm{S} 2$ & 1.37 & 0.857 & pole-on & -0.458 & 17.288 & 46.06 & 27.26 & 22.053 & 16.97 & 14.689 & 13.577 & 12.378 & 12.537 & 10.734 & 10.272 & 9.459 & 11.029 \\
\hline S2 & 1.37 & 0.928 & pole-on & -0.475 & 19.983 & 54.043 & 32.467 & 26.191 & 19.613 & 16.828 & 15.202 & 14.294 & 14.818 & 12.233 & 12.044 & 10.879 & 13.282 \\
\hline $\mathrm{S} 2$ & 1.37 & 1.0 & pole-on & -0.485 & 22.142 & 62.649 & 37.166 & 30.233 & 22.149 & 18.758 & 16.994 & 16.247 & 17.017 & 13.625 & 13.733 & 12.208 & 15.59 \\
\hline $\mathrm{S} 2$ & 1.01 & 0.0 & edge-on & 0.233 & 1.435 & 3.999 & 2.618 & 2.202 & 3.514 & 1.548 & 1.438 & 1.27 & 1.177 & 1.072 & 0.989 & 0.85 & 0.836 \\
\hline S2 & 1.01 & 0.071 & edge-on & 0.19 & 1.683 & 3.935 & 2.718 & 2.265 & 3.083 & 1.621 & 1.491 & 1.315 & 1.212 & 1.117 & 1.007 & 0.882 & 0.867 \\
\hline $\mathrm{S} 2$ & 1.01 & 0.143 & edge-on & 0.123 & 3.064 & 4.784 & 3.523 & 2.849 & 2.693 & 2.011 & 1.883 & 1.673 & 1.554 & 1.431 & 1.298 & 1.142 & 1.107 \\
\hline $\mathrm{S} 2$ & 1.01 & 0.215 & interm. & 0.049 & 13.351 & 6.561 & 4.746 & 3.925 & 3.033 & 2.866 & 2.892 & 2.558 & 2.441 & 2.446 & 2.138 & 2.001 & 1.979 \\
\hline S2 & 1.01 & 0.286 & interm. & -0.029 & 2.708 & 14.693 & 37.049 & 137.399 & 424.271 & 315.092 & 292.634 & 195.054 & 102.215 & 22.719 & 18.167 & 2.791 & 3.537 \\
\hline $\mathrm{S} 2$ & 1.01 & 0.357 & interm. & -0.101 & 1.467 & 259.545 & 15.297 & 9.403 & 20.985 & 5.215 & 4.626 & 4.006 & 3.961 & 3.462 & 3.374 & 2.827 & 2.767 \\
\hline S2 & 1.01 & 0.429 & interm. & \begin{tabular}{|l}
-0.162 \\
\end{tabular} & 2.962 & 22.369 & 12.518 & 9.851 & 14.56 & 6.406 & 5.8 & 5.225 & 4.998 & 4.43 & 4.242 & 3.726 & 3.792 \\
\hline S2 & 1.01 & 0.5 & interm. & -0.212 & 4.673 & 23.673 & 13.984 & 11.6 & 14.779 & 7.699 & 6.986 & 6.339 & 6.17 & 5.506 & 5.221 & 4.731 & 4.847 \\
\hline $\mathrm{S} 2$ & 1.01 & 0.571 & interm. & -0.255 & 6.527 & 27.975 & 16.77 & 13.581 & 16.429 & 9.157 & 8.442 & 7.587 & 7.498 & 6.737 & 6.375 & 5.769 & 6.144 \\
\hline $\mathrm{S} 2$ & 1.01 & 0.643 & pole-on & -0.29 & 8.683 & 32.796 & 20.262 & 16.511 & 18.859 & 10.956 & 10.112 & 9.1 & 9.094 & 8.14 & 7.592 & 6.997 & 7.802 \\
\hline
\end{tabular}


Table A.1 - continued.

\begin{tabular}{|c|c|c|c|c|c|c|c|c|c|c|c|c|c|c|c|c|c|}
\hline & & & $\tilde{\tau}_{\text {bu }}$ & & $\mathrm{bu}$ & 0.15 & 0.45 & 0.75 & 1.50 & 2.25 & 3.00 & 4.50 & 6.00 & 9.00 & 15.00 & 30.00 & $\infty$ \\
\hline Star & $\Sigma_{0}\left[\mathrm{~g} \mathrm{~cm}^{-2}\right]$ & $\cos i$ & ang. type & $\Delta B_{\mathrm{bu}}^{\infty}$ & $\xi_{\mathrm{bu}}^{B}$ & $\xi_{\mathrm{d}}^{B}$ & & & & & & & & & & & \\
\hline $\mathrm{S} 2$ & 1.01 & 0.714 & pole-on & -0.318 & 11.137 & 38.903 & 23.88 & 19.334 & 21.689 & 13.076 & 12.054 & 10.741 & 11.032 & 9.542 & 9.056 & 8.37 & 9.748 \\
\hline $\mathrm{S} 2$ & 1.01 & 0.786 & pole-on & -0.34 & 13.69 & 46.334 & 28.727 & 23.265 & 25.74 & 15.464 & 14.09 & 12.66 & 13.035 & 11.161 & 10.542 & 9.748 & 12.203 \\
\hline $\mathrm{S} 2$ & 1.01 & 0.857 & pole-on & -0.357 & 16.369 & 55.388 & 33.799 & 27.716 & 29.973 & 17.907 & 16.209 & 14.677 & 15.534 & 12.772 & 12.223 & 11.31 & 15.033 \\
\hline $\mathrm{S} 2$ & 1.01 & 0.928 & pole-on & $\mid-0.369$ & 19.178 & 65.621 & 40.794 & 33.008 & 35.623 & 20.254 & 18.139 & 17.2 & 18.52 & 14.462 & 14.033 & 13.021 & 18.471 \\
\hline $\mathrm{S} 2$ & 1.01 & 1.0 & pole-on & -0.374 & 21.168 & 75.637 & 46.756 & 38.126 & 40.767 & 22.643 & 20.087 & 19.373 & 21.644 & 16.098 & 15.748 & 14.639 & 21.924 \\
\hline S2 & 0.75 & 0.0 & edge-on & 0.184 & 1.576 & 4.89 & 3.246 & 2.566 & 2.012 & 1.779 & 1.611 & 1.425 & 1.324 & 1.212 & 1.058 & 0.964 & 0.929 \\
\hline S2 & 0.75 & 0.071 & edge-on & 0.154 & 2.571 & 4.94 & 3.303 & 2.669 & 2.126 & 1.878 & 1.727 & 1.533 & 1.385 & 1.292 & 1.13 & 1.035 & 0.992 \\
\hline S2 & 0.75 & 0.143 & edge-on & 0.104 & 3.154 & 6.076 & 4.101 & 3.486 & 2.775 & 2.503 & 2.313 & 2.059 & 1.908 & 1.773 & 1.544 & 1.413 & 1.395 \\
\hline $\mathrm{S} 2$ & 0.75 & 0.215 & interm. & 0.047 & 36.958 & 7.829 & 5.846 & 5.023 & 4.214 & 3.888 & 3.779 & 3.47 & 3.348 & 3.261 & 3.028 & 2.815 & 2.863 \\
\hline $\mathrm{S} 2$ & 0.75 & 0.286 & interm. & \begin{tabular}{|l|}
-0.009 \\
\end{tabular} & 86.537 & 27.113 & 22.479 & 61.17 & 217.786 & 292.935 & 346.778 & 324.308 & 291.774 & 328.82 & 266.675 & 285.246 & 200.053 \\
\hline S2 & 0.75 & 0.357 & interm. & -0.067 & 2.125 & 292.144 & 14.571 & 8.467 & 5.492 & 4.06 & 3.621 & 3.076 & 3.189 & 2.446 & 2.303 & 2.098 & 2.361 \\
\hline S2 & 0.75 & 0.429 & interm. & $\mid-0.116$ & 5.253 & 25.276 & 12.05 & 9.707 & 7.149 & 5.928 & 5.309 & 4.788 & 4.792 & 4.12 & 3.742 & 3.47 & 3.845 \\
\hline S2 & 0.75 & 0.5 & interm. & -0.156 & 4.331 & 24.282 & 14.08 & 11.723 & 8.936 & 7.719 & 7.026 & 6.461 & 6.322 & 5.504 & 5.178 & 4.763 & 5.283 \\
\hline S2 & 0.75 & 0.571 & interm. & -0.19 & 5.759 & 29.753 & 17.512 & 14.303 & 11.086 & 9.64 & 8.709 & 7.86 & 8.048 & 6.911 & 6.502 & 6.007 & 6.871 \\
\hline $\mathrm{S} 2$ & 0.75 & 0.643 & pole-on & -0.217 & 7.527 & 35.084 & 21.167 & 17.687 & 13.44 & 11.717 & 10.758 & 9.576 & 9.898 & 8.481 & 7.983 & 7.552 & 8.843 \\
\hline $\mathrm{S} 2$ & 0.75 & 0.714 & pole-on & -0.239 & 9.28 & 42.264 & 25.937 & 21.312 & 16.368 & 14.178 & 12.823 & 11.521 & 12.162 & 10.239 & 9.67 & 8.901 & 11.019 \\
\hline S2 & 0.75 & 0.786 & pole-on & -0.256 & 11.139 & 50.542 & 31.64 & 25.785 & 19.488 & 16.821 & 15.278 & 13.716 & 14.603 & 11.939 & 11.574 & 10.485 & 13.824 \\
\hline S2 & 0.75 & 0.857 & pole-on & -0.267 & 12.945 & 61.005 & 37.636 & 31.148 & 23.004 & 19.599 & 17.711 & 15.99 & 17.553 & 13.613 & 13.579 & 12.246 & 16.985 \\
\hline $\mathrm{S} 2$ & 0.75 & 0.928 & pole-on & -0.275 & 14.651 & 73.65 & 45.157 & 37.168 & 26.583 & 22.224 & 19.997 & 18.656 & 20.924 & 15.528 & 16.062 & 14.025 & 21.037 \\
\hline S2 & 0.75 & 1.0 & pole-on & -0.277 & 15.9 & 84.388 & 52.038 & 43.606 & 30.116 & 24.608 & 21.956 & 21.235 & 24.577 & 17.226 & 18.179 & 15.7 & 24.905 \\
\hline $\mathrm{S} 2$ & 0.56 & 0.0 & edge-on & 0.146 & 1.768 & 5.922 & 3.706 & 3.104 & 2.346 & 2.003 & 1.84 & 1.623 & 1.514 & 1.357 & 1.193 & 1.082 & 1.043 \\
\hline $\mathrm{S} 2$ & 0.56 & 0.071 & edge-on & 0.124 & 1.931 & 5.9 & 3.848 & 3.219 & 2.456 & 2.19 & 1.952 & 1.738 & 1.609 & 1.503 & 1.293 & 1.159 & 1.13 \\
\hline S2 & 0.56 & 0.143 & edge-on & 0.087 & 3.021 & 7.147 & 4.901 & 4.017 & 3.341 & 2.929 & 2.72 & 2.438 & 2.301 & 2.1 & 1.848 & 1.733 & 1.712 \\
\hline S2 & 0.56 & 0.215 & interm. & 0.043 & 7.945 & 10.513 & 7.025 & 5.996 & 5.401 & 4.972 & 5.062 & 4.526 & 4.406 & 4.017 & 3.875 & 3.916 & 3.637 \\
\hline $\mathrm{S} 2$ & 0.56 & 0.286 & interm. & 0.001 & 303.242 & 74.999 & 75.387 & 106.844 & 134.995 & 149.373 & 199.023 & 218.112 & 280.769 & 280.838 & 267.656 & 305.094 & 317.231 \\
\hline $\mathrm{S} 2$ & 0.56 & 0.357 & interm. & -0.045 & 1.33 & 277.759 & 114.138 & 9.369 & 3.832 & 3.162 & 2.696 & 2.393 & 2.311 & 1.819 & 1.718 & 1.518 & 1.564 \\
\hline S2 & 0.56 & 0.429 & interm. & -0.082 & 2.902 & 30.865 & 12.207 & 9.141 & 6.152 & 5.321 & 4.556 & 4.282 & 4.285 & 3.441 & 3.339 & 2.991 & 3.362 \\
\hline $\mathrm{S} 2$ & 0.56 & 0.5 & interm. & -0.113 & 4.26 & 25.641 & 14.412 & 11.764 & 8.35 & 7.305 & 6.768 & 6.002 & 6.135 & 5.052 & 4.83 & 4.333 & 5.184 \\
\hline $\mathrm{S} 2$ & 0.56 & 0.571 & interm. & -0.137 & 5.929 & 30.078 & 17.769 & 14.449 & 10.856 & 9.682 & 8.823 & 7.898 & 7.851 & 6.583 & 6.503 & 5.766 & 7.002 \\
\hline $\mathrm{S} 2$ & 0.56 & 0.643 & pole-on & -0.158 & 7.964 & 34.717 & 21.712 & 18.034 & 13.375 & 11.913 & 10.516 & 9.793 & 10.095 & 8.416 & 8.002 & 7.275 & 9.07 \\
\hline $\mathrm{S} 2$ & 0.56 & 0.714 & pole-on & $\mid-0.173$ & 10.048 & 42.396 & 26.576 & 21.915 & 16.57 & 14.466 & 13.073 & 11.701 & 12.423 & 10.25 & 9.819 & 8.848 & 11.322 \\
\hline S2 & 0.56 & 0.786 & pole-on & -0.184 & 12.688 & 52.645 & 32.977 & 27.353 & 20.128 & 17.127 & 15.673 & 14.229 & 15.276 & 11.996 & 11.875 & 10.564 & 14.514 \\
\hline $\mathrm{S} 2$ & 0.56 & 0.857 & pole-on & \begin{tabular}{|l}
-0.192 \\
\end{tabular} & 15.265 & 63.25 & 39.24 & 33.407 & 23.673 & 20.165 & 18.042 & 16.48 & 18.354 & 13.719 & 13.859 & 12.381 & 17.832 \\
\hline $\mathrm{S} 2$ & 0.56 & 0.928 & pole-on & -0.196 & 18.43 & 76.227 & 47.869 & 40.6 & 27.254 & 22.857 & 20.396 & 19.098 & 22.549 & 15.624 & 16.437 & 14.036 & 22.526 \\
\hline S2 & 0.56 & 1.0 & pole-on & -0.195 & 20.291 & 90.359 & 55.015 & 46.336 & 31.098 & 25.179 & 22.551 & 21.986 & 25.838 & 17.237 & 18.91 & 16.109 & 27.239 \\
\hline
\end{tabular}


Table A.1 - continued.

\begin{tabular}{|c|c|c|c|c|c|c|c|c|c|c|c|c|c|c|c|c|c|}
\hline & & & $\tilde{\tau}_{\mathrm{bu}}$ & & $\mathrm{bu}$ & 0.15 & 0.45 & 0.75 & 1.50 & 2.25 & 3.00 & 4.50 & 6.00 & 9.00 & 15.00 & 30.00 & $\infty$ \\
\hline Star & $\Sigma_{0}\left[\mathrm{~g} \mathrm{~cm}^{-2}\right]$ & $\cos i$ & ang. type & $\Delta B_{\mathrm{bu}}^{\infty}$ & $\xi_{\mathrm{bu}}^{B}$ & $\xi_{\mathrm{d}}^{B}$ & & & & & & & & & & & \\
\hline S2 & 0.41 & 0.0 & edge-on & 0.115 & 1.904 & 7.171 & 4.311 & 3.435 & 2.7 & 2.316 & 2.124 & 1.818 & 1.672 & 1.57 & 1.335 & 1.24 & 1.178 \\
\hline $\mathrm{S} 2$ & 0.41 & 0.071 & edge-on & 0.098 & 2.067 & 7.067 & 4.533 & 3.718 & 2.851 & 2.486 & 2.306 & 1.99 & 1.832 & 1.717 & 1.454 & 1.32 & 1.271 \\
\hline $\mathrm{S} 2$ & 0.41 & 0.143 & edge-on & 0.07 & 3.097 & 8.688 & 5.691 & 4.766 & 3.875 & 3.518 & 3.28 & 2.75 & 2.69 & 2.566 & 2.215 & 2.074 & 1.996 \\
\hline S2 & 0.41 & 0.215 & interm. & 0.036 & 8.048 & 14.475 & 8.436 & 7.527 & 6.394 & 6.246 & 6.334 & 5.479 & 5.337 & 5.536 & 4.914 & 4.872 & 4.674 \\
\hline $\mathrm{S} 2$ & 0.41 & 0.286 & interm. & 0.006 & 325.941 & 89.774 & 81.513 & 90.26 & 99.567 & 141.653 & 188.049 & 209.869 & 286.666 & 235.39 & 230.728 & 290.957 & 290.017 \\
\hline $\mathrm{S} 2$ & 0.41 & 0.357 & interm. & -0.028 & 10.088 & 287.946 & 242.767 & 102.114 & 7.584 & 3.114 & 5.681 & 2.252 & 2.275 & 1.467 & 1.46 & 1.366 & 1.348 \\
\hline S2 & 0.41 & 0.429 & interm. & -0.055 & 3.329 & 114.263 & 12.059 & 8.003 & 5.633 & 4.619 & 3.772 & 3.863 & 3.625 & 2.921 & 2.65 & 2.339 & 2.753 \\
\hline $\mathrm{S} 2$ & 0.41 & 0.5 & interm. & -0.077 & 4.307 & 29.424 & 13.731 & 10.788 & 7.741 & 6.513 & 5.69 & 5.379 & 5.472 & 4.579 & 4.078 & 3.838 & 4.58 \\
\hline $\mathrm{S} 2$ & 0.41 & 0.571 & interm. & -0.095 & 5.868 & 30.188 & 16.67 & 13.849 & 10.111 & 8.789 & 7.415 & 7.287 & 7.434 & 6.16 & 5.751 & 5.159 & 6.273 \\
\hline $\mathrm{S} 2$ & 0.41 & 0.643 & pole-on & \begin{tabular}{|l}
-0.109 \\
\end{tabular} & 7.822 & 35.267 & 21.863 & 17.339 & 13.074 & 11.052 & 9.856 & 9.108 & 9.634 & 7.692 & 7.469 & 6.853 & 8.54 \\
\hline $\mathrm{S} 2$ & 0.41 & 0.714 & pole-on & -0.12 & 10.025 & 44.073 & 26.144 & 20.859 & 16.166 & 13.69 & 12.224 & 11.079 & 11.898 & 9.47 & 9.263 & 8.399 & 11.015 \\
\hline $\mathrm{S} 2$ & 0.41 & 0.786 & pole-on & -0.128 & 12.318 & 52.375 & 33.204 & 26.75 & 19.56 & 16.682 & 14.793 & 13.212 & 14.833 & 11.13 & 11.143 & 10.25 & 14.394 \\
\hline $\mathrm{S} 2$ & 0.41 & 0.857 & pole-on & -0.132 & 15.307 & 64.978 & 40.873 & 33.01 & 23.509 & 19.348 & 17.585 & 15.595 & 18.522 & 13.11 & 13.34 & 11.903 & 17.927 \\
\hline $\mathrm{S} 2$ & 0.41 & 0.928 & pole-on & -0.134 & 18.504 & 77.366 & 50.087 & 40.975 & 27.268 & 22.705 & 18.944 & 18.613 & 22.076 & 14.894 & 15.82 & 13.596 & 22.54 \\
\hline $\mathrm{S} 2$ & 0.41 & 1.0 & pole-on & -0.132 & 20.159 & 91.097 & 56.338 & 46.712 & 31.317 & 25.464 & 22.132 & 21.343 & 26.003 & 16.783 & 18.339 & 15.431 & 26.966 \\
\hline $\mathrm{S} 2$ & 0.3 & 0.0 & edge-on & 0.089 & 2.091 & 9.205 & 5.304 & 4.089 & 3.018 & 2.581 & 2.365 & 2.155 & 1.888 & 1.737 & 1.482 & 1.388 & 1.3 \\
\hline $\mathrm{S} 2$ & 0.3 & 0.071 & edge-on & 0.076 & 2.22 & 9.892 & 5.421 & 4.307 & 3.248 & 2.934 & 2.562 & 2.257 & 2.087 & 1.861 & 1.593 & 1.497 & 1.407 \\
\hline $\mathrm{S} 2$ & 0.3 & 0.143 & edge-on & 0.054 & 3.422 & 13.301 & 6.616 & 5.706 & 4.384 & 4.092 & 3.74 & 3.487 & 3.051 & 2.875 & 2.513 & 2.439 & 2.388 \\
\hline $\mathrm{S} 2$ & 0.3 & 0.215 & interm. & 0.029 & 8.308 & 57.184 & 12.846 & 9.437 & 7.939 & 8.206 & 7.161 & 7.142 & 6.838 & 6.387 & 6.21 & 5.895 & 5.674 \\
\hline $\mathrm{S} 2$ & 0.3 & 0.286 & interm. & 0.007 & 307.856 & 203.145 & 124.363 & 155.981 & 171.274 & 197.938 & 198.094 & 237.045 & 252.117 & 219.426 & 262.68 & 265.888 & 275.838 \\
\hline $\mathrm{S} 2$ & 0.3 & 0.357 & interm. & -0.016 & 23.143 & 312.535 & 273.005 & 212.32 & 90.758 & 55.577 & 28.266 & 9.299 & 4.793 & 5.332 & 5.018 & 1.528 & 2.431 \\
\hline $\mathrm{S} 2$ & 0.3 & 0.429 & interm. & -0.037 & 2.777 & 207.001 & 18.72 & 8.347 & 5.176 & 3.951 & 3.775 & 3.405 & 3.355 & 2.88 & 2.696 & 2.484 & 2.452 \\
\hline $\mathrm{S} 2$ & 0.3 & 0.5 & interm. & -0.053 & 4.14 & 53.104 & 12.064 & 9.987 & 7.447 & 5.975 & 5.064 & 4.803 & 4.707 & 4.314 & 4.08 & 3.398 & 3.942 \\
\hline $\mathrm{S} 2$ & 0.3 & 0.571 & interm. & -0.065 & 5.834 & 41.648 & 16.446 & 13.099 & 9.3 & 7.795 & 7.088 & 6.629 & 6.822 & 5.761 & 5.22 & 4.76 & 5.514 \\
\hline $\mathrm{S} 2$ & 0.3 & 0.643 & pole-on & -0.075 & 7.744 & 34.31 & 19.568 & 16.838 & 11.785 & 10.028 & 8.938 & 8.274 & 8.57 & 6.841 & 6.979 & 6.219 & 7.64 \\
\hline $\mathrm{S} 2$ & 0.3 & 0.714 & pole-on & -0.082 & 10.174 & 44.878 & 24.973 & 20.991 & 15.308 & 12.632 & 11.7 & 10.075 & 10.94 & 8.871 & 8.431 & 7.646 & 9.809 \\
\hline $\mathrm{S} 2$ & 0.3 & 0.786 & pole-on & -0.087 & 12.535 & 54.745 & 30.6 & 25.89 & 18.027 & 15.717 & 14.676 & 12.441 & 13.39 & 10.553 & 10.663 & 9.16 & 12.86 \\
\hline $\mathrm{S} 2$ & 0.3 & 0.857 & pole-on & -0.09 & 15.468 & 61.628 & 38.204 & 32.028 & 22.615 & 18.307 & 16.882 & 14.675 & 16.241 & 12.438 & 12.865 & 11.296 & 16.315 \\
\hline $\mathrm{S} 2$ & 0.3 & 0.928 & pole-on & -0.09 & 18.484 & 82.989 & 49.643 & 40.948 & 26.098 & 21.175 & 18.687 & 17.448 & 21.523 & 14.221 & 15.183 & 12.587 & 21.251 \\
\hline $\mathrm{S} 2$ & 0.3 & 1.0 & pole-on & -0.087 & 20.741 & 101.189 & 58.898 & 44.898 & 30.347 & 24.686 & 21.457 & 20.741 & 25.81 & 15.901 & 17.759 & 15.213 & 26.203 \\
\hline S3 & 2.5 & 0.0 & edge-on & 0.441 & 1.268 & 2.346 & 1.728 & 1.501 & 1.245 & 1.116 & 1.02 & 0.918 & 0.851 & 0.773 & 0.671 & 0.612 & 0.599 \\
\hline S3 & 2.5 & 0.071 & edge-on & 0.335 & 1.647 & 2.367 & 1.717 & 1.505 & 1.235 & 1.089 & 1.004 & 0.897 & 0.827 & 0.75 & 0.648 & 0.587 & 0.569 \\
\hline S3 & 2.5 & 0.143 & edge-on & 0.176 & 4.705 & 3.127 & 2.178 & 1.826 & 1.396 & 1.19 & 1.071 & 0.94 & 0.868 & 0.762 & 0.708 & 0.58 & 0.572 \\
\hline S3 & 2.5 & 0.215 & interm. & 0.035 & 432.992 & 4.364 & 2.806 & 2.236 & 1.536 & 1.241 & 1.074 & 0.913 & 0.815 & 0.646 & 0.534 & 0.463 & 0.47 \\
\hline $\mathrm{S} 3$ & 2.5 & 0.286 & interm. & -0.1 & 0.445 & 6.067 & 327.9 & 54.278 & 12.528 & 8.669 & 6.986 & 5.667 & 4.825 & 4.15 & 3.349 & 3.085 & 2.92 \\
\hline
\end{tabular}


Table A.1 - continued.

\begin{tabular}{|c|c|c|c|c|c|c|c|c|c|c|c|c|c|c|c|c|c|}
\hline & & & $\tilde{\tau}_{\text {bu }}$ & & $\mathrm{bu}$ & 0.15 & 0.45 & 0.75 & 1.50 & 2.25 & 3.00 & 4.50 & 6.00 & 9.00 & 15.00 & 30.00 & $\infty$ \\
\hline Star & $\Sigma_{0}\left[\mathrm{~g} \mathrm{~cm}^{-2}\right]$ & $\cos i$ & ang. type & $\Delta B_{\mathrm{bu}}^{\infty}$ & $\xi_{\mathrm{bu}}^{B}$ & $\xi_{\mathrm{d}}^{B}$ & & & & & & & & & & & \\
\hline S3 & 2.5 & 0.357 & interm. & $\mid-0.208$ & 1.626 & 14.578 & 8.246 & 6.452 & 4.925 & 4.161 & 3.807 & 3.409 & 3.104 & 2.826 & 2.456 & 2.228 & 2.193 \\
\hline S3 & 2.5 & 0.429 & interm. & $\mid-0.299$ & 3.207 & 12.613 & 8.285 & 6.522 & 5.059 & 4.377 & 4.004 & 3.572 & 3.306 & 3.004 & 2.641 & 2.423 & 2.361 \\
\hline S3 & 2.5 & 0.5 & interm. & -0.371 & 5.155 & 14.692 & 8.967 & 7.245 & 5.573 & 4.825 & 4.42 & 3.977 & 3.704 & 3.376 & 2.965 & 2.762 & 2.715 \\
\hline S3 & 2.5 & 0.571 & interm. & -0.43 & 7.44 & 16.888 & 10.178 & 8.143 & 6.27 & 5.431 & 5.002 & 4.528 & 4.205 & 3.854 & 3.428 & 3.205 & 3.174 \\
\hline S3 & 2.5 & 0.643 & pole-on & $\mid-0.481$ & 9.84 & 19.358 & 11.605 & 9.245 & 7.108 & 6.222 & 5.705 & 5.177 & 4.844 & 4.44 & 3.957 & 3.736 & 3.743 \\
\hline S3 & 2.5 & 0.714 & pole-on & -0.522 & 12.448 & 22.405 & 13.144 & 10.546 & 8.113 & 7.058 & 6.51 & 5.926 & 5.596 & 5.148 & 4.609 & 4.354 & 4.401 \\
\hline S3 & 2.5 & 0.786 & pole-on & -0.555 & 15.02 & 25.833 & 15.065 & 12.031 & 9.207 & 8.036 & 7.465 & 6.771 & 6.479 & 5.908 & 5.367 & 5.068 & 5.185 \\
\hline S3 & 2.5 & 0.857 & pole-on & -0.582 & 17.654 & 30.114 & 17.272 & 13.73 & 10.526 & 9.173 & 8.505 & 7.812 & 7.531 & 6.834 & 6.249 & 5.868 & 6.143 \\
\hline S3 & 2.5 & 0.928 & pole-on & -0.605 & 20.1 & 35.058 & 20.046 & 15.866 & 12.118 & 10.528 & 9.711 & 9.011 & 8.765 & 7.825 & 7.251 & 6.817 & 7.274 \\
\hline S3 & 2.5 & 1.0 & pole-on & -0.62 & 22.173 & 40.392 & 22.95 & 18.203 & 13.758 & 11.855 & 10.965 & 10.266 & 10.141 & 8.857 & 8.319 & 7.779 & 8.479 \\
\hline S3 & 1.85 & 0.0 & edge-on & 0.35 & 1.369 & 2.767 & 1.994 & 1.716 & 1.397 & 1.214 & 1.156 & 1.565 & 1.025 & 0.888 & 0.754 & 0.688 & 0.668 \\
\hline S3 & 1.85 & 0.071 & edge-on & 0.277 & 1.65 & 2.689 & 2.067 & 1.77 & 1.429 & 1.231 & 1.173 & 1.397 & 1.014 & 0.9 & 0.77 & 0.698 & 0.673 \\
\hline S3 & 1.85 & 0.143 & edge-on & 0.163 & 3.84 & 3.571 & 2.677 & 2.263 & 1.723 & 1.451 & 1.393 & 1.318 & 1.149 & 1.044 & 0.883 & 0.799 & 0.782 \\
\hline S3 & 1.85 & 0.215 & interm. & 0.051 & 48.782 & 4.976 & 3.695 & 3.008 & 2.203 & 1.799 & 1.793 & 1.231 & 1.393 & 1.312 & 1.162 & 1.04 & 1.065 \\
\hline S3 & 1.85 & 0.286 & interm. & \begin{tabular}{|l}
-0.062 \\
\end{tabular} & 1.791 & 8.808 & 94.101 & 335.001 & 112.286 & 23.027 & 13.138 & 15.957 & 6.758 & 4.677 & 3.31 & 3.045 & 2.637 \\
\hline S3 & 1.85 & 0.357 & interm. & -0.156 & 1.45 & 39.382 & 8.783 & 6.684 & 5.19 & 4.834 & 4.063 & 5.185 & 3.571 & 3.113 & 2.645 & 2.512 & 2.391 \\
\hline S3 & 1.85 & 0.429 & interm. & -0.236 & 2.976 & 16.038 & 9.175 & 7.374 & 5.785 & 5.246 & 4.636 & 5.205 & 3.972 & 3.553 & 3.1 & 2.942 & 2.883 \\
\hline S3 & 1.85 & 0.5 & interm. & $\mid-0.301$ & 4.821 & 17.968 & 10.55 & 8.498 & 6.62 & 5.964 & 5.285 & 5.786 & 4.608 & 4.186 & 3.695 & 3.499 & 3.51 \\
\hline S3 & 1.85 & 0.571 & interm. & -0.354 & 6.942 & 20.867 & 12.231 & 9.85 & 7.706 & 6.895 & 6.275 & 6.565 & 5.341 & 4.925 & 4.38 & 4.154 & 4.243 \\
\hline S3 & 1.85 & 0.643 & pole-on & -0.4 & 9.326 & 24.407 & 14.312 & 11.464 & 8.899 & 7.988 & 7.333 & 7.575 & 6.259 & 5.81 & 5.207 & 4.958 & 5.178 \\
\hline S3 & 1.85 & 0.714 & pole-on & -0.436 & 11.877 & 28.481 & 16.569 & 13.46 & 10.337 & 9.303 & 8.545 & 8.726 & 7.328 & 6.785 & 6.175 & 5.823 & 6.157 \\
\hline S3 & 1.85 & 0.786 & pole-on & -0.466 & 14.551 & 33.419 & 19.445 & 15.617 & 12.002 & 10.752 & 9.941 & 10.086 & 8.475 & 7.917 & 7.212 & 6.835 & 7.366 \\
\hline S3 & 1.85 & 0.857 & pole-on & -0.489 & 17.291 & 39.165 & 22.883 & 18.296 & 13.981 & 12.479 & 11.468 & 11.769 & 9.922 & 9.076 & 8.5 & 7.982 & 8.924 \\
\hline S3 & 1.85 & 0.928 & pole-on & -0.507 & 19.97 & 46.229 & 26.82 & 21.489 & 16.244 & 14.247 & 13.101 & 13.796 & 11.623 & 10.387 & 9.972 & 9.236 & 10.653 \\
\hline S3 & 1.85 & 1.0 & pole-on & -0.519 & 22.005 & 53.872 & 31.133 & 25.052 & 18.479 & 16.08 & 14.706 & 16.156 & 13.352 & 11.73 & 11.433 & 10.512 & 12.609 \\
\hline S3 & 1.37 & 0.0 & edge-on & 0.278 & 1.509 & 3.667 & 2.399 & 1.967 & 1.594 & 1.399 & 1.292 & 1.15 & 1.067 & 0.972 & 0.844 & 0.772 & 0.747 \\
\hline S3 & 1.37 & 0.071 & edge-on & 0.227 & 1.781 & 3.719 & 2.47 & 2.088 & 1.677 & 1.475 & 1.354 & 1.229 & 1.119 & 1.019 & 0.886 & 0.822 & 0.787 \\
\hline S3 & 1.37 & 0.143 & edge-on & 0.144 & 3.543 & 4.607 & 3.289 & 2.695 & 2.153 & 1.886 & 1.716 & 1.549 & 1.411 & 1.288 & 1.134 & 1.045 & 1.013 \\
\hline S3 & 1.37 & 0.215 & interm. & 0.058 & 19.401 & 6.536 & 4.678 & 4.0 & 3.18 & 2.815 & 2.633 & 2.432 & 2.255 & 2.12 & 1.914 & 1.793 & 1.727 \\
\hline S3 & 1.37 & 0.286 & interm. & -0.031 & 5.526 & 9.847 & 9.498 & 76.933 & 342.854 & 290.933 & 221.112 & 81.665 & 7.668 & 3.727 & 1.951 & 1.158 & 1.109 \\
\hline $\mathrm{S} 3$ & 1.37 & 0.357 & interm. & -0.111 & 1.244 & 239.28 & 8.161 & 6.266 & 4.757 & 4.201 & 3.75 & 3.298 & 3.011 & 2.76 & 2.461 & 2.267 & 2.347 \\
\hline S3 & 1.37 & 0.429 & interm. & \begin{tabular}{|l}
-0.179 \\
\end{tabular} & 2.752 & 16.321 & 9.608 & 7.822 & 5.947 & 5.292 & 4.774 & 4.262 & 4.121 & 3.688 & 3.382 & 3.109 & 3.191 \\
\hline S3 & 1.37 & 0.5 & interm. & -0.234 & 4.53 & 19.63 & 11.424 & 9.421 & 7.361 & 6.456 & 5.961 & 5.318 & 5.087 & 4.645 & 4.24 & 3.969 & 4.115 \\
\hline $\mathrm{S} 3$ & 1.37 & 0.571 & interm. & -0.28 & 6.574 & 23.198 & 13.862 & 11.375 & 8.793 & 7.791 & 7.075 & 6.419 & 6.271 & 5.59 & 5.187 & 4.85 & 5.189 \\
\hline S3 & 1.37 & 0.643 & pole-on & -0.318 & 8.809 & 27.682 & 16.724 & 13.522 & 10.406 & 9.253 & 8.48 & 7.674 & 7.604 & 6.828 & 6.277 & 5.885 & 6.399 \\
\hline
\end{tabular}


Table A.1 - continued.

\begin{tabular}{|c|c|c|c|c|c|c|c|c|c|c|c|c|c|c|c|c|c|}
\hline & & & $\tilde{\tau}_{\mathrm{bu}}$ & & $\mathrm{bu}$ & 0.15 & 0.45 & 0.75 & 1.50 & 2.25 & 3.00 & 4.50 & 6.00 & 9.00 & 15.00 & 30.00 & $\infty$ \\
\hline Star & $\Sigma_{0}\left[\mathrm{~g} \mathrm{~cm}^{-2}\right]$ & $\cos i$ & ang. type & $\Delta B_{\mathrm{bu}}^{\infty}$ & $\xi_{\mathrm{bu}}^{B}$ & $\xi_{\mathrm{d}}^{B}$ & & & & & & & & & & & \\
\hline S3 & 1.37 & 0.714 & pole-on & -0.349 & 11.361 & 33.493 & 19.745 & 16.209 & 12.513 & 11.017 & 10.127 & 9.056 & 9.11 & 8.042 & 7.564 & 6.969 & 7.939 \\
\hline S3 & 1.37 & 0.786 & pole-on & -0.373 & 14.068 & 39.581 & 23.713 & 19.283 & 14.758 & 12.909 & 11.88 & 10.708 & 10.975 & 9.441 & 8.973 & 8.316 & 9.687 \\
\hline S3 & 1.37 & 0.857 & pole-on & -0.391 & 16.824 & 46.753 & 28.049 & 23.091 & 17.412 & 15.158 & 13.806 & 12.599 & 13.093 & 10.896 & 10.581 & 9.687 & 11.78 \\
\hline S3 & 1.37 & 0.928 & pole-on & -0.405 & 19.706 & 56.269 & 33.623 & 27.285 & 20.141 & 17.268 & 15.639 & 14.749 & 15.584 & 12.302 & 12.425 & 11.143 & 14.378 \\
\hline S3 & 1.37 & 1.0 & pole-on & -0.412 & 21.812 & 65.678 & 39.096 & 32.205 & 23.139 & 19.365 & 17.445 & 16.678 & 18.157 & 13.93 & 14.306 & 12.687 & 16.991 \\
\hline S3 & 1.01 & 0.0 & edge-on & 0.22 & 1.677 & 4.456 & 2.74 & 2.351 & 1.819 & 1.605 & 1.466 & 1.3 & 1.19 & 1.039 & 0.793 & 0.823 & 0.864 \\
\hline S3 & 1.01 & 0.071 & edge-on & 0.184 & 1.961 & 4.485 & 2.859 & 2.521 & 1.941 & 1.731 & 1.558 & 1.384 & 1.277 & 1.102 & 0.855 & 0.886 & 0.921 \\
\hline S3 & 1.01 & 0.143 & edge-on & 0.124 & 3.512 & 5.36 & 3.868 & 3.253 & 2.585 & 2.31 & 2.14 & 1.9 & 1.749 & 1.542 & 1.319 & 1.26 & 1.288 \\
\hline S3 & 1.01 & 0.215 & interm. & 0.059 & 12.199 & 8.03 & 5.565 & 5.322 & 4.385 & 3.942 & 3.58 & 3.406 & 3.215 & 3.081 & 3.024 & 2.724 & 2.653 \\
\hline S3 & 1.01 & 0.286 & interm. & -0.007 & 50.443 & 18.865 & 11.819 & 23.682 & 191.658 & 250.512 & 268.387 & 311.962 & 362.139 & 330.088 & 250.75 & 201.314 & 154.863 \\
\hline S3 & 1.01 & 0.357 & interm. & -0.075 & 1.477 & 293.44 & 11.987 & 4.559 & 3.631 & 3.23 & 2.811 & 2.524 & 2.503 & 2.235 & 2.489 & 1.765 & 1.719 \\
\hline S3 & 1.01 & 0.429 & interm. & $\mid-0.129$ & 2.572 & 18.672 & 10.325 & 7.458 & 5.553 & 4.987 & 4.639 & 4.135 & 3.942 & 3.735 & 3.811 & 2.947 & 2.883 \\
\hline S3 & 1.01 & 0.5 & interm. & -0.174 & 4.259 & 20.428 & 12.744 & 9.822 & 7.235 & 6.55 & 6.112 & 5.44 & 5.374 & 4.96 & 4.959 & 4.124 & 3.892 \\
\hline S3 & 1.01 & 0.571 & interm. & -0.211 & 6.237 & 24.792 & 15.623 & 12.157 & 9.01 & 8.377 & 7.606 & 6.74 & 6.786 & 6.223 & 6.212 & 5.355 & 5.116 \\
\hline S3 & 1.01 & 0.643 & pole-on & -0.242 & 8.386 & 29.898 & 19.064 & 14.83 & 10.973 & 10.178 & 9.302 & 8.311 & 8.37 & 7.653 & 7.624 & 6.695 & 6.59 \\
\hline S3 & 1.01 & 0.714 & pole-on & -0.266 & 10.74 & 37.261 & 23.199 & 18.138 & 15.06 & 12.152 & 11.279 & 10.0 & 10.222 & 9.126 & 8.965 & 8.226 & 8.612 \\
\hline S3 & 1.01 & 0.786 & pole-on & -0.284 & 13.437 & 44.864 & 27.835 & 22.421 & 15.774 & 14.693 & 13.278 & 11.941 & 12.5 & 10.668 & 10.452 & 9.757 & 11.393 \\
\hline S3 & 1.01 & 0.857 & pole-on & -0.298 & 16.18 & 53.436 & 33.472 & 26.436 & 18.62 & 17.092 & 15.517 & 14.032 & 15.117 & 12.4 & 12.266 & 11.358 & 14.909 \\
\hline S3 & 1.01 & 0.928 & pole-on & -0.307 & 19.276 & 64.504 & 40.153 & 31.813 & 21.555 & 19.6 & 17.597 & 16.373 & 18.226 & 13.911 & 14.077 & 13.209 & 19.288 \\
\hline S3 & 1.01 & 1.0 & pole-on & -0.31 & 21.398 & 74.777 & 47.218 & 37.514 & 24.851 & 21.688 & 19.626 & 18.809 & 21.265 & 15.603 & 15.978 & 14.93 & 23.966 \\
\hline S3 & 0.75 & 0.0 & edge-on & 0.176 & 1.815 & 5.542 & 3.426 & 2.764 & 2.108 & 1.824 & 1.684 & 1.476 & 1.34 & 1.235 & 1.078 & 0.976 & 0.935 \\
\hline S3 & 0.75 & 0.071 & edge-on & 0.149 & 2.087 & 5.436 & 3.521 & 2.875 & 2.243 & 1.932 & 1.793 & 1.561 & 1.428 & 1.326 & 1.162 & 1.054 & 1.013 \\
\hline S3 & 0.75 & 0.143 & edge-on & 0.104 & 3.648 & 6.419 & 4.71 & 3.853 & 3.056 & 2.705 & 2.467 & 2.246 & 2.08 & 1.94 & 1.69 & 1.576 & 1.512 \\
\hline S3 & 0.75 & 0.215 & interm. & 0.053 & 20.664 & 9.426 & 6.89 & 6.116 & 5.303 & 4.905 & 4.69 & 4.326 & 4.177 & 4.161 & 3.88 & 3.758 & 3.577 \\
\hline S3 & 0.75 & 0.286 & interm. & 0.004 & 356.927 & 18.097 & 16.503 & 25.969 & 65.733 & 92.01 & 79.814 & 197.844 & 250.789 & 246.588 & 281.701 & 261.543 & 309.774 \\
\hline S3 & 0.75 & 0.357 & interm. & -0.049 & 1.054 & 291.849 & 65.815 & 6.04 & 2.75 & 2.402 & 2.079 & 1.94 & 1.798 & 1.483 & 1.399 & 1.209 & 1.343 \\
\hline S3 & 0.75 & 0.429 & interm. & -0.092 & 2.474 & 18.485 & 8.929 & 6.928 & 5.317 & 4.493 & 3.939 & 3.613 & 3.573 & 3.086 & 2.831 & 2.399 & 2.889 \\
\hline S3 & 0.75 & 0.5 & interm. & -0.128 & 3.987 & 21.163 & 11.868 & 9.668 & 7.303 & 6.372 & 5.802 & 5.217 & 5.173 & 4.481 & 4.173 & 3.709 & 4.217 \\
\hline S3 & 0.75 & 0.571 & interm. & -0.156 & 5.628 & 24.693 & 15.043 & 12.451 & 9.213 & 8.29 & 7.385 & 6.687 & 6.83 & 5.9 & 5.515 & 5.038 & 5.771 \\
\hline S3 & 0.75 & 0.643 & pole-on & -0.181 & 26.476 & 31.885 & 18.944 & 15.527 & 11.707 & 10.267 & 9.353 & 8.366 & 8.67 & 7.477 & 6.882 & 6.358 & 7.677 \\
\hline S3 & 0.75 & 0.714 & pole-on & \begin{tabular}{|l}
-0.198 \\
\end{tabular} & 9.091 & 39.644 & 23.076 & 19.193 & 14.396 & 12.773 & 11.588 & 10.15 & 10.843 & 9.025 & 8.609 & 7.795 & 9.782 \\
\hline S3 & 0.75 & 0.786 & pole-on & -0.212 & 10.935 & 47.095 & 28.963 & 23.509 & 17.553 & 15.363 & 13.788 & 12.238 & 13.193 & 10.627 & 10.397 & 9.304 & 12.479 \\
\hline S3 & 0.75 & 0.857 & pole-on & -0.221 & 13.043 & 56.462 & 34.728 & 28.951 & 20.574 & 17.849 & 16.188 & 14.474 & 16.017 & 12.358 & 12.399 & 10.863 & 15.519 \\
\hline S3 & 0.75 & 0.928 & pole-on & -0.227 & 14.785 & 67.829 & 42.224 & 34.796 & 23.834 & 20.378 & 18.204 & 16.962 & 19.661 & 14.071 & 14.574 & 12.509 & 19.364 \\
\hline S3 & 0.75 & 1.0 & pole-on & -0.227 & 15.86 & 79.722 & 49.792 & 40.896 & 26.983 & 22.788 & 20.079 & 19.462 & 22.703 & 15.762 & 16.784 & 14.31 & 23.162 \\
\hline
\end{tabular}


Table A.1 - continued.

\begin{tabular}{|c|c|c|c|c|c|c|c|c|c|c|c|c|c|c|c|c|c|}
\hline & & & $\tilde{\tau}_{\text {bu }}$ & & $\mathrm{bu}$ & 0.15 & 0.45 & 0.75 & 1.50 & 2.25 & 3.00 & 4.50 & 6.00 & 9.00 & 15.00 & 30.00 & $\infty$ \\
\hline Star & $\Sigma_{0}\left[\mathrm{~g} \mathrm{~cm}^{-2}\right]$ & $\cos i$ & ang. type & $\Delta B_{\mathrm{bu}}^{\infty}$ & $\xi_{\mathrm{bu}}^{B}$ & $\xi_{\mathrm{d}}^{B}$ & & & & & & & & & & & \\
\hline S3 & 0.56 & 0.0 & edge-on & 0.141 & 2.13 & 6.563 & 3.973 & 3.212 & 2.439 & 2.104 & 1.918 & 1.631 & 1.525 & 1.412 & 1.197 & 1.112 & 1.047 \\
\hline S3 & 0.56 & 0.071 & edge-on & 0.121 & 2.349 & 6.243 & 4.0 & 3.327 & 2.65 & 2.219 & 2.049 & 1.768 & 1.612 & 1.506 & 1.291 & 1.185 & 1.138 \\
\hline S3 & 0.56 & 0.143 & edge-on & 0.086 & 3.704 & 7.507 & 5.086 & 4.428 & 3.581 & 3.125 & 2.872 & 2.607 & 2.405 & 2.305 & 2.009 & 1.807 & 1.742 \\
\hline S3 & 0.56 & 0.215 & interm. & 0.047 & 9.193 & 11.029 & 8.03 & 7.33 & 6.268 & 6.006 & 5.696 & 5.436 & 5.079 & 5.152 & 4.911 & 4.581 & 4.534 \\
\hline S3 & 0.56 & 0.286 & interm. & 0.009 & 365.41 & 55.16 & 50.157 & 40.616 & 95.565 & 98.997 & 143.33 & 119.122 & 192.469 & 186.694 & 218.694 & 263.552 & 246.734 \\
\hline S3 & 0.56 & 0.357 & interm. & -0.033 & 5.873 & 306.846 & 160.575 & 54.689 & 4.754 & 2.178 & 1.934 & 1.87 & 1.778 & 1.411 & 1.134 & 1.075 & 1.121 \\
\hline S3 & 0.56 & 0.429 & interm. & -0.065 & 2.332 & 57.008 & 8.829 & 6.539 & 4.503 & 3.938 & 3.546 & 3.239 & 3.119 & 2.647 & 2.468 & 2.144 & 2.406 \\
\hline S3 & 0.56 & 0.5 & interm. & -0.091 & 3.932 & 20.754 & 11.95 & 8.802 & 6.623 & 5.715 & 5.213 & 4.812 & 4.698 & 4.12 & 3.76 & 3.404 & 3.879 \\
\hline S3 & 0.56 & 0.571 & interm. & -0.113 & 5.651 & 24.769 & 15.281 & 11.985 & 8.883 & 7.81 & 6.894 & 6.29 & 6.459 & 5.454 & 5.016 & 4.717 & 5.352 \\
\hline S3 & 0.56 & 0.643 & pole-on & -0.13 & 7.79 & 31.581 & 18.711 & 15.627 & 11.369 & 9.764 & 8.936 & 8.169 & 8.211 & 6.969 & 6.59 & 6.041 & 7.351 \\
\hline S3 & 0.56 & 0.714 & pole-on & -0.143 & 9.964 & 38.52 & 23.66 & 19.245 & 14.121 & 12.299 & 11.304 & 9.748 & 10.581 & 8.687 & 8.306 & 7.567 & 9.732 \\
\hline S3 & 0.56 & 0.786 & pole-on & -0.153 & 12.53 & 47.235 & 29.41 & 23.755 & 17.327 & 15.158 & 13.366 & 12.055 & 12.795 & 10.069 & 9.945 & 8.912 & 12.361 \\
\hline S3 & 0.56 & 0.857 & pole-on & -0.159 & 15.582 & 57.173 & 36.377 & 28.742 & 20.667 & 17.83 & 15.517 & 14.186 & 16.289 & 11.727 & 11.777 & 10.668 & 15.485 \\
\hline S3 & 0.56 & 0.928 & pole-on & -0.162 & 18.334 & 70.888 & 44.058 & 36.161 & 24.178 & 20.218 & 17.568 & 16.626 & 19.451 & 13.529 & 14.135 & 12.24 & 19.463 \\
\hline S3 & 0.56 & 1.0 & pole-on & -0.16 & 20.801 & 81.44 & 50.908 & 41.672 & 27.224 & 22.355 & 19.287 & 19.053 & 22.702 & 15.114 & 16.404 & 13.912 & 23.57 \\
\hline S3 & 0.41 & 0.0 & edge-on & 0.112 & 2.263 & 8.008 & 4.447 & 3.848 & 2.793 & 2.355 & 2.163 & 1.884 & 1.706 & 1.559 & 1.345 & 1.228 & 1.178 \\
\hline S3 & 0.41 & 0.071 & edge-on & 0.096 & 2.491 & 7.714 & 4.608 & 4.092 & 2.897 & 2.502 & 2.292 & 1.986 & 1.853 & 1.677 & 1.45 & 1.32 & 1.28 \\
\hline S3 & 0.41 & 0.143 & edge-on & 0.068 & 3.902 & 9.475 & 5.886 & 4.98 & 3.872 & 3.497 & 3.319 & 2.93 & 2.691 & 2.459 & 2.192 & 2.045 & 2.017 \\
\hline S3 & 0.41 & 0.215 & interm. & 0.038 & 9.563 & 27.387 & 9.429 & 8.185 & 7.225 & 6.733 & 6.897 & 6.655 & 6.202 & 5.86 & 5.521 & 5.513 & 5.491 \\
\hline S3 & 0.41 & 0.286 & interm. & 0.01 & 320.824 & 83.257 & 83.327 & 117.251 & 119.065 & 150.311 & 151.839 & 149.581 & 221.303 & 203.425 & 245.279 & 262.177 & 279.242 \\
\hline S3 & 0.41 & 0.357 & interm. & -0.019 & 10.135 & 301.436 & 268.45 & 136.486 & 68.11 & 19.446 & 14.775 & 3.627 & 1.86 & 1.642 & 1.857 & 1.384 & 1.258 \\
\hline S3 & 0.41 & 0.429 & interm. & -0.044 & 2.279 & 141.313 & 9.649 & 6.459 & 4.745 & 3.814 & 3.211 & 3.103 & 3.012 & 2.477 & 2.431 & 2.023 & 2.131 \\
\hline S3 & 0.41 & 0.5 & interm. & -0.064 & 3.605 & 27.428 & 11.575 & 8.592 & 6.298 & 5.424 & 4.841 & 4.512 & 4.462 & 3.753 & 3.462 & 3.021 & 3.39 \\
\hline S3 & 0.41 & 0.571 & interm. & -0.079 & 5.382 & 28.304 & 14.146 & 11.459 & 8.327 & 7.415 & 6.694 & 6.007 & 5.945 & 5.185 & 4.686 & 4.322 & 4.959 \\
\hline S3 & 0.41 & 0.643 & pole-on & -0.092 & 7.559 & 33.025 & 18.585 & 14.861 & 10.802 & 9.393 & 8.421 & 7.612 & 7.946 & 6.458 & 6.248 & 5.745 & 7.539 \\
\hline S3 & 0.41 & 0.714 & pole-on & -0.1 & 9.811 & 38.678 & 23.148 & 18.36 & 13.754 & 11.435 & 10.557 & 9.342 & 9.891 & 8.191 & 7.892 & 6.907 & 9.005 \\
\hline S3 & 0.41 & 0.786 & pole-on & -0.107 & 12.368 & 49.245 & 28.926 & 22.453 & 17.287 & 14.174 & 12.725 & 11.167 & 12.382 & 9.863 & 9.673 & 8.36 & 11.935 \\
\hline S3 & 0.41 & 0.857 & pole-on & -0.11 & 15.427 & 59.898 & 35.694 & 29.841 & 20.596 & 17.185 & 15.365 & 13.712 & 15.07 & 11.365 & 11.201 & 10.002 & 14.717 \\
\hline S3 & 0.41 & 0.928 & pole-on & -0.111 & 18.374 & 72.148 & 44.098 & 34.626 & 24.072 & 19.39 & 17.568 & 15.598 & 18.814 & 13.051 & 13.835 & 11.407 & 18.667 \\
\hline S3 & 0.41 & 1.0 & pole-on & -0.109 & 20.701 & 85.31 & 50.408 & 41.701 & 27.209 & 21.072 & 19.652 & 18.874 & 22.257 & 14.288 & 15.637 & 12.966 & 22.916 \\
\hline S3 & 0.3 & 0.0 & edge-on & 0.086 & 2.51 & 10.071 & 5.338 & 4.146 & 3.095 & 2.704 & 2.356 & 2.102 & 1.917 & 1.775 & 1.507 & 1.354 & 1.374 \\
\hline S3 & 0.3 & 0.071 & edge-on & 0.074 & 2.799 & 10.38 & 5.336 & 4.229 & 3.38 & 2.82 & 2.523 & 2.225 & 1.972 & 1.887 & 1.6 & 1.43 & 1.418 \\
\hline S3 & 0.3 & 0.143 & edge-on & 0.054 & 7.093 & 19.248 & 6.661 & 5.439 & 4.555 & 4.105 & 3.76 & 3.19 & 3.09 & 2.749 & 2.363 & 2.333 & 2.271 \\
\hline S3 & 0.3 & 0.215 & interm. & 0.03 & 15.224 & 50.475 & 11.63 & 13.3 & 9.945 & 8.398 & 7.71 & 7.277 & 7.227 & 7.159 & 6.435 & 6.621 & 6.294 \\
\hline S3 & 0.3 & 0.286 & interm. & 0.009 & 275.284 & 212.519 & 132.991 & 149.15 & 215.419 & 218.448 & 224.952 & 270.133 & 250.52 & 234.845 & 244.373 & 277.698 & 270.83 \\
\hline
\end{tabular}


Table A.1 - continued.

\begin{tabular}{|c|c|c|c|c|c|c|c|c|c|c|c|c|c|c|c|c|c|}
\hline & & & 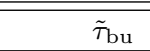 & & $\overline{\mathrm{bu}}$ & $\overline{0.15}$ & 0.45 & 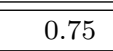 & 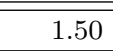 & 2.25 & 3.00 & 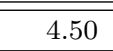 & 26.00 & 9.00 & 15.00 & 30.00 & $\bar{\infty}$ \\
\hline Star & $\Sigma_{0}\left[\mathrm{~g} \mathrm{~cm}^{-2}\right]$ & $\cos i$ & ang. type & $\Delta B_{\mathrm{bu}}^{\infty}$ & $\xi_{\mathrm{bu}}^{B}$ & $\xi_{\mathrm{d}}^{B}$ & & & & & & & & & & & \\
\hline S3 & 0.3 & 0.357 & interm. & -0.011 & 76.221 & 297.838 & 317.487 & 244.71 & 158.067 & 107.346 & 73.889 & 25.574 & 13.273 & 24.816 & 8.619 & 6.064 & 2.316 \\
\hline S3 & 0.3 & 0.429 & interm. & -0.031 & 2.524 & 245.67 & 42.41 & 8.272 & 5.767 & 3.87 & 3.641 & 3.162 & 3.287 & 2.587 & 2.399 & 2.142 & 2.114 \\
\hline S3 & 0.3 & 0.5 & interm. & -0.044 & 7.095 & 74.876 & 14.197 & 9.121 & 6.902 & 5.733 & 4.83 & 4.441 & 4.158 & 3.69 & 3.512 & 2.991 & 3.172 \\
\hline S3 & 0.3 & 0.571 & interm. & -0.055 & 5.344 & 31.969 & 14.738 & 11.345 & 8.041 & 7.136 & 6.592 & 5.914 & 5.589 & 4.772 & 4.654 & 4.112 & 4.643 \\
\hline S3 & 0.3 & 0.643 & pole-on & -0.064 & 7.244 & 33.166 & 18.436 & 14.348 & 10.288 & 8.967 & 8.136 & 7.332 & 7.632 & 6.331 & 5.917 & 5.444 & 6.053 \\
\hline S3 & 0.3 & 0.714 & pole-on & -0.07 & 9.471 & 40.31 & 22.668 & 18.742 & 12.826 & 11.489 & 10.443 & 8.738 & 9.355 & 7.954 & 7.102 & 6.689 & 8.549 \\
\hline S3 & 0.3 & 0.786 & pole-on & -0.074 & 12.379 & 47.613 & 27.649 & 22.859 & 16.0 & 14.289 & 12.948 & 10.607 & 11.86 & 9.141 & 9.079 & 8.072 & 10.714 \\
\hline S3 & 0.3 & 0.857 & pole-on & -0.076 & 15.545 & 62.145 & 35.322 & 28.246 & 19.235 & 16.176 & 14.603 & 13.57 & 14.625 & 10.332 & 11.2 & 9.238 & 14.182 \\
\hline S3 & 0.3 & 0.928 & pole-on & -0.076 & 19.46 & 70.216 & 42.11 & 35.428 & 21.956 & 19.208 & 16.643 & 15.213 & 18.835 & 12.603 & 13.076 & 11.288 & 17.93 \\
\hline S3 & 0.3 & 1.0 & pole-on & -0.074 & 21.146 & 94.419 & 52.726 & 42.628 & 25.751 & 21.395 & 19.229 & 18.697 & 21.384 & 14.09 & 15.347 & 12.911 & 22.254 \\
\hline
\end{tabular}


Table A.2 - Empirical law parameters for band $V$.

\begin{tabular}{|c|c|c|c|c|c|c|c|c|c|c|c|c|c|c|c|c|c|}
\hline & & & $\tilde{\tau}_{\mathrm{bu}}$ & & $\mathrm{bu}$ & 0.15 & 0.45 & 0.75 & 1.50 & 2.25 & 3.00 & 4.50 & 6.00 & 9.00 & 15.00 & 30.00 & $\infty$ \\
\hline Star & $\Sigma_{0}\left[\mathrm{~g} \mathrm{~cm}^{-2}\right]$ & $\cos i$ & ang. type & $\Delta V_{\mathrm{bu}}^{\infty}$ & $\xi_{\mathrm{bu}}^{V}$ & $\xi_{\mathrm{d}}^{V}$ & & & & & & & & & & & \\
\hline S1 & 2.5 & 0.0 & edge-on & 0.554 & 1.086 & 1.915 & 1.484 & 1.327 & 1.106 & 0.99 & 0.911 & 0.827 & 0.767 & 0.69 & 0.598 & 0.539 & 0.52 \\
\hline $\mathrm{S} 1$ & 2.5 & 0.071 & edge-on & 0.33 & 1.453 & 1.611 & 1.22 & 1.09 & 0.872 & 0.772 & 0.704 & 0.645 & 0.586 & 0.526 & 0.449 & 0.402 & 0.386 \\
\hline S1 & 2.5 & 0.143 & edge-on & 0.039 & 133.16 & 1.59 & 1.002 & 0.804 & 0.405 & 0.294 & 0.233 & 0.22 & 0.221 & 0.21 & 0.207 & 0.206 & 0.208 \\
\hline S1 & 2.5 & 0.215 & interm. & -0.197 & 0.662 & 133.572 & 22.539 & 10.466 & 6.261 & 4.714 & 4.261 & 3.261 & 3.012 & 2.813 & 2.431 & 2.128 & 2.189 \\
\hline S1 & 2.5 & 0.286 & interm. & -0.378 & 1.663 & 10.671 & 5.867 & 4.442 & 3.454 & 2.898 & 2.628 & 2.183 & 2.023 & 1.879 & 1.653 & 1.457 & 1.479 \\
\hline S1 & 2.5 & 0.357 & interm. & -0.521 & 2.869 & 8.264 & 5.079 & 3.984 & 3.109 & 2.637 & 2.403 & 2.032 & 1.878 & 1.749 & 1.547 & 1.368 & 1.388 \\
\hline S1 & 2.5 & 0.429 & interm. & -0.636 & 4.315 & 8.559 & 5.072 & 3.954 & 3.087 & 2.634 & 2.51 & 2.057 & 1.912 & 1.778 & 1.578 & 1.4 & 1.41 \\
\hline S1 & 2.5 & 0.5 & pole-on & -0.725 & 6.032 & 9.145 & 5.266 & 4.098 & 3.19 & 2.724 & 2.494 & 2.15 & 2.005 & 1.853 & 1.638 & 1.478 & 1.483 \\
\hline S1 & 2.5 & 0.571 & pole-on & -0.8 & 7.78 & 9.825 & 5.572 & 4.331 & 3.353 & 2.873 & 2.631 & 2.287 & 2.125 & 1.973 & 1.754 & 1.586 & 1.592 \\
\hline S1 & 2.5 & 0.643 & pole-on & -0.863 & 9.548 & 10.686 & 5.988 & 4.643 & 3.582 & 3.075 & 2.821 & 2.464 & 2.293 & 2.129 & 1.886 & 1.722 & 1.721 \\
\hline $\mathrm{S} 1$ & 2.5 & 0.714 & pole-on & -0.915 & 11.253 & 11.628 & 6.478 & 5.011 & 3.859 & 3.315 & 3.046 & 2.671 & 2.485 & 2.295 & 2.051 & 1.881 & 1.882 \\
\hline S1 & 2.5 & 0.786 & pole-on & -0.957 & 12.886 & 12.764 & 7.048 & 5.453 & 4.181 & 3.606 & 3.302 & 2.905 & 2.71 & 2.526 & 2.24 & 2.065 & 2.054 \\
\hline S1 & 2.5 & 0.857 & pole-on & \begin{tabular}{|l}
-0.992 \\
\end{tabular} & 14.471 & 14.03 & 7.71 & 5.948 & 4.561 & 3.921 & 3.606 & 3.181 & 2.97 & 2.763 & 2.455 & 2.262 & 2.235 \\
\hline $\mathrm{S} 1$ & 2.5 & 0.928 & pole-on & -1.02 & 15.985 & 15.516 & 8.439 & 6.509 & 4.975 & 4.288 & 3.933 & 3.482 & 3.255 & 3.016 & 2.668 & 13.603 & 2.433 \\
\hline S1 & 2.5 & 1.0 & pole-on & \begin{tabular}{|l}
-1.042 \\
\end{tabular} & 17.182 & 16.997 & 9.184 & 7.077 & 5.383 & 4.631 & 4.248 & 3.771 & 3.51 & 3.243 & 2.88 & 2.653 & 2.665 \\
\hline $\mathrm{S} 1$ & 1.85 & 0.0 & edge-on & 0.446 & 1.099 & 2.11 & 1.695 & 1.483 & 1.24 & 1.11 & 1.037 & 0.959 & 0.877 & 0.777 & 0.678 & 0.611 & 0.593 \\
\hline $\mathrm{S} 1$ & 1.85 & 0.071 & edge-on & 0.279 & 1.433 & 1.81 & 1.447 & 1.245 & 1.025 & 0.897 & 0.833 & 0.731 & 0.666 & 0.606 & 0.524 & 0.474 & 0.461 \\
\hline S1 & 1.85 & 0.143 & edge-on & 0.059 & 16.404 & 1.733 & 1.242 & 0.949 & 0.618 & 0.486 & 0.357 & 0.278 & 0.248 & 0.223 & 0.213 & 0.231 & 0.214 \\
\hline S1 & 1.85 & 0.215 & interm. & -0.14 & 0.524 & 21.848 & 225.622 & 27.261 & 11.375 & 8.674 & 7.176 & 5.569 & 5.526 & 4.633 & 4.047 & 3.51 & 3.286 \\
\hline $\mathrm{S} 1$ & 1.85 & 0.286 & interm. & -0.302 & 1.503 & 19.785 & 8.325 & 6.238 & 4.568 & 3.963 & 3.495 & 3.038 & 2.924 & 2.645 & 2.327 & 2.109 & 2.021 \\
\hline $\mathrm{S} 1$ & 1.85 & 0.357 & interm. & -0.434 & 2.648 & 12.049 & 6.789 & 5.354 & 4.103 & 3.526 & 3.176 & 2.816 & 2.675 & 2.426 & 2.152 & 1.958 & 1.902 \\
\hline $\mathrm{S} 1$ & 1.85 & 0.429 & interm. & -0.542 & 4.086 & 11.957 & 6.777 & 5.373 & 4.094 & 3.553 & 3.189 & 2.853 & 2.731 & 2.477 & 2.196 & 2.006 & 1.951 \\
\hline S1 & 1.85 & 0.5 & pole-on & -0.626 & 5.841 & 12.631 & 7.105 & 5.614 & 4.294 & 3.707 & 3.34 & 3.009 & 2.862 & 2.613 & 2.325 & 2.138 & 2.084 \\
\hline S1 & 1.85 & 0.571 & pole-on & -0.696 & 7.696 & 13.687 & 8.634 & 6.016 & 4.587 & 3.967 & 3.59 & 3.244 & 3.086 & 2.806 & 2.502 & 2.31 & 2.26 \\
\hline $\mathrm{S} 1$ & 1.85 & 0.643 & pole-on & -0.756 & 9.647 & 14.908 & 8.244 & 6.531 & 4.968 & 4.305 & 3.886 & 3.537 & 3.354 & 3.064 & 2.734 & 2.517 & 2.468 \\
\hline S1 & 1.85 & 0.714 & pole-on & -0.804 & 11.582 & 16.31 & 9.0 & 7.121 & 5.418 & 4.701 & 4.246 & 3.882 & 3.666 & 3.358 & 3.0 & 2.765 & 2.71 \\
\hline $\mathrm{S} 1$ & 1.85 & 0.786 & pole-on & -0.844 & 13.523 & 17.997 & 9.919 & 7.817 & 5.945 & 5.164 & 4.649 & 4.262 & 4.005 & 3.69 & 3.282 & 3.034 & 2.981 \\
\hline $\mathrm{S} 1$ & 1.85 & 0.857 & pole-on & -0.876 & 15.388 & 19.923 & 10.934 & 8.637 & 6.533 & 5.683 & 5.091 & 4.669 & 4.375 & 4.02 & 3.603 & 3.356 & 3.324 \\
\hline $\mathrm{S} 1$ & 1.85 & 0.928 & pole-on & -0.903 & 17.164 & 22.179 & 12.118 & 9.511 & 7.169 & 6.186 & 5.558 & 5.09 & 4.758 & 4.392 & 3.98 & 3.713 & 3.707 \\
\hline S1 & 1.85 & 1.0 & pole-on & -0.922 & 18.632 & 24.346 & 13.225 & 10.373 & 7.733 & 6.672 & 6.006 & 5.55 & 5.171 & 4.792 & 4.372 & 4.085 & 4.097 \\
\hline $\mathrm{S} 1$ & 1.37 & 0.0 & edge-on & 0.349 & 1.182 & 2.589 & 1.998 & 1.725 & 1.42 & 1.285 & 1.169 & 1.056 & 0.98 & 0.892 & 0.772 & 0.703 & 0.682 \\
\hline $\mathrm{S} 1$ & 1.37 & 0.071 & edge-on & 0.231 & 1.491 & 2.246 & 1.738 & 1.488 & 1.199 & 1.089 & 0.972 & 0.878 & 0.797 & 0.733 & 0.628 & 0.581 & 0.541 \\
\hline $\mathrm{S} 1$ & 1.37 & 0.143 & edge-on & 0.067 & 7.82 & 2.08 & 1.663 & 1.307 & 0.874 & 0.78 & 0.609 & 0.55 & 0.488 & 0.371 & 0.297 & 0.262 & 0.239 \\
\hline $\mathrm{S} 1$ & 1.37 & 0.215 & interm. & -0.097 & 0.385 & 35.384 & 181.665 & 293.433 & 41.206 & 20.395 & 15.691 & 11.083 & 8.905 & 8.882 & 7.118 & 6.27 & 6.269 \\
\hline S1 & 1.37 & 0.286 & interm. & -0.237 & 1.351 & 41.667 & 11.926 & 8.575 & 6.259 & 5.244 & 4.835 & 4.172 & 3.746 & 3.568 & 3.138 & 2.897 & 2.813 \\
\hline
\end{tabular}


Table A.2 - continued.

\begin{tabular}{|c|c|c|c|c|c|c|c|c|c|c|c|c|c|c|c|c|c|}
\hline & & & $\tilde{\tau}_{\mathrm{bu}}$ & & $\mathrm{bu}$ & 0.15 & 0.45 & 0.75 & 1.50 & 2.25 & 3.00 & 4.50 & 6.00 & 9.00 & 15.00 & 30.00 & $\infty$ \\
\hline Star & $\Sigma_{0}\left[\mathrm{~g} \mathrm{~cm}^{-2}\right]$ & $\cos i$ & ang. type & $\Delta V_{\mathrm{bu}}^{\infty}$ & $\xi_{\mathrm{bu}}^{V}$ & $\xi_{\mathrm{d}}^{V}$ & & & & & & & & & & & \\
\hline S1 & 1.37 & 0.357 & interm. & -0.355 & 2.488 & 17.615 & 8.93 & 7.063 & 5.419 & 4.642 & 4.322 & 3.778 & 3.481 & 3.297 & 2.886 & 2.668 & 2.594 \\
\hline $\mathrm{S} 1$ & 1.37 & 0.429 & interm. & -0.454 & 3.908 & 16.13 & 8.985 & 7.201 & 5.513 & 4.75 & 4.412 & 3.905 & 3.607 & 3.403 & 2.996 & 2.77 & 2.711 \\
\hline S1 & 1.37 & 0.5 & pole-on & -0.533 & 5.627 & 17.064 & 9.556 & 7.657 & 5.849 & 5.065 & 4.718 & 4.176 & 3.886 & 3.652 & 3.233 & 3.004 & 2.918 \\
\hline S1 & 1.37 & 0.571 & pole-on & -0.598 & 7.561 & 18.532 & 10.363 & 8.275 & 6.323 & 5.522 & 5.09 & 4.569 & 4.251 & 3.976 & 3.542 & 3.275 & 3.221 \\
\hline $\mathrm{S} 1$ & 1.37 & 0.643 & pole-on & -0.653 & 9.641 & 20.389 & 11.401 & 9.009 & 6.974 & 6.059 & 5.605 & 5.021 & 4.693 & 4.391 & 3.899 & 3.646 & 3.594 \\
\hline $\mathrm{S} 1$ & 1.37 & 0.714 & pole-on & -0.698 & 11.788 & 22.509 & 12.545 & 10.044 & 7.684 & 6.706 & 6.16 & 5.553 & 5.194 & 4.857 & 4.347 & 4.04 & 4.043 \\
\hline S1 & 1.37 & 0.786 & pole-on & -0.735 & 13.887 & 24.957 & 13.963 & 11.162 & 8.469 & 7.398 & 6.783 & 6.102 & 5.729 & 5.374 & 4.832 & 4.529 & 4.558 \\
\hline $\mathrm{S} 1$ & 1.37 & 0.857 & pole-on & -0.765 & 15.977 & 27.961 & 15.528 & 12.339 & 9.307 & 8.102 & 7.445 & 6.741 & 6.375 & 5.985 & 5.406 & 5.062 & 5.164 \\
\hline S1 & 1.37 & 0.928 & pole-on & -0.789 & 17.967 & 31.178 & 17.246 & 13.633 & 10.302 & 8.92 & 8.208 & 7.533 & 7.122 & 6.631 & 6.059 & 5.671 & 5.823 \\
\hline S1 & 1.37 & 1.0 & pole-on & -0.806 & 19.653 & 34.745 & 19.06 & 14.978 & 11.309 & 9.792 & 8.961 & 8.299 & 7.89 & 7.281 & 6.705 & 6.25 & 6.516 \\
\hline $\mathrm{S} 1$ & 1.01 & 0.0 & edge-on & 0.277 & 1.221 & 3.215 & 2.274 & 1.994 & 3.139 & 1.463 & 1.33 & 1.186 & 1.121 & 1.016 & 0.819 & 0.81 & 0.813 \\
\hline S1 & 1.01 & 0.071 & edge-on & 0.193 & 1.437 & 2.786 & 1.996 & 1.729 & 2.431 & 1.272 & 1.136 & 1.044 & 0.97 & 0.886 & 0.714 & 0.696 & 0.691 \\
\hline S1 & 1.01 & 0.143 & edge-on & 0.076 & 4.093 & 2.567 & 1.874 & 1.593 & 9.552 & 1.022 & 0.925 & 0.731 & 0.74 & 0.649 & 0.511 & 0.46 & 0.456 \\
\hline S1 & 1.01 & 0.215 & interm. & -0.051 & 0.308 & 24.353 & 65.201 & 149.974 & 419.396 & 240.774 & 85.372 & 41.539 & 29.832 & 22.875 & 18.312 & 16.394 & 14.361 \\
\hline S1 & 1.01 & 0.286 & interm. & \begin{tabular}{|l}
-0.169 \\
\end{tabular} & 1.315 & 193.793 & 21.198 & 13.531 & 15.254 & 7.395 & 6.508 & 5.96 & 5.173 & 4.853 & 4.363 & 3.901 & 3.66 \\
\hline S1 & 1.01 & 0.357 & interm. & -0.273 & 2.359 & 23.994 & 12.492 & 9.409 & 10.106 & 6.198 & 5.588 & 5.17 & 4.681 & 4.319 & 3.979 & 3.566 & 3.359 \\
\hline S1 & 1.01 & 0.429 & interm. & -0.363 & 3.636 & 21.137 & 12.184 & 9.565 & 9.683 & 6.382 & 5.832 & 5.337 & 4.932 & 4.511 & 4.227 & 3.77 & 3.572 \\
\hline S1 & 1.01 & 0.5 & pole-on & -0.435 & 5.24 & 22.067 & 12.906 & 10.183 & 9.894 & 6.895 & 6.333 & 5.802 & 5.396 & 4.958 & 4.636 & 4.179 & 3.956 \\
\hline S1 & 1.01 & 0.571 & pole-on & -0.494 & 7.103 & 24.186 & 14.102 & 11.266 & 10.543 & 7.566 & 6.962 & 6.405 & 5.978 & 5.483 & 5.18 & 4.663 & 4.469 \\
\hline $\mathrm{S} 1$ & 1.01 & 0.643 & pole-on & -0.545 & 9.181 & 26.897 & 15.65 & 12.485 & 11.27 & 8.387 & 7.725 & 7.105 & 6.665 & 6.216 & 5.785 & 5.276 & 5.171 \\
\hline $\mathrm{S} 1$ & 1.01 & 0.714 & pole-on & -0.587 & 11.34 & 30.176 & 17.383 & 13.917 & 12.223 & 9.356 & 8.582 & 7.908 & 7.524 & 6.95 & 6.459 & 5.942 & 6.034 \\
\hline $\mathrm{S} 1$ & 1.01 & 0.786 & pole-on & -0.62 & 13.511 & 33.946 & 19.403 & 15.519 & 13.294 & 10.5 & 9.603 & 8.846 & 8.501 & 7.866 & 7.18 & 6.704 & 7.02 \\
\hline $\mathrm{S} 1$ & 1.01 & 0.857 & pole-on & -0.647 & 15.677 & 38.601 & 21.891 & 17.351 & 14.496 & 11.706 & 10.671 & 9.93 & 9.599 & 8.877 & 8.015 & 7.512 & 8.261 \\
\hline $\mathrm{S} 1$ & 1.01 & 0.928 & pole-on & -0.669 & 17.747 & 43.814 & 24.518 & 19.626 & 15.769 & 12.981 & 11.778 & 11.093 & 10.862 & 9.929 & 8.917 & 8.413 & 9.686 \\
\hline S1 & 1.01 & 1.0 & pole-on & -0.683 & 19.516 & 48.695 & 27.39 & 21.806 & 16.998 & 14.16 & 12.849 & 12.236 & 12.109 & 10.93 & 9.795 & 9.245 & 11.125 \\
\hline $\mathrm{S} 1$ & 0.75 & 0.0 & edge-on & 0.214 & 1.337 & 4.101 & 2.699 & 2.309 & 1.908 & 1.666 & 1.53 & 1.383 & 1.72 & 1.164 & 1.024 & 0.933 & 0.905 \\
\hline $\mathrm{S} 1$ & 0.75 & 0.071 & edge-on & 0.156 & 1.546 & 3.566 & 2.376 & 2.037 & 1.697 & 1.487 & 1.374 & 1.225 & 1.133 & 1.045 & 0.912 & 0.833 & 0.795 \\
\hline S1 & 0.75 & 0.143 & edge-on & 0.071 & 4.218 & 3.445 & 2.248 & 1.946 & 1.527 & 1.345 & 1.258 & 1.089 & 0.997 & 0.887 & 0.782 & 0.707 & 0.633 \\
\hline $\mathrm{S} 1$ & 0.75 & 0.215 & interm. & -0.023 & 21.076 & 5.588 & 18.905 & 29.11 & 113.856 & 280.209 & 318.189 & 337.261 & 344.856 & 329.168 & 311.936 & 253.2 & 273.522 \\
\hline S1 & 0.75 & 0.286 & interm. & -0.121 & 3.789 & 371.885 & 46.003 & 24.051 & 13.451 & 10.945 & 9.278 & 7.996 & 7.152 & 6.749 & 5.695 & 5.121 & 5.317 \\
\hline S1 & 0.75 & 0.357 & interm. & -0.206 & 2.13 & 40.286 & 17.027 & 12.512 & 9.164 & 7.897 & 7.029 & 6.351 & 5.995 & 5.535 & 4.941 & 4.55 & 4.704 \\
\hline S1 & 0.75 & 0.429 & interm. & -0.283 & 3.239 & 27.996 & 15.585 & 12.197 & 9.297 & 8.147 & 7.423 & 6.72 & 6.293 & 5.872 & 5.281 & 4.922 & 5.064 \\
\hline $\mathrm{S} 1$ & 0.75 & 0.5 & pole-on & -0.346 & 4.539 & 28.169 & 16.502 & 13.055 & 10.054 & 8.965 & 8.031 & 7.343 & 7.036 & 6.518 & 5.909 & 5.496 & 5.756 \\
\hline $\mathrm{S} 1$ & 0.75 & 0.571 & pole-on & -0.398 & 5.938 & 30.234 & 18.131 & 14.571 & 11.251 & 9.921 & 8.96 & 8.274 & 7.923 & 7.328 & 6.675 & 6.284 & 6.638 \\
\hline S1 & 0.75 & 0.643 & pole-on & -0.443 & 7.376 & 34.678 & 20.437 & 16.347 & 12.632 & 11.184 & 10.244 & 9.351 & 9.077 & 8.327 & 7.573 & 7.139 & 7.68 \\
\hline
\end{tabular}


Table A.2 - continued.

\begin{tabular}{|c|c|c|c|c|c|c|c|c|c|c|c|c|c|c|c|c|c|}
\hline & & & 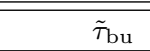 & & $\overline{\mathrm{bu}}$ & 0.15 & 0.45 & 0.75 & $\bar{~} 1.50$ & 2.25 & 3.00 & 4.50 & (6.00 & 9.00 & 15.00 & 30.00 & $\infty$ \\
\hline Star & $\Sigma_{0}\left[\mathrm{~g} \mathrm{~cm}^{-2}\right]$ & $\cos i$ & ang. type & $\Delta V_{\mathrm{bu}}^{\infty}$ & $\xi_{\mathrm{bu}}^{V}$ & $\xi_{\mathrm{d}}^{V}$ & & & & & & & & & & & \\
\hline S1 & 0.75 & 0.714 & pole-on & -0.479 & 8.781 & 38.989 & 22.943 & 18.565 & 14.352 & 12.645 & 11.54 & 10.54 & 10.353 & 9.379 & 8.663 & 8.115 & 8.88 \\
\hline S1 & 0.75 & 0.786 & pole-on & -0.508 & 10.153 & 44.306 & 26.022 & 21.175 & 16.264 & 14.28 & 13.05 & 11.869 & 11.828 & 10.543 & 9.784 & 9.139 & 10.321 \\
\hline S1 & 0.75 & 0.857 & pole-on & -0.531 & 11.42 & 51.093 & 29.734 & 23.994 & 18.346 & 15.928 & 14.571 & 13.406 & 13.479 & 11.68 & 11.122 & 10.264 & 11.932 \\
\hline S1 & 0.75 & 0.928 & pole-on & -0.548 & 12.654 & 58.104 & 33.636 & 27.524 & 20.404 & 17.732 & 15.947 & 14.872 & 15.264 & 12.862 & 12.526 & 11.387 & 13.761 \\
\hline S1 & 0.75 & 1.0 & pole-on & -0.558 & 13.455 & 64.339 & 37.9 & 30.867 & 22.416 & 19.197 & 17.25 & 16.433 & 17.137 & 13.914 & 13.82 & 12.434 & 15.555 \\
\hline S1 & 0.56 & 0.0 & edge-on & 0.168 & 1.448 & 5.049 & 3.392 & 2.807 & 2.241 & 1.934 & 1.801 & 1.587 & 1.475 & 1.336 & 1.166 & 1.074 & 1.045 \\
\hline S1 & 0.56 & 0.071 & edge-on & 0.127 & 1.574 & 4.636 & 2.975 & 2.476 & 2.047 & 1.788 & 1.639 & 1.481 & 1.343 & 1.249 & 1.076 & 0.989 & 0.955 \\
\hline S1 & 0.56 & 0.143 & edge-on & 0.067 & 2.954 & 4.545 & 3.086 & 2.565 & 2.021 & 1.788 & 1.612 & 1.44 & 1.293 & 1.248 & 1.013 & 0.973 & 0.916 \\
\hline S1 & 0.56 & 0.215 & interm. & -0.001 & 274.53 & 20.445 & 10.237 & 2.494 & 5.778 & 28.446 & 7.976 & 26.151 & 73.211 & 38.128 & 182.118 & 168.69 & 318.603 \\
\hline $\mathrm{S} 1$ & 0.56 & 0.286 & interm. & -0.079 & 1.075 & 383.395 & 289.324 & 65.948 & 25.425 & 17.385 & 14.724 & 11.369 & 10.861 & 8.899 & 8.333 & 6.985 & 7.464 \\
\hline S1 & 0.56 & 0.357 & interm. & -0.146 & 2.125 & 86.034 & 22.353 & 15.881 & 11.205 & 9.472 & 8.645 & 7.478 & 7.495 & 6.382 & 6.261 & 5.59 & 5.775 \\
\hline $\mathrm{S} 1$ & 0.56 & 0.429 & interm. & -0.207 & 3.255 & 37.889 & 18.478 & 14.81 & 11.094 & 9.405 & 8.836 & 7.93 & 7.801 & 6.909 & 6.471 & 5.997 & 6.32 \\
\hline $\mathrm{S} 1$ & 0.56 & 0.5 & pole-on & -0.259 & 4.515 & 35.683 & 19.43 & 15.837 & 12.006 & 10.523 & 9.915 & 8.82 & 8.749 & 7.738 & 7.355 & 6.799 & 7.331 \\
\hline S1 & 0.56 & 0.571 & pole-on & -0.302 & 5.978 & 37.039 & 21.971 & 17.649 & 13.687 & 11.989 & 11.072 & 9.999 & 9.945 & 8.768 & 8.373 & 7.824 & 8.586 \\
\hline S1 & 0.56 & 0.643 & pole-on & -0.339 & 7.729 & 41.26 & 24.895 & 20.054 & 15.582 & 13.677 & 12.726 & 11.385 & 11.501 & 10.099 & 9.628 & 8.915 & 10.037 \\
\hline S1 & 0.56 & 0.714 & pole-on & -0.368 & 9.62 & 47.656 & 28.452 & 23.145 & 17.848 & 15.58 & 14.434 & 12.961 & 13.242 & 11.425 & 10.978 & 10.178 & 11.854 \\
\hline S1 & 0.56 & 0.786 & pole-on & -0.392 & 11.628 & 54.714 & 32.704 & 26.812 & 20.532 & 17.731 & 16.506 & 14.616 & 15.357 & 12.876 & 12.506 & 11.518 & 13.985 \\
\hline $\mathrm{S} 1$ & 0.56 & 0.857 & pole-on & -0.41 & 13.757 & 62.445 & 37.738 & 30.75 & 23.118 & 20.034 & 18.356 & 16.49 & 17.441 & 14.278 & 14.091 & 12.822 & 16.28 \\
\hline S1 & 0.56 & 0.928 & pole-on & -0.422 & 15.999 & 71.541 & 43.121 & 35.285 & 25.841 & 21.893 & 19.934 & 18.425 & 19.926 & 15.667 & 15.891 & 14.204 & 19.013 \\
\hline S1 & 0.56 & 1.0 & pole-on & -0.428 & 17.632 & 78.909 & 48.439 & 39.859 & 28.093 & 23.624 & 21.38 & 20.334 & 22.466 & 16.985 & 17.431 & 15.332 & 21.573 \\
\hline S1 & 0.41 & 0.0 & edge-on & 0.13 & 1.649 & 6.44 & 4.274 & 3.442 & 2.707 & 2.314 & 2.083 & 1.877 & 1.731 & 1.578 & 1.399 & 1.279 & 1.229 \\
\hline S1 & 0.41 & 0.071 & edge-on & 0.102 & 2.735 & 6.249 & 3.815 & 3.058 & 2.483 & 2.186 & 1.983 & 1.768 & 1.624 & 1.48 & 1.314 & 1.179 & 1.129 \\
\hline S1 & 0.41 & 0.143 & edge-on & 0.06 & 2.817 & 7.0 & 4.117 & 3.367 & 2.571 & 2.396 & 2.183 & 1.849 & 1.799 & 1.649 & 1.431 & 1.346 & 1.245 \\
\hline S1 & 0.41 & 0.215 & interm. & 0.011 & 149.565 & 24.593 & 4.546 & 3.665 & 3.786 & 2.522 & 2.626 & 2.072 & 6.533 & 9.096 & 8.673 & 10.053 & 5.361 \\
\hline S1 & 0.41 & 0.286 & interm. & -0.045 & 1.4 & 362.898 & 353.755 & 335.245 & 154.378 & 55.241 & 35.387 & 22.913 & 19.432 & 14.82 & 11.265 & 9.655 & 10.924 \\
\hline S1 & 0.41 & 0.357 & interm. & -0.095 & 2.084 & 266.384 & 30.626 & 20.579 & 13.134 & 10.575 & 9.154 & 8.543 & 8.205 & 7.028 & 6.59 & 5.915 & 6.739 \\
\hline $\mathrm{S} 1$ & 0.41 & 0.429 & interm. & -0.14 & 3.072 & 49.409 & 22.049 & 17.001 & 12.397 & 10.641 & 9.745 & 8.854 & 8.677 & 7.691 & 6.9 & 6.598 & 7.319 \\
\hline S1 & 0.41 & 0.5 & pole-on & -0.179 & 4.333 & 38.625 & 22.612 & 18.227 & 13.841 & 11.691 & 10.915 & 9.934 & 9.939 & 8.738 & 8.109 & 7.542 & 8.561 \\
\hline S1 & 0.41 & 0.571 & pole-on & -0.212 & 5.651 & 41.764 & 25.018 & 20.124 & 15.512 & 13.565 & 12.513 & 11.419 & 11.54 & 10.189 & 9.395 & 8.877 & 10.161 \\
\hline S1 & 0.41 & 0.643 & pole-on & -0.24 & 7.337 & 46.138 & 28.836 & 23.234 & 17.95 & 15.819 & 14.497 & 13.122 & 13.335 & 11.762 & 10.911 & 10.099 & 12.29 \\
\hline S1 & 0.41 & 0.714 & pole-on & -0.262 & 9.056 & 55.028 & 33.633 & 27.206 & 20.807 & 18.329 & 16.794 & 14.948 & 15.775 & 13.46 & 12.555 & 11.731 & 14.627 \\
\hline S1 & 0.41 & 0.786 & pole-on & -0.279 & 11.081 & 61.276 & 38.619 & 31.802 & 24.266 & 20.892 & 19.41 & 17.151 & 18.247 & 14.991 & 14.34 & 13.229 & 17.448 \\
\hline $\mathrm{S} 1$ & 0.41 & 0.857 & pole-on & -0.292 & 13.461 & 71.761 & 44.683 & 36.85 & 27.585 & 23.203 & 21.374 & 19.241 & 21.233 & 16.671 & 16.23 & 14.847 & 20.764 \\
\hline S1 & 0.41 & 0.928 & pole-on & -0.3 & 15.833 & 83.217 & 51.511 & 42.796 & 30.327 & 25.717 & 22.897 & 21.368 & 23.936 & 18.116 & 18.344 & 16.247 & 24.2 \\
\hline S1 & 0.41 & 1.0 & pole-on & -0.302 & 17.488 & 92.261 & 57.278 & 47.959 & 33.244 & 27.31 & 24.507 & 23.434 & 26.94 & 19.379 & 20.06 & 17.618 & 27.455 \\
\hline
\end{tabular}


Table A.2 - continued.

\begin{tabular}{|c|c|c|c|c|c|c|c|c|c|c|c|c|c|c|c|c|c|}
\hline & & & $\tilde{\tau}_{\mathrm{bu}}$ & & $\mathrm{bu}$ & 0.15 & 0.45 & 0.75 & 1.50 & 2.25 & 3.00 & 4.50 & 6.00 & 9.00 & 15.00 & 30.00 & $\infty$ \\
\hline Star & $\Sigma_{0}\left[\mathrm{~g} \mathrm{~cm}^{-2}\right]$ & $\cos i$ & ang. type & $\Delta V_{\mathrm{bu}}^{\infty}$ & $\xi_{\mathrm{bu}}^{V}$ & $\xi_{\mathrm{d}}^{V}$ & & & & & & & & & & & \\
\hline S1 & 0.3 & 0.0 & edge-on & 0.1 & 1.936 & 9.085 & 4.943 & 4.025 & 3.134 & 2.834 & 2.498 & 2.206 & 2.01 & 1.865 & 1.604 & 1.51 & 1.396 \\
\hline $\mathrm{S} 1$ & 0.3 & 0.071 & edge-on & 0.081 & 1.996 & 9.436 & 4.634 & 3.536 & 2.979 & 2.634 & 2.447 & 2.08 & 1.883 & 1.783 & 1.543 & 1.4 & 1.356 \\
\hline S1 & 0.3 & 0.143 & edge-on & 0.051 & 6.703 & 10.1 & 4.801 & 4.039 & 3.445 & 3.059 & 2.67 & 2.461 & 2.281 & 2.176 & 1.864 & 1.776 & 1.639 \\
\hline S1 & 0.3 & 0.215 & interm. & 0.015 & 49.051 & 30.672 & 6.287 & 5.351 & 4.925 & 9.582 & 4.035 & 4.836 & 4.095 & 3.563 & 3.308 & 3.099 & 2.543 \\
\hline $\mathrm{S} 1$ & 0.3 & 0.286 & interm. & -0.022 & 23.392 & 291.483 & 331.434 & 331.024 & 345.051 & 280.35 & 325.328 & 255.225 & 233.685 & 169.548 & 159.107 & 65.214 & 66.312 \\
\hline S1 & 0.3 & 0.357 & interm. & -0.058 & 1.905 & 330.425 & 72.23 & 33.506 & 14.405 & 11.447 & 10.273 & 8.829 & 8.876 & 6.82 & 6.459 & 5.752 & 6.978 \\
\hline $\mathrm{S} 1$ & 0.3 & 0.429 & interm. & -0.09 & 2.982 & 92.594 & 25.331 & 19.548 & 12.272 & 10.391 & 9.688 & 8.971 & 9.128 & 7.513 & 7.054 & 6.504 & 7.852 \\
\hline S1 & 0.3 & 0.5 & pole-on & -0.119 & 4.059 & 47.283 & 24.352 & 19.359 & 14.043 & 12.162 & 11.205 & 10.236 & 10.5 & 8.823 & 8.449 & 7.942 & 9.348 \\
\hline S1 & 0.3 & 0.571 & pole-on & -0.142 & 5.246 & 45.722 & 27.135 & 22.115 & 16.395 & 14.185 & 12.957 & 11.723 & 12.333 & 10.577 & 9.924 & 9.166 & 11.142 \\
\hline S1 & 0.3 & 0.643 & pole-on & -0.161 & 6.867 & 50.497 & 30.993 & 25.762 & 18.863 & 17.161 & 15.249 & 13.772 & 14.588 & 12.244 & 11.616 & 10.655 & 13.587 \\
\hline $\mathrm{S} 1$ & 0.3 & 0.714 & pole-on & -0.177 & 8.518 & 57.053 & 35.669 & 30.168 & 22.572 & 19.609 & 17.511 & 15.877 & 17.155 & 14.08 & 13.701 & 12.547 & 16.381 \\
\hline S1 & 0.3 & 0.786 & pole-on & -0.189 & 10.423 & 68.1 & 41.975 & 35.065 & 26.279 & 23.283 & 20.405 & 18.126 & 20.024 & 15.922 & 15.587 & 14.197 & 19.926 \\
\hline S1 & 0.3 & 0.857 & pole-on & -0.196 & 12.489 & 78.97 & 48.703 & 40.943 & 30.174 & 25.503 & 22.935 & 20.738 & 23.127 & 17.355 & 17.587 & 15.708 & 23.647 \\
\hline S1 & 0.3 & 0.928 & pole-on & -0.202 & 14.776 & 91.468 & 57.457 & 47.5 & 33.024 & 27.77 & 25.216 & 23.18 & 26.876 & 19.308 & 20.126 & 17.622 & 28.346 \\
\hline S1 & 0.3 & 1.0 & pole-on & -0.201 & 16.328 & 100.854 & 63.168 & 52.547 & 35.603 & 29.669 & 26.208 & 25.382 & 29.634 & 20.219 & 21.455 & 18.659 & 32.012 \\
\hline $\mathrm{S} 2$ & 2.5 & 0.0 & edge-on & 0.491 & 1.068 & 1.95 & 1.512 & 1.397 & 1.178 & 1.055 & 0.979 & 0.871 & 0.812 & 0.738 & 0.646 & 0.583 & 0.566 \\
\hline $\mathrm{S} 2$ & 2.5 & 0.071 & edge-on & 0.31 & 1.411 & 1.646 & 1.285 & 1.187 & 0.977 & 0.855 & 0.793 & 0.694 & 0.647 & 0.581 & 0.504 & 0.45 & 0.429 \\
\hline $\mathrm{S} 2$ & 2.5 & 0.143 & edge-on & 0.066 & 16.148 & 1.603 & 1.132 & 0.926 & 0.634 & 0.473 & 0.4 & 0.3 & 0.273 & 0.225 & 0.208 & 0.207 & 0.209 \\
\hline S2 & 2.5 & 0.215 & interm. & -0.144 & 0.471 & 10.801 & 296.749 & 31.832 & 12.019 & 8.961 & 7.349 & 5.941 & 5.165 & 4.7 & 3.796 & 3.73 & 3.433 \\
\hline $\mathrm{S} 2$ & 2.5 & 0.286 & interm. & -0.306 & 1.43 & 23.954 & 8.599 & 6.159 & 4.427 & 3.815 & 3.451 & 3.013 & 2.74 & 2.524 & 2.156 & 2.076 & 1.976 \\
\hline $\mathrm{S} 2$ & 2.5 & 0.357 & interm. & -0.438 & 2.595 & 12.495 & 6.747 & 5.276 & 3.876 & 3.37 & 3.075 & 2.679 & 2.502 & 2.328 & 1.999 & 1.891 & 1.811 \\
\hline S2 & 2.5 & 0.429 & interm. & -0.545 & 4.04 & 11.887 & 6.702 & 5.22 & 3.908 & 3.379 & 3.101 & 2.758 & 2.542 & 2.351 & 2.054 & 1.925 & 1.859 \\
\hline $\mathrm{S} 2$ & 2.5 & 0.5 & pole-on & \begin{tabular}{|l}
-0.628 \\
\end{tabular} & 5.79 & 12.411 & 6.961 & 5.463 & 4.085 & 3.551 & 3.265 & 2.893 & 2.682 & 2.484 & 2.181 & 2.048 & 1.972 \\
\hline $\mathrm{S} 2$ & 2.5 & 0.571 & pole-on & -0.697 & 7.614 & 13.397 & 7.433 & 5.841 & 4.389 & 3.791 & 3.496 & 3.119 & 2.898 & 2.687 & 2.363 & 2.208 & 2.137 \\
\hline $\mathrm{S} 2$ & 2.5 & 0.643 & pole-on & -0.755 & 9.502 & 14.515 & 8.037 & 6.34 & 4.749 & 4.126 & 3.814 & 3.392 & 3.151 & 2.934 & 2.588 & 2.414 & 2.337 \\
\hline $\mathrm{S} 2$ & 2.5 & 0.714 & pole-on & -0.803 & 11.439 & 15.996 & 8.785 & 6.923 & 5.213 & 4.508 & 4.172 & 3.728 & 3.479 & 3.227 & 2.841 & 2.649 & 2.564 \\
\hline $\mathrm{S} 2$ & 2.5 & 0.786 & pole-on & -0.84 & 13.244 & 17.573 & 9.664 & 7.61 & 5.717 & 4.96 & 4.577 & 4.105 & 3.832 & 3.535 & 3.137 & 2.929 & 2.825 \\
\hline $\mathrm{S} 2$ & 2.5 & 0.857 & pole-on & -0.872 & 14.967 & 19.471 & 10.663 & 8.404 & 6.29 & 5.461 & 5.012 & 4.512 & 4.197 & 3.876 & 3.437 & 3.2 & 3.144 \\
\hline $\mathrm{S} 2$ & 2.5 & 0.928 & pole-on & -0.898 & 16.735 & 21.709 & 11.794 & 9.269 & 6.915 & 5.942 & 5.45 & 4.924 & 4.587 & 4.243 & 3.785 & 3.549 & 3.513 \\
\hline $\mathrm{S} 2$ & 2.5 & 1.0 & pole-on & -0.916 & 18.154 & 23.936 & 12.927 & 10.111 & 7.515 & 6.429 & 5.902 & 5.348 & 5.031 & 4.624 & 4.18 & 3.917 & 3.898 \\
\hline $\mathrm{S} 2$ & 1.85 & 0.0 & edge-on & 0.39 & 1.12 & 2.297 & 1.799 & 1.583 & 1.338 & 1.159 & 1.899 & 1.418 & 0.925 & 0.828 & 0.718 & 0.65 & 0.636 \\
\hline S2 & 1.85 & 0.071 & edge-on & 0.261 & 1.386 & 1.926 & 1.541 & 1.36 & 1.121 & 0.971 & 1.259 & 0.976 & 0.74 & 0.675 & 0.594 & 0.525 & 0.513 \\
\hline $\mathrm{S} 2$ & 1.85 & 0.143 & edge-on & 0.081 & 6.369 & 1.794 & 1.43 & 1.186 & 0.839 & 0.655 & 0.541 & 0.457 & 0.397 & 0.343 & 0.287 & 0.239 & 0.233 \\
\hline $\mathrm{S} 2$ & 1.85 & 0.215 & interm. & -0.094 & 0.381 & 7.409 & 82.173 & 327.703 & 41.561 & 24.369 & 40.595 & 19.877 & 11.885 & 9.505 & 7.688 & 7.105 & 6.591 \\
\hline $\mathrm{S} 2$ & 1.85 & 0.286 & interm. & -0.238 & 1.347 & 55.645 & 13.374 & 9.192 & 6.467 & 5.383 & 6.7 & 5.345 & 3.889 & 3.594 & 3.147 & 2.944 & 2.814 \\
\hline
\end{tabular}


Table A.2 - continued.

\begin{tabular}{|c|c|c|c|c|c|c|c|c|c|c|c|c|c|c|c|c|c|}
\hline & & & $\tilde{\tau}_{\mathrm{bu}}$ & & bu & 0.15 & 0.45 & 0.75 & 1.50 & 2.25 & 3.00 & 4.50 & 6.00 & 9.00 & 15.00 & 30.00 & $\infty$ \\
\hline Star & $\Sigma_{0}\left[\mathrm{~g} \mathrm{~cm}^{-2}\right]$ & $\cos i$ & ang. type & $\Delta V_{\mathrm{bu}}^{\infty}$ & $\xi_{\mathrm{bu}}^{V}$ & $\xi_{\mathrm{d}}^{V}$ & & & & & & & & & & & \\
\hline S2 & 1.85 & 0.357 & interm. & -0.359 & 2.444 & 18.396 & 9.231 & 7.124 & 5.331 & 4.653 & 5.366 & 4.462 & 3.481 & 3.211 & 2.824 & 2.639 & 2.544 \\
\hline S2 & 1.85 & 0.429 & interm. & -0.459 & 3.817 & 16.032 & 8.983 & 7.083 & 5.353 & 4.686 & 5.172 & 4.434 & 3.589 & 3.308 & 2.921 & 2.718 & 2.623 \\
\hline $\mathrm{S} 2$ & 1.85 & 0.5 & pole-on & -0.537 & 5.466 & 16.646 & 9.466 & 7.448 & 5.679 & 4.97 & 5.35 & 4.628 & 3.82 & 3.532 & 3.129 & 2.925 & 2.825 \\
\hline $\mathrm{S} 2$ & 1.85 & 0.571 & pole-on & -0.602 & 7.272 & 18.012 & 10.201 & 8.084 & 6.158 & 5.379 & 5.71 & 4.964 & 4.177 & 3.834 & 3.438 & 3.183 & 3.105 \\
\hline $\mathrm{S} 2$ & 1.85 & 0.643 & pole-on & -0.656 & 9.182 & 19.716 & 11.168 & 8.88 & 6.741 & 5.918 & 6.191 & 5.426 & 4.578 & 4.227 & 3.777 & 3.515 & 3.471 \\
\hline $\mathrm{S} 2$ & 1.85 & 0.714 & pole-on & -0.701 & 11.209 & 21.949 & 12.271 & 9.767 & 7.415 & 6.503 & 6.773 & 5.992 & 5.032 & 4.679 & 4.178 & 3.92 & 3.898 \\
\hline $\mathrm{S} 2$ & 1.85 & 0.786 & pole-on & -0.736 & 13.137 & 24.325 & 13.629 & 10.788 & 8.177 & 7.17 & 7.45 & 6.648 & 5.559 & 5.179 & 4.66 & 4.372 & 4.398 \\
\hline $\mathrm{S} 2$ & 1.85 & 0.857 & pole-on & -0.765 & 15.028 & 27.189 & 15.09 & 11.961 & 9.01 & 7.895 & 8.223 & 7.401 & 6.152 & 5.734 & 5.214 & 4.892 & 4.975 \\
\hline $\mathrm{S} 2$ & 1.85 & 0.928 & pole-on & -0.788 & 16.854 & 30.509 & 16.774 & 13.237 & 9.938 & 8.708 & 9.08 & 8.261 & 6.865 & 6.38 & 5.836 & 5.46 & 5.623 \\
\hline $\mathrm{S} 2$ & 1.85 & 1.0 & pole-on & -0.804 & 18.397 & 33.904 & 18.528 & 14.585 & 10.916 & 9.536 & 9.986 & 9.146 & 7.597 & 7.008 & 6.452 & 6.042 & 6.283 \\
\hline $\mathrm{S} 2$ & 1.37 & 0.0 & edge-on & 0.306 & 1.213 & 2.888 & 2.064 & 1.779 & 1.485 & 1.373 & 1.219 & 1.11 & 1.016 & 0.937 & 0.814 & 0.746 & 0.721 \\
\hline $\mathrm{S} 2$ & 1.37 & 0.071 & edge-on & 0.216 & 1.439 & 2.496 & 1.766 & 1.563 & 1.292 & 1.174 & 1.068 & 0.954 & 0.876 & 0.806 & 0.694 & 0.635 & 0.613 \\
\hline $\mathrm{S} 2$ & 1.37 & 0.143 & edge-on & 0.084 & 4.395 & 2.343 & 1.632 & 1.445 & 1.083 & 0.939 & 0.806 & 0.704 & 0.626 & 0.554 & 0.452 & 0.402 & 0.386 \\
\hline $\mathrm{S} 2$ & 1.37 & 0.215 & interm. & -0.051 & 18.068 & 6.4 & 28.129 & 70.457 & 389.707 & 322.05 & 204.138 & 70.283 & 46.205 & 37.748 & 25.417 & 20.498 & 18.642 \\
\hline $\mathrm{S} 2$ & 1.37 & 0.286 & interm. & -0.175 & 1.197 & 300.31 & 24.376 & 15.083 & 9.471 & 7.952 & 6.911 & 6.046 & 5.556 & 5.021 & 4.393 & 4.076 & 3.923 \\
\hline $\mathrm{S} 2$ & 1.37 & 0.357 & interm. & -0.28 & 2.256 & 26.285 & 12.681 & 9.629 & 7.139 & 6.154 & 5.573 & 4.991 & 4.66 & 4.27 & 3.766 & 3.507 & 3.451 \\
\hline $\mathrm{S} 2$ & 1.37 & 0.429 & interm. & -0.37 & 3.5 & 20.787 & 11.833 & 9.41 & 7.128 & 6.2 & 5.67 & 5.127 & 4.792 & 4.447 & 3.948 & 3.684 & 3.618 \\
\hline $\mathrm{S} 2$ & 1.37 & 0.5 & pole-on & -0.442 & 5.019 & 21.445 & 12.458 & 10.029 & 7.617 & 6.671 & 6.076 & 5.537 & 5.2 & 4.82 & 4.294 & 4.024 & 4.008 \\
\hline $\mathrm{S} 2$ & 1.37 & 0.571 & pole-on & -0.501 & 6.773 & 23.159 & 13.483 & 10.907 & 8.331 & 7.295 & 6.658 & 6.078 & 5.738 & 5.305 & 4.774 & 4.488 & 4.514 \\
\hline $\mathrm{S} 2$ & 1.37 & 0.643 & pole-on & -0.551 & 8.707 & 25.88 & 15.037 & 12.048 & 9.198 & 8.075 & 7.387 & 6.729 & 6.414 & 5.936 & 5.381 & 5.044 & 5.138 \\
\hline $\mathrm{S} 2$ & 1.37 & 0.714 & pole-on & -0.591 & 10.622 & 28.854 & 16.589 & 13.395 & 10.2 & 8.967 & 8.211 & 7.535 & 7.216 & 6.659 & 6.05 & 5.719 & 5.862 \\
\hline $\mathrm{S} 2$ & 1.37 & 0.786 & pole-on & -0.622 & 12.733 & 32.567 & 18.644 & 14.955 & 11.403 & 10.025 & 9.218 & 8.423 & 8.134 & 7.464 & 6.834 & 6.425 & 6.696 \\
\hline $\mathrm{S} 2$ & 1.37 & 0.857 & pole-on & -0.648 & 14.817 & 36.812 & 20.96 & 16.753 & 12.801 & 11.174 & 10.28 & 9.454 & 9.239 & 8.309 & 7.709 & 7.218 & 7.649 \\
\hline $\mathrm{S} 2$ & 1.37 & 0.928 & pole-on & -0.668 & 16.743 & 41.983 & 23.658 & 18.959 & 14.31 & 12.435 & 11.367 & 10.602 & 10.411 & 9.229 & 8.646 & 8.064 & 8.741 \\
\hline $\mathrm{S} 2$ & 1.37 & 1.0 & pole-on & -0.681 & 18.376 & 46.681 & 26.488 & 21.12 & 15.791 & 13.655 & 12.392 & 11.735 & 11.577 & 10.082 & 9.625 & 8.87 & 9.86 \\
\hline S2 & 1.01 & 0.0 & edge-on & 0.24 & 1.304 & 3.798 & 2.474 & 2.048 & 3.537 & 1.526 & 1.407 & 1.267 & 1.174 & 1.071 & 0.981 & 0.846 & 0.831 \\
\hline $\mathrm{S} 2$ & 1.01 & 0.071 & edge-on & 0.177 & 1.503 & 3.416 & 2.305 & 1.852 & 2.577 & 1.383 & 1.262 & 1.143 & 1.059 & 0.976 & 0.872 & 0.77 & 0.745 \\
\hline $\mathrm{S} 2$ & 1.01 & 0.143 & edge-on & 0.083 & 3.426 & 3.247 & 2.319 & 1.833 & 1.697 & 1.247 & 1.129 & 1.013 & 0.931 & 0.839 & 0.713 & 0.642 & 0.6 \\
\hline $\mathrm{S} 2$ & 1.01 & 0.215 & interm. & -0.018 & 15.939 & 4.34 & 2.491 & 1.754 & 453.771 & 120.727 & 120.839 & 313.361 & 354.819 & 351.847 & 391.27 & 338.331 & 342.653 \\
\hline $\mathrm{S} 2$ & 1.01 & 0.286 & interm. & -0.12 & 1.076 & 371.419 & 63.721 & 29.739 & 40.315 & 11.927 & 10.432 & 8.5 & 7.807 & 6.886 & 6.076 & 5.443 & 5.492 \\
\hline S2 & 1.01 & 0.357 & interm. & -0.207 & 2.117 & 41.955 & 16.721 & 12.137 & 18.522 & 7.683 & 6.987 & 6.252 & 5.813 & 5.433 & 4.865 & 4.471 & 4.427 \\
\hline $\mathrm{S} 2$ & 1.01 & 0.429 & interm. & -0.283 & 3.309 & 26.699 & 14.468 & 11.441 & 15.513 & 7.731 & 7.108 & 6.388 & 6.074 & 5.639 & 5.085 & 4.719 & 4.742 \\
\hline $\mathrm{S} 2$ & 1.01 & 0.5 & pole-on & -0.345 & 4.68 & 26.956 & 15.545 & 12.384 & 15.259 & 8.434 & 7.759 & 7.055 & 6.761 & 6.197 & 5.66 & 5.293 & 5.45 \\
\hline S2 & 1.01 & 0.571 & pole-on & -0.397 & 6.312 & 28.912 & 17.006 & 13.788 & 16.059 & 9.347 & 8.652 & 7.849 & 7.598 & 6.985 & 6.373 & 6.033 & 6.239 \\
\hline $\mathrm{S} 2$ & 1.01 & 0.643 & pole-on & -0.441 & 8.092 & 32.281 & 19.235 & 15.549 & 17.452 & 10.655 & 9.773 & 8.856 & 8.634 & 7.88 & 7.244 & 6.845 & 7.245 \\
\hline
\end{tabular}


Table A.2 - continued.

\begin{tabular}{|c|c|c|c|c|c|c|c|c|c|c|c|c|c|c|c|c|c|}
\hline & & & $\tilde{\tau}_{\mathrm{bu}}$ & & $\mathrm{bu}$ & 0.15 & 0.45 & 0.75 & 1.50 & 2.25 & 3.00 & 4.50 & 6.00 & 9.00 & 15.00 & 30.00 & $\infty$ \\
\hline Star & $\Sigma_{0}\left[\mathrm{~g} \mathrm{~cm}^{-2}\right]$ & $\cos i$ & ang. type & $\Delta V_{\mathrm{bu}}^{\infty}$ & $\xi_{\mathrm{bu}}^{V}$ & $\xi_{\mathrm{d}}^{V}$ & & & & & & & & & & & \\
\hline S2 & 1.01 & 0.714 & pole-on & -0.476 & 10.036 & 36.726 & 21.777 & 17.459 & 19.41 & 12.004 & 11.036 & 10.003 & 9.862 & 8.899 & 8.218 & 7.739 & 8.51 \\
\hline S2 & 1.01 & 0.786 & pole-on & -0.503 & 12.111 & 42.375 & 25.006 & 19.962 & 21.902 & 13.498 & 12.474 & 11.253 & 11.274 & 10.015 & 9.262 & 8.764 & 10.009 \\
\hline $\mathrm{S} 2$ & 1.01 & 0.857 & pole-on & -0.524 & 14.185 & 48.305 & 28.332 & 22.908 & 24.994 & 15.16 & 13.879 & 12.738 & 12.857 & 11.105 & 10.376 & 9.835 & 11.642 \\
\hline S2 & 1.01 & 0.928 & pole-on & -0.54 & 16.387 & 55.129 & 32.548 & 26.204 & 28.196 & 16.776 & 15.309 & 14.255 & 14.707 & 12.27 & 11.607 & 10.936 & 13.679 \\
\hline S2 & 1.01 & 1.0 & pole-on & -0.549 & 17.988 & 61.609 & 36.436 & 29.331 & 31.395 & 18.344 & 16.6 & 15.811 & 16.514 & 13.41 & 12.758 & 12.004 & 15.593 \\
\hline $\mathrm{S} 2$ & 0.75 & 0.0 & edge-on & 0.188 & 1.475 & 4.719 & 3.068 & 2.527 & 2.009 & 1.764 & 1.609 & 1.434 & 1.335 & 1.222 & 1.06 & 0.971 & 0.946 \\
\hline S2 & 0.75 & 0.071 & edge-on & 0.145 & 1.643 & 4.272 & 2.817 & 2.384 & 1.886 & 1.66 & 1.526 & 1.356 & 1.271 & 1.153 & 0.998 & 0.905 & 0.885 \\
\hline $\mathrm{S} 2$ & 0.75 & 0.143 & edge-on & 0.077 & 3.524 & 4.093 & 2.963 & 2.448 & 1.956 & 1.689 & 1.6 & 1.369 & 1.262 & 1.162 & 1.026 & 0.919 & 0.871 \\
\hline $\mathrm{S} 2$ & 0.75 & 0.215 & interm. & 0.005 & 336.352 & 7.907 & 2.756 & 2.229 & 1.52 & 5.069 & 3.137 & 5.304 & 22.756 & 22.214 & 19.381 & 43.606 & 79.95 \\
\hline $\mathrm{S} 2$ & 0.75 & 0.286 & interm. & -0.076 & 0.911 & 360.73 & 380.807 & 133.713 & 30.347 & 21.307 & 16.602 & 12.782 & 11.627 & 9.813 & 8.065 & 7.193 & 7.681 \\
\hline $\mathrm{S} 2$ & 0.75 & 0.357 & interm. & -0.146 & 1.92 & 106.683 & 22.127 & 15.675 & 10.69 & 9.169 & 8.144 & 7.271 & 6.952 & 6.113 & 5.629 & 5.175 & 5.582 \\
\hline $\mathrm{S} 2$ & 0.75 & 0.429 & interm. & $\mid-0.207$ & 3.062 & 34.684 & 17.588 & 13.851 & 10.118 & 8.972 & 8.4 & 7.379 & 7.257 & 6.497 & 5.919 & 5.572 & 6.026 \\
\hline $\mathrm{S} 2$ & 0.75 & 0.5 & pole-on & -0.258 & 4.209 & 31.707 & 18.142 & 14.664 & 11.239 & 9.802 & 9.055 & 8.294 & 8.105 & 7.322 & 6.826 & 6.322 & 6.863 \\
\hline $\mathrm{S} 2$ & 0.75 & 0.571 & pole-on & -0.301 & 5.56 & 34.468 & 20.188 & 16.555 & 12.629 & 11.186 & 10.363 & 9.354 & 9.297 & 8.373 & 7.734 & 7.262 & 8.028 \\
\hline $\mathrm{S} 2$ & 0.75 & 0.643 & pole-on & -0.337 & 6.991 & 38.566 & 22.962 & 18.828 & 14.42 & 12.811 & 11.862 & 10.637 & 10.782 & 9.505 & 8.885 & 8.312 & 9.44 \\
\hline $\mathrm{S} 2$ & 0.75 & 0.714 & pole-on & -0.365 & 8.476 & 43.447 & 26.419 & 21.764 & 16.694 & 14.765 & 13.548 & 12.116 & 12.444 & 10.843 & 10.232 & 9.548 & 11.126 \\
\hline $\mathrm{S} 2$ & 0.75 & 0.786 & pole-on & -0.387 & 9.962 & 50.305 & 30.65 & 24.973 & 19.28 & 16.831 & 15.41 & 13.882 & 14.364 & 12.199 & 11.594 & 10.757 & 13.082 \\
\hline S2 & 0.75 & 0.857 & pole-on & -0.404 & 11.385 & 58.831 & 35.411 & 28.925 & 21.565 & 18.939 & 17.149 & 15.606 & 16.435 & 13.479 & 13.252 & 12.11 & 15.297 \\
\hline $\mathrm{S} 2$ & 0.75 & 0.928 & pole-on & -0.415 & 12.799 & 66.737 & 40.793 & 33.686 & 24.281 & 20.955 & 18.804 & 17.544 & 18.935 & 14.849 & 14.869 & 13.396 & 17.881 \\
\hline $\mathrm{S} 2$ & 0.75 & 1.0 & pole-on & -0.42 & 13.822 & 74.687 & 45.528 & 37.618 & 26.695 & 22.653 & 20.292 & 19.308 & 21.404 & 16.074 & 16.4 & 14.626 & 20.385 \\
\hline $\mathrm{S} 2$ & 0.56 & 0.0 & edge-on & 0.149 & 1.686 & 6.276 & 3.666 & 3.005 & 2.383 & 2.08 & 1.89 & 1.678 & 1.535 & 1.423 & 1.231 & 1.104 & 1.079 \\
\hline S2 & 0.56 & 0.071 & edge-on & 0.119 & 1.814 & 5.289 & 3.424 & 2.855 & 2.265 & 2.008 & 1.829 & 1.604 & 1.492 & 1.354 & 1.171 & 1.084 & 1.053 \\
\hline $\mathrm{S} 2$ & 0.56 & 0.143 & edge-on & 0.071 & 3.1 & 5.646 & 3.817 & 3.165 & 2.523 & 2.272 & 2.082 & 1.829 & 1.686 & 1.559 & 1.372 & 1.265 & 1.237 \\
\hline S2 & 0.56 & 0.215 & interm. & 0.016 & 96.7 & 6.807 & 4.295 & 4.077 & 2.776 & 2.53 & 3.373 & 2.275 & 2.011 & 1.914 & 3.416 & 1.671 & 2.679 \\
\hline $\mathrm{S} 2$ & 0.56 & 0.286 & interm. & -0.044 & 0.854 & 294.461 & 358.954 & 349.209 & 234.876 & 124.04 & 58.212 & 22.779 & 22.118 & 13.555 & 12.199 & 9.6 & 11.248 \\
\hline $\mathrm{S} 2$ & 0.56 & 0.357 & interm. & -0.096 & 1.834 & 276.853 & 28.206 & 18.612 & 11.734 & 10.098 & 8.615 & 7.444 & 7.434 & 6.243 & 5.737 & 5.374 & 5.806 \\
\hline S2 & 0.56 & 0.429 & interm. & -0.144 & 2.989 & 40.893 & 19.31 & 15.547 & 11.21 & 9.516 & 8.623 & 7.762 & 7.864 & 6.743 & 6.317 & 5.917 & 6.502 \\
\hline $\mathrm{S} 2$ & 0.56 & 0.5 & pole-on & -0.183 & 4.195 & 36.276 & 20.238 & 16.843 & 12.157 & 10.494 & 9.794 & 8.99 & 8.878 & 7.64 & 7.328 & 6.908 & 7.704 \\
\hline $\mathrm{S} 2$ & 0.56 & 0.571 & pole-on & -0.217 & 5.581 & 38.007 & 22.14 & 18.401 & 14.021 & 12.236 & 11.439 & 10.234 & 10.316 & 9.033 & 8.531 & 7.948 & 9.153 \\
\hline S2 & 0.56 & 0.643 & pole-on & -0.244 & 7.255 & 42.508 & 26.094 & 21.462 & 16.262 & 14.216 & 13.117 & 11.831 & 12.129 & 10.425 & 9.88 & 9.285 & 10.958 \\
\hline $\mathrm{S} 2$ & 0.56 & 0.714 & pole-on & -0.265 & 9.073 & 48.724 & 29.948 & 24.825 & 19.046 & 16.428 & 15.136 & 13.574 & 14.105 & 11.892 & 11.525 & 10.624 & 13.17 \\
\hline S2 & 0.56 & 0.786 & pole-on & -0.282 & 11.105 & 56.865 & 35.139 & 29.018 & 22.024 & 18.904 & 17.529 & 15.597 & 16.48 & 13.638 & 13.122 & 12.035 & 15.641 \\
\hline S2 & 0.56 & 0.857 & pole-on & -0.294 & 13.326 & 64.931 & 40.622 & 33.786 & 24.823 & 21.416 & 19.497 & 17.408 & 19.176 & 14.997 & 15.061 & 13.523 & 18.541 \\
\hline $\mathrm{S} 2$ & 0.56 & 0.928 & pole-on & -0.302 & 15.445 & 75.752 & 47.371 & 38.849 & 27.468 & 23.444 & 21.241 & 19.686 & 22.299 & 16.427 & 16.805 & 15.097 & 21.976 \\
\hline $\mathrm{S} 2$ & 0.56 & 1.0 & pole-on & -0.304 & 17.164 & 84.639 & 52.681 & 44.164 & 30.159 & 25.278 & 22.968 & 21.495 & 24.875 & 17.673 & 18.6 & 16.22 & 25.0 \\
\hline
\end{tabular}


Table A.2 - continued.

\begin{tabular}{|c|c|c|c|c|c|c|c|c|c|c|c|c|c|c|c|c|c|}
\hline & & & 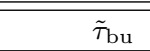 & & bu & 0.15 & 0.45 & 0.75 & $\bar{~} 1.50$ & 2.25 & 3.00 & 4.50 & (6.00 & 9.00 & 15.00 & 30.00 & $\infty$ \\
\hline Star & $\Sigma_{0}\left[\mathrm{~g} \mathrm{~cm}^{-2}\right]$ & $\cos i$ & ang. type & $\Delta V_{\mathrm{bu}}^{\infty}$ & $\xi_{\mathrm{bu}}^{V}$ & $\xi_{\mathrm{d}}^{V}$ & & & & & & & & & & & \\
\hline S2 & 0.41 & 0.0 & edge-on & 0.116 & 1.896 & 7.522 & 4.512 & 3.675 & 2.736 & 2.469 & 2.177 & 2.002 & 1.753 & 1.603 & 1.401 & 1.266 & 1.237 \\
\hline $\mathrm{S} 2$ & 0.41 & 0.071 & edge-on & 0.096 & 1.999 & 6.776 & 4.302 & 3.454 & 2.689 & 2.364 & 2.201 & 1.982 & 1.736 & 1.59 & 1.362 & 1.27 & 1.231 \\
\hline $\mathrm{S} 2$ & 0.41 & 0.143 & edge-on & 0.062 & 3.085 & 7.187 & 4.667 & 3.908 & 3.182 & 2.818 & 2.625 & 2.363 & 2.094 & 1.98 & 1.762 & 1.599 & 1.566 \\
\hline $\mathrm{S} 2$ & 0.41 & 0.215 & interm. & 0.021 & 37.804 & 10.442 & 6.215 & 4.929 & 4.314 & 4.323 & 3.883 & 3.944 & 3.167 & 3.175 & 3.062 & 2.888 & 2.575 \\
\hline $\mathrm{S} 2$ & 0.41 & 0.286 & interm. & -0.02 & 21.593 & 268.917 & 239.523 & 324.944 & 324.551 & 322.077 & 317.659 & 259.741 & 260.622 & 189.349 & 185.505 & 94.257 & 152.022 \\
\hline $\mathrm{S} 2$ & 0.41 & 0.357 & interm. & -0.059 & 4.249 & 302.072 & 82.821 & 26.328 & 11.901 & 8.77 & 7.702 & 6.549 & 7.074 & 5.593 & 5.272 & 4.485 & 5.875 \\
\hline $\mathrm{S} 2$ & 0.41 & 0.429 & interm. & -0.093 & 2.755 & 65.286 & 20.213 & 15.257 & 11.099 & 8.784 & 8.143 & 7.386 & 7.585 & 6.575 & 5.925 & 5.404 & 6.55 \\
\hline S2 & 0.41 & 0.5 & pole-on & -0.122 & 3.959 & 36.693 & 20.287 & 16.163 & 12.379 & 10.609 & 9.689 & 8.772 & 8.959 & 7.673 & 7.2 & 6.56 & 7.892 \\
\hline $\mathrm{S} 2$ & 0.41 & 0.571 & pole-on & -0.146 & 5.347 & 39.121 & 23.133 & 18.927 & 14.502 & 12.223 & 11.52 & 10.359 & 10.603 & 9.066 & 8.641 & 8.037 & 9.708 \\
\hline $\mathrm{S} 2$ & 0.41 & 0.643 & pole-on & -0.166 & 6.811 & 44.023 & 27.695 & 22.289 & 16.931 & 14.43 & 13.5 & 12.181 & 12.786 & 10.847 & 10.167 & 9.468 & 11.764 \\
\hline $\mathrm{S} 2$ & 0.41 & 0.714 & pole-on & -0.181 & 8.635 & 51.723 & 31.547 & 26.554 & 19.881 & 17.152 & 15.927 & 14.071 & 14.983 & 12.566 & 11.864 & 11.004 & 14.285 \\
\hline $\mathrm{S} 2$ & 0.41 & 0.786 & pole-on & -0.192 & 10.663 & 59.942 & 38.119 & 31.186 & 23.14 & 19.912 & 18.493 & 16.073 & 17.768 & 14.05 & 13.69 & 12.397 & 17.077 \\
\hline $\mathrm{S} 2$ & 0.41 & 0.857 & pole-on & -0.2 & 12.868 & 69.134 & 44.123 & 36.675 & 26.337 & 22.457 & 20.526 & 18.136 & 20.525 & 15.633 & 15.747 & 13.863 & 20.656 \\
\hline $\mathrm{S} 2$ & 0.41 & 0.928 & pole-on & -0.205 & 15.073 & 81.691 & 50.583 & 42.513 & 29.982 & 24.507 & 22.434 & 20.337 & 24.046 & 17.061 & 17.849 & 15.395 & 24.288 \\
\hline $\mathrm{S} 2$ & 0.41 & 1.0 & pole-on & -0.205 & 17.021 & 90.056 & 56.384 & 48.238 & 32.262 & 26.583 & 24.145 & 22.732 & 26.92 & 18.477 & 19.852 & 16.797 & 28.544 \\
\hline $\mathrm{S} 2$ & 0.3 & 0.0 & edge-on & 0.091 & 2.117 & 9.949 & 5.242 & 4.385 & 3.203 & 2.818 & 2.668 & 2.201 & 2.022 & 1.806 & 1.609 & 1.484 & 1.403 \\
\hline $\mathrm{S} 2$ & 0.3 & 0.071 & edge-on & 0.075 & 2.264 & 8.803 & 5.314 & 4.056 & 3.231 & 2.727 & 2.484 & 2.174 & 2.039 & 1.82 & 1.7 & 1.491 & 1.431 \\
\hline $\mathrm{S} 2$ & 0.3 & 0.143 & edge-on & 0.051 & 3.426 & 12.037 & 6.329 & 4.894 & 3.863 & 3.751 & 3.329 & 2.736 & 2.758 & 2.474 & 2.175 & 2.051 & 1.989 \\
\hline $\mathrm{S} 2$ & 0.3 & 0.215 & interm. & 0.022 & 27.543 & 35.64 & 9.342 & 7.595 & 6.311 & 6.958 & 6.545 & 5.195 & 4.698 & 4.581 & 4.67 & 4.16 & 4.247 \\
\hline $\mathrm{S} 2$ & 0.3 & 0.286 & interm. & -0.002 & 253.213 & 237.789 & 262.702 & 230.98 & 251.482 & 286.709 & 323.315 & 309.025 & 315.638 & 311.241 & 309.117 & 302.371 & 290.763 \\
\hline $\mathrm{S} 2$ & 0.3 & 0.357 & interm. & -0.033 & 3.904 & 289.436 & 253.746 & 106.557 & 32.994 & 12.027 & 6.712 & 6.082 & 7.019 & 4.564 & 4.09 & 3.624 & 4.194 \\
\hline $\mathrm{S} 2$ & 0.3 & 0.429 & interm. & -0.058 & 2.657 & 195.814 & 19.712 & 14.194 & 9.435 & 7.712 & 6.974 & 6.526 & 6.848 & 5.576 & 5.187 & 4.753 & 5.454 \\
\hline $\mathrm{S} 2$ & 0.3 & 0.5 & pole-on & -0.078 & 3.844 & 54.268 & 19.034 & 16.026 & 10.856 & 9.394 & 8.691 & 8.122 & 8.487 & 7.225 & 6.558 & 6.049 & 7.244 \\
\hline $\mathrm{S} 2$ & 0.3 & 0.571 & pole-on & -0.095 & 5.146 & 39.503 & 21.97 & 17.902 & 13.055 & 11.565 & 10.905 & 9.718 & 10.275 & 8.543 & 8.09 & 7.453 & 9.13 \\
\hline $\mathrm{S} 2$ & 0.3 & 0.643 & pole-on & -0.109 & 6.8 & 45.633 & 25.308 & 21.711 & 16.247 & 14.09 & 12.83 & 11.773 & 12.162 & 10.447 & 9.756 & 8.852 & 11.229 \\
\hline $\mathrm{S} 2$ & 0.3 & 0.714 & pole-on & -0.119 & 8.487 & 53.309 & 31.471 & 25.876 & 19.397 & 16.861 & 15.506 & 13.741 & 14.95 & 11.921 & 11.581 & 10.642 & 13.97 \\
\hline $\mathrm{S} 2$ & 0.3 & 0.786 & pole-on & -0.127 & 10.504 & 61.341 & 37.133 & 30.87 & 23.55 & 19.832 & 18.205 & 15.841 & 17.561 & 13.857 & 13.587 & 12.104 & 17.44 \\
\hline $\mathrm{S} 2$ & 0.3 & 0.857 & pole-on & -0.132 & 12.785 & 71.414 & 44.02 & 37.424 & 26.206 & 22.653 & 20.499 & 17.785 & 21.317 & 15.448 & 15.321 & 13.51 & 20.783 \\
\hline $\mathrm{S} 2$ & 0.3 & 0.928 & pole-on & -0.134 & 15.35 & 81.429 & 52.192 & 42.396 & 29.76 & 24.381 & 21.825 & 20.274 & 24.853 & 16.914 & 17.585 & 15.109 & 25.3 \\
\hline $\mathrm{S} 2$ & 0.3 & 1.0 & pole-on & -0.133 & 17.102 & 92.391 & 57.563 & 48.408 & 32.661 & 26.716 & 24.041 & 22.697 & 27.633 & 17.77 & 19.671 & 16.382 & 28.901 \\
\hline S3 & 2.5 & 0.0 & edge-on & 0.444 & 1.153 & 2.216 & 1.62 & 1.426 & 1.195 & 1.08 & 1.0 & 0.898 & 0.84 & 0.77 & 0.672 & 0.61 & 0.59 \\
\hline S3 & 2.5 & 0.071 & edge-on & 0.302 & 1.478 & 1.951 & 1.436 & 1.264 & 1.025 & 0.942 & 0.873 & 0.762 & 0.722 & 0.651 & 0.557 & 0.505 & 0.484 \\
\hline S3 & 2.5 & 0.143 & edge-on & 0.094 & 8.908 & 2.075 & 1.395 & 1.158 & 0.769 & 0.679 & 0.593 & 0.458 & 0.435 & 0.344 & 0.28 & 0.236 & 0.225 \\
\hline S3 & 2.5 & 0.215 & interm. & -0.095 & 0.301 & 1.993 & 17.955 & 363.272 & 54.149 & 24.363 & 17.5 & 13.647 & 10.592 & 9.513 & 7.373 & 6.569 & 6.082 \\
\hline S3 & 2.5 & 0.286 & interm. & -0.247 & 1.19 & 52.831 & 12.116 & 8.319 & 5.898 & 4.789 & 4.232 & 3.908 & 3.467 & 3.207 & 2.8 & 2.577 & 2.479 \\
\hline
\end{tabular}


Table A.2 - continued.

\begin{tabular}{|c|c|c|c|c|c|c|c|c|c|c|c|c|c|c|c|c|c|}
\hline & & & $\tilde{\tau}_{\mathrm{bu}}$ & & $\mathrm{bu}$ & 0.15 & 0.45 & 0.75 & 1.50 & 2.25 & 3.00 & 4.50 & 6.00 & 9.00 & 15.00 & 30.00 & $\infty$ \\
\hline Star & $\Sigma_{0}\left[\mathrm{~g} \mathrm{~cm}^{-2}\right]$ & $\cos i$ & ang. type & $\Delta V_{\mathrm{bu}}^{\infty}$ & $\xi_{\mathrm{bu}}^{V}$ & $\xi_{\mathrm{d}}^{V}$ & & & & & & & & & & & \\
\hline S3 & 2.5 & 0.357 & interm. & -0.371 & 2.283 & 15.031 & 8.191 & 6.317 & 4.806 & 4.069 & 3.679 & 3.377 & 3.032 & 2.821 & 2.471 & 2.289 & 2.2 \\
\hline S3 & 2.5 & 0.429 & interm. & -0.474 & 3.627 & 13.415 & 7.925 & 6.211 & 4.767 & 4.075 & 3.711 & 3.375 & 3.098 & 2.87 & 2.529 & 2.324 & 2.249 \\
\hline S3 & 2.5 & 0.5 & pole-on & -0.553 & 5.258 & 14.299 & 8.263 & 6.518 & 5.003 & 4.306 & 3.928 & 3.582 & 3.261 & 3.049 & 2.683 & 2.482 & 2.408 \\
\hline $\mathrm{S} 3$ & 2.5 & 0.571 & pole-on & -0.617 & 7.079 & 15.48 & 8.914 & 7.006 & 5.387 & 4.642 & 4.243 & 3.879 & 3.575 & 3.318 & 2.914 & 2.702 & 2.626 \\
\hline S3 & 2.5 & 0.643 & pole-on & -0.672 & 8.985 & 16.947 & 9.69 & 7.654 & 5.894 & 5.074 & 4.654 & 4.232 & 3.917 & 3.64 & 3.196 & 2.985 & 2.92 \\
\hline $\mathrm{S} 3$ & 2.5 & 0.714 & pole-on & -0.717 & 10.911 & 18.793 & 10.674 & 8.399 & 6.447 & 5.591 & 5.123 & 4.654 & 4.308 & 3.993 & 3.545 & 3.298 & 3.25 \\
\hline S3 & 2.5 & 0.786 & pole-on & -0.752 & 12.836 & 20.893 & 11.817 & 9.28 & 7.101 & 6.161 & 5.622 & 5.117 & 4.748 & 4.413 & 3.939 & 3.683 & 3.664 \\
\hline S3 & 2.5 & 0.857 & pole-on & -0.781 & 14.71 & 23.394 & 13.13 & 10.308 & 7.801 & 6.735 & 6.173 & 5.618 & 5.268 & 4.899 & 4.391 & 4.125 & 4.131 \\
\hline $\mathrm{S} 3$ & 2.5 & 0.928 & pole-on & -0.804 & 16.529 & 26.355 & 14.561 & 11.373 & 8.564 & 7.415 & 6.792 & 6.246 & 5.894 & 5.455 & 4.92 & 4.614 & 4.67 \\
\hline S3 & 2.5 & 1.0 & pole-on & -0.821 & 17.935 & 29.282 & 16.005 & 12.452 & 9.419 & 8.142 & 7.471 & 6.896 & 6.541 & 6.01 & 5.474 & 5.136 & 5.222 \\
\hline S3 & 1.85 & 0.0 & edge-on & 0.351 & 1.268 & 2.73 & 1.876 & 1.633 & 1.574 & 1.208 & 1.121 & 1.551 & 0.995 & 0.869 & 0.746 & 0.677 & 0.657 \\
\hline $\mathrm{S} 3$ & 1.85 & 0.071 & edge-on & 0.254 & 1.522 & 2.465 & 1.686 & 1.482 & 1.231 & 1.096 & 1.027 & 1.152 & 0.868 & 0.785 & 0.666 & 0.607 & 0.584 \\
\hline S3 & 1.85 & 0.143 & edge-on & 0.102 & 5.195 & 2.603 & 1.688 & 1.431 & 1.096 & 0.907 & 0.839 & 0.691 & 0.599 & 0.549 & 0.464 & 0.392 & 0.385 \\
\hline S3 & 1.85 & 0.215 & interm. & -0.048 & 0.263 & 2.551 & 1.19 & 12.635 & 349.911 & 346.199 & 285.015 & 157.802 & 233.58 & 35.652 & 24.057 & 19.565 & 17.572 \\
\hline S3 & 1.85 & 0.286 & interm. & -0.182 & 1.025 & 321.375 & 20.003 & 12.834 & 8.385 & 6.731 & 6.222 & 6.81 & 4.675 & 4.284 & 3.796 & 3.456 & 3.358 \\
\hline S3 & 1.85 & 0.357 & interm. & -0.293 & 2.093 & 19.991 & 10.545 & 8.203 & 6.195 & 5.33 & 4.891 & 5.281 & 3.938 & 3.658 & 3.245 & 2.98 & 2.918 \\
\hline S3 & 1.85 & 0.429 & interm. & -0.386 & 3.389 & 17.155 & 10.142 & 8.112 & 6.226 & 5.391 & 4.971 & 5.16 & 4.095 & 3.81 & 3.375 & 3.114 & 3.066 \\
\hline S3 & 1.85 & 0.5 & pole-on & -0.459 & 4.948 & 17.963 & 10.736 & 8.581 & 6.65 & 5.763 & 5.328 & 5.471 & 4.403 & 4.102 & 3.655 & 3.407 & 3.359 \\
\hline S3 & 1.85 & 0.571 & pole-on & -0.519 & 6.73 & 19.793 & 11.707 & 9.35 & 7.199 & 6.293 & 5.835 & 5.934 & 4.864 & 4.549 & 4.054 & 3.782 & 3.758 \\
\hline S3 & 1.85 & 0.643 & pole-on & -0.57 & 8.629 & 21.995 & 12.884 & 10.345 & 7.986 & 6.976 & 6.474 & 6.515 & 5.413 & 5.063 & 4.542 & 4.258 & 4.283 \\
\hline S3 & 1.85 & 0.714 & pole-on & -0.611 & 10.646 & 24.665 & 14.294 & 11.451 & 8.839 & 7.747 & 7.209 & 7.233 & 6.036 & 5.666 & 5.124 & 4.812 & 4.898 \\
\hline S3 & 1.85 & 0.786 & pole-on & -0.643 & 12.736 & 27.859 & 15.932 & 12.719 & 9.798 & 8.69 & 8.036 & 8.017 & 6.747 & 6.343 & 5.794 & 5.43 & 5.565 \\
\hline S3 & 1.85 & 0.857 & pole-on & -0.67 & 14.776 & 31.561 & 17.964 & 14.253 & 10.941 & 9.669 & 8.983 & 8.977 & 7.612 & 7.11 & 6.534 & 6.117 & 6.36 \\
\hline S3 & 1.85 & 0.928 & pole-on & -0.69 & 16.731 & 35.82 & 20.183 & 16.098 & 12.264 & 10.815 & 9.985 & 10.024 & 8.468 & 7.899 & 7.386 & 6.869 & 7.254 \\
\hline S3 & 1.85 & 1.0 & pole-on & -0.705 & 18.351 & 40.04 & 22.595 & 18.025 & 13.573 & 11.879 & 10.915 & 11.109 & 9.411 & 8.692 & 8.19 & 7.615 & 8.224 \\
\hline $\mathrm{S} 3$ & 1.37 & 0.0 & edge-on & 0.277 & 1.403 & 3.162 & 2.265 & 1.899 & 1.541 & 1.382 & 1.267 & 1.135 & 1.043 & 0.97 & 0.836 & 0.766 & 0.757 \\
\hline S3 & 1.37 & 0.071 & edge-on & 0.208 & 1.608 & 2.968 & 2.113 & 1.797 & 1.457 & 1.298 & 1.191 & 1.076 & 0.974 & 0.924 & 0.785 & 0.723 & 0.701 \\
\hline S3 & 1.37 & 0.143 & edge-on & 0.099 & 4.03 & 3.061 & 2.216 & 1.866 & 1.404 & 1.231 & 1.126 & 0.991 & 0.872 & 0.828 & 0.686 & 0.629 & 0.601 \\
\hline S3 & 1.37 & 0.215 & interm. & -0.013 & 27.074 & 3.112 & 2.066 & 1.435 & 21.489 & 12.787 & 71.901 & 124.609 & 271.485 & 330.026 & 364.861 & 351.673 & 336.885 \\
\hline S3 & 1.37 & 0.286 & interm. & -0.127 & 0.902 & 405.536 & 53.22 & 22.724 & 12.135 & 9.857 & 8.4 & 6.862 & 6.561 & 5.615 & 5.155 & 4.611 & 4.505 \\
\hline S3 & 1.37 & 0.357 & interm. & -0.221 & 1.944 & 33.399 & 13.449 & 10.247 & 7.553 & 6.554 & 5.901 & 5.344 & 5.068 & 4.536 & 4.146 & 3.791 & 3.848 \\
\hline S3 & 1.37 & 0.429 & interm. & \begin{tabular}{|l}
-0.302 \\
\end{tabular} & 3.147 & 22.111 & 12.511 & 9.991 & 7.632 & 6.691 & 6.09 & 5.495 & 5.27 & 4.739 & 4.358 & 4.048 & 4.106 \\
\hline S3 & 1.37 & 0.5 & pole-on & -0.368 & 4.617 & 22.742 & 13.302 & 10.694 & 8.259 & 7.184 & 6.705 & 6.077 & 5.776 & 5.284 & 4.841 & 4.532 & 4.639 \\
\hline S3 & 1.37 & 0.571 & pole-on & -0.421 & 6.315 & 24.75 & 14.733 & 11.863 & 9.188 & 8.041 & 7.436 & 6.766 & 6.533 & 5.939 & 5.466 & 5.103 & 5.321 \\
\hline S3 & 1.37 & 0.643 & pole-on & -0.466 & 8.132 & 28.027 & 16.507 & 13.312 & 10.384 & 9.066 & 8.4 & 7.638 & 7.379 & 6.714 & 6.237 & 5.836 & 6.152 \\
\hline
\end{tabular}


Table A.2 - continued.

\begin{tabular}{|c|c|c|c|c|c|c|c|c|c|c|c|c|c|c|c|c|c|}
\hline & & & $\tilde{\tau}_{\text {bu }}$ & & $\mathrm{bu}$ & 0.15 & 0.45 & 0.75 & 1.50 & 2.25 & 3.00 & 4.50 & 6.00 & 9.00 & 15.00 & 30.00 & $\infty$ \\
\hline Star & $\Sigma_{0}\left[\mathrm{~g} \mathrm{~cm}^{-2}\right]$ & $\cos i$ & ang. type & $\Delta V_{\mathrm{bu}}^{\infty}$ & $\xi_{\mathrm{bu}}^{V}$ & $\xi_{\mathrm{d}}^{V}$ & & & & & & & & & & & \\
\hline S3 & 1.37 & 0.714 & pole-on & -0.503 & 10.144 & 32.099 & 18.713 & 15.128 & 11.73 & 10.299 & 9.504 & 8.673 & 8.409 & 7.644 & 7.093 & 6.639 & 7.117 \\
\hline S3 & 1.37 & 0.786 & pole-on & -0.531 & 12.259 & 36.778 & 21.292 & 17.203 & 13.249 & 11.634 & 10.719 & 9.821 & 9.639 & 8.594 & 8.058 & 7.538 & 8.196 \\
\hline S3 & 1.37 & 0.857 & pole-on & -0.554 & 14.39 & 42.092 & 24.304 & 19.669 & 14.953 & 13.093 & 12.097 & 11.057 & 11.035 & 9.65 & 9.145 & 8.484 & 9.515 \\
\hline S3 & 1.37 & 0.928 & pole-on & -0.571 & 16.474 & 48.14 & 27.812 & 22.419 & 16.947 & 14.58 & 13.313 & 12.442 & 12.615 & 10.718 & 10.342 & 9.488 & 10.995 \\
\hline S3 & 1.37 & 1.0 & pole-on & -0.582 & 18.145 & 54.206 & 31.401 & 25.388 & 18.699 & 15.981 & 14.603 & 13.894 & 14.151 & 11.717 & 11.566 & 10.452 & 12.501 \\
\hline S3 & 1.01 & 0.0 & edge-on & 0.218 & 1.576 & 4.418 & 2.614 & 2.265 & 1.802 & 1.609 & 1.463 & 1.318 & 1.209 & 1.049 & 0.806 & 0.824 & 0.866 \\
\hline S3 & 1.01 & 0.071 & edge-on & 0.171 & 1.758 & 4.057 & 2.499 & 2.162 & 1.724 & 1.529 & 1.436 & 1.27 & 1.18 & 1.032 & 0.792 & 0.806 & 0.84 \\
\hline S3 & 1.01 & 0.143 & edge-on & 0.093 & 3.648 & 4.435 & 2.821 & 2.376 & 1.857 & 1.629 & 1.516 & 1.346 & 1.266 & 1.112 & 0.845 & 0.847 & 0.867 \\
\hline S3 & 1.01 & 0.215 & interm. & 0.012 & 368.035 & 5.059 & 3.065 & 2.411 & 1.808 & 1.482 & 1.293 & 2.427 & 1.067 & 2.74 & 54.434 & 3.144 & 32.643 \\
\hline S3 & 1.01 & 0.286 & interm. & -0.079 & 0.763 & 365.893 & 355.31 & 138.749 & 27.084 & 15.916 & 12.267 & 9.454 & 8.569 & 7.593 & 7.002 & 5.785 & 5.186 \\
\hline S3 & 1.01 & 0.357 & interm. & -0.155 & 1.794 & 60.869 & 17.66 & 12.351 & 8.776 & 7.521 & 6.872 & 6.038 & 5.735 & 5.357 & 5.256 & 4.458 & 4.142 \\
\hline S3 & 1.01 & 0.429 & interm. & -0.222 & 2.991 & 25.998 & 15.118 & 11.691 & 8.718 & 7.705 & 7.131 & 6.402 & 6.165 & 5.735 & 5.557 & 4.876 & 4.568 \\
\hline S3 & 1.01 & 0.5 & pole-on & -0.277 & 4.314 & 26.223 & 15.958 & 12.572 & 9.672 & 8.6 & 7.922 & 7.18 & 7.001 & 6.505 & 6.204 & 5.619 & 5.299 \\
\hline S3 & 1.01 & 0.571 & pole-on & \begin{tabular}{|l}
-0.322 \\
\end{tabular} & 5.9 & 28.863 & 17.821 & 14.218 & 10.868 & 9.793 & 9.067 & 8.188 & 8.071 & 7.437 & 7.106 & 6.488 & 6.239 \\
\hline S3 & 1.01 & 0.643 & pole-on & -0.36 & 7.722 & 32.97 & 20.694 & 16.536 & 12.39 & 11.223 & 10.366 & 9.473 & 9.37 & 8.558 & 8.169 & 7.537 & 7.467 \\
\hline S3 & 1.01 & 0.714 & pole-on & -0.391 & 9.659 & 38.52 & 23.468 & 18.785 & 14.172 & 12.987 & 11.868 & 10.743 & 10.828 & 9.767 & 9.27 & 8.706 & 9.034 \\
\hline S3 & 1.01 & 0.786 & pole-on & -0.414 & 11.833 & 44.117 & 27.324 & 21.8 & 16.209 & 14.702 & 13.613 & 12.253 & 12.576 & 11.02 & 10.519 & 9.976 & 11.108 \\
\hline S3 & 1.01 & 0.857 & pole-on & -0.432 & 14.002 & 51.365 & 31.783 & 25.114 & 18.481 & 16.653 & 15.23 & 13.825 & 14.418 & 12.285 & 11.795 & 11.226 & 13.67 \\
\hline S3 & 1.01 & 0.928 & pole-on & -0.445 & 16.22 & 59.757 & 36.474 & 29.143 & 20.893 & 18.58 & 16.8 & 15.692 & 16.651 & 13.584 & 13.126 & 12.577 & 16.906 \\
\hline S3 & 1.01 & 1.0 & pole-on & -0.452 & 17.888 & 67.128 & 41.234 & 33.068 & 23.281 & 20.175 & 18.139 & 17.356 & 18.705 & 14.675 & 14.355 & 13.873 & 19.944 \\
\hline S3 & 0.75 & 0.0 & edge-on & 0.173 & 2.556 & 5.438 & 3.358 & 2.718 & 2.097 & 1.83 & 1.677 & 1.481 & 1.363 & 1.238 & 1.073 & 0.986 & 0.947 \\
\hline S3 & 0.75 & 0.071 & edge-on & 0.139 & 13.39 & 4.981 & 3.121 & 2.628 & 2.1 & 1.822 & 1.636 & 1.466 & 1.357 & 1.23 & 1.083 & 0.985 & 0.951 \\
\hline S3 & 0.75 & 0.143 & edge-on & 0.084 & 3.864 & 5.301 & 3.713 & 3.011 & 2.454 & 2.122 & 1.914 & 1.727 & 1.622 & 1.516 & 1.268 & 1.169 & 1.136 \\
\hline S3 & 0.75 & 0.215 & interm. & 0.023 & 300.67 & 6.624 & 4.416 & 3.857 & 3.033 & 2.821 & 2.494 & 2.3 & 2.017 & 2.136 & 1.784 & 1.608 & 1.525 \\
\hline S3 & 0.75 & 0.286 & interm. & -0.045 & 23.252 & 139.143 & 321.736 & 315.791 & 244.091 & 88.21 & 30.455 & 15.025 & 12.459 & 7.941 & 7.003 & 5.882 & 6.743 \\
\hline S3 & 0.75 & 0.357 & interm. & -0.104 & 1.666 & 244.644 & 20.397 & 13.682 & 9.019 & 7.691 & 6.818 & 6.316 & 5.777 & 5.054 & 4.835 & 4.301 & 4.809 \\
\hline S3 & 0.75 & 0.429 & interm. & -0.157 & 2.787 & 32.002 & 15.727 & 12.859 & 9.372 & 8.14 & 7.384 & 6.709 & 6.601 & 5.77 & 5.504 & 4.999 & 5.582 \\
\hline S3 & 0.75 & 0.5 & pole-on & -0.201 & 3.992 & 29.357 & 17.281 & 13.685 & 10.782 & 9.371 & 8.637 & 7.769 & 7.781 & 6.805 & 6.399 & 6.01 & 6.64 \\
\hline S3 & 0.75 & 0.571 & pole-on & -0.238 & 5.261 & 32.846 & 19.224 & 16.054 & 12.333 & 10.921 & 10.021 & 9.103 & 9.048 & 8.047 & 7.514 & 7.055 & 7.977 \\
\hline S3 & 0.75 & 0.643 & pole-on & -0.268 & 6.709 & 37.497 & 22.645 & 18.535 & 14.254 & 12.635 & 11.68 & 10.465 & 10.666 & 9.34 & 8.85 & 8.251 & 9.613 \\
\hline S3 & 0.75 & 0.714 & pole-on & -0.292 & 8.188 & 43.906 & 26.726 & 21.693 & 16.818 & 14.835 & 13.518 & 12.147 & 12.54 & 10.766 & 10.301 & 9.541 & 11.554 \\
\hline S3 & 0.75 & 0.786 & pole-on & -0.31 & 9.851 & 50.87 & 31.1 & 25.726 & 19.521 & 17.013 & 15.469 & 13.945 & 14.666 & 12.286 & 11.878 & 10.883 & 13.768 \\
\hline S3 & 0.75 & 0.857 & pole-on & -0.323 & 11.326 & 58.962 & 36.305 & 30.156 & 22.229 & 19.418 & 17.455 & 15.84 & 17.023 & 13.586 & 13.498 & 12.187 & 16.328 \\
\hline S3 & 0.75 & 0.928 & pole-on & -0.332 & 12.817 & 68.713 & 42.653 & 34.944 & 25.119 & 21.332 & 19.245 & 17.886 & 19.77 & 14.947 & 15.411 & 13.651 & 19.373 \\
\hline S3 & 0.75 & 1.0 & pole-on & -0.335 & 13.793 & 76.675 & 47.536 & 39.373 & 27.598 & 23.178 & 20.904 & 19.86 & 22.407 & 16.233 & 17.076 & 14.882 & 22.104 \\
\hline
\end{tabular}


Table A.2 - continued.

\begin{tabular}{|c|c|c|c|c|c|c|c|c|c|c|c|c|c|c|c|c|c|}
\hline & & & $\tilde{\tau}_{\mathrm{bu}}$ & & $\mathrm{bu}$ & 0.15 & 0.45 & 0.75 & 1.50 & 2.25 & 3.00 & 4.50 & 6.00 & 9.00 & 15.00 & 30.00 & $\infty$ \\
\hline Star & $\Sigma_{0}\left[\mathrm{~g} \mathrm{~cm}^{-2}\right]$ & $\cos i$ & ang. type & $\Delta V_{\mathrm{bu}}^{\infty}$ & $\xi_{\text {bu }}^{V}$ & $\xi_{\mathrm{d}}^{V}$ & & & & & & & & & & & \\
\hline S3 & 0.56 & 0.0 & edge-on & 0.14 & 2.051 & 6.695 & 4.028 & 3.188 & 2.415 & 2.07 & 1.916 & 1.686 & 1.55 & 1.402 & 1.203 & 1.099 & 1.053 \\
\hline S3 & 0.56 & 0.071 & edge-on & 0.114 & 2.291 & 6.086 & 3.904 & 3.119 & 2.431 & 2.051 & 1.884 & 1.688 & 1.522 & 1.385 & 1.2 & 1.09 & 1.056 \\
\hline S3 & 0.56 & 0.143 & edge-on & 0.073 & 3.755 & 6.43 & 4.446 & 3.769 & 2.825 & 2.593 & 2.347 & 2.121 & 1.915 & 1.787 & 1.555 & 1.427 & 1.412 \\
\hline S3 & 0.56 & 0.215 & interm. & 0.028 & 21.886 & 12.265 & 5.962 & 5.161 & 4.335 & 3.897 & 3.732 & 3.592 & 3.465 & 3.281 & 2.901 & 2.729 & 2.655 \\
\hline S3 & 0.56 & 0.286 & interm. & -0.02 & 1.123 & 132.367 & 208.905 & 284.623 & 338.139 & 281.278 & 301.255 & 224.137 & 183.059 & 70.93 & 43.61 & 13.014 & 18.173 \\
\hline S3 & 0.56 & 0.357 & interm. & -0.067 & 14.537 & 325.208 & 25.652 & 14.935 & 8.075 & 6.663 & 5.807 & 5.252 & 5.196 & 4.32 & 4.145 & 3.826 & 4.015 \\
\hline S3 & 0.56 & 0.429 & interm. & -0.107 & 2.579 & 35.327 & 15.558 & 12.131 & 9.181 & 7.768 & 7.131 & 6.371 & 6.421 & 5.494 & 5.109 & 4.833 & 5.431 \\
\hline S3 & 0.56 & 0.5 & pole-on & -0.14 & 3.861 & 30.681 & 17.209 & 14.045 & 10.498 & 9.175 & 8.543 & 7.621 & 7.742 & 6.764 & 6.204 & 5.874 & 6.83 \\
\hline S3 & 0.56 & 0.571 & pole-on & -0.167 & 5.384 & 32.45 & 19.976 & 16.369 & 12.557 & 10.948 & 10.137 & 9.17 & 9.299 & 8.108 & 7.644 & 7.156 & 8.397 \\
\hline S3 & 0.56 & 0.643 & pole-on & -0.19 & 6.977 & 38.387 & 24.01 & 19.718 & 14.982 & 13.013 & 12.093 & 10.948 & 11.227 & 9.592 & 9.136 & 8.448 & 10.245 \\
\hline S3 & 0.56 & 0.714 & pole-on & -0.207 & 8.867 & 45.73 & 27.923 & 23.441 & 17.887 & 15.346 & 14.23 & 12.569 & 13.265 & 11.324 & 10.654 & 9.964 & 12.467 \\
\hline S3 & 0.56 & 0.786 & pole-on & -0.221 & 10.941 & 53.329 & 33.007 & 27.994 & 21.094 & 18.177 & 16.476 & 14.659 & 16.07 & 12.738 & 12.35 & 11.424 & 15.104 \\
\hline S3 & 0.56 & 0.857 & pole-on & -0.23 & 13.264 & 61.922 & 38.905 & 32.791 & 23.854 & 20.522 & 18.744 & 16.608 & 18.799 & 14.345 & 14.357 & 12.864 & 18.351 \\
\hline S3 & 0.56 & 0.928 & pole-on & -0.235 & 15.691 & 73.506 & 46.107 & 38.21 & 26.823 & 22.43 & 20.127 & 19.059 & 21.809 & 15.626 & 16.576 & 14.302 & 21.879 \\
\hline S3 & 0.56 & 1.0 & pole-on & -0.236 & 17.098 & 81.331 & 52.288 & 43.666 & 29.813 & 24.434 & 21.819 & 21.025 & 24.893 & 16.898 & 18.064 & 15.663 & 25.292 \\
\hline S3 & 0.41 & 0.0 & edge-on & 0.11 & 2.307 & 8.402 & 4.724 & 3.502 & 2.811 & 2.381 & 2.149 & 1.908 & 1.717 & 1.587 & 1.393 & 1.255 & 1.181 \\
\hline S3 & 0.41 & 0.071 & edge-on & 0.091 & 2.581 & 8.5 & 4.467 & 3.626 & 2.803 & 2.397 & 2.197 & 1.928 & 1.723 & 1.59 & 1.364 & 1.335 & 1.19 \\
\hline S3 & 0.41 & 0.143 & edge-on & 0.061 & 4.076 & 9.511 & 5.0 & 4.161 & 3.485 & 2.973 & 2.774 & 2.489 & 2.353 & 2.204 & 1.888 & 1.783 & 1.663 \\
\hline S3 & 0.41 & 0.215 & interm. & 0.028 & 16.843 & 15.085 & 8.089 & 6.132 & 5.852 & 4.982 & 5.451 & 4.914 & 4.324 & 4.763 & 4.29 & 4.11 & 3.858 \\
\hline S3 & 0.41 & 0.286 & interm. & -0.003 & 165.259 & 163.355 & 128.627 & 242.953 & 279.614 & 315.214 & 305.845 & 317.557 & 317.957 & 286.987 & 292.766 & 266.256 & 272.771 \\
\hline S3 & 0.41 & 0.357 & interm. & -0.039 & 1.251 & 316.693 & 208.97 & 40.881 & 11.084 & 6.14 & 5.084 & 4.59 & 3.886 & 3.443 & 2.812 & 2.865 & 3.281 \\
\hline S3 & 0.41 & 0.429 & interm. & -0.068 & 2.415 & 121.595 & 14.802 & 12.246 & 8.208 & 7.01 & 6.21 & 5.672 & 5.33 & 4.748 & 4.449 & 4.031 & 4.658 \\
\hline S3 & 0.41 & 0.5 & pole-on & \begin{tabular}{|l}
-0.092 \\
\end{tabular} & 3.621 & 29.185 & 16.456 & 13.794 & 10.021 & 8.564 & 7.475 & 7.37 & 7.256 & 6.181 & 5.708 & 5.19 & 6.238 \\
\hline S3 & 0.41 & 0.571 & pole-on & -0.111 & 5.155 & 32.787 & 20.024 & 17.018 & 12.244 & 10.504 & 9.427 & 8.651 & 8.954 & 7.554 & 7.205 & 6.666 & 7.947 \\
\hline S3 & 0.41 & 0.643 & pole-on & -0.128 & 6.744 & 37.29 & 24.2 & 19.609 & 14.946 & 12.735 & 11.718 & 10.591 & 11.148 & 9.402 & 8.779 & 8.22 & 9.941 \\
\hline S3 & 0.41 & 0.714 & pole-on & -0.14 & 8.523 & 44.255 & 27.631 & 23.75 & 17.846 & 15.182 & 14.067 & 12.313 & 13.34 & 10.744 & 10.568 & 9.738 & 12.595 \\
\hline S3 & 0.41 & 0.786 & pole-on & -0.149 & 10.889 & 54.078 & 34.564 & 28.505 & 21.059 & 17.896 & 16.621 & 14.292 & 16.042 & 12.706 & 12.318 & 11.005 & 15.46 \\
\hline S3 & 0.41 & 0.857 & pole-on & -0.155 & 13.142 & 61.87 & 40.27 & 33.843 & 24.676 & 20.581 & 18.863 & 16.441 & 18.88 & 14.143 & 14.338 & 12.47 & 19.055 \\
\hline S3 & 0.41 & 0.928 & pole-on & -0.158 & 15.791 & 73.549 & 48.385 & 39.504 & 27.946 & 23.108 & 20.361 & 19.169 & 22.539 & 15.413 & 16.342 & 14.131 & 22.916 \\
\hline S3 & 0.41 & 1.0 & pole-on & -0.157 & 17.771 & 84.497 & 54.619 & 45.637 & 29.64 & 24.655 & 22.602 & 20.852 & 25.033 & 16.86 & 18.137 & 15.468 & 26.368 \\
\hline S3 & 0.3 & 0.0 & edge-on & 0.086 & 2.521 & 10.8 & 5.439 & 4.289 & 3.21 & 2.821 & 2.452 & 2.204 & 1.92 & 1.811 & 1.556 & 1.445 & 1.345 \\
\hline S3 & 0.3 & 0.071 & edge-on & 0.073 & 7.165 & 10.862 & 5.372 & 4.291 & 3.083 & 2.724 & 2.484 & 2.17 & 1.951 & 1.816 & 1.574 & 1.438 & 1.376 \\
\hline S3 & 0.3 & 0.143 & edge-on & 0.05 & 6.508 & 13.379 & 6.101 & 5.22 & 4.057 & 3.692 & 3.482 & 3.043 & 2.661 & 2.578 & 2.102 & 2.103 & 1.976 \\
\hline S3 & 0.3 & 0.215 & interm. & 0.025 & 25.24 & 24.912 & 12.875 & 9.155 & 7.614 & 6.928 & 6.98 & 7.046 & 5.691 & 6.423 & 6.354 & 5.409 & 4.817 \\
\hline S3 & 0.3 & 0.286 & interm. & 0.004 & 305.739 & 243.11 & 214.845 & 207.806 & 263.286 & 259.612 & 295.337 & 276.393 & 293.276 & 298.49 & 347.519 & 319.183 & 302.918 \\
\hline
\end{tabular}


Table A.2 - continued.

\begin{tabular}{|c|c|c|c|c|c|c|c|c|c|c|c|c|c|c|c|c|c|}
\hline & & & $\tilde{\tau}_{\mathrm{bu}}$ & & $\mathrm{bu}$ & 0.15 & 0.45 & 0.75 & 1.50 & 2.25 & 3.00 & 4.50 & 6.00 & 9.00 & 15.00 & 30.00 & $\infty$ \\
\hline Star & $\Sigma_{0}\left[\mathrm{~g} \mathrm{~cm}^{-2}\right]$ & $\cos i$ & ang. type & $\Delta V_{\mathrm{bu}}^{\infty}$ & $\xi_{\mathrm{bu}}^{V}$ & $\xi_{\mathrm{d}}^{V}$ & & & & & & & & & & & \\
\hline S3 & 0.3 & 0.357 & interm. & -0.022 & 2.038 & 287.759 & 279.586 & 187.871 & 91.745 & 35.998 & 13.321 & 22.24 & 5.453 & 4.366 & 3.98 & 3.111 & 2.942 \\
\hline S3 & 0.3 & 0.429 & interm. & -0.042 & 7.086 & 221.119 & 25.216 & 11.154 & 8.325 & 6.259 & 5.886 & 4.82 & 5.048 & 4.057 & 3.884 & 3.473 & 3.753 \\
\hline S3 & 0.3 & 0.5 & pole-on & -0.06 & 3.525 & 65.868 & 16.802 & 11.967 & 8.927 & 7.957 & 7.169 & 6.136 & 6.572 & 5.471 & 5.234 & 4.784 & 5.633 \\
\hline S3 & 0.3 & 0.571 & pole-on & -0.073 & 5.035 & 34.597 & 19.538 & 15.025 & 11.271 & 9.958 & 8.932 & 8.138 & 8.293 & 6.831 & 6.767 & 6.052 & 7.316 \\
\hline S3 & 0.3 & 0.643 & pole-on & -0.084 & 6.787 & 36.945 & 23.551 & 18.826 & 13.68 & 12.122 & 10.846 & 9.519 & 10.393 & 8.487 & 8.209 & 7.622 & 9.1 \\
\hline S3 & 0.3 & 0.714 & pole-on & -0.092 & 8.636 & 43.626 & 27.443 & 22.363 & 16.717 & 14.751 & 13.157 & 11.816 & 12.639 & 10.315 & 9.863 & 8.922 & 12.157 \\
\hline S3 & 0.3 & 0.786 & pole-on & -0.099 & 10.605 & 52.726 & 33.155 & 27.676 & 20.166 & 17.492 & 15.517 & 13.556 & 15.333 & 11.625 & 11.969 & 10.618 & 14.494 \\
\hline S3 & 0.3 & 0.857 & pole-on & -0.102 & 13.583 & 68.489 & 40.698 & 33.467 & 23.345 & 19.788 & 17.628 & 15.847 & 18.444 & 12.777 & 13.564 & 11.88 & 18.513 \\
\hline S3 & 0.3 & 0.928 & pole-on & -0.104 & 15.989 & 77.767 & 47.803 & 39.676 & 27.285 & 22.232 & 19.516 & 18.357 & 21.986 & 14.389 & 15.775 & 13.365 & 22.467 \\
\hline S3 & 0.3 & 1.0 & pole-on & -0.103 & 17.978 & 84.642 & 54.665 & 44.383 & 29.413 & 24.037 & 21.045 & 20.382 & 24.704 & 16.015 & 17.795 & 14.361 & 26.463 \\
\hline
\end{tabular}


Table A.3 - Empirical law parameters for band $R$.

\begin{tabular}{|c|c|c|c|c|c|c|c|c|c|c|c|c|c|c|c|c|c|}
\hline & & & $\tilde{\tau}_{\mathrm{bu}}$ & & $\mathrm{bu}$ & 0.15 & 0.45 & 0.75 & 1.50 & 2.25 & 3.00 & 4.50 & 6.00 & 9.00 & 15.00 & 30.00 & $\infty$ \\
\hline Star & $\Sigma_{0}\left[\mathrm{~g} \mathrm{~cm}^{-2}\right]$ & $\cos i$ & ang. type & $\Delta R_{\mathrm{bu}}^{\infty}$ & $\xi_{\mathrm{bu}}^{R}$ & $\xi_{\mathrm{d}}^{R}$ & & & & & & & & & & & \\
\hline S1 & 2.5 & 0.0 & edge-on & 0.555 & 1.039 & 1.697 & 1.412 & 1.327 & 1.091 & 0.989 & 0.924 & 0.832 & 0.768 & 0.693 & 0.599 & 0.541 & 0.528 \\
\hline $\mathrm{S} 1$ & 2.5 & 0.071 & edge-on & 0.258 & 1.392 & 1.203 & 1.028 & 0.982 & 0.791 & 0.703 & 0.64 & 0.577 & 0.521 & 0.466 & 0.4 & 0.353 & 0.34 \\
\hline S1 & 2.5 & 0.143 & interm. & -0.115 & 0.31 & 2.224 & 123.391 & 206.692 & 11.032 & 6.508 & 5.421 & 3.892 & 3.449 & 3.056 & 2.593 & 2.141 & 2.17 \\
\hline S1 & 2.5 & 0.215 & interm. & -0.372 & 1.268 & 11.275 & 4.649 & 3.37 & 2.502 & 2.088 & 1.846 & 1.557 & 1.44 & 1.296 & 1.115 & 0.957 & 0.959 \\
\hline S1 & 2.5 & 0.286 & interm. & -0.565 & 2.251 & 6.222 & 3.723 & 2.899 & 2.182 & 1.846 & 1.655 & 1.403 & 1.295 & 1.173 & 1.009 & 0.876 & 0.873 \\
\hline S1 & 2.5 & 0.357 & interm. & -0.715 & 3.425 & 6.127 & 3.671 & 2.875 & 2.158 & 1.836 & 1.645 & 1.4 & 1.298 & 1.178 & 1.017 & 0.886 & 0.88 \\
\hline S1 & 2.5 & 0.429 & pole-on & -0.834 & 4.848 & 6.51 & 3.786 & 2.928 & 2.213 & 1.882 & 1.694 & 1.453 & 1.344 & 1.219 & 1.055 & 0.926 & 0.924 \\
\hline S1 & 2.5 & 0.5 & pole-on & -0.925 & 6.436 & 6.961 & 3.954 & 3.077 & 2.306 & 1.97 & 1.77 & 1.528 & 1.414 & 1.284 & 1.113 & 0.983 & 0.982 \\
\hline $\mathrm{S} 1$ & 2.5 & 0.571 & pole-on & -1.002 & 8.029 & 7.473 & 4.176 & 3.238 & 2.435 & 2.071 & 1.872 & 1.622 & 1.503 & 1.364 & 1.188 & 1.054 & 1.05 \\
\hline S1 & 2.5 & 0.643 & pole-on & -1.066 & 9.572 & 8.059 & 4.442 & 3.441 & 2.588 & 2.205 & 1.992 & 1.732 & 1.608 & 1.462 & 1.276 & 1.136 & 1.129 \\
\hline S1 & 2.5 & 0.714 & pole-on & -1.118 & 11.074 & 8.709 & 4.755 & 3.677 & 2.755 & 2.356 & 2.13 & 1.859 & 1.727 & 1.572 & 1.375 & 1.232 & 1.221 \\
\hline S1 & 2.5 & 0.786 & pole-on & -1.16 & 12.466 & 9.411 & 5.109 & 3.937 & 2.956 & 2.524 & 2.289 & 1.999 & 1.858 & 1.691 & 1.486 & 1.335 & 1.315 \\
\hline S1 & 2.5 & 0.857 & pole-on & -1.194 & 13.78 & 10.238 & 5.508 & 4.245 & 3.17 & 2.716 & 2.464 & 2.161 & 2.008 & 1.829 & 1.603 & 1.442 & 1.413 \\
\hline S1 & 2.5 & 0.928 & pole-on & -1.222 & 15.014 & 11.14 & 5.937 & 4.579 & 3.418 & 2.92 & 2.646 & 2.33 & 2.167 & 1.97 & 1.716 & 1.546 & 1.53 \\
\hline S1 & 2.5 & 1.0 & pole-on & -1.242 & 16.088 & 12.022 & 6.376 & 4.899 & 3.66 & 3.122 & 2.835 & 2.5 & 2.323 & 2.095 & 1.832 & 1.656 & 1.657 \\
\hline S1 & 1.85 & 0.0 & edge-on & 0.442 & 1.08 & 1.906 & 1.613 & 1.459 & 1.211 & 1.096 & 1.026 & 0.948 & 0.863 & 0.775 & 0.676 & 0.612 & 0.594 \\
\hline S1 & 1.85 & 0.071 & edge-on & 0.215 & 1.433 & 1.402 & 1.353 & 1.064 & 0.873 & 0.787 & 0.728 & 0.626 & 0.589 & 0.534 & 0.455 & 0.403 & 0.388 \\
\hline S1 & 1.85 & 0.143 & interm. & -0.079 & 0.281 & 31.897 & 64.645 & 160.186 & 150.699 & 28.03 & 33.604 & 9.09 & 8.317 & 6.593 & 5.355 & 4.037 & 3.889 \\
\hline S1 & 1.85 & 0.215 & interm. & -0.306 & 1.119 & 21.96 & 7.059 & 4.862 & 3.557 & 2.948 & 2.62 & 2.123 & 2.012 & 1.881 & 1.614 & 1.407 & 1.35 \\
\hline S1 & 1.85 & 0.286 & interm. & -0.484 & 2.027 & 9.758 & 5.07 & 3.884 & 2.967 & 2.503 & 2.242 & 1.909 & 1.798 & 1.654 & 1.432 & 1.274 & 1.217 \\
\hline $\mathrm{S} 1$ & 1.85 & 0.357 & interm. & -0.625 & 3.109 & 8.783 & 4.92 & 3.813 & 2.916 & 2.473 & 2.229 & 1.914 & 1.805 & 1.66 & 1.438 & 1.294 & 1.237 \\
\hline S1 & 1.85 & 0.429 & pole-on & -0.738 & 4.465 & 9.119 & 5.088 & 3.94 & 2.993 & 2.546 & 2.311 & 2.003 & 1.886 & 1.722 & 1.503 & 1.352 & 1.303 \\
\hline $\mathrm{S} 1$ & 1.85 & 0.5 & pole-on & -0.824 & 6.011 & 9.736 & 5.375 & 4.15 & 3.151 & 2.683 & 2.423 & 2.119 & 1.99 & 1.829 & 1.596 & 1.444 & 1.394 \\
\hline $\mathrm{S} 1$ & 1.85 & 0.571 & pole-on & -0.896 & 7.624 & 10.447 & 5.687 & 4.418 & 3.351 & 2.844 & 2.561 & 2.268 & 2.138 & 1.954 & 1.711 & 1.554 & 1.501 \\
\hline S1 & 1.85 & 0.643 & pole-on & -0.957 & 9.259 & 11.274 & 6.105 & 4.738 & 3.587 & 3.059 & 2.783 & 2.459 & 2.308 & 2.109 & 1.848 & 1.678 & 1.624 \\
\hline $\mathrm{S} 1$ & 1.85 & 0.714 & pole-on & -1.006 & 10.846 & 12.181 & 6.601 & 5.113 & 3.872 & 3.314 & 2.986 & 2.656 & 2.49 & 2.281 & 1.995 & 1.818 & 1.764 \\
\hline $\mathrm{S} 1$ & 1.85 & 0.786 & pole-on & -1.045 & 12.38 & 13.265 & 7.168 & 5.552 & 4.186 & 3.584 & 3.229 & 2.904 & 2.704 & 2.468 & 2.164 & 1.971 & 1.917 \\
\hline S1 & 1.85 & 0.857 & pole-on & -1.077 & 13.833 & 14.474 & 7.777 & 6.013 & 4.535 & 3.875 & 3.495 & 3.128 & 2.916 & 2.664 & 2.344 & 2.153 & 2.102 \\
\hline S1 & 1.85 & 0.928 & pole-on & -1.103 & 15.269 & 15.842 & 8.449 & 6.535 & 4.897 & 4.182 & 3.783 & 3.365 & 3.14 & 2.878 & 2.558 & 2.359 & 2.317 \\
\hline $\mathrm{S} 1$ & 1.85 & 1.0 & pole-on & -1.122 & 16.431 & 17.238 & 9.103 & 7.043 & 5.238 & 4.455 & 4.055 & 3.625 & 3.371 & 3.116 & 2.777 & 2.566 & 3.342 \\
\hline S1 & 1.37 & 0.0 & edge-on & 0.347 & 1.13 & 2.431 & 1.935 & 1.707 & 1.38 & 1.258 & 1.137 & 1.043 & 0.961 & 0.879 & 0.765 & 0.695 & 0.679 \\
\hline S1 & 1.37 & 0.071 & edge-on & 0.179 & 1.483 & 1.698 & 1.46 & 1.297 & 1.012 & 0.951 & 0.824 & 0.764 & 0.688 & 0.631 & 0.532 & 0.484 & 0.458 \\
\hline S1 & 1.37 & 0.143 & interm. & -0.047 & 0.217 & 53.941 & 0.809 & 17.997 & 274.593 & 289.029 & 327.173 & 143.024 & 35.083 & 23.924 & 16.595 & 12.093 & 12.666 \\
\hline S1 & 1.37 & 0.215 & interm. & -0.246 & 0.962 & 46.853 & 10.7 & 7.182 & 5.017 & 3.995 & 3.69 & 3.184 & 2.833 & 2.605 & 2.25 & 2.036 & 1.97 \\
\hline S1 & 1.37 & 0.286 & interm. & -0.407 & 1.797 & 14.751 & 6.672 & 5.229 & 3.979 & 3.297 & 3.077 & 2.679 & 2.429 & 2.243 & 1.952 & 1.789 & 1.724 \\
\hline
\end{tabular}


Table A.3 - continued.

\begin{tabular}{|c|c|c|c|c|c|c|c|c|c|c|c|c|c|c|c|c|c|}
\hline & & & $\tilde{\tau}_{\text {bu }}$ & & $\mathrm{bu}$ & 0.15 & 0.45 & 0.75 & 1.50 & 2.25 & 3.00 & 4.50 & 6.00 & 9.00 & 15.00 & 30.00 & $\infty$ \\
\hline Star & $\Sigma_{0}\left[\mathrm{~g} \mathrm{~cm}^{-2}\right]$ & $\cos i$ & ang. type & $\Delta R_{\mathrm{bu}}^{\infty}$ & $\xi_{\mathrm{bu}}^{R}$ & $\xi_{\mathrm{d}}^{R}$ & & & & & & & & & & & \\
\hline S1 & 1.37 & 0.357 & interm. & -0.538 & 2.812 & 12.204 & 6.48 & 5.112 & 3.933 & 3.293 & 3.081 & 2.696 & 2.456 & 2.277 & 1.982 & 1.819 & 1.756 \\
\hline S1 & 1.37 & 0.429 & pole-on & -0.642 & 4.106 & 12.34 & 6.758 & 5.334 & 4.088 & 3.461 & 3.209 & 2.825 & 2.575 & 2.408 & 2.101 & 1.924 & 1.864 \\
\hline S1 & 1.37 & 0.5 & pole-on & -0.724 & 5.645 & 13.247 & 7.181 & 5.693 & 4.349 & 3.709 & 3.427 & 3.049 & 2.771 & 2.576 & 2.253 & 2.075 & 2.015 \\
\hline S1 & 1.37 & 0.571 & pole-on & $\mid-0.792$ & 7.283 & 14.264 & 7.704 & 6.13 & 4.662 & 3.994 & 3.683 & 3.269 & 3.007 & 2.807 & 2.442 & 2.25 & 2.198 \\
\hline S1 & 1.37 & 0.643 & pole-on & -0.848 & 9.031 & 15.442 & 8.384 & 6.648 & 5.05 & 4.334 & 3.959 & 3.558 & 3.32 & 3.033 & 2.671 & 2.466 & 2.419 \\
\hline S1 & 1.37 & 0.714 & pole-on & -0.894 & 10.746 & 16.876 & 9.147 & 7.241 & 5.49 & 4.72 & 4.33 & 3.869 & 3.586 & 3.312 & 2.924 & 2.721 & 2.683 \\
\hline S1 & 1.37 & 0.786 & pole-on & -0.931 & 12.437 & 18.475 & 9.996 & 7.906 & 5.959 & 5.132 & 4.698 & 4.197 & 3.9 & 3.634 & 3.23 & 3.006 & 2.986 \\
\hline S1 & 1.37 & 0.857 & pole-on & -0.96 & 14.129 & 20.353 & 10.945 & 8.637 & 6.45 & 5.562 & 5.09 & 4.561 & 4.238 & 3.999 & 3.557 & 3.324 & 3.309 \\
\hline S1 & 1.37 & 0.928 & pole-on & -0.983 & 15.672 & 22.445 & 12.019 & 9.401 & 6.983 & 6.04 & 5.54 & 5.008 & 4.643 & 4.388 & 3.929 & 3.668 & 3.672 \\
\hline S1 & 1.37 & 1.0 & pole-on & -1.001 & 17.025 & 24.338 & 12.985 & 10.101 & 7.584 & 6.535 & 5.997 & 5.456 & 5.085 & 4.77 & 4.297 & 4.007 & 4.036 \\
\hline S1 & 1.01 & 0.0 & edge-on & 0.272 & 1.172 & 2.942 & 2.144 & 1.923 & 3.033 & 1.44 & 1.307 & 1.174 & 1.105 & 1.014 & 0.825 & 0.803 & 0.803 \\
\hline S1 & 1.01 & 0.071 & edge-on & 0.152 & 1.379 & 2.108 & 1.638 & 1.524 & 1.936 & 1.116 & 0.986 & 0.886 & 0.825 & 0.768 & 0.619 & 0.606 & 0.582 \\
\hline $\mathrm{S} 1$ & 1.01 & 0.143 & interm. & -0.011 & 116.039 & 33.347 & 6.766 & 0.708 & 360.643 & 13.046 & 51.753 & 175.924 & 175.217 & 327.419 & 289.309 & 319.12 & 294.908 \\
\hline S1 & 1.01 & 0.215 & interm. & -0.176 & 0.982 & 179.383 & 22.092 & 12.31 & 15.49 & 5.884 & 5.258 & 4.676 & 4.022 & 3.725 & 3.213 & 2.887 & 2.774 \\
\hline S1 & 1.01 & 0.286 & interm. & -0.316 & 1.772 & 22.44 & 9.882 & 7.18 & 8.113 & 4.452 & 4.059 & 3.706 & 3.29 & 2.997 & 2.72 & 2.41 & 2.31 \\
\hline S1 & 1.01 & 0.357 & interm. & -0.434 & 2.686 & 16.567 & 9.034 & 6.934 & 7.278 & 4.462 & 4.085 & 3.718 & 3.364 & 3.029 & 2.843 & 2.504 & 2.381 \\
\hline S1 & 1.01 & 0.429 & pole-on & -0.532 & 3.843 & 16.31 & 9.377 & 7.296 & 7.222 & 4.749 & 4.35 & 3.94 & 3.608 & 3.252 & 3.073 & 2.712 & 2.559 \\
\hline S1 & 1.01 & 0.5 & pole-on & $\mid-0.609$ & 5.288 & 17.49 & 10.039 & 7.834 & 7.473 & 5.132 & 4.696 & 4.278 & 3.919 & 3.549 & 3.372 & 2.977 & 2.796 \\
\hline S1 & 1.01 & 0.571 & pole-on & -0.671 & 6.971 & 19.111 & 10.828 & 8.5 & 7.853 & 5.594 & 5.116 & 4.659 & 4.307 & 3.877 & 3.731 & 3.303 & 3.088 \\
\hline S1 & 1.01 & 0.643 & pole-on & -0.724 & 8.72 & 20.955 & 11.845 & 9.291 & 8.335 & 6.097 & 5.593 & 5.104 & 4.724 & 4.335 & 4.119 & 3.697 & 3.45 \\
\hline S1 & 1.01 & 0.714 & pole-on & -0.767 & 10.518 & 23.021 & 12.996 & 10.202 & 8.954 & 6.672 & 6.124 & 5.633 & 5.241 & 4.819 & 4.559 & 4.127 & 3.889 \\
\hline S1 & 1.01 & 0.786 & pole-on & -0.801 & 12.32 & 25.616 & 14.29 & 11.193 & 9.598 & 7.362 & 6.724 & 6.214 & 5.815 & 5.381 & 5.04 & 4.605 & 4.411 \\
\hline S1 & 1.01 & 0.857 & pole-on & -0.829 & 14.155 & 28.382 & 15.676 & 12.271 & 10.311 & 8.127 & 7.418 & 6.888 & 6.458 & 6.042 & 5.559 & 5.114 & 5.054 \\
\hline S1 & 1.01 & 0.928 & pole-on & -0.85 & 15.824 & 31.6 & 17.251 & 13.568 & 11.042 & 8.925 & 8.12 & 7.577 & 7.176 & 6.743 & 6.103 & 5.656 & 5.755 \\
\hline S1 & 1.01 & 1.0 & pole-on & -0.865 & 17.281 & 34.698 & 18.881 & 14.86 & 11.822 & 9.697 & 8.815 & 8.3 & 7.899 & 7.391 & 6.638 & 6.193 & 6.533 \\
\hline S1 & 0.75 & 0.0 & edge-on & 0.211 & 1.297 & 3.817 & 2.61 & 2.206 & 1.832 & 1.646 & 1.522 & 1.377 & 1.266 & 1.154 & 1.018 & 0.939 & 0.912 \\
\hline $\mathrm{S} 1$ & 0.75 & 0.071 & edge-on & 0.124 & 45.219 & 2.836 & 1.995 & 1.681 & 1.43 & 1.296 & 1.209 & 1.074 & 0.989 & 0.905 & 0.79 & 0.72 & 0.682 \\
\hline S1 & 0.75 & 0.143 & interm. & 0.004 & 290.788 & 10.903 & 1.872 & 1.012 & 0.712 & 2.896 & 0.484 & 0.384 & 13.49 & 2.416 & 39.135 & 92.047 & 76.254 \\
\hline S1 & 0.75 & 0.215 & interm. & -0.127 & 0.855 & 380.042 & 53.847 & 28.987 & 13.626 & 10.343 & 8.487 & 7.054 & 6.371 & 5.537 & 4.731 & 4.136 & 4.309 \\
\hline $\mathrm{S} 1$ & 0.75 & 0.286 & interm. & -0.243 & 1.995 & 40.242 & 14.516 & 10.672 & 7.198 & 6.084 & 5.44 & 4.773 & 4.423 & 4.063 & 3.601 & 3.209 & 3.3 \\
\hline S1 & 0.75 & 0.357 & interm. & -0.345 & 2.404 & 23.79 & 12.11 & 9.389 & 6.893 & 5.901 & 5.308 & 4.789 & 4.493 & 4.168 & 3.688 & 3.356 & 3.451 \\
\hline S1 & 0.75 & 0.429 & pole-on & -0.432 & 3.416 & 22.201 & 12.275 & 9.681 & 7.263 & 6.303 & 5.744 & 5.196 & 4.882 & 4.516 & 4.041 & 3.72 & 3.82 \\
\hline S1 & 0.75 & 0.5 & pole-on & \begin{tabular}{|l}
-0.502 \\
\end{tabular} & 4.588 & 23.106 & 13.131 & 10.408 & 7.904 & 6.913 & 6.289 & 5.711 & 5.365 & 4.987 & 4.501 & 4.19 & 4.299 \\
\hline $\mathrm{S} 1$ & 0.75 & 0.571 & pole-on & -0.56 & 5.796 & 25.123 & 14.32 & 11.417 & 8.681 & 7.6 & 6.965 & 6.316 & 5.989 & 5.577 & 5.048 & 4.682 & 4.863 \\
\hline S1 & 0.75 & 0.643 & pole-on & -0.609 & 7.028 & 27.986 & 15.838 & 12.662 & 9.656 & 8.474 & 7.715 & 7.069 & 6.719 & 6.228 & 5.695 & 5.306 & 5.538 \\
\hline
\end{tabular}


Table A.3 - continued.

\begin{tabular}{|c|c|c|c|c|c|c|c|c|c|c|c|c|c|c|c|c|c|}
\hline & & & $\tilde{\tau}_{\mathrm{bu}}$ & & $\mathrm{bu}$ & 0.15 & 0.45 & 0.75 & 1.50 & 2.25 & 3.00 & 4.50 & 6.00 & 9.00 & 15.00 & 30.00 & $\infty$ \\
\hline Star & $\Sigma_{0}\left[\mathrm{~g} \mathrm{~cm}^{-2}\right]$ & $\cos i$ & ang. type & $\Delta R_{\mathrm{bu}}^{\infty}$ & $\xi_{\mathrm{bu}}^{R}$ & $\xi_{\mathrm{d}}^{R}$ & & & & & & & & & & & \\
\hline S1 & 0.75 & 0.714 & pole-on & \begin{tabular}{|l|}
-0.648 \\
\end{tabular} & 8.246 & 31.122 & 17.557 & 14.055 & 10.716 & 9.416 & 8.615 & 7.87 & 7.564 & 6.972 & 6.395 & 5.957 & 6.283 \\
\hline S1 & 0.75 & 0.786 & pole-on & -0.678 & 9.314 & 34.905 & 19.686 & 15.765 & 11.922 & 10.527 & 9.594 & 8.803 & 8.496 & 7.774 & 7.134 & 6.681 & 7.1 \\
\hline S1 & 0.75 & 0.857 & pole-on & -0.702 & 10.436 & 39.115 & 21.915 & 17.542 & 13.287 & 11.692 & 10.643 & 9.775 & 9.565 & 8.596 & 8.003 & 7.411 & 8.057 \\
\hline S1 & 0.75 & 0.928 & pole-on & -0.721 & 11.348 & 44.069 & 24.619 & 19.769 & 14.755 & 12.812 & 11.647 & 10.827 & 10.685 & 9.412 & 8.885 & 8.206 & 9.09 \\
\hline S1 & 0.75 & 1.0 & pole-on & -0.733 & 12.18 & 48.527 & 27.192 & 21.86 & 16.08 & 13.928 & 12.64 & 11.866 & 11.822 & 10.234 & 9.765 & 8.971 & 10.101 \\
\hline S1 & 0.56 & 0.0 & edge-on & 0.165 & 1.406 & 5.123 & 3.211 & 2.712 & 2.187 & 1.888 & 1.756 & 1.609 & 1.459 & 1.36 & 1.186 & 1.08 & 1.045 \\
\hline S1 & 0.56 & 0.071 & edge-on & 0.102 & 1.583 & 3.919 & 2.476 & 2.218 & 1.737 & 1.532 & 1.468 & 1.3 & 1.177 & 1.109 & 0.927 & 0.89 & 0.833 \\
\hline $\mathrm{S} 1$ & 0.56 & 0.143 & interm. & 0.015 & 47.45 & 17.431 & 2.455 & 1.456 & 1.08 & 0.895 & 0.834 & 0.738 & 0.907 & 0.591 & 0.354 & 1.107 & 0.392 \\
\hline S1 & 0.56 & 0.215 & interm. & -0.084 & 2.009 & 387.039 & 310.062 & 103.078 & 32.535 & 21.994 & 17.082 & 12.192 & 11.832 & 9.412 & 8.904 & 7.258 & 6.955 \\
\hline S1 & 0.56 & 0.286 & interm. & -0.176 & 10.93 & 95.752 & 22.097 & 14.374 & 9.84 & 8.011 & 7.214 & 6.213 & 5.967 & 5.302 & 4.963 & 4.393 & 4.401 \\
\hline $\mathrm{S} 1$ & 0.56 & 0.357 & interm. & -0.258 & 2.412 & 34.162 & 15.383 & 11.585 & 8.649 & 7.373 & 6.818 & 6.054 & 5.902 & 5.25 & 4.906 & 4.453 & 4.577 \\
\hline S1 & 0.56 & 0.429 & pole-on & -0.331 & 3.376 & 28.385 & 15.292 & 11.878 & 9.158 & 7.988 & 7.335 & 6.568 & 6.382 & 5.784 & 5.371 & 4.958 & 5.107 \\
\hline S1 & 0.56 & 0.5 & pole-on & -0.391 & 4.527 & 29.037 & 16.327 & 13.117 & 9.996 & 8.838 & 8.128 & 7.283 & 7.151 & 6.501 & 6.078 & 5.581 & 5.821 \\
\hline $\mathrm{S} 1$ & 0.56 & 0.571 & pole-on & -0.441 & 5.884 & 31.521 & 18.099 & 14.473 & 11.194 & 9.861 & 9.152 & 8.215 & 8.083 & 7.317 & 6.845 & 6.349 & 6.675 \\
\hline S1 & 0.56 & 0.643 & pole-on & -0.483 & 7.41 & 34.996 & 20.239 & 16.346 & 12.623 & 11.116 & 10.31 & 9.239 & 9.193 & 8.28 & 7.761 & 7.196 & 7.716 \\
\hline S1 & 0.56 & 0.714 & pole-on & -0.517 & 9.151 & 39.613 & 22.972 & 18.542 & 14.239 & 12.528 & 11.574 & 10.405 & 10.434 & 9.283 & 8.727 & 8.116 & 8.843 \\
\hline S1 & 0.56 & 0.786 & pole-on & -0.543 & 10.94 & 44.746 & 25.92 & 21.056 & 16.106 & 14.119 & 13.002 & 11.619 & 11.758 & 10.392 & 9.85 & 9.102 & 10.194 \\
\hline S1 & 0.56 & 0.857 & pole-on & -0.563 & 12.738 & 51.025 & 29.396 & 23.869 & 18.027 & 15.674 & 14.394 & 13.063 & 13.315 & 11.409 & 11.052 & 10.077 & 11.696 \\
\hline S1 & 0.56 & 0.928 & pole-on & -0.578 & 14.593 & 57.555 & 33.516 & 27.005 & 19.936 & 17.159 & 15.723 & 14.474 & 15.001 & 12.509 & 12.224 & 11.13 & 13.317 \\
\hline $\mathrm{S} 1$ & 0.56 & 1.0 & pole-on & -0.586 & 16.081 & 63.496 & 36.895 & 29.949 & 21.777 & 18.636 & 16.912 & 15.814 & 16.674 & 13.501 & 13.447 & 12.096 & 14.926 \\
\hline S1 & 0.41 & 0.0 & edge-on & 0.127 & 1.623 & 6.6 & 4.18 & 3.279 & 2.644 & 2.349 & 2.127 & 1.874 & 1.73 & 1.616 & 1.413 & 1.294 & 1.236 \\
\hline S1 & 0.41 & 0.071 & edge-on & 0.084 & 1.702 & 5.633 & 3.278 & 2.744 & 2.237 & 1.907 & 1.827 & 1.615 & 1.469 & 1.353 & 1.197 & 1.087 & 1.038 \\
\hline S1 & 0.41 & 0.143 & interm. & 0.022 & 13.014 & 13.282 & 5.113 & 2.182 & 1.668 & 1.544 & 1.45 & 1.225 & 1.097 & 1.105 & 0.894 & 0.757 & 0.718 \\
\hline $\mathrm{S} 1$ & 0.41 & 0.215 & interm. & -0.048 & 0.84 & 344.892 & 375.408 & 367.815 & 256.422 & 133.704 & 56.625 & 37.85 & 33.934 & 21.351 & 17.801 & 15.952 & 15.218 \\
\hline S1 & 0.41 & 0.286 & interm. & -0.116 & 1.586 & 293.954 & 38.255 & 22.373 & 13.907 & 10.425 & 9.169 & 8.15 & 7.557 & 6.556 & 5.846 & 5.507 & 5.73 \\
\hline S1 & 0.41 & 0.357 & interm. & -0.18 & 2.299 & 52.135 & 20.217 & 14.865 & 10.568 & 8.91 & 8.14 & 7.438 & 7.168 & 6.265 & 5.797 & 5.399 & 5.717 \\
\hline S1 & 0.41 & 0.429 & pole-on & -0.237 & 3.129 & 36.663 & 18.701 & 14.669 & 11.073 & 9.521 & 8.714 & 8.03 & 7.804 & 6.936 & 6.436 & 6.113 & 6.437 \\
\hline S1 & 0.41 & 0.5 & pole-on & -0.284 & 4.145 & 35.565 & 20.075 & 16.084 & 12.266 & 10.61 & 9.852 & 8.997 & 8.856 & 7.836 & 7.31 & 6.914 & 7.479 \\
\hline $\mathrm{S} 1$ & 0.41 & 0.571 & pole-on & -0.324 & 5.384 & 37.776 & 22.269 & 17.937 & 13.83 & 12.123 & 11.121 & 10.16 & 10.131 & 8.967 & 8.387 & 7.851 & 8.74 \\
\hline S1 & 0.41 & 0.643 & pole-on & -0.357 & 6.85 & 42.123 & 25.234 & 20.741 & 15.89 & 13.883 & 12.775 & 11.6 & 11.69 & 10.234 & 9.625 & 8.987 & 10.221 \\
\hline S1 & 0.41 & 0.714 & pole-on & -0.384 & 8.478 & 47.486 & 29.016 & 23.668 & 18.139 & 15.861 & 14.559 & 13.135 & 13.328 & 11.65 & 10.918 & 10.251 & 11.927 \\
\hline $\mathrm{S} 1$ & 0.41 & 0.786 & pole-on & -0.404 & 10.323 & 54.762 & 33.354 & 27.306 & 20.591 & 17.978 & 16.502 & 14.765 & 15.289 & 13.017 & 12.432 & 11.438 & 13.916 \\
\hline S1 & 0.41 & 0.857 & pole-on & \begin{tabular}{|l}
-0.419 \\
\end{tabular} & 12.229 & 61.699 & 38.021 & 31.162 & 23.196 & 20.001 & 18.248 & 16.533 & 17.449 & 14.257 & 13.944 & 12.743 & 16.209 \\
\hline S1 & 0.41 & 0.928 & pole-on & -0.43 & 14.242 & 71.018 & 43.389 & 35.243 & 25.709 & 21.8 & 19.731 & 18.294 & 19.767 & 15.666 & 15.52 & 13.941 & 18.685 \\
\hline S1 & 0.41 & 1.0 & pole-on & -0.434 & 15.782 & 78.434 & 47.884 & 39.436 & 28.054 & 23.368 & 21.113 & 20.079 & 22.136 & 16.711 & 16.969 & 15.109 & 21.18 \\
\hline
\end{tabular}


Table A.3 - continued.

\begin{tabular}{|c|c|c|c|c|c|c|c|c|c|c|c|c|c|c|c|c|c|}
\hline & & & $\tilde{\tau}_{\text {bu }}$ & & $\mathrm{bu}$ & 0.15 & 0.45 & 0.75 & 1.50 & 2.25 & 3.00 & 4.50 & 6.00 & 9.00 & 15.00 & 30.00 & $\infty$ \\
\hline Star & $\Sigma_{0}\left[\mathrm{~g} \mathrm{~cm}^{-2}\right]$ & $\cos i$ & ang. type & $\Delta R_{\mathrm{bu}}^{\infty}$ & $\xi_{\mathrm{bu}}^{R}$ & $\xi_{\mathrm{d}}^{R}$ & & & & & & & & & & & \\
\hline S1 & 0.3 & 0.0 & edge-on & 0.098 & 1.925 & 8.572 & 5.132 & 4.037 & 3.268 & 2.799 & 2.602 & 2.281 & 2.07 & 1.944 & 1.699 & 1.545 & 1.493 \\
\hline S1 & 0.3 & 0.071 & edge-on & 0.068 & 2.066 & 7.578 & 4.245 & 3.418 & 2.738 & 2.45 & 2.229 & 1.986 & 1.839 & 1.673 & 1.452 & 1.337 & 1.282 \\
\hline $\mathrm{S} 1$ & 0.3 & 0.143 & interm. & 0.025 & 33.485 & 29.979 & 3.789 & 3.139 & 2.682 & 2.426 & 2.106 & 1.909 & 1.805 & 1.738 & 1.482 & 1.396 & 1.218 \\
\hline S1 & 0.3 & 0.215 & interm. & -0.023 & 24.573 & 303.837 & 307.811 & 335.109 & 307.23 & 350.268 & 338.568 & 318.0 & 323.62 & 291.891 & 212.128 & 194.633 & 172.367 \\
\hline S1 & 0.3 & 0.286 & interm. & -0.072 & 1.463 & 332.586 & 174.486 & 44.976 & 17.801 & 13.797 & 11.546 & 9.183 & 9.535 & 7.39 & 6.669 & 5.91 & 6.583 \\
\hline S1 & 0.3 & 0.357 & interm. & -0.117 & 2.146 & 116.398 & 26.503 & 17.923 & 11.771 & 9.944 & 8.899 & 8.011 & 7.983 & 6.669 & 6.319 & 5.81 & 6.463 \\
\hline S1 & 0.3 & 0.429 & pole-on & -0.159 & 2.95 & 44.126 & 22.13 & 16.989 & 12.009 & 10.467 & 9.551 & 8.621 & 8.856 & 7.422 & 7.071 & 6.669 & 7.42 \\
\hline S1 & 0.3 & 0.5 & pole-on & -0.194 & 3.899 & 39.62 & 23.196 & 18.143 & 13.455 & 11.895 & 10.965 & 9.806 & 10.088 & 8.581 & 8.293 & 7.715 & 8.746 \\
\hline S1 & 0.3 & 0.571 & pole-on & -0.223 & 4.989 & 43.488 & 25.857 & 20.73 & 15.539 & 13.585 & 12.542 & 11.131 & 11.695 & 9.939 & 9.456 & 8.758 & 10.305 \\
\hline S1 & 0.3 & 0.643 & pole-on & -0.247 & 6.279 & 48.903 & 29.301 & 23.924 & 17.932 & 15.799 & 14.507 & 12.929 & 13.636 & 11.51 & 10.999 & 10.292 & 12.342 \\
\hline S1 & 0.3 & 0.714 & pole-on & -0.266 & 7.739 & 55.494 & 33.881 & 27.828 & 21.003 & 18.256 & 16.673 & 14.847 & 15.698 & 12.993 & 12.654 & 11.712 & 14.578 \\
\hline S1 & 0.3 & 0.786 & pole-on & -0.281 & 9.521 & 62.401 & 39.25 & 32.352 & 24.159 & 20.885 & 19.06 & 16.721 & 18.253 & 14.68 & 14.332 & 13.078 & 17.33 \\
\hline S1 & 0.3 & 0.857 & pole-on & -0.291 & 11.307 & 72.88 & 45.256 & 36.997 & 26.975 & 23.13 & 21.222 & 18.775 & 20.855 & 16.427 & 16.25 & 14.546 & 20.448 \\
\hline S1 & 0.3 & 0.928 & pole-on & -0.298 & 13.171 & 82.222 & 51.2 & 42.574 & 30.049 & 25.449 & 22.912 & 21.135 & 23.911 & 17.779 & 17.941 & 16.096 & 23.861 \\
\hline S1 & 0.3 & 1.0 & pole-on & \begin{tabular}{|l}
-0.299 \\
\end{tabular} & 14.651 & 90.028 & 56.237 & 47.015 & 32.122 & 27.265 & 24.288 & 22.909 & 26.665 & 18.899 & 20.027 & 17.339 & 27.151 \\
\hline $\mathrm{S} 2$ & 2.5 & 0.0 & edge-on & 0.482 & 1.05 & 1.853 & 1.481 & 1.338 & 1.157 & 1.041 & 0.968 & 0.871 & 0.808 & 0.733 & 0.636 & 0.577 & 0.561 \\
\hline $\mathrm{S} 2$ & 2.5 & 0.071 & edge-on & 0.243 & 1.482 & 1.355 & 1.099 & 0.997 & 0.854 & 0.73 & 0.682 & 0.611 & 0.549 & 0.488 & 0.427 & 0.376 & 0.356 \\
\hline S2 & 2.5 & 0.143 & interm. & -0.066 & 0.214 & 0.896 & 0.496 & 1.402 & 191.779 & 242.205 & 54.859 & 19.734 & 15.819 & 12.076 & 8.234 & 7.163 & 6.403 \\
\hline $\mathrm{S} 2$ & 2.5 & 0.215 & interm. & -0.305 & 0.931 & 35.152 & 8.647 & 5.709 & 3.772 & 3.188 & 2.877 & 2.43 & 2.234 & 2.084 & 1.755 & 1.627 & 1.546 \\
\hline $\mathrm{S} 2$ & 2.5 & 0.286 & interm. & -0.483 & 1.807 & 10.64 & 5.368 & 4.082 & 2.982 & 2.559 & 2.335 & 2.012 & 1.875 & 1.727 & 1.476 & 1.361 & 1.306 \\
\hline S2 & 2.5 & 0.357 & interm. & -0.622 & 2.874 & 9.055 & 5.085 & 3.933 & 2.884 & 2.494 & 2.272 & 1.97 & 1.844 & 1.698 & 1.454 & 1.347 & 1.294 \\
\hline $\mathrm{S} 2$ & 2.5 & 0.429 & pole-on & -0.733 & 4.163 & 9.195 & 5.18 & 4.033 & 2.956 & 2.561 & 2.326 & 2.038 & 1.905 & 1.755 & 1.515 & 1.4 & 1.34 \\
\hline $\mathrm{S} 2$ & 2.5 & 0.5 & pole-on & -0.818 & 5.657 & 9.758 & 5.394 & 4.218 & 3.118 & 2.691 & 2.458 & 2.15 & 2.006 & 1.852 & 1.607 & 1.483 & 1.428 \\
\hline S2 & 2.5 & 0.571 & pole-on & -0.888 & 7.19 & 10.427 & 5.752 & 4.49 & 3.317 & 2.866 & 2.613 & 2.297 & 2.141 & 1.977 & 1.724 & 1.582 & 1.531 \\
\hline S2 & 2.5 & 0.643 & pole-on & -0.948 & 8.746 & 11.306 & 6.172 & 4.82 & 3.558 & 3.072 & 2.813 & 2.476 & 2.318 & 2.134 & 1.864 & 1.716 & 1.656 \\
\hline S2 & 2.5 & 0.714 & pole-on & -0.995 & 10.27 & 12.264 & 6.665 & 5.211 & 3.849 & 3.322 & 3.044 & 2.68 & 2.512 & 2.315 & 2.024 & 1.852 & 1.796 \\
\hline S2 & 2.5 & 0.786 & pole-on & -1.033 & 11.75 & 13.33 & 7.226 & 5.644 & 4.172 & 3.6 & 3.304 & 2.915 & 2.727 & 2.51 & 2.187 & 2.011 & 1.952 \\
\hline $\mathrm{S} 2$ & 2.5 & 0.857 & pole-on & -1.063 & 13.157 & 14.617 & 7.857 & 6.132 & 4.521 & 3.905 & 3.569 & 3.176 & 2.947 & 2.7 & 2.362 & 2.196 & 2.128 \\
\hline $\mathrm{S} 2$ & 2.5 & 0.928 & pole-on & $\mid-1.088$ & 14.475 & 15.962 & 8.551 & 6.672 & 4.913 & 4.221 & 3.852 & 3.42 & 3.174 & 2.918 & 2.58 & 2.412 & 2.343 \\
\hline S2 & 2.5 & 1.0 & pole-on & -1.106 & 15.596 & 17.387 & 9.24 & 7.182 & 5.25 & 4.512 & 4.121 & 3.669 & 3.42 & 3.158 & 2.797 & 2.623 & 2.564 \\
\hline $\mathrm{S} 2$ & 1.85 & 0.0 & edge-on & 0.38 & 1.107 & 2.274 & 1.754 & 1.563 & 1.318 & 1.154 & 1.846 & 1.397 & 0.909 & 0.822 & 0.718 & 0.646 & 0.63 \\
\hline $\mathrm{S} 2$ & 1.85 & 0.071 & edge-on & 0.207 & 1.428 & 1.743 & 1.338 & 1.197 & 0.992 & 0.87 & 0.979 & 0.792 & 0.611 & 0.59 & 0.499 & 0.446 & 0.423 \\
\hline S2 & 1.85 & 0.143 & interm. & -0.026 & 0.228 & 1.373 & 0.741 & 0.568 & 4.992 & 52.513 & 451.067 & 428.242 & 349.056 & 350.952 & 73.585 & 38.014 & 35.516 \\
\hline $\mathrm{S} 2$ & 1.85 & 0.215 & interm. & -0.234 & 0.856 & 110.487 & 15.69 & 8.89 & 5.617 & 4.551 & 6.555 & 4.934 & 3.214 & 2.946 & 2.528 & 2.361 & 2.244 \\
\hline S2 & 1.85 & 0.286 & interm. & -0.396 & 1.686 & 16.208 & 7.581 & 5.581 & 4.073 & 3.495 & 4.443 & 3.544 & 2.61 & 2.364 & 2.045 & 1.895 & 1.833 \\
\hline
\end{tabular}


Table A.3 - continued.

\begin{tabular}{|c|c|c|c|c|c|c|c|c|c|c|c|c|c|c|c|c|c|}
\hline & & & $\tilde{\tau}_{\mathrm{bu}}$ & & $\mathrm{bu}$ & 0.15 & 0.45 & 0.75 & 1.50 & 2.25 & 3.00 & 4.50 & 6.00 & 9.00 & 15.00 & 30.00 & $\infty$ \\
\hline Star & $\Sigma_{0}\left[\mathrm{~g} \mathrm{~cm}^{-2}\right]$ & $\cos i$ & ang. type & $\Delta R_{\mathrm{bu}}^{\infty}$ & $\xi_{\mathrm{bu}}^{R}$ & $\xi_{\mathrm{d}}^{R}$ & & & & & & & & & & & \\
\hline S2 & 1.85 & 0.357 & interm. & -0.527 & 2.679 & 12.45 & 6.827 & 5.256 & 3.953 & 3.356 & 4.034 & 3.313 & 2.568 & 2.347 & 2.053 & 1.883 & 1.817 \\
\hline S2 & 1.85 & 0.429 & pole-on & -0.63 & 3.941 & 12.358 & 7.062 & 5.442 & 4.032 & 3.517 & 3.984 & 3.358 & 2.687 & 2.456 & 2.152 & 1.975 & 1.911 \\
\hline $\mathrm{S} 2$ & 1.85 & 0.5 & pole-on & -0.712 & 5.372 & 13.12 & 7.446 & 5.757 & 4.334 & 3.762 & 4.12 & 3.519 & 2.881 & 2.628 & 2.292 & 2.123 & 2.054 \\
\hline S2 & 1.85 & 0.571 & pole-on & -0.78 & 6.894 & 14.213 & 7.974 & 6.181 & 4.648 & 4.04 & 4.353 & 3.745 & 3.103 & 2.827 & 2.477 & 2.287 & 2.24 \\
\hline S2 & 1.85 & 0.643 & pole-on & -0.836 & 8.526 & 15.483 & 8.593 & 6.711 & 5.053 & 4.396 & 4.667 & 4.041 & 3.366 & 3.069 & 2.702 & 2.5 & 2.461 \\
\hline S2 & 1.85 & 0.714 & pole-on & -0.88 & 10.138 & 16.893 & 9.394 & 7.307 & 5.507 & 4.795 & 5.028 & 4.383 & 3.659 & 3.355 & 2.966 & 2.759 & 2.731 \\
\hline S2 & 1.85 & 0.786 & pole-on & -0.916 & 11.752 & 18.556 & 10.266 & 7.993 & 6.001 & 5.207 & 5.451 & 4.79 & 3.987 & 3.687 & 3.262 & 3.051 & 3.027 \\
\hline $\mathrm{S} 2$ & 1.85 & 0.857 & pole-on & -0.944 & 13.244 & 20.494 & 11.216 & 8.719 & 6.507 & 5.639 & 5.925 & 5.261 & 4.343 & 4.047 & 3.614 & 3.378 & 3.363 \\
\hline $\mathrm{S} 2$ & 1.85 & 0.928 & pole-on & -0.967 & 14.659 & 22.652 & 12.234 & 9.478 & 7.047 & 6.147 & 6.422 & 5.774 & 4.749 & 4.439 & 3.986 & 3.72 & 3.737 \\
\hline $\mathrm{S} 2$ & 1.85 & 1.0 & pole-on & -0.983 & 15.914 & 24.807 & 13.26 & 10.25 & 7.642 & 6.659 & 6.946 & 6.246 & 5.175 & 4.848 & 4.377 & 4.085 & 4.12 \\
\hline $\mathrm{S} 2$ & 1.37 & 0.0 & edge-on & 0.298 & 1.173 & 2.676 & 2.008 & 1.751 & 1.457 & 1.317 & 1.217 & 1.097 & 1.005 & 0.922 & 0.813 & 0.747 & 0.715 \\
\hline $\mathrm{S} 2$ & 1.37 & 0.071 & edge-on & 0.175 & 1.404 & 2.067 & 1.59 & 1.352 & 1.115 & 1.043 & 0.938 & 0.84 & 0.761 & 0.693 & 0.599 & 0.55 & 0.518 \\
\hline $\mathrm{S} 2$ & 1.37 & 0.143 & interm. & 0.003 & 328.395 & 2.085 & 0.993 & 0.75 & 0.492 & 0.461 & 0.366 & 0.462 & 12.359 & 16.67 & 81.543 & 166.375 & 190.78 \\
\hline $\mathrm{S} 2$ & 1.37 & 0.215 & interm. & -0.168 & 0.811 & 347.723 & 35.466 & 18.141 & 9.954 & 7.574 & 6.547 & 5.537 & 4.96 & 4.392 & 3.766 & 3.439 & 3.315 \\
\hline $\mathrm{S} 2$ & 1.37 & 0.286 & interm. & -0.31 & 1.597 & 28.54 & 10.68 & 7.946 & 5.663 & 4.768 & 4.345 & 3.842 & 3.546 & 3.212 & 2.806 & 2.564 & 2.516 \\
\hline $\mathrm{S} 2$ & 1.37 & 0.357 & interm. & -0.427 & 2.506 & 17.766 & 9.15 & 7.204 & 5.4 & 4.626 & 4.194 & 3.737 & 3.488 & 3.179 & 2.788 & 2.579 & 2.517 \\
\hline $\mathrm{S} 2$ & 1.37 & 0.429 & pole-on & -0.524 & 3.672 & 16.704 & 9.334 & 7.435 & 5.615 & 4.843 & 4.429 & 3.929 & 3.663 & 3.373 & 2.977 & 2.752 & 2.702 \\
\hline S2 & 1.37 & 0.5 & pole-on & -0.6 & 5.069 & 17.563 & 9.924 & 7.936 & 5.994 & 5.193 & 4.745 & 4.257 & 3.976 & 3.654 & 3.25 & 3.023 & 2.976 \\
\hline S2 & 1.37 & 0.571 & pole-on & -0.662 & 6.592 & 19.135 & 10.745 & 8.588 & 6.504 & 5.617 & 5.148 & 4.628 & 4.362 & 4.014 & 3.567 & 3.339 & 3.311 \\
\hline $\mathrm{S} 2$ & 1.37 & 0.643 & pole-on & -0.714 & 8.229 & 20.895 & 11.754 & 9.381 & 7.106 & 6.162 & 5.625 & 5.099 & 4.807 & 4.44 & 3.965 & 3.735 & 3.71 \\
\hline $\mathrm{S} 2$ & 1.37 & 0.714 & pole-on & -0.755 & 9.961 & 23.088 & 12.893 & 10.246 & 7.739 & 6.746 & 6.194 & 5.623 & 5.315 & 4.921 & 4.432 & 4.162 & 4.157 \\
\hline $\mathrm{S} 2$ & 1.37 & 0.786 & pole-on & -0.788 & 11.656 & 25.61 & 14.147 & 11.204 & 8.514 & 7.418 & 6.809 & 6.234 & 5.901 & 5.456 & 4.936 & 4.639 & 4.687 \\
\hline $\mathrm{S} 2$ & 1.37 & 0.857 & pole-on & -0.814 & 13.335 & 28.468 & 15.639 & 12.34 & 9.392 & 8.193 & 7.529 & 6.876 & 6.571 & 6.024 & 5.492 & 5.162 & 5.265 \\
\hline S2 & 1.37 & 0.928 & pole-on & -0.834 & 14.977 & 31.684 & 17.29 & 13.673 & 10.369 & 9.015 & 8.26 & 7.615 & 7.314 & 6.643 & 6.099 & 5.725 & 5.882 \\
\hline $\mathrm{S} 2$ & 1.37 & 1.0 & pole-on & -0.848 & 16.278 & 35.02 & 18.975 & 15.049 & 11.337 & 9.819 & 8.984 & 8.338 & 8.035 & 7.233 & 6.701 & 6.253 & 6.521 \\
\hline $\mathrm{S} 2$ & 1.01 & 0.0 & edge-on & 0.233 & 1.27 & 3.556 & 2.383 & 2.01 & 3.447 & 1.531 & 1.383 & 1.251 & 1.159 & 1.068 & 0.972 & 0.861 & 0.832 \\
\hline $\mathrm{S} 2$ & 1.01 & 0.071 & edge-on & 0.145 & 1.46 & 2.712 & 1.992 & 1.601 & 2.095 & 1.242 & 1.132 & 1.004 & 0.919 & 0.858 & 0.747 & 0.682 & 0.647 \\
\hline $\mathrm{S} 2$ & 1.01 & 0.143 & interm. & 0.02 & 17.791 & 2.369 & 1.465 & 1.08 & 0.555 & 0.661 & 0.554 & 0.418 & 0.353 & 0.311 & 5.289 & 0.246 & 0.229 \\
\hline $\mathrm{S} 2$ & 1.01 & 0.215 & interm. & -0.113 & 0.756 & 395.739 & 227.78 & 51.239 & 54.204 & 14.723 & 12.257 & 9.767 & 8.532 & 7.296 & 6.183 & 5.533 & 5.409 \\
\hline $\mathrm{S} 2$ & 1.01 & 0.286 & interm. & -0.23 & 1.531 & 50.323 & 15.738 & 11.113 & 18.602 & 6.429 & 5.821 & 5.171 & 4.817 & 4.335 & 3.831 & 3.465 & 3.417 \\
\hline $\mathrm{S} 2$ & 1.01 & 0.357 & interm. & -0.331 & 2.378 & 24.6 & 12.165 & 9.338 & 13.371 & 5.963 & 5.529 & 4.973 & 4.606 & 4.226 & 3.806 & 3.493 & 3.421 \\
\hline S2 & 1.01 & 0.429 & pole-on & -0.417 & 3.419 & 21.976 & 12.149 & 9.487 & 12.221 & 6.309 & 5.807 & 5.251 & 4.964 & 4.574 & 4.124 & 3.806 & 3.735 \\
\hline $\mathrm{S} 2$ & 1.01 & 0.5 & pole-on & -0.486 & 4.682 & 22.221 & 12.929 & 10.323 & 12.222 & 6.83 & 6.323 & 5.749 & 5.454 & 5.048 & 4.567 & 4.252 & 4.18 \\
\hline $\mathrm{S} 2$ & 1.01 & 0.571 & pole-on & -0.542 & 6.153 & 24.402 & 14.163 & 11.231 & 12.898 & 7.551 & 6.965 & 6.364 & 6.06 & 5.609 & 5.12 & 4.777 & 4.73 \\
\hline $\mathrm{S} 2$ & 1.01 & 0.643 & pole-on & -0.588 & 7.78 & 27.337 & 15.65 & 12.457 & 13.938 & 8.394 & 7.775 & 7.102 & 6.81 & 6.294 & 5.74 & 5.345 & 5.394 \\
\hline
\end{tabular}


Table A.3 - continued.

\begin{tabular}{|c|c|c|c|c|c|c|c|c|c|c|c|c|c|c|c|c|c|}
\hline & & & $\tilde{\tau}_{\text {bu }}$ & & $\mathrm{bu}$ & 0.15 & 0.45 & 0.75 & 1.50 & 2.25 & 3.00 & 4.50 & 6.00 & 9.00 & 15.00 & 30.00 & $\infty$ \\
\hline Star & $\Sigma_{0}\left[\mathrm{~g} \mathrm{~cm}^{-2}\right]$ & $\cos i$ & ang. type & $\Delta R_{\mathrm{bu}}^{\infty}$ & $\xi_{\mathrm{bu}}^{R}$ & $\xi_{\mathrm{d}}^{R}$ & & & & & & & & & & & \\
\hline $\mathrm{S} 2$ & 1.01 & 0.714 & pole-on & -0.626 & 9.506 & 30.328 & 17.488 & 13.912 & 15.279 & 9.379 & 8.679 & 7.916 & 7.645 & 7.005 & 6.423 & 6.035 & 6.158 \\
\hline $\mathrm{S} 2$ & 1.01 & 0.786 & pole-on & -0.655 & 11.276 & 34.165 & 19.581 & 15.593 & 16.98 & 10.486 & 9.656 & 8.865 & 8.578 & 7.804 & 7.148 & 6.74 & 7.061 \\
\hline $\mathrm{S} 2$ & 1.01 & 0.857 & pole-on & -0.678 & 13.049 & 38.679 & 22.028 & 17.532 & 18.955 & 11.645 & 10.7 & 9.857 & 9.642 & 8.635 & 7.977 & 7.512 & 8.113 \\
\hline $\mathrm{S} 2$ & 1.01 & 0.928 & pole-on & -0.695 & 14.807 & 43.413 & 24.654 & 19.636 & 21.319 & 12.843 & 11.782 & 10.952 & 10.807 & 9.498 & 8.827 & 8.318 & 9.34 \\
\hline $\mathrm{S} 2$ & 1.01 & 1.0 & pole-on & -0.705 & 16.244 & 48.376 & 27.596 & 21.888 & 23.518 & 13.971 & 12.738 & 12.012 & 11.967 & 10.314 & 9.646 & 9.116 & 10.575 \\
\hline $\mathrm{S} 2$ & 0.75 & 0.0 & edge-on & 0.183 & 7.558 & 4.753 & 2.989 & 2.497 & 1.994 & 1.783 & 1.638 & 1.44 & 1.365 & 1.236 & 1.077 & 0.994 & 0.965 \\
\hline $\mathrm{S} 2$ & 0.75 & 0.071 & edge-on & 0.121 & 1.545 & 3.854 & 2.448 & 2.057 & 1.663 & 1.501 & 1.395 & 1.239 & 1.133 & 1.067 & 0.921 & 0.852 & 0.801 \\
\hline S2 & 0.75 & 0.143 & interm. & 0.03 & 36.995 & 3.633 & 2.038 & 1.54 & 1.216 & 1.095 & 0.97 & 0.791 & 0.708 & 0.646 & 0.53 & 0.494 & 0.408 \\
\hline $\mathrm{S} 2$ & 0.75 & 0.215 & interm. & -0.07 & 2.467 & 332.743 & 380.503 & 334.162 & 102.391 & 41.729 & 30.877 & 21.607 & 19.333 & 14.513 & 11.794 & 10.574 & 10.219 \\
\hline $\mathrm{S} 2$ & 0.75 & 0.286 & interm. & \begin{tabular}{|l}
-0.162 \\
\end{tabular} & 1.467 & 219.94 & 26.915 & 16.655 & 10.54 & 8.511 & 7.582 & 6.598 & 6.234 & 5.529 & 4.938 & 4.558 & 4.602 \\
\hline S2 & 0.75 & 0.357 & interm. & -0.245 & 2.254 & 34.882 & 15.404 & 12.116 & 8.756 & 7.579 & 6.858 & 6.147 & 5.92 & 5.357 & 4.784 & 4.447 & 4.565 \\
\hline S2 & 0.75 & 0.429 & pole-on & -0.317 & 3.173 & 26.89 & 14.996 & 11.911 & 9.074 & 7.918 & 7.25 & 6.529 & 6.346 & 5.775 & 5.256 & 4.924 & 5.1 \\
\hline S2 & 0.75 & 0.5 & pole-on & -0.376 & 4.241 & 27.958 & 16.066 & 12.778 & 9.889 & 8.675 & 8.0 & 7.28 & 7.057 & 6.463 & 5.906 & 5.556 & 5.757 \\
\hline S2 & 0.75 & 0.571 & pole-on & -0.424 & 5.432 & 30.246 & 17.647 & 14.38 & 11.101 & 9.764 & 8.945 & 8.103 & 7.962 & 7.272 & 6.699 & 6.324 & 6.604 \\
\hline $\mathrm{S} 2$ & 0.75 & 0.643 & pole-on & -0.464 & 6.869 & 34.014 & 19.968 & 16.108 & 12.488 & 11.029 & 10.119 & 9.156 & 9.053 & 8.185 & 7.586 & 7.139 & 7.626 \\
\hline $\mathrm{S} 2$ & 0.75 & 0.714 & pole-on & -0.496 & 8.058 & 38.037 & 22.522 & 18.266 & 14.112 & 12.379 & 11.417 & 10.267 & 10.325 & 9.212 & 8.606 & 8.04 & 8.797 \\
\hline S2 & 0.75 & 0.786 & pole-on & -0.521 & 9.373 & 43.446 & 25.516 & 20.749 & 16.029 & 14.01 & 12.849 & 11.625 & 11.733 & 10.368 & 9.65 & 9.031 & 10.136 \\
\hline S2 & 0.75 & 0.857 & pole-on & -0.54 & 10.668 & 49.278 & 29.07 & 23.624 & 17.907 & 15.532 & 14.296 & 12.959 & 13.296 & 11.401 & 10.875 & 10.062 & 11.604 \\
\hline $\mathrm{S} 2$ & 0.75 & 0.928 & pole-on & -0.553 & 11.785 & 56.316 & 33.095 & 27.039 & 19.892 & 17.128 & 15.588 & 14.483 & 15.025 & 12.47 & 12.146 & 11.06 & 13.303 \\
\hline S2 & 0.75 & 1.0 & pole-on & -0.56 & 12.746 & 62.284 & 36.973 & 30.038 & 21.798 & 18.576 & 16.822 & 15.859 & 16.664 & 13.472 & 13.346 & 12.117 & 15.058 \\
\hline $\mathrm{S} 2$ & 0.56 & 0.0 & edge-on & 0.144 & 1.639 & 6.418 & 3.775 & 3.112 & 2.368 & 2.087 & 1.906 & 1.729 & 1.569 & 1.451 & 1.263 & 1.14 & 1.114 \\
\hline $\mathrm{S} 2$ & 0.56 & 0.071 & edge-on & 0.1 & 1.771 & 5.533 & 3.237 & 2.736 & 2.135 & 1.855 & 1.733 & 1.505 & 1.402 & 1.303 & 1.121 & 1.033 & 0.978 \\
\hline S2 & 0.56 & 0.143 & interm. & 0.036 & 4.695 & 4.977 & 2.84 & 2.363 & 1.882 & 1.597 & 1.57 & 1.329 & 1.186 & 1.156 & 0.947 & 0.853 & 0.793 \\
\hline S2 & 0.56 & 0.215 & interm. & -0.038 & 0.476 & 168.26 & 321.904 & 381.947 & 364.11 & 348.434 & 290.264 & 190.167 & 146.107 & 80.261 & 45.77 & 33.63 & 36.244 \\
\hline $\mathrm{S} 2$ & 0.56 & 0.286 & interm. & \begin{tabular}{|l}
-0.109 \\
\end{tabular} & 1.339 & 332.336 & 49.748 & 25.696 & 14.325 & 10.882 & 9.411 & 8.039 & 7.533 & 6.363 & 5.882 & 5.369 & 5.913 \\
\hline S2 & 0.56 & 0.357 & interm. & -0.173 & 2.126 & 49.841 & 19.5 & 14.391 & 10.286 & 8.658 & 7.806 & 7.009 & 6.803 & 5.993 & 5.531 & 5.185 & 5.575 \\
\hline $\mathrm{S} 2$ & 0.56 & 0.429 & pole-on & -0.229 & 3.045 & 33.688 & 17.666 & 13.822 & 10.274 & 9.062 & 8.29 & 7.6 & 7.347 & 6.54 & 6.048 & 5.757 & 6.174 \\
\hline $\mathrm{S} 2$ & 0.56 & 0.5 & pole-on & -0.276 & 4.119 & 32.485 & 18.958 & 15.102 & 11.589 & 10.074 & 9.296 & 8.627 & 8.385 & 7.49 & 6.968 & 6.514 & 7.175 \\
\hline $\mathrm{S} 2$ & 0.56 & 0.571 & pole-on & -0.316 & 5.319 & 35.056 & 20.903 & 17.069 & 13.175 & 11.548 & 10.586 & 9.579 & 9.585 & 8.509 & 8.028 & 7.474 & 8.39 \\
\hline $\mathrm{S} 2$ & 0.56 & 0.643 & pole-on & -0.348 & 6.761 & 39.338 & 23.824 & 19.263 & 15.015 & 13.147 & 12.21 & 10.881 & 11.056 & 9.734 & 9.125 & 8.58 & 9.76 \\
\hline $\mathrm{S} 2$ & 0.56 & 0.714 & pole-on & -0.373 & 8.375 & 44.447 & 27.369 & 22.382 & 17.171 & 15.09 & 13.716 & 12.435 & 12.71 & 11.041 & 10.482 & 9.811 & 11.465 \\
\hline S2 & 0.56 & 0.786 & pole-on & \begin{tabular}{|l|}
-0.393 \\
\end{tabular} & 10.095 & 51.756 & 31.581 & 25.894 & 19.615 & 17.103 & 15.746 & 13.958 & 14.674 & 12.451 & 11.836 & 10.991 & 13.369 \\
\hline $\mathrm{S} 2$ & 0.56 & 0.857 & pole-on & -0.408 & 11.843 & 59.323 & 36.152 & 29.457 & 22.097 & 19.049 & 17.454 & 15.677 & 16.789 & 13.654 & 13.376 & 12.203 & 15.577 \\
\hline $\mathrm{S} 2$ & 0.56 & 0.928 & pole-on & -0.417 & 13.755 & 67.92 & 41.301 & 33.715 & 24.572 & 21.032 & 18.979 & 17.6 & 18.946 & 14.985 & 15.007 & 13.504 & 18.064 \\
\hline $\mathrm{S} 2$ & 0.56 & 1.0 & pole-on & -0.421 & 15.236 & 75.21 & 46.288 & 37.788 & 26.924 & 22.651 & 20.495 & 19.369 & 21.282 & 16.149 & 16.478 & 14.594 & 20.515 \\
\hline
\end{tabular}


Table A.3 - continued.

\begin{tabular}{|c|c|c|c|c|c|c|c|c|c|c|c|c|c|c|c|c|c|}
\hline & & & $\tilde{\tau}_{\mathrm{bu}}$ & & $\mathrm{bu}$ & 0.15 & 0.45 & 0.75 & 1.50 & 2.25 & 3.00 & 4.50 & 6.00 & 9.00 & 15.00 & 30.00 & $\infty$ \\
\hline Star & $\Sigma_{0}\left[\mathrm{~g} \mathrm{~cm}^{-2}\right]$ & $\cos i$ & ang. type & $\Delta R_{\mathrm{bu}}^{\infty}$ & $\xi_{\mathrm{bu}}^{R}$ & $\xi_{\mathrm{d}}^{R}$ & & & & & & & & & & & \\
\hline S2 & 0.41 & 0.0 & edge-on & 0.112 & 1.935 & 8.472 & 4.609 & 3.763 & 2.921 & 2.519 & 2.327 & 2.029 & 1.881 & 1.732 & 1.505 & 1.343 & 1.296 \\
\hline $\mathrm{S} 2$ & 0.41 & 0.071 & edge-on & 0.082 & 2.067 & 9.228 & 3.995 & 3.494 & 2.642 & 2.298 & 2.1 & 1.87 & 1.702 & 1.571 & 1.349 & 1.256 & 1.222 \\
\hline $\mathrm{S} 2$ & 0.41 & 0.143 & interm. & 0.037 & 4.308 & 17.941 & 4.187 & 3.453 & 2.699 & 2.493 & 2.366 & 2.091 & 1.889 & 1.778 & 1.537 & 1.422 & 1.308 \\
\hline $\mathrm{S} 2$ & 0.41 & 0.215 & interm. & -0.011 & 44.0 & 93.927 & 67.974 & 48.985 & 227.074 & 269.978 & 281.241 & 331.199 & 354.934 & 355.519 & 372.308 & 336.071 & 355.621 \\
\hline S2 & 0.41 & 0.286 & interm. & -0.065 & 1.265 & 379.497 & 260.822 & 73.954 & 22.046 & 12.715 & 10.597 & 8.739 & 8.428 & 6.748 & 6.065 & 5.683 & 6.42 \\
\hline $\mathrm{S} 2$ & 0.41 & 0.357 & interm. & -0.112 & 1.994 & 145.965 & 22.583 & 15.451 & 10.908 & 8.712 & 7.855 & 6.927 & 7.057 & 6.095 & 5.827 & 5.237 & 5.995 \\
\hline S2 & 0.41 & 0.429 & pole-on & -0.154 & 2.832 & 38.705 & 19.132 & 14.814 & 11.014 & 9.345 & 8.544 & 7.798 & 7.979 & 6.939 & 6.449 & 6.04 & 6.861 \\
\hline S2 & 0.41 & 0.5 & pole-on & $\mid-0.188$ & 3.855 & 35.666 & 20.481 & 16.329 & 12.666 & 10.665 & 9.986 & 9.056 & 9.092 & 7.973 & 7.561 & 6.999 & 8.002 \\
\hline $\mathrm{S} 2$ & 0.41 & 0.571 & pole-on & -0.218 & 4.951 & 38.226 & 22.991 & 18.769 & 14.302 & 12.416 & 11.431 & 10.426 & 10.61 & 9.232 & 8.709 & 8.246 & 9.597 \\
\hline S2 & 0.41 & 0.643 & pole-on & -0.241 & 6.317 & 43.092 & 26.495 & 21.551 & 16.572 & 14.465 & 13.324 & 11.966 & 12.407 & 10.725 & 10.199 & 9.514 & 11.463 \\
\hline $\mathrm{S} 2$ & 0.41 & 0.714 & pole-on & -0.26 & 7.843 & 49.809 & 30.624 & 25.481 & 19.266 & 16.633 & 15.347 & 13.681 & 14.616 & 12.271 & 11.633 & 10.913 & 13.665 \\
\hline $\mathrm{S} 2$ & 0.41 & 0.786 & pole-on & -0.274 & 9.682 & 57.08 & 36.064 & 29.99 & 22.389 & 19.361 & 17.697 & 15.665 & 16.89 & 13.9 & 13.479 & 12.293 & 16.14 \\
\hline S2 & 0.41 & 0.857 & pole-on & -0.284 & 11.444 & 67.317 & 41.565 & 34.643 & 25.543 & 21.754 & 19.762 & 17.554 & 19.552 & 15.311 & 15.211 & 13.769 & 19.16 \\
\hline $\mathrm{S} 2$ & 0.41 & 0.928 & pole-on & -0.29 & 13.498 & 76.004 & 48.105 & 40.008 & 28.174 & 23.64 & 21.45 & 19.854 & 22.506 & 16.743 & 17.112 & 15.216 & 22.299 \\
\hline $\mathrm{S} 2$ & 0.41 & 1.0 & pole-on & -0.29 & 14.886 & 84.552 & 53.673 & 44.424 & 30.668 & 25.603 & 22.888 & 21.856 & 25.234 & 18.042 & 18.955 & 16.543 & 25.495 \\
\hline S2 & 0.3 & 0.0 & edge-on & 0.087 & 2.258 & 11.13 & 5.969 & 4.542 & 3.457 & 3.023 & 2.813 & 2.477 & 2.184 & 2.018 & 1.743 & 1.635 & 1.506 \\
\hline $\mathrm{S} 2$ & 0.3 & 0.071 & edge-on & 0.066 & 2.316 & 16.954 & 5.338 & 4.597 & 3.211 & 2.814 & 2.56 & 2.376 & 2.186 & 1.901 & 1.666 & 1.5 & 1.483 \\
\hline $\mathrm{S} 2$ & 0.3 & 0.143 & interm. & 0.035 & 4.542 & 25.531 & 7.858 & 4.961 & 3.962 & 3.392 & 3.189 & 3.049 & 2.739 & 2.589 & 2.178 & 2.132 & 1.993 \\
\hline S2 & 0.3 & 0.215 & interm. & 0.003 & 272.158 & 104.073 & 63.064 & 44.972 & 63.831 & 48.231 & 41.32 & 83.104 & 83.31 & 116.621 & 174.194 & 98.484 & 226.288 \\
\hline $\mathrm{S} 2$ & 0.3 & 0.286 & interm. & -0.035 & 14.722 & 301.471 & 332.21 & 300.923 & 133.822 & 36.703 & 21.968 & 7.545 & 8.942 & 5.506 & 5.245 & 4.14 & 5.116 \\
\hline S2 & 0.3 & 0.357 & interm. & -0.068 & 2.918 & 268.645 & 24.879 & 16.041 & 8.674 & 7.468 & 7.248 & 5.81 & 5.994 & 5.097 & 4.645 & 4.218 & 5.174 \\
\hline S2 & 0.3 & 0.429 & pole-on & -0.097 & 2.667 & 58.759 & 18.488 & 14.642 & 10.561 & 9.065 & 8.107 & 7.135 & 7.35 & 6.398 & 6.16 & 5.793 & 6.512 \\
\hline $\mathrm{S} 2$ & 0.3 & 0.5 & pole-on & -0.122 & 3.639 & 38.261 & 19.432 & 16.473 & 11.727 & 10.159 & 9.584 & 8.466 & 8.827 & 7.599 & 7.173 & 6.629 & 7.871 \\
\hline S2 & 0.3 & 0.571 & pole-on & -0.142 & 4.8 & 38.68 & 22.732 & 18.764 & 14.06 & 12.365 & 11.412 & 10.162 & 10.645 & 9.219 & 8.812 & 8.158 & 9.761 \\
\hline S2 & 0.3 & 0.643 & pole-on & -0.159 & 6.032 & 43.462 & 26.855 & 22.459 & 16.573 & 14.575 & 13.758 & 12.12 & 12.594 & 10.796 & 10.385 & 9.515 & 11.967 \\
\hline $\mathrm{S} 2$ & 0.3 & 0.714 & pole-on & -0.172 & 7.598 & 50.52 & 31.684 & 26.66 & 19.922 & 17.393 & 15.93 & 13.853 & 14.979 & 12.653 & 12.056 & 10.954 & 14.572 \\
\hline $\mathrm{S} 2$ & 0.3 & 0.786 & pole-on & -0.181 & 9.401 & 60.261 & 37.757 & 31.872 & 23.547 & 20.268 & 18.545 & 16.172 & 17.808 & 14.271 & 13.926 & 12.751 & 17.471 \\
\hline S2 & 0.3 & 0.857 & pole-on & $\mid-0.187$ & 11.213 & 70.29 & 44.027 & 37.071 & 27.372 & 22.709 & 20.584 & 18.292 & 20.926 & 15.823 & 16.121 & 14.356 & 21.131 \\
\hline $\mathrm{S} 2$ & 0.3 & 0.928 & pole-on & -0.191 & 13.449 & 80.831 & 52.382 & 42.001 & 30.407 & 26.019 & 22.562 & 21.024 & 24.384 & 17.298 & 18.013 & 16.001 & 25.077 \\
\hline S2 & 0.3 & 1.0 & pole-on & -0.19 & 14.975 & 90.335 & 58.125 & 48.582 & 33.396 & 27.529 & 24.032 & 23.027 & 27.135 & 18.889 & 20.091 & 17.509 & 29.158 \\
\hline S3 & 2.5 & 0.0 & edge-on & 0.43 & 1.111 & 2.038 & 1.578 & 1.394 & 1.197 & 1.07 & 1.011 & 0.9 & 0.845 & 0.767 & 0.671 & 0.61 & 0.596 \\
\hline S3 & 2.5 & 0.071 & edge-on & 0.248 & 1.465 & 1.635 & 1.252 & 1.103 & 0.929 & 0.839 & 0.786 & 0.685 & 0.647 & 0.576 & 0.485 & 0.436 & 0.418 \\
\hline S3 & 2.5 & 0.143 & interm. & -0.007 & 32.88 & 1.266 & 0.838 & 0.628 & 0.284 & 0.251 & 0.227 & 11.713 & 32.329 & 151.561 & 279.528 & 340.224 & 336.806 \\
\hline S3 & 2.5 & 0.215 & interm. & -0.228 & 0.761 & 258.086 & 16.731 & 9.416 & 5.833 & 4.677 & 4.053 & 3.546 & 3.133 & 2.891 & 2.521 & 2.27 & 2.177 \\
\hline S3 & 2.5 & 0.286 & interm. & -0.396 & 1.593 & 15.166 & 6.911 & 5.187 & 3.859 & 3.277 & 2.938 & 2.624 & 2.381 & 2.201 & 1.924 & 1.748 & 1.682 \\
\hline
\end{tabular}


Table A.3 - continued.

\begin{tabular}{|c|c|c|c|c|c|c|c|c|c|c|c|c|c|c|c|c|c|}
\hline & & & $\tilde{\tau}_{\text {bu }}$ & & $\mathrm{bu}$ & 0.15 & 0.45 & 0.75 & 1.50 & 2.25 & 3.00 & 4.50 & 6.00 & 9.00 & 15.00 & 30.00 & $\infty$ \\
\hline Star & $\Sigma_{0}\left[\mathrm{~g} \mathrm{~cm}^{-2}\right]$ & $\cos i$ & ang. type & $\Delta R_{\mathrm{bu}}^{\infty}$ & $\xi_{\mathrm{bu}}^{R}$ & $\xi_{\mathrm{d}}^{R}$ & & & & & & & & & & & \\
\hline S3 & 2.5 & 0.357 & interm. & $\mid-0.529$ & 2.591 & 11.102 & 6.194 & 4.846 & 3.652 & 3.148 & 2.825 & 2.553 & 2.317 & 2.139 & 1.876 & 1.707 & 1.651 \\
\hline S3 & 2.5 & 0.429 & pole-on & -0.635 & 3.813 & 11.164 & 6.288 & 4.94 & 3.734 & 3.218 & 2.901 & 2.627 & 2.395 & 2.21 & 1.938 & 1.775 & 1.718 \\
\hline S3 & 2.5 & 0.5 & pole-on & -0.716 & 5.308 & 11.743 & 6.599 & 5.185 & 3.932 & 3.395 & 3.076 & 2.771 & 2.549 & 2.351 & 2.062 & 1.886 & 1.834 \\
\hline S3 & 2.5 & 0.571 & pole-on & -0.784 & 6.795 & 12.699 & 7.066 & 5.54 & 4.196 & 3.633 & 3.302 & 2.973 & 2.739 & 2.529 & 2.219 & 2.031 & 1.98 \\
\hline S3 & 2.5 & 0.643 & pole-on & -0.841 & 8.376 & 13.783 & 7.646 & 5.979 & 4.534 & 3.925 & 3.582 & 3.223 & 2.98 & 2.741 & 2.41 & 2.211 & 2.154 \\
\hline S3 & 2.5 & 0.714 & pole-on & -0.886 & 9.9 & 15.084 & 8.32 & 6.503 & 4.925 & 4.272 & 3.892 & 3.505 & 3.235 & 2.976 & 2.625 & 2.417 & 2.373 \\
\hline S3 & 2.5 & 0.786 & pole-on & -0.921 & 11.448 & 16.578 & 9.069 & 7.104 & 5.366 & 4.651 & 4.226 & 3.803 & 3.524 & 3.241 & 2.868 & 2.653 & 2.633 \\
\hline S3 & 2.5 & 0.857 & pole-on & -0.95 & 12.967 & 18.247 & 9.941 & 7.757 & 5.827 & 5.031 & 4.568 & 4.118 & 3.826 & 3.54 & 3.154 & 2.935 & 2.908 \\
\hline S3 & 2.5 & 0.928 & pole-on & -0.973 & 14.381 & 20.174 & 10.873 & 8.451 & 6.3 & 5.439 & 4.945 & 4.496 & 4.199 & 3.889 & 3.474 & 3.237 & 3.231 \\
\hline S3 & 2.5 & 1.0 & pole-on & -0.99 & 15.534 & 22.03 & 11.811 & 9.124 & 6.801 & 5.883 & 5.36 & 4.899 & 4.592 & 4.238 & 3.809 & 3.552 & 3.558 \\
\hline S3 & 1.85 & 0.0 & edge-on & 0.338 & 1.208 & 2.562 & 1.793 & 1.574 & 1.323 & 1.183 & 1.119 & 1.539 & 0.977 & 0.866 & 0.748 & 0.675 & 0.672 \\
\hline S3 & 1.85 & 0.071 & edge-on & 0.211 & 1.45 & 2.099 & 1.491 & 1.284 & 1.101 & 0.967 & 0.919 & 0.99 & 0.734 & 0.689 & 0.595 & 0.532 & 0.52 \\
\hline S3 & 1.85 & 0.143 & interm. & 0.021 & 144.362 & 1.807 & 1.085 & 0.81 & 0.566 & 0.424 & 0.32 & 2.919 & 3.128 & 0.252 & 0.233 & 13.478 & 0.298 \\
\hline S3 & 1.85 & 0.215 & interm. & -0.162 & 0.679 & 376.35 & 43.653 & 20.109 & 10.055 & 7.149 & 6.321 & 7.18 & 4.812 & 4.262 & 3.69 & 3.34 & 3.193 \\
\hline S3 & 1.85 & 0.286 & interm. & -0.311 & 1.476 & 24.349 & 9.811 & 7.333 & 5.226 & 4.39 & 3.993 & 4.441 & 3.275 & 2.966 & 2.584 & 2.378 & 2.284 \\
\hline S3 & 1.85 & 0.357 & interm. & -0.433 & 2.407 & 15.051 & 8.264 & 6.593 & 4.899 & 4.189 & 3.823 & 4.093 & 3.185 & 2.903 & 2.542 & 2.343 & 2.269 \\
\hline S3 & 1.85 & 0.429 & pole-on & -0.532 & 3.57 & 14.577 & 8.443 & 6.712 & 5.073 & 4.366 & 3.976 & 4.162 & 3.339 & 3.049 & 2.676 & 2.475 & 2.399 \\
\hline S3 & 1.85 & 0.5 & pole-on & $\mid-0.609$ & 4.983 & 15.563 & 8.908 & 7.127 & 5.387 & 4.674 & 4.264 & 4.377 & 3.589 & 3.283 & 2.886 & 2.686 & 2.603 \\
\hline S3 & 1.85 & 0.571 & pole-on & -0.671 & 6.526 & 16.784 & 9.62 & 7.675 & 5.819 & 5.047 & 4.629 & 4.695 & 3.891 & 3.578 & 3.161 & 2.949 & 2.89 \\
\hline S3 & 1.85 & 0.643 & pole-on & -0.723 & 8.185 & 18.52 & 10.52 & 8.358 & 6.331 & 5.516 & 5.058 & 5.09 & 4.255 & 3.926 & 3.5 & 3.27 & 3.218 \\
\hline S3 & 1.85 & 0.714 & pole-on & -0.765 & 9.902 & 20.37 & 11.501 & 9.164 & 6.901 & 6.029 & 5.545 & 5.569 & 4.692 & 4.328 & 3.892 & 3.63 & 3.608 \\
\hline S3 & 1.85 & 0.786 & pole-on & -0.798 & 11.618 & 22.67 & 12.656 & 10.03 & 7.557 & 6.65 & 6.085 & 6.108 & 5.139 & 4.791 & 4.324 & 4.055 & 4.04 \\
\hline S3 & 1.85 & 0.857 & pole-on & -0.825 & 13.265 & 25.2 & 13.944 & 11.001 & 8.307 & 7.28 & 6.74 & 6.701 & 5.684 & 5.31 & 4.807 & 4.511 & 4.542 \\
\hline S3 & 1.85 & 0.928 & pole-on & -0.846 & 14.796 & 28.136 & 15.326 & 12.117 & 9.166 & 8.024 & 7.425 & 7.337 & 6.264 & 5.847 & 5.36 & 5.003 & 5.074 \\
\hline S3 & 1.85 & 1.0 & pole-on & -0.86 & 16.103 & 31.026 & 16.863 & 13.281 & 10.019 & 8.765 & 8.111 & 8.006 & 6.832 & 6.403 & 5.907 & 5.512 & 5.637 \\
\hline S3 & 1.37 & 0.0 & edge-on & 0.265 & 1.36 & 3.109 & 2.181 & 1.883 & 1.537 & 1.347 & 1.255 & 1.131 & 1.048 & 0.97 & 0.839 & 0.764 & 0.753 \\
\hline S3 & 1.37 & 0.071 & edge-on & 0.176 & 1.539 & 2.597 & 1.731 & 1.584 & 1.311 & 1.148 & 1.061 & 0.968 & 0.894 & 0.82 & 0.709 & 0.65 & 0.631 \\
\hline S3 & 1.37 & 0.143 & interm. & 0.037 & 12.814 & 2.133 & 1.502 & 1.15 & 0.888 & 0.693 & 0.594 & 0.486 & 0.446 & 0.397 & 0.29 & 0.256 & 0.301 \\
\hline S3 & 1.37 & 0.215 & interm. & \begin{tabular}{|l}
-0.109 \\
\end{tabular} & 0.57 & 361.024 & 322.719 & 66.478 & 22.642 & 15.591 & 12.597 & 9.501 & 8.447 & 7.243 & 6.228 & 5.454 & 5.111 \\
\hline S3 & 1.37 & 0.286 & interm. & -0.234 & 1.364 & 50.044 & 14.073 & 10.047 & 6.93 & 5.856 & 5.398 & 4.634 & 4.307 & 3.882 & 3.527 & 3.187 & 3.109 \\
\hline S3 & 1.37 & 0.357 & interm. & -0.341 & 2.232 & 21.112 & 10.796 & 8.434 & 6.295 & 5.47 & 5.029 & 4.444 & 4.145 & 3.804 & 3.414 & 3.151 & 3.123 \\
\hline S3 & 1.37 & 0.429 & pole-on & -0.43 & 3.316 & 18.737 & 10.671 & 8.621 & 6.522 & 5.706 & 5.275 & 4.715 & 4.434 & 4.081 & 3.645 & 3.408 & 3.376 \\
\hline S3 & 1.37 & 0.5 & pole-on & -0.5 & 4.641 & 19.875 & 11.523 & 9.274 & 7.074 & 6.191 & 5.703 & 5.117 & 4.841 & 4.468 & 4.039 & 3.786 & 3.766 \\
\hline S3 & 1.37 & 0.571 & pole-on & -0.557 & 6.156 & 21.846 & 12.677 & 10.114 & 7.755 & 6.79 & 6.264 & 5.657 & 5.406 & 4.973 & 4.499 & 4.226 & 4.239 \\
\hline S3 & 1.37 & 0.643 & pole-on & -0.605 & 7.8 & 24.275 & 13.935 & 11.149 & 8.546 & 7.469 & 6.943 & 6.304 & 6.004 & 5.526 & 5.055 & 4.749 & 4.806 \\
\hline
\end{tabular}


Table A.3 - continued.

\begin{tabular}{|c|c|c|c|c|c|c|c|c|c|c|c|c|c|c|c|c|c|}
\hline & & & $\tilde{\tau}_{\mathrm{bu}}$ & & $\mathrm{bu}$ & 0.15 & 0.45 & 0.75 & 1.50 & 2.25 & 3.00 & 4.50 & 6.00 & 9.00 & 15.00 & 30.00 & $\infty$ \\
\hline Star & $\Sigma_{0}\left[\mathrm{~g} \mathrm{~cm}^{-2}\right]$ & $\cos i$ & ang. type & $\Delta R_{\mathrm{bu}}^{\infty}$ & $\xi_{\mathrm{bu}}^{R}$ & $\xi_{\mathrm{d}}^{R}$ & & & & & & & & & & & \\
\hline S3 & 1.37 & 0.714 & pole-on & -0.644 & 9.487 & 27.03 & 15.474 & 12.395 & 9.495 & 8.334 & 7.74 & 7.017 & 6.736 & 6.195 & 5.677 & 5.34 & 5.461 \\
\hline S3 & 1.37 & 0.786 & pole-on & -0.674 & 11.309 & 30.658 & 17.252 & 13.729 & 10.569 & 9.307 & 8.624 & 7.822 & 7.563 & 6.898 & 6.365 & 5.98 & 6.188 \\
\hline S3 & 1.37 & 0.857 & pole-on & -0.697 & 13.057 & 34.3 & 19.377 & 15.436 & 11.816 & 10.331 & 9.566 & 8.73 & 8.484 & 7.657 & 7.13 & 6.686 & 7.015 \\
\hline S3 & 1.37 & 0.928 & pole-on & -0.716 & 14.718 & 38.9 & 21.721 & 17.271 & 13.142 & 11.436 & 10.508 & 9.727 & 9.531 & 8.454 & 7.943 & 7.42 & 7.913 \\
\hline S3 & 1.37 & 1.0 & pole-on & -0.728 & 16.189 & 42.935 & 24.058 & 19.24 & 14.467 & 12.49 & 11.473 & 10.723 & 10.574 & 9.216 & 8.807 & 8.159 & 8.872 \\
\hline S3 & 1.01 & 0.0 & edge-on & 0.208 & 1.536 & 4.228 & 2.508 & 2.244 & 1.757 & 1.558 & 1.435 & 1.3 & 1.182 & 1.042 & 0.809 & 0.825 & 0.869 \\
\hline S3 & 1.01 & 0.071 & edge-on & 0.144 & 1.691 & 3.596 & 2.254 & 1.926 & 1.555 & 1.392 & 1.276 & 1.152 & 1.037 & 0.94 & 0.732 & 0.739 & 0.768 \\
\hline S3 & 1.01 & 0.143 & interm. & 0.045 & 6.267 & 3.031 & 2.149 & 1.686 & 1.247 & 1.089 & 1.01 & 0.832 & 0.741 & 0.693 & 0.552 & 0.511 & 0.45 \\
\hline S3 & 1.01 & 0.215 & interm. & -0.065 & 0.435 & 151.384 & 347.381 & 358.071 & 221.179 & 62.808 & 39.44 & 27.073 & 21.717 & 16.41 & 12.843 & 11.273 & 11.489 \\
\hline S3 & 1.01 & 0.286 & interm. & -0.164 & 1.276 & 228.21 & 23.412 & 14.452 & 9.193 & 7.747 & 6.787 & 6.086 & 5.695 & 5.246 & 4.944 & 4.208 & 3.908 \\
\hline S3 & 1.01 & 0.357 & interm. & -0.253 & 2.117 & 29.897 & 14.193 & 10.606 & 7.812 & 6.84 & 6.301 & 5.635 & 5.355 & 5.01 & 4.694 & 4.135 & 3.822 \\
\hline S3 & 1.01 & 0.429 & pole-on & -0.328 & 3.144 & 24.063 & 13.666 & 10.777 & 8.058 & 7.195 & 6.6 & 6.035 & 5.73 & 5.376 & 5.09 & 4.543 & 4.205 \\
\hline S3 & 1.01 & 0.5 & pole-on & -0.39 & 4.346 & 24.963 & 14.47 & 11.659 & 8.816 & 7.91 & 7.29 & 6.647 & 6.428 & 6.007 & 5.654 & 5.131 & 4.755 \\
\hline S3 & 1.01 & 0.571 & pole-on & -0.441 & 5.768 & 27.239 & 16.323 & 12.962 & 9.74 & 8.819 & 8.199 & 7.481 & 7.191 & 6.718 & 6.336 & 5.846 & 5.432 \\
\hline S3 & 1.01 & 0.643 & pole-on & -0.482 & 7.331 & 30.281 & 18.426 & 14.544 & 10.946 & 9.979 & 9.224 & 8.377 & 8.162 & 7.628 & 7.126 & 6.68 & 6.275 \\
\hline S3 & 1.01 & 0.714 & pole-on & -0.516 & 9.023 & 34.865 & 20.674 & 16.473 & 12.254 & 11.276 & 10.393 & 9.443 & 9.29 & 8.52 & 8.033 & 7.577 & 7.368 \\
\hline S3 & 1.01 & 0.786 & pole-on & -0.541 & 10.963 & 39.279 & 23.536 & 18.679 & 13.753 & 12.666 & 11.704 & 10.604 & 10.533 & 9.528 & 8.946 & 8.56 & 8.619 \\
\hline S3 & 1.01 & 0.857 & pole-on & -0.562 & 12.755 & 44.847 & 26.765 & 21.383 & 15.488 & 14.127 & 13.011 & 11.909 & 11.967 & 10.561 & 9.931 & 9.596 & 10.343 \\
\hline S3 & 1.01 & 0.928 & pole-on & -0.576 & 14.559 & 50.898 & 30.291 & 24.111 & 17.183 & 15.636 & 14.286 & 13.236 & 13.48 & 11.582 & 10.997 & 10.695 & 12.486 \\
\hline S3 & 1.01 & 1.0 & pole-on & -0.584 & 16.079 & 56.76 & 33.892 & 27.139 & 19.095 & 16.996 & 15.521 & 14.613 & 15.097 & 12.597 & 12.002 & 11.686 & 14.84 \\
\hline S3 & 0.75 & 0.0 & edge-on & 0.165 & 1.775 & 5.494 & 3.369 & 2.7 & 2.136 & 1.821 & 1.665 & 1.492 & 1.357 & 1.259 & 1.08 & 1.005 & 0.952 \\
\hline S3 & 0.75 & 0.071 & edge-on & 0.119 & 2.456 & 4.959 & 2.993 & 2.441 & 1.918 & 1.685 & 1.532 & 1.376 & 1.254 & 1.162 & 0.989 & 0.912 & 0.887 \\
\hline S3 & 0.75 & 0.143 & interm. & 0.047 & 72.016 & 5.388 & 2.872 & 2.386 & 1.847 & 1.573 & 1.436 & 1.286 & 1.146 & 1.073 & 0.904 & 0.875 & 0.759 \\
\hline S3 & 0.75 & 0.215 & interm. & -0.032 & 0.685 & 17.847 & 77.842 & 149.225 & 325.394 & 365.041 & 331.854 & 289.993 & 262.962 & 204.098 & 97.153 & 52.742 & 64.379 \\
\hline S3 & 0.75 & 0.286 & interm. & \begin{tabular}{|l}
-0.109 \\
\end{tabular} & 1.136 & 345.504 & 41.878 & 23.186 & 12.506 & 9.728 & 8.656 & 7.193 & 6.914 & 6.044 & 5.33 & 4.98 & 5.139 \\
\hline S3 & 0.75 & 0.357 & interm. & -0.179 & 1.948 & 43.177 & 16.252 & 12.497 & 8.97 & 7.897 & 7.24 & 6.4 & 6.239 & 5.558 & 5.167 & 4.745 & 5.053 \\
\hline S3 & 0.75 & 0.429 & pole-on & -0.24 & 2.887 & 28.932 & 15.466 & 12.545 & 9.535 & 8.418 & 7.746 & 6.99 & 6.845 & 6.187 & 5.65 & 5.304 & 5.681 \\
\hline S3 & 0.75 & 0.5 & pole-on & -0.291 & 3.921 & 29.464 & 16.81 & 13.661 & 10.721 & 9.386 & 8.665 & 7.875 & 7.684 & 6.974 & 6.492 & 6.06 & 6.592 \\
\hline S3 & 0.75 & 0.571 & pole-on & -0.333 & 5.038 & 32.02 & 18.914 & 15.51 & 12.029 & 10.694 & 9.853 & 9.001 & 8.833 & 8.027 & 7.397 & 6.964 & 7.643 \\
\hline S3 & 0.75 & 0.643 & pole-on & -0.368 & 6.354 & 35.716 & 21.497 & 17.722 & 13.815 & 12.139 & 11.267 & 10.241 & 10.217 & 9.13 & 8.506 & 8.01 & 8.944 \\
\hline S3 & 0.75 & 0.714 & pole-on & -0.394 & 7.659 & 41.145 & 24.85 & 20.467 & 15.744 & 13.961 & 12.855 & 11.634 & 11.682 & 10.342 & 9.714 & 9.065 & 10.43 \\
\hline S3 & 0.75 & 0.786 & pole-on & -0.415 & 9.066 & 47.395 & 28.637 & 23.681 & 18.128 & 15.857 & 14.599 & 13.137 & 13.464 & 11.629 & 11.075 & 10.245 & 12.214 \\
\hline S3 & 0.75 & 0.857 & pole-on & -0.431 & 10.391 & 54.232 & 33.036 & 27.19 & 20.428 & 17.723 & 16.25 & 14.728 & 15.468 & 12.974 & 12.448 & 11.464 & 14.259 \\
\hline S3 & 0.75 & 0.928 & pole-on & -0.441 & 11.596 & 62.61 & 38.185 & 31.331 & 22.754 & 19.621 & 17.781 & 16.492 & 17.603 & 14.177 & 14.04 & 12.637 & 16.49 \\
\hline $\mathrm{S} 3$ & 0.75 & 1.0 & pole-on & -0.446 & 12.551 & 69.219 & 42.721 & 34.972 & 24.963 & 21.146 & 19.222 & 18.238 & 19.792 & 15.284 & 15.522 & 13.819 & 18.775 \\
\hline
\end{tabular}


Table A.3 - continued.

\begin{tabular}{|c|c|c|c|c|c|c|c|c|c|c|c|c|c|c|c|c|c|}
\hline & & & $\tilde{\tau}_{\text {bu }}$ & & $\mathrm{bu}$ & 0.15 & 0.45 & 0.75 & 1.50 & 2.25 & 3.00 & 4.50 & 6.00 & 9.00 & 15.00 & 30.00 & $\infty$ \\
\hline Star & $\Sigma_{0}\left[\mathrm{~g} \mathrm{~cm}^{-2}\right]$ & $\cos i$ & ang. type & $\Delta R_{\mathrm{bu}}^{\infty}$ & $\xi_{\mathrm{bu}}^{R}$ & $\xi_{\mathrm{d}}^{R}$ & & & & & & & & & & & \\
\hline S3 & 0.56 & 0.0 & edge-on & 0.133 & 2.1 & 6.909 & 4.034 & 3.269 & 2.46 & 2.172 & 1.958 & 1.729 & 1.549 & 1.415 & 1.241 & 1.112 & 1.12 \\
\hline S3 & 0.56 & 0.071 & edge-on & 0.099 & 2.323 & 5.869 & 3.63 & 2.973 & 2.262 & 1.961 & 1.841 & 1.596 & 1.416 & 1.324 & 1.149 & 1.079 & 1.022 \\
\hline S3 & 0.56 & 0.143 & interm. & 0.047 & 5.323 & 6.27 & 3.823 & 3.064 & 2.564 & 2.174 & 2.046 & 1.782 & 1.633 & 1.465 & 1.314 & 1.17 & 1.137 \\
\hline S3 & 0.56 & 0.215 & interm. & -0.006 & 98.48 & 48.064 & 32.346 & 18.991 & 42.695 & 131.568 & 101.633 & 177.814 & 325.464 & 273.318 & 306.937 & 326.084 & 373.702 \\
\hline S3 & 0.56 & 0.286 & interm. & -0.068 & 0.981 & 328.351 & 275.117 & 50.522 & 15.956 & 11.161 & 9.296 & 7.426 & 7.616 & 5.605 & 4.98 & 4.885 & 5.381 \\
\hline S3 & 0.56 & 0.357 & interm. & -0.12 & 1.845 & 126.827 & 19.059 & 13.57 & 9.561 & 8.011 & 7.282 & 6.532 & 6.56 & 5.664 & 5.161 & 4.765 & 5.429 \\
\hline S3 & 0.56 & 0.429 & pole-on & $\mid-0.167$ & 2.715 & 32.915 & 17.008 & 13.465 & 10.08 & 8.868 & 8.113 & 7.374 & 7.344 & 6.424 & 5.999 & 5.654 & 6.194 \\
\hline S3 & 0.56 & 0.5 & pole-on & -0.207 & 3.784 & 32.283 & 18.408 & 15.034 & 11.622 & 9.911 & 9.269 & 8.435 & 8.506 & 7.481 & 7.037 & 6.566 & 7.439 \\
\hline S3 & 0.56 & 0.571 & pole-on & -0.239 & 4.983 & 34.506 & 20.734 & 17.3 & 13.187 & 11.722 & 10.798 & 9.734 & 9.922 & 8.661 & 8.151 & 7.665 & 8.782 \\
\hline S3 & 0.56 & 0.643 & pole-on & -0.265 & 6.506 & 39.788 & 24.248 & 20.008 & 15.64 & 13.616 & 12.572 & 11.355 & 11.621 & 10.078 & 9.502 & 8.942 & 10.536 \\
\hline S3 & 0.56 & 0.714 & pole-on & -0.286 & 8.105 & 44.793 & 28.466 & 23.614 & 17.874 & 15.787 & 14.458 & 12.903 & 13.746 & 11.585 & 11.012 & 10.262 & 12.46 \\
\hline S3 & 0.56 & 0.786 & pole-on & $\mid-0.301$ & 9.968 & 54.121 & 33.28 & 27.307 & 20.79 & 18.063 & 16.659 & 14.915 & 15.71 & 13.025 & 12.526 & 11.637 & 14.831 \\
\hline S3 & 0.56 & 0.857 & pole-on & -0.313 & 11.856 & 61.256 & 38.49 & 32.436 & 23.579 & 20.393 & 18.695 & 16.754 & 18.111 & 14.394 & 14.281 & 13.066 & 17.49 \\
\hline S3 & 0.56 & 0.928 & pole-on & -0.32 & 13.922 & 70.824 & 44.232 & 37.067 & 26.438 & 22.53 & 20.286 & 18.873 & 20.888 & 15.957 & 16.164 & 14.451 & 20.506 \\
\hline S3 & 0.56 & 1.0 & pole-on & $\mid-0.321$ & 15.411 & 79.81 & 49.902 & 41.553 & 28.885 & 24.547 & 22.101 & 20.728 & 23.474 & 17.186 & 17.873 & 15.712 & 23.55 \\
\hline S3 & 0.41 & 0.0 & edge-on & 0.105 & 2.427 & 8.698 & 4.803 & 3.771 & 2.964 & 2.414 & 2.245 & 1.981 & 1.757 & 1.638 & 1.413 & 1.304 & 1.214 \\
\hline S3 & 0.41 & 0.071 & edge-on & 0.08 & 2.71 & 7.933 & 4.574 & 3.501 & 2.755 & 2.301 & 2.111 & 1.854 & 1.712 & 1.528 & 1.357 & 1.241 & 1.17 \\
\hline S3 & 0.41 & 0.143 & interm. & 0.043 & 7.445 & 10.59 & 4.949 & 4.085 & 3.28 & 2.735 & 2.519 & 2.333 & 2.201 & 2.01 & 1.766 & 1.552 & 1.555 \\
\hline S3 & 0.41 & 0.215 & interm. & 0.007 & 333.075 & 59.533 & 17.523 & 10.113 & 19.306 & 18.251 & 43.639 & 44.982 & 47.865 & 36.603 & 45.399 & 97.2 & 128.88 \\
\hline S3 & 0.41 & 0.286 & interm. & -0.036 & 1.278 & 267.83 & 329.431 & 251.665 & 89.12 & 33.193 & 8.92 & 6.602 & 6.563 & 4.511 & 3.538 & 3.435 & 4.308 \\
\hline S3 & 0.41 & 0.357 & interm. & -0.074 & 1.731 & 251.3 & 21.281 & 14.2 & 8.071 & 7.318 & 6.136 & 5.604 & 5.601 & 4.801 & 4.267 & 3.859 & 4.731 \\
\hline S3 & 0.41 & 0.429 & pole-on & -0.108 & 2.579 & 39.259 & 16.665 & 13.197 & 9.651 & 8.31 & 7.476 & 6.735 & 6.959 & 5.956 & 5.462 & 5.043 & 5.949 \\
\hline S3 & 0.41 & 0.5 & pole-on & -0.136 & 3.614 & 32.946 & 17.615 & 14.937 & 11.161 & 9.844 & 9.137 & 8.122 & 8.172 & 7.207 & 6.807 & 6.195 & 7.421 \\
\hline S3 & 0.41 & 0.571 & pole-on & -0.159 & 4.768 & 35.288 & 21.558 & 18.008 & 13.371 & 11.717 & 10.635 & 9.721 & 9.995 & 8.772 & 8.182 & 7.561 & 9.213 \\
\hline S3 & 0.41 & 0.643 & pole-on & -0.178 & 6.198 & 40.287 & 24.982 & 20.951 & 15.965 & 13.964 & 12.866 & 11.551 & 12.067 & 10.519 & 9.543 & 9.117 & 11.116 \\
\hline S3 & 0.41 & 0.714 & pole-on & -0.193 & 7.859 & 46.985 & 30.09 & 24.682 & 18.787 & 16.835 & 15.104 & 13.226 & 14.173 & 11.819 & 11.318 & 10.544 & 13.699 \\
\hline S3 & 0.41 & 0.786 & pole-on & -0.204 & 9.64 & 54.519 & 35.35 & 29.793 & 22.211 & 18.767 & 17.637 & 15.292 & 16.683 & 13.502 & 13.028 & 12.066 & 16.398 \\
\hline S3 & 0.41 & 0.857 & pole-on & -0.211 & 11.548 & 64.428 & 41.225 & 34.702 & 25.58 & 21.807 & 19.61 & 17.421 & 19.65 & 15.194 & 14.98 & 13.736 & 19.8 \\
\hline S3 & 0.41 & 0.928 & pole-on & -0.215 & 13.925 & 75.523 & 49.161 & 40.439 & 28.423 & 24.155 & 21.648 & 19.874 & 23.044 & 16.652 & 16.903 & 15.248 & 23.614 \\
\hline S3 & 0.41 & 1.0 & pole-on & -0.215 & 15.405 & 84.909 & 54.794 & 46.141 & 31.872 & 26.266 & 23.612 & 22.097 & 26.136 & 18.345 & 18.971 & 16.761 & 27.18 \\
\hline S3 & 0.3 & 0.0 & edge-on & 0.083 & 2.738 & 11.146 & 5.776 & 4.479 & 3.536 & 2.827 & 2.522 & 2.266 & 2.023 & 1.836 & 1.588 & 1.478 & 1.405 \\
\hline S3 & 0.3 & 0.071 & edge-on & 0.065 & 2.946 & 11.526 & 5.577 & 4.296 & 3.334 & 2.714 & 2.506 & 2.195 & 1.965 & 1.755 & 1.585 & 1.431 & 1.352 \\
\hline S3 & 0.3 & 0.143 & interm. & 0.038 & 6.151 & 32.57 & 6.733 & 5.037 & 3.919 & 3.443 & 3.298 & 3.081 & 2.627 & 2.57 & 2.436 & 2.136 & 2.05 \\
\hline S3 & 0.3 & 0.215 & interm. & 0.011 & 258.681 & 108.756 & 35.583 & 27.638 & 28.321 & 10.69 & 28.17 & 12.691 & 20.98 & 24.884 & 47.17 & 38.056 & 33.244 \\
\hline S3 & 0.3 & 0.286 & interm. & -0.016 & 36.733 & 285.486 & 351.892 & 289.83 & 302.828 & 255.101 & 163.407 & 102.901 & 66.299 & 63.9 & 27.776 & 17.91 & 15.12 \\
\hline
\end{tabular}


Table A.3 - continued.

\begin{tabular}{|c|c|c|c|c|c|c|c|c|c|c|c|c|c|c|c|c|c|}
\hline & & & $\tilde{\tau}_{\mathrm{bu}}$ & & bu & 0.15 & 0.45 & 0.75 & 1.50 & 2.25 & 3.00 & 4.50 & 6.00 & 9.00 & 15.00 & 30.00 & $\infty$ \\
\hline Star & $\Sigma_{0}\left[\mathrm{~g} \mathrm{~cm}^{-2}\right]$ & $\cos i$ & ang. type & $\Delta R_{\mathrm{bu}}^{\infty}$ & $\xi_{\mathrm{bu}}^{R}$ & $\xi_{\mathrm{d}}^{R}$ & & & & & & & & & & & \\
\hline S3 & 0.3 & 0.357 & interm. & -0.044 & 6.538 & 299.593 & 89.591 & 16.511 & 6.79 & 6.159 & 4.706 & 4.767 & 4.52 & 3.574 & 3.429 & 2.947 & 3.449 \\
\hline S3 & 0.3 & 0.429 & pole-on & -0.068 & 2.34 & 102.95 & 15.375 & 11.219 & 8.182 & 7.11 & 5.985 & 5.7 & 5.962 & 4.873 & 4.494 & 4.36 & 5.056 \\
\hline S3 & 0.3 & 0.5 & pole-on & -0.087 & 3.465 & 33.147 & 16.447 & 13.274 & 9.85 & 8.817 & 7.88 & 7.414 & 7.474 & 6.363 & 6.12 & 5.672 & 6.49 \\
\hline S3 & 0.3 & 0.571 & pole-on & -0.102 & 4.59 & 33.046 & 19.643 & 16.391 & 12.28 & 11.167 & 9.785 & 9.102 & 9.416 & 7.866 & 7.668 & 7.049 & 8.585 \\
\hline S3 & 0.3 & 0.643 & pole-on & -0.116 & 6.123 & 40.372 & 24.034 & 20.057 & 15.284 & 13.374 & 12.032 & 10.924 & 11.483 & 9.675 & 9.183 & 8.747 & 10.611 \\
\hline S3 & 0.3 & 0.714 & pole-on & -0.126 & 7.57 & 47.655 & 30.016 & 24.782 & 17.844 & 16.102 & 14.574 & 13.131 & 13.679 & 11.363 & 10.898 & 10.273 & 13.022 \\
\hline S3 & 0.3 & 0.786 & pole-on & -0.133 & 9.682 & 57.568 & 35.664 & 29.712 & 21.445 & 19.029 & 17.342 & 15.256 & 16.567 & 12.954 & 12.82 & 11.781 & 16.479 \\
\hline S3 & 0.3 & 0.857 & pole-on & -0.137 & 11.867 & 67.902 & 42.214 & 35.192 & 25.556 & 22.08 & 19.388 & 17.999 & 19.594 & 14.977 & 15.107 & 13.587 & 20.005 \\
\hline S3 & 0.3 & 0.928 & pole-on & -0.14 & 13.972 & 78.273 & 49.504 & 41.57 & 28.276 & 24.006 & 21.262 & 20.408 & 23.851 & 16.182 & 17.133 & 14.933 & 24.394 \\
\hline S3 & 0.3 & 1.0 & pole-on & -0.138 & 15.822 & 91.102 & 57.142 & 47.459 & 31.915 & 26.726 & 23.673 & 23.09 & 26.81 & 18.255 & 19.193 & 17.09 & 28.878 \\
\hline
\end{tabular}


Table A.4 - Empirical law parameters for band $I$.

\begin{tabular}{|c|c|c|c|c|c|c|c|c|c|c|c|c|c|c|c|c|c|}
\hline & & & 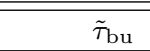 & & $\overline{\mathrm{bu}}$ & $\overline{0.15}$ & 0.45 & 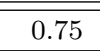 & 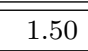 & 2.25 & 3.00 & 4.50 & 6.00 & 9.00 & 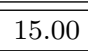 & 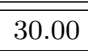 & $\bar{\infty}$ \\
\hline Star & $\Sigma_{0}\left[\mathrm{~g} \mathrm{~cm}^{-2}\right]$ & $\cos i$ & ang. type & $\Delta I_{\mathrm{bu}}^{\infty}$ & $\xi_{\mathrm{bu}}^{I}$ & $\xi_{\mathrm{d}}^{I}$ & & & & & & & & & & & \\
\hline S1 & 2.5 & 0.0 & edge-on & 0.454 & 1.063 & 1.4 & 1.252 & 1.121 & 0.938 & 0.839 & 0.784 & 0.697 & 0.648 & 0.579 & 0.5 & 0.45 & 0.432 \\
\hline S1 & 2.5 & 0.071 & edge-on & 0.12 & 21.853 & 1.984 & 0.539 & 0.486 & 0.388 & 0.314 & 0.307 & 0.263 & 0.232 & 0.215 & 0.212 & 0.207 & 0.203 \\
\hline S1 & 2.5 & 0.143 & interm. & -0.293 & 1.018 & 15.196 & 5.665 & 4.093 & 2.891 & 2.515 & 2.124 & 1.799 & 1.665 & 1.441 & 1.226 & 1.063 & 1.043 \\
\hline $\mathrm{S} 1$ & 2.5 & 0.215 & interm. & -0.569 & 1.8 & 6.039 & 3.318 & 2.664 & 2.013 & 1.753 & 1.532 & 1.303 & 1.21 & 1.063 & 0.911 & 0.79 & 0.78 \\
\hline S1 & 2.5 & 0.286 & interm. & -0.77 & 2.737 & 5.139 & 3.12 & 2.5 & 1.9 & 1.638 & 1.446 & 1.234 & 1.141 & 1.007 & 0.868 & 0.756 & 0.868 \\
\hline S1 & 2.5 & 0.357 & pole-on & -0.925 & 3.853 & 5.263 & 3.152 & 2.514 & 1.902 & 1.632 & 1.448 & 1.243 & 1.135 & 1.015 & 0.877 & 0.765 & 0.753 \\
\hline S1 & 2.5 & 0.429 & pole-on & -1.043 & 5.234 & 5.594 & 3.253 & 2.572 & 1.943 & 1.666 & 1.482 & 1.275 & 1.175 & 1.047 & 0.904 & 0.795 & 0.781 \\
\hline S1 & 2.5 & 0.5 & pole-on & -1.138 & 6.636 & 5.966 & 3.384 & 2.676 & 2.012 & 1.723 & 1.537 & 1.324 & 1.221 & 1.094 & 0.946 & 0.832 & 0.814 \\
\hline S1 & 2.5 & 0.571 & pole-on & -1.215 & 8.027 & 6.356 & 3.551 & 2.789 & 2.1 & 1.798 & 1.606 & 1.386 & 1.279 & 1.148 & 0.996 & 0.879 & 0.858 \\
\hline S1 & 2.5 & 0.643 & pole-on & -1.278 & 9.42 & 6.795 & 3.744 & 2.937 & 2.21 & 1.887 & 1.69 & 1.463 & 1.349 & 1.214 & 1.054 & 0.934 & 0.911 \\
\hline $\mathrm{S} 1$ & 2.5 & 0.714 & pole-on & -1.329 & 10.785 & 7.3 & 3.975 & 3.099 & 2.333 & 1.991 & 1.784 & 1.552 & 1.432 & 1.29 & 1.118 & 0.994 & 0.968 \\
\hline $\mathrm{S} 1$ & 2.5 & 0.786 & pole-on & -1.369 & 11.997 & 7.798 & 4.221 & 3.293 & 2.47 & 2.112 & 1.897 & 1.647 & 1.523 & 1.371 & 1.187 & 1.06 & 1.242 \\
\hline S1 & 2.5 & 0.857 & pole-on & \begin{tabular}{|l}
-1.402 \\
\end{tabular} & 13.18 & 8.419 & 4.494 & 3.499 & 2.62 & 2.239 & 2.009 & 1.755 & 1.621 & 1.461 & 1.258 & 1.124 & 1.11 \\
\hline S1 & 2.5 & 0.928 & pole-on & -1.428 & 14.278 & 9.06 & 4.806 & 3.727 & 2.785 & 2.38 & 2.14 & 1.871 & 1.725 & 1.545 & 1.34 & 1.194 & 1.206 \\
\hline S1 & 2.5 & 1.0 & pole-on & -1.447 & 15.159 & 9.688 & 5.102 & 3.948 & 2.954 & 2.52 & 2.264 & 1.978 & 1.82 & 1.631 & 1.421 & 1.277 & 1.269 \\
\hline S1 & 1.85 & 0.0 & edge-on & 0.376 & 1.057 & 1.527 & 1.391 & 1.221 & 1.051 & 0.946 & 0.875 & 0.804 & 0.748 & 0.645 & 0.558 & 0.504 & 0.487 \\
\hline $\mathrm{S} 1$ & 1.85 & 0.071 & edge-on & 0.11 & 1.211 & 13.371 & 0.598 & 0.558 & 0.456 & 0.394 & 0.361 & 0.316 & 0.281 & 0.225 & 0.209 & 0.205 & 0.206 \\
\hline $\mathrm{S} 1$ & 1.85 & 0.143 & interm. & -0.23 & 0.98 & 27.056 & 9.613 & 6.069 & 4.224 & 3.401 & 3.017 & 2.374 & 2.253 & 2.178 & 1.89 & 1.599 & 1.543 \\
\hline S1 & 1.85 & 0.215 & interm. & -0.477 & 1.698 & 9.667 & 4.557 & 3.536 & 2.688 & 2.266 & 2.034 & 1.698 & 1.613 & 1.5 & 1.296 & 1.132 & 1.086 \\
\hline S1 & 1.85 & 0.286 & interm. & -0.666 & 2.549 & 7.51 & 4.16 & 3.266 & 2.502 & 2.119 & 1.92 & 1.617 & 1.526 & 1.41 & 1.218 & 1.079 & 1.039 \\
\hline S1 & 1.85 & 0.357 & pole-on & -0.813 & 3.598 & 7.418 & 4.19 & 3.3 & 2.505 & 2.126 & 1.922 & 1.651 & 1.547 & 1.422 & 1.228 & 1.096 & 1.055 \\
\hline $\mathrm{S} 1$ & 1.85 & 0.429 & pole-on & -0.928 & 4.876 & 7.79 & 4.335 & 3.399 & 2.573 & 2.187 & 1.982 & 1.715 & 1.602 & 1.457 & 1.273 & 1.135 & 1.096 \\
\hline $\mathrm{S} 1$ & 1.85 & 0.5 & pole-on & -1.017 & 6.34 & 8.279 & 4.547 & 3.56 & 2.684 & 2.287 & 2.075 & 1.803 & 1.681 & 1.534 & 1.335 & 1.191 & 1.152 \\
\hline $\mathrm{S} 1$ & 1.85 & 0.571 & pole-on & -1.09 & 7.787 & 8.817 & 4.815 & 3.741 & 2.82 & 2.404 & 2.184 & 1.91 & 1.775 & 1.617 & 1.407 & 1.265 & 1.22 \\
\hline $\mathrm{S} 1$ & 1.85 & 0.643 & pole-on & -1.151 & 9.238 & 9.437 & 5.109 & 3.974 & 2.995 & 2.548 & 2.319 & 2.031 & 1.89 & 1.722 & 1.506 & 1.343 & 1.306 \\
\hline $\mathrm{S} 1$ & 1.85 & 0.714 & pole-on & -1.2 & 10.659 & 10.138 & 5.44 & 4.242 & 3.185 & 2.722 & 2.472 & 2.18 & 2.014 & 1.834 & 1.592 & 1.434 & 1.393 \\
\hline $\mathrm{S} 1$ & 1.85 & 0.786 & pole-on & -1.239 & 11.945 & 10.899 & 5.835 & 4.534 & 3.403 & 2.906 & 2.642 & 2.329 & 2.152 & 1.952 & 1.702 & 1.542 & 1.503 \\
\hline $\mathrm{S} 1$ & 1.85 & 0.857 & pole-on & -1.27 & 13.224 & 11.762 & 6.251 & 4.867 & 3.649 & 3.113 & 2.821 & 2.483 & 2.294 & 2.085 & 1.834 & 1.665 & 1.624 \\
\hline $\mathrm{S} 1$ & 1.85 & 0.928 & pole-on & -1.295 & 14.452 & 12.72 & 6.743 & 5.22 & 3.895 & 3.312 & 3.012 & 2.656 & 2.453 & 2.237 & 1.982 & 1.8 & 1.758 \\
\hline $\mathrm{S} 1$ & 1.85 & 1.0 & pole-on & -1.313 & 15.43 & 13.716 & 7.204 & 5.565 & 4.121 & 3.496 & 3.194 & 2.834 & 2.618 & 2.402 & 2.128 & 1.939 & 1.897 \\
\hline $\mathrm{S} 1$ & 1.37 & 0.0 & edge-on & 0.301 & 1.077 & 1.777 & 1.637 & 1.427 & 1.198 & 1.06 & 0.964 & 0.88 & 0.812 & 0.734 & 0.637 & 0.574 & 0.562 \\
\hline S1 & 1.37 & 0.071 & edge-on & 0.097 & 1.136 & 5.749 & 0.748 & 0.69 & 0.547 & 0.499 & 0.409 & 0.363 & 0.336 & 0.295 & 0.239 & 0.228 & 0.211 \\
\hline $\mathrm{S} 1$ & 1.37 & 0.143 & interm. & -0.177 & 0.966 & 49.409 & 15.458 & 9.703 & 6.163 & 4.976 & 4.504 & 3.984 & 3.513 & 3.179 & 2.777 & 2.453 & 2.414 \\
\hline S1 & 1.37 & 0.215 & interm. & -0.398 & 1.562 & 15.069 & 6.292 & 4.759 & 3.553 & 2.99 & 2.801 & 2.457 & 2.207 & 2.046 & 1.796 & 1.594 & 1.548 \\
\hline S1 & 1.37 & 0.286 & interm. & -0.571 & 2.324 & 10.896 & 5.49 & 4.308 & 3.297 & 2.785 & 2.613 & 2.288 & 2.072 & 1.921 & 1.66 & 1.514 & 1.465 \\
\hline
\end{tabular}


Table A.4 - continued.

\begin{tabular}{|c|c|c|c|c|c|c|c|c|c|c|c|c|c|c|c|c|c|}
\hline & & & $\tilde{\tau}_{\mathrm{bu}}$ & & $\mathrm{bu}$ & 0.15 & 0.45 & 0.75 & 1.50 & 2.25 & 3.00 & 4.50 & 6.00 & 9.00 & 15.00 & 30.00 & $\infty$ \\
\hline Star & $\Sigma_{0}\left[\mathrm{~g} \mathrm{~cm}^{-2}\right]$ & $\cos i$ & ang. type & $\Delta I_{\mathrm{bu}}^{\infty}$ & $\xi_{\mathrm{bu}}^{I}$ & $\xi_{\mathrm{d}}^{I}$ & & & & & & & & & & & \\
\hline S1 & 1.37 & 0.357 & pole-on & -0.709 & 3.299 & 10.117 & 5.51 & 4.366 & 3.33 & 2.825 & 2.643 & 2.318 & 2.107 & 1.947 & 1.689 & 1.539 & 1.493 \\
\hline $\mathrm{S} 1$ & 1.37 & 0.429 & pole-on & -0.817 & 4.551 & 10.475 & 5.758 & 4.556 & 3.468 & 2.951 & 2.742 & 2.422 & 2.197 & 2.029 & 1.766 & 1.611 & 1.563 \\
\hline S1 & 1.37 & 0.5 & pole-on & -0.902 & 5.972 & 11.121 & 6.086 & 4.813 & 3.652 & 3.113 & 2.884 & 2.552 & 2.332 & 2.144 & 1.863 & 1.702 & 1.672 \\
\hline S1 & 1.37 & 0.571 & pole-on & -0.972 & 7.447 & 11.951 & 6.466 & 5.12 & 3.872 & 3.318 & 3.059 & 2.714 & 2.487 & 2.287 & 1.992 & 1.818 & 1.797 \\
\hline $\mathrm{S} 1$ & 1.37 & 0.643 & pole-on & -1.029 & 8.994 & 12.839 & 6.946 & 5.489 & 4.156 & 3.565 & 3.271 & 2.902 & 2.67 & 2.449 & 2.147 & 1.968 & 1.95 \\
\hline $\mathrm{S} 1$ & 1.37 & 0.714 & pole-on & -1.075 & 10.506 & 13.896 & 7.482 & 5.907 & 4.446 & 3.834 & 3.497 & 3.109 & 2.87 & 2.651 & 2.33 & 2.142 & 2.122 \\
\hline S1 & 1.37 & 0.786 & pole-on & -1.112 & 11.955 & 15.041 & 8.086 & 6.374 & 4.761 & 4.116 & 3.739 & 3.34 & 3.08 & 2.873 & 2.532 & 2.334 & 2.32 \\
\hline $\mathrm{S} 1$ & 1.37 & 0.857 & pole-on & -1.141 & 13.452 & 16.35 & 8.745 & 6.882 & 5.094 & 4.774 & 4.023 & 3.618 & 3.316 & 3.125 & 2.758 & 2.548 & 2.625 \\
\hline S1 & 1.37 & 0.928 & pole-on & -1.164 & 14.701 & 17.828 & 9.432 & 7.363 & 5.482 & 4.722 & 4.348 & 3.904 & 3.599 & 3.388 & 3.008 & 2.782 & 2.775 \\
\hline S1 & 1.37 & 1.0 & pole-on & -1.181 & 15.853 & 19.246 & 10.073 & 7.866 & 5.89 & 5.081 & 4.666 & 4.204 & 3.89 & 3.66 & 3.258 & 3.013 & 3.034 \\
\hline $\mathrm{S} 1$ & 1.01 & 0.0 & edge-on & 0.244 & 1.061 & 2.177 & 1.833 & 1.608 & 2.685 & 1.223 & 1.099 & 0.997 & 0.926 & 0.85 & 0.688 & 0.67 & 0.668 \\
\hline S1 & 1.01 & 0.071 & edge-on & 0.09 & 0.958 & 6.421 & 0.772 & 0.77 & 3.086 & 0.593 & 0.506 & 0.451 & 0.432 & 0.392 & 0.32 & 0.306 & 0.276 \\
\hline S1 & 1.01 & 0.143 & interm. & -0.12 & 1.188 & 194.001 & 35.226 & 20.488 & 312.754 & 8.72 & 7.534 & 6.487 & 5.675 & 5.62 & 3.921 & 3.932 & 4.218 \\
\hline S1 & 1.01 & 0.215 & interm. & -0.304 & 1.642 & 24.275 & 9.95 & 7.013 & 7.88 & 4.182 & 3.811 & 3.415 & 3.098 & 2.873 & 2.46 & 2.227 & 2.167 \\
\hline S1 & 1.01 & 0.286 & interm. & -0.458 & 2.326 & 15.77 & 7.939 & 5.983 & 6.394 & 3.807 & 3.475 & 3.145 & 2.85 & 2.607 & 2.349 & 2.075 & 2.016 \\
\hline S1 & 1.01 & 0.357 & pole-on & -0.585 & 3.201 & 14.069 & 7.762 & 6.012 & 6.035 & 3.866 & 3.546 & 3.19 & 2.931 & 2.639 & 2.448 & 2.144 & 2.065 \\
\hline S1 & 1.01 & 0.429 & pole-on & -0.689 & 4.321 & 14.238 & 8.061 & 6.26 & 6.018 & 4.07 & 3.724 & 3.364 & 3.071 & 2.771 & 2.621 & 2.282 & 2.188 \\
\hline S1 & 1.01 & 0.5 & pole-on & -0.768 & 5.733 & 15.057 & 8.507 & 6.652 & 6.191 & 4.332 & 3.963 & 3.578 & 3.287 & 2.963 & 2.833 & 2.48 & 2.344 \\
\hline S1 & 1.01 & 0.571 & pole-on & -0.834 & 7.235 & 16.231 & 9.119 & 7.126 & 6.456 & 4.627 & 4.254 & 3.837 & 3.532 & 3.181 & 3.083 & 2.702 & 2.536 \\
\hline $\mathrm{S} 1$ & 1.01 & 0.643 & pole-on & -0.888 & 8.849 & 17.579 & 9.815 & 7.679 & 6.776 & 4.995 & 4.578 & 4.167 & 3.835 & 3.486 & 3.364 & 2.973 & 2.769 \\
\hline $\mathrm{S} 1$ & 1.01 & 0.714 & pole-on & -0.932 & 10.459 & 19.158 & 10.64 & 8.291 & 7.192 & 5.433 & 4.952 & 4.544 & 4.196 & 3.831 & 3.688 & 3.275 & 3.048 \\
\hline S1 & 1.01 & 0.786 & pole-on & -0.967 & 12.031 & 20.903 & 11.497 & 8.933 & 7.625 & 5.896 & 5.386 & 4.951 & 4.586 & 4.218 & 4.036 & 3.613 & 3.347 \\
\hline $\mathrm{S} 1$ & 1.01 & 0.857 & pole-on & -0.995 & 13.586 & 22.838 & 12.447 & 9.707 & 8.092 & 6.425 & 5.868 & 5.409 & 5.028 & 4.656 & 4.404 & 3.97 & 3.715 \\
\hline $\mathrm{S} 1$ & 1.01 & 0.928 & pole-on & -1.017 & 15.027 & 25.077 & 13.568 & 10.567 & 8.608 & 7.001 & 6.386 & 5.896 & 5.506 & 5.132 & 4.785 & 4.344 & 4.118 \\
\hline S1 & 1.01 & 1.0 & pole-on & \begin{tabular}{|l}
-1.032 \\
\end{tabular} & 16.238 & 27.287 & 14.704 & 11.484 & 9.101 & 7.565 & 6.885 & 6.381 & 6.004 & 5.626 & 5.205 & 4.736 & 4.562 \\
\hline $\mathrm{S} 1$ & 0.75 & 0.0 & edge-on & 0.19 & 1.18 & 2.62 & 2.032 & 1.781 & 1.519 & 1.353 & 1.261 & 1.121 & 1.046 & 0.953 & 0.834 & 0.755 & 0.741 \\
\hline $\mathrm{S} 1$ & 0.75 & 0.071 & edge-on & 0.074 & 12.03 & 17.98 & 0.979 & 0.87 & 0.773 & 0.683 & 0.652 & 0.568 & 0.511 & 0.469 & 0.406 & 0.371 & 0.315 \\
\hline S1 & 0.75 & 0.143 & interm. & -0.085 & 1.082 & 350.767 & 105.624 & 51.595 & 26.323 & 19.517 & 15.252 & 12.489 & 10.898 & 9.179 & 8.009 & 6.858 & 7.292 \\
\hline $\mathrm{S} 1$ & 0.75 & 0.215 & interm. & -0.235 & 1.5 & 41.868 & 16.12 & 11.146 & 7.482 & 6.187 & 5.402 & 4.787 & 4.41 & 4.006 & 3.494 & 3.201 & 3.194 \\
\hline S1 & 0.75 & 0.286 & interm. & -0.366 & 2.102 & 24.097 & 11.232 & 8.452 & 6.08 & 5.222 & 4.688 & 4.216 & 3.904 & 3.593 & 3.166 & 2.907 & 2.933 \\
\hline S1 & 0.75 & 0.357 & pole-on & -0.479 & 2.891 & 19.926 & 10.418 & 8.131 & 6.025 & 5.248 & 4.725 & 4.291 & 3.984 & 3.672 & 3.272 & 2.987 & 3.083 \\
\hline S1 & 0.75 & 0.429 & pole-on & -0.572 & 3.848 & 19.543 & 10.738 & 8.43 & 6.323 & 5.524 & 5.003 & 4.535 & 4.241 & 3.928 & 3.527 & 3.237 & 3.334 \\
\hline $\mathrm{S} 1$ & 0.75 & 0.5 & pole-on & -0.647 & 4.963 & 20.183 & 11.386 & 8.959 & 6.765 & 5.912 & 5.386 & 4.894 & 4.601 & 4.288 & 3.876 & 3.56 & 3.66 \\
\hline S1 & 0.75 & 0.571 & pole-on & \begin{tabular}{|l}
-0.709 \\
\end{tabular} & 6.074 & 21.805 & 12.272 & 9.673 & 7.296 & 6.428 & 5.853 & 5.347 & 5.017 & 4.698 & 4.251 & 3.939 & 4.059 \\
\hline S1 & 0.75 & 0.643 & pole-on & -0.76 & 7.184 & 23.721 & 13.318 & 10.553 & 7.998 & 7.037 & 6.419 & 5.874 & 5.554 & 5.178 & 4.71 & 4.367 & 4.508 \\
\hline
\end{tabular}


Table A.4 - continued.

\begin{tabular}{|c|c|c|c|c|c|c|c|c|c|c|c|c|c|c|c|c|c|}
\hline & & & $\tilde{\tau}_{\mathrm{bu}}$ & & $\mathrm{bu}$ & 0.15 & 0.45 & 0.75 & 1.50 & 2.25 & 3.00 & 4.50 & 6.00 & 9.00 & 15.00 & 30.00 & $\infty$ \\
\hline Star & $\Sigma_{0}\left[\mathrm{~g} \mathrm{~cm}^{-2}\right]$ & $\cos i$ & ang. type & $\Delta I_{\mathrm{bu}}^{\infty}$ & $\xi_{\mathrm{bu}}^{I}$ & $\xi_{\mathrm{d}}^{I}$ & & & & & & & & & & & \\
\hline S1 & 0.75 & 0.714 & pole-on & -0.801 & 8.261 & 26.17 & 14.523 & 11.528 & 8.762 & 7.733 & 7.056 & 6.467 & 6.137 & 5.692 & 5.195 & 4.827 & 5.001 \\
\hline S1 & 0.75 & 0.786 & pole-on & -0.833 & 9.254 & 28.757 & 15.994 & 12.675 & 9.628 & 8.49 & 7.769 & 7.179 & 6.781 & 6.271 & 5.745 & 5.354 & 5.602 \\
\hline S1 & 0.75 & 0.857 & pole-on & -0.858 & 10.177 & 31.976 & 17.593 & 14.019 & 10.591 & 9.314 & 8.538 & 7.818 & 7.492 & 6.88 & 6.332 & 5.906 & 6.188 \\
\hline S1 & 0.75 & 0.928 & pole-on & -0.877 & 11.04 & 35.398 & 19.436 & 15.508 & 11.642 & 10.151 & 9.291 & 8.585 & 8.26 & 7.496 & 6.98 & 6.455 & 6.876 \\
\hline S1 & 0.75 & 1.0 & pole-on & -0.89 & 11.737 & 38.964 & 21.295 & 16.981 & 12.647 & 10.982 & 10.011 & 9.352 & 9.026 & 8.104 & 7.611 & 7.023 & 7.566 \\
\hline S1 & 0.56 & 0.0 & edge-on & 0.15 & 1.225 & 3.699 & 2.573 & 2.222 & 1.806 & 1.55 & 1.445 & 1.312 & 1.213 & 1.101 & 0.977 & 0.883 & 0.853 \\
\hline S1 & 0.56 & 0.071 & edge-on & 0.066 & 1.019 & 7.318 & 1.449 & 1.196 & 0.969 & 0.849 & 0.79 & 0.715 & 0.634 & 0.608 & 0.49 & 0.461 & 0.41 \\
\hline S1 & 0.56 & 0.143 & interm. & -0.051 & 1.928 & 381.264 & 376.479 & 318.794 & 140.186 & 63.793 & 43.878 & 34.391 & 30.578 & 22.931 & 22.407 & 17.355 & 17.879 \\
\hline S1 & 0.56 & 0.215 & interm. & -0.169 & 1.753 & 76.124 & 25.621 & 17.641 & 11.45 & 9.317 & 8.209 & 7.028 & 6.62 & 5.953 & 5.379 & 4.908 & 4.769 \\
\hline S1 & 0.56 & 0.286 & interm. & -0.276 & 2.23 & 36.449 & 15.365 & 11.395 & 8.156 & 7.095 & 6.375 & 5.645 & 5.381 & 4.882 & 4.482 & 4.096 & 4.082 \\
\hline S1 & 0.56 & 0.357 & pole-on & -0.37 & 2.938 & 26.9 & 13.474 & 10.485 & 7.873 & 6.861 & 6.332 & 5.633 & 5.412 & 4.911 & 4.563 & 4.212 & 4.204 \\
\hline S1 & 0.56 & 0.429 & pole-on & -0.451 & 3.856 & 24.868 & 13.617 & 10.793 & 8.291 & 7.261 & 6.708 & 6.009 & 5.809 & 5.317 & 4.931 & 4.553 & 4.619 \\
\hline S1 & 0.56 & 0.5 & pole-on & -0.518 & 4.976 & 25.507 & 14.452 & 11.627 & 8.929 & 7.887 & 7.26 & 6.547 & 6.34 & 5.819 & 5.395 & 5.014 & 5.123 \\
\hline S1 & 0.56 & 0.571 & pole-on & -0.572 & 6.317 & 27.646 & 15.744 & 12.676 & 9.81 & 8.63 & 8.038 & 7.251 & 7.028 & 6.437 & 6.025 & 5.563 & 5.744 \\
\hline S1 & 0.56 & 0.643 & pole-on & -0.617 & 7.779 & 30.497 & 17.428 & 13.986 & 10.843 & 9.631 & 8.851 & 8.009 & 7.795 & 7.143 & 6.659 & 6.221 & 6.452 \\
\hline $\mathrm{S} 1$ & 0.56 & 0.714 & pole-on & -0.654 & 9.35 & 33.969 & 19.37 & 15.662 & 12.038 & 10.638 & 9.811 & 8.858 & 8.684 & 7.923 & 7.39 & 6.884 & 7.269 \\
\hline S1 & 0.56 & 0.786 & pole-on & -0.682 & 11.087 & 37.998 & 21.666 & 17.489 & 13.429 & 11.789 & 10.847 & 9.821 & 9.686 & 8.747 & 8.217 & 7.608 & 8.145 \\
\hline S1 & 0.56 & 0.857 & pole-on & -0.704 & 12.776 & 42.524 & 24.218 & 19.527 & 14.819 & 12.987 & 11.954 & 10.859 & 10.792 & 9.562 & 9.06 & 8.382 & 9.139 \\
\hline $\mathrm{S} 1$ & 0.56 & 0.928 & pole-on & -0.72 & 14.442 & 47.534 & 27.139 & 21.745 & 16.371 & 14.201 & 12.943 & 11.906 & 12.031 & 10.408 & 9.997 & 9.144 & 10.252 \\
\hline S1 & 0.56 & 1.0 & pole-on & -0.73 & 15.886 & 52.197 & 29.865 & 24.07 & 17.791 & 15.26 & 13.935 & 12.984 & 13.188 & 11.188 & 10.878 & 9.93 & 11.384 \\
\hline S1 & 0.41 & 0.0 & edge-on & 0.118 & 1.42 & 5.001 & 3.209 & 2.676 & 2.154 & 1.908 & 1.74 & 2.565 & 1.439 & 1.33 & 1.114 & 1.057 & 1.005 \\
\hline S1 & 0.41 & 0.071 & edge-on & 0.058 & 1.26 & 7.226 & 1.847 & 1.485 & 1.222 & 1.203 & 1.07 & 0.923 & 0.817 & 0.803 & 0.663 & 0.587 & 0.56 \\
\hline S1 & 0.41 & 0.143 & interm. & -0.024 & 19.26 & 369.438 & 357.644 & 393.141 & 351.139 & 357.482 & 353.622 & 302.004 & 283.801 & 265.512 & 222.534 & 194.568 & 207.02 \\
\hline S1 & 0.41 & 0.215 & interm. & -0.113 & 1.817 & 312.347 & 52.736 & 32.552 & 20.401 & 15.359 & 13.298 & 11.684 & 10.861 & 9.077 & 8.346 & 7.607 & 7.534 \\
\hline S1 & 0.41 & 0.286 & interm. & -0.195 & 2.225 & 62.018 & 23.286 & 16.296 & 11.517 & 9.453 & 8.617 & 7.674 & 7.429 & 6.519 & 5.956 & 5.614 & 5.702 \\
\hline S1 & 0.41 & 0.357 & pole-on & -0.268 & 2.85 & 39.753 & 18.258 & 13.866 & 10.402 & 8.815 & 8.155 & 7.416 & 7.347 & 6.356 & 5.985 & 5.602 & 5.784 \\
\hline $\mathrm{S} 1$ & 0.41 & 0.429 & pole-on & -0.334 & 3.667 & 32.578 & 17.605 & 13.909 & 10.72 & 9.227 & 8.532 & 7.793 & 7.626 & 6.759 & 6.419 & 5.984 & 6.278 \\
\hline $\mathrm{S} 1$ & 0.41 & 0.5 & pole-on & -0.388 & 4.654 & 33.179 & 18.63 & 14.903 & 11.606 & 10.062 & 9.345 & 8.523 & 8.372 & 7.472 & 7.052 & 6.735 & 7.01 \\
\hline S1 & 0.41 & 0.571 & pole-on & -0.434 & 5.824 & 34.908 & 20.25 & 16.527 & 12.853 & 11.167 & 10.364 & 9.421 & 9.33 & 8.364 & 7.878 & 7.391 & 7.928 \\
\hline S1 & 0.41 & 0.643 & pole-on & -0.471 & 7.189 & 38.581 & 22.517 & 18.37 & 14.289 & 12.5 & 11.578 & 10.535 & 10.51 & 9.327 & 8.772 & 8.256 & 9.063 \\
\hline S1 & 0.41 & 0.714 & pole-on & -0.502 & 8.75 & 43.351 & 25.333 & 20.882 & 16.095 & 13.988 & 12.959 & 11.715 & 11.757 & 10.32 & 9.842 & 9.174 & 10.328 \\
\hline $\mathrm{S} 1$ & 0.41 & 0.786 & pole-on & -0.525 & 10.491 & 48.54 & 28.75 & 23.443 & 18.084 & 15.607 & 14.47 & 13.023 & 13.349 & 11.457 & 10.943 & 10.194 & 11.702 \\
\hline S1 & 0.41 & 0.857 & pole-on & -0.543 & 12.243 & 54.903 & 32.614 & 26.456 & 20.038 & 17.202 & 15.854 & 14.426 & 14.888 & 12.536 & 12.111 & 11.167 & 13.415 \\
\hline S1 & 0.41 & 0.928 & pole-on & -0.555 & 14.107 & 61.094 & 36.506 & 29.774 & 22.042 & 18.768 & 17.249 & 15.899 & 16.683 & 13.608 & 13.382 & 12.205 & 15.182 \\
\hline S1 & 0.41 & 1.0 & pole-on & -0.561 & 15.566 & 67.4 & 40.623 & 32.816 & 24.04 & 20.264 & 18.406 & 17.321 & 18.492 & 14.572 & 14.667 & 13.166 & 17.017 \\
\hline
\end{tabular}


Table A.4 - continued.

\begin{tabular}{|c|c|c|c|c|c|c|c|c|c|c|c|c|c|c|c|c|c|}
\hline & & & $\tilde{\tau}_{\mathrm{bu}}$ & & $\mathrm{bu}$ & 0.15 & 0.45 & 0.75 & 1.50 & 2.25 & 3.00 & 4.50 & 6.00 & 9.00 & 15.00 & 30.00 & $\infty$ \\
\hline Star & $\Sigma_{0}\left[\mathrm{~g} \mathrm{~cm}^{-2}\right]$ & $\cos i$ & ang. type & $\Delta I_{\mathrm{bu}}^{\infty}$ & $\xi_{\mathrm{bu}}^{I}$ & $\xi_{\mathrm{d}}^{I}$ & & & & & & & & & & & \\
\hline S1 & 0.3 & 0.0 & edge-on & 0.092 & 1.707 & 7.568 & 4.105 & 3.496 & 2.656 & 2.303 & 2.062 & 1.894 & 1.741 & 1.532 & 1.327 & 1.267 & 1.21 \\
\hline $\mathrm{S} 1$ & 0.3 & 0.071 & edge-on & 0.049 & 1.586 & 8.412 & 2.515 & 2.172 & 1.697 & 1.519 & 1.328 & 1.226 & 1.121 & 0.977 & 0.887 & 0.809 & 0.739 \\
\hline S1 & 0.3 & 0.143 & interm. & -0.006 & 262.998 & 257.732 & 207.881 & 87.268 & 147.275 & 153.166 & 130.709 & 269.441 & 209.981 & 190.875 & 284.33 & 292.273 & 354.845 \\
\hline S1 & 0.3 & 0.215 & interm. & -0.069 & 1.859 & 396.074 & 236.68 & 110.909 & 41.262 & 31.431 & 24.993 & 21.607 & 19.627 & 16.916 & 14.718 & 12.524 & 14.065 \\
\hline $\mathrm{S} 1$ & 0.3 & 0.286 & interm. & -0.129 & 2.179 & 215.382 & 37.138 & 24.661 & 15.45 & 12.908 & 11.356 & 10.226 & 9.904 & 8.659 & 7.837 & 7.128 & 7.936 \\
\hline S1 & 0.3 & 0.357 & pole-on & -0.183 & 2.751 & 60.082 & 24.264 & 17.647 & 12.707 & 10.745 & 10.037 & 8.913 & 8.908 & 7.856 & 7.28 & 6.734 & 7.325 \\
\hline S1 & 0.3 & 0.429 & pole-on & -0.232 & 3.488 & 43.245 & 22.176 & 16.803 & 12.704 & 11.053 & 10.214 & 9.237 & 9.257 & 8.209 & 7.724 & 7.192 & 7.91 \\
\hline $\mathrm{S} 1$ & 0.3 & 0.5 & pole-on & -0.273 & 4.304 & 40.066 & 22.831 & 17.971 & 13.596 & 11.966 & 11.141 & 10.156 & 10.269 & 9.011 & 8.435 & 7.918 & 8.907 \\
\hline S1 & 0.3 & 0.571 & pole-on & -0.307 & 5.368 & 41.783 & 24.477 & 19.909 & 15.235 & 13.367 & 12.394 & 11.355 & 11.433 & 10.063 & 9.526 & 8.862 & 10.097 \\
\hline S1 & 0.3 & 0.643 & pole-on & -0.336 & 6.594 & 46.368 & 27.482 & 22.245 & 17.147 & 15.059 & 13.931 & 12.499 & 12.951 & 11.282 & 10.618 & 10.028 & 11.656 \\
\hline $\mathrm{S} 1$ & 0.3 & 0.714 & pole-on & -0.359 & 7.96 & 51.09 & 31.072 & 25.5 & 19.415 & 16.995 & 15.611 & 13.963 & 14.822 & 12.564 & 11.967 & 11.101 & 13.472 \\
\hline S1 & 0.3 & 0.786 & pole-on & -0.377 & 9.498 & 57.514 & 35.559 & 29.133 & 22.032 & 19.194 & 17.58 & 15.74 & 16.833 & 13.897 & 13.356 & 12.313 & 15.538 \\
\hline S1 & 0.3 & 0.857 & pole-on & -0.39 & 11.176 & 64.91 & 40.502 & 33.482 & 24.631 & 21.068 & 19.246 & 17.429 & 18.969 & 15.252 & 14.897 & 13.509 & 18.006 \\
\hline S1 & 0.3 & 0.928 & pole-on & -0.398 & 12.903 & 73.11 & 46.018 & 37.939 & 26.893 & 22.956 & 20.727 & 19.14 & 21.514 & 16.277 & 16.478 & 14.759 & 20.714 \\
\hline S1 & 0.3 & 1.0 & pole-on & -0.4 & 14.353 & 80.199 & 50.037 & 41.993 & 29.199 & 24.651 & 22.154 & 20.954 & 23.719 & 17.483 & 18.02 & 15.85 & 23.311 \\
\hline $\mathrm{S} 2$ & 2.5 & 0.0 & edge-on & 0.427 & 1.019 & 1.518 & 1.332 & 1.2 & 1.034 & 0.917 & 0.842 & 0.752 & 0.703 & 0.638 & 0.56 & 0.502 & 0.476 \\
\hline $\mathrm{S} 2$ & 2.5 & 0.071 & edge-on & 0.145 & 1.35 & 0.716 & 0.688 & 0.624 & 0.514 & 0.448 & 0.394 & 0.337 & 0.315 & 0.268 & 0.238 & 0.217 & 0.208 \\
\hline S2 & 2.5 & 0.143 & interm. & -0.211 & 0.698 & 53.819 & 15.003 & 8.81 & 5.323 & 4.468 & 3.792 & 3.331 & 2.968 & 2.705 & 2.199 & 2.056 & 1.997 \\
\hline $\mathrm{S} 2$ & 2.5 & 0.215 & interm. & -0.467 & 1.428 & 11.553 & 4.895 & 3.771 & 2.776 & 2.435 & 2.176 & 1.921 & 1.756 & 1.61 & 1.352 & 1.249 & 1.209 \\
\hline $\mathrm{S} 2$ & 2.5 & 0.286 & interm. & -0.656 & 2.271 & 8.026 & 4.256 & 3.352 & 2.507 & 2.201 & 1.969 & 1.741 & 1.601 & 1.464 & 1.236 & 1.145 & 1.109 \\
\hline $\mathrm{S} 2$ & 2.5 & 0.357 & pole-on & -0.801 & 3.303 & 7.676 & 4.258 & 3.337 & 2.488 & 2.169 & 1.96 & 1.73 & 1.591 & 1.451 & 1.234 & 1.141 & 1.103 \\
\hline S2 & 2.5 & 0.429 & pole-on & -0.912 & 4.599 & 7.945 & 4.373 & 3.427 & 2.558 & 2.222 & 2.015 & 1.759 & 1.635 & 1.493 & 1.329 & 1.176 & 1.136 \\
\hline $\mathrm{S} 2$ & 2.5 & 0.5 & pole-on & -1.002 & 5.953 & 8.399 & 4.574 & 3.566 & 2.663 & 2.313 & 2.094 & 1.846 & 1.711 & 1.562 & 1.337 & 1.23 & 1.191 \\
\hline S2 & 2.5 & 0.571 & pole-on & -1.075 & 7.326 & 8.902 & 4.831 & 3.773 & 2.803 & 2.438 & 2.206 & 1.943 & 1.796 & 1.647 & 1.416 & 1.302 & 1.258 \\
\hline $\mathrm{S} 2$ & 2.5 & 0.643 & pole-on & -1.134 & 8.725 & 9.54 & 5.144 & 4.008 & 2.976 & 2.594 & 2.34 & 2.06 & 1.92 & 1.758 & 1.513 & 1.384 & 1.339 \\
\hline $\mathrm{S} 2$ & 2.5 & 0.714 & pole-on & -1.181 & 10.101 & 10.265 & 5.511 & 4.281 & 3.178 & 2.758 & 2.501 & 2.208 & 2.054 & 1.872 & 1.615 & 1.481 & 1.43 \\
\hline $\mathrm{S} 2$ & 2.5 & 0.786 & pole-on & -1.218 & 11.375 & 11.046 & 5.911 & 4.59 & 3.398 & 2.953 & 2.677 & 2.369 & 2.199 & 2.001 & 1.727 & 1.592 & 1.536 \\
\hline $\mathrm{S} 2$ & 2.5 & 0.857 & pole-on & -1.248 & 12.603 & 11.931 & 6.343 & 4.939 & 3.644 & 3.158 & 2.87 & 2.532 & 2.348 & 2.134 & 1.844 & 1.718 & 1.657 \\
\hline $\mathrm{S} 2$ & 2.5 & 0.928 & pole-on & -1.272 & 13.723 & 12.941 & 6.844 & 5.305 & 3.911 & 3.382 & 3.055 & 2.699 & 2.5 & 2.28 & 1.984 & 1.859 & 1.795 \\
\hline S2 & 2.5 & 1.0 & pole-on & -1.288 & 14.698 & 13.931 & 7.344 & 5.661 & 4.164 & 3.576 & 3.237 & 2.866 & 2.669 & 2.452 & 2.137 & 2.003 & 1.937 \\
\hline $\mathrm{S} 2$ & 1.85 & 0.0 & edge-on & 0.344 & 1.04 & 1.745 & 1.472 & 1.345 & 1.144 & 1.019 & 1.649 & 1.212 & 0.784 & 0.712 & 0.614 & 0.561 & 0.537 \\
\hline $\mathrm{S} 2$ & 1.85 & 0.071 & edge-on & 0.135 & 1.175 & 0.867 & 0.8 & 0.743 & 0.618 & 0.53 & 0.563 & 0.471 & 0.373 & 0.341 & 0.275 & 0.251 & 0.239 \\
\hline $\mathrm{S} 2$ & 1.85 & 0.143 & interm. & -0.146 & 0.769 & 309.93 & 38.392 & 18.141 & 9.437 & 6.731 & 9.322 & 6.918 & 4.602 & 4.285 & 3.672 & 3.306 & 3.169 \\
\hline $\mathrm{S} 2$ & 1.85 & 0.215 & interm. & -0.371 & 1.444 & 18.92 & 7.449 & 5.391 & 3.836 & 3.199 & 4.182 & 3.443 & 2.386 & 2.238 & 1.941 & 1.749 & 1.692 \\
\hline $\mathrm{S} 2$ & 1.85 & 0.286 & interm. & -0.547 & 2.192 & 11.748 & 5.9 & 4.584 & 3.399 & 2.902 & 3.528 & 2.885 & 2.176 & 2.012 & 1.761 & 1.589 & 1.548 \\
\hline
\end{tabular}


Table A.4 - continued.

\begin{tabular}{|c|c|c|c|c|c|c|c|c|c|c|c|c|c|c|c|c|c|}
\hline & & & 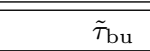 & & $\overline{\mathrm{bu}}$ & $\overline{0.15}$ & 0.45 & 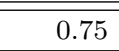 & 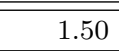 & 2.25 & 3.00 & 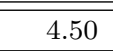 & 26.00 & 9.00 & 15.00 & 30.00 & $\bar{\infty}$ \\
\hline Star & $\Sigma_{0}\left[\mathrm{~g} \mathrm{~cm}^{-2}\right]$ & $\cos i$ & ang. type & $\Delta I_{\mathrm{bu}}^{\infty}$ & $\xi_{\mathrm{bu}}^{I}$ & $\xi_{\mathrm{d}}^{I}$ & & & & & & & & & & & \\
\hline S2 & 1.85 & 0.357 & pole-on & -0.685 & 3.181 & 10.582 & 5.785 & 4.54 & 3.382 & 2.892 & 3.358 & 2.793 & 2.188 & 2.016 & 1.76 & 1.597 & 1.55 \\
\hline S2 & 1.85 & 0.429 & pole-on & -0.793 & 4.367 & 10.744 & 5.992 & 4.682 & 3.49 & 2.999 & 3.361 & 2.835 & 2.28 & 2.094 & 1.826 & 1.679 & 1.611 \\
\hline $\mathrm{S} 2$ & 1.85 & 0.5 & pole-on & -0.878 & 5.705 & 11.346 & 6.259 & 4.926 & 3.686 & 3.163 & 3.462 & 2.947 & 2.409 & 2.195 & 1.924 & 1.751 & 1.706 \\
\hline $\mathrm{S} 2$ & 1.85 & 0.571 & pole-on & -0.948 & 7.124 & 12.113 & 6.667 & 5.223 & 3.916 & 3.364 & 3.624 & 3.105 & 2.566 & 2.341 & 2.05 & 1.87 & 1.823 \\
\hline $\mathrm{S} 2$ & 1.85 & 0.643 & pole-on & -1.005 & 8.556 & 13.01 & 7.144 & 5.606 & 4.201 & 3.625 & 3.842 & 3.321 & 2.758 & 2.508 & 2.201 & 2.015 & 1.98 \\
\hline $\mathrm{S} 2$ & 1.85 & 0.714 & pole-on & -1.051 & 9.995 & 14.082 & 7.684 & 6.019 & 4.511 & 3.897 & 4.094 & 3.563 & 2.955 & 2.704 & 2.377 & 2.19 & 2.156 \\
\hline $\mathrm{S} 2$ & 1.85 & 0.786 & pole-on & -1.086 & 11.38 & 15.284 & 8.298 & 6.507 & 4.86 & 4.194 & 4.384 & 3.831 & 3.18 & 2.931 & 2.585 & 2.386 & 2.358 \\
\hline $\mathrm{S} 2$ & 1.85 & 0.857 & pole-on & -1.115 & 12.68 & 16.616 & 8.979 & 7.009 & 5.212 & 4.494 & 4.7 & 4.142 & 3.434 & 3.187 & 2.818 & 2.608 & 2.583 \\
\hline $\mathrm{S} 2$ & 1.85 & 0.928 & pole-on & -1.137 & 13.931 & 18.143 & 9.691 & 7.517 & 5.583 & 4.835 & 5.065 & 4.483 & 3.715 & 3.463 & 3.085 & 2.849 & 2.834 \\
\hline $\mathrm{S} 2$ & 1.85 & 1.0 & pole-on & -1.153 & 14.941 & 19.676 & 10.375 & 8.058 & 5.984 & 5.213 & 5.413 & 4.836 & 4.0 & 3.751 & 3.331 & 3.094 & 3.093 \\
\hline S2 & 1.37 & 0.0 & edge-on & 0.276 & 1.064 & 2.222 & 1.669 & 1.493 & 1.284 & 1.16 & 1.071 & 0.953 & 0.889 & 0.808 & 0.693 & 0.634 & 0.619 \\
\hline $\mathrm{S} 2$ & 1.37 & 0.071 & edge-on & 0.119 & 1.11 & 1.164 & 0.905 & 0.812 & 0.706 & 0.626 & 0.596 & 0.505 & 0.45 & 0.425 & 0.347 & 0.307 & 0.295 \\
\hline $\mathrm{S} 2$ & 1.37 & 0.143 & interm. & -0.098 & 0.773 & 360.567 & 214.455 & 58.226 & 23.045 & 16.313 & 13.143 & 10.171 & 9.481 & 8.067 & 6.891 & 6.106 & 5.662 \\
\hline $\mathrm{S} 2$ & 1.37 & 0.215 & interm. & -0.287 & 1.383 & 31.803 & 11.997 & 8.246 & 5.581 & 4.756 & 4.238 & 3.719 & 3.435 & 3.127 & 2.778 & 2.527 & 2.441 \\
\hline $\mathrm{S} 2$ & 1.37 & 0.286 & interm. & -0.443 & 2.088 & 17.436 & 8.372 & 6.418 & 4.638 & 4.046 & 3.66 & 3.251 & 3.007 & 2.755 & 2.424 & 2.232 & 2.158 \\
\hline $\mathrm{S} 2$ & 1.37 & 0.357 & pole-on & -0.569 & 2.971 & 14.45 & 7.879 & 6.206 & 4.615 & 4.022 & 3.651 & 3.271 & 3.028 & 2.765 & 2.439 & 2.252 & 2.184 \\
\hline $\mathrm{S} 2$ & 1.37 & 0.429 & pole-on & -0.671 & 4.107 & 14.473 & 8.108 & 6.412 & 4.791 & 4.195 & 3.798 & 3.407 & 3.161 & 2.892 & 2.563 & 2.369 & 2.312 \\
\hline $\mathrm{S} 2$ & 1.37 & 0.5 & pole-on & -0.751 & 5.429 & 15.193 & 8.548 & 6.774 & 5.083 & 4.436 & 4.031 & 3.614 & 3.374 & 3.099 & 2.744 & 2.55 & 2.498 \\
\hline $\mathrm{S} 2$ & 1.37 & 0.571 & pole-on & -0.817 & 6.825 & 16.312 & 9.151 & 7.258 & 5.434 & 4.724 & 4.305 & 3.899 & 3.635 & 3.346 & 2.972 & 2.778 & 2.727 \\
\hline $\mathrm{S} 2$ & 1.37 & 0.643 & pole-on & -0.87 & 8.393 & 17.691 & 9.854 & 7.799 & 5.837 & 5.091 & 4.643 & 4.197 & 3.945 & 3.645 & 3.256 & 3.052 & 2.995 \\
\hline $\mathrm{S} 2$ & 1.37 & 0.714 & pole-on & -0.913 & 9.908 & 19.269 & 10.701 & 8.415 & 6.297 & 5.523 & 5.046 & 4.585 & 4.305 & 3.989 & 3.575 & 3.354 & 3.322 \\
\hline $\mathrm{S} 2$ & 1.37 & 0.786 & pole-on & -0.946 & 11.45 & 21.055 & 11.574 & 9.087 & 6.825 & 5.994 & 5.493 & 4.996 & 4.721 & 4.373 & 3.93 & 3.691 & 3.653 \\
\hline $\mathrm{S} 2$ & 1.37 & 0.857 & pole-on & -0.972 & 12.917 & 23.01 & 12.53 & 9.893 & 7.45 & 6.56 & 5.981 & 5.477 & 5.171 & 4.787 & 4.318 & 4.074 & 4.035 \\
\hline $\mathrm{S} 2$ & 1.37 & 0.928 & pole-on & -0.993 & 14.277 & 25.369 & 13.635 & 10.825 & 8.14 & 7.138 & 6.526 & 5.975 & 5.678 & 5.219 & 4.742 & 4.448 & 4.458 \\
\hline S2 & 1.37 & 1.0 & pole-on & -1.007 & 15.437 & 27.722 & 14.893 & 11.762 & 8.826 & 7.713 & 7.035 & 6.491 & 6.174 & 5.662 & 5.152 & 4.846 & 4.879 \\
\hline $\mathrm{S} 2$ & 1.01 & 0.0 & edge-on & 0.217 & 1.134 & 2.859 & 2.026 & 1.702 & 3.005 & 1.33 & 1.199 & 1.078 & 1.012 & 0.917 & 0.823 & 0.729 & 0.725 \\
\hline $\mathrm{S} 2$ & 1.01 & 0.071 & edge-on & 0.103 & 1.154 & 1.475 & 1.198 & 1.041 & 1.211 & 0.795 & 0.714 & 0.628 & 0.575 & 0.51 & 0.431 & 0.389 & 0.386 \\
\hline $\mathrm{S} 2$ & 1.01 & 0.143 & interm. & -0.058 & 0.85 & 368.933 & 364.626 & 340.066 & 167.139 & 60.072 & 42.972 & 33.082 & 27.503 & 22.452 & 16.792 & 15.109 & 15.449 \\
\hline $\mathrm{S} 2$ & 1.01 & 0.215 & interm. & -0.21 & 1.442 & 61.551 & 19.531 & 12.999 & 20.885 & 7.07 & 6.239 & 5.619 & 5.118 & 4.608 & 4.024 & 3.691 & 3.595 \\
\hline $\mathrm{S} 2$ & 1.01 & 0.286 & interm. & -0.343 & 2.053 & 27.42 & 11.779 & 8.7 & 12.837 & 5.469 & 5.007 & 4.487 & 4.166 & 3.816 & 3.362 & 3.117 & 3.015 \\
\hline S2 & 1.01 & 0.357 & pole-on & -0.454 & 2.849 & 20.41 & 10.599 & 8.214 & 10.723 & 5.325 & 4.906 & 4.44 & 4.141 & 3.878 & 3.411 & 3.154 & 3.058 \\
\hline $\mathrm{S} 2$ & 1.01 & 0.429 & pole-on & -0.547 & 3.887 & 19.243 & 10.733 & 8.444 & 10.186 & 5.592 & 5.133 & 4.664 & 4.395 & 4.044 & 3.647 & 3.383 & 3.291 \\
\hline $\mathrm{S} 2$ & 1.01 & 0.5 & pole-on & -0.621 & 5.13 & 20.075 & 11.409 & 8.95 & 10.271 & 5.965 & 5.508 & 5.03 & 4.74 & 4.39 & 3.978 & 3.697 & 3.592 \\
\hline S2 & 1.01 & 0.571 & pole-on & -0.68 & 6.524 & 21.679 & 12.272 & 9.7 & 10.745 & 6.488 & 5.978 & 5.489 & 5.209 & 4.825 & 4.373 & 4.078 & 3.972 \\
\hline $\mathrm{S} 2$ & 1.01 & 0.643 & pole-on & -0.73 & 8.055 & 23.65 & 13.359 & 10.582 & 11.463 & 7.134 & 6.564 & 6.034 & 5.725 & 5.295 & 4.831 & 4.514 & 4.411 \\
\hline
\end{tabular}


Table A.4 - continued.

\begin{tabular}{|c|c|c|c|c|c|c|c|c|c|c|c|c|c|c|c|c|c|}
\hline & & & $\tilde{\tau}_{\mathrm{bu}}$ & & $\mathrm{bu}$ & 0.15 & 0.45 & 0.75 & 1.50 & 2.25 & 3.00 & 4.50 & 6.00 & 9.00 & 15.00 & 30.00 & $\infty$ \\
\hline Star & $\Sigma_{0}\left[\mathrm{~g} \mathrm{~cm}^{-2}\right]$ & $\cos i$ & ang. type & $\Delta I_{\mathrm{bu}}^{\infty}$ & $\xi_{\mathrm{bu}}^{I}$ & $\xi_{\mathrm{d}}^{I}$ & & & & & & & & & & & \\
\hline S2 & 1.01 & 0.714 & pole-on & -0.77 & 9.65 & 26.017 & 14.681 & 11.561 & 12.465 & 7.823 & 7.223 & 6.633 & 6.315 & 5.902 & 5.321 & 5.002 & 4.931 \\
\hline $\mathrm{S} 2$ & 1.01 & 0.786 & pole-on & -0.8 & 11.272 & 28.77 & 16.168 & 12.747 & 13.686 & 8.613 & 7.949 & 7.291 & 6.988 & 6.435 & 5.877 & 5.53 & 5.534 \\
\hline $\mathrm{S} 2$ & 1.01 & 0.857 & pole-on & -0.824 & 12.854 & 31.991 & 17.866 & 14.15 & 15.111 & 9.477 & 8.723 & 8.028 & 7.728 & 7.058 & 6.472 & 6.083 & 6.226 \\
\hline S2 & 1.01 & 0.928 & pole-on & -0.843 & 14.343 & 35.663 & 19.893 & 15.677 & 16.932 & 10.364 & 9.503 & 8.806 & 8.537 & 7.71 & 7.085 & 6.685 & 6.987 \\
\hline $\mathrm{S} 2$ & 1.01 & 1.0 & pole-on & -0.854 & 15.648 & 39.201 & 21.883 & 17.245 & 18.547 & 11.255 & 10.268 & 9.636 & 9.367 & 8.338 & 7.706 & 7.27 & 7.806 \\
\hline $\mathrm{S} 2$ & 0.75 & 0.0 & edge-on & 0.172 & 1.277 & 3.82 & 2.488 & 2.158 & 1.722 & 1.529 & 1.417 & 1.259 & 1.162 & 1.057 & 0.934 & 0.859 & 0.831 \\
\hline S2 & 0.75 & 0.071 & edge-on & 0.089 & 1.417 & 2.289 & 1.449 & 1.354 & 1.096 & 0.974 & 0.909 & 0.804 & 0.731 & 0.681 & 0.574 & 0.544 & 0.474 \\
\hline $\mathrm{S} 2$ & 0.75 & 0.143 & interm. & -0.027 & 17.157 & 340.71 & 341.251 & 334.517 & 357.927 & 327.209 & 368.398 & 315.749 & 334.291 & 289.413 & 172.27 & 131.98 & 135.304 \\
\hline $\mathrm{S} 2$ & 0.75 & 0.215 & interm. & -0.148 & 1.407 & 232.811 & 37.709 & 24.071 & 14.534 & 11.516 & 9.96 & 8.57 & 8.04 & 7.02 & 6.203 & 5.661 & 5.562 \\
\hline $\mathrm{S} 2$ & 0.75 & 0.286 & interm. & -0.255 & 1.974 & 41.962 & 16.955 & 12.329 & 8.672 & 7.481 & 6.762 & 6.011 & 5.677 & 5.157 & 4.619 & 4.293 & 4.338 \\
\hline $\mathrm{S} 2$ & 0.75 & 0.357 & pole-on & -0.349 & 2.674 & 28.193 & 13.75 & 10.753 & 8.107 & 7.062 & 6.457 & 5.816 & 5.548 & 5.067 & 4.589 & 4.317 & 4.339 \\
\hline $\mathrm{S} 2$ & 0.75 & 0.429 & pole-on & -0.429 & 3.559 & 25.072 & 13.798 & 10.886 & 8.417 & 7.376 & 6.753 & 6.121 & 5.891 & 5.396 & 4.931 & 4.652 & 4.712 \\
\hline $\mathrm{S} 2$ & 0.75 & 0.5 & pole-on & -0.495 & 4.584 & 25.659 & 14.54 & 11.628 & 9.011 & 7.965 & 7.341 & 6.659 & 6.447 & 5.911 & 5.413 & 5.134 & 5.228 \\
\hline $\mathrm{S} 2$ & 0.75 & 0.571 & pole-on & -0.548 & 5.723 & 27.46 & 15.814 & 12.736 & 9.867 & 8.753 & 8.066 & 7.35 & 7.127 & 6.532 & 6.002 & 5.677 & 5.824 \\
\hline $\mathrm{S} 2$ & 0.75 & 0.643 & pole-on & -0.591 & 6.953 & 30.174 & 17.486 & 14.101 & 10.93 & 9.749 & 8.943 & 8.107 & 7.911 & 7.238 & 6.668 & 6.32 & 6.568 \\
\hline $\mathrm{S} 2$ & 0.75 & 0.714 & pole-on & -0.627 & 8.177 & 33.836 & 19.513 & 15.727 & 12.187 & 10.763 & 9.93 & 9.037 & 8.851 & 8.049 & 7.436 & 7.026 & 7.41 \\
\hline $\mathrm{S} 2$ & 0.75 & 0.786 & pole-on & -0.654 & 9.391 & 38.041 & 21.756 & 17.565 & 13.521 & 11.941 & 10.958 & 9.999 & 9.89 & 8.85 & 8.27 & 7.772 & 8.351 \\
\hline $\mathrm{S} 2$ & 0.75 & 0.857 & pole-on & -0.674 & 10.542 & 42.526 & 24.516 & 19.727 & 15.058 & 13.172 & 12.121 & 11.077 & 11.041 & 9.734 & 9.167 & 8.58 & 9.396 \\
\hline $\mathrm{S} 2$ & 0.75 & 0.928 & pole-on & -0.689 & 11.6 & 47.892 & 27.505 & 22.127 & 16.661 & 14.47 & 13.167 & 12.219 & 12.29 & 10.595 & 10.15 & 9.396 & 10.532 \\
\hline $\mathrm{S} 2$ & 0.75 & 1.0 & pole-on & -0.698 & 12.437 & 52.744 & 30.506 & 24.585 & 18.215 & 15.638 & 14.213 & 13.314 & 13.601 & 11.426 & 11.107 & 10.179 & 11.778 \\
\hline $\mathrm{S} 2$ & 0.56 & 0.0 & edge-on & 0.136 & 1.463 & 5.058 & 3.071 & 2.595 & 2.027 & 1.8 & 1.658 & 1.493 & 1.337 & 1.241 & 1.085 & 0.989 & 0.956 \\
\hline $\mathrm{S} 2$ & 0.56 & 0.071 & edge-on & 0.077 & 1.44 & 3.237 & 2.052 & 1.668 & 1.364 & 1.22 & 1.154 & 1.016 & 0.902 & 0.843 & 0.743 & 0.671 & 0.63 \\
\hline $\mathrm{S} 2$ & 0.56 & 0.143 & interm. & -0.005 & 272.023 & 138.955 & 130.118 & 21.999 & 57.785 & 125.824 & 102.085 & 158.814 & 160.852 & 183.146 & 207.901 & 261.9 & 384.925 \\
\hline $\mathrm{S} 2$ & 0.56 & 0.215 & interm. & -0.097 & 1.471 & 375.635 & 129.786 & 49.43 & 28.347 & 21.308 & 17.846 & 14.542 & 13.798 & 11.709 & 10.261 & 9.194 & 9.309 \\
\hline $\mathrm{S} 2$ & 0.56 & 0.286 & interm. & -0.18 & 1.99 & 87.426 & 25.367 & 17.413 & 12.022 & 10.03 & 9.002 & 7.965 & 7.731 & 6.733 & 6.143 & 5.68 & 5.987 \\
\hline $\mathrm{S} 2$ & 0.56 & 0.357 & pole-on & -0.255 & 2.65 & 40.046 & 18.238 & 13.968 & 10.337 & 8.877 & 8.144 & 7.306 & 7.128 & 6.423 & 5.88 & 5.535 & 5.867 \\
\hline $\mathrm{S} 2$ & 0.56 & 0.429 & pole-on & -0.321 & 3.46 & 33.582 & 17.286 & 13.596 & 10.495 & 9.215 & 8.406 & 7.719 & 7.474 & 6.768 & 6.296 & 5.917 & 6.242 \\
\hline $\mathrm{S} 2$ & 0.56 & 0.5 & pole-on & -0.375 & 4.467 & 31.951 & 17.893 & 14.568 & 11.366 & 9.979 & 9.16 & 8.347 & 8.237 & 7.415 & 6.925 & 6.518 & 6.961 \\
\hline $\mathrm{S} 2$ & 0.56 & 0.571 & pole-on & -0.419 & 5.687 & 34.471 & 19.78 & 16.044 & 12.459 & 11.026 & 10.217 & 9.265 & 9.146 & 8.229 & 7.703 & 7.237 & 7.834 \\
\hline $\mathrm{S} 2$ & 0.56 & 0.643 & pole-on & -0.456 & 7.055 & 37.507 & 21.955 & 18.024 & 13.907 & 12.295 & 11.416 & 10.343 & 10.306 & 9.221 & 8.601 & 8.137 & 8.92 \\
\hline $\mathrm{S} 2$ & 0.56 & 0.714 & pole-on & -0.485 & 8.537 & 42.563 & 24.785 & 20.22 & 15.601 & 13.819 & 12.75 & 11.51 & 11.582 & 10.245 & 9.649 & 9.055 & 10.13 \\
\hline $\mathrm{S} 2$ & 0.56 & 0.786 & pole-on & -0.508 & 10.179 & 47.488 & 28.023 & 22.966 & 17.601 & 15.509 & 14.207 & 12.833 & 13.056 & 11.318 & 10.815 & 10.038 & 11.625 \\
\hline $\mathrm{S} 2$ & 0.56 & 0.857 & pole-on & -0.525 & 11.885 & 53.992 & 31.842 & 26.088 & 19.687 & 17.125 & 15.708 & 14.191 & 14.644 & 12.441 & 11.983 & 11.061 & 13.281 \\
\hline $\mathrm{S} 2$ & 0.56 & 0.928 & pole-on & -0.536 & 13.631 & 60.335 & 35.983 & 29.353 & 21.696 & 18.724 & 16.94 & 15.731 & 16.524 & 13.519 & 13.315 & 12.057 & 15.093 \\
\hline $\mathrm{S} 2$ & 0.56 & 1.0 & pole-on & -0.541 & 15.049 & 66.842 & 40.098 & 32.975 & 23.739 & 20.177 & 18.348 & 17.271 & 18.294 & 14.501 & 14.537 & 13.105 & 16.986 \\
\hline
\end{tabular}


Table A.4 - continued.

\begin{tabular}{|c|c|c|c|c|c|c|c|c|c|c|c|c|c|c|c|c|c|}
\hline & & & 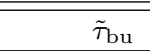 & & $\overline{\mathrm{bu}}$ & 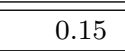 & 0.45 & 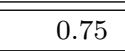 & 1.50 & 2.25 & 3.00 & 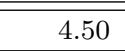 & 26.00 & 9.00 & 15.00 & 30.00 & $\infty$ \\
\hline Star & $\Sigma_{0}\left[\mathrm{~g} \mathrm{~cm}^{-2}\right]$ & $\cos i$ & ang. type & $\Delta I_{\mathrm{bu}}^{\infty}$ & $\xi_{\mathrm{bu}}^{I}$ & $\xi_{\mathrm{d}}^{I}$ & & & & & & & & & & & \\
\hline S2 & 0.41 & 0.0 & edge-on & 0.107 & 1.778 & 7.37 & 4.022 & 3.151 & 2.389 & 2.147 & 1.919 & 1.731 & 1.63 & 1.508 & 1.27 & 1.162 & 1.118 \\
\hline $\mathrm{S} 2$ & 0.41 & 0.071 & edge-on & 0.066 & 1.839 & 6.877 & 2.895 & 2.19 & 1.763 & 1.561 & 1.405 & 1.245 & 1.165 & 1.066 & 0.926 & 0.852 & 0.777 \\
\hline $\mathrm{S} 2$ & 0.41 & 0.143 & interm. & 0.006 & 218.244 & 173.68 & 8.838 & 10.744 & 2.003 & 2.321 & 2.345 & 2.753 & 6.179 & 1.137 & 3.384 & 13.625 & 9.71 \\
\hline $\mathrm{S} 2$ & 0.41 & 0.215 & interm. & -0.056 & 2.272 & 402.819 & 352.642 & 294.352 & 127.967 & 65.303 & 41.882 & 34.841 & 32.814 & 25.119 & 20.787 & 18.907 & 21.096 \\
\hline $\mathrm{S} 2$ & 0.41 & 0.286 & interm. & -0.117 & 1.853 & 277.276 & 42.278 & 28.17 & 17.432 & 13.957 & 11.6 & 10.538 & 9.747 & 8.697 & 7.977 & 7.242 & 7.94 \\
\hline $\mathrm{S} 2$ & 0.41 & 0.357 & pole-on & -0.172 & 2.478 & 53.924 & 23.783 & 17.247 & 12.426 & 10.93 & 9.617 & 8.752 & 8.624 & 7.642 & 7.006 & 6.63 & 7.24 \\
\hline $\mathrm{S} 2$ & 0.41 & 0.429 & pole-on & -0.221 & 3.246 & 39.901 & 20.792 & 16.536 & 12.459 & 10.8 & 9.738 & 9.049 & 8.898 & 7.928 & 7.403 & 6.959 & 7.709 \\
\hline $\mathrm{S} 2$ & 0.41 & 0.5 & pole-on & -0.262 & 4.157 & 37.358 & 21.374 & 17.364 & 13.253 & 11.663 & 10.578 & 9.818 & 9.797 & 8.706 & 8.151 & 7.702 & 8.626 \\
\hline $\mathrm{S} 2$ & 0.41 & 0.571 & pole-on & -0.297 & 5.213 & 39.017 & 23.635 & 19.008 & 14.771 & 12.977 & 11.873 & 10.913 & 10.95 & 9.677 & 9.125 & 8.617 & 9.827 \\
\hline $\mathrm{S} 2$ & 0.41 & 0.643 & pole-on & -0.325 & 6.512 & 43.192 & 26.29 & 21.513 & 16.468 & 14.689 & 13.369 & 12.236 & 12.47 & 10.938 & 10.287 & 9.684 & 11.341 \\
\hline $\mathrm{S} 2$ & 0.41 & 0.714 & pole-on & -0.347 & 7.937 & 48.595 & 29.959 & 24.594 & 18.873 & 16.58 & 15.117 & 13.743 & 14.195 & 12.152 & 11.534 & 10.913 & 13.131 \\
\hline $\mathrm{S} 2$ & 0.41 & 0.786 & pole-on & -0.364 & 9.559 & 54.779 & 34.303 & 28.491 & 21.424 & 18.851 & 17.02 & 15.178 & 16.164 & 13.538 & 12.951 & 12.031 & 15.199 \\
\hline $\mathrm{S} 2$ & 0.41 & 0.857 & pole-on & -0.377 & 11.071 & 61.677 & 39.221 & 32.179 & 24.063 & 20.767 & 18.599 & 17.103 & 18.414 & 14.944 & 14.492 & 13.323 & 17.557 \\
\hline $\mathrm{S} 2$ & 0.41 & 0.928 & pole-on & -0.384 & 13.059 & 70.288 & 44.618 & 36.932 & 26.353 & 22.574 & 20.434 & 18.896 & 20.945 & 16.049 & 16.04 & 14.503 & 20.22 \\
\hline $\mathrm{S} 2$ & 0.41 & 1.0 & pole-on & -0.386 & 14.374 & 77.664 & 49.56 & 40.97 & 28.772 & 24.192 & 21.735 & 20.644 & 23.298 & 17.128 & 17.553 & 15.75 & 22.806 \\
\hline $\mathrm{S} 2$ & 0.3 & 0.0 & edge-on & 0.085 & 2.094 & 9.2 & 5.088 & 3.7 & 2.924 & 2.54 & 2.404 & 1.989 & 1.867 & 1.691 & 1.493 & 1.337 & 1.305 \\
\hline $\mathrm{S} 2$ & 0.3 & 0.071 & edge-on & 0.056 & 2.069 & 22.387 & 3.663 & 2.774 & 2.169 & 1.896 & 1.706 & 1.556 & 1.426 & 1.335 & 1.131 & 1.027 & 0.983 \\
\hline $\mathrm{S} 2$ & 0.3 & 0.143 & interm. & 0.015 & 28.663 & 86.052 & 22.89 & 3.341 & 1.782 & 1.562 & 1.544 & 2.58 & 1.766 & 0.987 & 0.784 & 0.74 & 1.279 \\
\hline $\mathrm{S} 2$ & 0.3 & 0.215 & interm. & -0.026 & 15.087 & 312.214 & 385.12 & 376.617 & 349.31 & 344.988 & 304.981 & 282.656 & 268.111 & 266.812 & 170.255 & 118.163 & 134.329 \\
\hline $\mathrm{S} 2$ & 0.3 & 0.286 & interm. & -0.069 & 1.763 & 319.228 & 166.416 & 54.149 & 24.306 & 18.569 & 15.181 & 13.252 & 13.048 & 10.681 & 9.368 & 8.708 & 9.934 \\
\hline $\mathrm{S} 2$ & 0.3 & 0.357 & pole-on & -0.108 & 2.414 & 114.346 & 31.085 & 21.486 & 13.671 & 11.434 & 10.591 & 9.304 & 9.526 & 8.277 & 7.605 & 7.0 & 8.001 \\
\hline $\mathrm{S} 2$ & 0.3 & 0.429 & pole-on & -0.143 & 3.078 & 46.652 & 23.809 & 18.574 & 13.094 & 11.268 & 10.452 & 9.506 & 9.761 & 8.472 & 7.893 & 7.346 & 8.589 \\
\hline $\mathrm{S} 2$ & 0.3 & 0.5 & pole-on & -0.172 & 3.931 & 41.249 & 23.628 & 19.105 & 14.199 & 12.577 & 11.315 & 10.38 & 10.77 & 9.374 & 8.662 & 8.147 & 9.507 \\
\hline $\mathrm{S} 2$ & 0.3 & 0.571 & pole-on & -0.197 & 4.914 & 43.395 & 25.467 & 21.194 & 16.051 & 13.756 & 12.85 & 11.564 & 11.996 & 10.542 & 9.847 & 9.172 & 11.013 \\
\hline $\mathrm{S} 2$ & 0.3 & 0.643 & pole-on & -0.217 & 6.158 & 46.093 & 28.819 & 24.136 & 17.94 & 15.803 & 14.617 & 13.281 & 13.771 & 11.839 & 11.045 & 10.481 & 12.846 \\
\hline $\mathrm{S} 2$ & 0.3 & 0.714 & pole-on & -0.233 & 7.516 & 51.475 & 33.141 & 27.749 & 20.786 & 18.108 & 16.677 & 14.801 & 15.686 & 13.442 & 12.63 & 11.843 & 15.162 \\
\hline $\mathrm{S} 2$ & 0.3 & 0.786 & pole-on & -0.244 & 9.166 & 59.337 & 38.604 & 32.206 & 24.24 & 20.574 & 18.749 & 16.682 & 18.146 & 14.874 & 14.262 & 13.21 & 17.694 \\
\hline $\mathrm{S} 2$ & 0.3 & 0.857 & pole-on & -0.253 & 10.858 & 68.774 & 43.889 & 36.467 & 26.768 & 23.182 & 20.859 & 18.712 & 20.718 & 16.298 & 15.995 & 14.649 & 20.951 \\
\hline $\mathrm{S} 2$ & 0.3 & 0.928 & pole-on & -0.258 & 12.746 & 77.298 & 50.416 & 42.083 & 29.42 & 24.822 & 22.427 & 20.735 & 23.688 & 17.571 & 17.844 & 15.793 & 24.438 \\
\hline $\mathrm{S} 2$ & 0.3 & 1.0 & pole-on & -0.257 & 14.215 & 85.198 & 54.973 & 46.554 & 32.295 & 27.125 & 23.932 & 22.913 & 26.258 & 18.895 & 19.601 & 17.212 & 27.863 \\
\hline S3 & 2.5 & 0.0 & edge-on & 0.394 & 1.031 & 1.557 & 1.371 & 1.239 & 1.037 & 0.961 & 0.904 & 0.802 & 0.737 & 0.679 & 0.604 & 0.545 & 0.516 \\
\hline S3 & 2.5 & 0.071 & edge-on & 0.172 & 1.311 & 0.891 & 0.798 & 0.703 & 0.61 & 0.575 & 0.538 & 0.469 & 0.413 & 0.362 & 0.297 & 0.271 & 0.248 \\
\hline S3 & 2.5 & 0.143 & interm. & -0.131 & 0.505 & 355.747 & 111.373 & 32.372 & 12.431 & 9.071 & 7.634 & 6.227 & 5.662 & 4.734 & 4.174 & 3.626 & 3.637 \\
\hline S3 & 2.5 & 0.215 & interm. & -0.367 & 1.247 & 18.304 & 7.083 & 5.165 & 3.749 & 3.169 & 2.837 & 2.556 & 2.494 & 2.111 & 1.867 & 1.674 & 1.658 \\
\hline S3 & 2.5 & 0.286 & interm. & -0.547 & 2.03 & 10.622 & 5.459 & 4.227 & 3.199 & 2.725 & 2.469 & 2.234 & 2.068 & 1.856 & 1.639 & 1.478 & 1.461 \\
\hline
\end{tabular}


Table A.4 - continued.

\begin{tabular}{|c|c|c|c|c|c|c|c|c|c|c|c|c|c|c|c|c|c|}
\hline & & & $\tilde{\tau}_{\mathrm{bu}}$ & & $\mathrm{bu}$ & 0.15 & 0.45 & 0.75 & 1.50 & 2.25 & 3.00 & 4.50 & 6.00 & 9.00 & 15.00 & 30.00 & $\infty$ \\
\hline Star & $\Sigma_{0}\left[\mathrm{~g} \mathrm{~cm}^{-2}\right]$ & $\cos i$ & ang. type & $\Delta I_{\mathrm{bu}}^{\infty}$ & $\xi_{\mathrm{bu}}^{I}$ & $\xi_{\mathrm{d}}^{I}$ & & & & & & & & & & & \\
\hline S3 & 2.5 & 0.357 & pole-on & -0.687 & 2.991 & 9.48 & 5.289 & 4.139 & 3.135 & 2.686 & 2.439 & 2.204 & 2.039 & 1.837 & 1.62 & 1.455 & 1.443 \\
\hline S3 & 2.5 & 0.429 & pole-on & -0.796 & 4.206 & 9.721 & 5.427 & 4.262 & 3.211 & 2.768 & 2.505 & 2.263 & 2.098 & 1.898 & 1.668 & 1.518 & 1.489 \\
\hline S3 & 2.5 & 0.5 & pole-on & -0.88 & 5.552 & 10.248 & 5.672 & 4.458 & 3.361 & 2.9 & 2.639 & 2.373 & 2.195 & 1.991 & 1.752 & 1.593 & 1.56 \\
\hline S3 & 2.5 & 0.571 & pole-on & -0.951 & 6.935 & 10.89 & 6.021 & 4.72 & 3.556 & 3.063 & 2.799 & 2.52 & 2.336 & 2.116 & 1.858 & 1.69 & 1.658 \\
\hline S3 & 2.5 & 0.643 & pole-on & -1.008 & 8.339 & 11.722 & 6.444 & 5.041 & 3.795 & 3.278 & 2.994 & 2.682 & 2.497 & 2.256 & 1.979 & 1.807 & 1.775 \\
\hline S3 & 2.5 & 0.714 & pole-on & -1.054 & 9.706 & 12.649 & 6.907 & 5.419 & 4.075 & 3.512 & 3.207 & 2.889 & 2.679 & 2.428 & 2.129 & 1.943 & 1.927 \\
\hline S3 & 2.5 & 0.786 & pole-on & -1.089 & 11.053 & 13.719 & 7.459 & 5.844 & 4.422 & 3.789 & 3.453 & 3.096 & 2.872 & 2.599 & 2.291 & 2.105 & 2.087 \\
\hline S3 & 2.5 & 0.857 & pole-on & -1.118 & 12.331 & 14.939 & 8.077 & 6.311 & 4.728 & 4.063 & 3.699 & 3.317 & 3.078 & 2.801 & 2.486 & 2.29 & 2.276 \\
\hline S3 & 2.5 & 0.928 & pole-on & -1.14 & 13.495 & 16.308 & 8.739 & 6.799 & 5.048 & 4.367 & 3.957 & 3.573 & 3.324 & 3.04 & 2.708 & 2.5 & 2.491 \\
\hline S3 & 2.5 & 1.0 & pole-on & -1.156 & 14.511 & 17.669 & 9.397 & 7.271 & 5.381 & 4.646 & 4.249 & 3.845 & 3.598 & 3.286 & 2.933 & 2.716 & 2.712 \\
\hline S3 & 1.85 & 0.0 & edge-on & 0.312 & 1.133 & 1.908 & 1.61 & 1.406 & 1.193 & 1.059 & 0.992 & 1.385 & 0.874 & 0.766 & 0.658 & 0.597 & 0.586 \\
\hline S3 & 1.85 & 0.071 & edge-on & 0.154 & 1.282 & 0.995 & 0.994 & 0.876 & 0.755 & 0.662 & 0.606 & 0.604 & 0.462 & 0.442 & 0.381 & 0.343 & 0.33 \\
\hline S3 & 1.85 & 0.143 & interm. & -0.08 & 9.907 & 386.921 & 337.555 & 328.99 & 51.106 & 25.672 & 18.63 & 22.235 & 13.597 & 10.571 & 8.5 & 7.333 & 7.036 \\
\hline S3 & 1.85 & 0.215 & interm. & -0.28 & 1.219 & 37.36 & 11.504 & 8.17 & 5.306 & 4.379 & 4.063 & 4.426 & 3.224 & 2.974 & 2.669 & 2.416 & 2.347 \\
\hline S3 & 1.85 & 0.286 & interm. & -0.442 & 1.945 & 16.631 & 7.75 & 5.982 & 4.332 & 3.672 & 3.424 & 3.63 & 2.8 & 2.564 & 2.304 & 2.079 & 2.02 \\
\hline S3 & 1.85 & 0.357 & pole-on & -0.572 & 2.844 & 13.631 & 7.239 & 5.752 & 4.24 & 3.658 & 3.369 & 3.519 & 2.802 & 2.561 & 2.263 & 2.063 & 2.018 \\
\hline S3 & 1.85 & 0.429 & pole-on & -0.677 & 3.97 & 13.443 & 7.433 & 5.884 & 4.369 & 3.787 & 3.479 & 3.578 & 2.906 & 2.664 & 2.355 & 2.157 & 2.094 \\
\hline S3 & 1.85 & 0.5 & pole-on & -0.756 & 5.315 & 14.024 & 7.785 & 6.197 & 4.643 & 4.019 & 3.682 & 3.738 & 3.088 & 2.824 & 2.495 & 2.283 & 2.243 \\
\hline S3 & 1.85 & 0.571 & pole-on & -0.822 & 6.754 & 14.928 & 8.297 & 6.591 & 4.947 & 4.287 & 3.934 & 3.969 & 3.302 & 3.026 & 2.685 & 2.464 & 2.428 \\
\hline S3 & 1.85 & 0.643 & pole-on & -0.876 & 8.25 & 16.175 & 8.942 & 7.081 & 5.324 & 4.629 & 4.232 & 4.257 & 3.563 & 3.29 & 2.914 & 2.708 & 2.658 \\
\hline S3 & 1.85 & 0.714 & pole-on & -0.919 & 9.746 & 17.523 & 9.678 & 7.663 & 5.749 & 4.989 & 4.582 & 4.602 & 3.858 & 3.583 & 3.193 & 2.954 & 2.956 \\
\hline S3 & 1.85 & 0.786 & pole-on & -0.952 & 11.239 & 19.085 & 10.525 & 8.269 & 6.198 & 5.385 & 4.975 & 4.96 & 4.183 & 3.913 & 3.497 & 3.247 & 3.225 \\
\hline S3 & 1.85 & 0.857 & pole-on & -0.979 & 12.654 & 20.888 & 11.388 & 8.917 & 6.697 & 5.865 & 5.437 & 5.369 & 4.569 & 4.273 & 3.84 & 3.582 & 3.558 \\
\hline S3 & 1.85 & 0.928 & pole-on & -1.0 & 13.975 & 22.869 & 12.356 & 9.683 & 7.291 & 6.382 & 5.932 & 5.825 & 4.958 & 4.665 & 4.213 & 3.922 & 3.928 \\
\hline S3 & 1.85 & 1.0 & pole-on & -1.014 & 15.114 & 24.925 & 13.372 & 10.519 & 7.921 & 6.916 & 6.444 & 6.274 & 5.354 & 5.066 & 4.597 & 4.276 & 4.308 \\
\hline S3 & 1.37 & 0.0 & edge-on & 0.247 & 1.275 & 2.364 & 1.865 & 1.606 & 1.37 & 1.204 & 1.098 & 0.986 & 0.922 & 0.843 & 0.732 & 0.671 & 0.658 \\
\hline S3 & 1.37 & 0.071 & edge-on & 0.132 & 1.319 & 1.415 & 1.182 & 1.029 & 0.915 & 0.794 & 0.71 & 0.636 & 0.587 & 0.547 & 0.465 & 0.421 & 0.398 \\
\hline S3 & 1.37 & 0.143 & interm. & -0.042 & 0.363 & 329.683 & 187.713 & 333.113 & 358.463 & 304.895 & 221.239 & 144.276 & 64.324 & 42.353 & 31.543 & 25.272 & 23.788 \\
\hline S3 & 1.37 & 0.215 & interm. & -0.206 & 1.179 & 69.821 & 20.301 & 13.347 & 8.078 & 6.77 & 6.111 & 5.282 & 4.918 & 4.398 & 3.888 & 3.599 & 3.432 \\
\hline S3 & 1.37 & 0.286 & interm. & -0.346 & 1.85 & 24.566 & 10.684 & 8.233 & 5.896 & 5.078 & 4.713 & 4.137 & 3.886 & 3.554 & 3.138 & 2.923 & 2.812 \\
\hline S3 & 1.37 & 0.357 & pole-on & \begin{tabular}{|l}
-0.462 \\
\end{tabular} & 2.656 & 18.497 & 9.642 & 7.589 & 5.647 & 4.963 & 4.581 & 4.065 & 3.827 & 3.507 & 3.116 & 2.907 & 2.829 \\
\hline S3 & 1.37 & 0.429 & pole-on & -0.558 & 3.728 & 17.508 & 9.783 & 7.83 & 5.862 & 5.136 & 4.759 & 4.258 & 4.003 & 3.688 & 3.287 & 3.081 & 3.059 \\
\hline S3 & 1.37 & 0.5 & pole-on & -0.632 & 5.009 & 18.242 & 10.354 & 8.29 & 6.226 & 5.471 & 5.063 & 4.565 & 4.298 & 3.963 & 3.559 & 3.357 & 3.309 \\
\hline S3 & 1.37 & 0.571 & pole-on & -0.694 & 6.39 & 19.518 & 11.165 & 8.888 & 6.701 & 5.891 & 5.463 & 4.937 & 4.658 & 4.332 & 3.911 & 3.674 & 3.64 \\
\hline S3 & 1.37 & 0.643 & pole-on & -0.744 & 7.933 & 21.439 & 12.087 & 9.64 & 7.261 & 6.413 & 5.962 & 5.405 & 5.136 & 4.746 & 4.288 & 4.059 & 4.037 \\
\hline
\end{tabular}


Table A.4 - continued.

\begin{tabular}{|c|c|c|c|c|c|c|c|c|c|c|c|c|c|c|c|c|c|}
\hline & & & $\tilde{\tau}_{\mathrm{bu}}$ & & $\mathrm{bu}$ & 0.15 & 0.45 & 0.75 & 1.50 & 2.25 & 3.00 & 4.50 & 6.00 & 9.00 & 15.00 & 30.00 & $\infty$ \\
\hline Star & $\Sigma_{0}\left[\mathrm{~g} \mathrm{~cm}^{-2}\right]$ & $\cos i$ & ang. type & $\Delta I_{\mathrm{bu}}^{\infty}$ & $\xi_{\mathrm{bu}}^{I}$ & $\xi_{\mathrm{d}}^{I}$ & & & & & & & & & & & \\
\hline S3 & 1.37 & 0.714 & pole-on & -0.785 & 9.493 & 23.375 & 13.14 & 10.459 & 7.969 & 7.041 & 6.537 & 5.931 & 5.643 & 5.217 & 4.745 & 4.487 & 4.485 \\
\hline S3 & 1.37 & 0.786 & pole-on & -0.815 & 11.123 & 25.708 & 14.434 & 11.487 & 8.769 & 7.742 & 7.159 & 6.512 & 6.215 & 5.733 & 5.243 & 4.95 & 4.997 \\
\hline S3 & 1.37 & 0.857 & pole-on & -0.84 & 12.647 & 28.512 & 15.864 & 12.664 & 9.646 & 8.501 & 7.871 & 7.173 & 6.868 & 6.3 & 5.783 & 5.455 & 5.559 \\
\hline S3 & 1.37 & 0.928 & pole-on & -0.859 & 14.168 & 31.713 & 17.555 & 14.001 & 10.617 & 9.317 & 8.616 & 7.895 & 7.599 & 6.896 & 6.378 & 6.036 & 6.173 \\
\hline S3 & 1.37 & 1.0 & pole-on & -0.871 & 15.442 & 35.148 & 19.376 & 15.356 & 11.62 & 10.146 & 9.313 & 8.617 & 8.341 & 7.487 & 6.985 & 6.556 & 6.823 \\
\hline S3 & 1.01 & 0.0 & edge-on & 0.196 & 1.415 & 3.302 & 2.157 & 1.902 & 1.549 & 1.339 & 1.285 & 1.118 & 1.043 & 0.92 & 0.705 & 0.719 & 0.766 \\
\hline S3 & 1.01 & 0.071 & edge-on & 0.112 & 1.461 & 2.114 & 1.548 & 1.222 & 1.053 & 0.933 & 0.894 & 0.771 & 0.712 & 0.636 & 0.521 & 0.492 & 0.511 \\
\hline S3 & 1.01 & 0.143 & interm. & -0.011 & 86.852 & 66.19 & 53.3 & 90.994 & 126.957 & 159.528 & 97.415 & 177.626 & 275.382 & 371.677 & 371.207 & 368.948 & 330.415 \\
\hline S3 & 1.01 & 0.215 & interm. & -0.141 & 1.18 & 327.171 & 37.047 & 24.943 & 14.889 & 11.385 & 9.6 & 8.568 & 7.83 & 6.831 & 5.936 & 5.627 & 5.423 \\
\hline S3 & 1.01 & 0.286 & interm. & -0.255 & 1.807 & 39.77 & 15.474 & 11.677 & 8.258 & 7.087 & 6.289 & 5.67 & 5.32 & 4.918 & 4.537 & 4.089 & 3.855 \\
\hline S3 & 1.01 & 0.357 & pole-on & -0.353 & 2.583 & 25.148 & 12.802 & 10.081 & 7.487 & 6.649 & 5.951 & 5.469 & 5.136 & 4.863 & 4.492 & 4.043 & 3.83 \\
\hline S3 & 1.01 & 0.429 & pole-on & -0.438 & 3.516 & 22.711 & 12.746 & 10.162 & 7.729 & 6.846 & 6.278 & 5.73 & 5.448 & 5.155 & 4.781 & 4.373 & 4.088 \\
\hline S3 & 1.01 & 0.5 & pole-on & -0.505 & 4.702 & 23.627 & 13.59 & 10.885 & 8.265 & 7.362 & 6.763 & 6.206 & 5.943 & 5.65 & 5.228 & 4.79 & 4.436 \\
\hline S3 & 1.01 & 0.571 & pole-on & -0.56 & 6.058 & 25.221 & 14.686 & 11.87 & 8.98 & 8.081 & 7.428 & 6.853 & 6.518 & 6.192 & 5.757 & 5.341 & 4.905 \\
\hline S3 & 1.01 & 0.643 & pole-on & -0.606 & 7.571 & 27.813 & 16.306 & 13.03 & 9.78 & 8.927 & 8.218 & 7.566 & 7.261 & 6.875 & 6.365 & 5.989 & 5.489 \\
\hline S3 & 1.01 & 0.714 & pole-on & -0.642 & 9.181 & 31.029 & 18.019 & 14.427 & 10.794 & 9.914 & 9.093 & 8.378 & 8.092 & 7.58 & 7.041 & 6.729 & 6.17 \\
\hline S3 & 1.01 & 0.786 & pole-on & -0.67 & 10.841 & 34.783 & 20.208 & 16.12 & 11.883 & 10.959 & 10.067 & 9.284 & 8.971 & 8.371 & 7.777 & 7.469 & 7.007 \\
\hline S3 & 1.01 & 0.857 & pole-on & $\mid-0.692$ & 12.524 & 38.939 & 22.644 & 18.099 & 13.143 & 12.134 & 11.112 & 10.291 & 10.042 & 9.175 & 8.549 & 8.283 & 8.052 \\
\hline S3 & 1.01 & 0.928 & pole-on & -0.708 & 14.154 & 43.886 & 25.404 & 20.246 & 14.528 & 13.31 & 12.146 & 11.327 & 11.172 & 10.011 & 9.388 & 9.137 & 9.414 \\
\hline S3 & 1.01 & 1.0 & pole-on & -0.718 & 15.515 & 48.891 & 28.279 & 22.471 & 15.84 & 14.46 & 13.149 & 12.415 & 12.334 & 10.828 & 10.162 & 9.978 & 11.02 \\
\hline S3 & 0.75 & 0.0 & edge-on & 0.156 & 3.06 & 4.352 & 2.816 & 2.335 & 1.811 & 1.581 & 1.469 & 1.293 & 1.171 & 1.08 & 0.932 & 0.862 & 0.82 \\
\hline S3 & 0.75 & 0.071 & edge-on & 0.095 & 1.735 & 3.088 & 1.939 & 1.674 & 1.327 & 1.159 & 1.066 & 0.968 & 0.854 & 0.804 & 0.686 & 0.626 & 0.577 \\
\hline S3 & 0.75 & 0.143 & interm. & 0.006 & 331.307 & 36.647 & 11.183 & 10.463 & 0.617 & 18.53 & 1.38 & 3.534 & 6.625 & 17.973 & 20.537 & 14.236 & 14.528 \\
\hline S3 & 0.75 & 0.215 & interm. & -0.091 & 3.438 & 391.969 & 228.459 & 57.025 & 29.26 & 22.56 & 18.249 & 14.852 & 13.612 & 11.928 & 10.424 & 9.155 & 9.466 \\
\hline S3 & 0.75 & 0.286 & interm. & -0.18 & 1.715 & 82.52 & 23.872 & 15.958 & 11.141 & 9.349 & 8.628 & 7.495 & 7.107 & 6.382 & 5.943 & 5.42 & 5.605 \\
\hline S3 & 0.75 & 0.357 & pole-on & -0.26 & 2.424 & 35.567 & 16.747 & 12.595 & 9.568 & 8.305 & 7.621 & 6.956 & 6.629 & 6.088 & 5.621 & 5.222 & 5.442 \\
\hline S3 & 0.75 & 0.429 & pole-on & -0.33 & 3.277 & 29.32 & 16.037 & 12.611 & 9.802 & 8.653 & 8.018 & 7.293 & 7.053 & 6.446 & 5.983 & 5.593 & 5.841 \\
\hline S3 & 0.75 & 0.5 & pole-on & -0.387 & 4.246 & 29.025 & 16.736 & 13.529 & 10.544 & 9.386 & 8.658 & 7.973 & 7.694 & 7.062 & 6.563 & 6.178 & 6.524 \\
\hline S3 & 0.75 & 0.571 & pole-on & -0.434 & 5.361 & 31.471 & 18.512 & 14.868 & 11.637 & 10.388 & 9.559 & 8.778 & 8.611 & 7.82 & 7.276 & 6.837 & 7.31 \\
\hline S3 & 0.75 & 0.643 & pole-on & -0.473 & 6.581 & 34.816 & 20.699 & 16.659 & 13.022 & 11.626 & 10.716 & 9.799 & 9.662 & 8.75 & 8.181 & 7.671 & 8.272 \\
\hline S3 & 0.75 & 0.714 & pole-on & -0.504 & 7.768 & 39.064 & 23.126 & 18.917 & 14.626 & 12.986 & 12.017 & 10.91 & 10.849 & 9.759 & 9.122 & 8.538 & 9.491 \\
\hline S3 & 0.75 & 0.786 & pole-on & -0.528 & 9.07 & 44.233 & 26.264 & 21.328 & 16.791 & 14.515 & 13.348 & 12.209 & 12.188 & 10.78 & 10.221 & 9.489 & 10.772 \\
\hline S3 & 0.75 & 0.857 & pole-on & -0.546 & 10.241 & 50.048 & 29.805 & 24.179 & 18.407 & 16.055 & 14.738 & 13.523 & 13.715 & 11.791 & 11.392 & 10.459 & 12.308 \\
\hline S3 & 0.75 & 0.928 & pole-on & -0.558 & 11.376 & 56.329 & 33.654 & 27.469 & 20.423 & 17.598 & 16.122 & 14.937 & 15.502 & 12.834 & 12.598 & 11.494 & 13.966 \\
\hline S3 & 0.75 & 1.0 & pole-on & -0.564 & 12.272 & 62.403 & 37.599 & 30.712 & 22.353 & 19.05 & 17.358 & 16.426 & 17.222 & 13.898 & 13.888 & 12.498 & 15.763 \\
\hline
\end{tabular}


Table A.4 - continued.

\begin{tabular}{|c|c|c|c|c|c|c|c|c|c|c|c|c|c|c|c|c|c|}
\hline & & & $\tilde{\tau}_{\mathrm{bu}}$ & & $\mathrm{bu}$ & 0.15 & 0.45 & 0.75 & 1.50 & 2.25 & 3.00 & 4.50 & 6.00 & 9.00 & 15.00 & 30.00 & $\infty$ \\
\hline Star & $\Sigma_{0}\left[\mathrm{~g} \mathrm{~cm}^{-2}\right]$ & $\cos i$ & ang. type & $\Delta I_{\mathrm{bu}}^{\infty}$ & $\xi_{\mathrm{bu}}^{I}$ & $\xi_{\mathrm{d}}^{I}$ & & & & & & & & & & & \\
\hline S3 & 0.56 & 0.0 & edge-on & 0.126 & 2.039 & 5.87 & 3.326 & 2.869 & 2.127 & 1.883 & 1.682 & 1.462 & 1.339 & 1.237 & 1.078 & 0.956 & 0.932 \\
\hline S3 & 0.56 & 0.071 & edge-on & 0.082 & 2.254 & 4.444 & 2.372 & 2.039 & 1.61 & 1.435 & 1.265 & 1.113 & 1.008 & 0.954 & 0.821 & 0.731 & 0.703 \\
\hline S3 & 0.56 & 0.143 & interm. & 0.016 & 88.597 & 10.385 & 5.566 & 1.319 & 0.866 & 0.867 & 0.729 & 0.606 & 0.496 & 0.474 & 0.444 & 0.429 & 0.741 \\
\hline S3 & 0.56 & 0.215 & interm. & -0.052 & 1.001 & 331.716 & 353.645 & 346.738 & 186.359 & 58.832 & 46.865 & 33.787 & 33.434 & 25.692 & 22.568 & 18.609 & 19.871 \\
\hline S3 & 0.56 & 0.286 & interm. & -0.119 & 1.659 & 305.531 & 40.924 & 25.651 & 15.7 & 12.316 & 11.183 & 9.521 & 9.304 & 7.879 & 7.299 & 6.729 & 7.169 \\
\hline S3 & 0.56 & 0.357 & pole-on & -0.181 & 2.324 & 51.874 & 21.578 & 16.007 & 11.623 & 9.898 & 9.281 & 8.258 & 8.006 & 7.133 & 6.58 & 6.279 & 6.702 \\
\hline S3 & 0.56 & 0.429 & pole-on & -0.235 & 3.167 & 37.218 & 19.567 & 14.896 & 11.753 & 10.168 & 9.309 & 8.561 & 8.381 & 7.555 & 7.04 & 6.67 & 7.132 \\
\hline S3 & 0.56 & 0.5 & pole-on & -0.28 & 4.148 & 34.691 & 20.093 & 16.212 & 12.568 & 11.031 & 10.197 & 9.351 & 9.27 & 8.299 & 7.809 & 7.36 & 8.092 \\
\hline S3 & 0.56 & 0.571 & pole-on & -0.318 & 5.295 & 36.448 & 21.982 & 17.807 & 14.014 & 12.299 & 11.49 & 10.342 & 10.375 & 9.297 & 8.805 & 8.166 & 9.186 \\
\hline S3 & 0.56 & 0.643 & pole-on & -0.349 & 6.627 & 40.531 & 24.59 & 20.25 & 15.69 & 13.867 & 12.907 & 11.673 & 11.811 & 10.429 & 9.85 & 9.277 & 10.586 \\
\hline S3 & 0.56 & 0.714 & pole-on & -0.374 & 8.191 & 45.631 & 27.912 & 23.035 & 17.697 & 15.579 & 14.538 & 13.065 & 13.456 & 11.708 & 11.046 & 10.365 & 12.216 \\
\hline S3 & 0.56 & 0.786 & pole-on & -0.393 & 9.822 & 51.88 & 31.928 & 26.225 & 20.343 & 17.673 & 16.274 & 14.571 & 15.286 & 12.893 & 12.362 & 11.438 & 14.172 \\
\hline S3 & 0.56 & 0.857 & pole-on & -0.406 & 11.636 & 58.829 & 36.48 & 30.108 & 22.547 & 19.582 & 17.989 & 16.305 & 17.288 & 14.139 & 13.803 & 12.756 & 16.334 \\
\hline S3 & 0.56 & 0.928 & pole-on & -0.415 & 13.412 & 66.617 & 41.572 & 34.426 & 25.089 & 21.41 & 19.482 & 17.994 & 19.628 & 15.318 & 15.428 & 13.912 & 18.872 \\
\hline S3 & 0.56 & 1.0 & pole-on & -0.418 & 14.816 & 73.54 & 46.783 & 38.435 & 27.107 & 23.03 & 20.936 & 19.662 & 21.882 & 16.644 & 16.996 & 15.019 & 21.586 \\
\hline S3 & 0.41 & 0.0 & edge-on & 0.102 & 2.41 & 7.767 & 4.348 & 3.249 & 2.571 & 2.171 & 1.897 & 1.676 & 1.564 & 1.412 & 1.251 & 1.094 & 1.033 \\
\hline S3 & 0.41 & 0.071 & edge-on & 0.07 & 2.7 & 6.353 & 3.331 & 2.458 & 1.877 & 1.702 & 1.512 & 1.308 & 1.232 & 1.117 & 0.942 & 0.864 & 0.829 \\
\hline S3 & 0.41 & 0.143 & interm. & 0.023 & 22.766 & 27.121 & 2.725 & 2.133 & 1.581 & 1.429 & 1.233 & 1.071 & 0.963 & 0.859 & 0.725 & 0.613 & 0.62 \\
\hline S3 & 0.41 & 0.215 & interm. & -0.024 & 5.515 & 255.471 & 264.821 & 315.4 & 339.96 & 361.019 & 322.734 & 305.578 & 272.437 & 296.26 & 245.389 & 191.993 & 162.864 \\
\hline S3 & 0.41 & 0.286 & interm. & -0.071 & 1.534 & 360.395 & 172.611 & 44.942 & 23.101 & 18.209 & 14.909 & 12.309 & 11.041 & 9.989 & 8.92 & 8.02 & 9.064 \\
\hline S3 & 0.41 & 0.357 & pole-on & -0.115 & 2.221 & 134.63 & 25.941 & 18.958 & 12.722 & 11.282 & 9.922 & 8.974 & 8.719 & 7.658 & 7.313 & 6.739 & 7.588 \\
\hline S3 & 0.41 & 0.429 & pole-on & -0.154 & 3.017 & 44.534 & 20.907 & 16.705 & 12.745 & 10.855 & 10.037 & 9.245 & 9.08 & 8.2 & 7.58 & 7.127 & 8.05 \\
\hline S3 & 0.41 & 0.5 & pole-on & -0.187 & 3.975 & 37.972 & 22.308 & 17.761 & 13.599 & 12.081 & 11.017 & 10.127 & 10.053 & 9.028 & 8.471 & 8.009 & 9.116 \\
\hline S3 & 0.41 & 0.571 & pole-on & -0.215 & 5.026 & 40.4 & 23.967 & 20.05 & 15.302 & 13.682 & 12.391 & 11.337 & 11.433 & 10.13 & 9.606 & 9.019 & 10.589 \\
\hline S3 & 0.41 & 0.643 & pole-on & -0.237 & 6.393 & 44.407 & 26.933 & 22.864 & 17.527 & 15.375 & 14.137 & 12.791 & 13.283 & 11.482 & 10.82 & 10.25 & 12.267 \\
\hline S3 & 0.41 & 0.714 & pole-on & -0.256 & 7.937 & 50.186 & 31.271 & 26.031 & 20.105 & 17.714 & 16.285 & 14.442 & 15.187 & 12.919 & 12.336 & 11.51 & 14.464 \\
\hline S3 & 0.41 & 0.786 & pole-on & -0.269 & 9.679 & 57.877 & 36.552 & 30.103 & 22.993 & 20.237 & 18.311 & 16.329 & 17.438 & 14.444 & 13.915 & 12.99 & 16.952 \\
\hline S3 & 0.41 & 0.857 & pole-on & -0.278 & 11.432 & 65.73 & 42.375 & 34.617 & 25.723 & 22.479 & 20.271 & 18.403 & 20.189 & 15.81 & 15.548 & 14.185 & 19.832 \\
\hline S3 & 0.41 & 0.928 & pole-on & -0.284 & 13.457 & 73.564 & 48.068 & 39.927 & 28.529 & 24.25 & 21.98 & 20.266 & 22.96 & 16.997 & 17.477 & 15.478 & 23.259 \\
\hline S3 & 0.41 & 1.0 & pole-on & -0.284 & 14.72 & 83.029 & 53.384 & 45.304 & 31.218 & 26.106 & 23.527 & 22.286 & 25.72 & 18.47 & 19.202 & 16.934 & 26.69 \\
\hline S3 & 0.3 & 0.0 & edge-on & 0.082 & 15.313 & 10.277 & 4.996 & 3.731 & 3.003 & 2.563 & 2.208 & 1.96 & 1.745 & 1.601 & 1.434 & 1.24 & 1.206 \\
\hline S3 & 0.3 & 0.071 & edge-on & 0.059 & 2.989 & 10.465 & 4.166 & 3.066 & 2.405 & 2.09 & 1.778 & 1.638 & 1.447 & 1.271 & 1.139 & 1.044 & 0.983 \\
\hline S3 & 0.3 & 0.143 & interm. & 0.026 & 12.967 & 27.117 & 11.664 & 3.557 & 2.306 & 2.025 & 1.8 & 1.586 & 1.395 & 1.321 & 1.092 & 1.009 & 0.905 \\
\hline S3 & 0.3 & 0.215 & interm. & -0.003 & 209.896 & 118.636 & 129.681 & 114.352 & 97.154 & 150.596 & 218.312 & 226.937 & 268.823 & 281.904 & 297.38 & 325.499 & 370.601 \\
\hline S3 & 0.3 & 0.286 & interm. & -0.038 & 1.358 & 285.498 & 358.619 & 231.412 & 104.761 & 46.5 & 38.687 & 16.196 & 18.594 & 12.336 & 10.262 & 8.416 & 11.358 \\
\hline
\end{tabular}


Table A.4 - continued.

\begin{tabular}{|c|c|c|c|c|c|c|c|c|c|c|c|c|c|c|c|c|c|}
\hline & & & $\overline{\tilde{\tau}_{\mathrm{bu}}}$ & & $\mathrm{bu}$ & 0.15 & $\overline{0.45}$ & 0.75 & 1.50 & 2.25 & 3.00 & 4.50 & 6.00 & 9.00 & 15.00 & 30.00 & $\infty$ \\
\hline Star & $\Sigma_{0}\left[\mathrm{~g} \mathrm{~cm}^{-2}\right]$ & $\cos i$ & ang. type & $\Delta I_{\mathrm{bu}}^{\infty}$ & $\xi_{\mathrm{bu}}^{I}$ & $\xi_{\mathrm{d}}^{I}$ & & & & & & & & & & & \\
\hline S3 & 0.3 & 0.357 & pole-on & -0.068 & 2.107 & 276.818 & 38.972 & 24.38 & 13.669 & 11.446 & 9.822 & 9.063 & 8.924 & 7.653 & 6.878 & 6.39 & 7.563 \\
\hline S3 & 0.3 & 0.429 & pole-on & -0.096 & 2.858 & 55.426 & 22.347 & 17.028 & 12.664 & 10.858 & 10.102 & 8.911 & 9.09 & 7.852 & 7.357 & 6.837 & 7.876 \\
\hline S3 & 0.3 & 0.5 & pole-on & -0.119 & 3.761 & 39.24 & 21.958 & 18.275 & 13.603 & 11.713 & 11.088 & 9.909 & 10.013 & 8.833 & 8.269 & 7.808 & 9.392 \\
\hline S3 & 0.3 & 0.571 & pole-on & -0.138 & 4.843 & 38.879 & 24.442 & 20.42 & 15.403 & 13.508 & 12.489 & 11.317 & 11.876 & 10.378 & 9.437 & 8.92 & 10.902 \\
\hline S3 & 0.3 & 0.643 & pole-on & -0.154 & 6.292 & 44.199 & 28.503 & 23.322 & 18.059 & 15.605 & 14.594 & 12.808 & 13.629 & 11.708 & 11.192 & 10.414 & 13.009 \\
\hline S3 & 0.3 & 0.714 & pole-on & -0.167 & 7.657 & 51.276 & 33.098 & 27.639 & 20.555 & 17.934 & 16.442 & 14.873 & 15.678 & 13.241 & 12.558 & 11.727 & 15.46 \\
\hline S3 & 0.3 & 0.786 & pole-on & -0.175 & 9.429 & 59.954 & 39.476 & 32.346 & 24.34 & 20.823 & 19.089 & 16.714 & 18.258 & 14.774 & 14.494 & 13.208 & 18.527 \\
\hline S3 & 0.3 & 0.857 & pole-on & -0.182 & 11.312 & 67.738 & 45.228 & 37.07 & 27.192 & 23.547 & 21.288 & 18.963 & 21.26 & 16.293 & 16.22 & 14.616 & 22.089 \\
\hline S3 & 0.3 & 0.928 & pole-on & -0.185 & 13.282 & 78.588 & 51.55 & 43.563 & 30.5 & 25.321 & 23.048 & 21.081 & 24.774 & 17.634 & 18.403 & 16.014 & 26.138 \\
\hline S3 & 0.3 & 1.0 & pole-on & -0.184 & 14.856 & 88.985 & 57.125 & 48.268 & 32.583 & 27.649 & 24.818 & 23.477 & 27.958 & 19.201 & 20.303 & 17.894 & 30.033 \\
\hline
\end{tabular}




\section{Appendix B}

\section{Examples of fittings of the empirical laws}

Here we give examples of synthetic light curves fitted with Eqs. (4.3.7) and (4.3.8), with the $\eta$ 's given by Table 4.4. The figures on this appendix have 8 panels disposed in 4 columns and 2 lines. The panels for the BVRI filters are, respectively, the panels of the first, second, third and forth columns. In the panels from the first line, the synthetic light curves generated by HDUST are the dots connected by dashed segments and the best fit of the empirical law to the synthetic light curves are the solid curves. The panels from the second line show the residuals of the fit from the respective above panels.

In the following sections, each light curve is defined by the vector (Star, $i, \Sigma_{0}, \tilde{\tau}_{\mathrm{bu}}$ ) (corresponding to one element of each column of Table 4.1). For simplicity, we decided to show only light curves of Star 2 (the light curves of the other stars are qualitatively similar).

\section{B.1 $\left(\right.$ Star $\left., i, \Sigma_{0}, \tilde{\tau}_{\text {bu }}\right)=\left(\operatorname{Star} 2, *, 1.37 \mathbf{g ~ c m}^{-2}, *\right)$}

In this section, we fix $\Sigma_{0}$ and, varying $i$, we show three examples of fittings for each $i$ (the examples are for $\tilde{\tau}_{\mathrm{bu}}=\mathrm{bu}, 1.5,6$ ). 


\section{B.1.1 (Star, $\left.i, \Sigma_{0}, \tilde{\tau}_{\mathrm{bu}}\right)=\left(\operatorname{Star} 2,90 \mathrm{deg}, 1.37 \mathrm{~g} \mathrm{~cm}^{-2}, *\right)$}

Three examples of fittings for $i=90 \mathrm{deg}$.
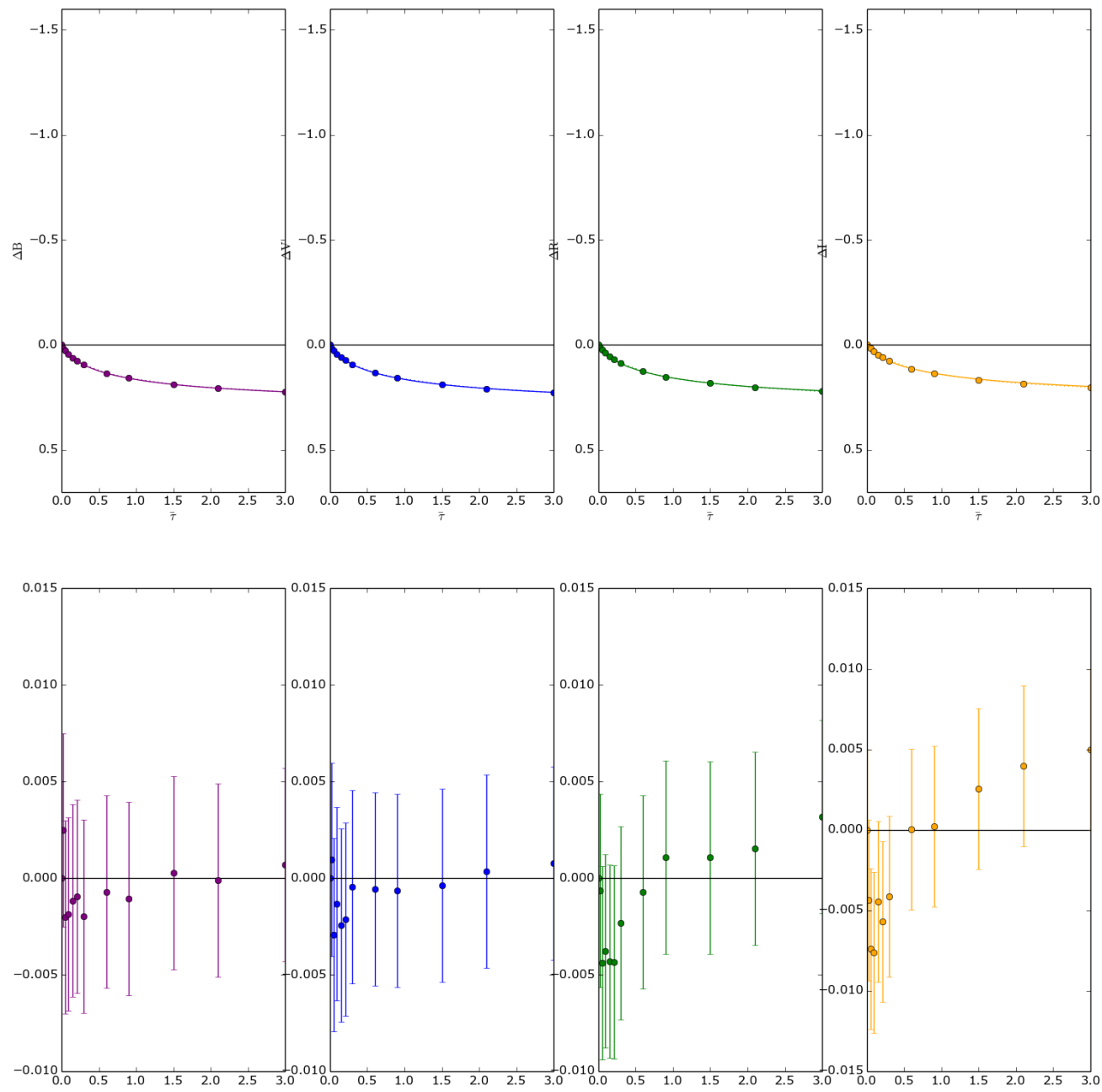

Figure B.1: $\left(\operatorname{Star}, i, \Sigma_{0}, \tilde{\tau}_{\mathrm{bu}}\right)=\left(\operatorname{Star} 2,90 \mathrm{deg}, 1.37 \mathrm{~g} \mathrm{~cm}^{-2}, \mathbf{b u}\right)$ 

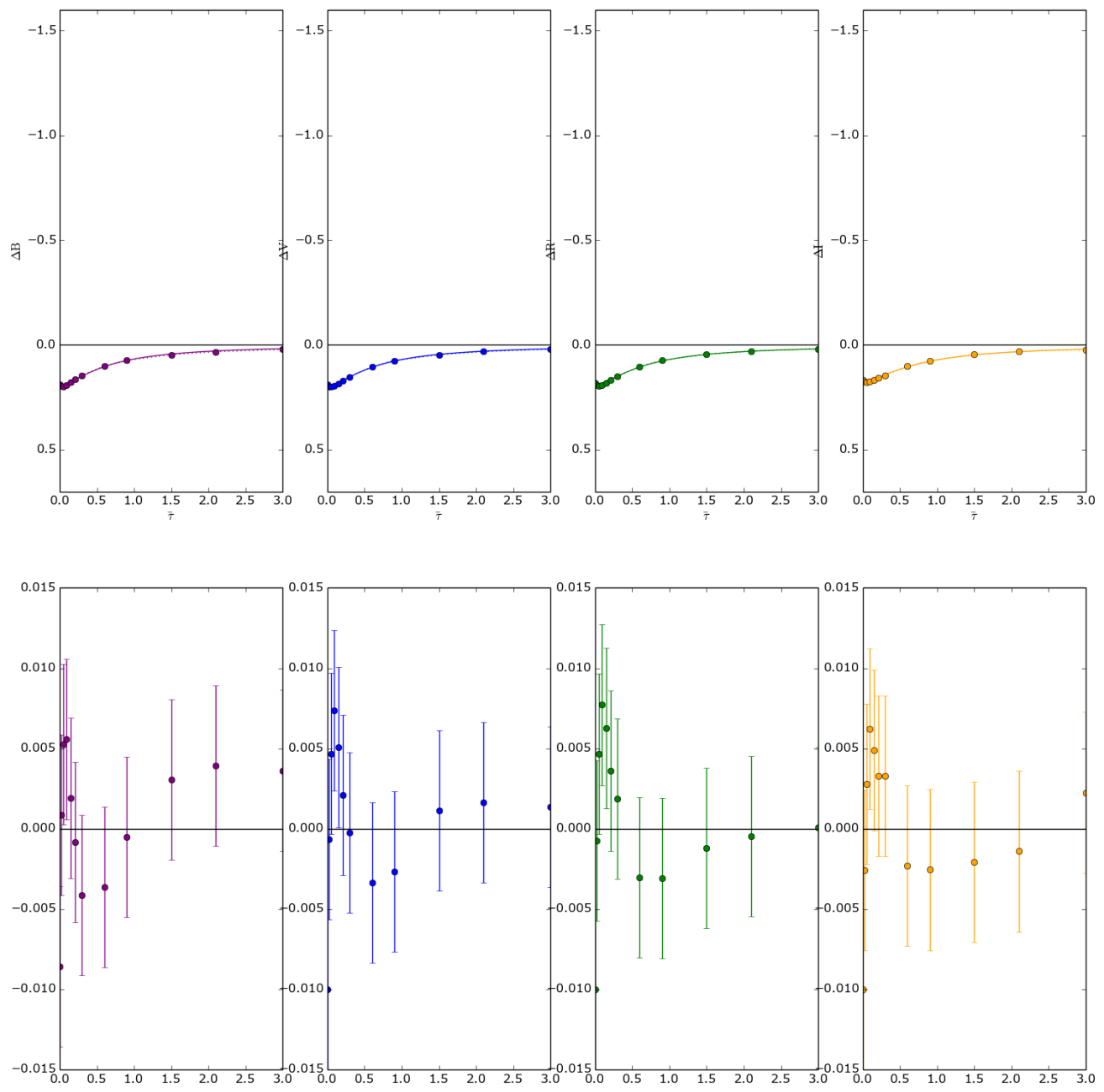

Figure B.2: $\left(\operatorname{Star}, i, \Sigma_{0}, \tilde{\tau}_{\mathrm{bu}}\right)=\left(\operatorname{Star} 2,90 \mathrm{deg}, 1.37 \mathrm{~g} \mathrm{~cm}^{-2}, \mathbf{1 . 5}\right)$ 

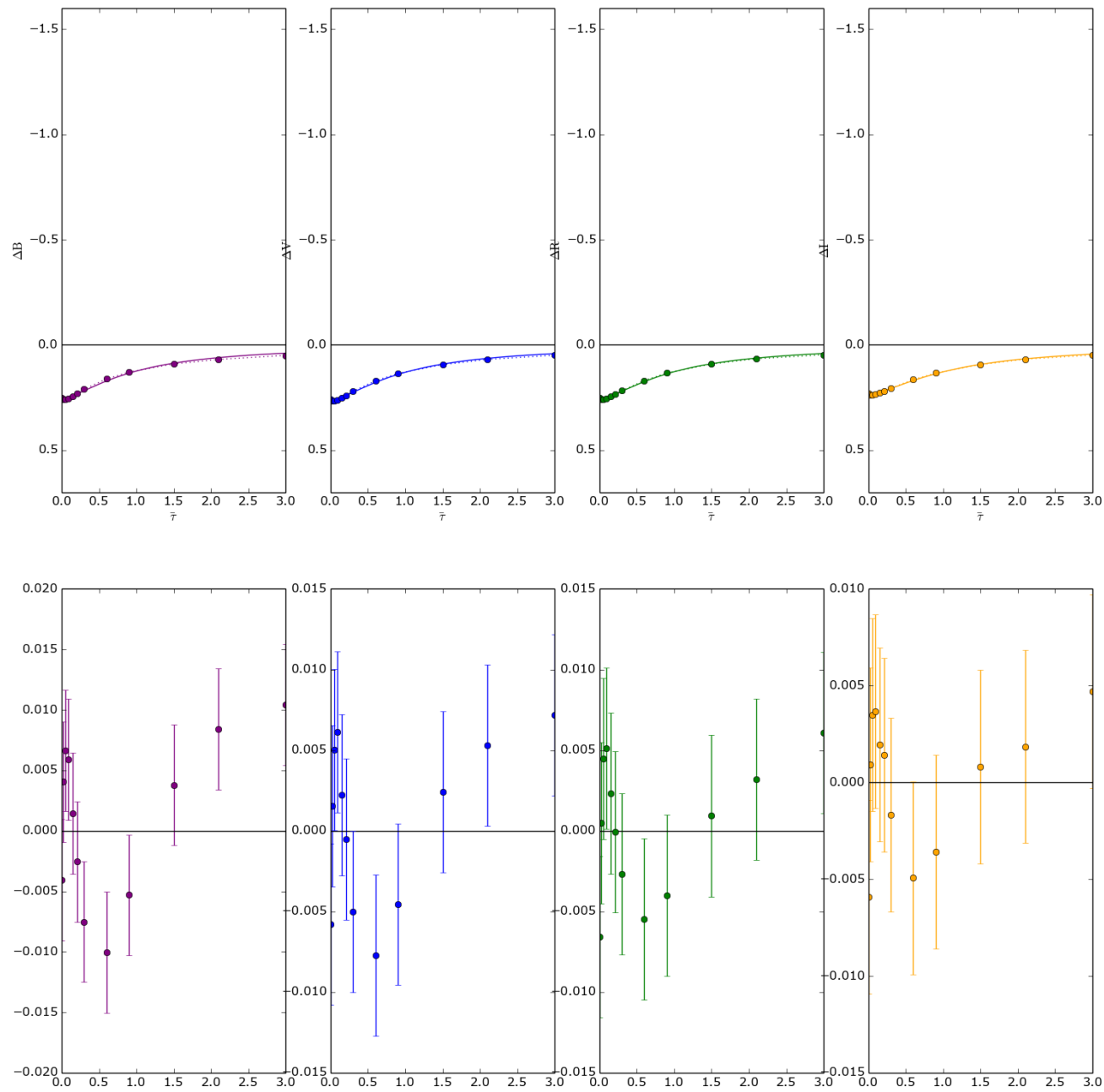

Figure B.3: $\left(\operatorname{Star}, i, \Sigma_{0}, \tilde{\mathrm{r}}_{\mathrm{bu}}\right)=\left(\operatorname{Star} 2,90 \mathrm{deg}, 1.37 \mathrm{~g} \mathrm{~cm}^{-2}, \mathbf{6}\right)$ 


\section{B.1.2 (Star, $\left.i, \Sigma_{0}, \tilde{\tau}_{\mathrm{bu}}\right)=\left(\operatorname{Star} 2,69.1 \mathrm{deg}, 1.37 \mathrm{~g} \mathrm{~cm}^{-2}, *\right)$}

Three examples of fittings for $i=69.1 \mathrm{deg}$.
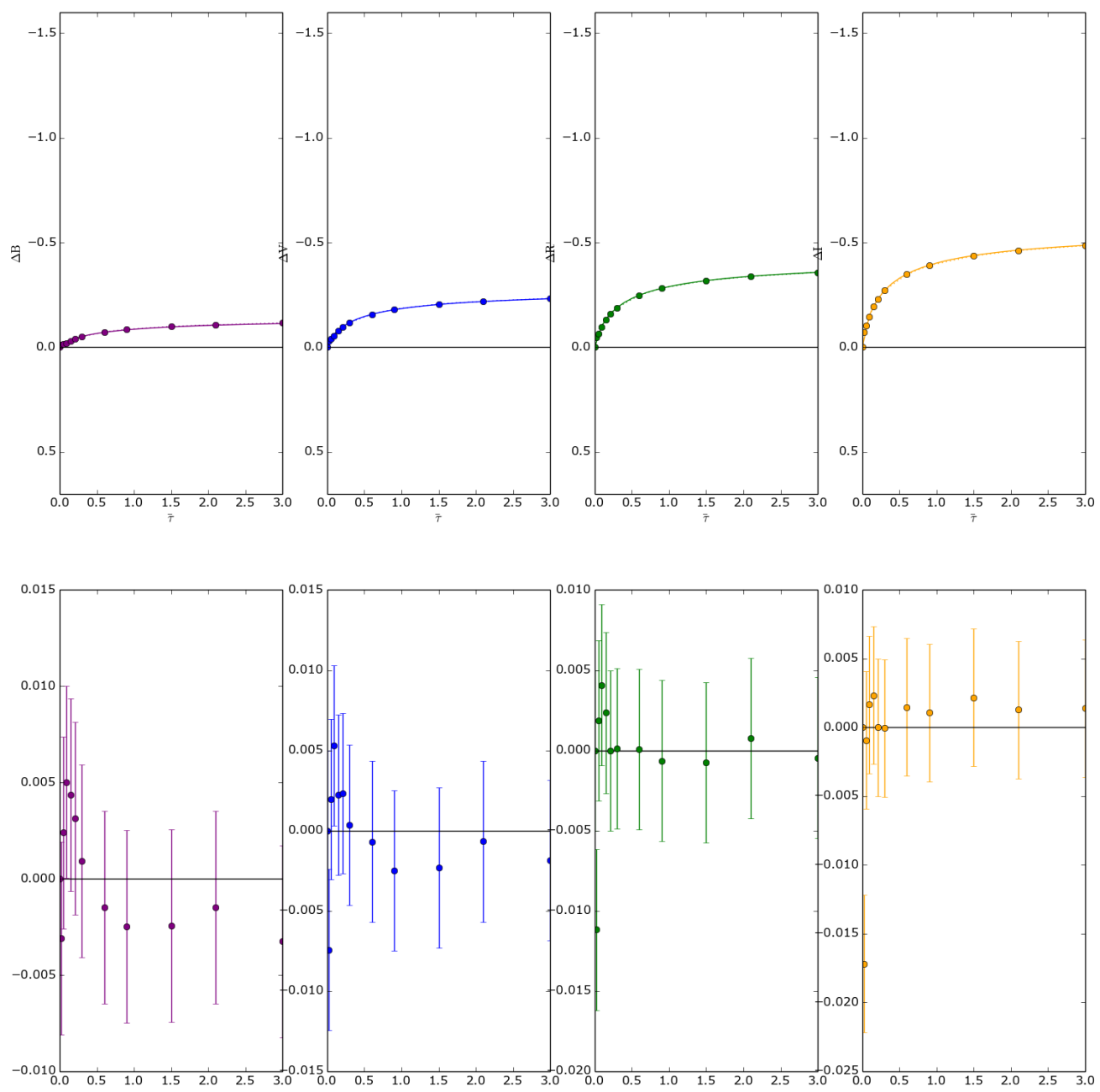

Figure B.4: $\left(\operatorname{Star}, i, \Sigma_{0}, \tilde{\tau}_{\mathrm{bu}}\right)=\left(\operatorname{Star} 2,69.1 \mathrm{deg}, 1.37 \mathrm{~g} \mathrm{~cm}^{-2}, \mathbf{b u}\right)$ 

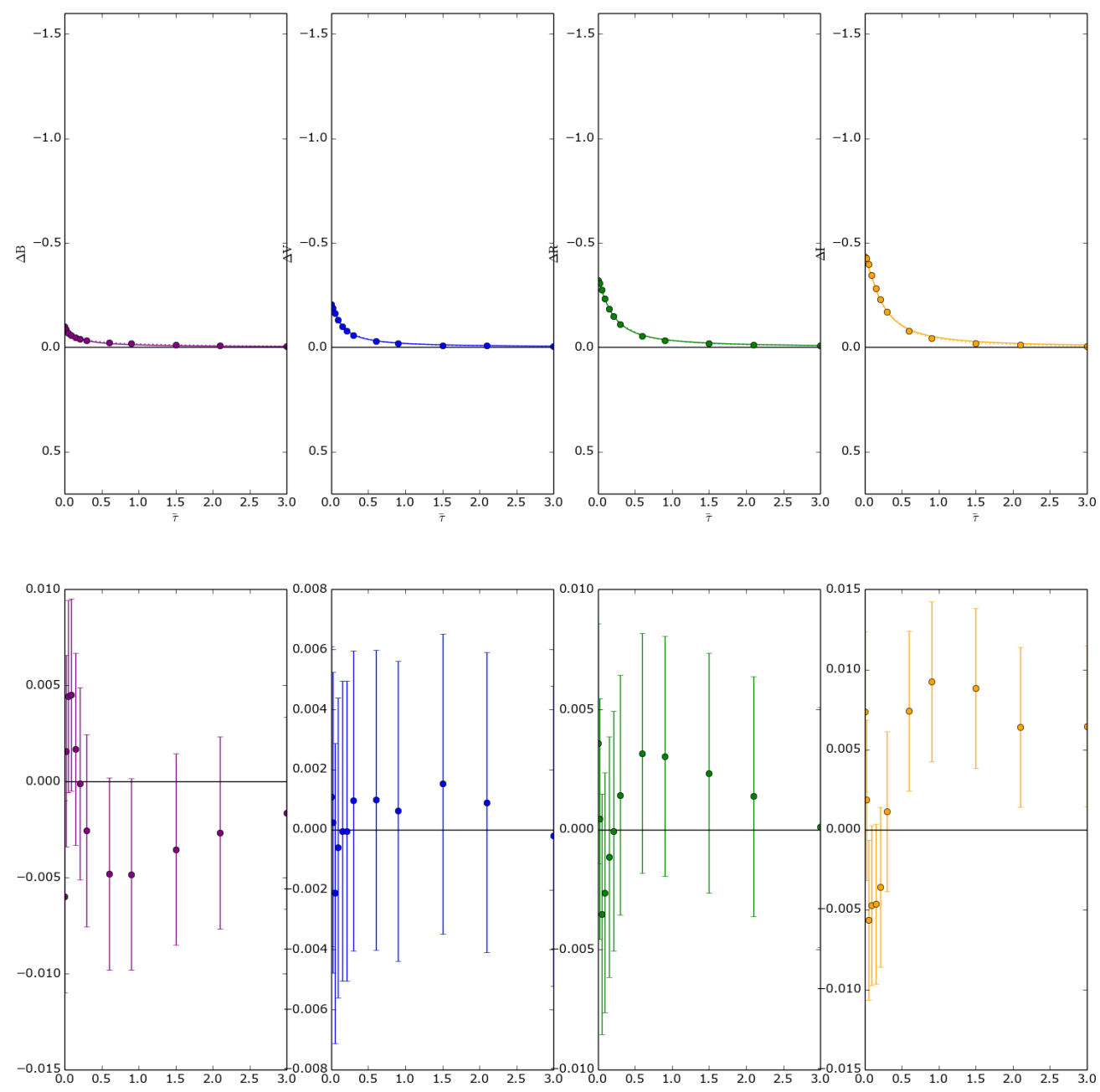

Figure B.5: $\left(\operatorname{Star}, i, \Sigma_{0}, \tilde{\tau}_{\mathrm{bu}}\right)=\left(\operatorname{Star} 2,69.1 \mathrm{deg}, 1.37 \mathrm{~g} \mathrm{~cm}^{-2}, \mathbf{1 . 5}\right)$ 

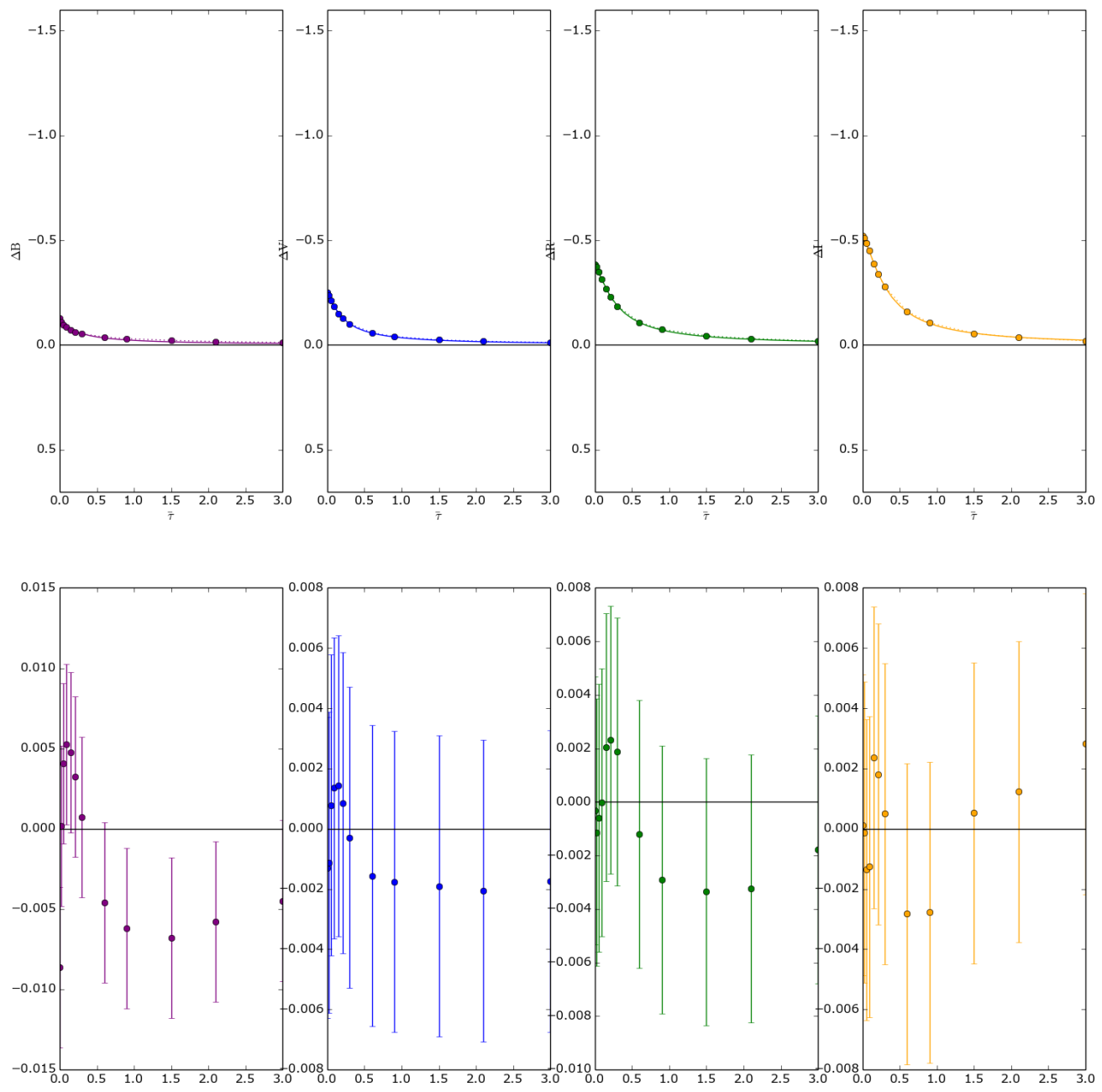

Figure B.6: $\left(\operatorname{Star}, i, \Sigma_{0}, \tilde{\tau}_{\mathrm{bu}}\right)=\left(\operatorname{Star} 2,69.1 \mathrm{deg}, 1.37 \mathrm{~g} \mathrm{~cm}^{-2}, \mathbf{6}\right)$ 


\section{B.1.3 (Star, $\left.i, \Sigma_{0}, \tilde{\tau}_{\mathrm{bu}}\right)=\left(\operatorname{Star} 2,0 \mathrm{deg}, 1.37 \mathrm{~g} \mathrm{~cm}^{-2}, *\right)$}

Three examples of fittings for $i=0 \mathrm{deg}$.
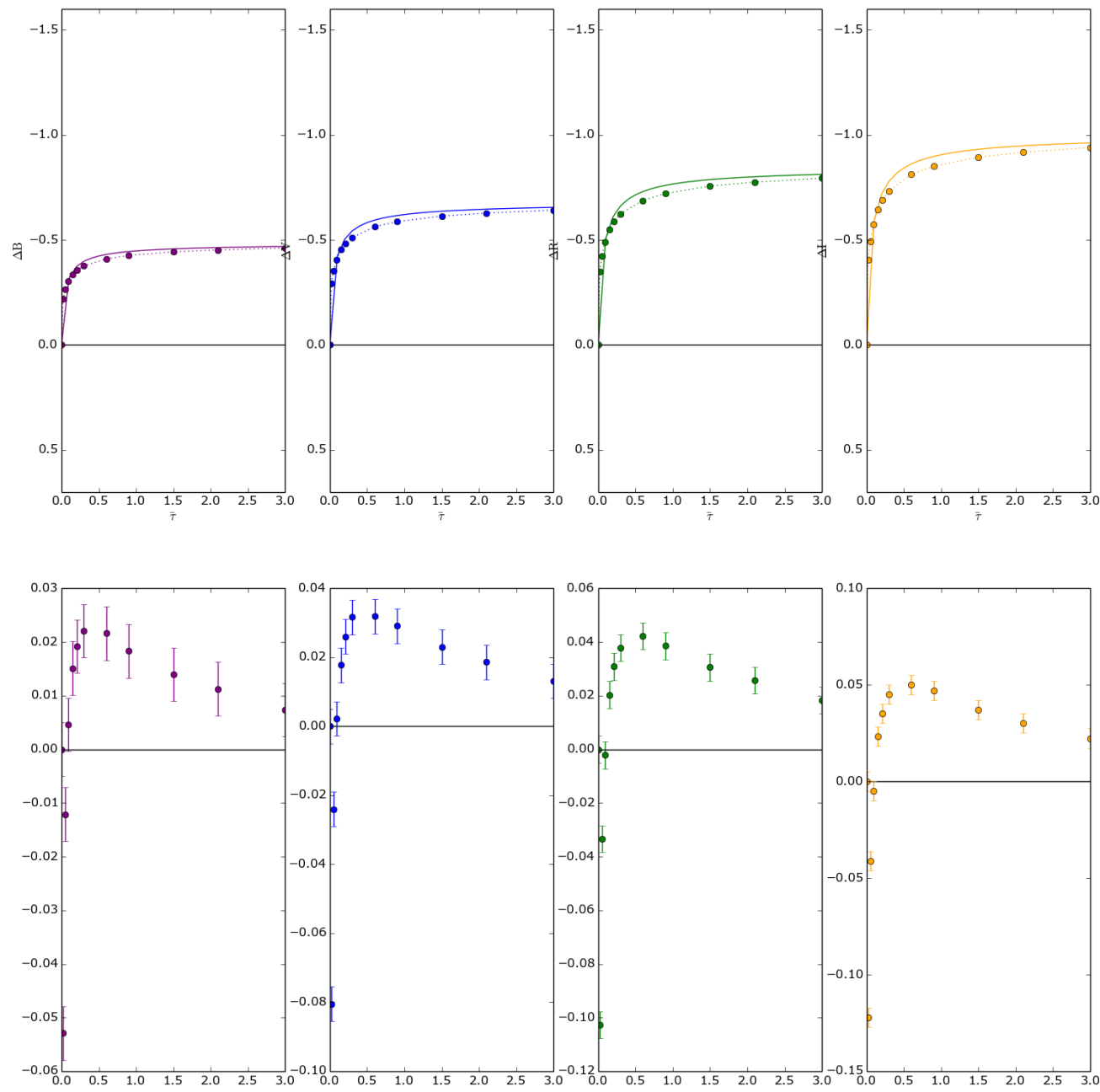

Figure B.7: $\left(\operatorname{Star}, i, \Sigma_{0}, \tilde{\tau}_{\mathrm{bu}}\right)=\left(\operatorname{Star} 2,0 \mathrm{deg}, 1.37 \mathrm{~g} \mathrm{~cm}^{-2}, \mathbf{b u}\right)$ 

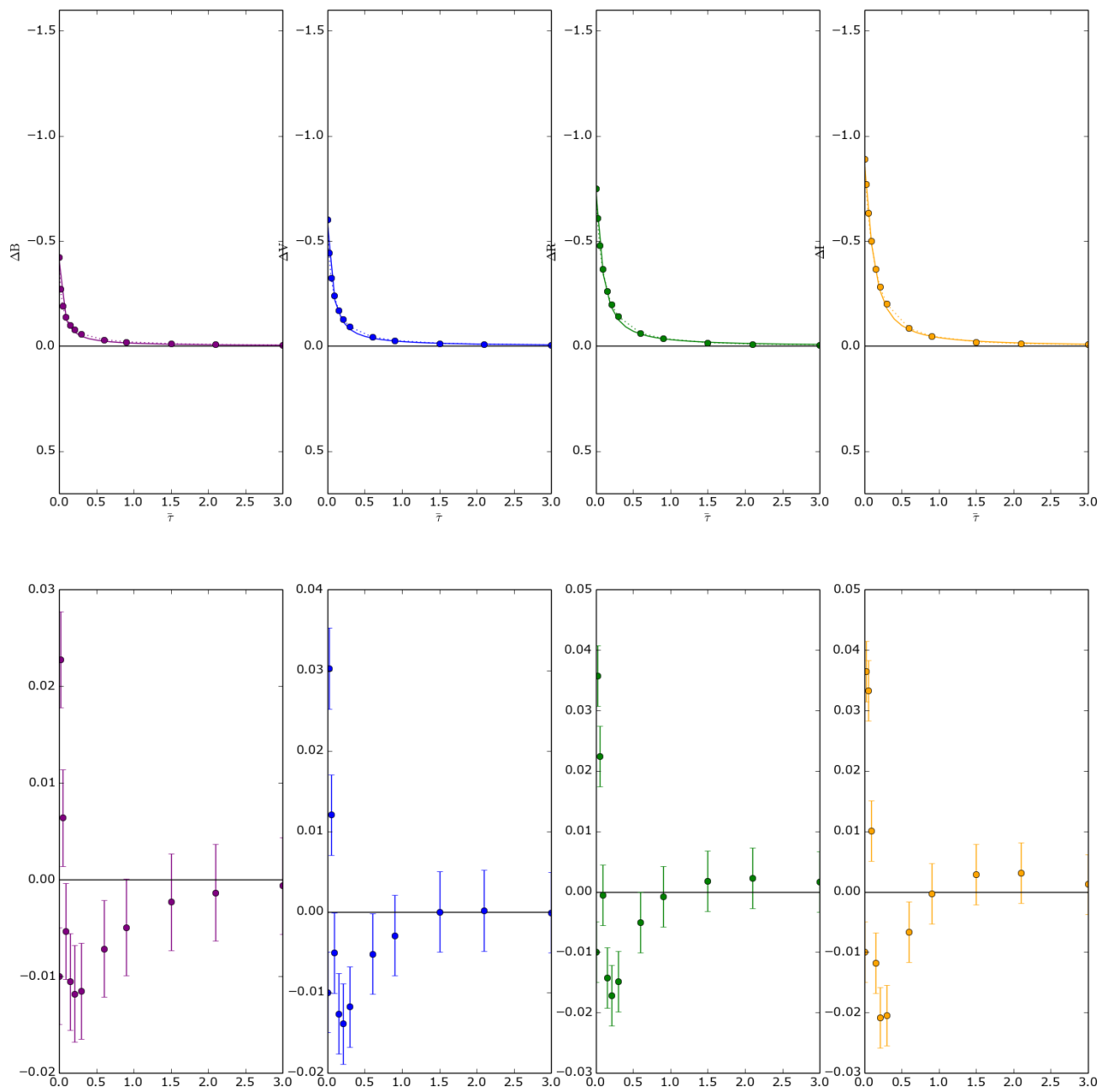

Figure B.8: $\left(\operatorname{Star}, i, \Sigma_{0}, \tilde{\tau}_{\mathrm{bu}}\right)=\left(\operatorname{Star} 2,0 \mathrm{deg}, 1.37 \mathrm{~g} \mathrm{~cm}^{-2}, \mathbf{1 . 5}\right)$ 

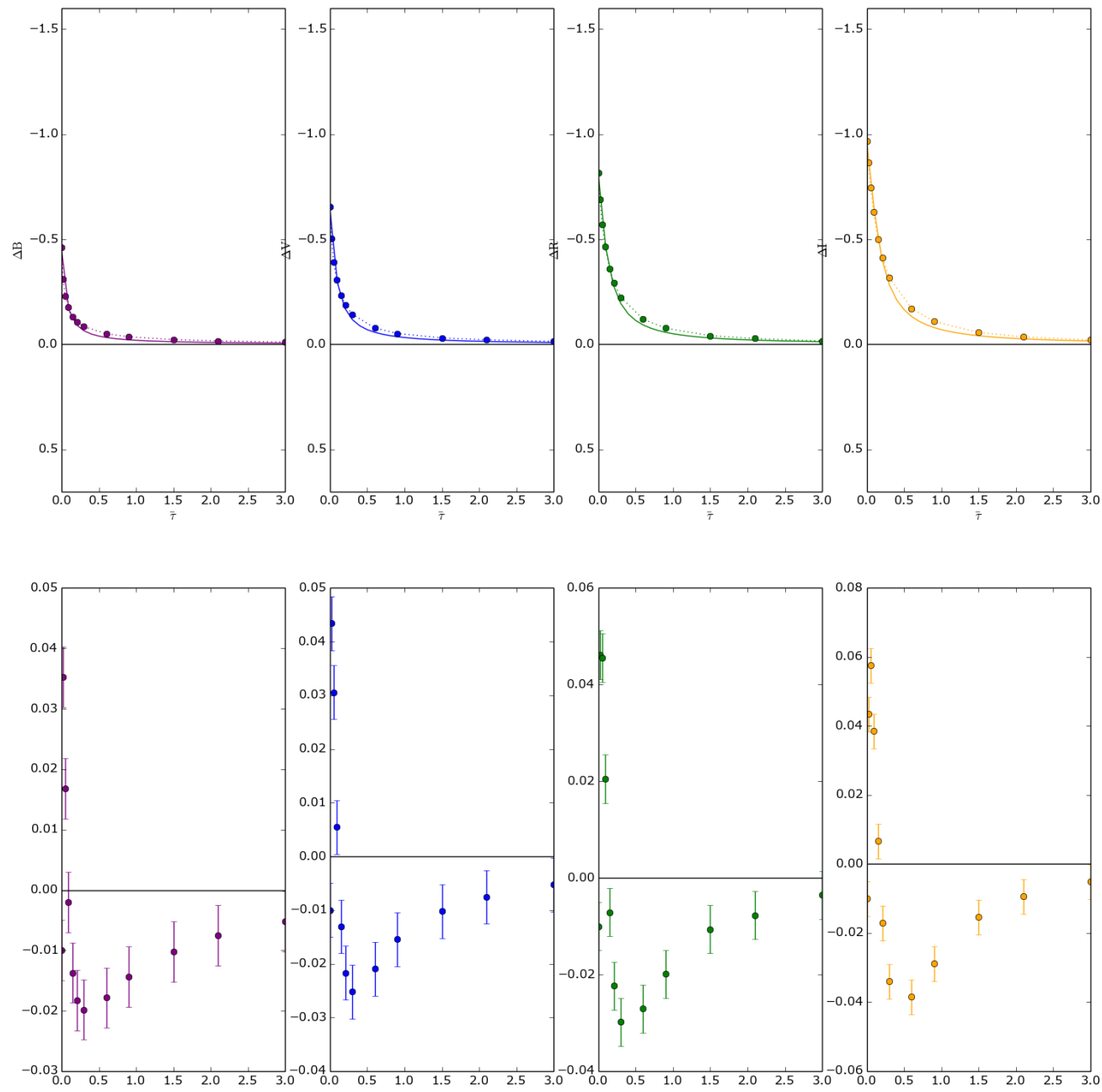

Figure B.9: $\left(\operatorname{Star}, i, \Sigma_{0}, \tilde{\tau}_{\mathrm{bu}}\right)=\left(\operatorname{Star} 2,0 \mathrm{deg}, 1.37 \mathrm{~g} \mathrm{~cm}^{-2}, \mathbf{6}\right)$ 


\section{B.2 $\left(\right.$ Star, $\left.i, \Sigma_{0}, \tilde{\tau}_{\mathrm{bu}}\right)=(\operatorname{Star} 2,38.2 \mathrm{deg}, *, *)$}

In this section, we fix $i$ and, varying $\Sigma_{0}$, we show three examples of fittings for each $\Sigma_{0}$ (the examples are for $\tilde{\tau}_{\mathrm{bu}}=\mathrm{bu}, 1.5,6$ ). 


\section{B.2.1 (Star, $\left.i, \Sigma_{0}, \tilde{\tau}_{\mathrm{bu}}\right)=\left(\operatorname{Star} 2,38.2 \mathrm{deg}, \mathbf{2 . 5 0} \mathbf{g ~ c m}^{-\mathbf{2}}, *\right)$}

Three examples of fittings for $\Sigma_{0}=2.50 \mathrm{~g} \mathrm{~cm}^{-2}$.
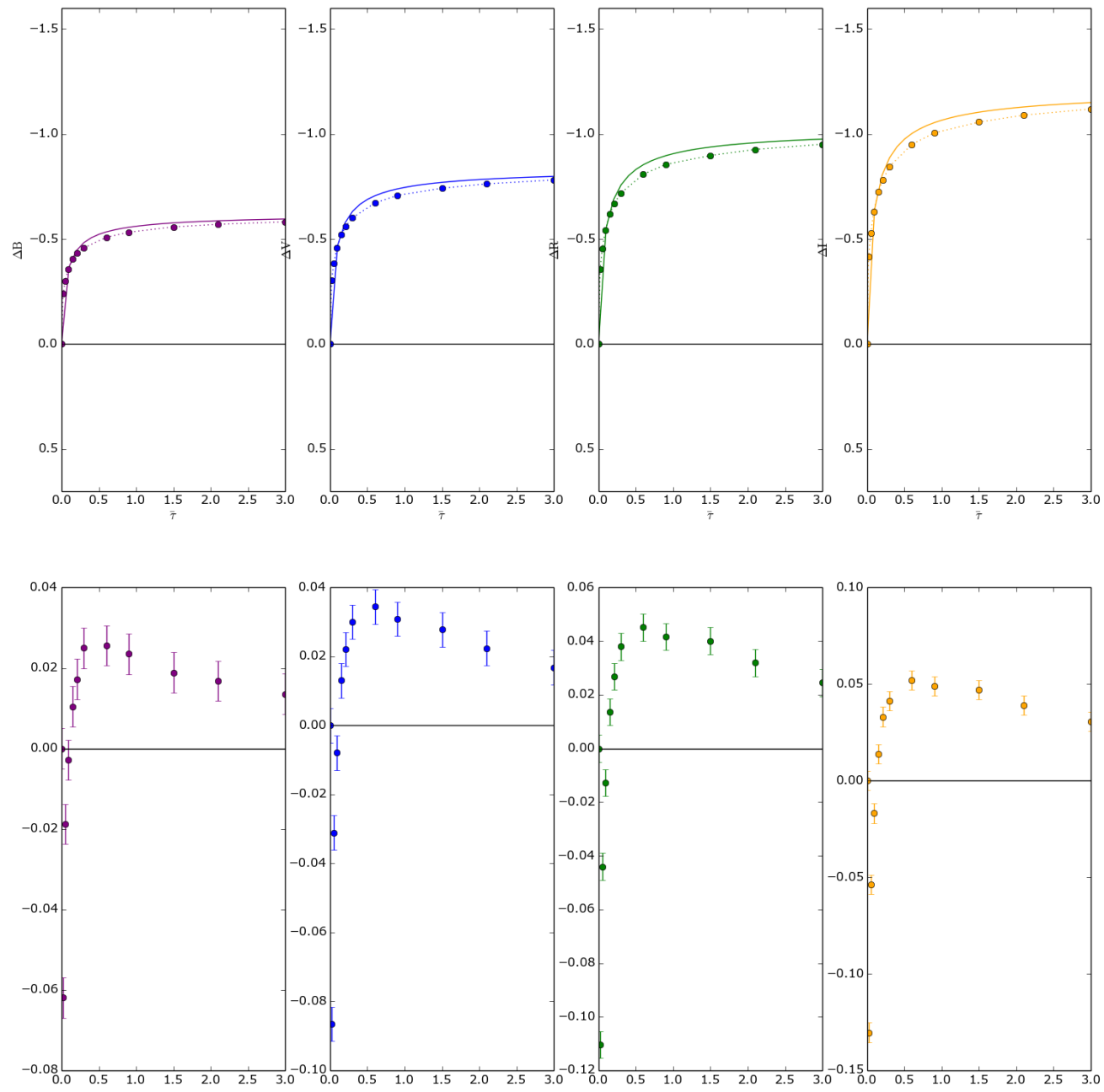

Figure B.10: $\left(\operatorname{Star}, i, \Sigma_{0}, \tilde{\tau}_{\mathrm{bu}}\right)=\left(\operatorname{Star} 2,38.2 \mathrm{deg}, 2.50 \mathrm{~g} \mathrm{~cm}^{-2}, \mathbf{b u}\right)$ 

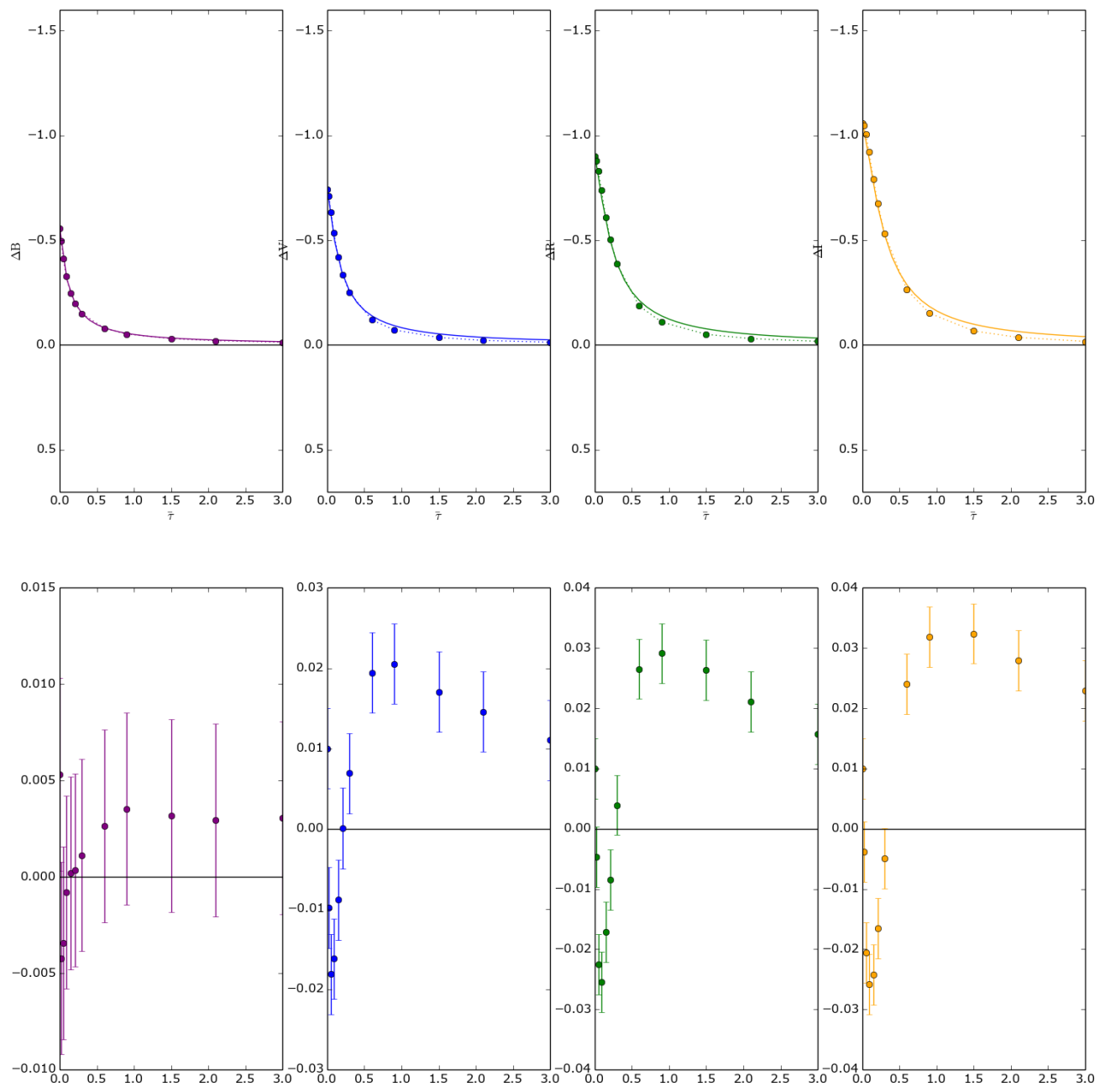

Figure B.11: $\left(\operatorname{Star}, i, \Sigma_{0}, \tilde{\mathrm{t}}_{\mathrm{bu}}\right)=\left(\operatorname{Star} 2,38.2 \mathrm{deg}, 2.50 \mathrm{~g} \mathrm{~cm}^{-2}, \mathbf{1 . 5}\right)$ 

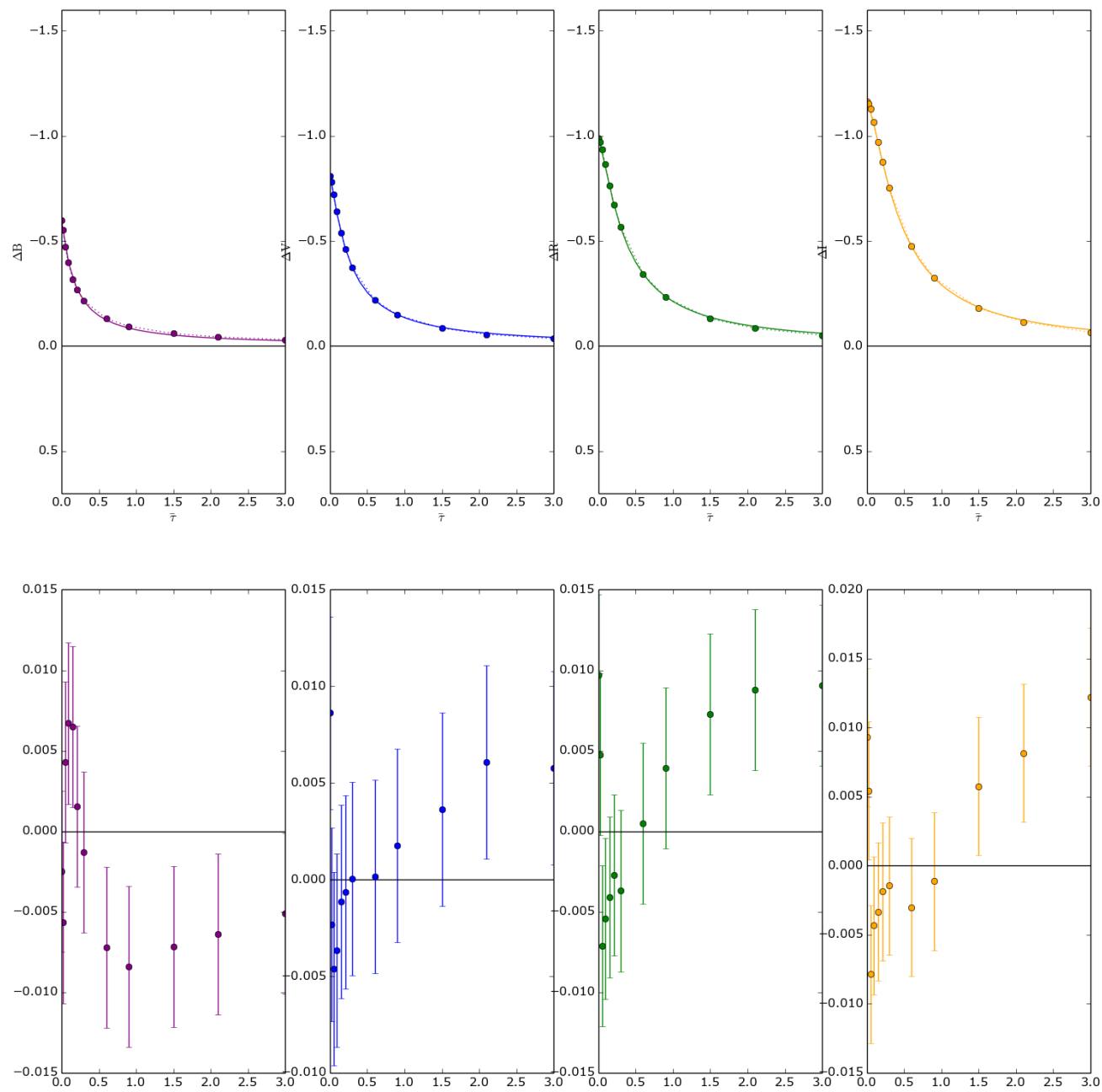

Figure B.12: $\left(\operatorname{Star}, i, \Sigma_{0}, \tilde{\tau}_{\mathrm{bu}}\right)=\left(\operatorname{Star} 2,38.2 \mathrm{deg}, 2.50 \mathrm{~g} \mathrm{~cm}^{-2}, \mathbf{6}\right)$ 


\section{B.2.2 (Star, $\left.i, \Sigma_{0}, \tilde{\tau}_{\mathrm{bu}}\right)=\left(\operatorname{Star} 2,38.2 \mathrm{deg}, \mathbf{1 . 0 1} \mathbf{g ~ c m}^{-\mathbf{2}}, *\right)$}

Three examples of fittings for $\Sigma_{0}=1.01 \mathrm{~g} \mathrm{~cm}^{-2}$.
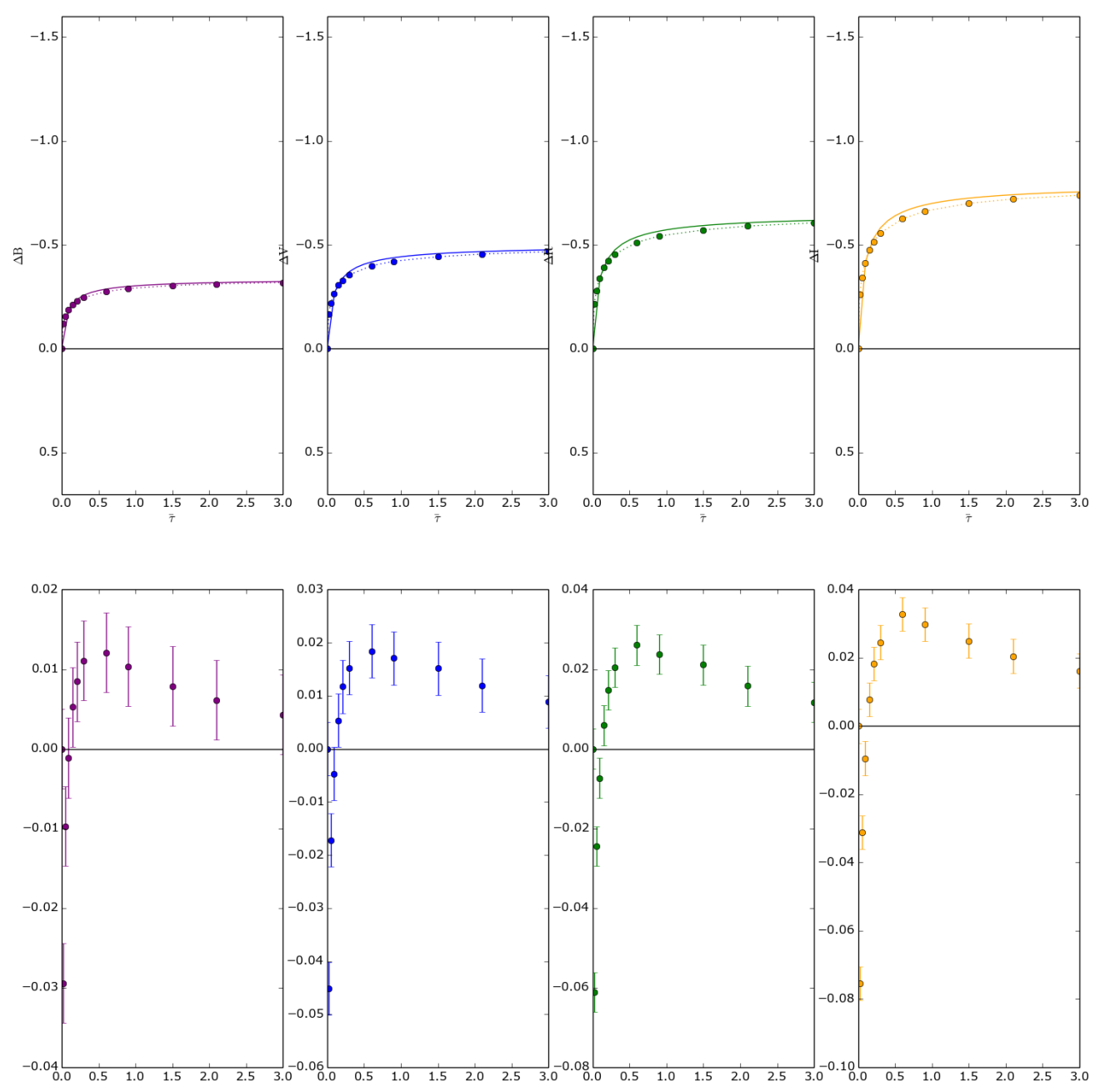

Figure B.13: $\left(\operatorname{Star}, i, \Sigma_{0}, \tilde{\mathrm{r}}_{\mathrm{bu}}\right)=\left(\operatorname{Star} 2,38.2 \mathrm{deg}, 1.01 \mathrm{~g} \mathrm{~cm}^{-2}, \mathbf{b u}\right)$ 

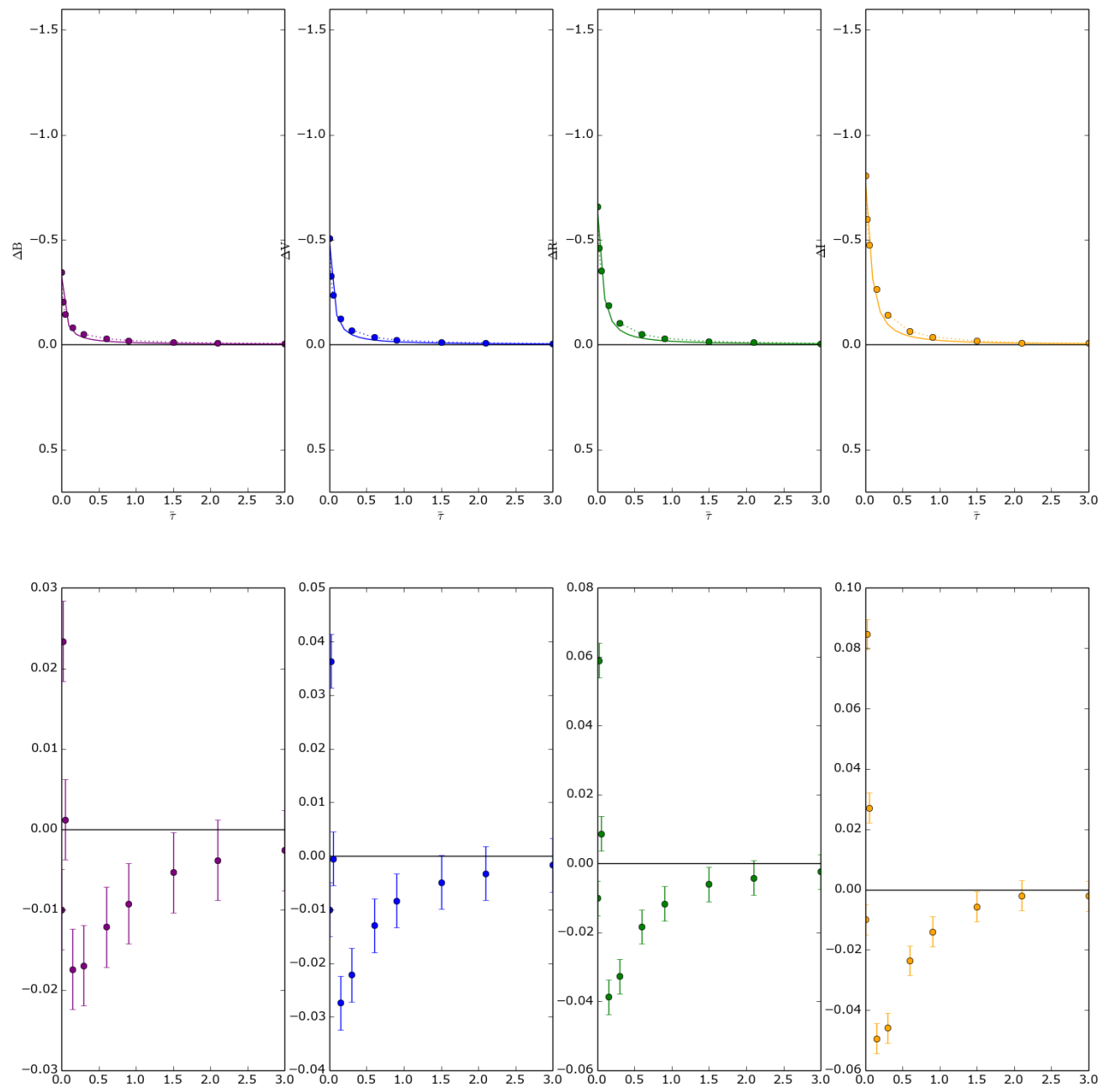

Figure B.14: $\left(\operatorname{Star}, i, \Sigma_{0}, \tilde{\tau}_{\mathrm{bu}}\right)=\left(\operatorname{Star} 2,38.2 \mathrm{deg}, 1.01 \mathrm{~g} \mathrm{~cm}^{-2}, \mathbf{1 . 5}\right)$ 

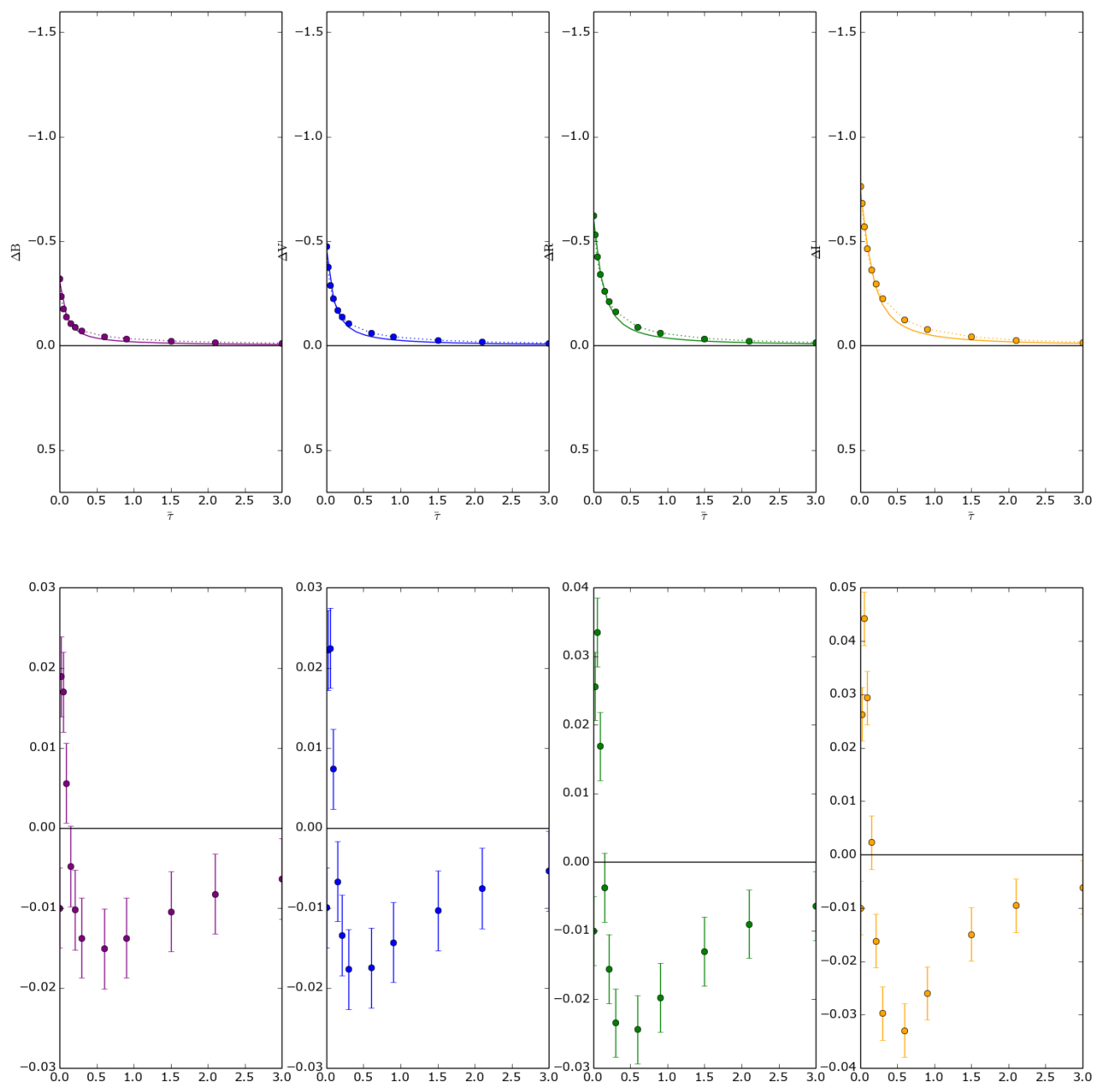

Figure B.15: $\left(\operatorname{Star}, i, \Sigma_{0}, \tilde{\tau}_{\mathrm{bu}}\right)=\left(\operatorname{Star} 2,38.2 \mathrm{deg}, 1.01 \mathrm{~g} \mathrm{~cm}^{-2}, \mathbf{6}\right)$ 


\section{B.2.3 (Star, $\left.i, \Sigma_{0}, \tilde{\tau}_{\mathrm{bu}}\right)=\left(\operatorname{Star} 2,38.2 \mathrm{deg}, \mathbf{0 . 4 1} \mathbf{g} \mathbf{~ c m}^{-\mathbf{2}}, *\right)$}

Three examples of fittings for $\Sigma_{0}=0.41 \mathrm{~g} \mathrm{~cm}^{-2}$.
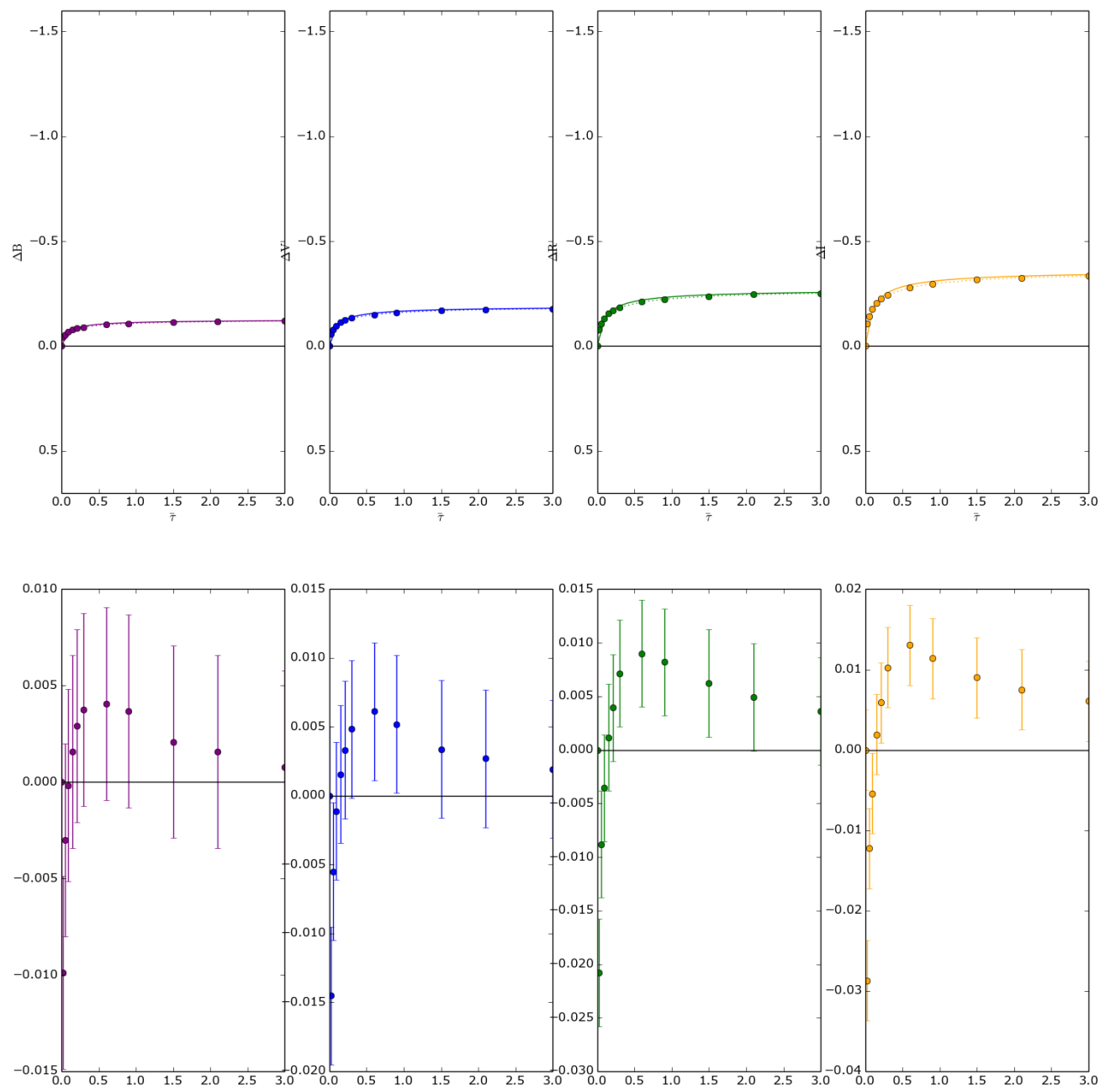

Figure B.16: $\left(\operatorname{Star}, i, \Sigma_{0}, \tilde{\tau}_{\mathrm{bu}}\right)=\left(\operatorname{Star} 2,38.2 \mathrm{deg}, 0.41 \mathrm{~g} \mathrm{~cm}^{-2}, \mathbf{b u}\right)$ 

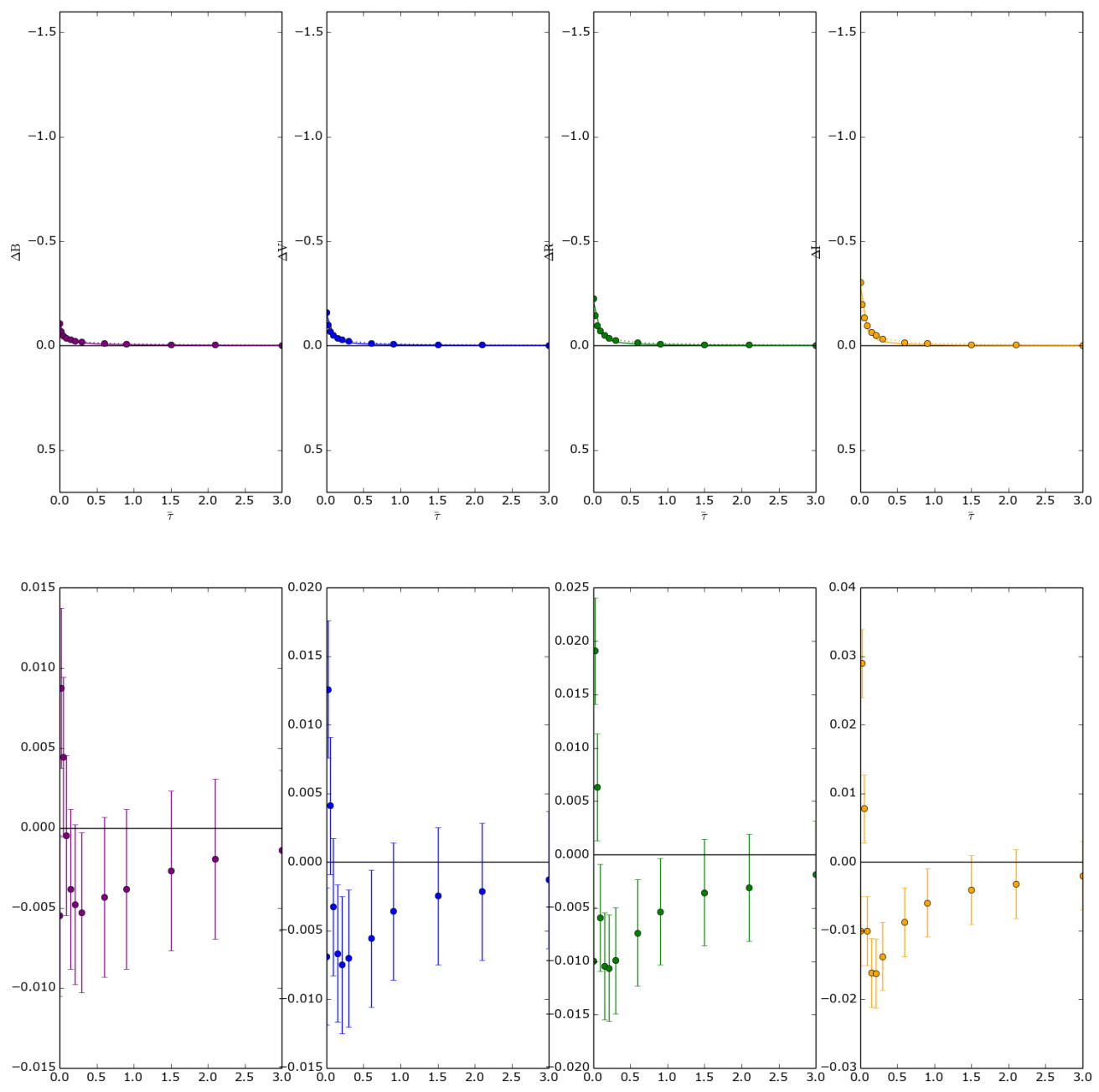

Figure B.17: $\left(\operatorname{Star}, i, \Sigma_{0}, \tilde{\mathrm{t}}_{\mathrm{bu}}\right)=\left(\operatorname{Star} 2,38.2 \mathrm{deg}, 0.41 \mathrm{~g} \mathrm{~cm}^{-2}, \mathbf{1 . 5}\right)$ 

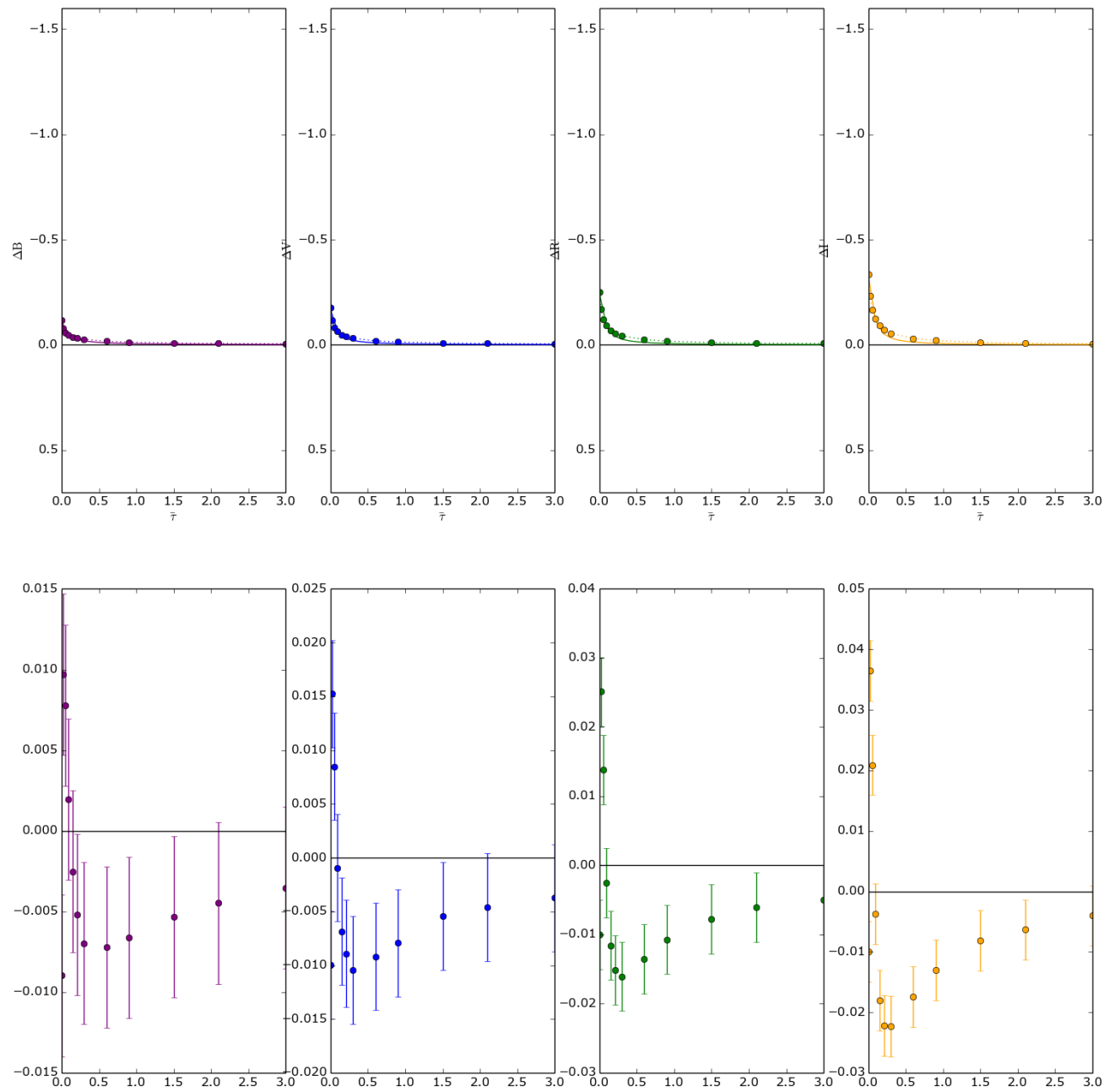

Figure B.18: $\left(\operatorname{Star}, i, \Sigma_{0}, \tilde{\tau}_{\mathrm{bu}}\right)=\left(\operatorname{Star} 2,38.2 \mathrm{deg}, 0.41 \mathrm{~g} \mathrm{~cm}^{-2}, \mathbf{6}\right)$ 
Appendix C

Scientific paper 


\title{
The life cycles of Be viscous decretion discs: fundamental disc parameters of 54 SMC Be stars
}

\author{
L. R. Rímulo, ${ }^{1 \star}$ A. C. Carciofi, ${ }^{1}$ R. G. Vieira,${ }^{1}$ Th. Rivinius,${ }^{2}$ D. M. Faes, ${ }^{1}$ \\ M. R. Ghoreyshi, ${ }^{1}$ J. E. Bjorkman, ${ }^{3}$ I. Soszyński ${ }^{4}$ \\ ${ }^{1}$ Instituto de Astronomia, Geofísica e Ciências Atmosféricas, Universidade de São Paulo, \\ Rua do Matão 1226, Cidade Universitária, 05508-900 São Paulo, SP, Brazil \\ ${ }^{2}$ ESO, European Organization for Astronomical Research in the Southern Hemisphere, Chile \\ ${ }^{3}$ Ritter Observatory, Department of Physics \& Astronomy, Mail Stop 113, University of Toledo, Toledo, OH 43606, US \\ ${ }^{4}$ Warsaw University Observatory, Al. Ujazdowskie 4, 00-478 Warszawa, Poland
}

Accepted XXX. Received YYY; in original form ZZZ

\begin{abstract}
Be stars are main-sequence massive stars with emission features in their spectrum, which originates from a circumstellar gaseous disc. Even though the viscous decretion disc (VDD) model can satisfactorily explain most observations, two important physical ingredients, namely the magnitude of the viscosity $(\alpha)$ and the disk mass injection rate, remain poorly constrained. The light curves of Be stars that undergo events of disc formation and dissipation offer an opportunity to constrain these quantities. A pipeline was developed to model these events that uses a grid of synthetic light curves, computed from detailed hydrodynamic simulations combined with radiative transfer calculations. Comparison between model and data was made possible by empirical laws that closely match the photometric behaviour of the events. A sample of $54 \mathrm{Be}$ stars from the OGLE survey of the Small Magellanic Cloud (SMC) was selected for this study. Because of the way our sample was selected (bright stars with clear disc events), it likely represents the densest discs found in the SMC. Like their siblings in the Galaxy, the mass of the disc in the SMC increases with the mass of the star. The typical mass and angular momentum loss rates associated with the disk events are of the order of $\sim 10^{-10} M_{\odot} \mathrm{yr}^{-1}$ and $\sim 5 \times 10^{36} \mathrm{~g} \mathrm{~cm}^{2} \mathrm{~s}^{-2}$, respectively. The values of $\alpha$ found in this work are typically of a few tenths, consistent with recent results in the literature and with the ones found in dwarf novae, but larger than the current theory predicts. Considering the sample as a whole, the viscosity parameter is roughly two times larger at build-up $\left(\left\langle\alpha_{\text {bu }}\right\rangle=0.63\right)$ than at dissipation $\left(\left\langle\alpha_{\mathrm{d}}\right\rangle=0.29\right)$. Further work is necessary to verify whether this trend is real or a result of some of the model assumptions. If real, this is a phenomenon worth further investigation, as it may lead to clues as to the origin of anomalous viscosity in astrophysical discs.
\end{abstract}

Key words: circumstellar matter - radiative transfer - stars: emission-line, Be - stars: massloss - techniques: photometric - hydrodynamics

\section{INTRODUCTION}

In a classical, observational, and quite broad definition, a Be star is a hot, massive star, with a B spectral type (mass ranging roughly from 3 to $17 M_{\odot}$ ), non-supergiant, whose spectrum has, or had at some time, one or more Balmer lines in emission (Jaschek et al. 1981; Collins 1987). In a more modern and theoretically-oriented definition, a Be star is a very rapidly rotating and non-radially pulsating B star that forms a geometrically thin viscous decretion disc (VDD) composed of an outwardly diffusing, viscosity driven gaseous Keplerian disc that is fed by mass ejected from the central

^ E-mail:1rrimulo@usp.br star (Rivinius et al. 2013), and a possibly non-negligible line-driven wind (Kee et al. 2016). There is no evidence of large scale magnetic fields in Be stars (Rivinius et al. 2013). Fast stellar rotation lowers the effective gravity near the stellar equator and a second mechanism, likely to be stellar pulsation (Rivinius et al. 1998; Baade et al. 2016; Rivinius et al. 2016), is responsible for pushing this near equatorial matter into orbit in the inner disc. Once in orbit, a viscous mechanism takes place, diffusing matter and angular momentum outwards, thus making the disc grow.

Be stars are usually quite variable in all observables and in several timescales (days, weeks, months or even years). The variability observed in Be stars indicates that the injection of matter and angular momentum into the disc is frequently quite erratic, with sudden 
outbursts of mass injection and periods of no or negligible mass injection. Rivinius et al. (2016) propose a terminology, which will be used here, in which a star that possesses a disc is said to be active and, conversely, when there is no detectable disc, the star is inactive. Two additional terms are used to distinguish the phases of active disc formation (outbursting Be star) and dissipation (dissipating Be star).

It has been demonstrated (e.g., Haubois et al. 2012) that a Be disc fed roughly at a constant rate, and for a sufficiently long time (a few to several years, depending on the value of the viscosity), reaches a quasi-steady state in which the density is nearly constant in time. If the gas temperature is properly taken into consideration, the radial density profile is typically a complicated function of the distance from the star (e.g., Carciofi \& Bjorkman 2008). However, a usual approximation is to consider the gas to be isothermal, in which case the density profile assumes a power-law form. This simple steadystate VDD has been successful in describing the main observed features of individual Be discs (Carciofi et al. 2006, 2007; Jones et al. 2008b; Carciofi et al. 2009; Klement et al. 2015, ress) and samples of Be stars (Silaj et al. 2010; Touhami et al. 2011; Vieira et al. 2017). However, despite its great success, there are several open and intriguing theoretical questions about the VDD model. Besides the fact that a good description of the mechanism responsible for putting stellar material into orbit is still needed, a complete physical understanding of the forces that drive these discs is also lacking. It has been commonly assumed that the forces operating on the discs are the gravity from the central star and the forces that come from the gradient of pressure and from viscosity, the latter being the one capable of producing torque. Recently, Kee et al. (2016) showed that radiative line forces may also generate a non-negligible torque, at least for gaseous discs with Solar metallicity. In this work we will proceed with the assumption that line-driven forces are negligible. We mitigate this potential issue by choosing to study Be stars in the SMC, whose low metallicity will greatly decrease the strength of the line forces.

In the alpha-disc formalism, the kinematic viscosity is scaled with the $\alpha$ parameter, defined such that the $R \phi$ component of the stress tensor is proportional to the gas pressure: $\tau_{R \phi}=-\alpha P$. The most reliable and direct way of estimating $\alpha$ is to study the timedependent disc behaviour, where the diffusive effect of viscosity will have clear observational counterparts. Therefore, light curves of long temporal coverage, such as the ones given by microlensing or planetary transit surveys, are excellent instruments to study the dynamical processes in action on the disc, as it builds-up and dissipates.

Dynamical studies of Be star viscous discs are still quite scarce. Jones et al. (2008a), using a 1-D time-dependent treatment of the alpha-disc and a non-LTE radiative transfer code, studied the temperature and density profiles of a dynamical disc and their respective $\mathrm{H} \alpha$ line profiles. Haubois et al. (2012) studied the theoretical photometric effects of time variable mass injection rates on the structure of the disc also using a 1-D time-dependent treatment of the alphadisc, associated with the Monte Carlo radiative transfer code HDUST (Carciofi \& Bjorkman 2006, 2008). Carciofi et al. (2012), by fitting these dynamical models to a dissipating portion in the light curve of the Be star $28 \mathrm{CMa}$ (which passed from an outbursting phase, that lasted from 2001 to 2003, to a dissipating phase at the end of 2003), estimated the value of the $\alpha$ parameter for the Be disc of $28 \mathrm{CMa}$ to be $\alpha=1.0 \pm 0.2$. Later, however, it was realised that a proper consideration of the previous history of the disc must be taken into consideration even when fitting the dissipating portion of the light curve, and this quite high value has been revisited to be closer to $\alpha=0.2$ (Ghoreyshi \& Carciofi 2017).

Another intriguing issue regarding Be stars is how they acquired such high rotation rates (typically $80 \%$ of break-up, Rivinius et al. 2013). As the rotating B star evolves, core contraction and internal angular momentum redistribution generally tends to enhance surface angular rotation (Ekström et al. 2008; Granada et al. 2013). Another scenario (e.g., Pols et al. 1991) would involve a past masstransfer phase in a binary system, during which the primary donates mass and angular momentum to the secondary. The left-over of such a system would be a fast-spinning Be star (the former mass gainer) and a subdwarf $\mathrm{O}$ or $\mathrm{B}$ star ( $\mathrm{sdO} / \mathrm{sdB}$, the former mass donor). Regardless of how they were spun-up, it has been proposed (Krtička et al. 2011) that the discs of Be stars may provide natural mechanisms for removing large quantities of angular momentum from the fast rotating stars, preventing them to reach the rotation critical limit. The evolutionary models of Granada et al. (2013) assumed the appearance of completely formed viscous discs every time their models reached a near-critical rotation. The mass density and the rate of angular momentum loss of their discs were roughly similar to the ones estimated by Vieira et al. (2017), who modelled the spectral energy distribution (SED) of 80 Be stars using the VDD model, provided that values of $\alpha$ of at least a few tenths were assumed in both approaches.

The main objective of this paper is to build upon the previous dynamical studies of Be discs and provide, for the first time, a detailed study of the temporal evolution of a large sample of Be stars from the Small Magellanic Cloud (SMC). By studying a large sample of stars, we may begin to answer several open questions related to Be stars and their discs, namely: i) What is the typical value of the viscosity in these discs? ii) Is there any significant evidence for a dependence of $\alpha$ with parameters such as the density of the disc, the spectral type of the star, etc.? iii) What are the typical rates of mass and angular momentum loss in these stars? To reach these goals we developed a new method for modelling the light curves of Be stars, described in Sects. 2 and 3. The sample of studied light curves is described in Sect. 4, and the model results are discussed in Sect. 5, followed by the conclusions.

\section{VISCOUS DECRETION DISCS AROUND BE STARS}

The optical light curves of early-type Be stars (with spectral type ranging roughly from B0 to B4) are usually quite variable in timescales of days to years, with amplitudes of up to tenths of mag (Rivinius et al. 2013). The majority of them show very irregular variability. Most present clear single bump-like features, characterised by a fast rise in brightness followed by a slower fading. Frequently, between the brightening and the fading phases some sort of plateau of nearly constant brightness is seen. Sometimes these bumps are reversed, so that an initial fast fading is followed by a slow recovering of the stellar brightness. Two examples of light curves showing these dips are presented in Fig. 1, taken from OGLE-II (Udalski et al. 1997) and OGLE-III (Udalski et al. 2008) data, for two Be star candidates from the SMC, based on the selection made by Mennickent et al. (2002). Object SMC_SC1 75701 shows two bumps, while SMC_SC6 128831 shows a dip.

These bumps and dips resemble the photometric features shown in Haubois et al. (2012, e.g., their Fig. 14), where a circumstellar viscous disc builds up as a result of a mass injection into the disc at a constant rate, and then dissipates after the mass injection ceases. Haubois et al. (2012) studied several disc feeding scenarios 

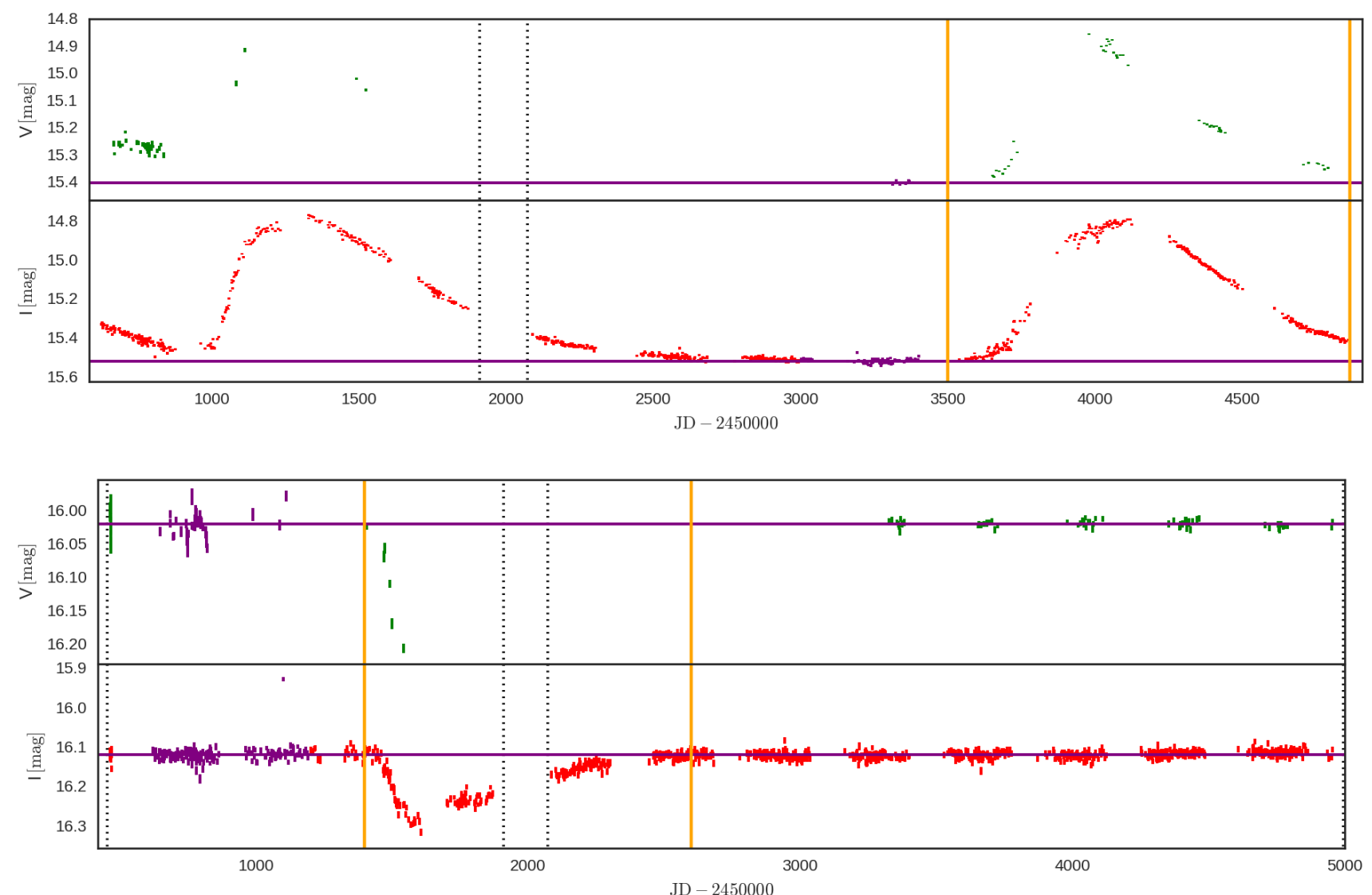

Figure 1. Two light curves, in photometric bands $V$ (green), and $I$ (red), selected from the OGLE-II and OGLE-III photometric surveys. Above: light curve of SMC_SC1 75701, showing two bumps. Below: light curve of SMC_SC6 128831, showing a dip. The pair of vertical dotted straight lines near JD $-2450000=2000$ separates OGLE-II from OGLE-III data. The measurements shown in purple are assumed to represent the inactive (discless) brightness level of the Be star. Their mean is given by the horizontal purple straight lines. The pairs of vertical orange straight lines bracket our visually selected bumps. These bumps are modelled in Figs. 8 and 9.

(constant, cyclic, and outburst) and their photometric counterparts, and demonstrated that the bumps are disc formation/dissipation events of active Be stars seen at near pole-on inclination angles $(i \lesssim 70 \mathrm{deg})$, while the dips are associated with near edge-on Be stars (often called shell stars, $i \gtrsim 70 \mathrm{deg}$ ). The inclination angle plays an important role on how the stellar brightness is modified by the presence of disc because, in the second case (edge-on), the disc is seen projected against the stellar disc, thus causing an attenuation of the stellar radiation. This attenuation does not happen for the pole-on case, where the net effect of the disc is to increase the optical brightness as a result of free-bound and free-free radiation from the gas (Gehrz et al. 1974; Vieira et al. 2015).

In this section, we describe the basic hydrodynamical concepts of gaseous VVDs, with a focus on how to model the aforementioned events of disc construction and dissipation responsible for the bumps seen in the light curves of active Be stars. The evolution of the surface density,

$\Sigma(R, t)=\int_{-\infty}^{\infty} \rho(R, z, t) \mathrm{d} z$,

of thin circumstellar axisymmetric discs of Be stars is described by the following equation (Papaloizou \& Lin 1995)

$$
\frac{\partial \Sigma}{\partial t}+\frac{1}{R} \frac{\partial}{\partial R}\left(R \Sigma v_{R}\right)=S_{\Sigma},
$$

where $R$ and $z$ are cylindrical coordinates, $R \Sigma v_{R}$ is the mass flux crossing radius $R$ per azimuthal angle, $S_{\Sigma}$ represents the sources and sinks of mass in the disc per unit of area (see below), and $\rho$ is the mass density. In Be discs with azimuthal symmetric, as assumed in this work, the mass flux at a given radius $\left(2 \pi R \Sigma v_{R}\right)$ can switch between a positive (decretion) and negative (accretion) value, in response to changes in $S_{\Sigma}$. In the circumstellar alpha-disc formulation, the mass flux is given by

$2 \pi R \Sigma v_{R}=-4 \pi\left(\frac{R}{G M}\right)^{\frac{1}{2}} \frac{\partial}{\partial R}\left(R^{2} \alpha c_{s}^{2} \Sigma\right)$,

where $c_{s}^{2}=k T_{\text {disc }} / \mu m_{H}$ is the isothermal sound speed and $\alpha$ is the viscosity parameter.

The orbital velocity of the disc, $v_{\phi}$, is assumed to be Keplerian $\left(v_{\phi}=v_{K}=v_{\text {orb }} \tilde{R}^{-1 / 2}\right.$, with $v_{\text {orb }}=\left(G M / R_{\mathrm{eq}}\right)^{1 / 2}$ and $\tilde{R}=R / R_{\mathrm{eq}}$, where $R_{\mathrm{eq}}$ is the stellar equatorial radius). This assumption holds as long as the gravitational force is much larger than the force due to the pressure gradient, which is generally true for distances from 
the star of dozens to a few hundreds of stellar radii (Okazaki 2001), as long as $\left(c_{S} / v_{K}\right)^{2} \ll 1$.

The function $S_{\Sigma}$ is the rate of mass injected into (or removed from) the disc per unit of area. It represents the variable mass exchange between the star, the disc and the outer medium. We consider that, during outbursts, mass is put into orbit along a very thin ring of radius $R_{\text {inj }}$ close to the stellar equator. Therefore, we assume

$S_{\Sigma}=\dot{M}_{\mathrm{inj}}(t) \frac{\delta\left(R-R_{\mathrm{inj}}\right)}{2 \pi R}+$ boundaries,

where $\dot{M}_{\mathrm{inj}}(t)$ is the mass injection rate from the star into the disc at $R_{\text {inj. }}$.

In addition, mass can flow away from the disc through its boundaries. Mass can fall back into the star through the inner boundary at $R_{\text {eq }}$, or it can leave the system at an outer boundary, $R_{\text {out }}$. The outer boundary can be interpreted as the limiting radius of the disc due to a binary companion (e.g., Okazaki et al. 2002) or due to the photoevaporation of the disc (e.g., Okazaki 2001). We consider that all mass that eventually reaches the stellar equator $R_{\text {eq }}$ is totally absorbed. The same is assumed for the mass that eventually reaches an outer radius $R_{\text {out }}$ (see below for the definition of $R_{\text {out }}$ ). Therefore, the boundaries consist of $\Sigma\left(R_{\mathrm{eq}}, t\right)=\Sigma\left(R_{\text {out }}, t\right)=0$.

For the hydrodynamical simulations, we assume the disc to be isothermal, with $T_{\text {disc }}=0.6 T_{\text {eff }}$, following Carciofi \& Bjorkman (2006). We further assume that the $\alpha$ parameter is constant with $R$, but we allow it to possibly be time-dependent, as there is evidence that it might happen in discs of Be stars (Ghoreyshi \& Carciofi 2017). Consequently, Eqs. (2)-(4) can be scaled in the following way

$\frac{\partial \Sigma}{\partial t}=\frac{1}{\tau}\left\{\frac{2}{\tilde{R}} \frac{\partial}{\partial \tilde{R}}\left[\tilde{R}^{\frac{1}{2}} \frac{\partial}{\partial \tilde{R}}\left(\tilde{R}^{2} \Sigma\right)\right]+\tau S_{\Sigma}\right\}$,

where we introduce the timescale parameter, $\tau(t)$, given by

$\tau(t)=\frac{1}{\alpha(t)}\left(\frac{R_{\mathrm{eq}}^{3}}{G M}\right)^{\frac{1}{2}} \frac{v_{\mathrm{orb}}^{2}}{c_{s}^{2}}$,

and distances from the star are expressed by the dimensionless parameter $\tilde{R}=R / R_{\mathrm{eq}}$. The timescale parameter is proportional to the viscous timescale at the stellar equator, which is given by $t_{\text {vis }}=R^{2} / v$, where the viscosity $v$ is given by $v=(2 / 3) \alpha c_{S}^{2} R / v_{K}$. Since we are in the thin disc limit $\left(c_{S}^{2} / v_{K}^{2} \ll 1\right)$, we see that $\tau$ is much larger than the orbital period at the stellar equator, given by $2 \pi\left(R_{\mathrm{eq}}^{3} / G M\right)^{1 / 2}$.

The solution of Eq. 5 is scaled in time by the timescale parameter, which controls how fast matter is distributed throughout the disc and, consequently, its observational counterparts. It follows that by fitting observed light curves of Be stars, this parameter can be estimated, and once other parameters are known $\left(R_{\mathrm{eq}}, M, T_{\mathrm{disc}}\right)$, the $\alpha$ value can be determined (e.g., Carciofi et al. 2012).

In order to generate models that do not depend on the timedependent form of $\alpha(t)$ and also on the parameters $R_{\mathrm{eq}}, M$ and $T_{\mathrm{disc}}$, which are related to the central star, we define a time parameter, $\tilde{\tau}(t)$, such that

$\mathrm{d} \tilde{\tau}=\frac{\mathrm{d} t}{\tau(t)}$,

which allows us to solve Eq. (5) in terms of $\tilde{\tau}$ instead of the physical time $t$. The advantage of using this new variable is that it separates the problem of the time dependency of $\alpha(t)$ from the problem of solving Eq. (5). Consequently, it allows us to create a grid of solutions of that equation that is independent of $\alpha(t), R_{\mathrm{eq}}, M$ and $T_{\text {disc }}$ (see Sect. 3)
In this work, Eq. (5) is solved numerically by the 1-D thin-disc code SINGLEBE (Okazaki 2007). At selected time parameters $\tilde{\tau}$, the solution $\Sigma(\tilde{R}, \tilde{\tau})$ enters as input for the Monte Carlo 3-D radiative transfer code HDUST (Carciofi et al. 2004; Carciofi \& Bjorkman 2006,2008 ) that calculates the emergent spectrum of the star+disc system. To convert between surface density and mass density (Eq. 1), it is assumed that the disc is vertically sustained by hydrostatic pressure, in which case the vertical density profile is a Gaussian, and

$\rho(\tilde{R}, z, \tilde{\tau})=\frac{\sum(\tilde{R}, \tilde{\tau})}{\sqrt{2 \pi} H} e^{-\frac{z^{2}}{2 H^{2}}}$,

where

$\frac{H}{R_{\mathrm{eq}}}=\left(\frac{c_{s}}{v_{\text {orb }}}\right) \tilde{R}^{\frac{3}{2}}$

is the scale height.

Before moving on to the modelling of bump-like events such as the ones of Fig. 1, we introduce in the next section some important parameters of the dynamical discs implied in the equations shown above.

\subsection{Dynamical disc parameters}

The radius of mass injection divides the disc in two regions: a narrow region between the inner boundary and the radius of mass injection $\left(1 \leq \tilde{R} \leq \tilde{R}_{\text {inj }}\right)$, and the much wider region between the radius of mass injection and the outer boundary $\left(\tilde{R}_{\text {inj }} \leq \tilde{R} \leq \tilde{R}_{\text {out }}\right)$. The steady-state solution of Eq. (5) corresponds to the limiting case of a disc that has been fed at a constant rate for an infinitely long time. It is obtained by setting $\partial \Sigma / \partial t=0$ and assuming that $\alpha$ and $\dot{M}_{\text {inj }}$ are time-independent. The surface density in the steady-state is given by

$$
\Sigma_{\text {steady }}(\tilde{R})=\left\{\begin{array}{cl}
\frac{\Sigma_{0}}{\tilde{R}^{2}}\left(\frac{\tilde{R}^{\frac{1}{2}}-1}{\tilde{R}_{\text {inj }}^{\frac{1}{2}}-1}\right) \Upsilon, & 1 \leq \tilde{R}<\tilde{R}_{\text {inj }} \\
\left.\frac{\Sigma_{0}\left(\tilde{R}_{\text {out }}^{\frac{1}{2}}-\tilde{R}^{\frac{1}{2}}\right.}{\tilde{R}^{2}}\right), & \tilde{R}_{\text {inj }} \leq \tilde{R} \leq \tilde{R}_{\text {out }}
\end{array},\right.
$$

where $\Upsilon=\left(\tilde{R}_{\text {out }}^{1 / 2}-\tilde{R}_{\text {inj }}^{1 / 2}\right) /\left(\tilde{R}_{\text {out }}^{1 / 2}-1\right)$ is a number usually very close to 1 for any Be disc.

The physical quantity $\Sigma_{0}$ represents the surface density at $R_{\mathrm{eq}}$, obtained by extrapolating $\Sigma_{\text {steady }}$ in the domain $\tilde{R}_{\text {inj }} \leq \tilde{R} \leq \tilde{R}_{\text {out }}$ to $R_{\text {eq. }}$. We will refer to this physical quantity as the asymptotic surface density, as it is the asymptotic value reached after an infinitely long disc build-up under a constant $\dot{M}_{\text {inj }}$. It is easily shown that $\Sigma_{0}$ is related to $\dot{M}_{\text {inj }}$ by the following equation

$2 \pi R_{\mathrm{eq}} \Sigma_{0}\left(\frac{R_{\mathrm{eq}}}{\tau}\right)=\dot{M}_{\mathrm{inj}}\left(\tilde{R}_{\mathrm{inj}}^{\frac{1}{2}}-1\right) \equiv\left(-\frac{\partial M}{\partial t}\right)_{\mathrm{typ}}$,

where $(\partial M / \partial t)_{\text {typ }}$ is defined below.

We may extend Eq. (11) to the general case of a time-dependent $\dot{M}_{\text {inj }}(\tilde{\tau})$, which would define, by the same equation, a time dependent asymptotic surface density, $\Sigma_{0}(\tilde{\tau})$. The function $\Sigma_{0}(\tilde{\tau})$, therefore, is just another way of specifying the history of mass injection from the star into the disc. It has, however, the advantage of being a surface density, which is a quantity that may be determined from, e.g, SED analyses, in contrast to the mass injection rate and the radius of mass injection, which are parameters that cannot be observationally determined.

The steady-state solution (Eq. 10) shows that, in the wider 
domain $\tilde{R}_{\text {inj }} \leq \tilde{R} \leq \tilde{R}_{\text {out }}$, the density profile of the disc is not altered if $\tilde{R}_{\text {inj }}$ is changed, provided that $\dot{M}_{\text {inj }}$ is also changed in order to maintain $\Sigma_{0}$ fixed, according to Eq. (11). In fact, we verified that the time-dependent solutions of Eq. (5) in the domain $\tilde{R}_{\text {inj }} \leq \tilde{R} \leq \tilde{R}_{\text {out }}$ are negligibly affected by the particular choice of $\tilde{R}_{\mathrm{inj}}$ or $\dot{M}_{\mathrm{inj}}(\tilde{\tau})$, as long as the quantity $\Sigma_{0}(\tilde{\tau})$ is kept fixed. This is a consequence of the fact that the dynamical solutions reach a near steady-state very quickly in the vicinity of the injection radius (Haubois et al. 2012). Furthermore, provided that mass is injected not too far from the stellar photosphere (i.e., assuming $\tilde{R}_{\text {inj }} \gtrsim 1$ ), the domain $1 \leq$ $\tilde{R} \leq \tilde{R}_{\text {inj }}$ is much narrower than the region where the continuum visual flux of Be stars is generated (Carciofi 2011), which means that the emission from this region can be ignored. Consequently, we conclude that $\Sigma_{0}(\tilde{\tau})$ (with the assumption that $\tilde{R}_{\text {inj }} \gtrsim 1$ ) is a much better parameter for describing the mass injection history of the disc than the pair of parameters $\dot{M}_{\text {inj }}(\tilde{\tau})$ and $\tilde{R}_{\text {inj }}$.

The time-dependent solutions of Eq. (5) generally show that, for Be stars dynamically feeding the disc but still far from steadystate, the mass flux close to $\tilde{R}_{\mathrm{inj}}$ has absolute values of the order of $(-\partial M / \partial t)_{\mathrm{typ}}$, defined by Eq. (11). Therefore, we refer to this quantity as the typical decretion rate, which depends on parameters relatively easy to estimate from SEDs of Be stars.

In our simulations, since the values of $\dot{M}_{\text {inj }}$ and $\tilde{R}_{\text {inj }}$ are of no interest, and the value of $\tilde{R}_{\text {out }}$ is quite uncertain, we arbitrarily chose $\tilde{R}_{\text {inj }}=1.017$ and $\tilde{R}_{\text {out }}=1000$ (we discuss below how this choice of $\tilde{R}_{\text {out }}$ might affect our results). Eq. 11, therefore, shows that the typical decretion rate is much smaller than the mass injection rate $\dot{M}_{\mathrm{inj}}(\tilde{\tau})$. In our case, the typical decretion rate is only $8.46 \times 10^{-3} \dot{M}_{\text {inj. }}$ This means that the majority of the injected mass flows inwards and is absorbed by the inner boundary at the stellar equator, and only a small remaining fraction of the injected mass is responsible for the growth of the disc. These results were first obtained from SPH simulations of Be discs by Okazaki et al. (2002), who found that only about $0.1 \%$ of the injected material flows outward, as a direct result of their choice for $R_{\text {inj }}$.

It can be shown, by substitution of Eqs. (10) and (11) in Eq. (3) that, in steady-state, only the fraction of the injected mass given by $\dot{M}_{\text {inj }}(1-\Upsilon)$ is flowing outwards through the disc and crossing the outer radius $R_{\text {out }}$, thus leaving the system. For our assumed values for $\tilde{R}_{\text {inj }}$ and $\tilde{R}_{\text {out }}, 1-\Upsilon=2.84 \times 10^{-4}$. Since the mass of the disc is not varying in steady-state, the mass flux given by $\dot{M}_{\text {inj }}(1-\Upsilon)$ is actually the rate of mass being lost by the star, which we will indicate by $(-\partial M / \partial t)_{\text {steady. }}$. It is easily seen that it is related to the typical decretion rate by the following equation

$\left(-\frac{\partial M}{\partial t}\right)_{\text {steady }}=\frac{\Lambda}{\tilde{R}_{\text {out }}^{\frac{1}{2}}}\left(-\frac{\partial M}{\partial t}\right)_{\text {typ }}$,

where $\Lambda=1 /\left(1-\tilde{R}_{\text {out }}^{-1 / 2}\right)$ is a number very close to 1 for any Be disc in general.

In steady-state, the angular momentum escaping the system at the outer boundary (and also being lost by the star) is $\left(G M R_{\text {out }}\right)^{1 / 2} \dot{M}_{\text {inj }}(1-\Upsilon)$, and is written as

$\left(-\frac{\partial J}{\partial t}\right)_{\text {steady }}=\Lambda\left(G M R_{\mathrm{eq}}\right)^{\frac{1}{2}}\left(-\frac{\partial M}{\partial t}\right)_{\mathrm{typ}}$.

From Eq. (12) we see that knowing $\tilde{R}_{\text {out }}$ is essential for estimating the rate of mass being lost by the star. Interestingly, this is not the case for the rate of angular momentum being lost by the star, given by Eq. (13).

\subsection{The Mass Reservoir Effect}

It is important to stress that the solution $\Sigma(\tilde{R}, \tilde{\tau})$ is shaped not just by the mass injection rate $\Sigma_{0}(\tilde{\tau})$ at the specific instant $\tilde{\tau}$, but by the whole mass injection history before the instant $\tilde{\tau}$. Therefore, the advantage of studying the relatively isolated bumps like the ones exemplified in Fig. 1, which started after a clear inactive phase, is that there is no disc present when the bump starts developing; thus, no previous history of mass injection has to be taken into account in the beginning of their modelling.

The light curves of several Be stars show that the duration of the build-up phase, which we refer to as the build-up time, is variable between Be stars and even between different bumps from the same star, ranging from a few days to years. The following phase of disc dissipation, however, contrary to the build-up phase, depends of the previous history of mass injection. For this reason, the modelling of the dissipation phase must not be disconnected from the modelling of the build-up phase that happened before it.

One of the main consequences of this fact is the mass reservoir effect (see also Ghoreyshi \& Carciofi 2017). Basically, discs that had a longer build-up phase necessarily transported more matter and angular momentum outwards and created a larger external reservoir of mass and angular momentum in its outer regions, which usually extend far beyond the first few stellar radii where the visible photometric observables are formed. It is common, for instance, that some bumps reach plateaus during the build-up phase. The plateau indicates that the density in the inner disc has reached near-steadystate values and, consequently, there is little photometric variation in the visible wavelengths. The outer disc, however, will likely be far from steady-state and thus will continue to increase in density and mass. When mass injection ceases, the dissipation phase begins. Re-accretion occurs and, due to the more massive outer disc, the inner disc remains relatively denser for a longer time. This makes the dissipation of the disc to appear slower in the observed light curves. Conversely, a disc that had a small build-up time would dissipate much faster.

The importance of the mass reservoir effect can be assessed by the reevaluation of the $\alpha$ parameter in $28 \mathrm{CMa}$ by Ghoreyshi \& Carciofi (2017). Carciofi et al. (2012) modelled the 2003 dissipation phase of $28 \mathrm{CMa}$ by considering a very long previous build-up time, and found that a high value of $\alpha$ was necessary $(1.0 \pm 0.2)$ to match the observed dissipation rate. Ghoreyshi \& Carciofi have shown that when the previous build-up phase is properly accounted for in the modelling, the value of $\alpha$ required to match the dissipation rate is much smaller $(0.21 \pm 0.05)$.

\section{A MODEL GRID OF DISC FORMATION AND DISSIPATION EVENTS}

In this section we describe the method we developed for fitting the light curves associated with events of disc formation and dissipation. The method consists of precomputing a large grid of dynamical models of the time-dependent disc structure, covering the entire range of observed scenarios (Sect. 3.1) and performing the radiative transfer in these models to produce synthetic light curves (Sect. 3.2). The observed light curves are then fitted by the synthetic one using the procedure described in Sects. 3.3 and Sect. 3.4. 


\subsection{Dynamical model grid}

For building a comprehensive grid of dynamical models that are solutions of Eq. 5, we used the definitions of Sect. 2 that allow us to write the solution $\Sigma(\tilde{R}, \tilde{\tau})$ in terms of $\Sigma_{0}(\tilde{\tau})$ and the dimensionless parameter $\tilde{\tau}$.

As discussed in Sect. 2.2, the advantage of studying relatively isolated bumps like the ones exemplified in Fig. 1, which started after a clear inactive phase, is that there is no previous history of mass injection to be taken into account for the modelling, so that during build-up the shape of the curve is controlled solely by $\Sigma_{0}(\tilde{\tau})$ and $\tilde{\tau}$, while for dissipation the previous disc build-up time should also be considered (Sect. 2.2). By using the time parameter (Eq. 7) instead of the physical time, our dynamical models are independent of the specific physical parameters $M, T_{\mathrm{eff}}, R_{\mathrm{eq}}$ and $\alpha(t)$ of the Be star under consideration (Eq. 6). Also, from the linearity of Eq. 5, it follows that multiplying $\Sigma_{0}(\tilde{\tau})$ by some constant results in the solution $\Sigma(\tilde{R}, \tilde{\tau})$ multiplied by the same constant. Consequently, only one value of $\Sigma_{0}$ during the build-up phase is necessary.

For our grid of dynamical models, we therefore assume that our Be stars start discless. At instant $\tilde{\tau}=0$, mass injection into the disc begins at an arbitrary constant rate $\left(\Sigma_{0}>0\right)$ that lasts until $\tilde{\tau}=\tilde{\tau}_{\mathrm{bu}}$, which we refer to as the scaled build-up time, since it is related to the above mentioned build-up time, but scaled by the timescale parameter. After that $\left(\tilde{\tau}>\tilde{\tau}_{\text {bu }}\right.$ ), mass injection no longer occurs $\left(\Sigma_{0}=0\right)$ and the disc dissipates. In Appendix A, we further discuss the properties of these dynamical models.

We chose 11 values of $\tilde{\tau}_{\mathrm{bu}}$, listed in Table 1 . Since the timescale parameter (Eq. 6) is roughly given by $\sim(100-200) / \alpha$ days for early Be stars in the main sequence with $\alpha \lesssim 1$, these values correspond to real build-up times of at least 15 days, which brackets the observed build-up times of the sample described below (Sect. 4, Table 5). In this study, we decided not to model the bumps with observed buildup times lower than about 15 days, usually referred to as flickers (Keller et al. 2002)

\subsection{Radiative transfer models}

Having selected a set of suitable hydrodynamic bump models, the next step is to produce photometric light curves of these models. The radiative transfer part of the problem requires a stellar model, which will be the primary source of radiation. The stellar model depends on the physical parameters $M, R_{\mathrm{eq}}$ and $T_{\mathrm{eff}}$, which were left unspecified in the dynamical model grid. In addition, three other parameters must be specified: the viewing angle, $i(i=0$ means pole-on orientation), the distance to the star, $d$, and the interstellar reddening.

One important feature of the central stars of Be stars is that they are fast rotators. Fast rotation causes the star to be oblate, with hotter poles and colder equatorial regions. Rotation is specified by the ratio of the rotation velocity at the equator to the Keplerian velocity at the equator, $W=v_{\text {eq }} / v_{\text {orb }}$. The ratio between the equatorial radius to the polar radius is given by $R_{\text {eq }} / R_{\text {pole }}=1+W^{2} / 2$ for a Roche-shaped star. All these parameters evolve in time as a consequence of stellar evolution, and Be stars can be found in luminosity classes from $\mathrm{V}$ to III (Rivinius et al. 2013). We adopt the Geneva evolutionary tracks (Georgy et al. 2013) to determine $R_{\text {eq }}$ and $T_{\text {eff }}$ given $M$ and the age in the main sequence, $t_{\mathrm{MS}}$.

The current version of HDUST allows for a spheroidal rotationally oblate star, with the latitude-dependent surface temperature being given by $T_{\text {surf }} \propto g_{\text {eff }}^{\beta}$ (Carciofi et al. 2008). Here, the coefficient $\beta(W)$ is calculated by fitting a straight line to the gradient
Table 1. Parameters of the grid of photometric models of bumps

\begin{tabular}{rrrr}
\hline Star & $i[\mathrm{deg}]$ & $\Sigma_{0}\left[\mathrm{~g} \mathrm{~cm}^{-2}\right]$ & $\tilde{\tau}_{\text {bu }}$ \\
\hline Star 1 & 00.0 & 0.30 & 00.15 \\
Star 2 & 21.8 & 0.41 & 00.45 \\
Star 3 & 31.0 & 0.56 & 00.75 \\
& 38.2 & 0.75 & 01.50 \\
& 44.4 & 1.01 & 02.25 \\
& 50.0 & 1.37 & 03.00 \\
& 55.2 & 1.85 & 04.50 \\
& 60.0 & 2.50 & 06.00 \\
& 64.6 & & 09.00 \\
& 69.1 & & 15.00 \\
& 73.4 & & 30.00 \\
& 77.6 & & \\
& 81.8 & & \\
& 85.9 & & \\
& 90.0 & & \\
\hline
\end{tabular}

Table 2. Parameters of the stellar models of Table 1

\begin{tabular}{rrrrrr}
\hline Star & $Z$ & $M\left[M_{\odot}\right]$ & $W$ & $t / t_{\mathrm{MS}}$ & $\alpha \tau[\mathrm{d}]$ \\
\hline Star 1 & 0.002 & 7 & 0.81 & 0.5 & 90.4 \\
Star 2 & 0.002 & 11 & 0.81 & 0.5 & 103.3 \\
Star 3 & 0.002 & 15 & 0.81 & 0.5 & 118.9 \\
\hline
\end{tabular}

$\partial \ln T_{\text {surf }} / \partial \ln g_{\text {eff }}$ given by the flux theory of Espinosa Lara \& Rieutord (2011). For the disc scale height (Eq. 9), we assume an isothermal disc with $T_{\text {disc }}=0.6 T_{\text {eff }}$, where $T_{\text {eff }}$ is the effective temperature of the star, defined by $T_{\mathrm{eff}}=\left(L_{*}\right)^{1 / 4}\left(\sigma S_{*}\right)^{-1 / 4}$, with $S_{*}$ being the surface area of the star.

In order to generate synthetic absolute magnitudes from the computed SEDs, we used the standard BV RI Johnson-Cousins passbands from Bessell (1990) and the Vega flux from Castelli \& Kurucz (1994) as standard of calibration.

A grid of model light curves was computed using the $11 \mathrm{dy}-$ namical models described in Sect. 3.1, with 8 different values of $\Sigma_{0}$ (third column of Table 1). For each of these disc models, radiative transfer models were calculated with HDUST at 17 different time parameters (not shown in the table) and 15 equally-spaced values of $\cos i$ (second column). This whole process was done for 3 different stellar models ("Star 1", "Star 2" and "Star 3", first column of Table 1). Details on the stellar models are given in Table 2. They were chosen to represent early B-type stars from the SMC $(Z=0.002)$, in the middle of their life in the main sequence, with the rotation parameter given by the mean value obtained for Be stars $(W=0.81$, Rivinius et al. 2006). In the sixth column of Table 2, we present the values of $\alpha \tau$ (Eq. 6) for the discs of these stars (with the assumption that $T_{\text {disc }}=0.6 T_{\text {eff }}$ ). In short, a single light curve is specified by taking one element of each column of Table 1 . The end result was a grid of $3 \times 15 \times 8 \times 11=3960$ light curves, for each of the $B V R I$ bands.

A grid of inactive (discless) stellar models was also calculated. Because these models can be computed much faster than the bump models, we were able to cover a much finer grid of stellar parameters (Table 3), aiming at a better determination of the stellar parameters. The grid is composed by models for 12 different masses (second column), 6 different rotation rates (third column), 5 equally spaced values for the age in the main sequence (forth column) and 10 equally spaced values of $\cos i$ (fifth column), resulting in a total of 
$[! t]$

Table 3. Parameters of the grid of photometric models of discless stars

\begin{tabular}{rrrrr}
\hline$Z$ & $M / M_{\odot}$ & $W$ & $t / t_{\mathrm{MS}}$ & $i[\mathrm{deg}]$ \\
\hline 0.002 & 2.50 & 0.600 & 0.00 & 00.0 \\
& 3.68 & 0.699 & 0.25 & 27.3 \\
4.85 & 0.770 & 0.50 & 38.9 \\
6.00 & 0.833 & 0.75 & 48.2 \\
7.15 & 0.901 & 1.00 & 56.3 \\
8.29 & 0.990 & & 63.6 \\
9.42 & & & 70.5 \\
10.54 & & & 77.2 \\
& 11.66 & & & 83.6 \\
12.78 & & & 90.0 \\
& 13.89 & & & \\
15.00 & & & \\
\hline
\end{tabular}

$12 \times 6 \times 5 \times 10=3600$ photometric models for each of the $B V R I$ bands.

\subsection{Empirical law}

In order to facilitate the comparison of the synthetic light curves (Sect. 3.2) with the observed ones (Sect. 4), we developed two empirical laws that match quite closely the synthetic light curves for build-up and dissipation. The usefulness of these formulae will become clear in the next section.

In our discussion of the features of the light curves, it is useful to separate them in three groups: (i) pole-on light curves, of stars seen at small inclination angles $(0 \leq i \lesssim 70 \mathrm{deg})$, which should statistically correspond to the majority of the observed light curves; (ii) edge-on light curves, of shell stars $(i \approx 90 \mathrm{deg})$; and, (iii) intermediate light curves, of stars seen at intermediate angles (70 $\lesssim i 85 \mathrm{deg}-$ the extension of this intermediate region varies depending the photometric band under consideration and will be defined below). Pole-on light curves show an increase in apparent brightness, due to the additional flux coming from the disc. Conversely, edge-on light curves show a decrease in apparent brightness, due to obscuration of the star by the disc. The intermediate case shows the smallest variations in apparent brightness, and frequently the light curve has a more complicated shape, as it is influenced by variable amounts of disc emission/absorption.

A computed light curve is a sequence of absolute magnitudes for a set of time parameters, in a given photometric band $X$, given by

$$
M_{X}(\tilde{\tau})=M_{X *}+\Delta X(\tilde{\tau}),
$$

where $M_{X *}$ is the absolute magnitude of the inactive Be star at band $X$, and $\Delta X(\tilde{\tau})$ is the magnitude difference caused by the disc ( $\triangle X(\tilde{\tau})$ can be either positive or negative). $M_{X *}$ can be estimated from the light curve during the inactive phase (e.g., the purple points of Fig. 1). A build-up light curve (for $\tilde{\tau}-\tilde{\tau}_{\text {bu }}<0$ ) is denoted by $\Delta X_{\text {bu }}(\tilde{\tau})$. Its limiting value, if the build-up phase were to have an infinite duration, is denoted by $\Delta X_{\mathrm{bu}}^{\infty}$ - the photometric excess of the disc when in steady-state. A dissipation light curve (for $\tilde{\tau}-\tilde{\tau}_{\mathrm{bu}} \geq 0$ ) is denoted by $\Delta X_{\mathrm{d}}(\tilde{\tau})$. Its value at the beginning of the dissipation $\left(\tilde{\tau}=\tilde{\tau}_{\mathrm{bu}}\right)$ is given by $\Delta X_{\mathrm{d}}^{0}$. Since every dynamical model starts from a discless state and asymptotically ends at a discless state, it follows that, regardless of the viewing angle, for every build-up light curve the quantity $\Delta X_{\mathrm{bu}}(\tilde{\tau}) / \Delta X_{\mathrm{bu}}^{\infty}$ is a function that goes from 0 to 1 as $\tilde{\tau}$ goes from 0 to $\infty$, and for every dissipation light curve, the quantity
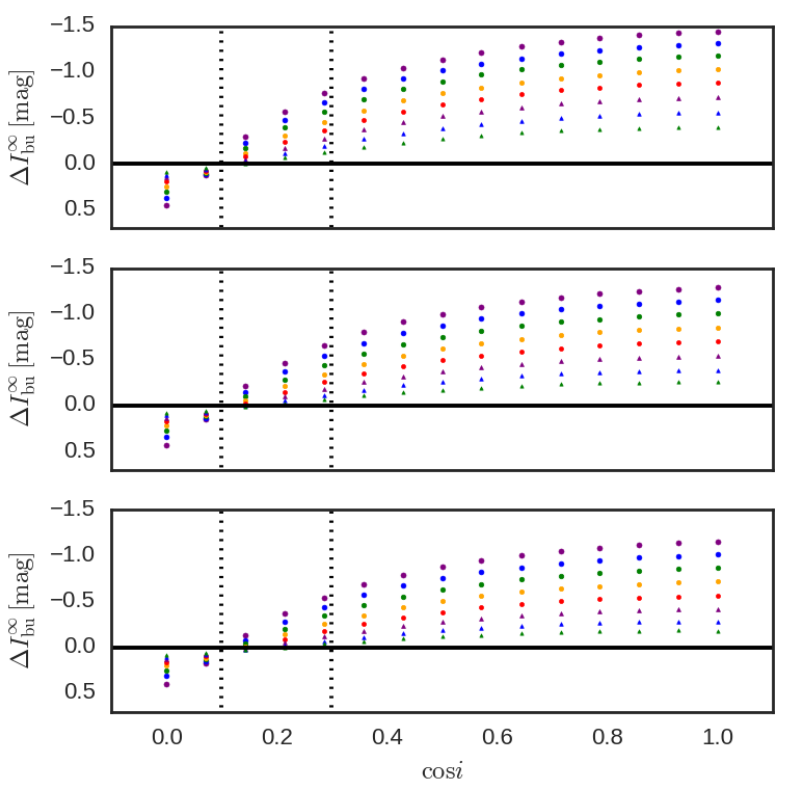

Figure 2. The values of $\Delta I_{\text {bu }}^{\infty}$ versus cos $i$ for our grid. From top to bottom, the results are for Star 1, Star 2 and Star 3, respectively. Purple, blue, green, orange and red circles correspond to $\Sigma_{0}=2.50,1.85,1.37,1.01$, $0.75 \mathrm{~g} \mathrm{~cm}^{-2}$. Purple, blue and green triangles correspond to $\Sigma_{0}=0.56$, $0.41,0.30 \mathrm{~g} \mathrm{~cm}^{-2}$. Vertical dotted lines define the region of intermediate angles for the $I$-band $(73 \lesssim i \lesssim 84 \mathrm{deg}$ ).

$\Delta X_{\mathrm{d}}(\tilde{\tau}) / \Delta X_{\mathrm{d}}^{0}$ is a function that goes from 1 to 0 as $\tilde{\tau}-\tilde{\tau}_{\mathrm{bu}}$ goes from 0 to $\infty$. In Appendix B, we show examples of light curves that accompany the conclusions drawn on this section.

The values of $\Delta I_{\mathrm{bu}}^{\infty}$ for our grid are shown in Fig. 2, plotted against $\cos i$. The values for the $B V R$ bands show qualitatively similar patterns to the ones presented in this figure. Each panel shows the results for a different star, and all 8 values of $\Sigma_{0}$ (Table 1) are represented in the figure by different colours and symbols. The curves monotonically increase with $\cos i$, starting with negative values at edge-on orientation and reaching a maximum for pole-on viewing. The angle for which $\Delta X_{\mathrm{bu}}^{\infty}=0$, where the disc excess emission is exactly matched by the absorption of photospheric light by the disc, depends both on the density scale (as shown in the figure) and (most importantly) on the band pass.

An analysis of our model grid allowed us to determine the ranges in inclination angle for which the light curve displays the intermediate behaviour described above. They were determined by visual inspection of our model grid, as the angles for which the light curves present more complex shapes (see, e.g., the $I$-band light curves seen at $i=81.8 \mathrm{deg}$ and $i=77.6 \mathrm{deg}$ in Fig. B1 of Appendix B). Their adopted values are 53-78 deg, 60-78 deg, 66$84 \mathrm{deg}$ and 73-84 deg for the $B V R I$ bands, respectively.

For pole-on orientations, the observed excess is given by $\Delta X \approx$ $-2.5 \log \left(1+F_{\text {disc }} / F_{*}\right) \approx-F_{\text {disc }} / F_{*}$. Vieira et al. (2015) studied the continuum emission from gaseous discs, and showed that it can be approximated by the sum of the flux coming from an optically thick inner part (the so-called pseudophotosphere) with the contribution from an optically thin outer part, i.e.,

$F_{\text {disc }} \propto F_{\text {thick }} \cos i+F_{\text {thin }}$.

If the contribution of the optically thin part of the disc were negli- 

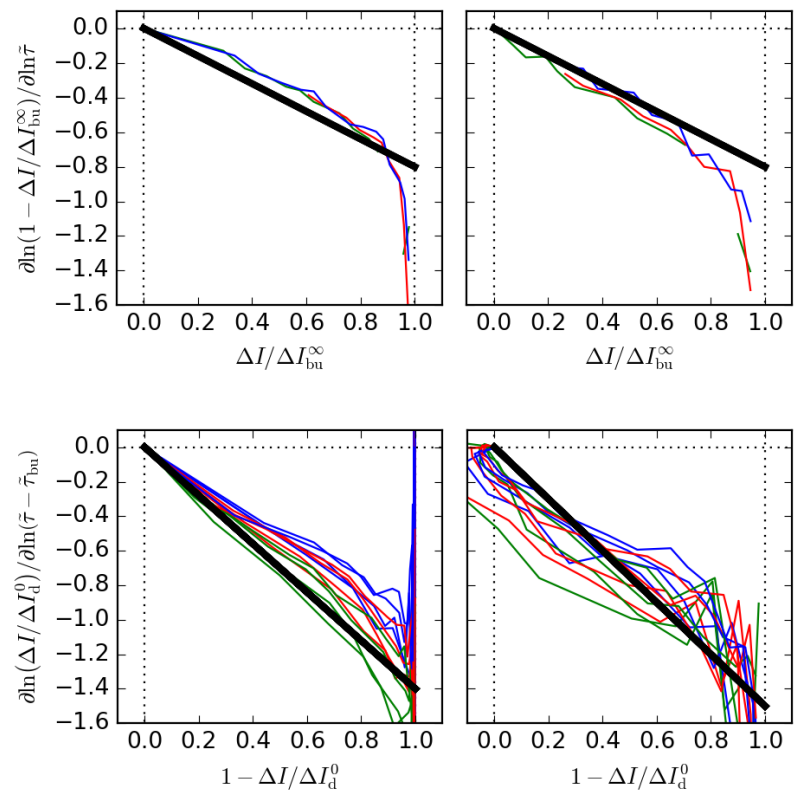

Figure 3. Comparison of the empirical law (black straight lines) with the computed light curves for build-up and dissipation. Top panels: $\log -\log$ derivative of $1-\Delta I_{\text {bu }}(\tilde{\tau}) / \Delta I_{\text {bu }}^{\infty}$ vs. $\Delta I_{\text {bu }}(\tilde{\tau}) / \Delta I_{\text {bu }}^{\infty}$. Bottom panels: $\log -\log$ derivative of $\Delta I_{\mathrm{d}} / \Delta I_{\mathrm{d}}^{0}$ vs. $1-\Delta I_{\mathrm{d}} / \Delta I_{\mathrm{d}}^{0}$, for values of $\tilde{\tau}_{\text {bu }}$ equal to $0.45,1.5$, 6 and 30. The results are shown for Star 2 at two inclination angles: $i=0 \mathrm{deg}$ (left), and $i=90 \mathrm{deg}$ (right). The green, red and blue curves correspond to $\Sigma_{0}$ equal to $1.37,0.75$ and $0.41 \mathrm{~g} \mathrm{~cm}^{-2}$.

gible and the stellar flux, $F_{*}$, did not depend on $\cos i, \Delta X$ would be a linear function of $\cos i$. Clearly this is not the case for the entire range of $\cos i$, and both the optically thin and thick parts of the disc contributes to observed behaviour of $\Delta X$ vs. $\cos i$. This pseudophotosphere concept will be important to understand the growth and decay rates of the light curves, discussed below.

Figure 2 also shows that the excesses increase a little when moving from a low- to a high-mass star, for discs with the same other features. This is a consequence of the fact that the stellar flux relative to the disc flux increases with the luminosity of the star.

Both the pole-on and edge-on light curves have functions $\Delta X_{\mathrm{bu}}(\tilde{\tau}) / \Delta X_{\mathrm{bu}}^{\infty}$ and $\Delta X_{\mathrm{d}}(\tilde{\tau}) / \Delta X_{\mathrm{d}}^{0}$ that are qualitatively similar to each other, suggesting that they could be approximated by simple and general formulas of $\tilde{\tau}$. This is illustrated in Fig. 3, where we compare the values of $\Delta I_{\mathrm{bu}}(\tilde{\tau}) / \Delta I_{\mathrm{bu}}^{\infty}$ and $\Delta I_{\mathrm{d}}(\tilde{\tau}) / \Delta I_{\mathrm{d}}^{0}$ (in the horizontal axis) with their $\log -\log$ derivatives (in the vertical axis). As the panels exemplify, the curves are similar to each other in a wide range parameters $\left(\cos i, \Sigma_{0}\right.$ and $\left.\tilde{\tau}_{\mathrm{bu}}\right)$, and they can be roughly approximated by straight lines (shown in black). Therefore, the build-up and dissipation light curves (for inclinations not in the intermediate region) should roughly obey the following differential equations:

$\frac{\partial \ln }{\partial \ln \tilde{\tau}}\left(1-\frac{\Delta X_{\mathrm{bu}}}{\Delta X_{\mathrm{bu}}^{\infty}}\right) \approx-\eta_{\mathrm{bu}} \frac{\Delta X_{\mathrm{bu}}}{\Delta X_{\mathrm{bu}}^{\infty}}$,

and

$\frac{\partial \ln }{\partial \ln \left(\tilde{\tau}-\tilde{\tau}_{\mathrm{bu}}\right)} \frac{\Delta X_{\mathrm{d}}}{\Delta X_{\mathrm{d}}^{0}} \approx-\eta_{\mathrm{d}}\left(1-\frac{\Delta X_{\mathrm{d}}}{\Delta X_{\mathrm{d}}^{0}}\right)$,
Table 4. Adopted values for the $\eta$ exponent of Eqs. 17 and 18

\begin{tabular}{cccccc}
\hline & & $B$ & $V$ & $R$ & $I$ \\
\hline$\eta_{\text {bu }}$ & (edge-on) & 0.8 & 0.8 & 0.8 & 0.8 \\
& (pole-on) & 0.8 & 0.8 & 0.8 & 0.8 \\
\hline \multirow{2}{*}{$\eta_{\mathrm{d}}$} & (edge-on) & 1.5 & 1.5 & 1.5 & 1.5 \\
& (pole-on) & 1.1 & 1.2 & 1.3 & 1.4 \\
\hline
\end{tabular}
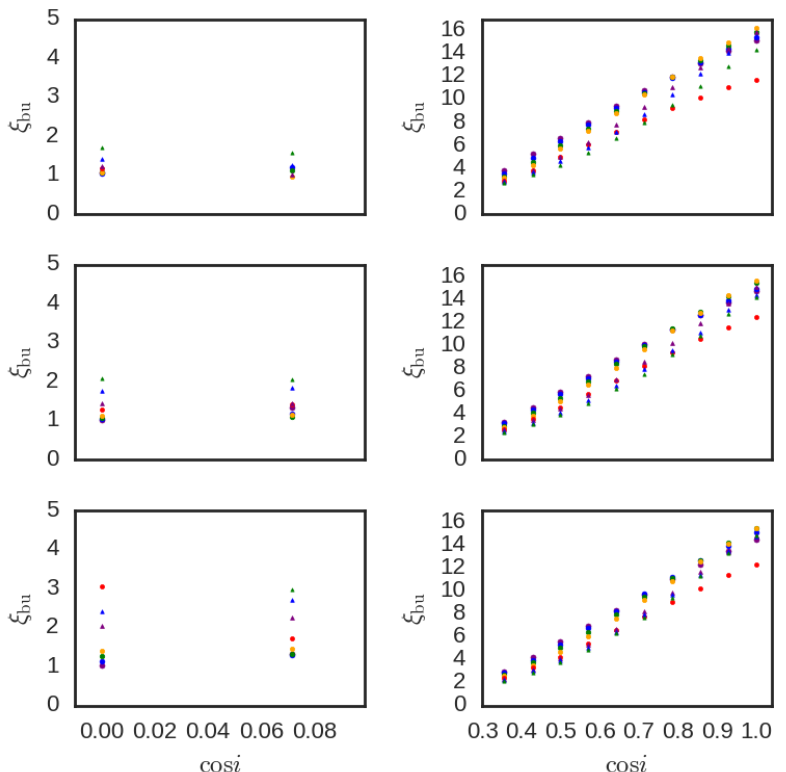

Figure 4. The $I$-band values of $\xi_{\text {bu }}$ for our grid vs. $\cos i$. Left: Edge-on models. Right: Pole-on models. From top to bottom, the results for Star 1, 2, and 3, respectively. The markers are the same as in Fig. 2.

whose solutions are, respectively,

$\Delta X_{\mathrm{bu}}=\Delta X_{\mathrm{bu}}^{\infty}\left[m 1-\frac{1}{1+\left(\xi_{\mathrm{bu}} \tilde{\tau}\right)^{\eta_{\mathrm{bu}}}}\right]$,

and

$\Delta X_{\mathrm{d}}=\Delta X_{\mathrm{d}}^{0}\left[\frac{1}{1+\left(\xi_{\mathrm{d}}\left(\tilde{\tau}-\tilde{\tau}_{\mathrm{bu}}\right)\right)^{\eta_{\mathrm{d}}}}\right]$.

The continuity condition requires

$\Delta X_{\mathrm{d}}^{0}=\Delta X_{\mathrm{bu}}^{\infty}\left[1-\frac{1}{1+\left(\xi_{\mathrm{bu}} \tilde{\mathrm{t}}_{\mathrm{bu}}\right)^{\eta_{\mathrm{bu}}}}\right]$.

The parameters $\xi_{\text {bu }}$ and $\xi_{\mathrm{d}}$ are constants of integration whose values must be determined by fitting the above formulae to the computed light curves. The values of the exponents $\eta_{\mathrm{bu}}$ and $\eta_{\mathrm{d}}$ were empirically determined to best match the model light curves (Table 4). It was found that good fits for the whole set of parameters $\left(\cos i, \Sigma_{0}\right.$ and $\tilde{\tau}_{\mathrm{bu}}$ ) could be obtained for certain fixed values of $\eta_{\mathrm{bu}}$ and $\eta_{\mathrm{d}}$ for each photometric band.

Representative $I$-band values of $\xi_{\mathrm{bu}}$ and $\xi_{\mathrm{d}}$ for our grid are shown in Figs. 4, 5 and 6 (the values of $\xi_{\text {bu }}$ and $\xi_{\mathrm{d}}$ for the $B V R$ bands show qualitatively similar patterns to the ones presented in these figures). Each row shows the results for a different star. The left (right) panels are for edge-on (pole-on) models. The values of $\xi$ 

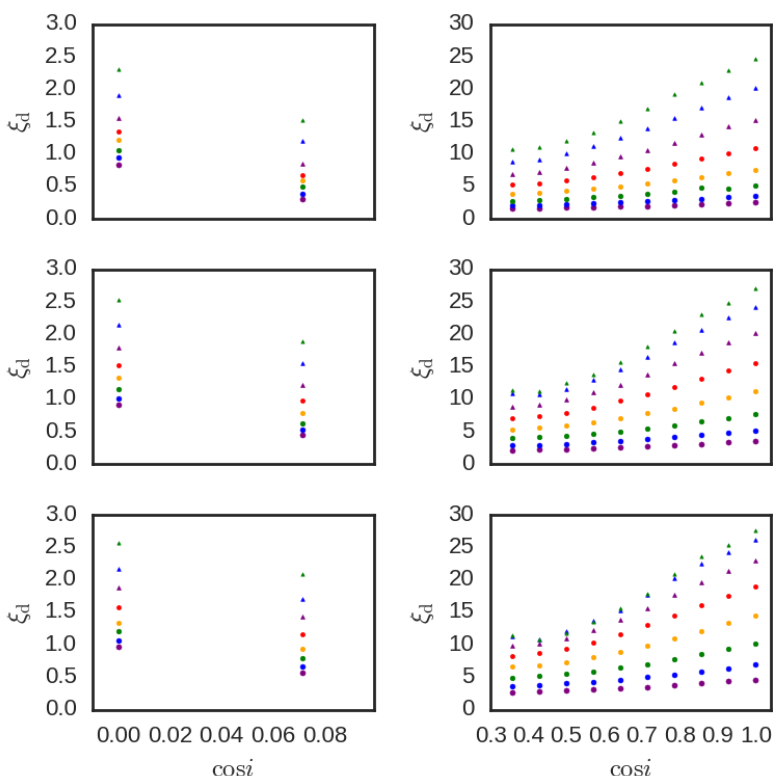

$\cos i$

Figure 5. Selected $I$-band values of $\xi_{\mathrm{d}}$ for our grid vs. $\cos i$. The scaled build-up time was fixed to $\tilde{\tau}_{\text {bu }}=2.25$. Left: Edge-on models. Right: Pole-on models. From top to bottom, the results for Star 1, 2, and 3, respectively. The markers are the same as in Fig. 2.
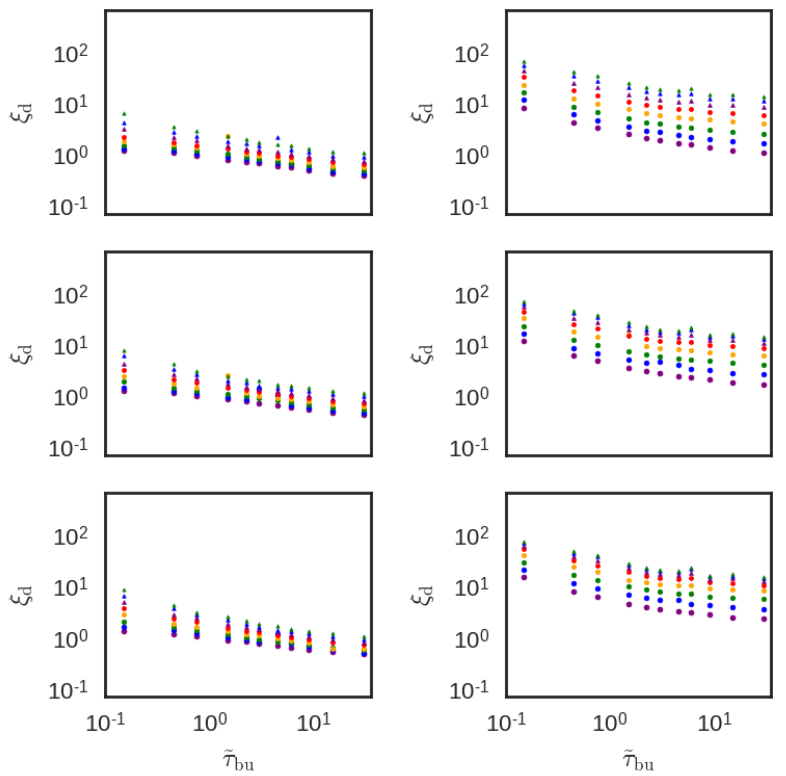

Figure 6. Selected $I$-band values of $\xi_{\mathrm{d}}$ for our grid vs. $\tilde{\tau}_{\text {bu }}$. Left: Edge-on models with $i=90 \mathrm{deg}$. Right: Pole-on models with $i=0 \mathrm{deg}$. From top to bottom, the results for Star 1, 2, and 3, respectively. The markers are the same as in Fig. 2. are directly related to the rate of photometric variations: the smaller the $\xi$, the slower the variation (see Eqs. 17 and 18).

Figure 4 exemplifies the strong variation of $\xi_{\text {bu }}$ with $i$, for the pole-on case. This is probably due to the fact that, in the build-up process, the density grows from the inside out (see Appendix A), which means that the optically thick part of the disc (an expanding pseudo-photosphere) forms first, and the optically thin part takes longer to be built. Since the optical excess of the disc is given by $\Delta X \propto F_{\text {thick }} \cos i+F_{\text {thin }}$, it follows that, as we move from pole-on to edge-on angles, only the optically thick contribution (proportional to $\cos i$ ) varies. As a consequence, the observed rate of increase in flux moves from being more to less optically thick dominated.

In the dissipation process, the density rapidly adjusts to a selfsimilar dissipation pattern in the inner disc (Appendix A). Therefore, the flux from the disc is the result of the decrease and disappearance of the optically thick region - transformed into an optically thin region - and the diminishing of the whole optically thin region. The pole-on values of $\xi_{\mathrm{d}}$ (right panels of Fig. 5) are affected by $\cos i$ to a less extent, when compared to the values of $\xi_{\mathrm{bu}}$. In the dissipation process, by the same reasoning applied to the build-up process, since the optically thick emission is attenuated by the effect of $\cos i$, its disappearance has a reduced effect for more inclined discs and, therefore, the disc should apparently disappear at a slower rate.

The values of $\xi_{\mathrm{d}}$ also show great variation with the asymptotic surface density (Figs. 5 and 6). More specifically, increasing $\Sigma_{0}$ results in a light curve with a slower decay rate. This is probably due to different levels of saturation in the optically thick region. The denser the optically thick region, the bigger its optical depth and the greater the amount of time for it to turn into an optically thin region.

In addition, Fig. 6 shows that $\xi_{\text {d }}$ strongly depends on the scaled build-up time. As expected from the mass reservoir effect (Sect. 2.2), increasing $\tilde{\tau}_{\mathrm{bu}}$ results in smaller values of $\xi_{\mathrm{d}}$, which implies slower decay rates.

From the above an important conclusion can be drawn: viscosity is not the only parameter affecting the rate of photometric variations in a Be light curve. The stellar parameters, the asymptotic surface density, as well as the inclination angle, all affect the observed shape of the light curve. Thus, extracting $\alpha$ from light curves, one of the main goals of this paper, cannot be done without some knowledge about these parameters.

\subsection{Fitting pipeline}

So far, our model light curves were given in terms of the adimensional time parameter, $\tilde{\tau}$. Thus, an equation is necessary to transform from the physical time $t$ to $\tilde{\tau}$, in order to connect the real light curves to our simulated ones.

A variation in the time parameter, $\mathrm{d} \tilde{\tau}$, is related to a variation in physical time by $\mathrm{d} \tilde{\tau}=\mathrm{d} t / \tau(t)$, where $\tau(t)$ depends on 3 stellar parameters $\left(M, R_{\mathrm{eq}}, T_{\mathrm{eff}}\right)$ and the viscous parameter $\alpha(t)$ (Eq. 6). For a given Be star, the build-up phase starts at $t_{1}$, and ends at $t_{2}$, when dissipation begins. Thus, the build-up time is given by $t_{2}-t_{1}$. In this work, following the results of Ghoreyshi \& Carciofi (2017), we explore the possibility that the viscosity parameter may be different at build-up $\left(\alpha(t)=\alpha_{\text {bu }}\right.$, for $\left.t_{1} \leq t<t_{2}\right)$ and dissipation $\left(\alpha(t)=\alpha_{\mathrm{d}}\right.$, for $\left.t \geq t_{2}\right)$. Therefore, the transformation equation from $t$ to $\tilde{\tau}$ is

$\tilde{\tau}=\left\{\begin{array}{ll}\alpha_{\mathrm{bu}} \frac{t-t_{1}}{\alpha \tau}, & t_{1} \leq t<t_{2} \\ \alpha_{\mathrm{bu}} \frac{t_{2}-t_{1}}{\alpha \tau}+\alpha_{\mathrm{d}} \frac{t-t_{2}}{\alpha \tau}, & t \geq t_{2}\end{array}\right.$,

which is such that, as $t$ goes from $t_{1}$ to $t_{2}, \tilde{\tau}$ goes from 0 to $\tilde{\tau}_{\mathrm{bu}}=$ $\alpha_{\mathrm{bu}}\left(t_{2}-t_{1}\right) / \alpha \tau$, and for $t$ larger than $t_{2}$, we see that $\tilde{\tau}>\tilde{\tau}_{\mathrm{bu}}$. Recall 
that $\alpha \tau$, defined in Eq. 6, is a quantity dependent only on the stellar parameters and the disc temperature.

Substitution of Eq. (20) into Eqs. (17), (18) and (19), gives the following equation for fitting an observed bump

$\Delta X(t)= \begin{cases}\Delta X_{\mathrm{bu}}^{\infty}\left(1-\frac{1}{1+\left[C_{\mathrm{bu}}\left(t-t_{1}\right)\right]^{\eta_{\mathrm{bu}}}}\right), & t_{1} \leq t<t_{2} \\ \Delta X_{\mathrm{bu}}^{\infty}\left(1-\frac{1}{1+\left[C_{\mathrm{bu}}\left(t_{2}-t_{1}\right)\right]^{\eta_{\mathrm{bu}}}}\right) \frac{1}{1+\left[C_{\mathrm{d}}\left(t-t_{2}\right)\right]^{\eta \mathrm{d}}}, & t \geq t_{2}\end{cases}$

where

$C_{\mathrm{bu}}=\alpha_{\mathrm{bu}} \frac{\xi_{\mathrm{bu}}}{\alpha \tau}$,

and

$C_{\mathrm{d}}=\alpha_{\mathrm{d}} \frac{\xi_{\mathrm{d}}}{\alpha \tau}$

are coefficients related to the rate of photometric variations. The values of the parameters $\Delta X_{\mathrm{bu}}^{\infty}, \xi_{\mathrm{bu}}$ and $\xi_{\mathrm{d}}$ were tabulated in Sect. 3.3, by fitting the respective empirical laws to the model grid.

Our goal is to fit an observed light curve with Eq. 21, in order to obtain, in a self-consistent way, all the stellar and disc parameters of interest. For that, the following chain of procedures is adopted:

(i) Find a light curve of a Be star that contains at least one clear inactive phase and one complete photometric bump.

(ii) Obtain the magnitudes $X_{*}$ at the inactive phase. Subtract these magnitudes from the light curve and obtain the excesses $\Delta X(t)$.

Without a clear inactive phase, it is not possible to obtain the pure photospheric level (e.g., the horizontal purple straight lines in Fig. 1) and, consequently, it is not possible to know how much of the observed bumps represent the disc contribution to the total flux. In addition, the photometric bump must contain a completely identified build-up phase, from which the instants $t_{1}$ and $t_{2}$ can be extracted, and a considerable extension of the dissipation phase.

(iii) Fit Eq. (21) to the selected bumps, obtaining the coefficients $\Delta X_{\mathrm{bu}}^{\infty}, C_{\mathrm{bu}}$ and $C_{\mathrm{d}}$, as well as the times $t_{1}$ and $t_{2}$ for the onsets of build-up and dissipation.

(iv) Transform the magnitudes at the inactive phase, $X_{*}$, to absolute magnitudes, $M_{X^{*}}$, by correcting for the distance to the star and reddening at each observed band.

Given the theoretical dependence of the coefficients in Eq. 21 on the stellar parameters, the absolute magnitudes are required to estimate the stellar parameters $\left(M, W\right.$ and $\left.t / t_{\mathrm{MS}}\right)$. From them, the parameter $\alpha \tau$ (see Eq. 6) can be estimated. Clearly, if the stellar parameters are known from some other way (e.g., by spectroscopic analysis), this requirement is no longer necessary. Unfortunately, this is not the case for our sample.

(v) Estimate the stellar parameters, the geometric parameter ( $\cos i)$ and the bump parameters $\left(\Sigma_{0}, \alpha_{\text {bu }}\right.$ and $\alpha_{\mathrm{d}}$, for each bump) that best reproduce the fitted stellar $\left(M_{X_{*} *}\right)$ and bump $\left(\Delta X_{\mathrm{bu}}^{\infty}, C_{\mathrm{bu}}\right.$ and $C_{\mathrm{d}}$ ) parameters (see Eqs. 22 and 23 and the parameters $\Delta X_{\mathrm{bu}}^{\infty}$, $\xi_{\text {bu }}$ and $\xi_{\mathrm{d}}$ from Sect. 3.3).

In practice, the above process involves several complications (e.g., estimating the goodness of the fit) that are described in the next section.

\subsection{Fitting using a MCMC sampling}

The task of fitting the measured stellar absolute magnitude $\left(M_{X *}\right)$ and bump parameters $\left(\Delta X_{\mathrm{bu}}^{\infty}, C_{\mathrm{bu}}\right.$ and $\left.C_{\mathrm{d}}\right)$ for estimating the model parameters - step 5, above - was done using the Markov-Chain Monte Carlo (MCMC) sampling technique. We used the Python MCMC sampler emcee (Foreman-Mackey et al. 2013). The code samples a large collection of models by varying all model parameters within a pre-specified range. The sampler provides a distripatipn of model parameters according to a posterior distribution $p$ (model $\mid$ data) $\propto L$ (data $\mid$ model) $\pi$ (model), where $L$ and $\pi$ are the likelihood and the prior distributions, respectively.

In our fitting procedure, there are $4+5 N_{\text {bumps }}$ model parameters for each light curve containing $N_{\text {bumps }}$ identified bumps. There are 3 stellar parameters $\left(M, t / t_{\mathrm{MS}}, W\right)$ and one geometric parameter ( $\cos i$ ), and, for each bump in the light curve, there are 5 parameters: the initial times of the build-up and dissipation phases ( $t_{1}$ and $\left.t_{2}\right)$, the asymptotic surface density $\left(\Sigma_{0}\right)$, and the viscosity parameters during the build-up and dissipation phases ( $\alpha_{\text {bu }}$ and $\alpha_{\mathrm{d}}$ ).

We assume that the errors of the observations follow a Gaussian distribution and, therefore, the likelihood of a Be star with certain model parameters, given the observed data, is given by

$L($ data $\mid$ model $) \propto e^{-\frac{1}{2} \chi^{2}}$,

where

$\chi^{2}=\chi_{\text {discless }}^{2}+\chi_{\text {bump }}^{2}$,

and

$\chi_{\text {discless }}^{2}=\sum_{\text {bands }} \frac{\left(M_{X *}^{\text {model }}-M_{X *}^{\text {obs }}\right)^{2}}{\sigma^{2}\left(M_{X *}^{\mathrm{obs}}\right)}$,

and

$\chi_{\text {bump }}^{2}=\sum_{\text {bands bumps }} \sum_{N_{t}} \sum_{i=1}^{N_{t}} \frac{\left(\Delta X_{i}^{\text {model }}-\Delta X_{i}^{\text {obs }}\right)^{2}}{\sigma^{2}\left(\Delta X_{i}^{\text {obs }}\right)}$,

where $N_{t}$ is the number of data points for a given bump at a given photometric band.

The prior distribution $\pi$ represents our prior knowledge of the distribution of Be stars. We assume it to be

$\pi($ model $) \propto M^{-2.3} f_{\mathrm{Be}}(M) e^{-\frac{(W-\langle W\rangle)^{2}}{2 \sigma_{W}^{2}}}$,

where the factor $M^{-2.3}$ is the initial mass function (IMF) of Kroupa (2001), and $f_{\mathrm{Be}}(M)$ represents the fraction of Be stars relative to the number of B stars, estimated by Martayan et al. (2007a, their Fig. 6). Finally, the Gaussian factor comes from the distribution of rotational velocities in the sample of Be stars, here estimated from Rivinius et al. (2006), assuming $\langle W\rangle=0.81$ and $\sigma_{W}=0.12$.

For the parameter sampling, we have chosen hundreds of "walkers" , proportional to the number of $4+5 N_{\text {bumps }}$ model parameters. For a randomly chosen set of parameters sampled by emcee in the course of the simulation, the corresponding stellar and bump observables are calculated by a multidimensional linear interpolation of the model grid. During the raffle of parameters the prior probability was set to zero if one of the values were sampled outside of the allowed range of a given parameter (Tables 1 and 3). The simulation consists of two steps, the so-called "burn-in phase" and the sampling phase. We verified that 1000 iterations in the burnin phase were sufficient for the convergence of all our models. For each parameter, the best-fitting values were chosen to be the median of distribution of the posterior probabilities, with upper (lower)

1 Each walker can be viewed as a separated Markov Chain in the sample, although the walkers influence each another (Foreman-Mackey et al. 2013). 


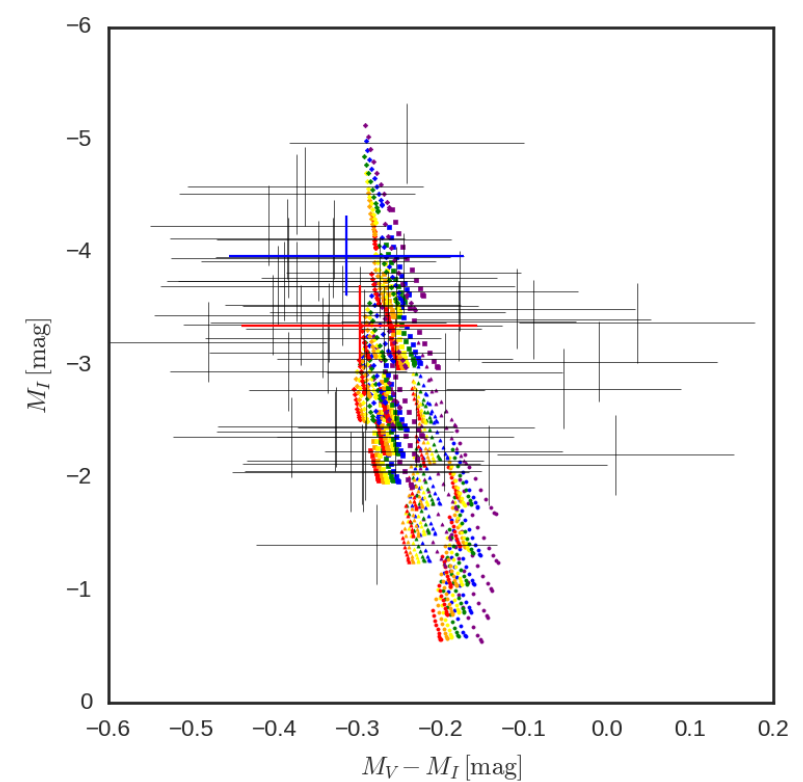

Figure 7. Color-magnitude diagram of simulated discless stars (Table 3). Circles, triangles, squares and diamonds correspond to stellar models with $M=6.0,8.3,11.7,15.0 M_{\odot}$, respectively. The colours red, orange, yellow, green, blue and purple correspond, respectively, to the 6 values of $W$ in increasing order. The 5 different stellar ages are seen as the groups of points move in the upper-right direction. For each star, the effect of going from $90 \mathrm{deg}$ to $0 \mathrm{deg}$ is to move in the upper-left direction. Also shown, as error bars, is the position of our selected stars (see eighth and ninth columns of Table 5). The blue and red error bars mark the positions of SMC_SC1 75701 and SMC_SC6 128831, respectively.

uncertainties estimated from the differences between $84 \%(16 \%)$ of the sample and the median

In Sect. 4 we present a selection of light curves of Be stars from the SMC, and measure their stellar and bump quantities (steps 1 to 4 of the pipeline). Later, in Sect. 5, we apply step 5 of the pipeline, as described in this section, in order to estimate the relevant parameters of the selected Be stars.

\section{OGLE LIGHT CURVES OF BE STAR CANDIDATES}

Mennickent et al. (2002) selected roughly one thousand Be star candidates from the SMC, by studying light curve variations using the OGLE-II database (Udalski et al. 1997). They classified the morphologies found in the light curves into four categories. The majority of the light curves ( $\sim 65 \%$ ) belonged to their type- 4 category, composed by the light curves showing irregular and non-periodic variations. These light curves should correspond to Be stars showing episodes of mass injection more complicated than the simple build-up followed by dissipation scenario described in Sect. 3 .

Most interesting for us is the type-1 group ( $13 \%$ of the sample), composed by light curves that show single sharp or humplike bumps, like the bumps of the light curve of SMC_SC1 75701 (Fig. 1). These bumps should be the result of single nearly continuous episodes of mass injection followed by dissipation of the disc, like the theoretical scenario explored in Sect. 3.

The type-2 group ( $\sim 14 \%$ of the sample), containing light curves showing high and low plateaus, also has some interesting cases for our purposes. High plateaus are usually the photometric result of a longer build-up process in which a near steady-state has been reached in the inner disc. The low plateaus are frequently the portions of the light curve during inactive phases.

Sabogal et al. (2005) selected roughly two thousand Be star candidates from the LMC and classified their light curves into the same four categories described by Mennickent et al. (2002). Previously, Keller et al. (2002) also studied light curves from the LMC using the MACHO survey. They spectroscopically analysed a subsample of their Be star candidates and found that $90 \%$ of them were Be stars. They also classified morphologically their light curves in a slightly different manner. Their so called "bumper events" and "flicker events" more or less correspond to the bumps of type-1 light curves, but also to features of the more irregular type-4 light curves. The bumpers have duration of a few hundred days, while the flicker events are faster, with durations of a few dozens of days. Dips like the one exemplified by the light curve of SMC_SC6 128831 (Fig. 1) were called "fading events". The frequency of these events was quite smaller than the bumpers, in accordance to the picture that fading events are associated with the less numerous shell stars.

Paul et al. (2012) studied the spectral properties of stars from the catalogues of Mennickent et al. (2002) and Sabogal et al. (2005). For the candidates from the SMC, they found that the majority of type-1 and type-2 light curves belong to early B type stars with emission features characteristic of circumstellar material (Paul et al. 2012). Therefore, these light curves are very likely to be from Be stars.

In this work, we selected light curves from the catalogue of Be star candidates from the SMC of Mennickent et al. (2002). In order to have light curves of a longer time baseline, we combined OGLEII data with OGLE-III (Udalski et al. 2008) Due to a calibration issue between OGLE-II and OGLE-III, namely a shift in the zero points present in some of the light curves, it was necessary to find inactivity intervals in both the OGLE-II and OGLE-III portions of these light curves to measure and correct the problem.

The light curves were visually inspected according to the criteria of item 1, Sect. 3.4, i.e., light curves with at least one clear inactive phase and one bump. In this initial work we focussed on well-behaved light curves with clear bumps. We also avoided the short events (flickers, with build-up times $\lesssim 15$ days), due to the fact that most of them are poorly sampled. The end result was a sample of 54 stars, containing 81 selected bumps, shown in Table 5 . In the table, horizontal lines separate the data for each of the 54 stars. Each row in the table contain the data for each of the 81 selected bumps. The fifth and sixth columns in the table contain the beginning and ending of the selected inactive interval for the light curve. The seventh, eighth and ninth columns contain the $B_{*} V_{*} I_{*}$ magnitudes obtained at the inactive phase for the light curve. Due to the nature of the OGLE survey, the $B_{*} V_{*}$ are not available for all sources. The eleventh column contains the bands that were considered in the fitting process of the specific bumps, depending on the availability of measurements in each band. The last two columns are initial visual estimates of $t_{1}$ and $t_{2}$, which were used as input for emcee.

As explained in Sect. 3.4, the magnitudes at the inactive phase are necessary to set the baseline level of the light curves and to provide an estimate of stellar parameters. In order to do the latter, these apparent magnitudes must be colour-corrected and converted to absolute magnitudes by the standard formula $M_{X *}=X_{*}-(5 \log d-5)-A_{X}$. We adopted as the distance to the SMC $d=60.3 \pm 3.8 \mathrm{kpc}$ from Hilditch et al. (2005). The mean $V$-band reddening of the bright stars over the whole SMC 
is $A_{V}=0.470 \pm 0.326$ (Zaritsky et al. 2004). The reddening in the other bands were obtained by the relations $A_{X} / A_{V}$ given by Gordon et al. (2003).

The colour-magnitude diagram (CMD) of Fig. 7 compares the grid of discless models (Table 3) to our sample of Be stars. Most of the stars lie in the upper right corner of the CMD, which means that the majority of our sample is comprised of early-type Be stars, as further discussed in Sect. 5.2.1.

\section{RESULTS}

In this section, the results obtained by applying the pipeline to the stars and bumps of our sample are described. Initially, the results for the two objects of Fig. 1 are examined in detail (Sect. 5.1), followed by an analysis of the results obtained for the entire sample (Sect. 5.2)

\subsection{SMC_SC1 75701 and SMC_SC6 128831}

The results for SMC_SC1 75701 and SMC_SC6 128831 are shown in Figs. 8 and 9, respectively. For SMC_SC1 75701 there was enough data for both the $I$ and $V$ bands to allow these two light curves to be fitted simultaneously. For SMC_SC6 128831, however, only $I$-band data was available. The times for the beginning of the build-up $\left(t_{1}\right)$ and dissipation $\left(t_{2}\right)$ are fitted quantities in the pipeline, but an initial estimate for them is provided to emcee by graphically analysing each light curve. These estimates are shown as the purple and orange segments in the horizontal straight lines in the figure. In the MCMC sampling, after a sufficient number of iterations, a stationary sample is obtained, for which the model parameters are more concentrated in the regions of higher posterior probability. In the plots we show 100 sets of randomly selected model curves obtained after the stationary sample was reached. The dispersion of the curves gives a visual measure of the goodness of the fits.

The goodness of the fit can be quantitatively assessed from the distribution of the posterior probabilities of each fitted parameter shown in Figs. 8 and 9. The main diagonal of the triangular diagram plots the distributions of the stellar $\left(M, t / t_{\mathrm{MS}}\right.$ and $\left.W\right)$, geometrical ( $\cos i)$, and bump $\left(\Sigma_{0}, \alpha_{\mathrm{bu}}\right.$ and $\left.\alpha_{\mathrm{d}}\right)$ parameters, and they can be used to assess how well-constrained each parameter is. The images below the diagonal show how the parameters correlate with each other.

The stellar parameters $\left(M, t / t_{\mathrm{MS}}\right.$ and $\left.W\right)$ are mainly constrained by the magnitudes at the inactive phase. In Fig. 8, the three leftmost histograms along the diagonal have broad distributions, which means that these parameters are not well constrained. The first histogram shows that SMC_SC1 75701 is an early Be star, probably even more massive than the available stellar models (Table 3). This agrees with the position of the star in the CMD (Fig. 7). The mass is anti-correlated with the main sequence age (see $t / t_{\mathrm{MS}} \times M$ plane), as expected from the fact that a less massive but more evolved star can have a similar absolute magnitude of a younger, more massive star.

The bump parameters $\left(\Sigma_{0}, \alpha_{\text {bu }}\right.$ and $\left.\alpha_{\mathrm{d}}\right)$ are mainly constrained by the shape of the observed bump. Roughly, the amplitude of the bump depends mostly on $\Sigma_{0}$ (and $\cos i$, see Fig. 2), while the value of viscosity parameter in each phase controls the rate of brightness variation. For SMC_SC1 75701, $\Sigma_{0}$ has a broad distribution peaking around $\sim 1.5 \mathrm{~g} \mathrm{~cm}^{-2}$, indicating a quite dense disc, close to the densest cases in the sample of Vieira et al. (2017) for the same spectral type. This fact can also be inferred from Fig. 2, given the large observed $\Delta I_{\mathrm{bu}}^{\infty}$. The best-fit viscosity parameters are $\alpha_{\mathrm{bu}}=0.24_{-0.08}^{+0.18}$ and $\alpha_{\mathrm{d}}=0.11_{-0.05}^{+0.08}$.

Of the three bump parameters derived for SMC_SC1 75701, $\Sigma_{0}$ and $\alpha_{\mathrm{d}}$ clearly anti-correlate with $\cos i$, while $\alpha_{\mathrm{bu}}$ shows no signs of correlation. In fact, an anti-correlation of these three parameters with $\cos i$ is expected, as a consequence of the dependency of $\Delta X_{\mathrm{bu}}^{\infty}$, $\xi_{\text {bu }}$ and $\xi_{\text {d }}$, defined in Sect. 3.3, on $\cos i$. Fig. 2 shows that, if the star is seen more pole-on (higher values of $\cos i$ ), smaller values of $\Sigma_{0}$ are required in order to obtain the fitted $\Delta X_{\mathrm{bu}}^{\infty}$, hence the strong anti-correlation seen in the $\Sigma_{0} \times \cos i$ plane. Eq. (22) shows that the fitted coefficient $C_{\mathrm{bu}}$ is proportional to the product of $\alpha_{\mathrm{bu}}$ and $\xi_{\text {bu }}$, and it was shown (Fig. 4) that discs seen more pole-on (higher values of $\cos i$ ) appear to build-up faster (having higher values of $\left.\xi_{\mathrm{bu}}\right)$. Therefore, for higher values of $\cos i$, smaller values of $\alpha_{\mathrm{bu}}$ are required to obtain the fitted $C_{\mathrm{bu}}$, which explains the anticorrelation in the $\alpha_{\mathrm{bu}} \times \cos i$ plane. Finally, Eq. (23) shows that the $C_{\mathrm{d}}$ is proportional to the product of $\alpha_{\mathrm{d}}$ and $\xi_{\mathrm{d}}$, and it was shown in Fig. 5 that the more pole-on and the less dense the disc, the faster the rate of brightness variation in the dissipation, thus the anticorrelation expected in the $\alpha_{\mathrm{d}} \times \cos i$ plane. Even though this last anti-correlation was not observed for SMC_SC1 75701, it is clearly seen for many objects of our sample, for instance, SMC_SC3 71445 (Fig. C11) and SMC_SC3 125899 (Fig. C13).

The results for SMC_SC6 128831 (Fig. 9) point to a less massive $\operatorname{star}\left(M=12.2_{-2.2}^{+2.7} M_{\odot}\right)$ surrounded by a much more massive disc $\left(\Sigma_{0}=2.6_{-0.6}^{+0.5} \mathrm{~g} \mathrm{~cm}^{-2}\right)$. SMC_SC6 128831 is an example of a dip, which means that this Be star is seen at a near edgeon angles. The very steep build-up phase of SMC_SC6 128831 hints to large mass injection rate and viscosity during build-up, as confirmed by the fifth and sixth histograms along the diagonal of Fig. 9. The viscosity parameter during dissipation was found to be $\alpha_{\mathrm{d}}=0.69_{-0.27}^{+0.34}$. The plane $\alpha_{\mathrm{d}} \times \Sigma_{0}$ shows a correlation, just as for the case of SMC_SC1 75701, which is a consequence of the decrescent relationship between $\xi_{\mathrm{d}}$ with $\Sigma_{0}$, also expected for near-edge-on inclinations (see Fig. 5).

For SMC_SC6 128831 a positive correlation between $\Sigma_{0}, \alpha_{\text {bu }}$ and $\alpha_{\mathrm{d}}$ with $\cos i$ was observed. Fig. 2 shows that, if the star moves away from the edge-on case $(\cos i=0)$, bigger values of $\Sigma_{0}$ are required in order to obtain the fitted $\Delta X_{\text {bu }}^{\infty}$, hence the correlation seen in the $\Sigma_{0} \times \cos i$ plane. Fig. 4 shows that,for the edge-on case there is no strong variation of $\xi_{\mathrm{bu}}$ with $\cos i$. However, there is the trend that a more tenuous discs appear to build-up faster, specially for a hotter star. Therefore, since Eq. 22 shows that $C_{\mathrm{bu}} \propto \alpha_{\mathrm{bu}} \xi_{\mathrm{bu}}$, it follows that, with the increase of $\Sigma_{0}$ with $\cos i$, the function $\xi_{\text {bu }}$ decreases and, hence, $\alpha_{\text {bu }}$ increases. Finally, Eq. 23 shows that $C_{\mathrm{d}} \propto \alpha_{\mathrm{d}} \xi_{\mathrm{d}}$ and Fig. 6 shows that $\xi_{\mathrm{d}}$ decreases with $\cos i$ and $\Sigma_{0}$. Therefore, as $\Sigma_{0}$ increases with $\cos i$, it follows that $\xi_{\mathrm{d}}$ decreases and, hence, $\alpha_{\mathrm{d}}$ must increase. Similar trends were found for the other two edge-on stars in our sample (SMC_SC1 92262, Figs. C4 and C5, and SMC_SC4 179053 (Fig. C34).

In general, the histograms of Fig. 9 are broader than the ones in Fig. 8, indicating that parameters are worse constrained than for SMC_SC1 75701. There are two main reasons for this. First, only $I$ band data was available for this star, which has a negative impact on the pipeline's ability to constrain the stellar parameters. The MCMC method ensures that the uncertainties in the stellar parameters are properly propagated into the other model parameters. Second, the fact that the dips have smaller amplitudes than the bumps of poleon stars, even for higher values of $\Sigma_{0}$, is a great disadvantage, 

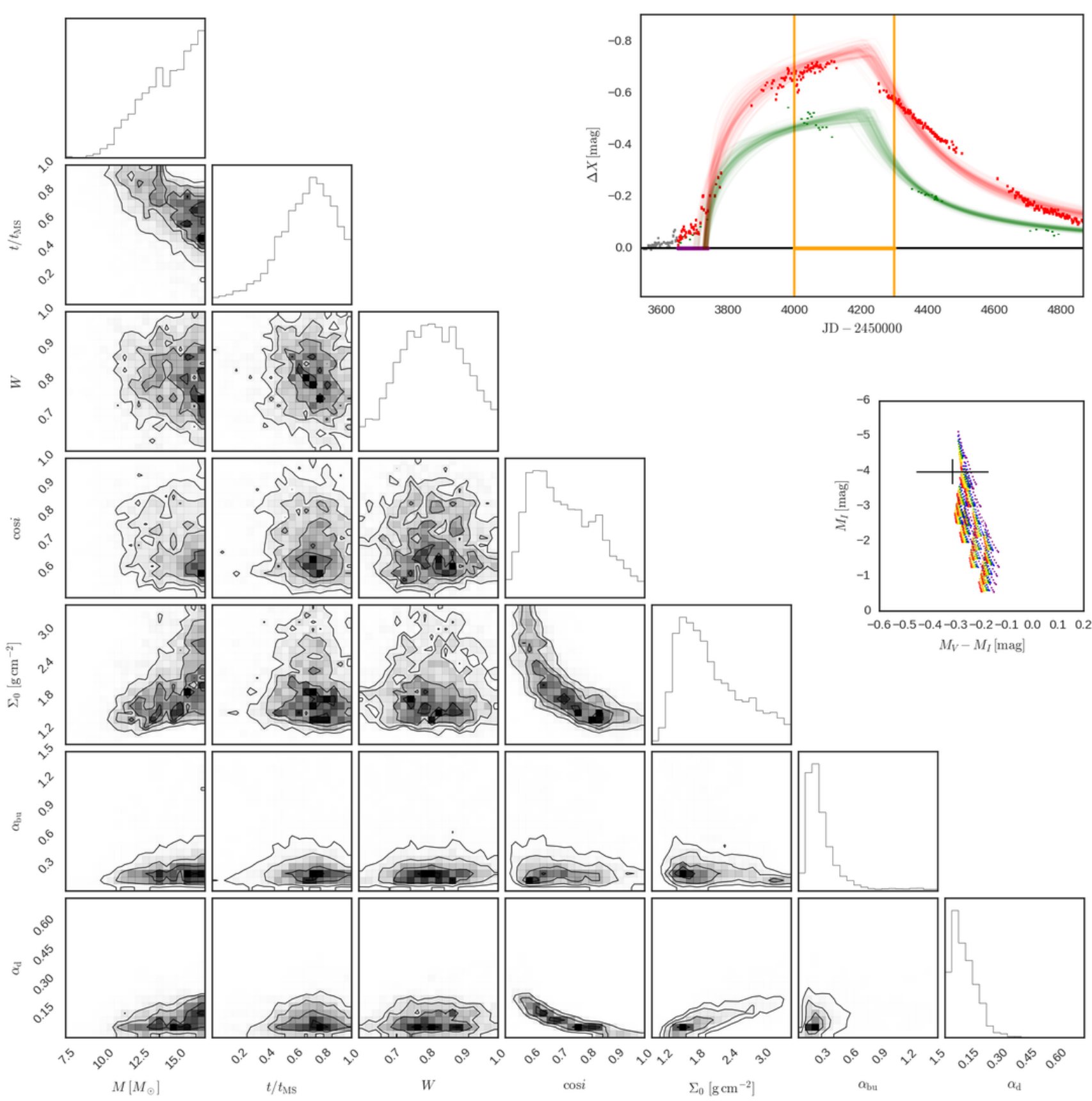

Figure 8. Upper-right: Light curve of SMC_SC1 75701. Thin lines: 100 model curves randomly selected from the stationary sample of the emcee code. The red (green) colour indicates the $I(V)$ band. The purple and orange time intervals marked in the horizontal straight lines are the allowed intervals for the model parameters $t_{1}$ and $t_{2}$, respectively. Middle-right: CMD displaying the model grid of inactive Be stars and the position of SMC_SC1 75701 (see Fig. 7 for details). Below: Results of the emcee run for SMC_SC1 75701. Histogram distributions of the posterior probabilities (top panels) and two-by-two correlations of the stellar $\left(M, t / t_{\mathrm{MS}}\right.$ and $\left.W\right)$, geometrical (cos $\left.i\right)$, and bump $\left(\Sigma_{0}, \alpha_{\mathrm{bu}}\right.$ and $\left.\alpha_{\mathrm{d}}\right)$ parameters (off-diagonal panels). The parameters $t_{1}$ and $t_{2}$ were not shown for convenience. The normalised density levels shown in the off-diagonal panels are $12 \%, 39 \%, 68 \%, 87 \%$ of the peak probability.

because the bump amplitude is much closer to the noise level of the measurements.

The results of the pipeline for all stars in Table 5 are shown in Figs. C1 to C79, available electronically only. The best-fit model parameters for all stars and bumps are listed in Table 6 .

\subsection{Results for the whole sample}

As seen previously, the scarcity of information about the central star (one, two, or at most 3 photometric bands only) causes a poor determination of its fundamental stellar parameters, which, owing to the nature of the MCMC method, propagates onto the disc parameters. The main result of this work, therefore, does not lie on 


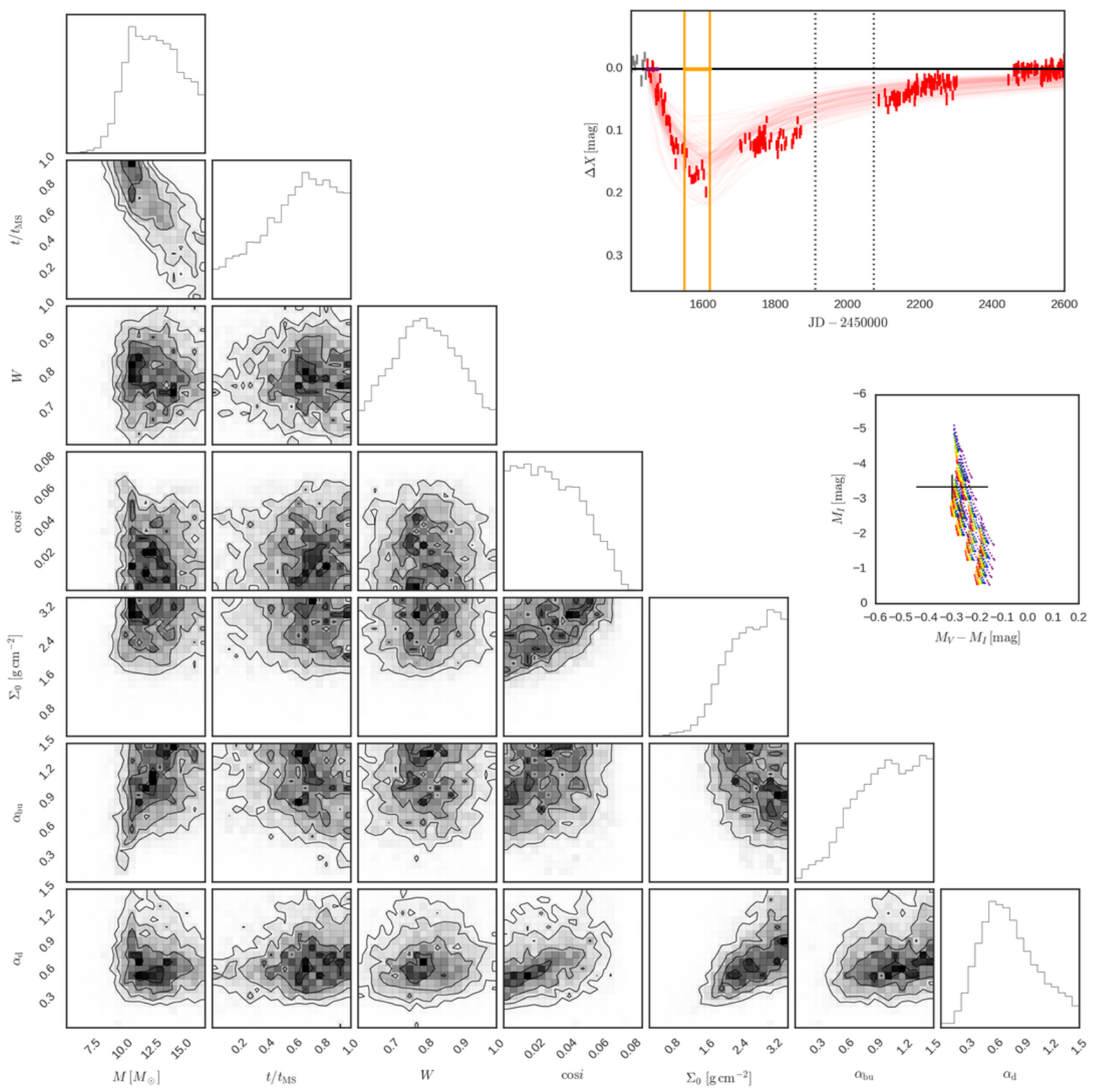

Figure 9. Same as Fig. 8 for SMC_SC6 128831.

the individual determination of the bump parameters, but on the statistical properties of the sample as a whole.

\subsubsection{Mass distribution}

Let us initially discuss the properties of our sample, in order to determine whether it represents a typical population of Be stars in SMC, or whether one or more selection biases where introduced.

Our selection of stars and bumps comes from the catalogue of visual photometric Be star candidates of Mennickent et al. (2002), where the candidate stars were selected according to the expected location of Be stars in colour-magnitude diagrams and according to the observed variability in the light curves. In Fig. 10, we show the sum of the posterior probabilities of parameter $M$ for all our sample of stars (solid line). Clearly, most of our stars are early-type Be stars, in agreement to the position of our sample in the CMD (Fig. 7). We also show the factor $\propto M^{-2.3} f_{\mathrm{Be}}(M)$ of Eq. 28 (dashed line). We recall that this factor was assumed as a prior in the MCMC fitting, and it represents our current knowledge about the populations of Be stars in the SMC. The green curve shows that, although the fraction of Be stars over B stars $\left(f_{\mathrm{Be}}(M)\right.$, estimated by Martayan et al. (2007a) from a cluster of the SMC) generally increases with 


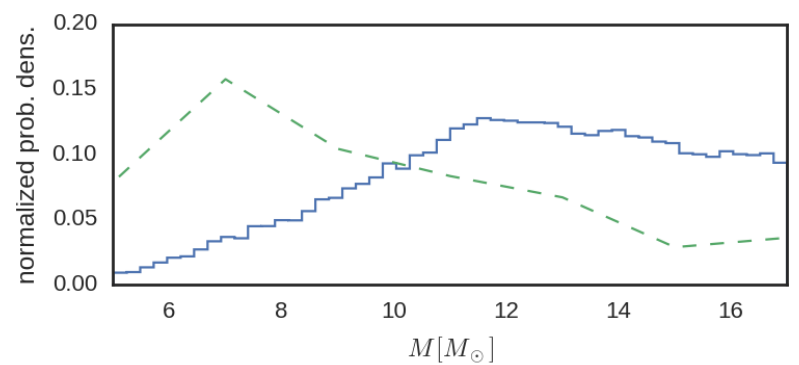

Figure 10. Solid line: Histogram of the sum of the posterior probabilities of parameter $M$ for all stars in our sample. Dashed line: IMF of Kroupa (2001) weighed by the fraction of Be stars over B stars of Martayan et al. (2007a), given by the factor $M^{-2.3} f_{\mathrm{Be}}(\boldsymbol{M})$ in Eq. (28).

$M$, the higher probability of the formation of less massive stars expressed in the IMF of Kroupa (2001) results in a bigger incidence of late type over early type Be stars. Our sample, therefore, is biased towards more massive stars.

This bias likely have several reasons:

(i) The typical apparent $I$-band magnitudes of a B0 and B9 star in the SMC are $\sim 15.5$ and $\sim 19.5$, with rms uncertainties given by $\gtrsim 0.005$ and $\gtrsim 0.15$, respectively (Wyrzykowski et al. 2009). Therefore, the threshold of detectability of a good bump increases for late type Be stars.

(ii) Late-type Be stars tend to have more tenuous discs than early-type ones (Vieira et al. 2017, see also next subsections), and therefore should develop bumps with much smaller amplitudes.

(iii) Late-type Be stars tend to show less variability (Rivinius et al. 2013, see also next subsections), which would make it less probable to identify bumps in their light curves.

\subsubsection{Asymptotic surface density}

In the upper panel of Fig. 11 we show how $\Sigma_{0}$ varies with stellar mass in our sample, demonstrating a clear tendency of denser discs around the more massive stars. Vieira et al. (2017) have shown that, for the Be stars in the Galaxy, the incidence of denser discs increases with the mass of the stars. Comparison of our results with the ones of Vieira et al. are done in Fig. 12. While our sample is biased towards large masses, their sample is more evenly distributed in mass. Another difference is that our results are all concentrated in a region of high disc density, while theirs cover a much wider range of densities for all spectral types. The reason for this lies in fact that for this initial study we selected light curves with large and well-defined bumps, disregarding low-amplitude and short-duration ones. In fact, the detection of tenuous discs by Vieira et al. was only possible because they studied the SED in the IR (typically between 9 and $60 \mu \mathrm{m}$ ), where the disc emission is much stronger than in the visible range. Therefore, all but the most dense of their discs would be too tenuous to generate appreciable photometric excesses in visual photometric bands, suitable for our fitting procedure.

We conclude that our sample of visual bumps should represent the upper limit for the densities found in the discs of SMC Be stars. In the Galaxy, these large densities are only found in early type Be stars. The median of the $\Sigma_{0}$ for our sample is $\left\langle\Sigma_{0}\right\rangle=1.44_{-0.75}^{+1.01} \mathrm{~g} \mathrm{~cm}^{-2}$. Furthermore, there may be some indication that the Be stars in the SMC may have more massive discs, on average, than their
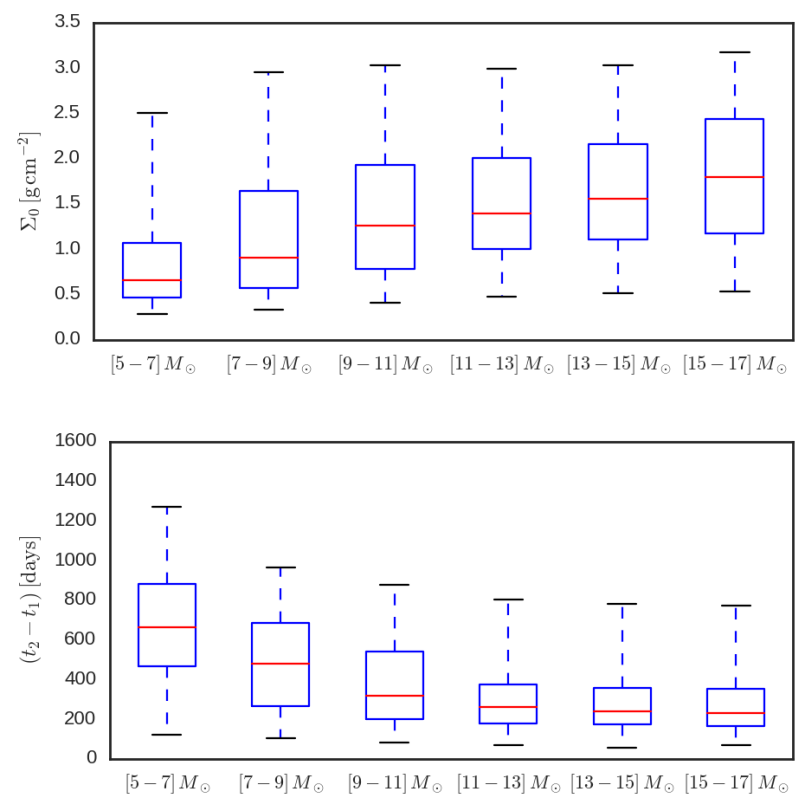

Figure 11. Boxplots of $\Sigma_{0}$ (above) and $t_{2}-t_{1}$ (below) for the summed posterior probabilities of our sample of bumps, separated in six equal intervals of mass, ranging from 5 to 17 solar masses. The middle line of the boxes mark the median $(50 \%)$ of the samples. The lower and upper ends of the boxes mark $25 \%$ and $75 \%$ of the samples. The lower and upper whiskers mark $5 \%$ and $95 \%$ of the samples.

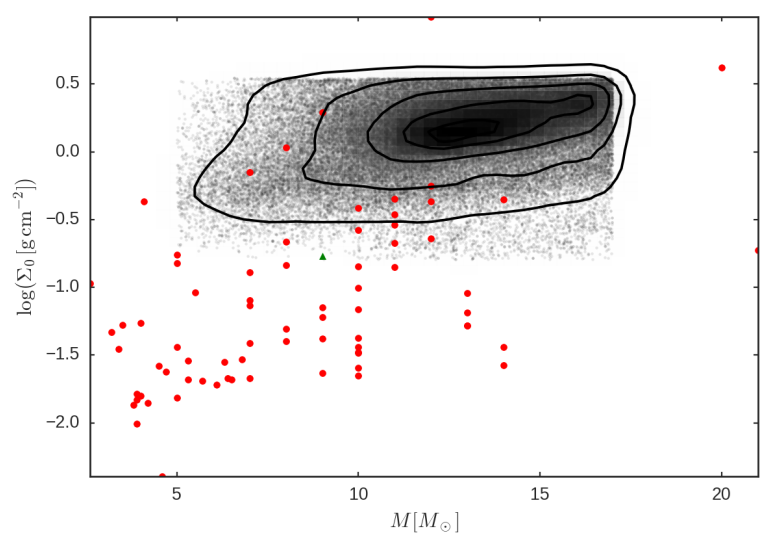

Figure 12. Distribution of the parameters $M$ and $\Sigma_{0}$ for our sample. The contour levels are the same as in Fig. 8. The red dots correspond to the surface densities at the base of the disc of Galactic Be stars, measured by Vieira et al. (2017). The green triangle corresponds to the initial state of the ablating disc model of Kee et al. (2016) for a B2e star.

galactic counterparts, in line with results from the literature that report higher $\mathrm{H} \alpha$ equivalent widths in the SMC Be stars than in the Galaxy (Martayan et al. 2007b). This last point, however, should be view with some caution given the large biases present in our sample. 

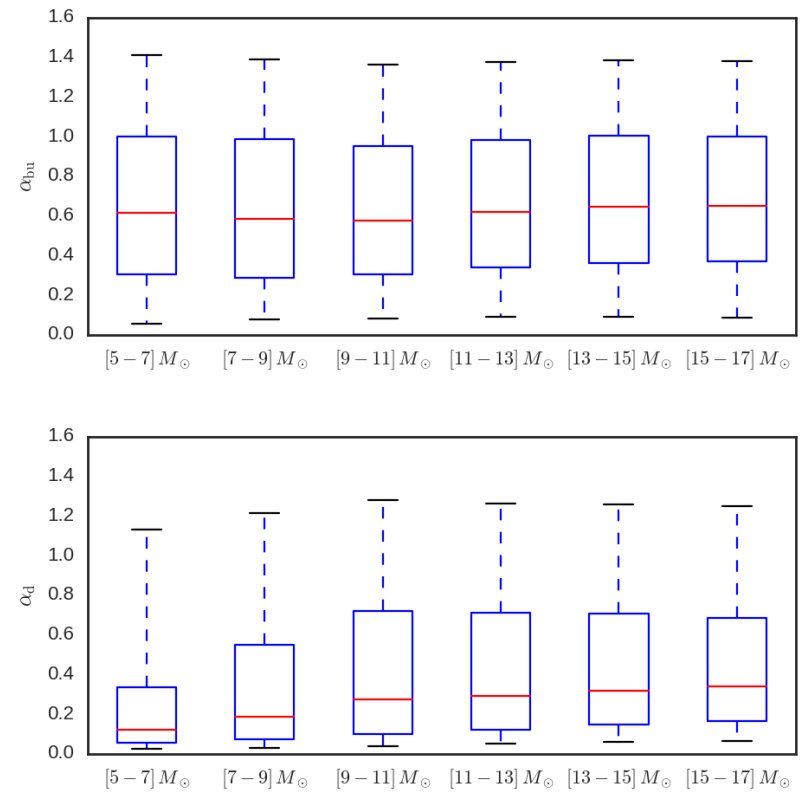

Figure 13. Same as Fig. 11 for $\alpha_{\text {bu }}$ (above) and $\alpha_{\mathrm{d}}$ (below).

\subsubsection{Disc life cycles}

In the lower panel of Fig. 11, we plot the distribution of the build-up time, $t_{2}-t_{1}$, versus the stellar mass. We see that the duration of the bump is much shorter for massive stars, which indicates that these stars are much more variable than their late type siblings. Similar findings were reported in the Galaxy (e.g. Rivinius et al. 2013). For a complete characterisation the disc life cycles it would be required a census of the number of bumps present during the timespan of OGLE-II and OGLE-III observations (roughly 12 years). Unfortunately, this cannot be done for our sample because in this study we focused only on the well-defined bumps. The median of the build-up time for our sample is $\left\langle t_{2}-t_{1}\right\rangle=305_{-171}^{+351}$ days.

\subsubsection{Viscosity parameter}

The majority of our determinations of the viscosity parameter had broad uncertainty distributions, and it was found that the errors in the determinations of $\alpha_{\text {bu }}$ were generally greater than those of $\alpha_{\mathrm{d}}$. Considering the sample as a whole, we find that there is no variation of $\alpha_{\mathrm{bu}}$ with the stellar mass (Fig. 13, top), but there is a slight hint that $\alpha_{\mathrm{d}}$ may grow with the stellar mass (Fig. 13, bottom). Furthermore, we find that on average the viscosity parameter is roughly two times larger at build-up than at dissipation $\left(\left\langle\alpha_{\mathrm{bu}}\right\rangle=\right.$ $0.63_{-0.39}^{+0.52}$ vs. $\left\langle\alpha_{\mathrm{d}}\right\rangle=0.29_{-0.20}^{+0.61}$ ).

Lamers et al. (1995) identified two bi-stability jumps in stellar winds, one strong jump at $T_{\text {eff }} \approx 22,000 \mathrm{~K}$, and a weaker one at $T_{\text {eff }} \approx 10,000 \mathrm{~K}$. These jumps are associated with rapid changes of the ionisation and excitation state of the gas in stellar winds around these temperatures, which in turn modify the line opacity. If radiative ablation were a relevant disc driving mechanism, we would expect that the jumps would cause changes in the line forces, thus affecting disc ablation and the observed timescales of disc dissipation. The absence of any structure around $T_{\text {eff }} \approx 22,000 \mathrm{~K}$ (roughly $10 M_{\odot}$ ) in Fig. 13 argues against ablation as a strong driving mechanism in the discs of our sample.

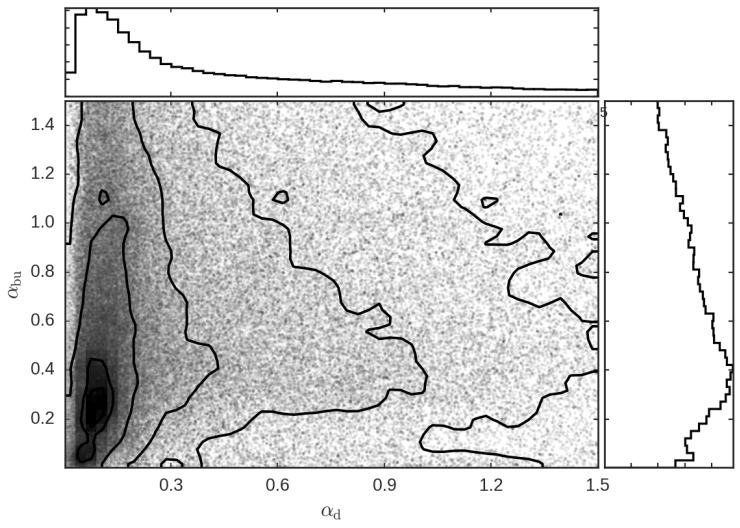

Figure 14. Distribution of the two viscosity parameters $\alpha_{\mathrm{bu}}$ and $\alpha_{\mathrm{d}}$. The histograms above and on the right are the projections for $\alpha_{\mathrm{d}}$ and $\alpha_{\mathrm{bu}}$, respectively. The contour levels are the same as in Fig. 8.

The estimated values of $\alpha$ in the range of a few tenths to one is in agreement with the usual values obtained for the hot and variable discs of dwarf novae (King et al. 2007; Kotko \& Lasota 2012), as well as with the values obtained for the Be star $28 \mathrm{CMa}$ by Ghoreyshi \& Carciofi (2017). They are, however, an order of magnitude or more above the usual values obtained in magnetohydrodynamic (MHD) simulations, where the magnetorotational instability (MRI, Balbus \& Hawley 1991) is the main theoretical assumption for the mechanism that generates the necessary viscosity (King et al. 2007).

In Fig. 14, we show the distributions of $\alpha_{\text {bu }}$ (right) and $\alpha_{\mathrm{d}}$ (above) and the distribution in the $\alpha_{\text {bu }} \times \alpha_{\mathrm{d}}$ plane. We found that, for most of the bumps, there was a correlation between $\alpha_{\mathrm{bu}}$ and $\alpha_{\mathrm{d}}$, with values of $\alpha_{\mathrm{bu}}$ greater than values of $\alpha_{\mathrm{d}}$ being more likely. This trend can be seen in the darker areas of the $\alpha_{\text {bu }} \times \alpha_{\mathrm{d}}$ plane. Ghoreyshi \& Carciofi (2017) found the same trend for 28 CMa.

It is unclear whether the the higher likelihood of $\alpha_{\text {bu }}>\alpha_{\mathrm{d}}$ is real a phenomenon or a result of the approximations employed in this work. One key approximation made in our model is that the hydrodynamical equations are solved assuming that the entire disc is isothermal. Earlier studies (e.g., Jones et al. 2004; Carciofi \& Bjorkman 2006) have shown the disc to be highly non-isothermal, which means that $c_{s}$ in Eq. 3 is a complicated function both of $R$ and time. Another approximation is that the possible effects of line forces were neglected. Recently, Kee et al. (2016) simulated the effects of a line-driven wind from early B-type stars over a non-viscous gaseous disc of solar metallicity and typical density. They showed that the line-driven wind was able to destroy the disc in timescales compatible with the observed large-amplitude photometric variations of Be stars. They argued that the presence of the line-driven wind might be the cause of the apparent abnormal value estimated for $\alpha$ by Carciofi et al. (2012), Ghoreyshi \& Carciofi (2017), and this work. Future work must explore if the viscous force and the line force working together can produce the variability of Be stars with smaller values of $\alpha$.

The line force of an outwardly diffusing near-Keplerian optically thin disc under irradiation by the hot star will have a positive component in the azimuthal direction, giving angular momentum to the gas (Gayley et al. 2001). It is possible that this additional source of angular momentum may help viscosity in pushing material outwards during the build-up phase and oppose to the reaccretion of the inner disc in the dissipation, thus contributing to the observed 
trend of $\alpha_{\mathrm{bu}}>\alpha_{\mathrm{d}}$. We expect, however, that for our low-metallicity SMC Be stars, the possible effect of the line force will be greatly diminished. Furthermore, so far all studies of the effects of line forces in gaseous Keplerian discs assumed that the gas is optically thin, which is not the case for our inner discs near the disc plane. The green triangle in Fig. 12 marks the mass and density at the stellar equator of the initial state of the ablating disc model of Kee et al. (2016) for a B2e star. Our calculations show that their initial state would generate only a modest excess $\Delta I=-0.1 \mathrm{mag}$, if seen pole-on.

On the other hand, it is possible that an opposite scenario might happen. The line force might operate ablating the tenuous material above the disc plane. These regions would receive radiation from the stellar surface and radiation reprocessed by the optically thick disc, behaving as a sink of mass and angular momentum of the disc (e.g. Krtička et al. 2011). In that case, the line-driven wind would actually slow down the build-up phase, because it would take a longer time for the disc to reach a near steady-state, and would speed up the dissipation phase. If the above were true, that would result in $\alpha_{\mathrm{bu}}<\alpha_{\mathrm{d}}$, contrary to the results of Ghoreyshi \& Carciofi (2017) and in this work.

We end this section by speculating about another possible cause for the observed trend $\alpha_{\mathrm{bu}}>\alpha_{\mathrm{d}}$. The disc formation is probably a mechanically violent process, with outbursts of matter injecting mechanical energy into the disc that is likely to disrupt its hydrostatic equilibrium and induce further turbulent motion. The dissipation, on the other hand, is expected to be a more gentle process, much less perturbed by stellar activity. The mechanically-driven turbulence during outburst might account for the larger values of $\alpha$ at these phases.

\subsubsection{Mass and angular momentum loss}

Although the discs of Be stars in our sample are generally not in steady-state, their steady-state mass and angular momentum loss rates (Eqs. 12 and 13) are useful estimates of the actual quantities that are lost by the star after the bump event ends (see Appendix A). The panels of Fig. 15 show our distributions of the steady-state mass (above) and angular momentum (below) loss rates. For the calculation of $(-\partial M / \partial t)_{\text {steady }}$, we considered the radius of the outer boundary to be given by the radius at which the disc outflow becomes angular momentum conserving (which can be seen as an outer radius of the viscous disc), estimated as $\tilde{R}_{\text {out }}=0.3\left(v_{\text {orb }} / c_{s}\right)^{2}$ by Krtička et al. (2011). The red dots (upper panel) are the estimates of $(-\partial M / \partial t)_{\text {steady }}$ made from the results of Vieira et al. (2017) for Galactic Be stars, assuming that $\alpha=1$.

The steady-state mass and angular momentum loss rates for our densest bumps are of the order of $\sim 10^{-10} M_{\odot} \mathrm{yr}^{-1}$ and $\sim 5 \times 10^{36} \mathrm{~g} \mathrm{~cm}^{2} \mathrm{~s}^{-2}$, respectively. The typical decretion rate, which estimates the flux of mass in the disc near the stellar equator, is an order of magnitude higher than $(-\partial M / \partial t)_{\text {steady }}$, being of the order of $\sim 10^{-9} M_{\odot} \mathrm{yr}^{-1}$, which also corresponds to the upper limit of the observed wind mass loss rate of B stars (Snow 1981; Puls et al. 2008). Values of the typical decretion rate and steady-state angular momentum loss rate are given in the eleventh and twelfth columns of Table 6 , respectively. The total angular momentum lost by the star as a consequence of the bump, $-\Delta J_{*}$, is given by $(-\partial J / \partial t)_{\text {steady }}$ times the build-up time (Eq. A4), and the total mass lost is simply $-\Delta M_{*}=-\Delta J_{*} /\left(G M R_{\text {out }}\right)^{1 / 2}$, if we still approximate the VDD as a Keplerian disc at $R_{\text {out }}$. If we consider that a typical bump has a build-up time of roughly one year, then the mass and angular
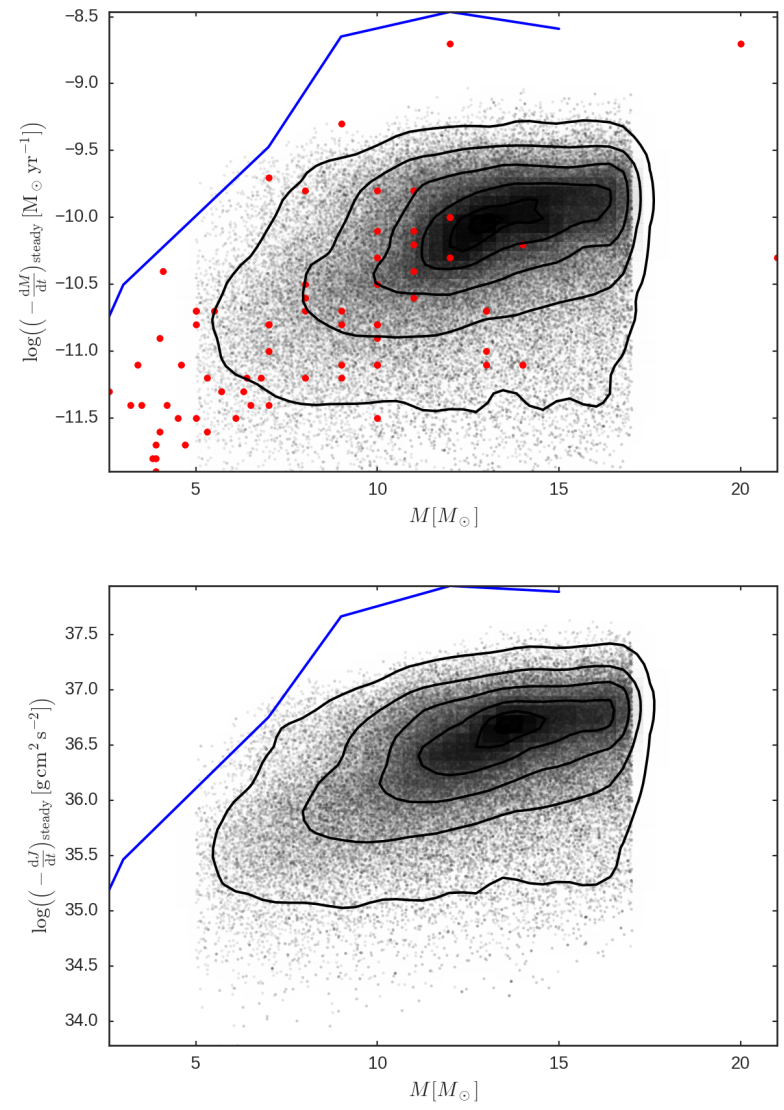

Figure 15. Distributions of the steady-state mass (above) and angular momentum (below) loss rates for our sample. The contour levels are the same as in Fig. 8. The red dots (upper panel) are the values of $(-\partial M / \partial t)_{\text {steady, }}$, calculated from the results of Vieira et al. (2017) for galactic Be stars. The blue curves are the estimations made by Granada et al. (2013) of the steadystate mass and angular momentum loss of their $Z=0.002$ stars during their episodes of disc formation.

momentum lost by the star as a consequence of one dense complete bump are of the order of $\sim 10^{-10} M_{\odot}$ and $\sim 10^{44} \mathrm{~g} \mathrm{~cm}^{2} \mathrm{~s}^{-1}$ (or $\sim 0.01$ Moon masses and $\sim 10^{-3}$ times Earth's orbital angular momentum around the Sun, respectively). The VDD is, thus, a physical mechanism capable of extracting a large quantity of angular momentum from the outer layers of the star, without requiring the loss of too much mass. It is, therefore, a breaking mechanism of the outer layers. The angular momentum lost by the star for each individual bump is given in the thirteenth column of Table 6 .

It was proposed (e.g., Krtička et al. 2011) that, with the evolution of the star, the formation of the VDD might be a natural mechanism to extract angular momentum from the outer layers of the star, preventing it to exceed the break up velocity. Granada et al. (2013) assumed the appearance of a steady-state VDD in the Geneva stellar evolution code every time the outer layers of the star reached $W>0.88$ (or $\omega>0.99$, in their notation, where $\left.\omega=\Omega /\left(8 G M / 27 R_{\text {pole }}^{3}\right)^{1 / 2}\right)$. The blue curves in Fig. 15 are the estimates made by Granada et al. (2013) of the steady-state mass and angular momentum loss of their $Z=0.002$ stars during their episodes of disc formation. The fact that the curves of Granada et al. (2013) lie up to one order of magnitude above our results and the 
ones of Vieira et al. suggests that their assumed discs were made much too dense to be able to remove the needed angular momentum.

Although the Be phenomenon is probably a powerful velocity breaking mechanism for the outer layers of the star, its effect on the star as a whole is expected to be modest. Our determinations of angular momentum loss show that, even if the Be phenomenon happened during $\sim 30 \%$ of the stellar main sequence lifetime, and the integrated time of all the build-up phases was $\sim 30 \%$ of that time, that would lead to the removal of $\sim 1 \%$ of the initial angular momentum of the star, which, for fast rotating stars of masses from $7 M_{\odot}$ to $15 M_{\odot}$ is of $8-30 \times 10^{51} \mathrm{~g} \mathrm{~cm}^{2} \mathrm{~s}^{-1}$ (Granada et al. 2013).

In conclusion, light-curve modelling offers a reliable way to measure directly the amount of angular momentum lost by disc formation events in Be stars. Coupling this information with future studies about the fraction of the main-sequence lifetime a Be star spends in outburst will allow us to estimate the total amount of angular momentum that is lost by the disc during the main sequence. To obtain the total angular momentum lost we must sum the amount lost by the stellar wind. This quantity, hitherto unknown, will provide an essential constraint on stellar evolution models, in effect allowing for calibration of the core-surface angular momentum coupling of a star.

\section{CONCLUSIONS}

We present a new method to model the light curves of Be stars with the goal of extracting quantitative information about the fundamental parameters of their discs, such as the viscosity parameter, $\alpha$, and the asymptotic surface density $\left(\Sigma_{0}\right.$, which is related to the disc mass injection rate). The method uses a large pre-computed grid of synthetic Be light curves, calculated using detailed hydrodynamic calculations coupled with three-dimensional NLTE radiative transfer calculations. The comparison between the model grid and the observed light curves was made possible by the identification of two empirical laws, that consists of simple formulas that closely match the photometric behaviour during disc build-up and dissipation.

An initial analysis of our model grid allowed us to draw important conclusions about the properties of viscous decretion discs (VDD) around Be stars, and their effect on the stellar SED as they evolve in time:

- The viscosity parameter $\alpha$ is the most important parameter controlling the observed rate of photometric variations in Be light curves, but it is not the only one. Stellar parameters (mass, radius and effective temperature), as well as the disc viewing angle and density level, all concur to change the rate of brightness variations in complicated ways, which means that if meaningful information about $\alpha$ is to be extracted from the data, these parameters must be somehow estimated;

- We identified a previously unknown effect, dubbed massreservoir effect, which also controls the rate of photometric variations during phases of disc dissipation. This effect is a consequence of the fact that VDDs build a mass reservoir at their outer regions, which is unnoticed at short wavelengths (e.g., visible). The longer the build-up phase of a disc, the bigger its mass reservoir. When mass injection from the star stops and reaccretion occurs, the reservoir feeds the inner disc with mass. Clearly, the larger the reservoir, the longer it will be able to supply mass to the inner disc, and the slower the photometric dissipation will appear.

We applied our fitting pipeline to a sample of light curves of 54 Be star candidates from the SMC (Mennickent et al. 2002) containing 81 clearly identified events of disc formation/dissipation (here referred to as bumps). The light curves come from OGLE-II and OGLE-III data, covering roughly 12 years. A Markov Chain Monte Carlo technique was used to properly estimate the posterior probabilities of each fitted parameter.

It was found that our sample is biased towards early type Be stars, likely because these stars are more variable and their discs are denser, resulting in clearer bumps. Also, photometric uncertainties increase for late type Be stars. Since our sample was selected based on the appearance of their bumps, we conclude that our Be discs must be among the densest found for Be stars in the SMC. We verified an increase of $\Sigma_{0}$ with the stellar mass and the median for our whole sample is $\left\langle\Sigma_{0}\right\rangle=1.44_{-0.75}^{+1.01} \mathrm{~g} \mathrm{~cm}^{-2}$. Our results may suggest that Be discs in the SMC are denser than their siblings in the Galaxy, in line with $\mathrm{H} \alpha$ surveys that found stronger line emission in the SMC than in our Galaxy.

The durations of the build-up phases become shorter for more massive stars, which indicates that, as it was already observed in the Galaxy, late-type Be stars are less variable than early-type ones. The median of the build-up time for our sample is $\left\langle t_{2}-t_{1}\right\rangle=305_{-171}^{+351}$ days.

We obtained, for the first time, estimates of $\alpha$ for a statistically significant sample of Be stars. In our work, we explored the possibility that the viscosity parameter might be different at build-up $\left(\alpha_{\mathrm{bu}}\right)$ and dissipation $\left(\alpha_{\mathrm{d}}\right)$. We found no significant variation of $\alpha_{\mathrm{bu}}$ with the stellar mass (Fig. 13, top), but some evidence points to a correlation between $\alpha_{\mathrm{d}}$ with $M$ (Fig. 13, bottom). Furthermore, we find that on average the viscosity parameter is sensibly larger at build-up than at dissipation. Our medians of the two viscosity parameters are $\left\langle\alpha_{\text {bu }}\right\rangle=0.63_{-0.39}^{+0.52}$ and $\left\langle\alpha_{\mathrm{d}}\right\rangle=0.29_{-0.20}^{+0.61}$. These values are in agreement in magnitude with the determinations of Carciofi et al. (2012) and Ghoreyshi \& Carciofi (2017) for the Galactic Be star $28 \mathrm{CMa}$. They are also similar to the values of $\alpha$ usually found in cataclysmic variables (King et al. 2007; Kotko \& Lasota 2012).

The trend that $\alpha_{\mathrm{bu}}>\alpha_{\mathrm{d}}$ was also seen by Ghoreyshi \& Carciofi (2017) in the different cycles of activity of the Be star 28 CMa. Further work is necessary to establish whether this trend is real or simply a result of our model assumptions. In particular, two important physical effects were ignored in this work, namely the fact that Be discs are non-isothermal and the line forces known to act on the disc material. This last point, however, is likely of little importance for Be stars in the SMC, given their low metallicity.

It must be further emphasised that in this work what is really measured are the timescales for disc build-up and dissipation. Under the assumption that viscosity is the only driving mechanism operating on the disc, these timescales can in turn be converted to estimates of the viscosity parameter. The presence of other driving mechanisms (such as the aforementioned ablation) might affect the determination of $\alpha$ in unpredictable ways.

The steady-state mass and angular momentum loss rates for the studied bumps are of the order of $\sim 10^{-10} M_{\odot} \mathrm{yr}^{-1}$ and $\sim 5 \times$ $10^{36} \mathrm{~g} \mathrm{~cm}^{2} \mathrm{~s}^{-2}$, respectively. The typical decretion rate is of the order of $\sim 10^{-9} M_{\odot} \mathrm{yr}^{-1}$. These values are in agreement with the upper limit of the observed wind mass loss rate of B stars (Snow 1981; Puls et al. 2008). In addition, these values roughly agree in magnitude with the work of Vieira et al. (2017), who studied a sample of 80 Galactic Be stars.

Future perspectives for this work are threefold. First, an effort must be made to remove the biases of our current sample, by including late-type Be stars and smaller-amplitude bumps. Second, the availability of a great number of past and current automated surveys (e.g., MACHO - Alcock et al. 1997, EROS - Aubourg et al. 1993, 
ASAS - Pojmanski 1997, VISTA-VVV - Minniti et al. 2010, KELT - Pepper et al. 2007) will allow us not only to greatly increase the number of Be stars studied, but also to explore the Be phenomenon and associated disc properties in other metallicities (Galaxy, LMC, etc.). Finally, also important is to obtain a better estimate of the central star properties, e.g., via spectroscopic modelling or using stars belonging to clusters with known age. Our current analysis was bound by the limited amount of information available on the central stars.

The $\alpha$ determinations made in this work should help investigating the physical mechanisms originating the anomalous viscosity in circumstellar discs environments. In addition, the estimates of the net mass and angular momentum loss rates are important for understanding the conditions in which the Be phenomenon appears, and its consequences for the evolution of B-type stars. 
Table 5. List of Be stars and their respective bumps selected for this study

\begin{tabular}{|c|c|c|c|c|c|c|c|c|c|c|c|c|}
\hline \multirow{2}{*}{$\begin{array}{r}\text { Field } \\
\text { SMC_SC1 }\end{array}$} & \multirow{2}{*}{$\begin{array}{r}\text { OGLE-II } \\
\text { ID } \\
7612\end{array}$} & \multicolumn{2}{|c|}{\begin{tabular}{l}
\multicolumn{2}{r}{ OGLE-III } \\
Field $\quad$ ID
\end{tabular}} & \multicolumn{2}{|c|}{$\begin{array}{r}\text { diskless interval } \\
(\mathrm{JD}-2450000)\end{array}$} & \multirow{2}{*}{$\begin{array}{r}B_{*} \\
0.01\end{array}$} & \multirow{2}{*}{$\begin{array}{r}V_{*} \\
16.164 \pm 0.009\end{array}$} & \multirow{2}{*}{$\begin{array}{r}I_{*} \\
16.443 \pm 0.009\end{array}$} & \multirow{2}{*}{$\begin{array}{r}\text { Bump } \\
\text { ID } \\
01 \\
02\end{array}$} & \multirow{2}{*}{$\begin{array}{r}\text { Bands } \\
I \\
I\end{array}$} & \multicolumn{2}{|c|}{$\begin{array}{l}\text { Bump interval } \\
\text { (JD-2450000) }\end{array}$} \\
\hline & & SMC133.4 & 8877 & 600 & 1300 & & & & & & $\begin{array}{l}1420 \\
3250\end{array}$ & $\begin{array}{l}2000 \\
3800\end{array}$ \\
\hline SMC_SC1 & 60553 & SMC128.6 & 57 & 3000 & 3500 & - & $15.418 \pm 0.003$ & $15.601 \pm 0.007$ & 01 & $V I$ & 3500 & 5000 \\
\hline SMC_SC1 & 75701 & SMC125.7 & 20383 & 3000 & 3500 & - & $15.397 \pm 0.003$ & $15.51 \pm 0.006$ & 01 & $V I$ & 3650 & 5000 \\
\hline SMC_SC1 & 92262 & SMC128.6 & 147 & 3500 & 3800 & - & $15.623 \pm 0.003$ & $15.811 \pm 0.007$ & $\begin{array}{l}01 \\
02\end{array}$ & $\begin{array}{r}I \\
V I\end{array}$ & $\begin{array}{l}2600 \\
3900\end{array}$ & $\begin{array}{l}3100 \\
5000\end{array}$ \\
\hline SMC_SC2 & 94939 & SMC125.3 & 52 & 1000 & 1100 & $15.832 \pm 0.008$ & $15.991 \pm 0.013$ & $16.126 \pm 0.007$ & 01 & $I$ & 1100 & 2000 \\
\hline SMC_SC3 & 5719 & SMC125.1 & 20231 & 2980 & 3020 & - & - & $16.233 \pm 0.01$ & 01 & $I$ & 1200 & 4500 \\
\hline SMC_SC3 & 15970 & SMC125.2 & 28056 & 700 & 750 & $15.282 \pm 0.01$ & $15.412 \pm 0.006$ & $15.541 \pm 0.006$ & $\begin{array}{l}01 \\
02 \\
03\end{array}$ & $\begin{array}{r}B V I \\
I \\
V I\end{array}$ & $\begin{array}{c}750 \\
2200 \\
4000\end{array}$ & $\begin{array}{l}2000 \\
3500 \\
5000\end{array}$ \\
\hline SMC_SC3 & 71445 & SMC125.2 & 34818 & 700 & 800 & $16.227 \pm 0.01$ & $16.425 \pm 0.009$ & $16.608 \pm 0.012$ & $\begin{array}{l}01 \\
02\end{array}$ & $\begin{array}{l}I \\
I\end{array}$ & $\begin{array}{l}1450 \\
4400\end{array}$ & $\begin{array}{l}2400 \\
4700\end{array}$ \\
\hline SMC_SC3 & 125899 & SMC125.2 & 6200 & 3500 & 3750 & - & $15.837 \pm 0.004$ & $15.956 \pm 0.008$ & 01 & $I$ & 2320 & 3400 \\
\hline SMC_SC3 & 197941 & SMC125.3 & 25034 & 4000 & 4060 & - & $15.671 \pm 0.003$ & $15.724 \pm 0.006$ & 01 & $V I$ & 2350 & 4000 \\
\hline SMC_SC4 & 22859 & SMC125.4 & 22723 & 700 & 1200 & $17.068 \pm 0.013$ & $17.133 \pm 0.012$ & $17.129 \pm 0.015$ & 01 & $V I$ & 1200 & 3500 \\
\hline SMC_SC4 & 71499 & SMC100.7 & 34896 & 1000 & 1450 & $15.414 \pm 0.011$ & $15.553 \pm 0.007$ & $15.597 \pm 0.006$ & $\begin{array}{l}01 \\
02\end{array}$ & $\begin{array}{ll}V & I \\
V & I\end{array}$ & $\begin{array}{l}1430 \\
2900\end{array}$ & $\begin{array}{l}2400 \\
4400\end{array}$ \\
\hline SMC_SC4 & 120783 & SMC100.6 & 7129 & 4250 & 4500 & - & $14.401 \pm 0.003$ & $14.442 \pm 0.005$ & 01 & $V I$ & 2750 & 4500 \\
\hline SMC_SC4 & 127840 & SMC100.6 & 38372 & 3500 & 4500 & - & $14.853 \pm 0.003$ & $15.026 \pm 0.006$ & 01 & $I$ & 2800 & 3500 \\
\hline SMC_SC4 & 156248 & SMC100.8 & 14683 & 1000 & 1200 & $15.91 \pm 0.01$ & $15.964 \pm 0.007$ & $15.852 \pm 0.006$ & 01 & $I$ & 1320 & 2300 \\
\hline SMC_SC4 & 156251 & SMC100.8 & 14642 & 600 & 680 & $14.881 \pm 0.005$ & $15.13 \pm 0.007$ & $15.338 \pm 0.005$ & $\begin{array}{l}01 \\
02 \\
03\end{array}$ & $\begin{array}{r}B V I \\
V I \\
V I\end{array}$ & $\begin{array}{c}650 \\
3170 \\
3770\end{array}$ & $\begin{array}{l}1200 \\
3600 \\
4600\end{array}$ \\
\hline SMC_SC4 & 159829 & SMC100.8 & 37214 & 4450 & 4500 & - & $15.895 \pm 0.005$ & $15.959 \pm 0.009$ & $\begin{array}{l}01 \\
02\end{array}$ & $\begin{array}{r}I \\
V I\end{array}$ & $\begin{array}{l}2600 \\
3430\end{array}$ & $\begin{array}{l}3000 \\
4500\end{array}$ \\
\hline SMC_SC4 & 159857 & SMC100.8 & 45127 & 3000 & 3500 & - & $15.626 \pm 0.004$ & $15.8 \pm 0.008$ & $\begin{array}{l}01 \\
02\end{array}$ & $\begin{array}{r}I \\
V I\end{array}$ & $\begin{array}{c}780 \\
3600\end{array}$ & $\begin{array}{l}1400 \\
4300\end{array}$ \\
\hline SMC_SC4 & 163828 & SMC100.7 & 8813 & 600 & 700 & $17.171 \pm 0.009$ & $17.164 \pm 0.009$ & $16.953 \pm 0.013$ & 01 & $B V I$ & 700 & 2400 \\
\hline SMC_SC4 & 167554 & SMC100.7 & 51098 & 3000 & 4500 & - & $17.258 \pm 0.009$ & $17.2 \pm 0.016$ & $\begin{array}{l}01 \\
02\end{array}$ & $\begin{array}{r}I \\
V I\end{array}$ & $\begin{array}{l}1620 \\
4700\end{array}$ & $\begin{array}{l}1900 \\
5000\end{array}$ \\
\hline
\end{tabular}


Table 5 - continued List of Be stars and their respective bumps selected for this study

\begin{tabular}{|c|c|c|c|c|c|c|c|c|c|c|c|c|}
\hline \multirow{2}{*}{$\begin{array}{r}\text { Field } \\
\text { SMC_SC4 }\end{array}$} & \multirow{2}{*}{$\begin{array}{r}\begin{array}{r}\text { OGLE-II } \\
\text { ID }\end{array} \\
171253\end{array}$} & \multicolumn{2}{|c|}{ OGLE-III } & \multicolumn{2}{|c|}{$\begin{array}{r}\text { diskless interval } \\
\text { (JD-2450000) }\end{array}$} & $B_{*}$ & $V_{*}$ & $I_{*}$ & & Bands & \multicolumn{2}{|c|}{$\begin{array}{l}\text { Bump interval } \\
\text { (JD-2450000) }\end{array}$} \\
\hline & & SMC100.7 & 42620 & 700 & 1200 & $15.674 \pm 0.01$ & $15.714 \pm 0.009$ & $15.691 \pm 0.006$ & $\begin{array}{l}01 \\
02\end{array}$ & $\begin{array}{r}I \\
V I\end{array}$ & $\begin{array}{l}1410 \\
3245\end{array}$ & $\begin{array}{l}1900 \\
4000\end{array}$ \\
\hline SMC_SC4 & 175272 & SMC100.6 & 7362 & 3950 & 4000 & - & $16.579 \pm 0.005$ & $16.431 \pm 0.009$ & 01 & $I$ & 2600 & 3800 \\
\hline SMC_SC4 & 179053 & SMC100.6 & 38443 & 0 & 1000 & $16.304 \pm 0.009$ & $16.339 \pm 0.009$ & $16.148 \pm 0.008$ & 01 & I & 1300 & 2400 \\
\hline SMC_SC5 & 11453 & SMC100.8 & 14734 & 3300 & 3400 & - & $15.871 \pm 0.004$ & $15.779 \pm 0.008$ & 01 & $I$ & 3400 & 5000 \\
\hline SMC_SC5 & 21117 & SMC100.8 & 52883 & 3650 & 3750 & - & $16.051 \pm 0.004$ & $16.143 \pm 0.009$ & $\begin{array}{l}01 \\
02 \\
03 \\
04\end{array}$ & $\begin{array}{r}V I \\
I \\
I \\
V I \\
V I\end{array}$ & $\begin{array}{c}970 \\
2200 \\
2900 \\
3850\end{array}$ & $\begin{array}{l}2000 \\
2800 \\
3400 \\
5000\end{array}$ \\
\hline SMC_SC5 & 21134 & SMC100.8 & 45175 & 1000 & 1500 & $15.994 \pm 0.013$ & $16.023 \pm 0.006$ & $16.091 \pm 0.007$ & 01 & $I$ & 1600 & 2000 \\
\hline SMC_SC5 & 32377 & SMC100.7 & 50838 & 4050 & 4100 & - & $15.844 \pm 0.003$ & $15.941 \pm 0.008$ & $\begin{array}{l}01 \\
02\end{array}$ & $\begin{array}{l}I \\
I\end{array}$ & $\begin{array}{l}3030 \\
4350\end{array}$ & $\begin{array}{l}3400 \\
5000\end{array}$ \\
\hline SMC_SC5 & 43650 & SMC100.6 & 15248 & 1400 & 2000 & $17.177 \pm 0.013$ & $17.214 \pm 0.01$ & $17.305 \pm 0.017$ & $\begin{array}{l}01 \\
02\end{array}$ & $\begin{array}{l}V I \\
V I\end{array}$ & $\begin{array}{c}750 \\
2550\end{array}$ & $\begin{array}{l}2000 \\
5000\end{array}$ \\
\hline SMC_SC5 & 54851 & SMC100.5 & 14725 & 600 & 850 & $16.264 \pm 0.01$ & $16.311 \pm 0.009$ & $16.366 \pm 0.008$ & $\begin{array}{l}01 \\
02 \\
03\end{array}$ & $\begin{array}{r}B V I \\
I \\
V I\end{array}$ & $\begin{array}{c}850 \\
2120 \\
3650\end{array}$ & $\begin{array}{l}2000 \\
3600 \\
5000\end{array}$ \\
\hline SMC_SC5 & 65500 & SMC101.8 & 21127 & 1000 & 1500 & $16.034 \pm 0.011$ & $15.981 \pm 0.007$ & $15.959 \pm 0.006$ & 01 & $B V I$ & 600 & 1000 \\
\hline SMC_SC5 & 129535 & SMC100.6 & 53957 & 4700 & 4800 & - & $16.923 \pm 0.008$ & $16.953 \pm 0.013$ & 01 & $V I$ & 3300 & 5000 \\
\hline SMC_SC5 & 145724 & SMC101.8 & 21370 & 3000 & 3200 & - & - & $17.116 \pm 0.019$ & 01 & $I$ & 3230 & 5000 \\
\hline SMC_SC5 & 180034 & SMC100.1 & 27826 & 3900 & 4100 & - & $16.436 \pm 0.005$ & $16.431 \pm 0.009$ & 01 & $V I$ & 4120 & 5000 \\
\hline SMC_SC5 & 260841 & SMC100.1 & 36050 & 800 & 900 & $15.858 \pm 0.011$ & $16.013 \pm 0.008$ & $16.182 \pm 0.008$ & $\begin{array}{l}01 \\
02\end{array}$ & $\begin{array}{r}I \\
V I\end{array}$ & $\begin{array}{l}1500 \\
3800\end{array}$ & $\begin{array}{l}2200 \\
4800\end{array}$ \\
\hline SMC_SC5 & 260957 & SMC100.1 & 36101 & 1200 & 1700 & $16.747 \pm 0.009$ & $16.917 \pm 0.009$ & $17.043 \pm 0.015$ & 01 & $V I$ & 3620 & 5000 \\
\hline SMC_SC5 & 266088 & SMC100.2 & 9240 & 700 & 750 & $17.227 \pm 0.012$ & $17.315 \pm 0.01$ & $17.408 \pm 0.016$ & 01 & $B V I$ & 750 & 2000 \\
\hline SMC_SC5 & 276982 & SMC100.3 & 9403 & 4400 & 4500 & - & $15.993 \pm 0.005$ & $15.757 \pm 0.008$ & 01 & $V I$ & 3030 & 4500 \\
\hline SMC_SC5 & 282963 & SMC100.3 & 9408 & 1000 & 1500 & $15.431 \pm 0.009$ & $15.591 \pm 0.006$ & $15.665 \pm 0.005$ & 01 & $I$ & 1600 & 4300 \\
\hline SMC_SC6 & 11085 & SMC100.1 & 36096 & 700 & 1100 & $15.52 \pm 0.01$ & $15.667 \pm 0.007$ & $15.863 \pm 0.006$ & $\begin{array}{l}01 \\
02\end{array}$ & $\begin{array}{l}V I \\
V I \\
V I\end{array}$ & $\begin{array}{l}1400 \\
3900\end{array}$ & $\begin{array}{l}2000 \\
5000\end{array}$ \\
\hline SMC_SC6 & 17538 & SMC100.2 & 9240 & 0 & 750 & $17.213 \pm 0.012$ & $17.315 \pm 0.013$ & $17.424 \pm 0.015$ & 01 & $V I$ & 800 & 3000 \\
\hline SMC_SC6 & 42440 & SMC100.3 & 56046 & 700 & 1100 & $16.867 \pm 0.012$ & $17.01 \pm 0.011$ & $17.19 \pm 0.017$ & 01 & I & 1400 & 3500 \\
\hline
\end{tabular}


Table 5 - continued List of Be stars and their respective bumps selected for this study

\begin{tabular}{|c|c|c|c|c|c|c|c|c|c|c|c|c|}
\hline \multirow{2}{*}{$\begin{array}{r}\text { Field } \\
\text { SMC_SC6 }\end{array}$} & \multirow{2}{*}{$\begin{array}{r}\text { OGLE-II } \\
\text { ID } \\
99991\end{array}$} & \multicolumn{2}{|c|}{\begin{tabular}{rr}
\multicolumn{2}{r}{ OGLE-III } \\
Field & ID
\end{tabular}} & \multicolumn{2}{|c|}{$\begin{array}{r}\text { diskless interval } \\
(\text { JD-2450000) }\end{array}$} & \multirow{2}{*}{$\begin{array}{c}B_{*} \\
.007\end{array}$} & \multirow{2}{*}{$\begin{array}{r}V_{*} \\
.007\end{array}$} & \multirow{2}{*}{$\begin{array}{r}I_{*} \\
16.126 \pm 0.009\end{array}$} & \multirow{2}{*}{$\begin{array}{r}\text { Bump } \\
\text { ID } \\
01 \\
02 \\
03 \\
04\end{array}$} & \multirow{2}{*}{$\begin{array}{r}\text { Bands } \\
I \\
I \\
I \\
I\end{array}$} & \multicolumn{2}{|c|}{$\begin{array}{l}\text { Bump interval } \\
\text { (JD-2450000) }\end{array}$} \\
\hline & & SMC100.1 & 43700 & 1300 & 1800 & & & & & & $\begin{array}{c}650 \\
1800 \\
3150 \\
4200\end{array}$ & $\begin{array}{l}1400 \\
2500 \\
4000 \\
5000\end{array}$ \\
\hline SMC_SC6 & 105368 & SMC100.2 & 17645 & 600 & 1200 & $16.462 \pm 0.011$ & $16.592 \pm 0.008$ & $16.681 \pm 0.011$ & $\begin{array}{l}01 \\
02\end{array}$ & $\begin{array}{r}V I \\
I\end{array}$ & $\begin{array}{l}1150 \\
2700\end{array}$ & $\begin{array}{l}2000 \\
4000\end{array}$ \\
\hline SMC_SC6 & 116294 & SMC100.2 & 49901 & 0 & 900 & $16.775 \pm 0.012$ & $16.958 \pm 0.01$ & $17.085 \pm 0.013$ & 01 & $I$ & 985 & 2000 \\
\hline SMC_SC6 & 128831 & SMC100.3 & 55954 & 600 & 1200 & $15.849 \pm 0.009$ & $16.018 \pm 0.007$ & $16.116 \pm 0.007$ & 01 & $I$ & 1445 & 2600 \\
\hline SMC_SC6 & 199611 & SMC100.3 & 29080 & 600 & 1300 & $15.265 \pm 0.011$ & $15.447 \pm 0.008$ & $15.594 \pm 0.006$ & 01 & $I$ & 1500 & 2000 \\
\hline SMC_SC6 & 272665 & SMC106.6 & 26640 & 1000 & 1500 & $17.784 \pm 0.016$ & $17.962 \pm 0.016$ & $18.04 \pm 0.029$ & 01 & $I$ & 1620 & 4500 \\
\hline SMC_SC7 & 57131 & SMC105.6 & 33029 & 1200 & 2000 & $16.037 \pm 0.015$ & $16.127 \pm 0.008$ & $16.269 \pm 0.008$ & 01 & $I$ & 2780 & 3500 \\
\hline SMC_SC8 & 183240 & SMC105.2 & 32029 & 3000 & 4000 & - & $14.783 \pm 0.003$ & $14.946 \pm 0.005$ & 01 & $V I$ & 4150 & 4800 \\
\hline SMC_SC9 & 105383 & SMC110.6 & 114 & 1000 & 1300 & $16.115 \pm 0.01$ & $16.264 \pm 0.007$ & $16.4 \pm 0.009$ & $\begin{array}{l}01 \\
02\end{array}$ & $\begin{array}{l}V I \\
V I\end{array}$ & $\begin{array}{l}1240 \\
3780\end{array}$ & $\begin{array}{l}3500 \\
4800\end{array}$ \\
\hline SMC_SC9 & 168422 & SMC113.7 & 6330 & 4700 & 4850 & - & $17.002 \pm 0.009$ & $17.057 \pm 0.014$ & 01 & $V I$ & 2700 & 4850 \\
\hline SMC_SC10 & 8906 & SMC110.6 & 22338 & 4500 & 5000 & - & $15.253 \pm 0.003$ & $15.382 \pm 0.006$ & $\begin{array}{l}01 \\
02\end{array}$ & $\begin{aligned} & I \\
& V I\end{aligned}$ & $\begin{array}{l}2935 \\
3650\end{array}$ & $\begin{array}{l}3300 \\
3710\end{array}$ \\
\hline SMC_SC11 & 28090 & SMC113.2 & 4458 & 4500 & 5000 & - & $15.248 \pm 0.004$ & $15.433 \pm 0.006$ & 01 & $V I$ & 2300 & 4500 \\
\hline SMC_SC11 & 46587 & SMC110.3 & 16096 & 600 & 1000 & $17.087 \pm 0.013$ & $17.248 \pm 0.01$ & $17.343 \pm 0.021$ & 01 & $I$ & 1110 & 4500 \\
\hline
\end{tabular}




\begin{tabular}{|c|c|c|c|c|c|c|c|c|c|c|c|c|}
\hline OGLE-II ID & $\begin{array}{r}\text { Bump } \\
\text { ID }\end{array}$ & $M\left[M_{\odot}\right]$ & $t / t_{\mathrm{MS}}$ & $W$ & $\cos i$ & $\begin{array}{r}\Sigma_{0} \\
{\left[\mathrm{~g} \mathrm{~cm}^{-2}\right]}\end{array}$ & $\alpha_{\mathrm{bu}}$ & $\alpha_{\mathrm{d}}$ & $\tilde{\tau}_{\text {bu }}$ & $\begin{array}{r}\left(-\frac{\partial M}{\partial t}\right)_{\text {typ }} \\
{\left[10^{-9} \times\right.} \\
\left.M_{\odot} \mathrm{yr}^{-1}\right]\end{array}$ & $\begin{array}{r}\left(-\frac{\partial J}{\partial t}\right)_{\mathrm{std}} \\
{\left[10^{36} \times\right.} \\
\left.\mathrm{g} \mathrm{cm}^{2} \mathrm{~s}^{-2}\right]\end{array}$ & $\begin{array}{r}-\Delta J_{*} \\
{\left[10^{44} \times\right.} \\
\left.\mathrm{g} \mathrm{cm}^{2} \mathrm{~s}^{-1}\right]\end{array}$ \\
\hline SMC_SC1 7612 & $\begin{array}{l}01 \\
02\end{array}$ & $12.8_{-2.6}^{+2.6}$ & $0.5_{-0.3}^{+0.3}$ & $0.81_{-0.1}^{+0.11}$ & $0.36_{-0.04}^{+0.11}$ & $\begin{array}{l}1.1_{-0.6}^{+1.3} \\
1.3_{-0.7}^{+12.2}\end{array}$ & $\begin{array}{l}0.33_{-0.24}^{+0.68} \\
0.38_{-0.27}^{+0.86}\end{array}$ & $\begin{array}{l}0.65_{-0.41}^{+0.54} \\
0.62_{-0.4}^{+0.53}\end{array}$ & $\begin{array}{l}0.15_{-0.12}^{+0.32} \\
0.15_{-0.1}^{+0.28}\end{array}$ & $\begin{array}{l}0.62_{-0.38}^{+0.65} \\
0.77_{-0.45}^{+8.8}\end{array}$ & $\begin{array}{l}1.09_{-0.68}^{+1.35} \\
1.35_{-0.81}^{+1.66}\end{array}$ & $\begin{array}{l}0.07_{-0.84}^{+0.08} \\
0.07_{-0.04}^{+8.88}\end{array}$ \\
\hline SMC_SC1 60553 & 01 & $12.7_{-1.9}^{+2.8}$ & $0.7_{-0.3}^{+0.2}$ & $0.81_{-0.11}^{+0.1}$ & $0.66_{-0.1}^{+0.12}$ & $1.6_{-0.4}^{+0.7}$ & $1.25_{-0.25}^{+0.18}$ & $0.09_{-0.03}^{+0.04}$ & $2.76_{-0.57}^{+0.5}$ & $4.32_{-1.54}^{+2.2}$ & $8.24_{-3.31}^{+5.62}$ & $2.63_{-1.05}^{+1.73}$ \\
\hline SMC_SC1 75701 & 01 & $14.7_{-2.3}^{+1.7}$ & $0.7_{-0.2}^{+0.2}$ & $0.81_{-0.1}^{+0.1}$ & $0.7_{-0.1}^{+0.15}$ & $1.9_{-0.4}^{+0.8}$ & $0.24_{-0.08}^{+0.18}$ & $0.11_{-0.05}^{+0.08}$ & $0.66_{-0.22}^{+0.48}$ & $1.15_{-0.52}^{+1.06}$ & $2.47_{-1.24}^{+2.62}$ & $1.03_{-0.52}^{+1.08}$ \\
\hline SMC_SC1 92262 & $\begin{array}{l}01 \\
02\end{array}$ & $12.7_{-1.8}^{+2.5}$ & $0.6_{-0.3}^{+0.2}$ & $0.8_{-0.09}^{+0.11}$ & $0.03_{-0.02}^{+0.03}$ & $\begin{array}{l}1.5_{-0.9}^{+1.0} \\
2.4_{-0.5}^{+0.4}\end{array}$ & $\begin{array}{l}0.47_{-0.29}^{+0.6} \\
0.99_{-0.23}^{+0.26}\end{array}$ & $\begin{array}{l}0.84_{-0.38}^{+0.4} \\
0.94_{-0.3}^{+0.35}\end{array}$ & $\begin{array}{r}0.53_{-0.34}^{+0.63} \\
1.2_{-0.37}^{2+38.38}\end{array}$ & $\begin{array}{l}1.07_{-0.56}^{+1.62} \\
4.42_{-1.42}^{2+72.72}\end{array}$ & $\begin{array}{l}2.01_{-1.06}^{+2.83} \\
8.36_{-3.24}^{6+38}\end{array}$ & $\begin{array}{l}0.31_{-0}^{+0.4} \\
1.51_{-0.62}^{+1.06}\end{array}$ \\
\hline SMC_SC2 94939 & 01 & $12.2_{-2.3}^{+2.7}$ & $0.6_{-0.3}^{+0.2}$ & $0.81_{-0.11}^{+0.1}$ & $0.45_{-0.07}^{+0.13}$ & $2.4_{-0.8}^{+0.7}$ & $0.88_{-0.45}^{+0.42}$ & $0.69_{-0.33}^{+0.43}$ & $1.15_{-0.56}^{+0.53}$ & $3.44_{-1.75}^{+2.61}$ & $6.05_{-3.17}^{+5.42}$ & $1.06_{-0.56}^{+0.92}$ \\
\hline SMC_SC3 5719 & 01 & $12.3_{-2.3}^{+2.7}$ & $0.6_{-0.3}^{+0.3}$ & $0.81_{-0.11}^{+0.1}$ & $0.38_{-0.05}^{+0.11}$ & $2.6_{-0.8}^{+0.5}$ & $0.72_{-0.33}^{+0.42}$ & $0.47_{-0.22}^{+0.36}$ & $3.83_{-1.72}^{+2.41}$ & $3.25_{-1.77}^{+2.3}$ & $\begin{array}{l}5.73_{-3.18}^{+4.5} \\
\end{array}$ & $4.09_{-2.27}^{+3.25}$ \\
\hline SMC_SC3 15970 & $\begin{array}{l}01 \\
02 \\
03\end{array}$ & $13.5_{-1.3}^{+1.5}$ & $0.8_{-0.2}^{+0.1}$ & $0.87_{-0.09}^{+0.07}$ & $0.63_{-0.03}^{+0.05}$ & $\begin{array}{l}1.1_{-0.1}^{+0.2} \\
1.4_{-0.2}^{+0.2} \\
1.2_{-0.1}^{+0.2}\end{array}$ & $\begin{array}{c}0.89_{-0.45}^{+0.31} \\
0.85_{-0.31}^{+0: 29} \\
1.1_{-0.3}^{+0.25} \\
\end{array}$ & $\begin{array}{l}0.13_{-0.04}^{+0.05} \\
0.17_{-0.04}^{+0.04} \\
0.12_{-0.05}^{+0.05}\end{array}$ & $\begin{array}{l}1.64_{-0.79}^{+0.67} \\
1.92_{-0.77}^{+0.77} \\
2.62_{-0.9}^{+0.83}\end{array}$ & $\begin{array}{l}2.52_{-1.14}^{+1.15} \\
2.89_{-1.38}^{+1.03} \\
3.27_{-1.12}^{+1.37}\end{array}$ & $\begin{array}{l}5.31_{-2.54}^{+3.1} \\
6.17_{-2}^{+3.61} \\
6.97_{-2.73}^{+3.75}\end{array}$ & $\begin{array}{l}1.55_{-0.74}^{+0.95} \\
2.24_{-0.92}^{+1.22} \\
2.62_{-1.18}^{+1.66}\end{array}$ \\
\hline SMC_SC3 71445 & $\begin{array}{l}01 \\
02 \\
\end{array}$ & $11.2_{-2.3}^{+2.7}$ & $0.5_{-0.3}^{+0.3}$ & $0.81_{-0.1}^{+0.1}$ & $0.43_{-0.06}^{+0.09}$ & $\begin{array}{l}2.5_{-0.6}^{+0.6} \\
1.4_{-0.5}^{+0.7} \\
\end{array}$ & $\begin{array}{l}0.57_{-0.25}^{+0.41} \\
0.65_{-0.34}^{+0.53} \\
\end{array}$ & $\begin{array}{l}0.75_{-0.31}^{+0.37} \\
1.05_{-0.38}^{+0.31} \\
\end{array}$ & $\begin{array}{l}1.11_{-0.48}^{+0.8} \\
0.47_{-0.24}^{+0.41} \\
\end{array}$ & $\begin{array}{l}2.14_{-1.0}^{+1.74} \\
1.38_{-0.61}^{+0.91} \\
\end{array}$ & $\begin{array}{r}3.42_{-1}^{+3.2} \\
2.19_{-1.02}^{+1.74} \\
\end{array}$ & $\begin{array}{r}0.83_{-0.41}^{+0.77} \\
0.2_{-0.09}^{+0.117} \\
\end{array}$ \\
\hline SMC_SC3 125899 & 01 & $13.4_{-2.4}^{+2.3}$ & $0.6_{-0.3}^{+0.2}$ & $0.82_{-0.11}^{+0.1}$ & $0.37_{-0.06}^{+0.17}$ & $1.7_{-0.7}^{+0.9}$ & $0.7_{-0.44}^{+0.54}$ & $0.41_{-0.22}^{+0.33}$ & $0.89_{-0.53}^{+0.7}$ & $2.14_{-1.32}^{+2.11}$ & $4.04_{-2.55}^{+4.74}$ & $0.73_{-0.46}^{+0.88}$ \\
\hline SMC_SC3 197941 & 01 & $12.0_{-1.8}^{+2.5}$ & $0.8_{-0.2}^{+0.2}$ & $0.82_{-0.11}^{+0.1}$ & $0.57_{-0.05}^{+0.15}$ & $0.8_{-0.2}^{+0.3}$ & $0.4_{-0.23}^{+0.52}$ & $0.1_{-0.04}^{+0.04}$ & $1.43_{-0.81}^{+1.79}$ & $0.69_{-0.38}^{+0.82}$ & $1.3_{-0.75}^{+1.69}$ & $0.66_{-0.38}^{+0.85}$ \\
\hline SMC_SC4 22859 & 01 & $9.5_{-2.1}^{+2.5}$ & $0.4_{-0.3}^{+0.3}$ & $0.81_{-0.11}^{+0.1}$ & $0.64_{-0.1}^{+0.19}$ & $0.4_{-0.1}^{+0.2}$ & $0.66_{-0.51}^{+0.57}$ & $0.04_{-0.02}^{+0.64}$ & $3.5_{-2.71}^{+3.15}$ & $0.27_{-0.19}^{+0.26}$ & $0.35_{-0.25}^{+0.42}$ & $0.21_{-0.15}^{+0.25}$ \\
\hline SMC_SC4 71499 & $\begin{array}{l}01 \\
02\end{array}$ & $14.5_{-2.2}^{+1.8}$ & $0.7_{-0.2}^{+0.2}$ & $0.82_{-0.09}^{+0.1}$ & $0.54_{-0.03}^{+0.07}$ & $\begin{array}{l}1.3_{-0.3}^{+0.5} \\
1.5_{-0.3}^{+0.5}\end{array}$ & $\begin{array}{r}0.7_{-0.35}^{+0.46} \\
0.64_{-0.34}^{+0.447}\end{array}$ & $\begin{array}{l}0.25_{-0.08}^{+0.11} \\
0.19_{-0.06}^{+0.06}\end{array}$ & $\begin{array}{l}0.96_{-0.47}^{+0.66} \\
1.16_{-0.63}^{+0.83}\end{array}$ & $\begin{array}{l}2.15_{-0.94}^{+1.3} \\
2.18_{-1.0}^{+1.97}\end{array}$ & $\begin{array}{r}4.6_{-2.26}^{+3.3} \\
4.59_{-2.23}^{+4.38}\end{array}$ & $\begin{array}{l}0.94_{-0.46}^{+0.67} \\
1.26_{-0.61}^{+1.19}\end{array}$ \\
\hline SMC_SC4 120783 & 01 & $15.2_{-1.8}^{+1.2}$ & $0.9_{-0.1}^{+0.1}$ & $0.84_{-0.1}^{+0.09}$ & $0.64_{-0.11}^{+0.2}$ & $0.7_{-0.2}^{+0.3}$ & $0.63_{-0.43}^{+0.54}$ & $0.11_{-0.05}^{+0.06}$ & $1.56_{-1.06}^{+1.33}$ & $1.47_{-0.93}^{+1.37}$ & $3.67_{-2.41}^{+3.63}$ & $1.53_{-1.01}^{+1.46}$ \\
\hline SMC_SC4 127840 & 01 & $14.3_{-1.8}^{+1.7}$ & $0.9_{-0.2}^{+0.1}$ & $0.83_{-0.11}^{+0.09}$ & $0.6_{-0.09}^{+0.15}$ & $2.6_{-0.6}^{+0.5}$ & $0.4_{-0.15}^{+0.23}$ & $0.85_{-0.41}^{+0.4}$ & $0.54_{-0.19}^{+0.3}$ & $3.19_{-1.53}^{+2.4}$ & $7.35_{-3.72}^{+6.28}$ & $1.6_{-0.8}^{+1.3}$ \\
\hline SMC_SC4 156248 & 01 & $12.3_{-1.9}^{+2.8}$ & $0.7_{-0.3}^{+0.2}$ & $0.81_{-0.1}^{+0.09}$ & $0.07_{-0.04}^{+0.02}$ & $1.3_{-0.9}^{+1.4}$ & $0.5_{-0.34}^{+0.73}$ & $0.78_{-0.41}^{+0.43}$ & $1.44_{-1.0}^{+1.98}$ & $0.83_{-0.56}^{+3.31}$ & $1.6_{-1.07}^{+6.2}$ & $0.63_{-0.43}^{+2.39}$ \\
\hline SMC_SC4 156251 & $\begin{array}{l}01 \\
02 \\
03\end{array}$ & $15.3_{-1.7}^{+1.2}$ & $0.7_{-0.2}^{+0.1}$ & $0.83_{-0.1}^{+0.1}$ & $0.76_{-0.07}^{+0.08}$ & $\begin{array}{l}2.2_{-0.4}^{+0.4} \\
1.5_{-0.2}^{+0.2} \\
2.5_{-0.4}^{+0.4}\end{array}$ & $\begin{array}{r}0.4_{-0.11}^{+0.16} \\
0.65_{-0.18}^{+0.29} \\
0.8_{-0.33}^{+0.42} \\
\end{array}$ & $\begin{array}{c}0.78_{-0.33}^{+0.4} \\
0.61_{-0.35}^{+0.35} \\
0.49_{-0.13}^{+0.18} \\
\end{array}$ & $\begin{array}{c}0.37_{-0.14}^{+0.14} \\
0.62_{-0.25}^{+0.25} \\
0.96_{-0.36}^{+0.49} \\
\end{array}$ & $\begin{array}{r}2.52_{-0.91}^{+1.17} \\
2.7_{-0.46}^{+1.48} \\
5.27_{-2.16}^{+2.84} \\
\end{array}$ & $\begin{array}{c}5.83_{-2.42}^{+3.14} \\
6.27_{-2.6}^{+3.88} \\
11.98_{-5.13}^{+7.6} \\
{ }^{+7}\end{array}$ & $\begin{array}{r}0.86_{-0.37}^{+0.48} \\
0.94_{-0.39}^{+0.37} \\
2.31_{-0.92}^{+1.35} \\
\end{array}$ \\
\hline SMC_SC4 159829 & $\begin{array}{l}01 \\
02\end{array}$ & $13.1_{-2.3}^{+2.6}$ & $0.6_{-0.3}^{+0.3}$ & $0.8_{-0.1}^{+0.11}$ & $0.61_{-0.07}^{+0.1}$ & $\begin{array}{l}2.0_{-0.5}^{+0.7} \\
2.1_{-0.4}^{+0.6}\end{array}$ & $\begin{array}{l}1.07_{-0.34}^{+0.28} \\
0.96_{-0.36}^{+0.363}\end{array}$ & $\begin{array}{l}0.25_{-0.08}^{+0.1} \\
0.67_{-0.24}^{+0.31}\end{array}$ & $\begin{array}{l}1.47_{-0.48}^{+0.48} \\
2.26_{-0.77}^{+0.84}\end{array}$ & $\begin{array}{l}3.91_{-1.44}^{+1.91} \\
3.64_{-1.56}^{+1.99}\end{array}$ & $\begin{array}{l}7.21_{-2.97}^{+4.56} \\
6.74_{-3.15}^{+4.54}\end{array}$ & $\begin{array}{l}1.37_{-0.56}^{+0.84} \\
2.18_{-1.0}^{+1.49}\end{array}$ \\
\hline SMC_SC4 159857 & $\begin{array}{l}01 \\
02\end{array}$ & $13.7_{-2.3}^{+1.9}$ & $0.7_{-0.3}^{+0.2}$ & $0.81_{-0.1}^{+0.1}$ & $0.55_{-0.03}^{+0.08}$ & $\begin{array}{l}0.6_{-0.2}^{+1.0} \\
1.0_{-0.2}^{+0.6}\end{array}$ & $\begin{array}{r}0.42_{-0.32}^{+0.64} \\
0.7_{-0.43}^{+0.48}\end{array}$ & $\begin{array}{l}0.66_{-0.35}^{+0.46} \\
0.34_{-0.16}^{+0.56}\end{array}$ & $\begin{array}{l}0.28_{-0.22}^{+0.45} \\
0.42_{-0.27}^{+0.28}\end{array}$ & $\begin{array}{l}0.62_{-0.36}^{+0.5} \\
1.38_{-0.65}^{+0.94}\end{array}$ & $\begin{array}{r}1.22_{-0.75}^{+1.15} \\
2.7_{-1.36}^{+2.24}\end{array}$ & $\begin{array}{l}0.12_{-0.07}^{+0.11} \\
0.22_{-0.11}^{+0.19}\end{array}$ \\
\hline SMC_SC4 163828 & 01 & $9.7_{-2.0}^{+2.4}$ & $0.4_{-0.3}^{+0.4}$ & $0.79_{-0.1}^{+0.11}$ & $0.71_{-0.08}^{+0.14}$ & $0.7_{-0.1}^{+0.2}$ & $0.53_{-0.33}^{+0.58}$ & $0.06_{-0.02}^{+0.05}$ & $3.42_{-2.13}^{+3.83}$ & $0.43_{-0.25}^{+0.5}$ & $0.59_{-0.36}^{+0.78}$ & $0.44_{-0.26}^{+0.58}$ \\
\hline SMC_SC4 167554 & $\begin{array}{l}01 \\
02\end{array}$ & $9.9_{-2.1}^{+2.4}$ & $0.4_{-0.2}^{+0.3}$ & $0.82_{-0.1}^{+0.1}$ & $0.63_{-0.09}^{+0.12}$ & $\begin{array}{l}0.9_{-0.3}^{+0.7} \\
1.6_{-0.5}^{+0.7}\end{array}$ & $\begin{array}{l}0.54_{-0.36}^{+0.51} \\
0.42_{-0.21}^{+0.474}\end{array}$ & $\begin{array}{r}0.6_{-0.29}^{+0.45} \\
0.86_{-0.37}^{+0.41}\end{array}$ & $\begin{array}{r}0.45_{-0.3}^{+0.42} \\
0.42_{-0.2}^{+0.5}\end{array}$ & $\begin{array}{l}0.59_{-0.31}^{+0.46} \\
0.86_{-0.41}^{+0.82}\end{array}$ & $\begin{array}{l}0.82_{-0.46}^{+0.76} \\
1.19_{-0.61}^{+1.44}\end{array}$ & $\begin{array}{l}0.08_{-0.04}^{+0.08} \\
0.14_{-0.07}^{+0.15}\end{array}$ \\
\hline
\end{tabular}


Table 6 - continued Results of the pipeline for each star and bump of the sample

\begin{tabular}{|c|c|c|c|c|c|c|c|c|c|c|c|c|}
\hline OGLE-II ID & $\begin{array}{r}\text { ump } \\
\text { ID }\end{array}$ & {$\left[M_{\odot}\right]$} & $t / t_{\mathrm{MS}}$ & $W$ & $\cos i$ & $\begin{array}{r}\Sigma_{0} \\
{\left[\mathrm{~g} \mathrm{~cm}^{-2}\right]}\end{array}$ & $\alpha_{\text {bu }}$ & $\alpha_{\mathrm{d}}$ & $\tilde{\tau}_{\text {bu }}$ & $\begin{array}{r}\left(-\frac{\partial M}{\partial t}\right)_{\text {typ }} \\
{\left[10^{-9} \times\right.} \\
\left.M_{\odot} \mathrm{yr}^{-1}\right]\end{array}$ & $\begin{array}{r}\left(-\frac{\partial J}{\partial t}\right)_{\text {std }} \\
{\left[10^{36} \times\right.} \\
\left.\mathrm{g} \mathrm{cm}^{2} \mathrm{~s}^{-2}\right]\end{array}$ & $\begin{array}{r}-\Delta J_{*} \\
{\left[10^{44} \times\right.} \\
\left.\mathrm{g} \mathrm{cm}^{2} \mathrm{~s}^{-1}\right]\end{array}$ \\
\hline 71253 & $\begin{array}{l}01 \\
02 \\
\end{array}$ & $14.3_{-2.5}^{+1.9}$ & $0.6_{-0.3}^{+0.2}$ & $.81_{-0.11}^{+0.11}$ & $58+0.13$ & $\begin{array}{l}0.3_{-0.1}^{+1.2} \\
0.3_{-0.1}^{+0.8} \\
\end{array}$ & $\begin{array}{l}0.29_{-0.28}^{+0.74} \\
0.48_{-0.44}^{+0.66} \\
\end{array}$ & $\begin{array}{l}0.81_{-0.49}^{+0.44} \\
0.64_{-0.42}^{+0.52} \\
\end{array}$ & $\begin{array}{l}24_{-0.23}^{+0.6} \\
31_{-0.29}^{+0.45} \\
\end{array}$ & $\begin{array}{l}0.16_{-0.12}^{+0.3} \\
0.29_{-0.2}^{+0.32}\end{array}$ & $\begin{array}{r}0.32_{-0.23}^{+0.65} \\
0.58_{-0.4}^{+0.7} \\
\end{array}$ & $\begin{array}{l}0.04_{-0.83}^{+0.07} \\
0.05_{-0.04}^{+0.86} \\
\end{array}$ \\
\hline SMC_SC4 175272 & 01 & $12.1_{-2.4}^{+2.8}$ & $0.5_{-0.3}^{+0.3}$ & $0.81_{-0.1}^{+0.1}$ & $88_{-0.06}^{+0.12}$ & $1.8_{-0.7}^{+0.8}$ & $0.75_{-0.39}^{+0.49}$ & $0.48_{-0.23}^{+0.29}$ & $2.31_{-1.21}^{+1.52}$ & $2.07_{-1.16}^{+1.73}$ & $3.52_{-2.05}^{+3.33}$ & $1.37_{-0.8}^{+1.31}$ \\
\hline SMC_SC4 179053 & 01 & $12.6_{-2.3}^{+2.6}$ & $0.6_{-0.4}^{+0.3}$ & $0.8_{-0.11}^{+0.11}$ & $0.02_{-0.02}^{+0.02}$ & $2.1_{-0.6}^{+0.7}$ & $0.96_{-0.52}^{+0.37}$ & $1.1_{-0.32}^{+0.27}$ & $2.14_{-1.16}^{+1.11}$ & $3.1_{-1.32}^{+1.84}$ & $\begin{array}{r}5.5_{-2.58}^{+4.29} \\
\end{array}$ & $1.74_{-0.86}^{+1.19}$ \\
\hline SMC_S & 01 & $3.6_{-2.3}^{+2.2}$ & $0.7_{-0.3}^{+0.2}$ & $0.81_{-0.11}^{+0.11}$ & $.38_{-0.06}^{+0.12}$ & $1.8_{-0.7}^{+0.7}$ & $0.9_{-0.4}^{+0.38}$ & $0.26_{-0.11}^{+0.15}$ & $17_{-1.74}^{+1.82}$ & $3.3_{-1.81}^{+2.78}$ & $6.49_{-3.7}^{+6.33}$ & $4.41_{-2.51}^{+4.36}$ \\
\hline SMC $_{-}$ & $\begin{array}{l}01 \\
02 \\
03 \\
04 \\
\end{array}$ & $13.5_{-1.7}^{+1.9}$ & $0.7_{-0.2}^{+0.1}$ & $0.84_{-0.1}^{+0.09}$ & $0.52^{+0.04}$ & $\begin{array}{l}1.3_{-0.2}^{+0.3} \\
1.8_{-0.4}^{+0.5} \\
1.9_{-0.5}^{+0.6} \\
1.3_{-0.2}^{+0.2} \\
\end{array}$ & $\begin{array}{c}0.5_{-0.17}^{+0.32} \\
0.58_{-0.26}^{+0: 4^{2}} \\
0.57_{-0.19}^{+0.28} \\
0.87_{-0.3}^{+0.36} \\
\end{array}$ & $\begin{array}{l}0.61_{-0.32}^{+0.48} \\
0.17_{-0.05}^{+0.08} \\
0.35_{-0.13}^{+0.23} \\
0.16_{-0.05}^{+0.07} \\
\end{array}$ & $\begin{array}{l}0.64_{-0.33}^{+0.39} \\
0.85_{-0.38}^{+0.53} \\
0.57_{-0.18}^{+0.28} \\
3.11_{-1.03}^{+1.26} \\
\end{array}$ & $\begin{array}{l}1.46_{-0.57}^{+1.04} \\
2.44_{-1.04}^{+1.63} \\
2.53_{-1.01}^{+1.34} \\
2.65_{-1.09}^{+1.36} \\
\end{array}$ & $\begin{array}{l}2.97_{-1.34}^{+2.63} \\
4.99_{-2.39}^{+3.88} \\
5.13_{-2.37}^{+3.52} \\
5.36_{-2.46}^{+3.51} \\
\end{array}$ & $\begin{array}{l}0.56_{-0.38}^{+0.47} \\
1.08_{-0.51}^{+0.78} \\
0.76_{-0.35}^{+0.54} \\
2.83_{-1.29}^{+1.95} \\
\end{array}$ \\
\hline 521134 & 01 & $12.7_{-2.4}^{+2.5}$ & $0.6_{-0.3}^{+0.2}$ & $0.8_{-0.09}^{+0.11}$ & $0.38_{-0.06}^{+0.15}$ & $0.8_{-0.4}^{+1.4}$ & $0.4_{-0.33}^{+0.68}$ & $0.18_{-0.11}^{+0.26}$ & $0.34_{-0.28}^{+0.59}$ & $0.54_{-0.36}^{+0.58}$ & $0.97_{-0.66}^{+1.22}$ & $0.12_{-0.08}^{+0.15}$ \\
\hline SMC_S & $\begin{array}{l}01 \\
02 \\
\end{array}$ & $13.3_{-2.2}^{+2.3}$ & $0.7_{-0.3}^{+0.2}$ & $0.81_{-0.11}^{+0.1}$ & $0.34^{+0.06}$ & $\begin{array}{l}1.9_{-0.7}^{+0.9} \\
2.2_{-0.6}^{+0.8} \\
\end{array}$ & $\begin{array}{l}0.71_{-0.45}^{+0.45} \\
0.51_{-0.24}^{+0.41} \\
\end{array}$ & $\begin{array}{l}0.66_{-0.3}^{+0.48} \\
0.32_{-0.12}^{+0.15} \\
\end{array}$ & $\begin{array}{r}0.3_{-0.17}^{+0.28} \\
0.84_{-0.38}^{+0.69} \\
\end{array}$ & $\begin{array}{l}2.53_{-1.38}^{+2.18} \\
2.31_{-1.12}^{+1.89} \\
\end{array}$ & $\begin{array}{l}4.93_{-2}^{+4.81} \\
4.49_{-2.34}^{+4.36} \\
\end{array}$ & $\begin{array}{l}0.31_{-0.17}^{+0.3} \\
1.08_{-0.56}^{+1.03} \\
\end{array}$ \\
\hline SMC & $\begin{array}{l}01 \\
02 \\
\end{array}$ & $10.7_{-2.6}^{+3.0}$ & $0.4_{-0.3}^{+0.3}$ & $81_{-0.11}^{+0.1}$ & $55^{+0.09}$ & $\begin{array}{l}0.5_{-0.2}^{+1.2} \\
0.6_{-0.2}^{+0.2}\end{array}$ & $\begin{array}{l}0.35_{-0.3}^{+0.75} \\
0.72_{-0.53}^{+0.33} \\
\end{array}$ & $\begin{array}{l}0.39_{-0.3}^{+0.71} \\
0.53_{-0.42}^{+0.67} \\
\end{array}$ & $\begin{array}{l}0.61_{-0.53}^{+1.32} \\
6.35_{-4.65}^{+4.94} \\
\end{array}$ & $\begin{array}{r}0.24_{-0.16}^{+0.33} \\
0.5_{-0.33}^{+0.45} \\
\end{array}$ & $\begin{array}{l}0.35_{-8.23}^{+0.59} \\
0.71_{-0.46}^{+8.83} \\
\end{array}$ & $\begin{array}{l}0.07_{-0.95}^{+0.12} \\
0.74_{-0.47}^{+0.92} \\
\end{array}$ \\
\hline SMC & $\begin{array}{l}01 \\
02 \\
03\end{array}$ & $11.7_{-1.5}^{+1.6}$ & $0.7_{-0.1}^{+0.1}$ & $\begin{array}{l}+0.1 \\
-0.08\end{array}$ & $2+0.04$ & $\begin{array}{l}1.3_{-0.2}^{+0.3} \\
1.3_{-0.2}^{+0.2} \\
1.1_{-0.1}^{+0.2} \\
\end{array}$ & $\begin{array}{c}0.38_{-0}^{+0.35} \\
0.48_{-0.21}^{+0.42} \\
0.51_{-0.2}^{+0.25} \\
\end{array}$ & $\begin{array}{l}0.11_{-0.03}^{+0.04} \\
0.12_{-0.03}^{+0.04} \\
0.05_{-0.01}^{+0.81} \\
\end{array}$ & $\begin{array}{l}0.82_{-0.3}^{+0.73} \\
1.19_{-0.54}^{+0.99} \\
2.11_{-0.8}^{+0.99} \\
\end{array}$ & $\begin{array}{l}1.06_{-0.42}^{+0.78} \\
1.28_{-0.58}^{+1.07} \\
1.12_{-0.49}^{+0.7^{+1}} \\
{ }^{-0.4}\end{array}$ & $\begin{array}{l}1.95_{-0.9}^{+1.69} \\
2.33_{-1.18}^{+2.28} \\
2.04_{-0.97}^{+1.52} \\
\end{array}$ & $\begin{array}{r}0.59_{-0.28}^{+0.5} \\
0.8_{-0.49}^{+0.79} \\
1.19_{-0.57}^{+0.88} \\
\end{array}$ \\
\hline $\mathrm{SMC}_{-}$ & 01 & $12.1_{-2.4}^{+2.9}$ & $0.6_{-0.3}^{+0.2}$ & $0.81_{-0.1}^{+0.1}$ & $71_{-0.08}^{+0.13}$ & $1.7_{-0.4}^{+0.6}$ & $0.7_{-0.34}^{+0.38}$ & $0.26_{-0.1}^{+0.13}$ & $0.44_{-0.19}^{+0.24}$ & $2.07_{-1.02}^{+1.66}$ & $3.7_{-2.05}^{+3.62}$ & $0.32_{-0.17}^{+0.31}$ \\
\hline SMC & 0 & $9.4_{-2.0}^{+2.2}$ & $0.5_{-0.3}^{+0.3}$ & $0.81_{-0.11}^{+0.1}$ & $58_{-0.06}^{+0.13}$ & $0.7_{-0.2}^{+0.2}$ & $0.65_{-0.37}^{+0.52}$ & $0.25_{-0.13}^{+0.36}$ & $3.88_{-2.29}^{+3.02}$ & $0.58_{-0.32}^{+0.54}$ & $0.8_{-0.46}^{+0.86}$ & $0.56_{-0.33}^{+0.6}$ \\
\hline SMC_SC5 145724 & 01 & $9.2_{-1.8}^{+2.1}$ & $0.5_{-0.3}^{+0.3}$ & $0.81_{-0.1}^{+0.1}$ & $0.4_{-0.06}^{+0.13}$ & $2.6_{-1.0}^{+0.7}$ & $0.88_{-0.46}^{+0.4}$ & $0.16_{-0.08}^{+0.19}$ & $3.87_{-1.98}^{+1.89}$ & $2.6_{-1.55}^{+2.03}$ & $3.46_{-2.12}^{+3.23}$ & $1.79_{-1.11}^{+1.7}$ \\
\hline SMC & 0 & $12.6_{-2.6}^{+2.6}$ & $0.4_{-0.3}^{+0.3}$ & $0.8_{-0.1}^{+0.1}$ & $62+0.17$ & $1.0_{-0.3}^{+0.5}$ & $0.4^{+0.65}$ & $22_{-0.09}^{+0.17}$ & $0.97_{-0.57}^{+1.54}$ & $0.68_{-0.4}^{+0.81}$ & $1.17_{-0.71}^{+1.57}$ & $0.36_{-0.22}^{+0.47}$ \\
\hline SMC & $\begin{array}{l}01 \\
02\end{array}$ & $12.7_{-2.0}^{+2.4}$ & $0.6_{-0.3}^{+0.2}$ & $0.83_{-0.1}^{+0.09}$ & $0.54_{-}^{+}$ & $\begin{array}{l}0.6_{-0.1}^{+0.4} \\
0.5_{-0.1}^{+0.2} \\
\end{array}$ & $\begin{array}{r}0.8_{-0.49}^{+0.42} \\
0.78_{-0.41}^{+0.4}\end{array}$ & $\begin{array}{c}0.07_{-0.03}^{+0.58} \\
0.08_{-0.04}^{+0.78} \\
\end{array}$ & $\begin{array}{l}1.42_{-0.93}^{+0.81} \\
2.49_{-1.38}^{+1.47} \\
\end{array}$ & $\begin{array}{l}0.86_{-0.45}^{+0.71} \\
0.78_{-0.41}^{+0.6}\end{array}$ & $\begin{array}{r}1.58_{-0.88}^{+1.61} \\
1.44_{-0.82}^{+1.39} \\
\end{array}$ & $\begin{array}{l}0.38_{-0.22}^{+0.38} \\
0.64_{-0.36}^{+0.64} \\
\end{array}$ \\
\hline SMC_SC5 260957 & 01 & $9.4_{-1.9}^{+2.5}$ & $0.5_{-0.3}^{+0.3}$ & $0.82_{-0.11}^{+0.1}$ & $0.3 \delta_{-0.06}$ & $1.3_{-0.3}^{+0.4}$ & $0.64_{-0.3}^{+0.46}$ & $0.05_{-0.02}^{+0.02}$ & $2.41_{-1.1}^{+1.92}$ & $1.07_{-0.57}^{+0.89}$ & $1.48_{-0.85}^{+1.49}$ & $0.66_{-0.38}^{+0.68}$ \\
\hline SMC_SC5 266088 & 01 & $8.8_{-2.1}^{+2.4}$ & $0.4_{-0.3}^{+0.4}$ & $0.8_{-0.1}^{+0.1}$ & $0.71_{-0.08}^{+0.14}$ & $0.7_{-0.2}^{+0.4}$ & $0.29_{-0.22}^{+0.73}$ & $0.07_{-0.03}^{+0.07}$ & $1.53_{-1.17}^{+3.83}$ & $0.23_{-0.15}^{+0.46}$ & $0.31_{-0.21}^{+0.58}$ & $0.18_{-0.12}^{+0.34}$ \\
\hline SMC & 0 & $15.2_{-2.0}^{+1.3}$ & $0.5_{-0.2}^{+0.2}$ & $0.81_{-0.11}^{+0.1}$ & $0.78_{-0.11}^{+0.11}$ & $1.0_{-0.3}^{+0.4}$ & $0.05_{-0.03}^{+0.18}$ & $0.06_{-0.02}^{+0.05}$ & $0.25_{-0.13}^{+0.8}$ & $0.13_{-0.06}^{+0.26}$ & $0.26_{-0.14}^{+0.57}$ & $0.17_{-0.09}^{+0.34}$ \\
\hline SMC_SC5 282963 & 01 & $13.8_{-2.4}^{+2.1}$ & $0.7_{-0.3}^{+0.2}$ & $0.82_{-0.11}^{+0.1}$ & $0.37_{-0.05}^{+0.11}$ & $1.5_{-0.5}^{+0.7}$ & $0.69_{-0.35}^{+0.47}$ & $0.44_{-0.19}^{+0.29}$ & $5.52_{-2.75}^{+3.69}$ & $2.46_{-1.47}^{+1.98}$ & $5.05_{-3.2}^{+4.7}$ & $6.01_{-3.82}^{+5.53}$ \\
\hline SMC & $\begin{array}{l}01 \\
02 \\
\end{array}$ & -2.0 & $0.6_{-0.3}^{+0.2}$ & $0.82_{-0.1}^{+0.09}$ & $0.69_{-0.07}^{+0.07}$ & $\begin{array}{l}2.3_{-0.4}^{+0.6} \\
2.5_{-0.6}^{+0.6} \\
\end{array}$ & $\begin{array}{l}0.63_{-0.26}^{+0.47} \\
0.65_{-0.24}^{+0.43} \\
\end{array}$ & $\begin{array}{r}0.16_{-0.05}^{+0.09} \\
0.34_{-0.1}^{+0.2} \\
\end{array}$ & $\begin{array}{l}0.87_{-0.36}^{+0.63} \\
1.04_{-0.36}^{+0.63} \\
\end{array}$ & $\begin{array}{l}3.17_{-1.35}^{+2.3} \\
3.66_{-1.49}^{+2.39} \\
\end{array}$ & $\begin{array}{l}6.76_{-3.13}^{+5.89} \\
7.79_{-3.49}^{+6.18} \\
\end{array}$ & $\begin{array}{l}1.39_{-0.65}^{+1.2} \\
1.82_{-0.8}^{+1.43} \\
\end{array}$ \\
\hline 617538 & 01 & $8.8_{-2.1}^{+2.5}$ & $0.3_{-0.2}^{+0.3}$ & $0.81_{-0.1}^{+0.1}$ & $0.62_{-0.1}^{+0.17}$ & $0.8_{-0.2}^{+0.4}$ & $0.63_{-0.45}^{+0.55}$ & $0.1_{-0.05}^{+0.12}$ & $3.15_{-2.3}^{+2.77}$ & $0.5_{-0.29}^{+0.49}$ & $\begin{array}{l}0.61_{-0.36}^{+0.74} \\
\end{array}$ & $0.33_{-0.2}^{+0.41}$ \\
\hline SMC_SC6 42440 & 01 & $9.4_{-2.1}^{+2.6}$ & $0.4_{-0.3}^{+0.4}$ & $0.8_{-0.11}^{+0.11}$ & $0.37_{-0.06}^{+0.14}$ & $1.8_{-0.8}^{+0.8}$ & $0.76_{-0.41}^{+0.49}$ & $0.2_{-0.11}^{+0.16}$ & $2.4_{-1.29}^{+1.65}$ & $1.5_{-0.83}^{+1.6}$ & $2.01_{-1.15}^{+2.62}$ & $0.75_{-0.44}^{+0.97}$ \\
\hline
\end{tabular}




\begin{tabular}{|c|c|c|c|c|c|c|c|c|c|c|c|c|}
\hline SMC_SC6 99991 & $\begin{array}{l}01 \\
02 \\
03 \\
04\end{array}$ & $13.0_{-2.2}^{+2.2}$ & $0.7_{-0.2}^{+0.2}$ & $0.82_{-0.1}^{+0.1}$ & $0.34_{-0.03}^{+0.04}$ & $\begin{array}{l}1.6_{-0.5}^{+0.6} \\
1.8_{-0.4}^{+0.7} \\
1.8_{-0.4}^{+0.5} \\
1.7_{-0.5}^{+0.6} \\
\end{array}$ & $\begin{array}{l}0.53_{-0.23}^{+0.44} \\
0.67_{-0.32}^{+0.42} \\
0.51_{-0.22}^{+0.4} \\
0.42_{-0.18}^{+0.33} \\
\end{array}$ & $\begin{array}{l}0.94_{-0.28}^{+0.33} \\
0.75_{-0.26}^{+0.31} \\
0.82_{-0.23}^{+0.33} \\
0.94_{-0.26}^{+0.28} \\
\end{array}$ & $\begin{array}{l}0.64_{-0.27}^{+0.51} \\
1.07_{-0.49}^{+0.73} \\
0.81_{-0.34}^{+0.63} \\
0.78_{-0.36}^{+1.04} \\
\end{array}$ & $\begin{array}{l}1.82_{-0.72}^{+1.03} \\
2.42_{-1.06}^{+1.77^{2}} \\
1.89_{-0.77}^{+1.26} \\
1.56_{-0.66}^{+1.05} \\
\end{array}$ & $\begin{array}{l}3.54_{-1.6}^{+2.38} \\
4.68_{-2.24}^{+3.84} \\
3.64_{-1.65}^{+2.95} \\
3.04_{-1.44}^{+2.29} \\
\end{array}$ & $\begin{array}{r}0.61_{-0.27}^{+0.41} \\
1.09_{-0.52}^{+0.85} \\
0.82_{-0.36}^{+0.65} \\
0.8_{-0.37}^{+0.61} \\
\end{array}$ \\
\hline SMC_SC6 105368 & $\begin{array}{l}01 \\
02\end{array}$ & $12.4_{-2.5}^{+2.7}$ & $0.5_{-0.3}^{+0.2}$ & $0.82_{-0.1}^{+0.11}$ & $0.53_{-0.03}^{+0.05}$ & $\begin{array}{l}1.0_{-0.2}^{+0.4} \\
1.5_{-0.5}^{+0.7}\end{array}$ & $\begin{array}{l}0.56_{-0.32}^{+0.5} \\
0.24_{-0.12}^{+0.45}\end{array}$ & $\begin{array}{r}0.2_{-0.07}^{+0.12} \\
0.22_{-0.09}^{+0.27}\end{array}$ & $\begin{array}{l}1.03_{-0.99}^{+0.9} \\
0.56_{-0.27}^{+1.02}\end{array}$ & $\begin{array}{l}0.89_{-0.45}^{+0.79} \\
0.65_{-0.31}^{+8.76}\end{array}$ & $\begin{array}{l}1.52_{-0.81}^{+1.66} \\
1.17_{-0.63}^{+1.33}\end{array}$ & $\begin{array}{l}0.36_{-8.19}^{+0.39} \\
0.34_{-0.18}^{+0.83}\end{array}$ \\
\hline SMC_SC6 116294 & 01 & $9.2_{-1.8}^{+2.3}$ & $0.5_{-0.3}^{+0.3}$ & $0.81_{-0.11}^{+0.1}$ & $0.4_{-0.06}^{+0.14}$ & $2.6_{-1.0}^{+0.7}$ & $0.89_{-0.38}^{+0.38}$ & $0.18_{-0.09}^{+0.19}$ & $2.39_{-1.09}^{+1.03}$ & $2.67_{-1.5}^{+2.3}$ & $3.63_{-2.09}^{+3.66}$ & $1.13_{-0.65}^{+1.13}$ \\
\hline SMC_SC6 128831 & 01 & $12.2_{-2.2}^{+2.7}$ & $0.6_{-0.3}^{+0.2}$ & $0.8_{-0.09}^{+0.11}$ & $0.03_{-0.02}^{+0.02}$ & $2.6_{-0.6}^{+0.5}$ & $0.98_{-0.4}^{+0.35}$ & $0.69_{-0.27}^{+0.34}$ & $0.82_{-0.36}^{+0.32}$ & $4.18_{-1.72}^{+2.2}$ & $7.47_{-3.44}^{+4.69}$ & $0.84_{-0.4}^{+0.61}$ \\
\hline SMC_SC6 199611 & 01 & $13.8_{-2.0}^{+2.1}$ & $0.8_{-0.3}^{+0.2}$ & $0.81_{-0.1}^{+0.1}$ & $0.37_{-0.06}^{+0.12}$ & $1.4_{-0.7}^{+1.4}$ & $0.47_{-0.33}^{+0.62}$ & $0.34_{-0.18}^{+0.34}$ & $0.35_{-0.25}^{+0.48}$ & $1.48_{-0.91}^{+1.51}$ & $3.03_{-1.93}^{+3.73}$ & $0.34_{-0.21}^{+0.42}$ \\
\hline SMC_SC6 272665 & 01 & $7.0_{-1.2}^{+1.8}$ & $0.4_{-0.3}^{+0.3}$ & $0.8_{-0.1}^{+0.11}$ & $0.37_{-0.05}^{+0.14}$ & $0.7_{-0.2}^{+0.4}$ & $0.68_{-0.46}^{+0.54}$ & $0.08_{-0.05}^{+0.25}$ & $5.97_{-4.09}^{+5.43}$ & $0.37_{-0.22}^{+0.37}$ & $0.4_{-0.24}^{+0.46}$ & $0.37_{-0.24}^{+0.42}$ \\
\hline SMC_SC7 57131 & 01 & $12.1_{-2.2}^{+2.6}$ & $0.6_{-0.3}^{+0.3}$ & $0.81_{-0.1}^{+0.1}$ & $0.39_{-0.06}^{+0.12}$ & $2.0_{-0.7}^{+0.7}$ & $0.78_{-0.39}^{+0.45}$ & $0.74_{-0.35}^{+0.41}$ & $1.69_{-0.81}^{+0.98}$ & $2.48_{-1.36}^{+1.97}$ & $4.32_{-2.46}^{+3.9}$ & $1.26_{-0.73}^{+1.11}$ \\
\hline SMC_SC8 183240 & 01 & $14.7_{-1.8}^{+1.6}$ & $0.8_{-0.2}^{+0.1}$ & $0.82_{-0.11}^{+0.1}$ & $0.64_{-0.11}^{+0.18}$ & $0.7_{-0.2}^{+0.3}$ & $0.63_{-0.38}^{+0.51}$ & $0.25_{-0.12}^{+0.21}$ & $1.1_{-0.63}^{+0.86}$ & $1.37_{-0.8}^{+1.24}$ & $3.2_{-1.94}^{+3.22}$ & $0.92_{-0.54}^{+0.89}$ \\
\hline SMC_SC9 105383 & $\begin{array}{l}01 \\
02 \\
\end{array}$ & $13.2_{-2.5}^{+2.6}$ & $0.5_{-0.3}^{+0.2}$ & $0.83_{-0.1}^{+0.09}$ & $0.56_{-0.05}^{+0.08}$ & $\begin{array}{l}1.9_{-0.5}^{+0.8} \\
1.8_{-0.4}^{+0.5} \\
\end{array}$ & $\begin{array}{l}0.52_{-0.25}^{+0.5} \\
0.86_{-0.39}^{+0.38} \\
\end{array}$ & $\begin{array}{l}0.1_{-0.03}^{+0.04} \\
0.1_{-0.03}^{+0.05} \\
\end{array}$ & $\begin{array}{l}1.17_{-0.58}^{+1.2} \\
1.76_{-0.75}^{+8.79} \\
\end{array}$ & $\begin{array}{r}1.82_{-0.86}^{+1.58} \\
2.58_{-1.15}^{+1.8} \\
\end{array}$ & $\begin{array}{r}3.36_{-1}^{+3.43} \\
4.75_{-2.38}^{+4.76} \\
\end{array}$ & $\begin{array}{r}1.01_{-0.51}^{+1.07} \\
1.3_{-0.62}^{+1.12} \\
\end{array}$ \\
\hline SMC_SC9 168422 & 01 & $9.4_{-2.0}^{+2.3}$ & $0.4_{-0.3}^{+0.3}$ & $0.82_{-0.11}^{+0.1}$ & $0.61_{-0.08}^{+0.16}$ & $0.5_{-0.2}^{+0.4}$ & $0.56_{-0.45}^{+0.62}$ & $0.08_{-0.04}^{+0.12}$ & $2.15_{-1.71}^{+2.35}$ & $0.33_{-0.22}^{+0.41}$ & $0.44_{-0.3}^{+0.63}$ & $0.2_{-0.13}^{+0.29}$ \\
\hline SMC_SC10 8906 & $\begin{array}{l}01 \\
02\end{array}$ & $13.9_{-2.1}^{+2.0}$ & $0.8_{-0.2}^{+0.1}$ & $0.81_{-0.1}^{+0.11}$ & $0.58_{-0.05}^{+0.1}$ & $\begin{array}{l}2.0_{-0.7}^{+1.0} \\
1.8_{-0.6}^{+0.7} \\
\end{array}$ & $\begin{array}{l}0.79_{-0.34}^{+0.44} \\
0.81_{-0.35}^{+0.35} \\
\end{array}$ & $\begin{array}{l}0.47_{-0.18}^{+0.26} \\
0.92_{-0.32}^{+0.34} \\
\end{array}$ & $\begin{array}{l}0.16_{-0.87}^{+0.1} \\
0.06_{-0.02}^{+0.83} \\
\end{array}$ & $\begin{array}{l}3.88_{-1.57}^{+2.17} \\
3.39_{-1.5}^{+2.05} \\
\end{array}$ & $\begin{array}{r}8.2_{-3.71}^{+5.63} \\
7.26_{-3.55}^{+5}: 01 \\
\end{array}$ & $\begin{array}{l}0.26_{-0.02}^{+0.19} \\
0.09_{-0.04}^{+8.06}\end{array}$ \\
\hline SMC_SC11 28090 & 01 & $13.7_{-2.1}^{+2.1}$ & $0.8_{-0.2}^{+0.1}$ & $0.83_{-0.11}^{+0.09}$ & $0.56_{-0.05}^{+0.12}$ & $1.7_{-0.5}^{+0.8}$ & $0.1_{-0.05}^{+0.1}$ & $0.12_{-0.05}^{+0.05}$ & $0.41_{-0.18}^{+0.37}$ & $0.48_{-0.23}^{+0.41}$ & $1.02_{-0.54}^{+1.01}$ & $0.64_{-0.34}^{+0.64}$ \\
\hline SMC_SC11 46587 & 01 & $9.0_{-2.1}^{+2.5}$ & $0.4_{-0.3}^{+0.4}$ & $0.81_{-0.11}^{+0.1}$ & $0.39_{-0.07}^{+0.19}$ & $0.9_{-0.4}^{+0.7}$ & $0.6_{-0.43}^{+0.57}$ & $0.36_{-0.22}^{+0.51}$ & $4.02_{-2.89}^{+3.88}$ & $0.55_{-0.34}^{+0.58}$ & $0.72_{-0.47}^{+0.91}$ & $0.55_{-0.36}^{+0.7}$ \\
\hline
\end{tabular}




\section{ACKNOWLEDGEMENTS}

This work made use of the computing facilities of the Laboratory of Astroinformatics (IAG/USP, NAT/Unicsul), whose purchase was made possible by the Brazilian agency FAPESP (grant 2009/540064) and the INCT-A. L. R. R. acknowledges the support from FAPESP (grant 2012/21518-5) and from CNPq (grant 142411/2011-6). A. C. C. acknowledges the support from CNPq (grant 307594/20157) and FAPESP (grant 2015/17967-7). D. M. F. acknowledges the support from FAPESP (grant 2016/16844-1). R. G. V. acknowledges the support from FAPESP (grant 2012/20364-4). M. R. G. acknowledges the support from CAPES PROEX Programa Astronomia. J. E. B. was supported by NSF grant AST-1412135. The OGLE project has received funding from the National Science Centre, Poland, grant MAESTRO 2014/14/A/ST9/00121.

\section{REFERENCES}

Alcock C., et al., 1997, ApJ, 486, 697

Aubourg E., et al., 1993, Nature, 365, 623

Baade D., et al., 2016, A\&A, 588, A56

Balbus S. A., Hawley J. F., 1991, ApJ, 376, 214

Bessell M. S., 1990, PASP, 102, 1181

Carciofi A. C., 2011, in Neiner C., Wade G., Meynet G., Peters G., eds, IAU Symposium Vol. 272, Active OB Stars: Structure, Evolution, Mass Loss, and Critical Limits. pp 325-336 (arXiv:1009.3969), doi:10.1017/S1743921311010738

Carciofi A. C., Bjorkman J. E., 2006, ApJ, 639, 1081

Carciofi A. C., Bjorkman J. E., 2008, ApJ, 684, 1374

Carciofi A. C., Bjorkman J. E., Magalhães A. M., 2004, ApJ, 604, 238

Carciofi A. C., et al., 2006, ApJ, 652, 1617

Carciofi A. C., Magalhães A. M., Leister N. V., Bjorkman J. E., Levenhagen R. S., 2007, ApJ, 671, L49

Carciofi A. C., Domiciano de Souza A., Magalhães A. M., Bjorkman J. E., Vakili F., 2008, ApJ, 676, L41

Carciofi A. C., Okazaki A. T., Le Bouquin J.-B., Štefl S., Rivinius T., Baade D., Bjorkman J. E., Hummel C. A., 2009, A\&A, 504, 915

Carciofi A. C., Bjorkman J. E., Otero S. A., Okazaki A. T., Štefl S., Rivinius T., Baade D., Haubois X., 2012, ApJ, 744, L15

Castelli F., Kurucz R. L., 1994, A\&A, 281, 817

Collins II G. W., 1987, in Slettebak A., Snow T. P., eds, IAU Colloq. 92: Physics of Be Stars. pp 3-19

Ekström S., Meynet G., Maeder A., Barblan F., 2008, A\&A, 478, 467

Espinosa Lara F., Rieutord M., 2011, A\&A, 533, A43

Foreman-Mackey D., Hogg D. W., Lang D., Goodman J., 2013, PASP, 125, 306

Gayley K. G., Ignace R., Owocki S. P., 2001, ApJ, 558, 802

Gehrz R. D., Hackwell J. A., Jones T. W., 1974, ApJ, 191, 675

Georgy C., Ekström S., Granada A., Meynet G., Mowlavi N., Eggenberger P., Maeder A., 2013, A\&A, 553, A24

Ghoreyshi M. R., Carciofi A. C., 2017, in Miroshnichenko A., Zharikov S., Korčáková D., Wolf M., eds, Astronomical Society of the Pacific Conference Series Vol. 508, Astronomical Society of the Pacific Conference Series. p. 323 (arXiv: 1702.06982)

Gordon K. D., Clayton G. C., Misselt K. A., Landolt A. U., Wolff M. J., 2003, ApJ, 594, 279

Granada A., Ekström S., Georgy C., Krtička J., Owocki S., Meynet G., Maeder A., 2013, A\&A, 553, A25

Haubois X., Carciofi A. C., Rivinius T., Okazaki A. T., Bjorkman J. E., 2012, ApJ, 756, 156

Hilditch R. W., Howarth I. D., Harries T. J., 2005, MNRAS, 357, 304

Jaschek M., Slettebak A., Jaschek C., 1981, Be star terminology., Be Star Newsletter

Jones C. E., Sigut T. A. A., Marlborough J. M., 2004, MNRAS, 352, 841

Jones C. E., Sigut T. A. A., Porter J. M., 2008a, MNRAS, 386, 1922
Jones C. E., Tycner C., Sigut T. A. A., Benson J. A., Hutter D. J., 2008b, ApJ, 687, 598

Kee N. D., Owocki S., Sundqvist J. O., 2016, MNRAS, 458, 2323

Keller S. C., Bessell M. S., Cook K. H., Geha M., Syphers D., 2002, AJ, 124, 2039

King A. R., Pringle J. E., Livio M., 2007, MNRAS, 376, 1740

Klement R., et al., 2015, A\&A, 584, A85

Klement R., Carciofi A. C., Rivinius T., in press, MNRAS

Kotko I., Lasota J.-P., 2012, A\&A, 545, A115

Kroupa P., 2001, MNRAS, 322, 231

Krtička J., Owocki S. P., Meynet G., 2011, A\&A, 527, A84

Lamers H. J. G. L. M., Snow T. P., Lindholm D. M., 1995, ApJ, 455, 269

Martayan C., Frémat Y., Hubert A.-M., Floquet M., Zorec J., Neiner C., 2007a, A\&A, 462, 683

Martayan C., Floquet M., Hubert A. M., Gutiérrez-Soto J., Fabregat J., Neiner C., Mekkas M., 2007b, A\&A, 472, 577

Mennickent R. E., Pietrzyński G., Gieren W., Szewczyk O., 2002, A\&A, 393,887

Minniti D., et al., 2010, New Astron., 15, 433

Okazaki A. T., 2001, PASJ, 53, 119

Okazaki A. T., 2007, in Okazaki A. T., Owocki S. P., Stefl S., eds, Astronomical Society of the Pacific Conference Series Vol. 361, Active OB-Stars: Laboratories for Stellare and Circumstellar Physics. p. 230

Okazaki A. T., Bate M. R., Ogilvie G. I., Pringle J. E., 2002, MNRAS, 337, 967

Papaloizou J. C. B., Lin D. N. C., 1995, ARA\&A, 33, 505

Paul K. T., Subramaniam A., Mathew B., Mennickent R. E., Sabogal B., 2012, MNRAS, 421, 3622

Pepper J., et al., 2007, PASP, 119, 923

Pojmanski G., 1997, Acta Astron., 47, 467

Pols O. R., Cote J., Waters L. B. F. M., Heise J., 1991, A\&A, 241, 419

Puls J., Vink J. S., Najarro F., 2008, A\&ARv, 16, 209

Rivinius T., Baade D., Stefl S., Stahl O., Wolf B., Kaufer A., 1998, A\&A, 333,125

Rivinius T., Štefl S., Baade D., 2006, A\&A, 459, 137

Rivinius T., Carciofi A. C., Martayan C., 2013, A\&ARv, 21, 69

Rivinius T., Baade D., Carciofi A. C., 2016, A\&A, 593, A106

Sabogal B. E., Mennickent R. E., Pietrzyński G., Gieren W., 2005, MNRAS, 361,1055

Silaj J., Jones C. E., Tycner C., Sigut T. A. A., Smith A. D., 2010, ApJS, 187,228

Snow Jr. T. P., 1981, ApJ, 251, 139

Touhami Y., Gies D. R., Schaefer G. H., 2011, ApJ, 729, 17

Udalski A., Kubiak M., Szymanski M., 1997, Acta Astron., 47, 319

Udalski A., Szymanski M. K., Soszynski I., Poleski R., 2008, Acta Astron., 58,69

Vieira R. G., Carciofi A. C., Bjorkman J. E., 2015, MNRAS, 454, 2107

Vieira R. G., Carciofi A. C., Bjorkman J. E., Rivinius T., Baade D., Rímulo L. R., 2017, MNRAS, 464, 3071

Wyrzykowski Ł., et al., 2009, MNRAS, 397, 1228

Zaritsky D., Harris J., Thompson I. B., Grebel E. K., 2004, AJ, 128, 1606

\section{APPENDIX A: PROPERTIES OF THE HYDRODYNAMICAL SOLUTIONS OF THE VDD MODEL}

A particular bump model (Sect. 3.1) with arbitrary density $\Sigma_{0}$ and scaled build-up time $\tilde{\tau}_{\mathrm{bu}}=30$ is used to illustrate features of the bump models in Fig. A1. The top panel shows the amount of angular momentum in the disc, given by

$J_{\mathrm{disc}}=\int(G M R)^{\frac{1}{2}} \Sigma 2 \pi R \mathrm{~d} R$,

and the amount of angular momentum that is lost by the star, given by the angular momentum that is injected at the radius of mass injection minus the angular momentum that falls back to the stellar equator. By the continuity of angular momentum, the difference 

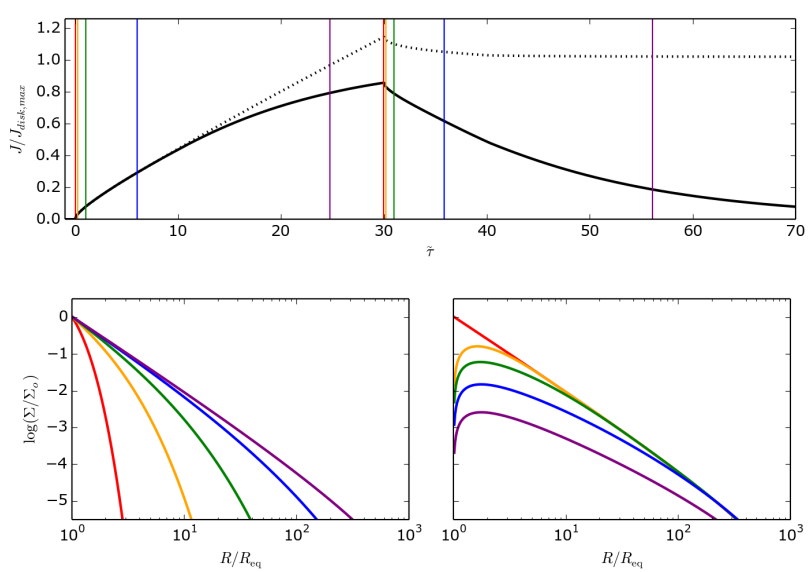

Figure A1. Dynamical bump model with $\tilde{\tau}_{\text {bu }}=30$. Top: the amount of angular momentum in the disc (solid black curve) and the amount of angular momentum that is lost by the star (dotted black curve). Colored vertical straight lines mark the 5 instants $\tilde{\tau}=0,0.2,1,6,25$ (during the build-up phase), and the 5 instants $\tilde{\tau}=30,30.2,31,36,55$ (during the dissipation phase). Surface density profiles are shown at the first 5 instants (bottom left) and at the last 5 instants (bottom right).

between the dotted and the solid curves is the angular momentum that escapes the system through the outer boundary at $R_{\text {out }}$. All values were scaled by the maximum angular momentum supported by the disc, which is

$J_{\text {disc, } \max }=\int(G M R)^{\frac{1}{2}} \Sigma_{\text {steady }} 2 \pi R \mathrm{~d} R$.

The plot shows that, as the build-up process occurs, the disc mass and angular momentum content increase continuously. Eventually (in our example, roughly after $\tilde{\tau}=10$ ), a non-negligible amount of angular momentum starts to reach the outer radius $R_{\mathrm{out}}=1000 R_{\mathrm{eq}}$, leaving the system through the outer boundary. After the end of the build-up phase (which, in our example, happens at $\tilde{\tau}=30$ ), the disc starts to dissipate: the black curve shows that the disc loses angular momentum until it reaches zero. However, as the dotted curve shows, only a fraction of the angular momentum of the disc returns to the star by re-accretion. The dotted curve tends to a nonzero value, which is the angular momentum that was lost by the star in the whole process. This non-zero total angular momentum lost by the star was verified in our simulations to be given exactly by

$-\Delta J_{*}=\int_{0}^{\tilde{\tau}_{\mathrm{bu}}} \tau(t)\left(-\frac{\partial J}{\partial t}\right)_{\text {steady }} \mathrm{d} \tilde{\tau}$,

or, in the case of a constant $\alpha$ in time during build-up (Eq. 20),

$-\Delta J_{*}=\left(-\frac{\partial J}{\partial t}\right)_{\text {steady }}\left(t_{2}-t_{1}\right)$.

This quantity, therefore, is nearly independent of $R_{\text {out }}$.

The bottom panels of Fig. A1 show surface density radial profiles at the specify instants marked in the top panel. During build-up, the disc grows in an inside out pattern, with the inner regions reaching a near stationary regime earlier than the outer parts. During dissipation, however, the disc becomes less and less dense as a whole, more or less self-similar way, because the entire disc is coupled by viscous forces.

\section{APPENDIX B: EXAMPLES OF MODEL LIGHT CURVES}

Figure B1 shows examples of $I$-band light curves from our grid (see Table 1 and Sects. 3.2 and 3.3). The dashed black curves correspond to $\Delta I_{\mathrm{bu}} / \Delta I_{\mathrm{bu}}^{\infty}$ versus $\tilde{\tau}$. The solid curves correspond to $\Delta I_{\mathrm{d}} / \Delta I_{\mathrm{d}}^{0}$ versus $\tilde{\tau}-\tilde{\tau}_{\mathrm{bu}}$ for four dissipating light curves with increasing scaled build-up times. Since $\Delta I_{\text {bu }}^{\infty}$ is the limiting magnitude of the build-up light curves and $\Delta I_{\mathrm{d}}^{0}$ is the magnitude at the instant of the beginning of dissipation, it follows that all $\Delta I_{\mathrm{bu}} / \Delta I_{\mathrm{bu}}^{\infty}$ curves go from 0 to 1 and all $\Delta I_{\mathrm{d}} / \Delta I_{\mathrm{d}}^{0}$ curves go from 1 to 0 .

The build-up and dissipation light curves of the edge-on (upperleft panel) and nearly-pole-on cases (right panels) can be approximated by the mathematical formulae given by Eqs. 17 and 18, respectively. The light curves at intermediate angles like the ones of the middle-left and lower-left panels show more complex forms that cannot be described by Eqs. 17 and 18. The light curves show that, at $\tilde{\tau} \approx 1$, the simulated bump has reached a significant fraction of its limiting value, and, at $\tilde{\tau}-\tilde{\tau}_{\mathrm{bu}} \approx 1$, the bump has already fallen considerably from its previous magnitude before the beginning of the dissipation.

Furthermore, dissipating curves with larger scaled build-up times dissipate at slower rates, as a result of the mass reservoir effect (Sect. 2.2). Also, as discussed in Sect. 3.3, both the dissipation and growth rates depend on the viewing angle.

\section{APPENDIX C: ONLINE FIGURES}



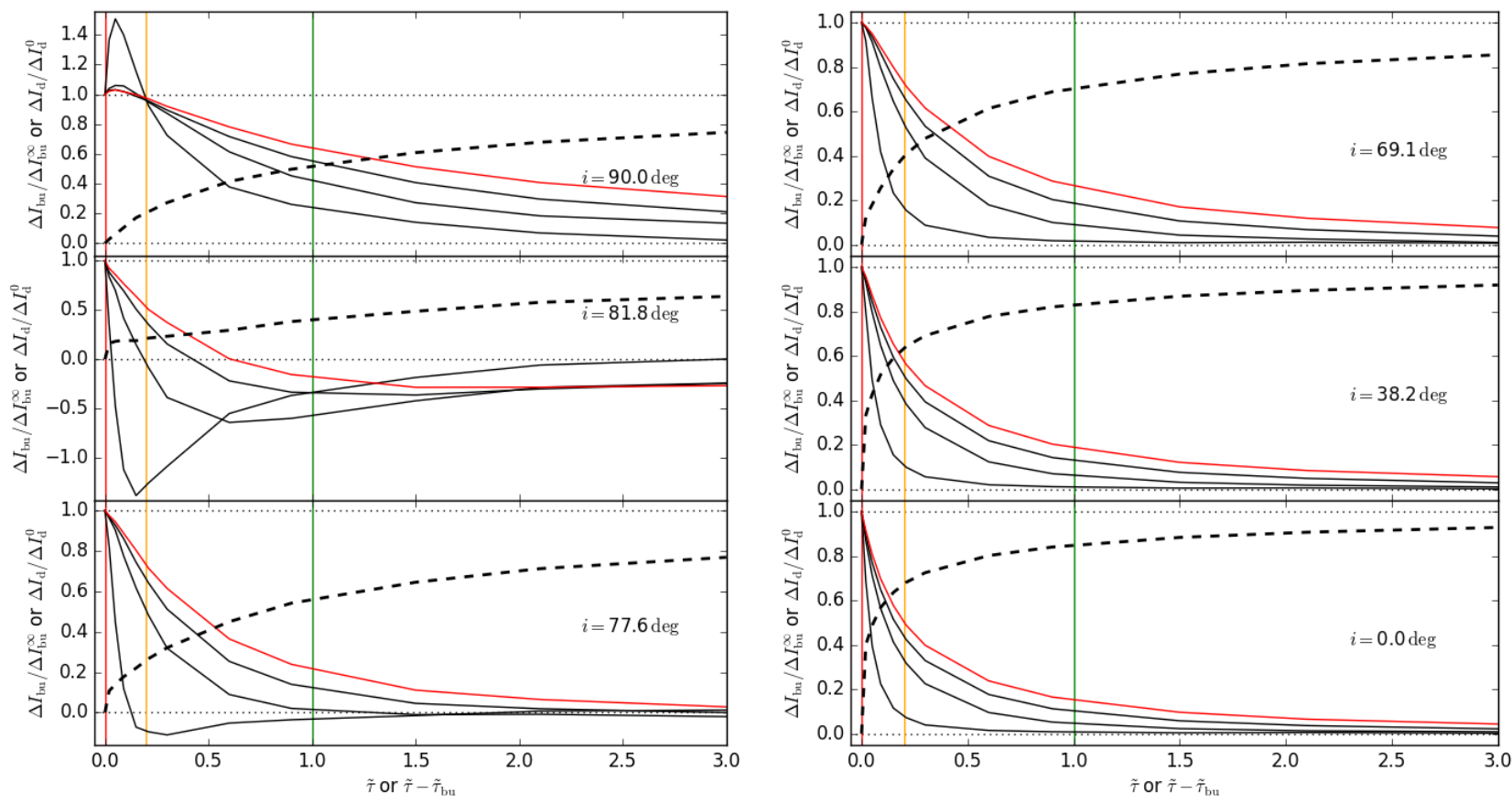

Figure B1. Examples of model $I$-band light curves. Each panel shows the results for different inclination angles, as indicated. The dashed black curves correspond to $\Delta I_{\mathrm{bu}} / \Delta I_{\mathrm{bu}}^{\infty}$ versus $\tilde{\tau}$, and the solid curves correspond to $\Delta I_{\mathrm{d}} / \Delta I_{\mathrm{d}}^{0}$ versus $\tilde{\tau}-\tilde{\tau}_{\mathrm{bu}}$, for four dissipating light curves with scaled build-up times given by $\tilde{\tau}_{\text {bu }}=0.15,1.5,6$ (in black) and $\tilde{\tau}_{\text {bu }}=30$ (in red). Vertical colored straight lines mark the instants $\tilde{\tau}$ or $\tilde{\tau}-\tilde{\tau}_{\text {bu }}=0,0.2,1$ (same color-code as in Fig. A1). All light curves are from Star 2 and $\Sigma_{0}=1.37 \mathrm{~g} \mathrm{~cm}^{-2}$. 


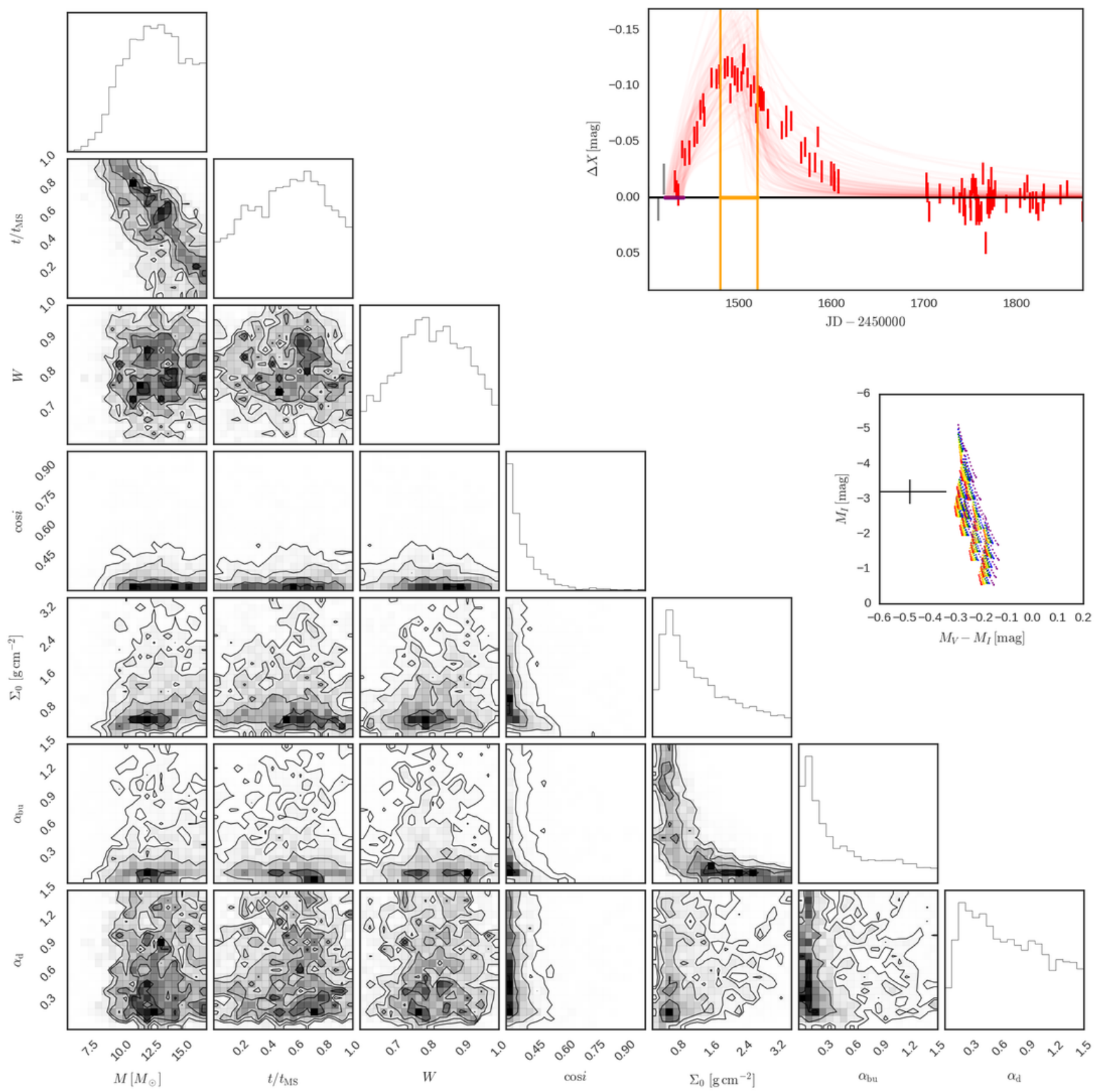

Figure C1. Same as Fig. 8 for SMC_SC1 7612 and bump ID 01. 


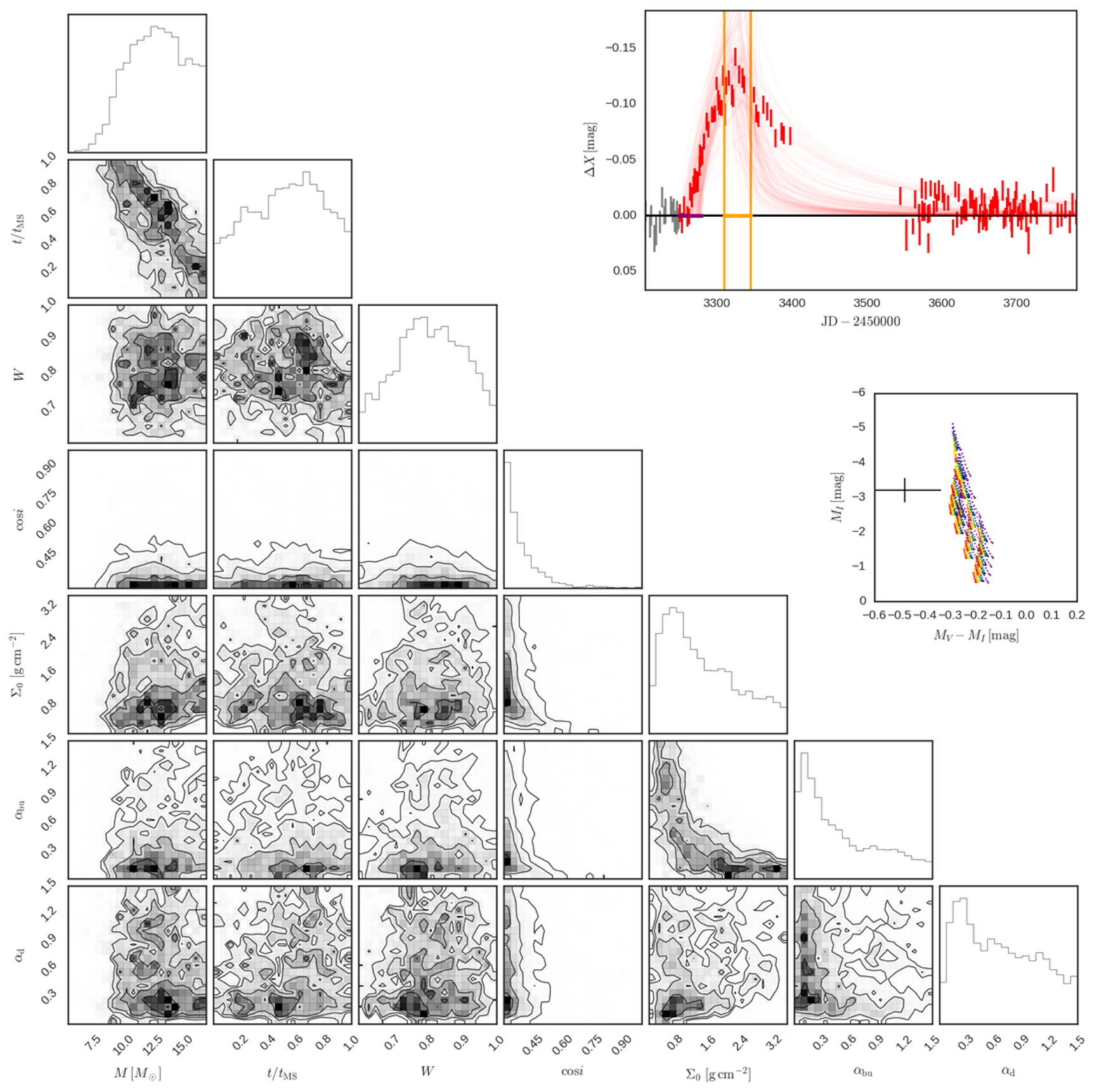

Figure C2. Same as Fig. 8 for SMC_SC1 7612 and bump ID 02. 

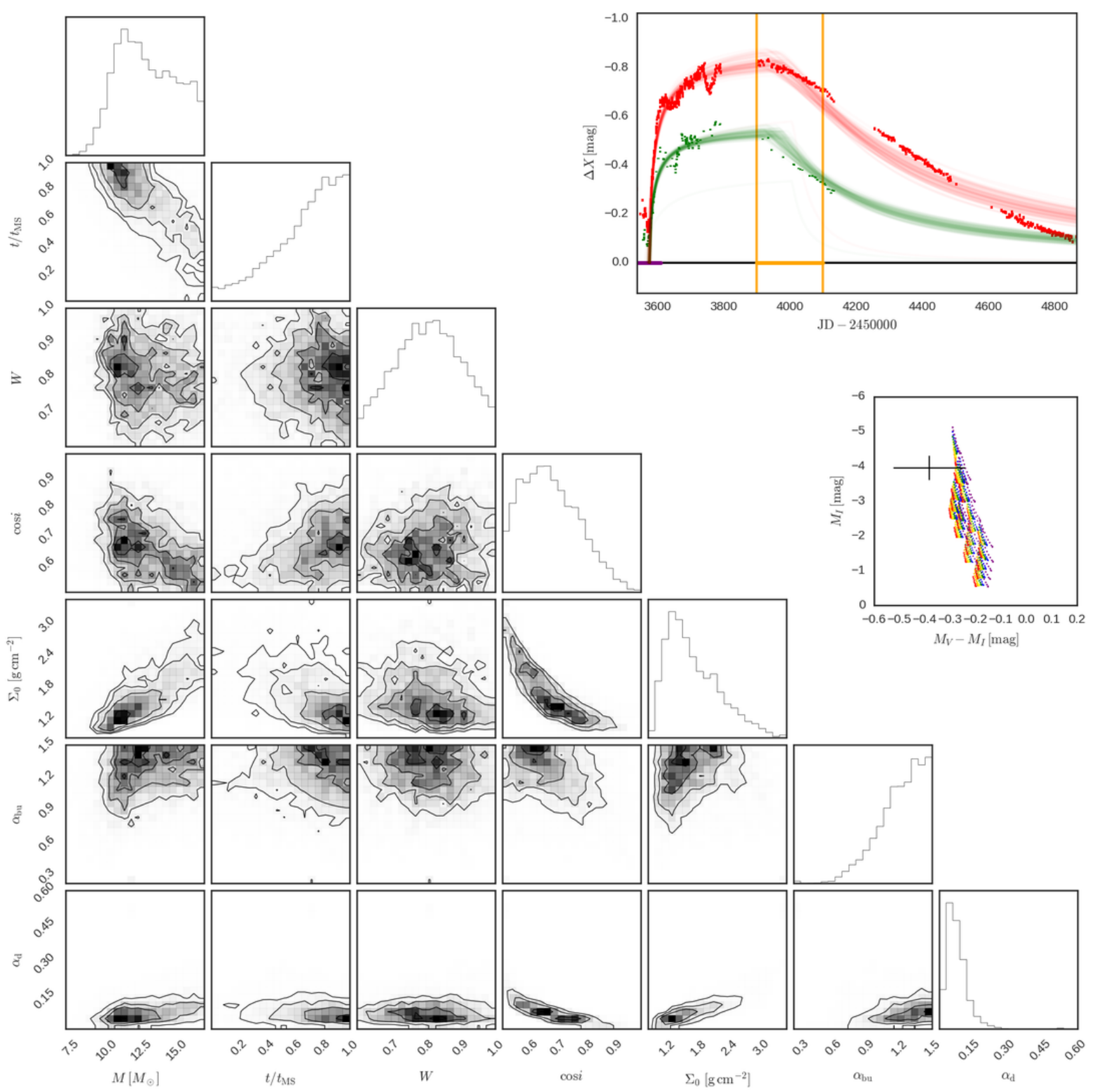

$$
M_{V}-M_{I}[\mathrm{mag}]
$$

Figure C3. Same as Fig. 8 for SMC_SC1 60553 and bump ID 01. 


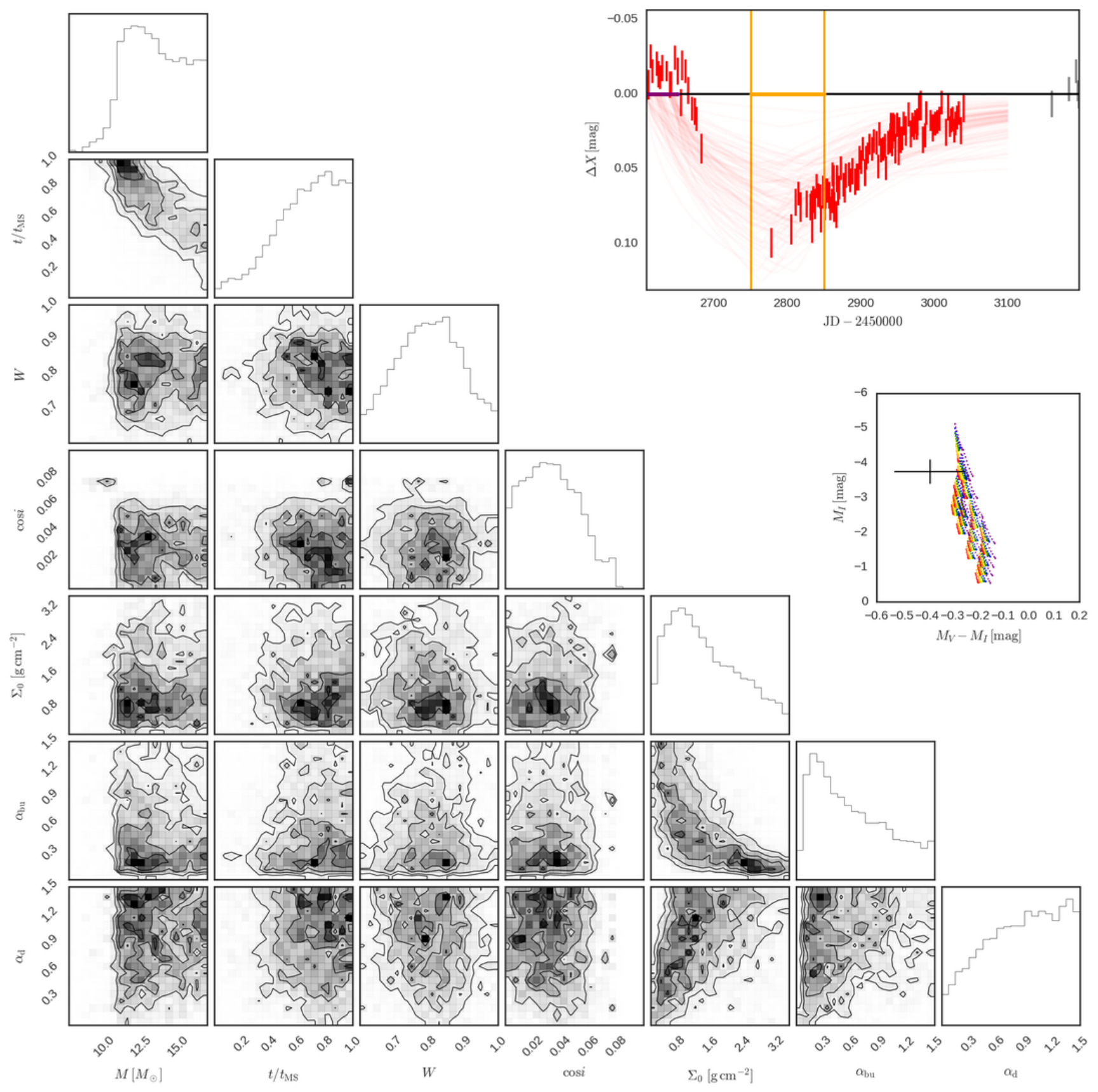

Figure C4. Same as Fig. 8 for SMC_SC1 92262 and bump ID 01. 

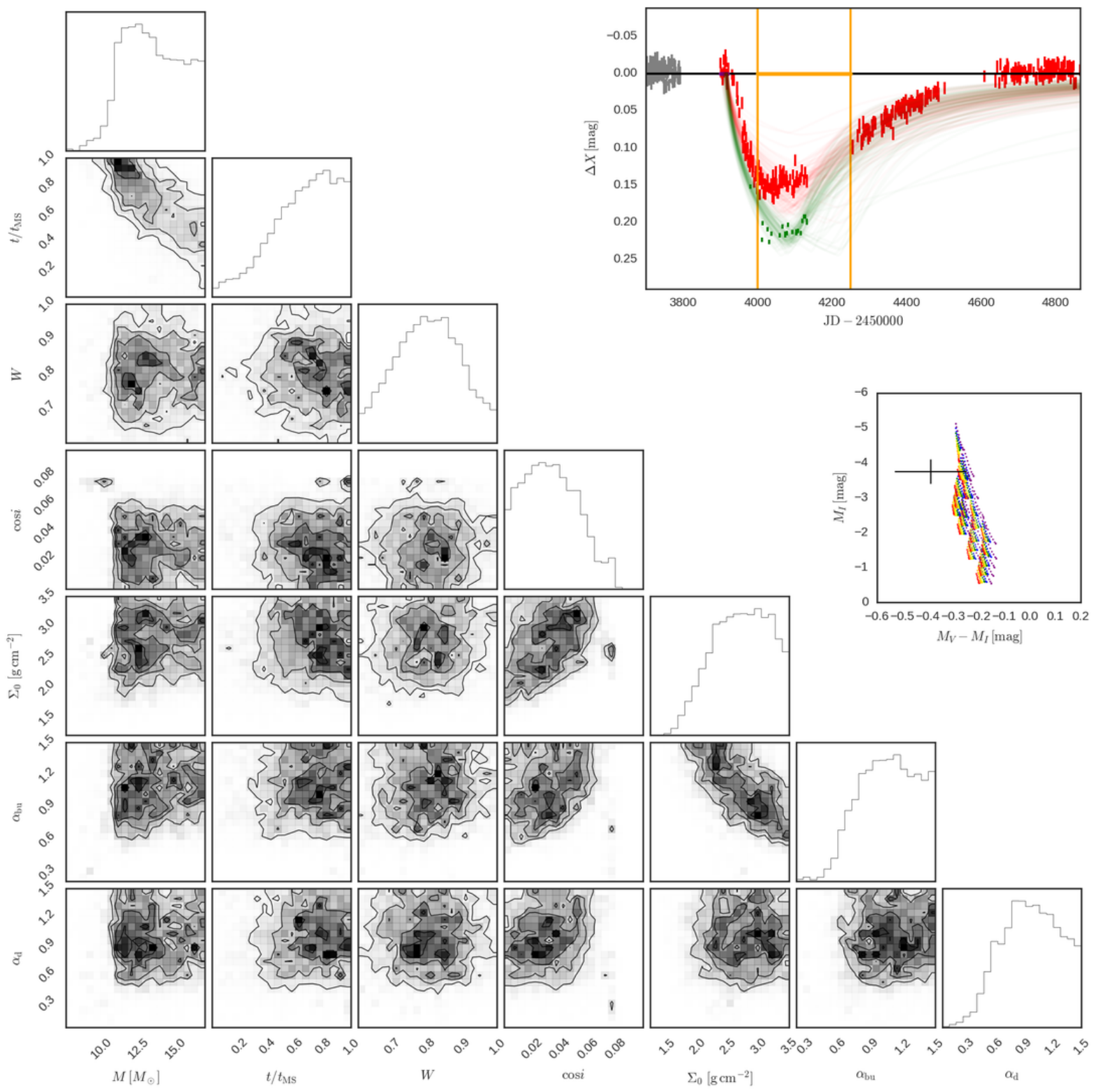

Figure C5. Same as Fig. 8 for SMC_SC1 92262 and bump ID 02. 


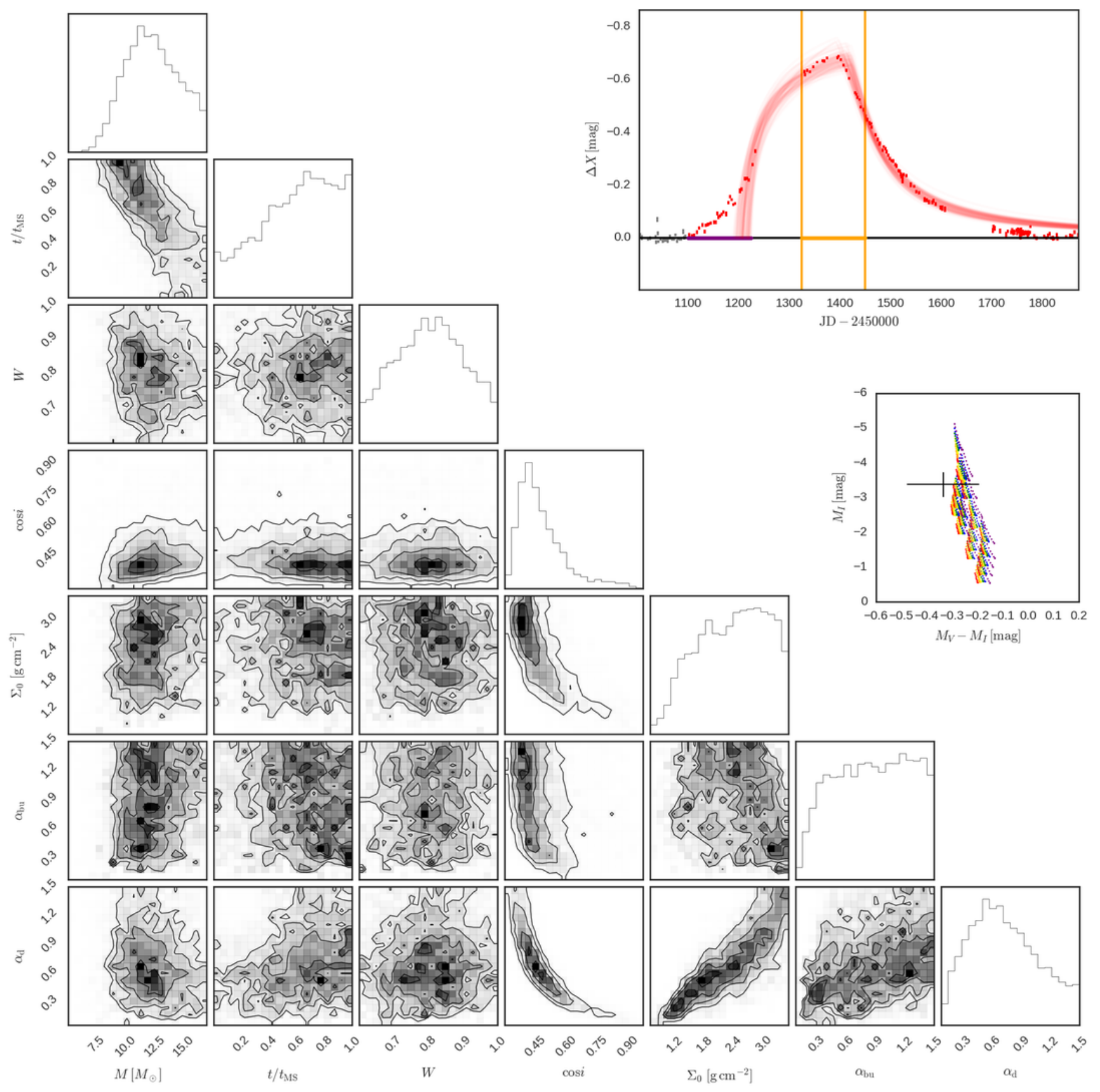

Figure C6. Same as Fig. 8 for SMC_SC2 94939 and bump ID 01. 


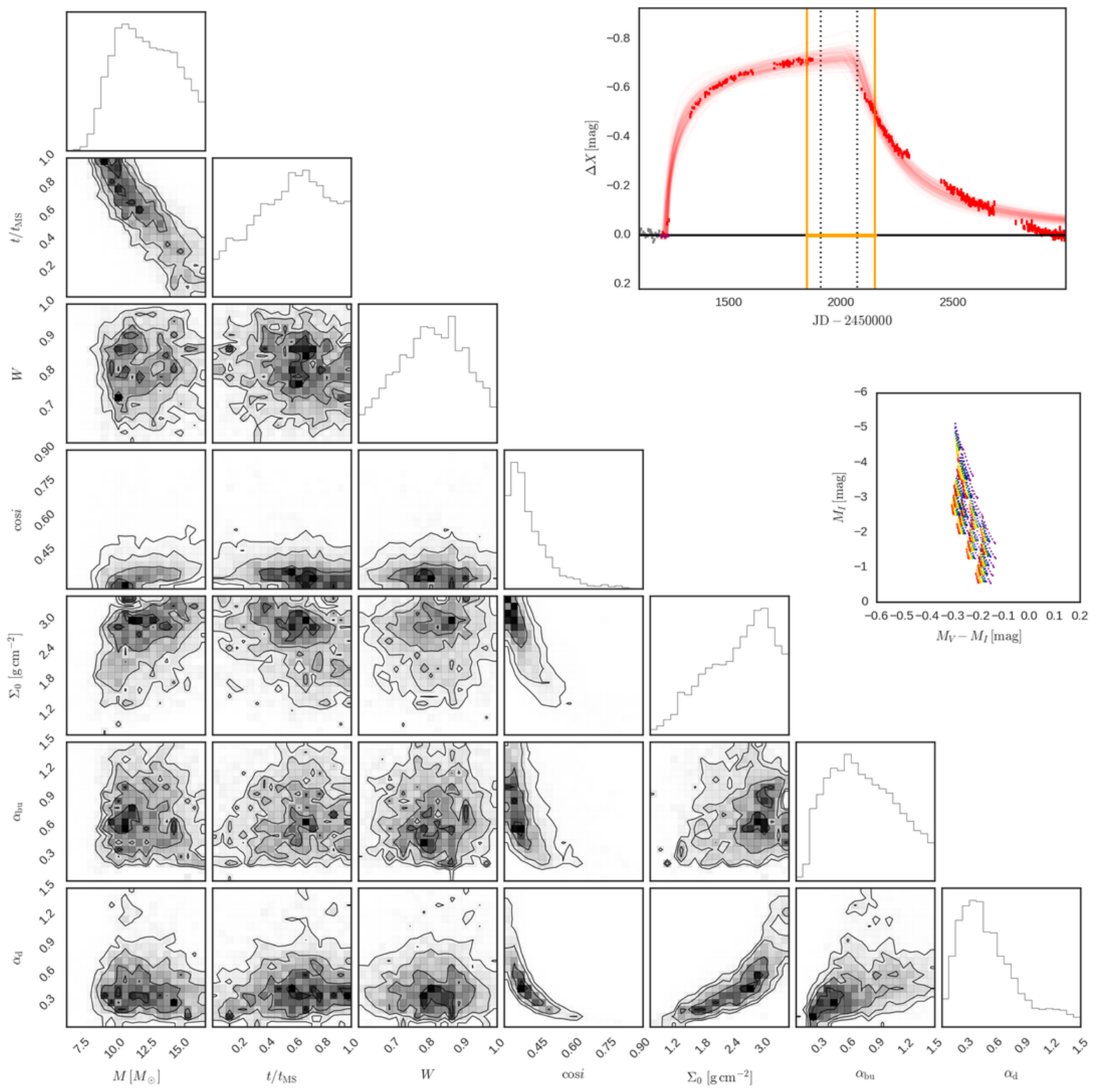

Figure C7. Same as Fig. 8 for SMC_SC3 5719 and bump ID 01. 


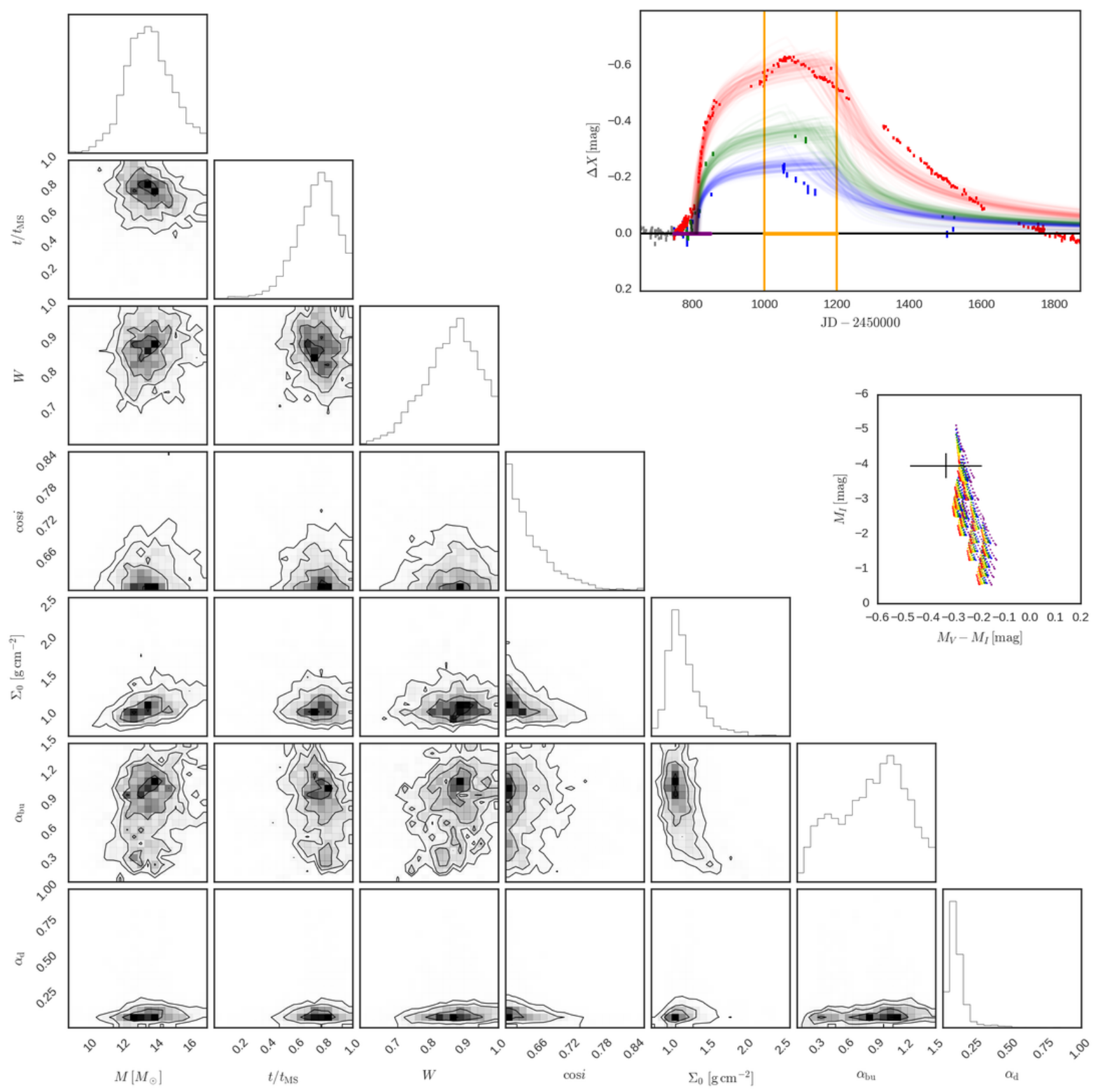

Figure C8. Same as Fig. 8 for SMC_SC3 15970 and bump ID 01. 


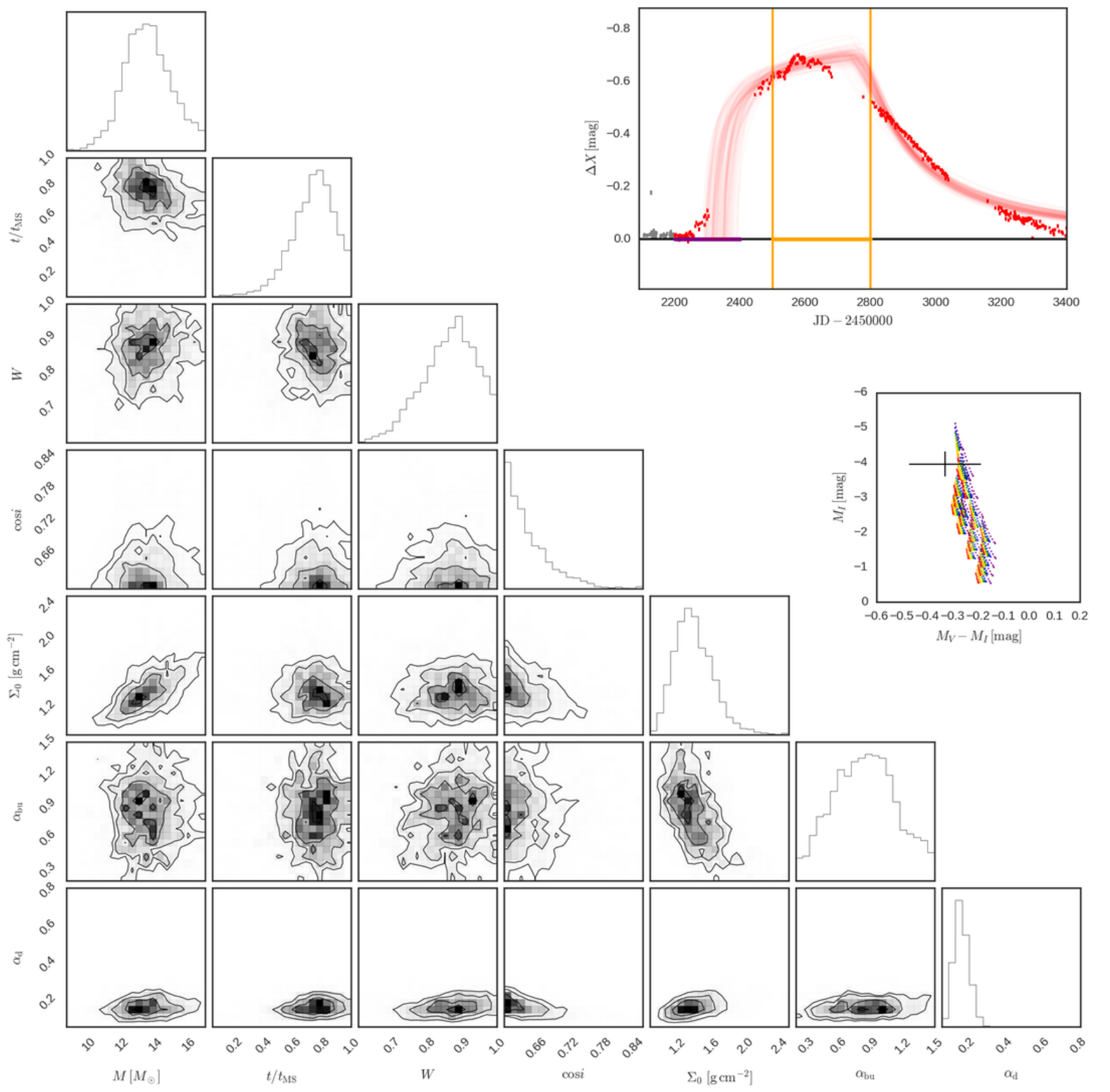

Figure C9. Same as Fig. 8 for SMC_SC3 15970 and bump ID 02 . 


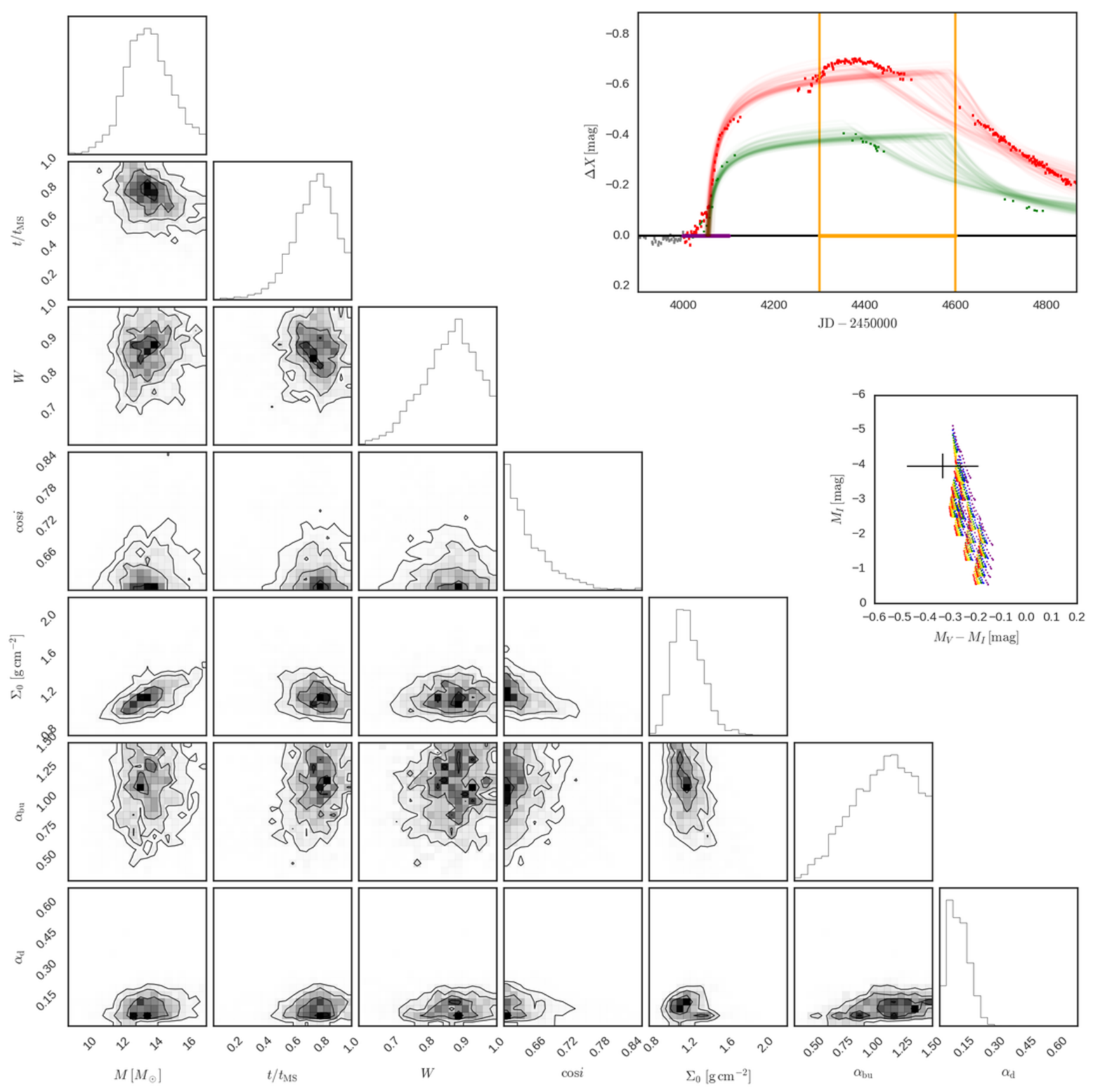

Figure C10. Same as Fig. 8 for SMC_SC3 15970 and bump ID 03. 

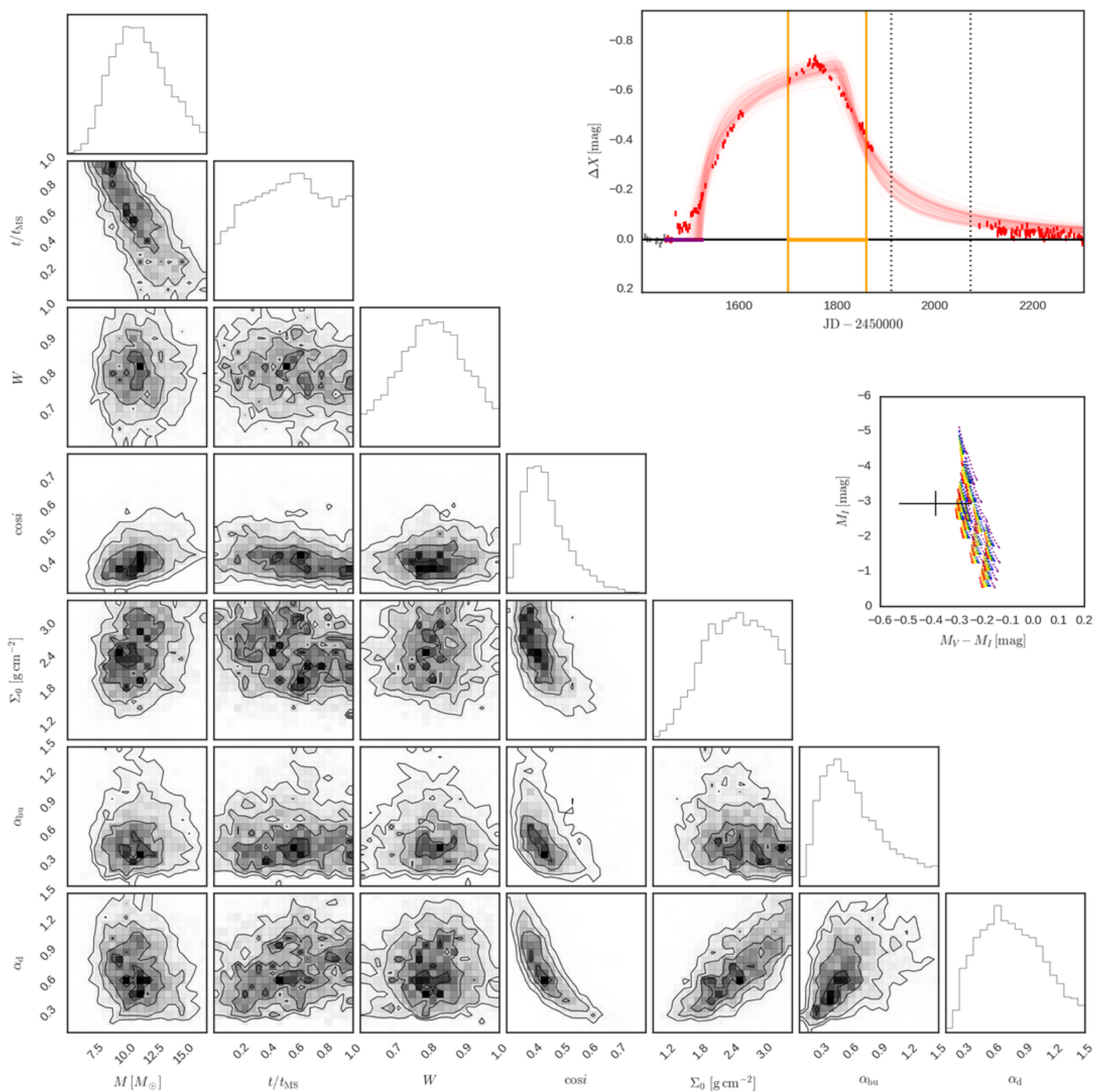

$$
M_{V}-M_{I}[\mathrm{mag}]
$$
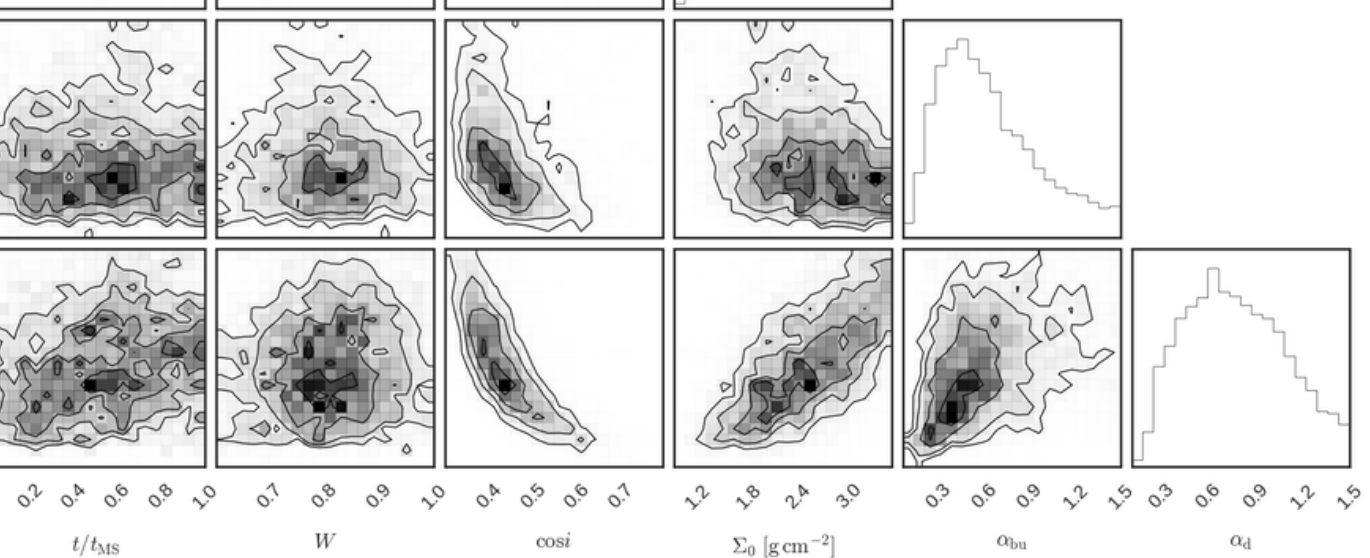

Figure C11. Same as Fig. 8 for SMC_SC3 71445 and bump ID 01. 


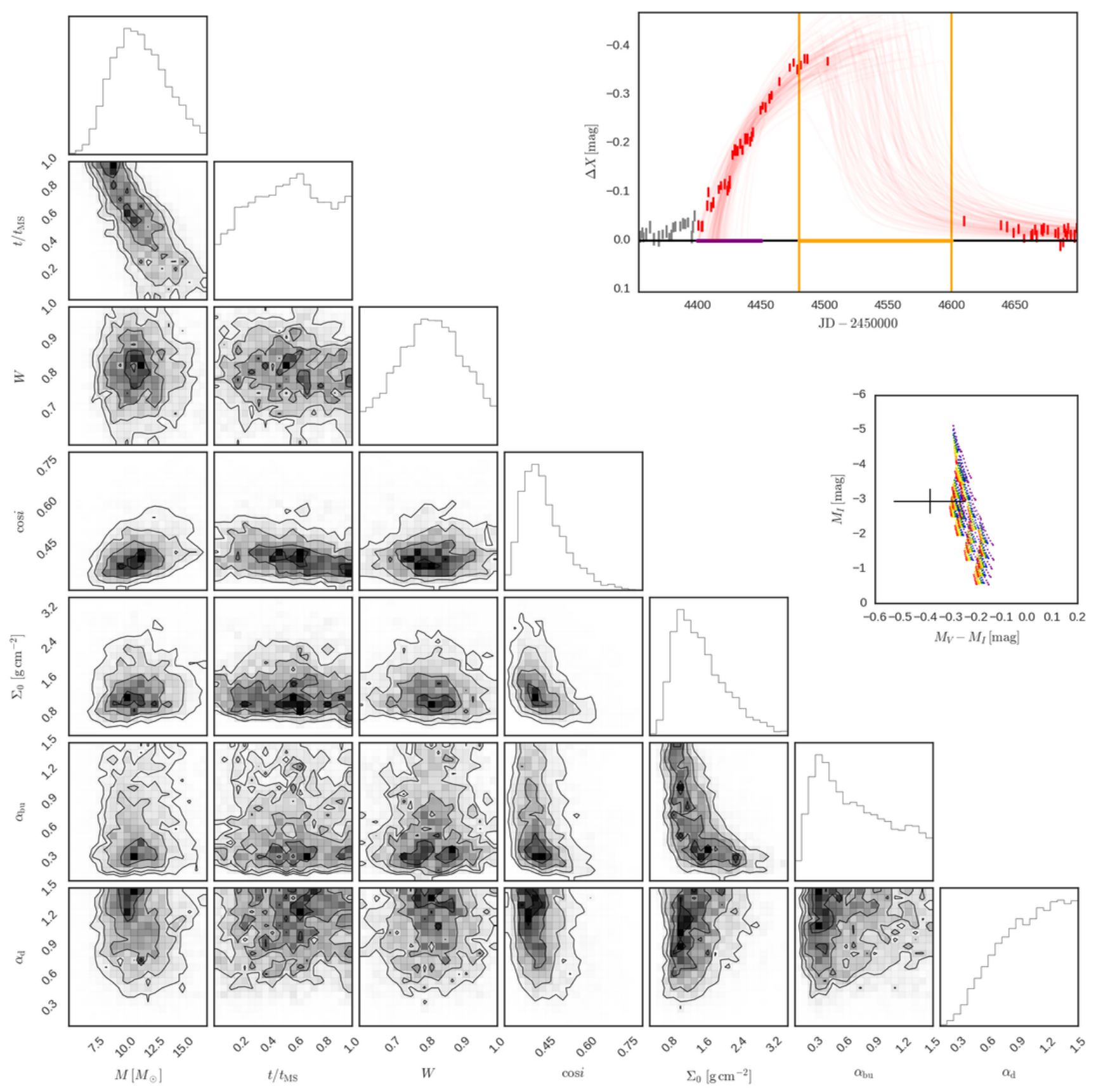

Figure C12. Same as Fig. 8 for SMC_SC3 71445 and bump ID 02. 


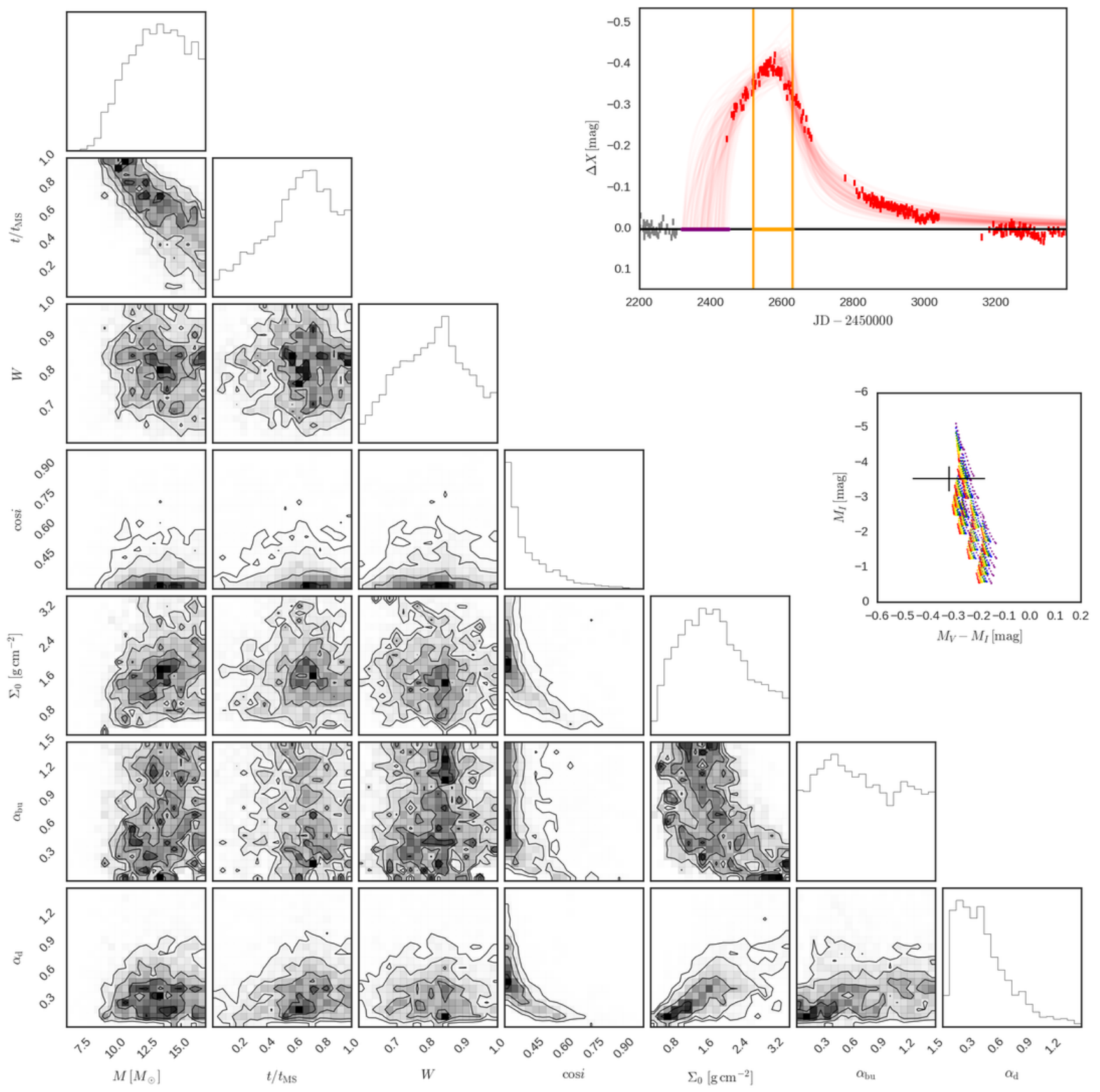

Figure C13. Same as Fig. 8 for SMC_SC3 125899 and bump ID 01. 


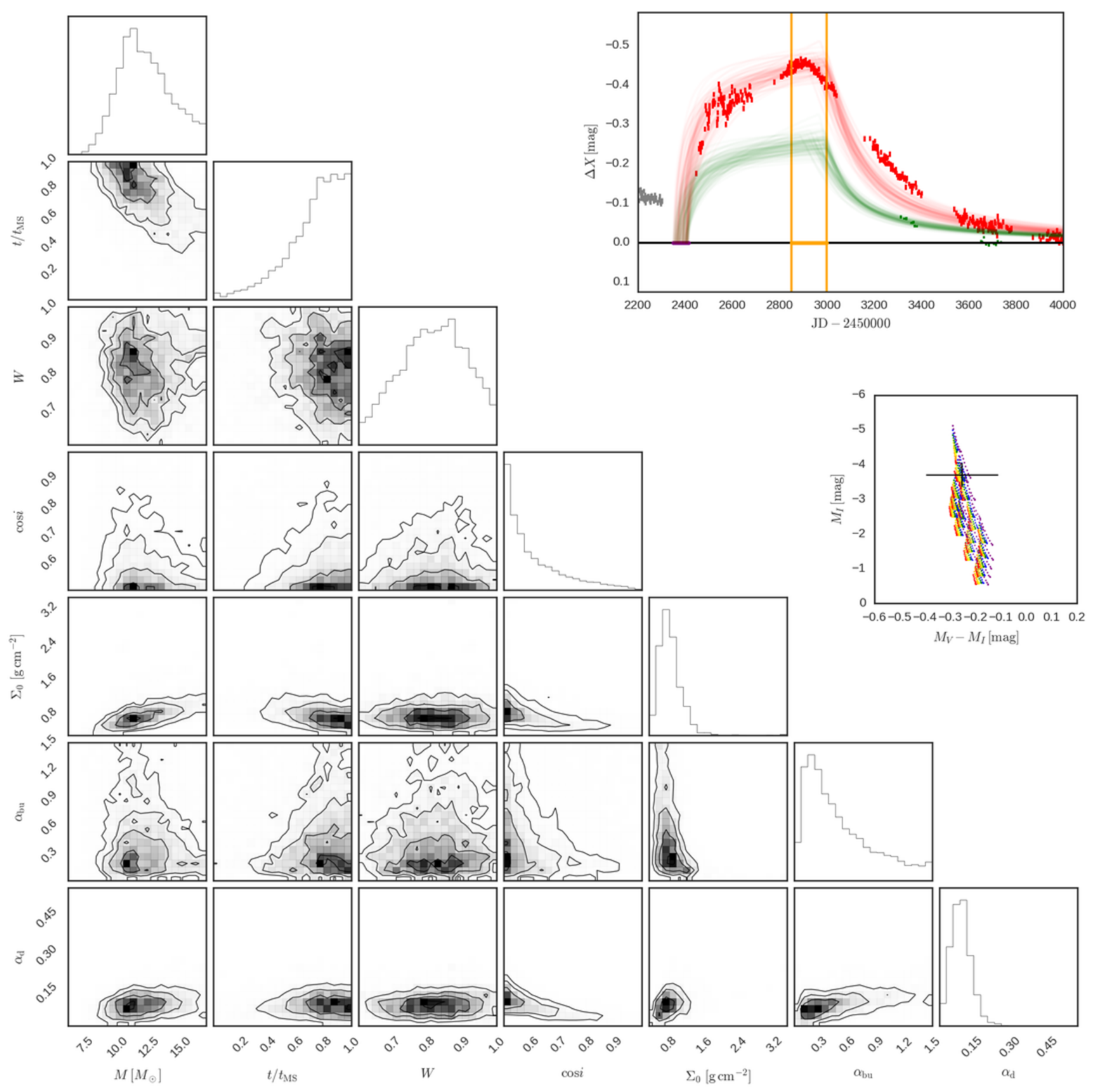

Figure C14. Same as Fig. 8 for SMC_SC3 197941 and bump ID 01. 


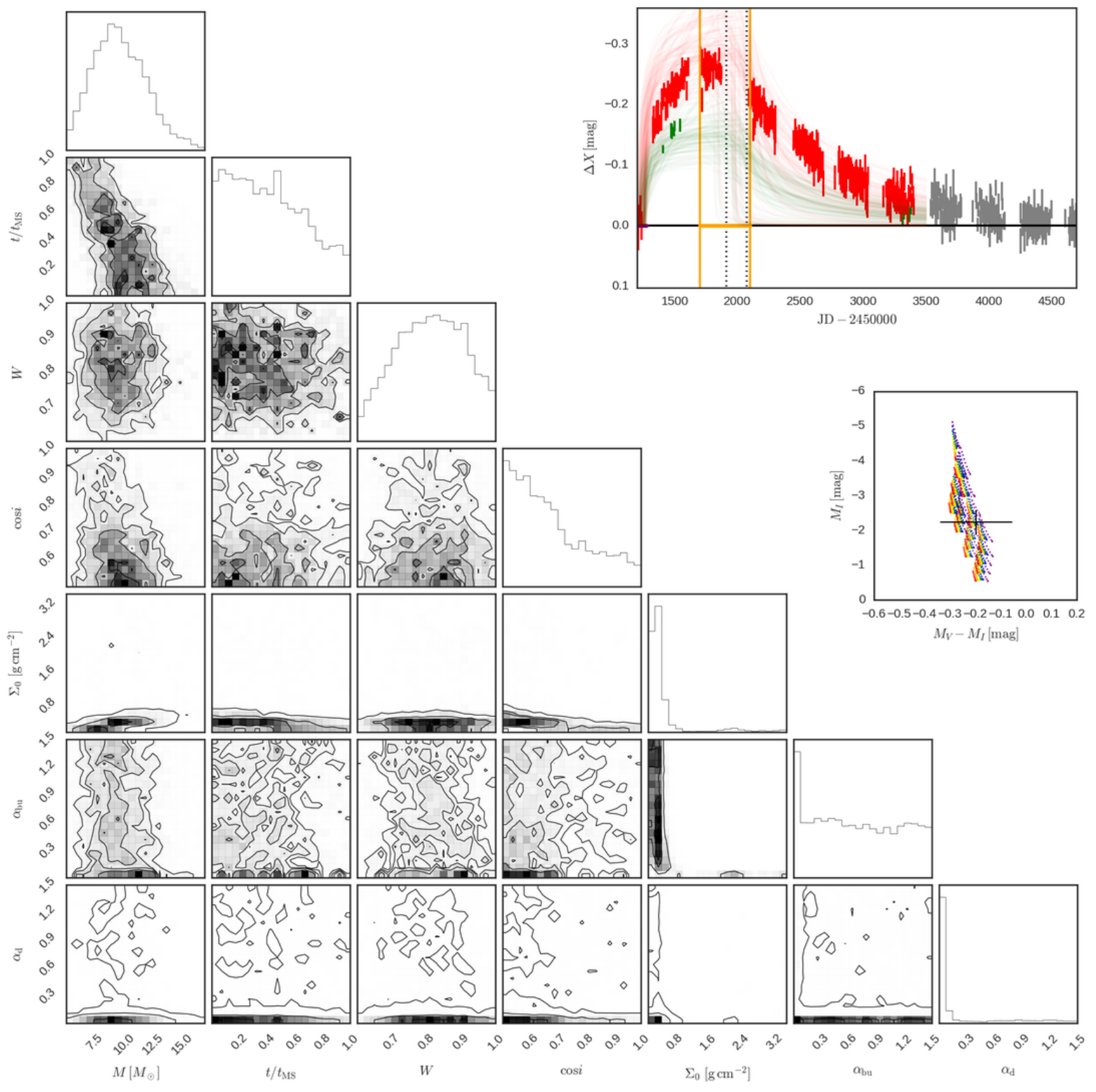

Figure C15. Same as Fig. 8 for SMC_SC4 22859 and bump ID 01. 


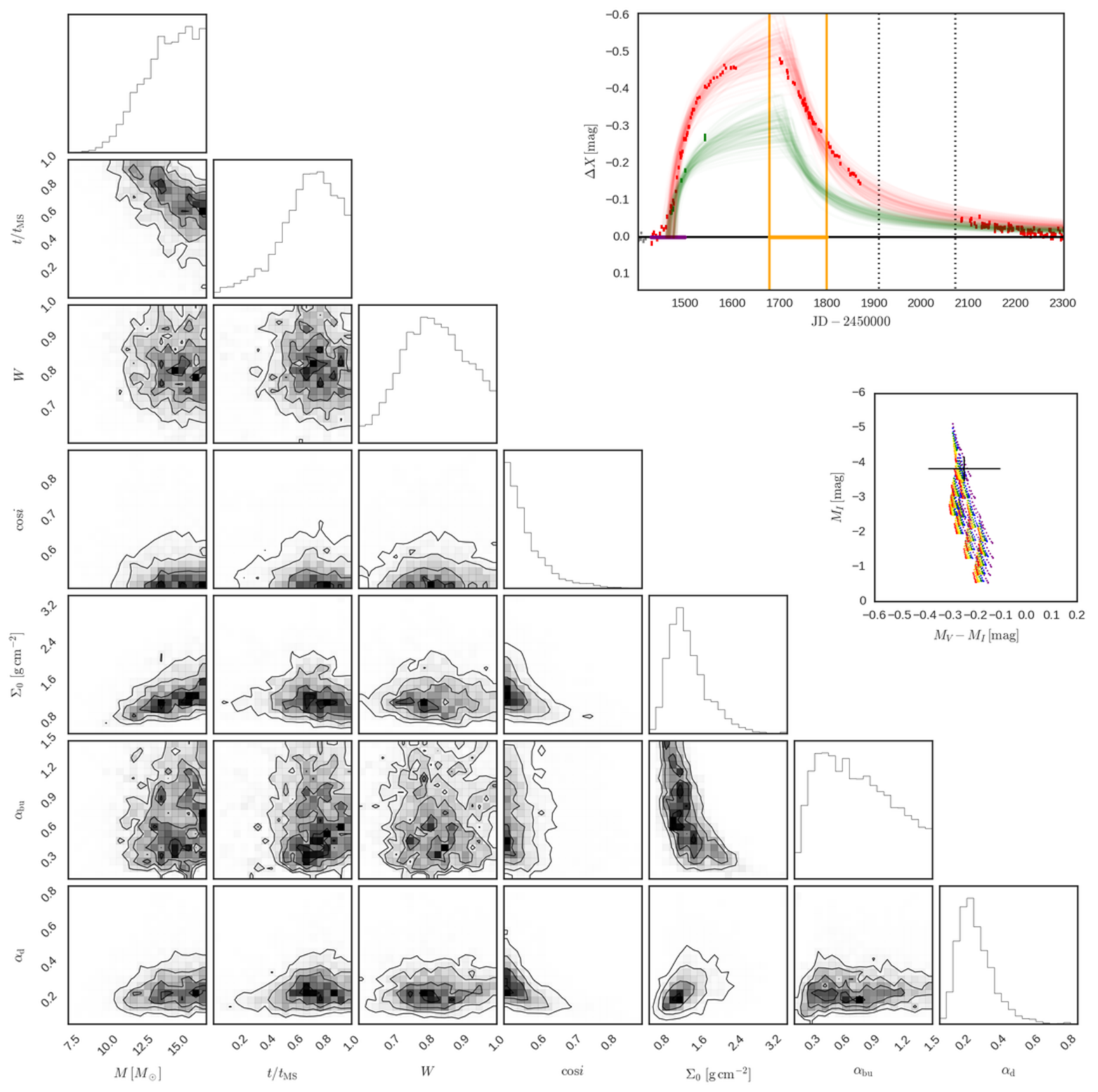

Figure C16. Same as Fig. 8 for SMC_SC4 71499 and bump ID 01. 

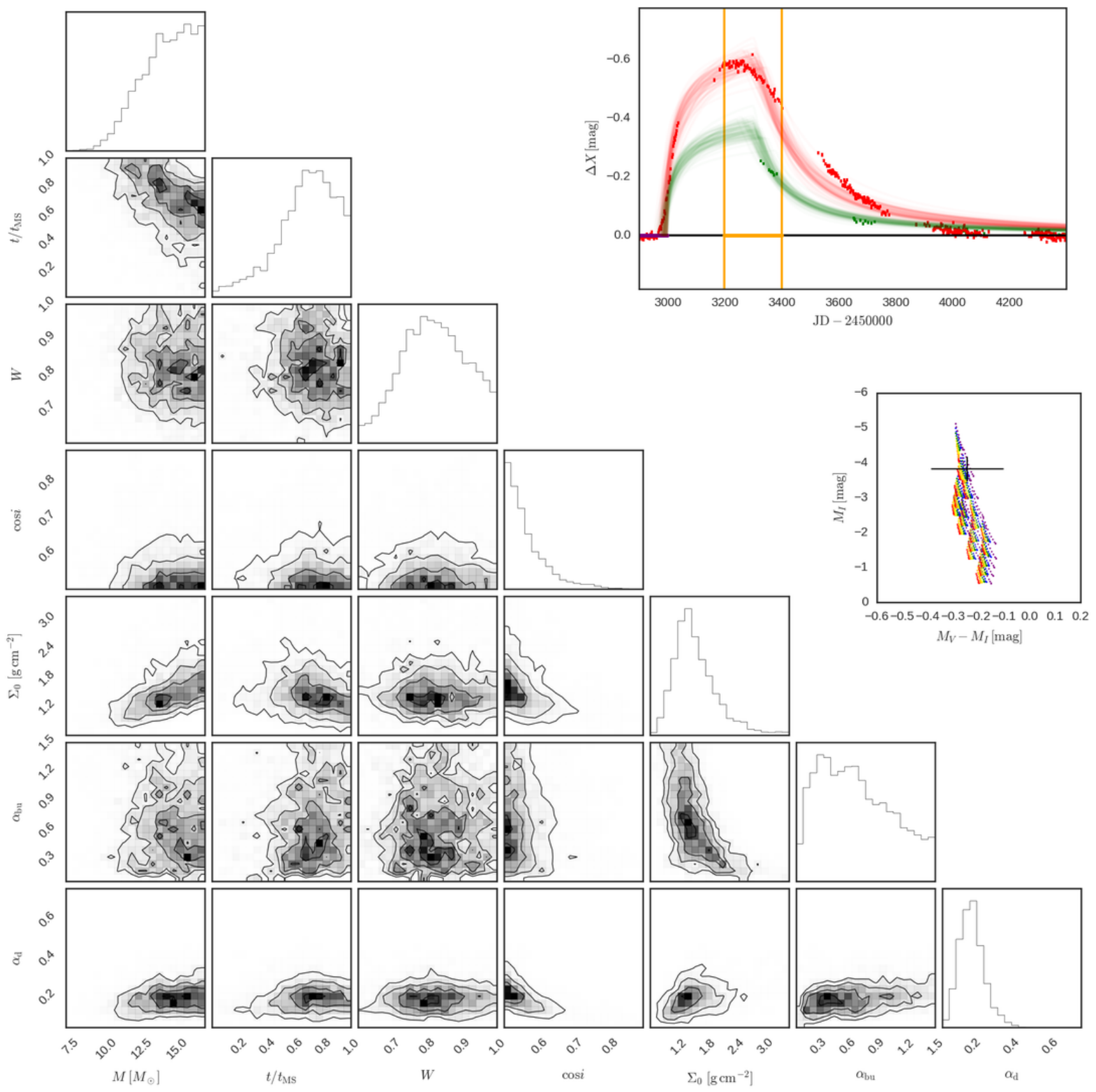

$$
M_{V}-M_{I}[\mathrm{mag}]
$$
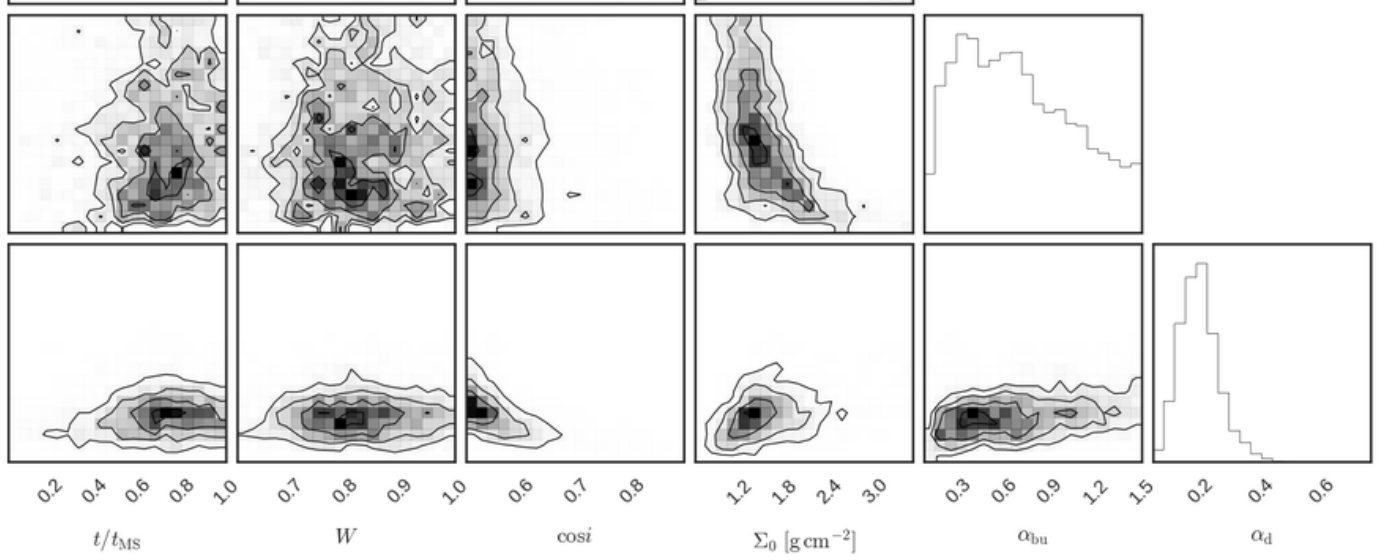

Figure C17. Same as Fig. 8 for SMC_SC4 71499 and bump ID 02. 


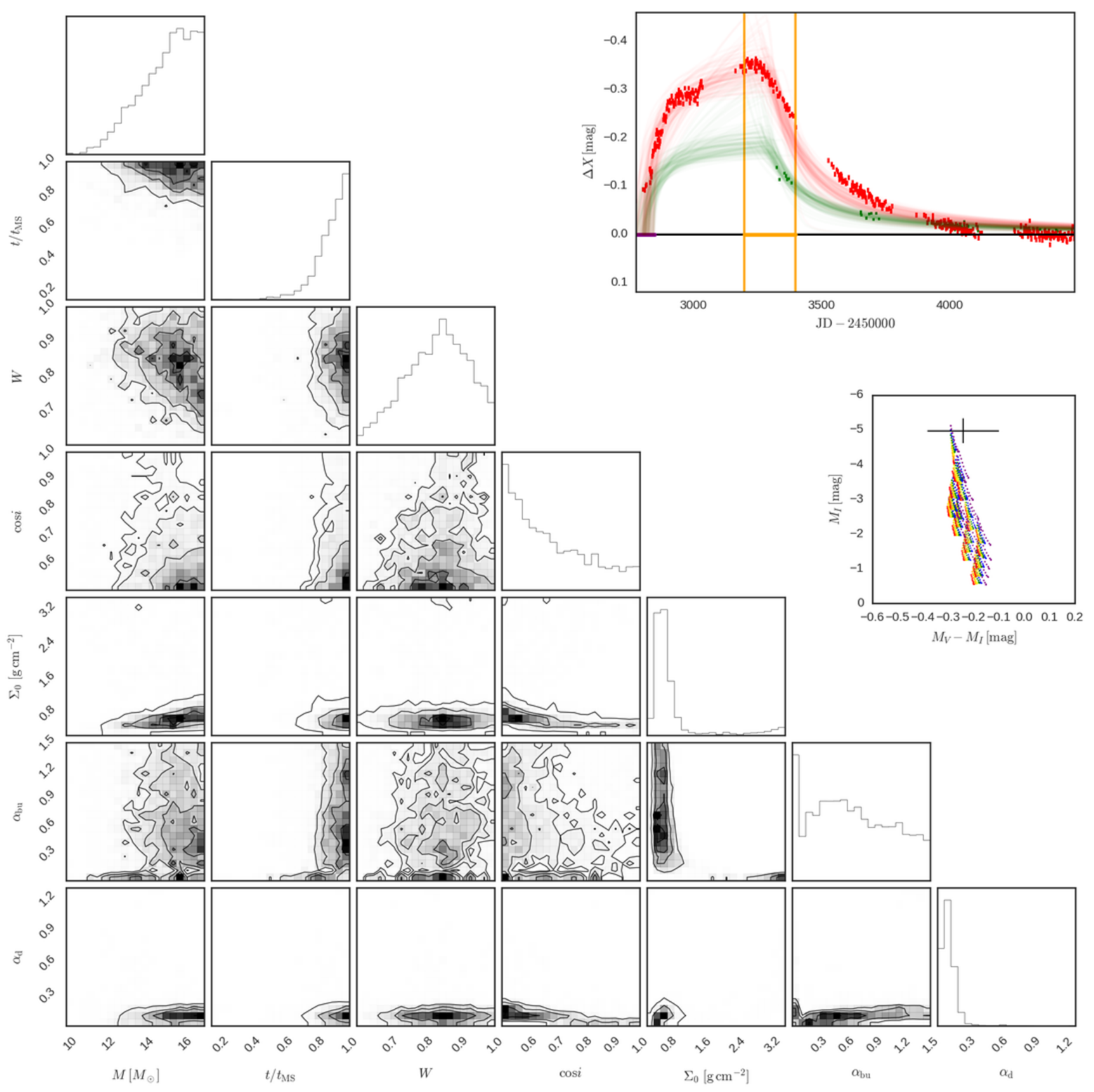

Figure C18. Same as Fig. 8 for SMC_SC4 120783 and bump ID 01. 


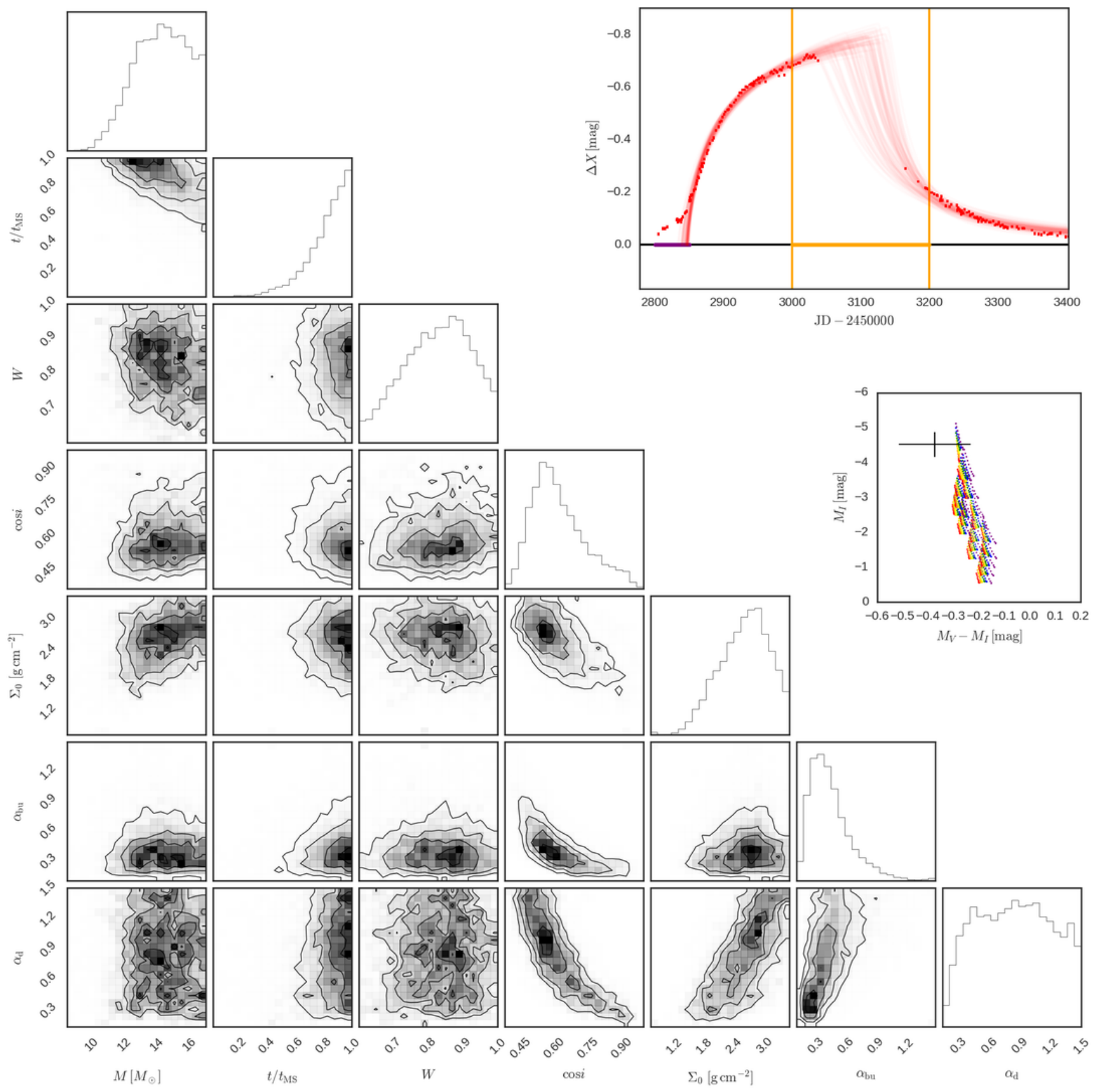

Figure C19. Same as Fig. 8 for SMC_SC4 127840 and bump ID 01. 


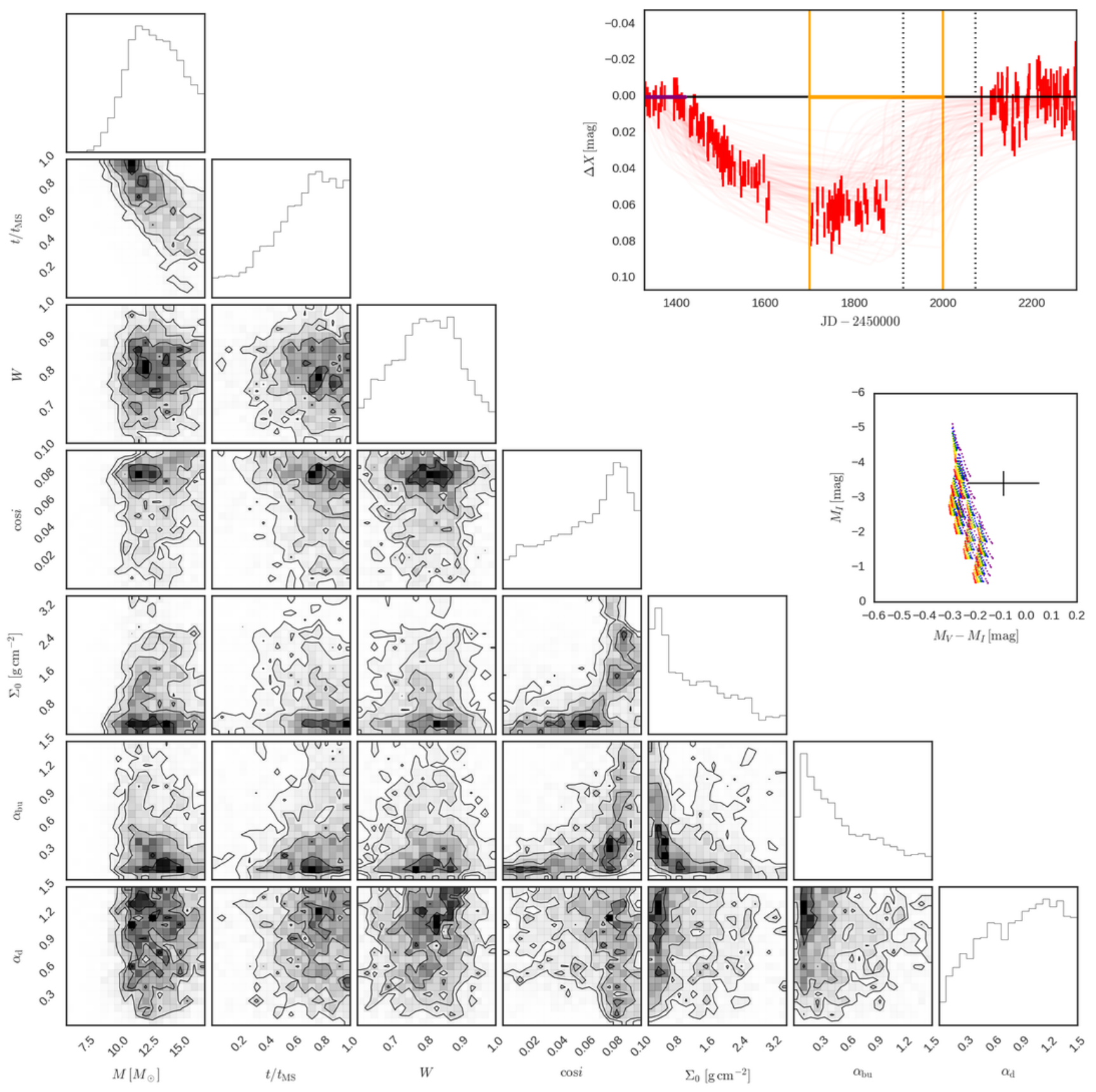

Figure C20. Same as Fig. 8 for SMC_SC4 156248 and bump ID 01. 


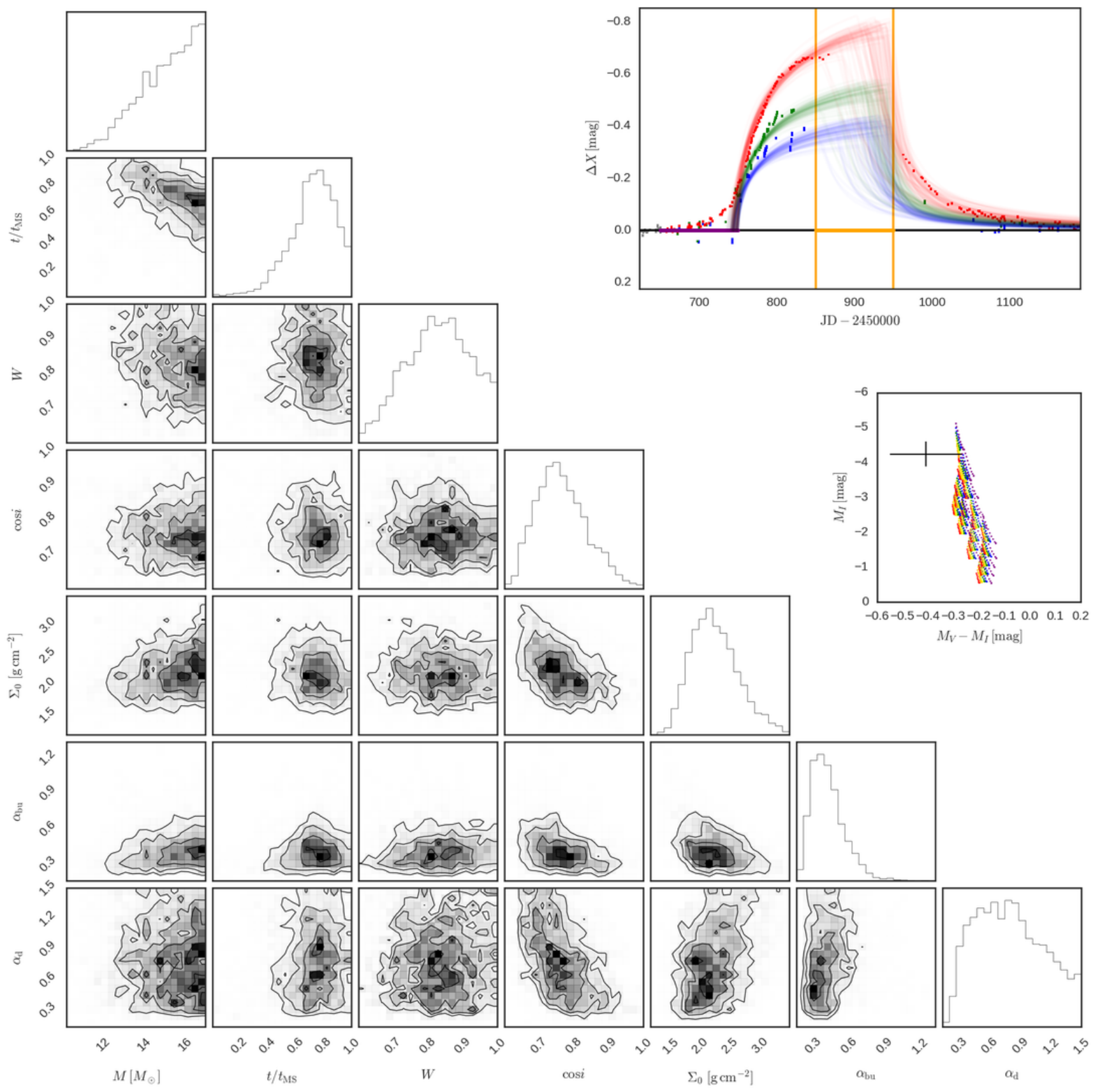

Figure C21. Same as Fig. 8 for SMC_SC4 156251 and bump ID 01. 


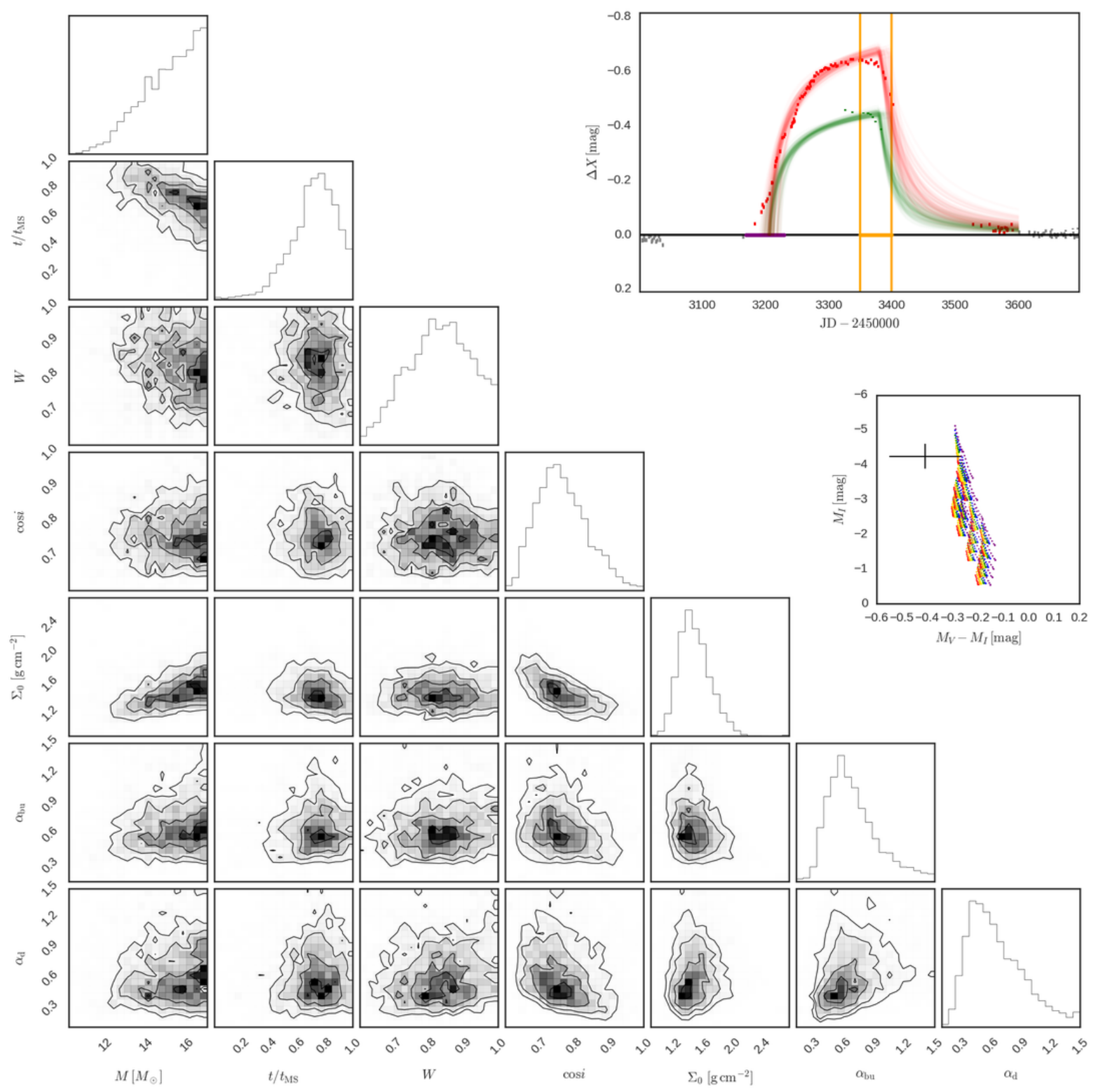

Figure C22. Same as Fig. 8 for SMC_SC4 156251 and bump ID 02. 

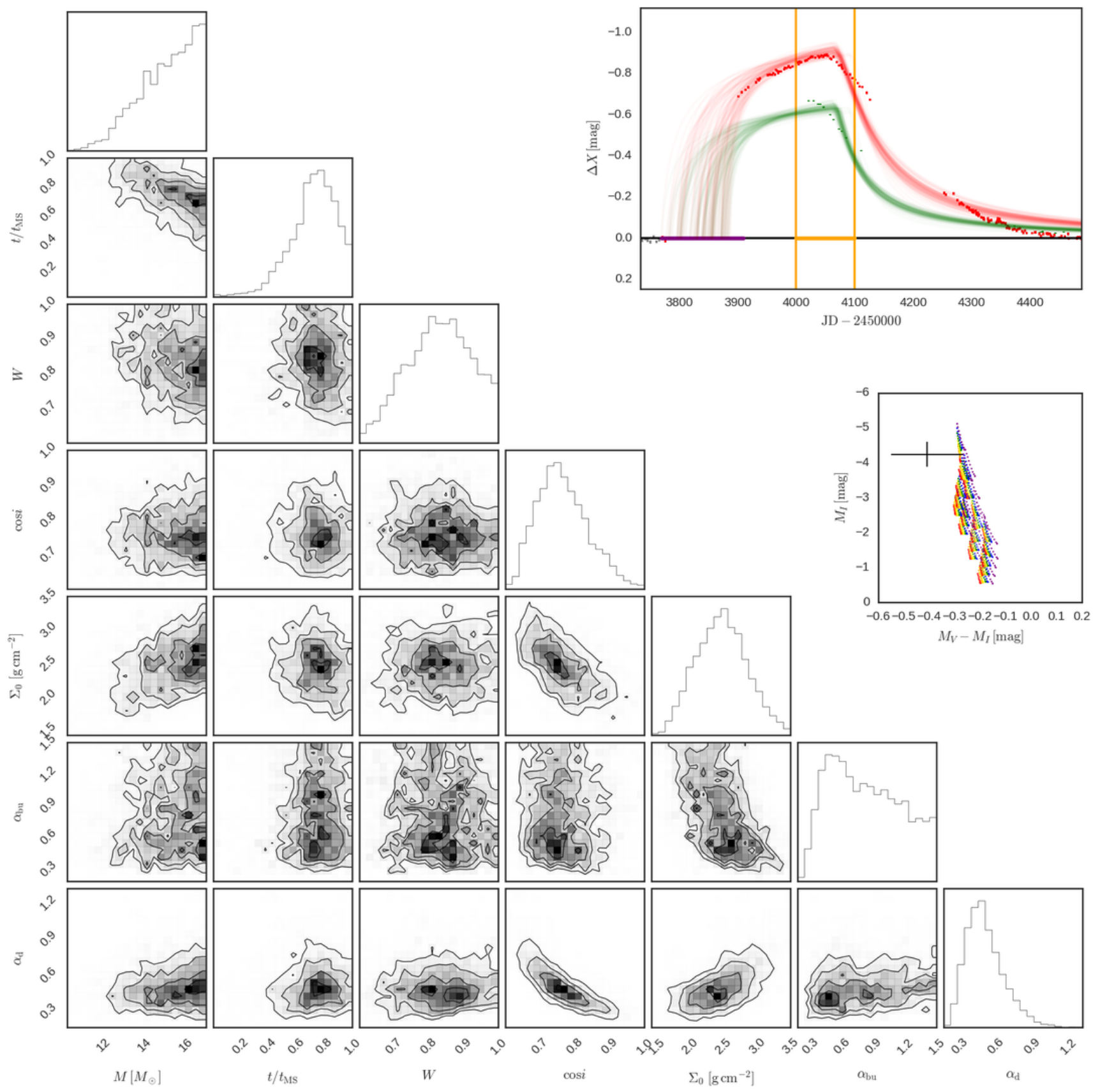

$$
M_{V}-M_{I}[\mathrm{mag}]
$$
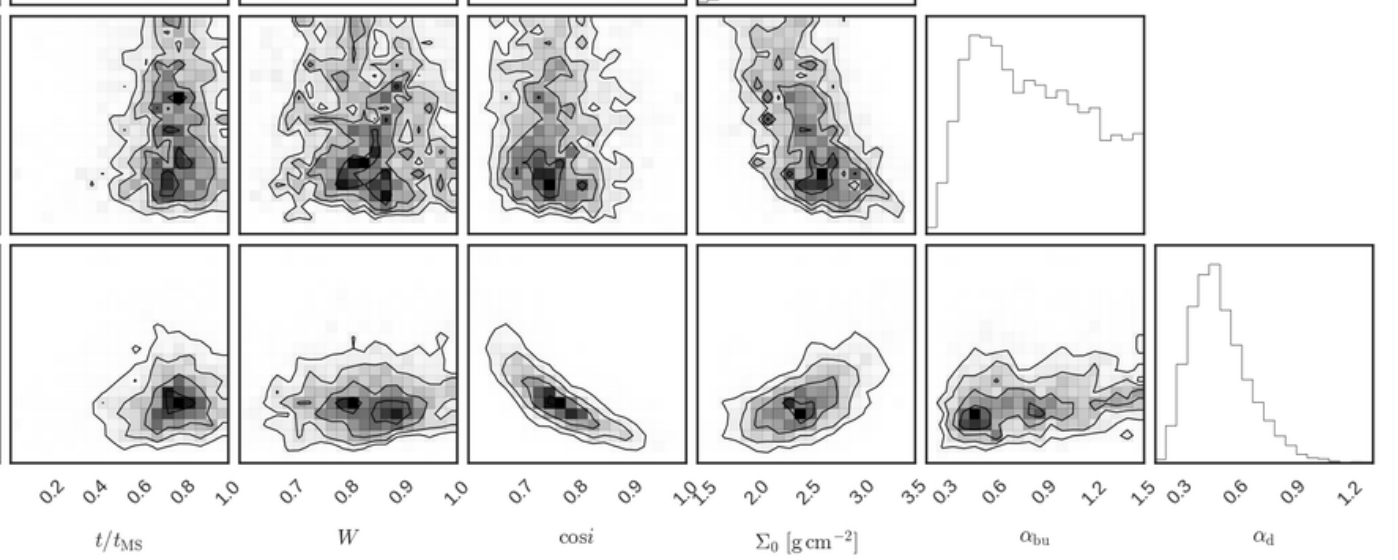

Figure C23. Same as Fig. 8 for SMC_SC4 156251 and bump ID 03. 


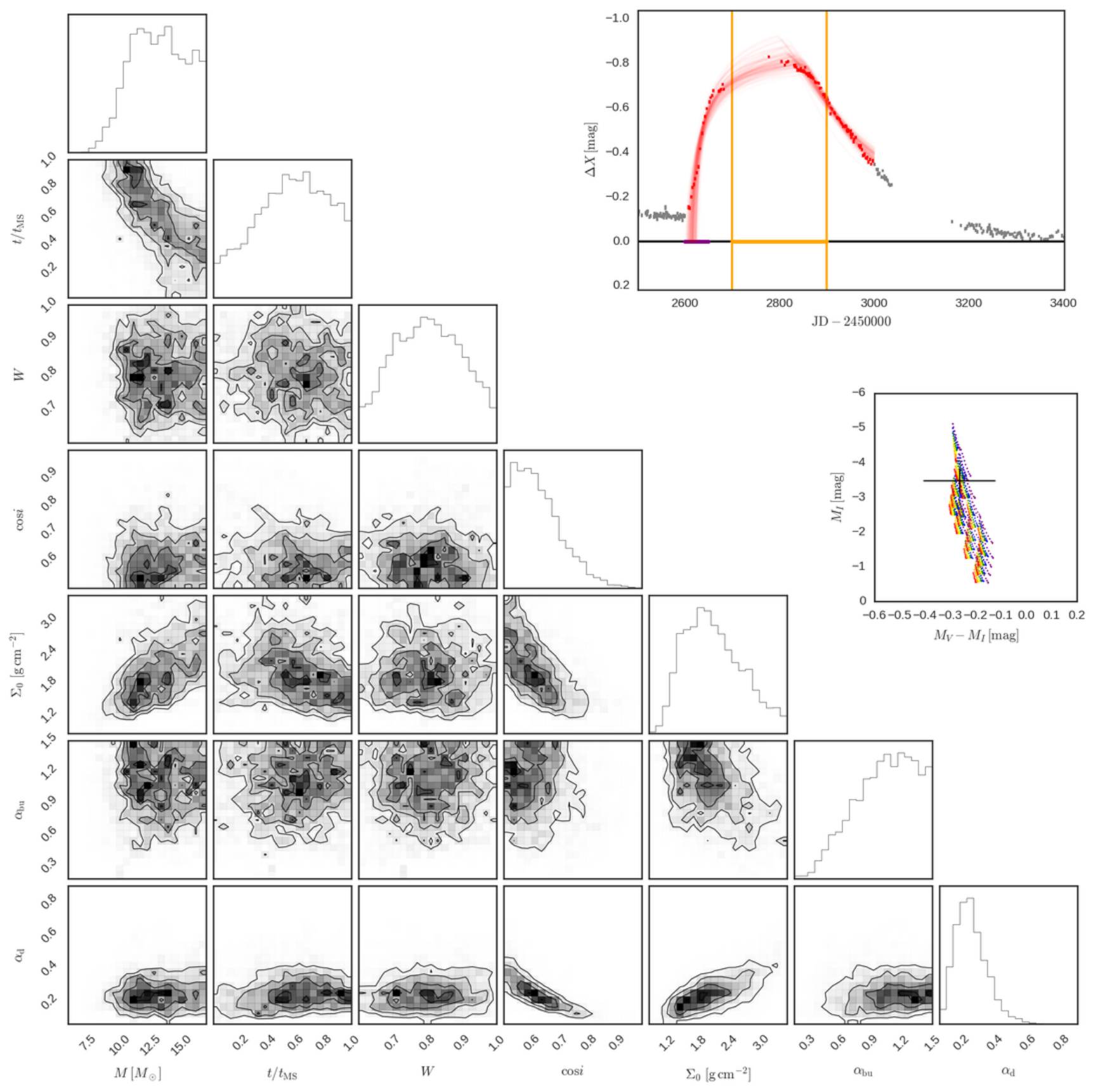

Figure C24. Same as Fig. 8 for SMC_SC4 159829 and bump ID 01. 

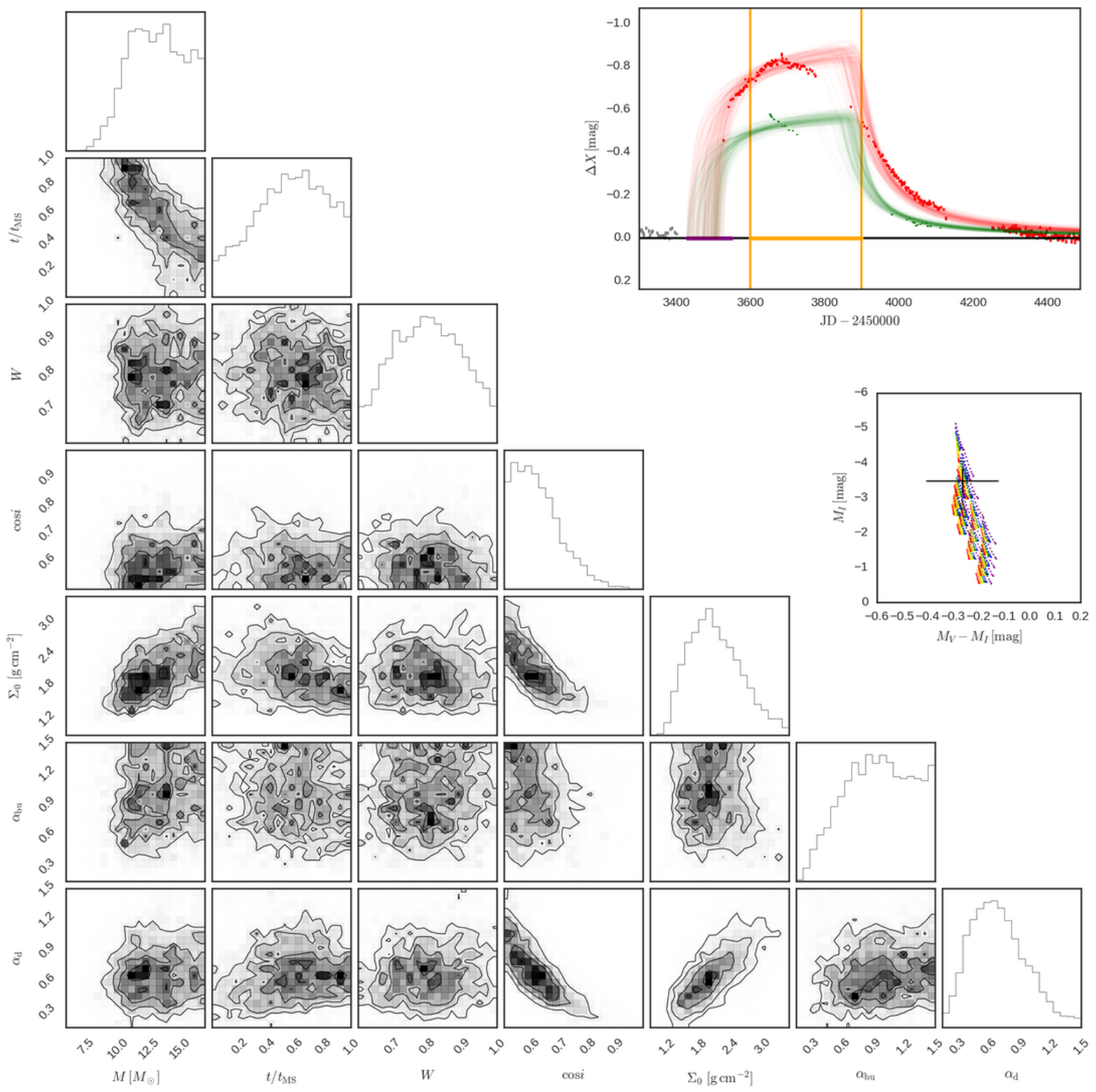

$$
M_{V}-M_{I}[\mathrm{mag}]
$$
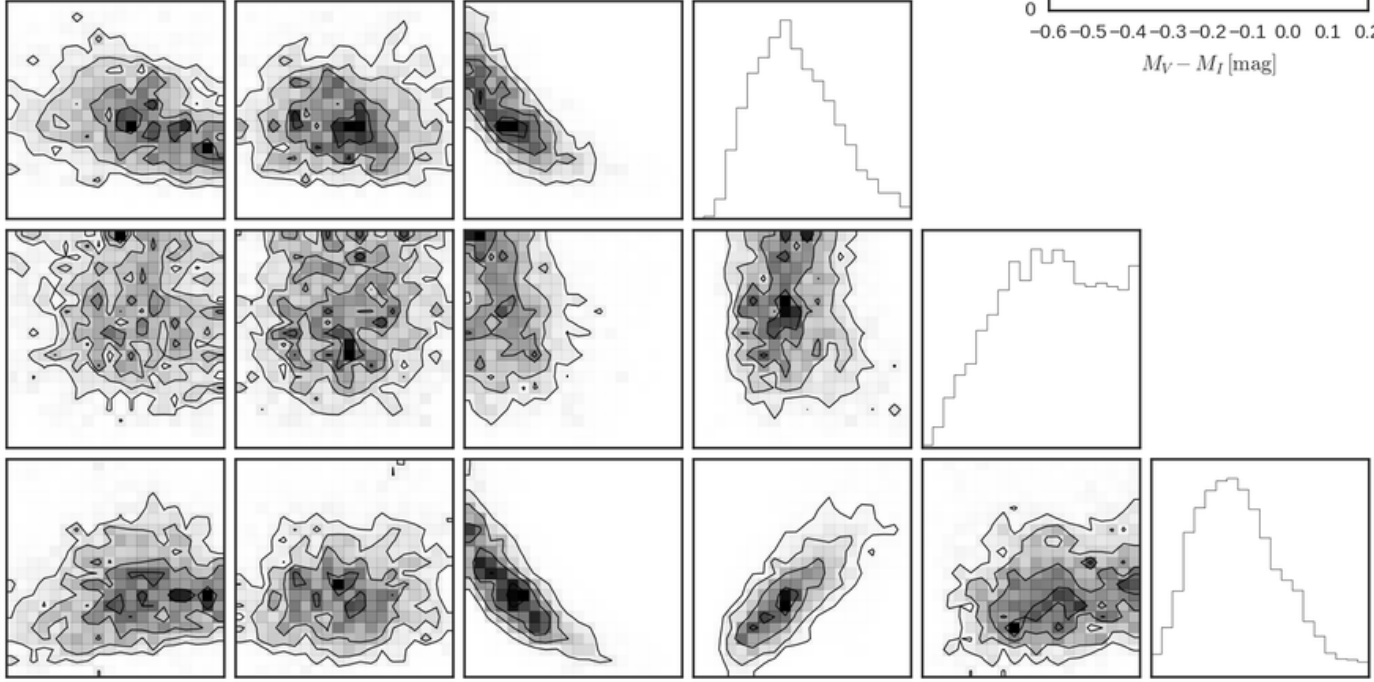

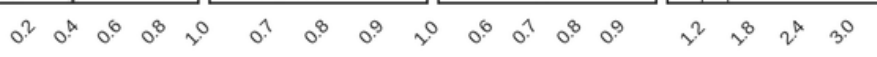

$\operatorname{cosi}$

$\Sigma_{0}\left[\mathrm{~g} \mathrm{~cm}^{-2}\right]$

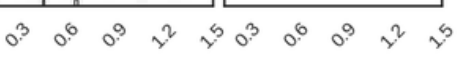

Figure C25. Same as Fig. 8 for SMC_SC4 159829 and bump ID 02. 


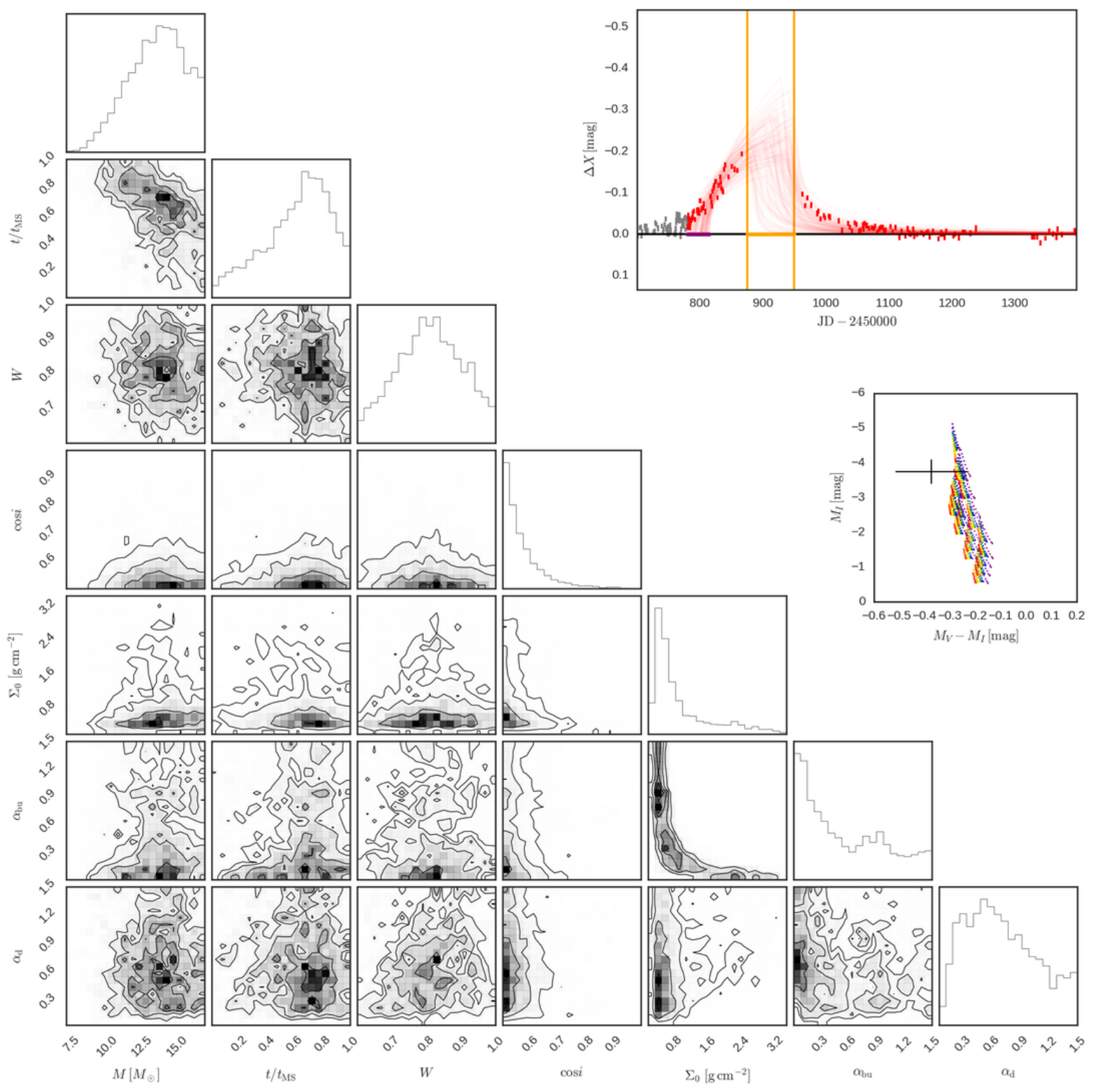

Figure C26. Same as Fig. 8 for SMC_SC4 159857 and bump ID 01. 

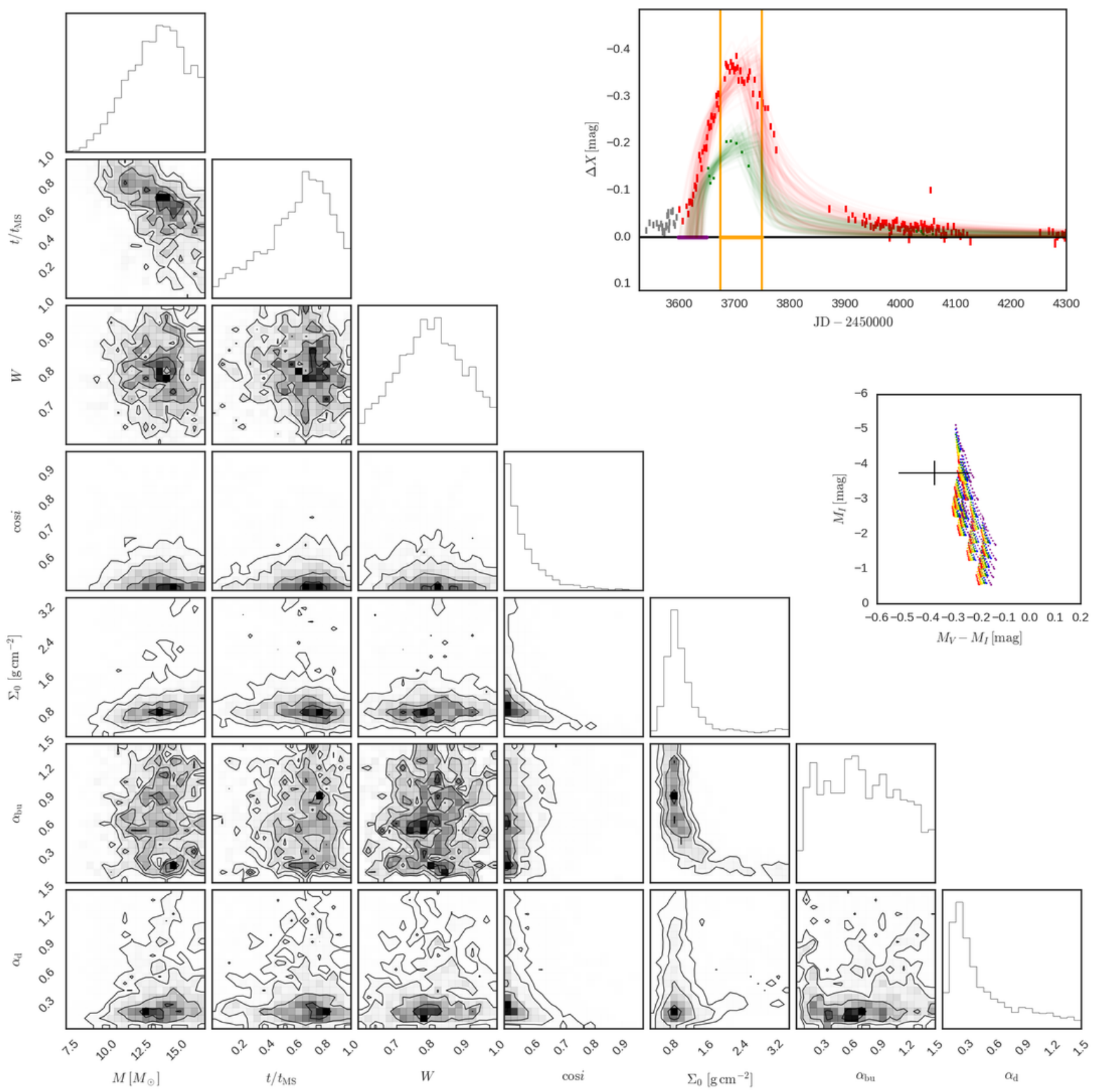

Figure C27. Same as Fig. 8 for SMC_SC4 159857 and bump ID 02. 


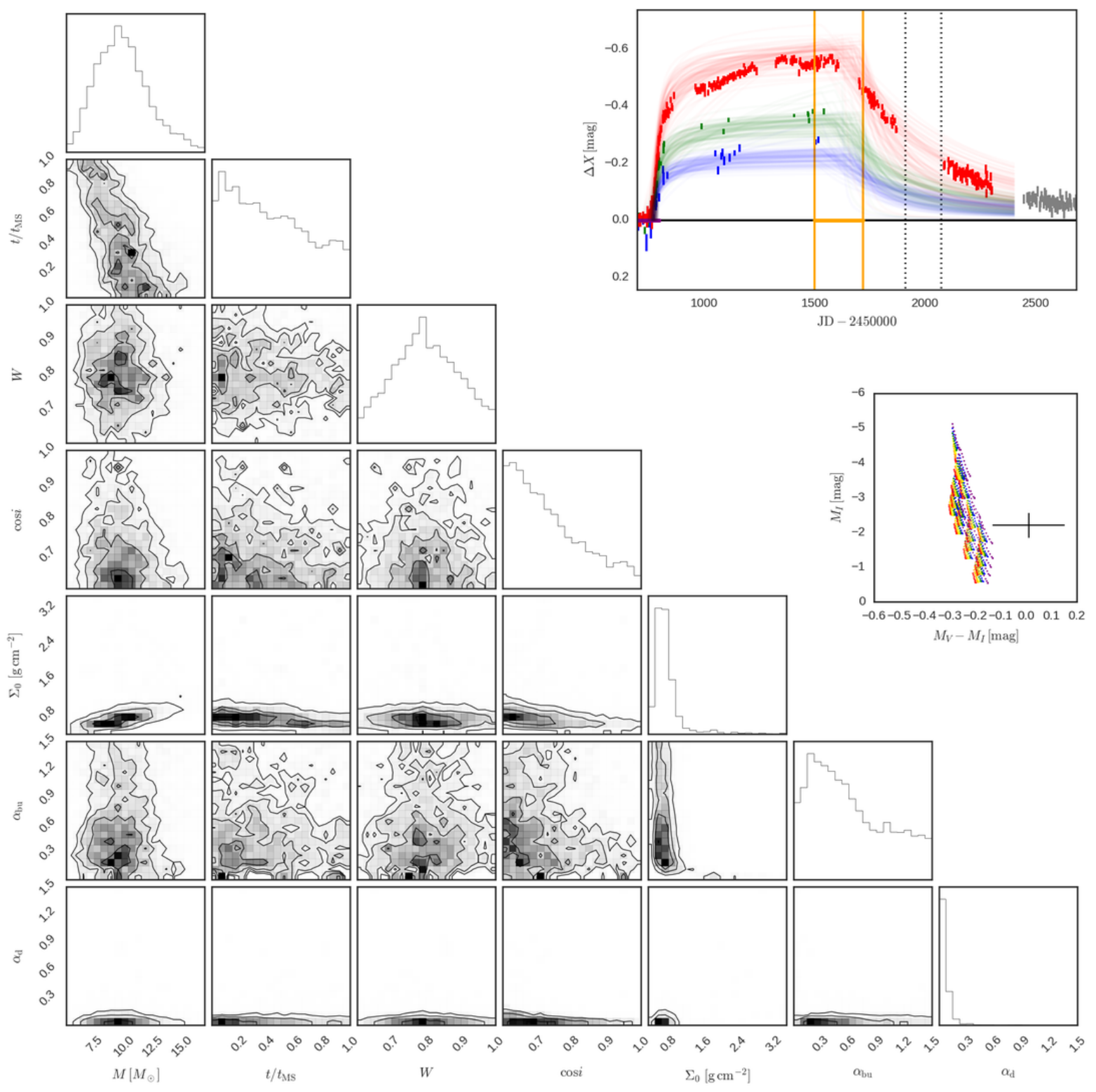

Figure C28. Same as Fig. 8 for SMC_SC4 163828 and bump ID 01. 

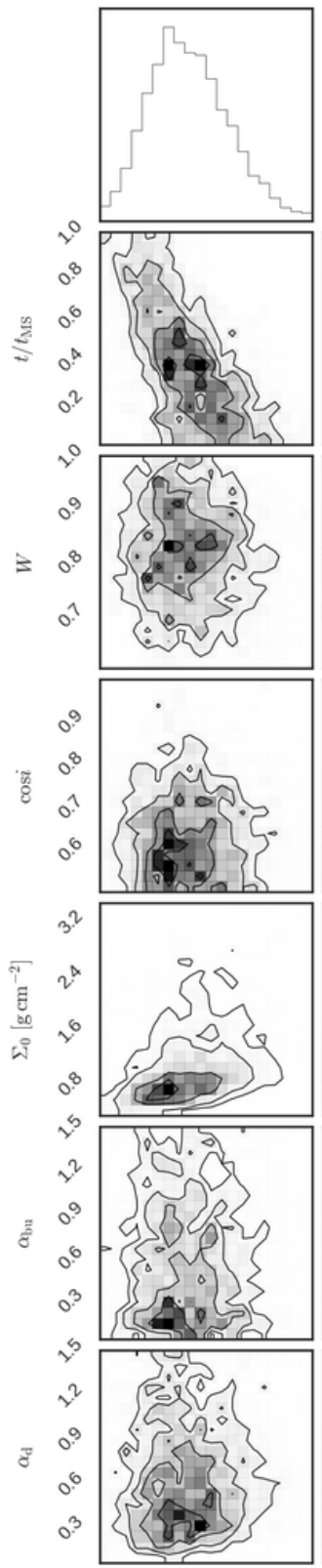

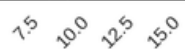
$M\left[M_{\odot}\right]$
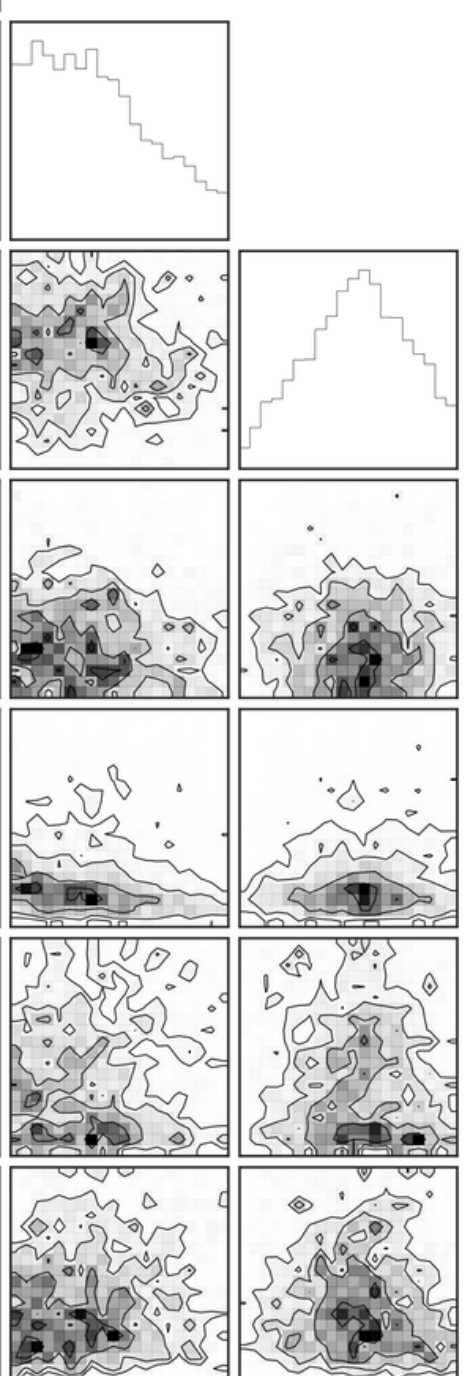

$0^{2} 0^{2} 0^{0} 0^{8} 2^{0}$

$t / t_{\mathrm{MS}}$
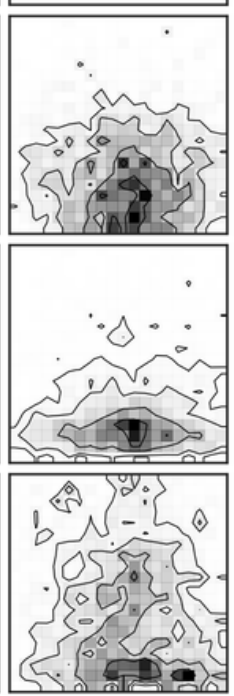
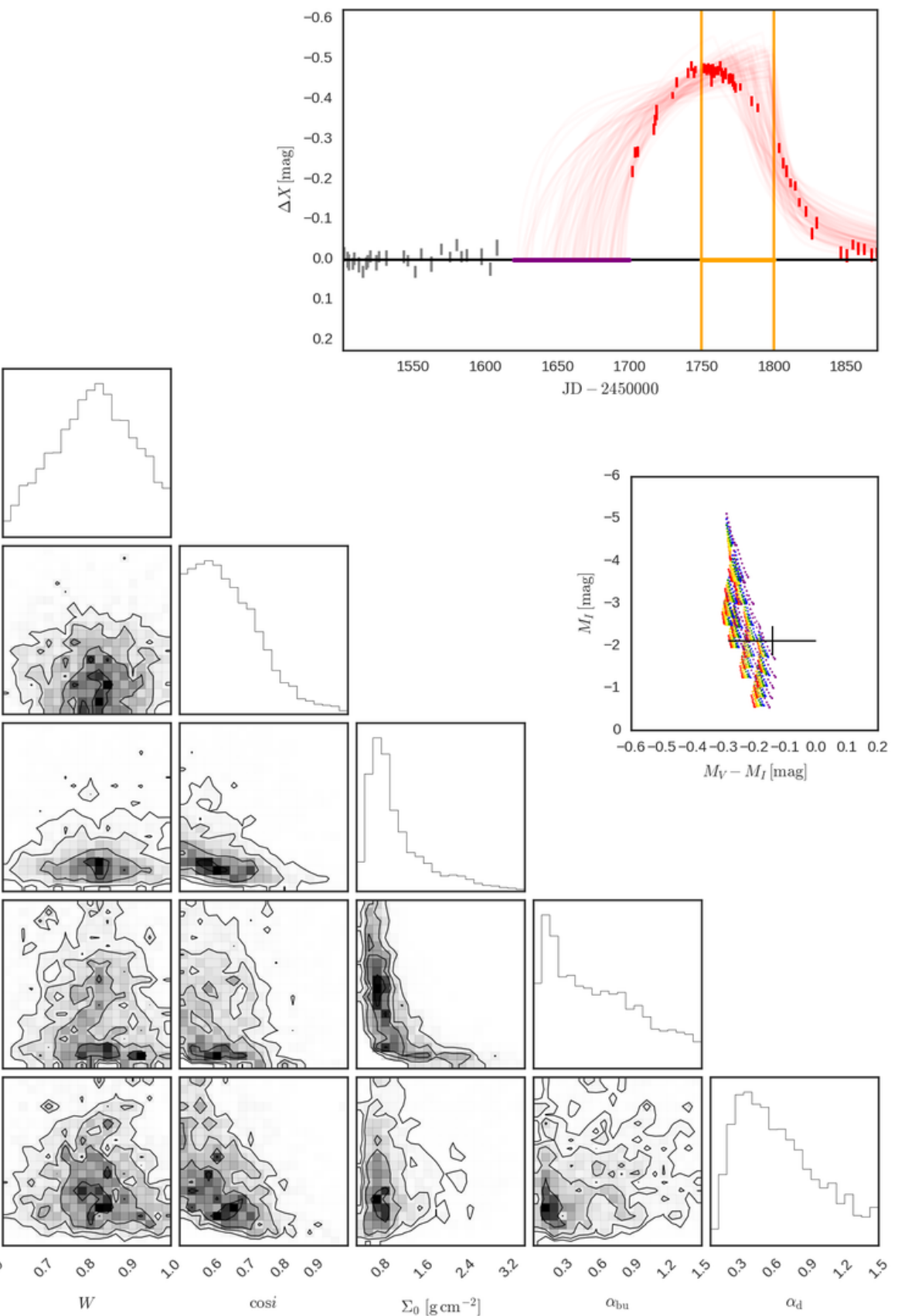

Figure C29. Same as Fig. 8 for SMC_SC4 167554 and bump ID 01. 


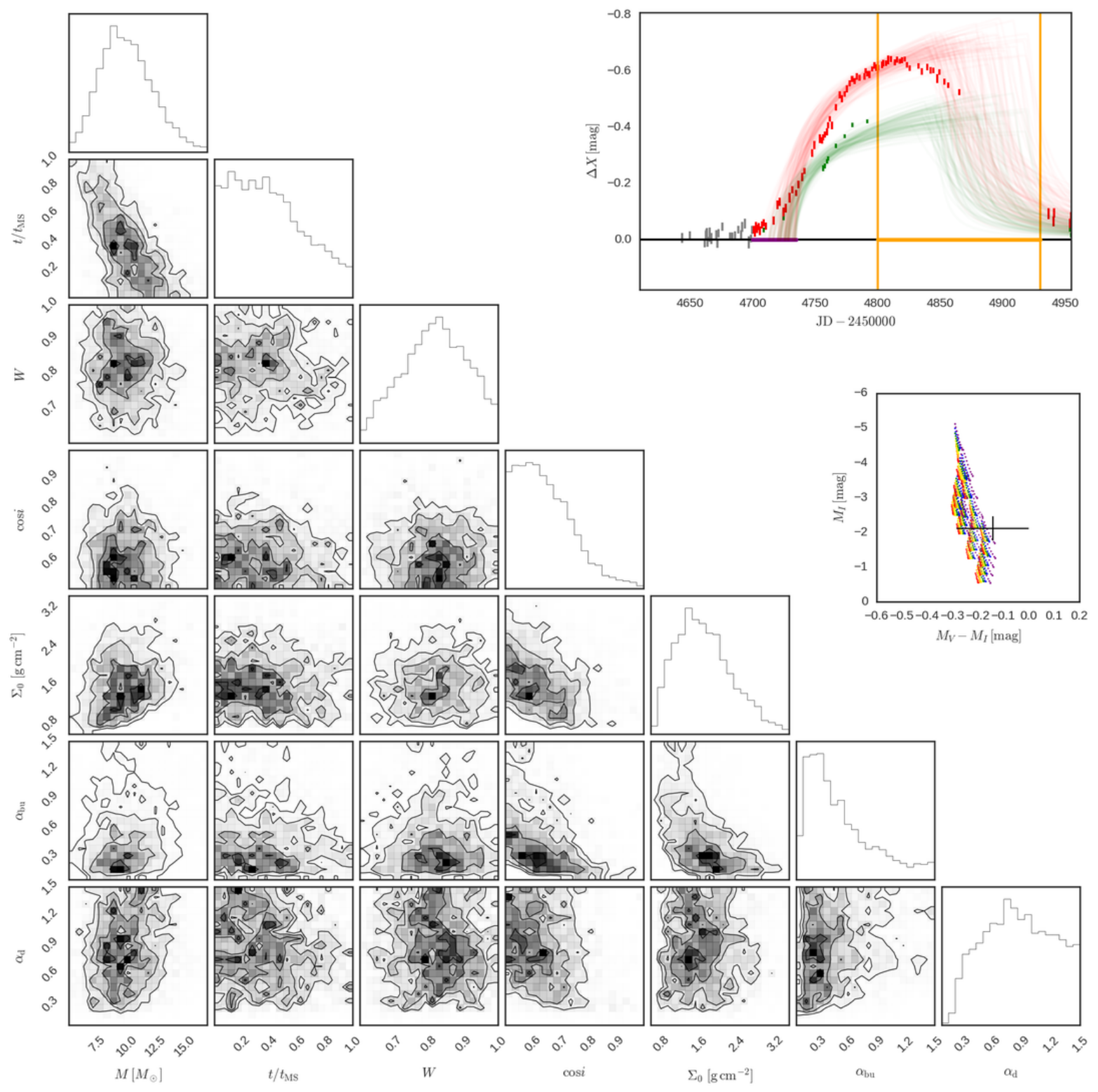

Figure C30. Same as Fig. 8 for SMC_SC4 167554 and bump ID 02. 

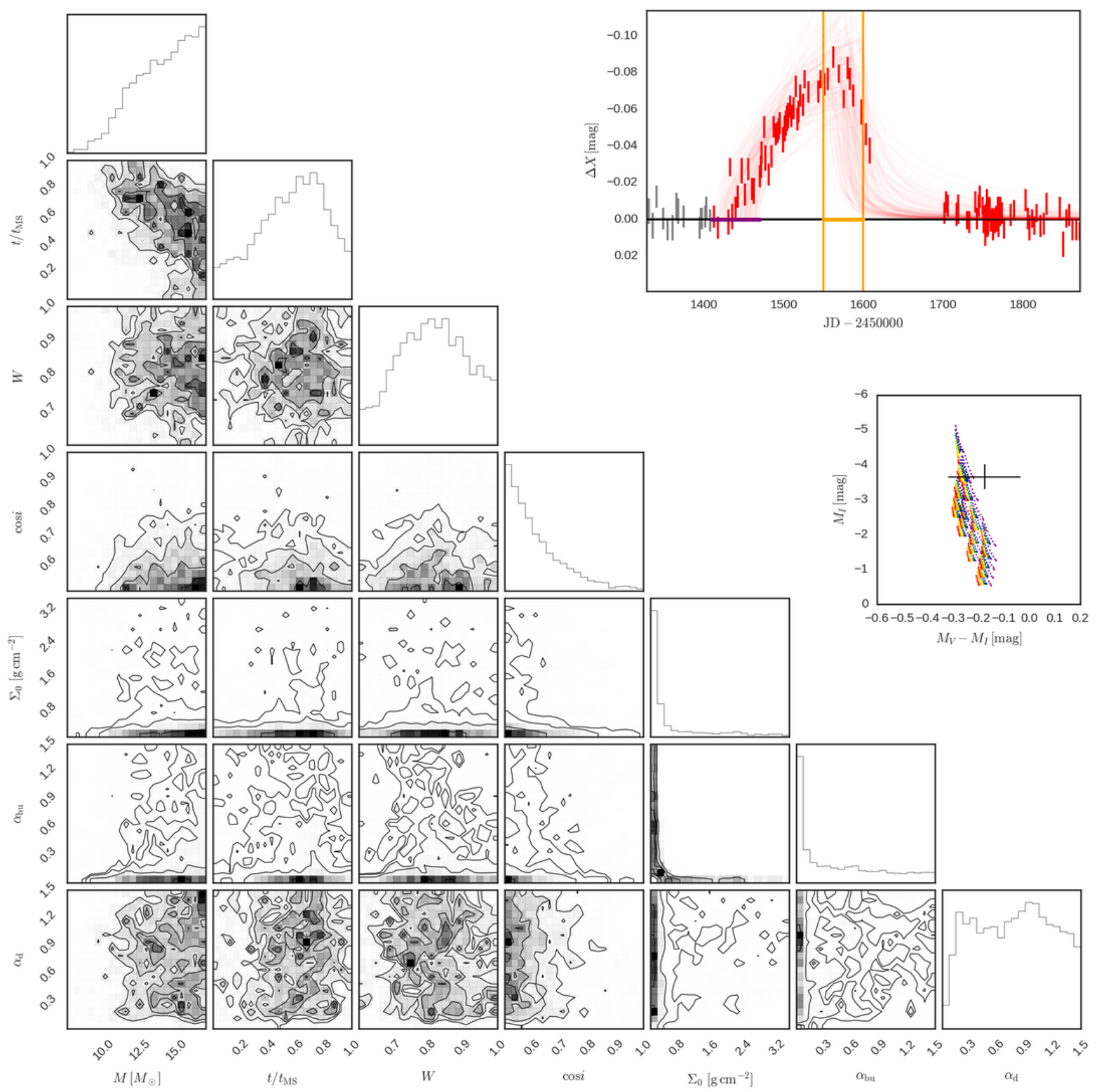

$$
M_{V}-M_{I}[\mathrm{mag}]
$$

Figure C31. Same as Fig. 8 for SMC_SC4 171253 and bump ID 01. 


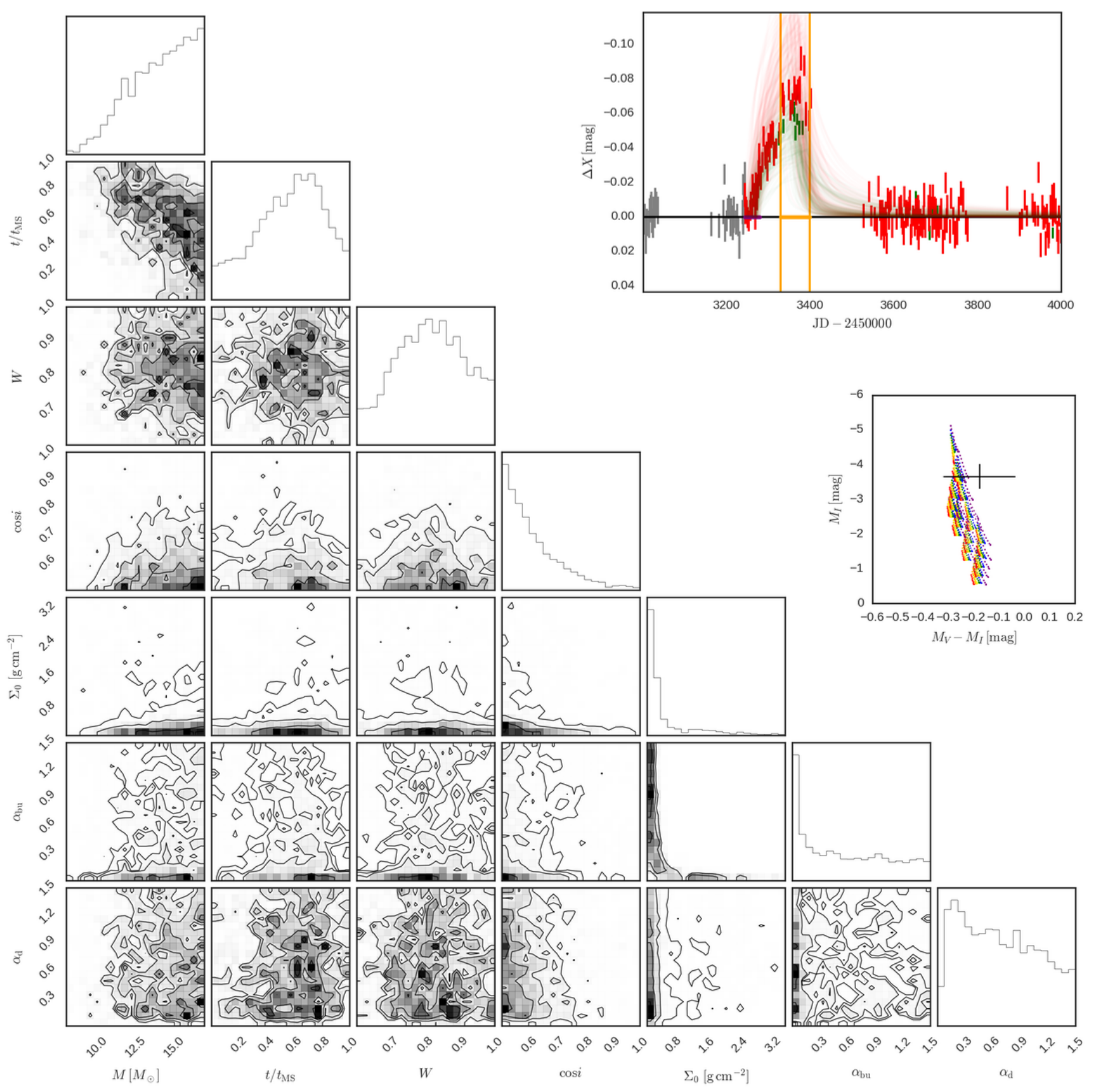

Figure C32. Same as Fig. 8 for SMC_SC4 171253 and bump ID 02. 

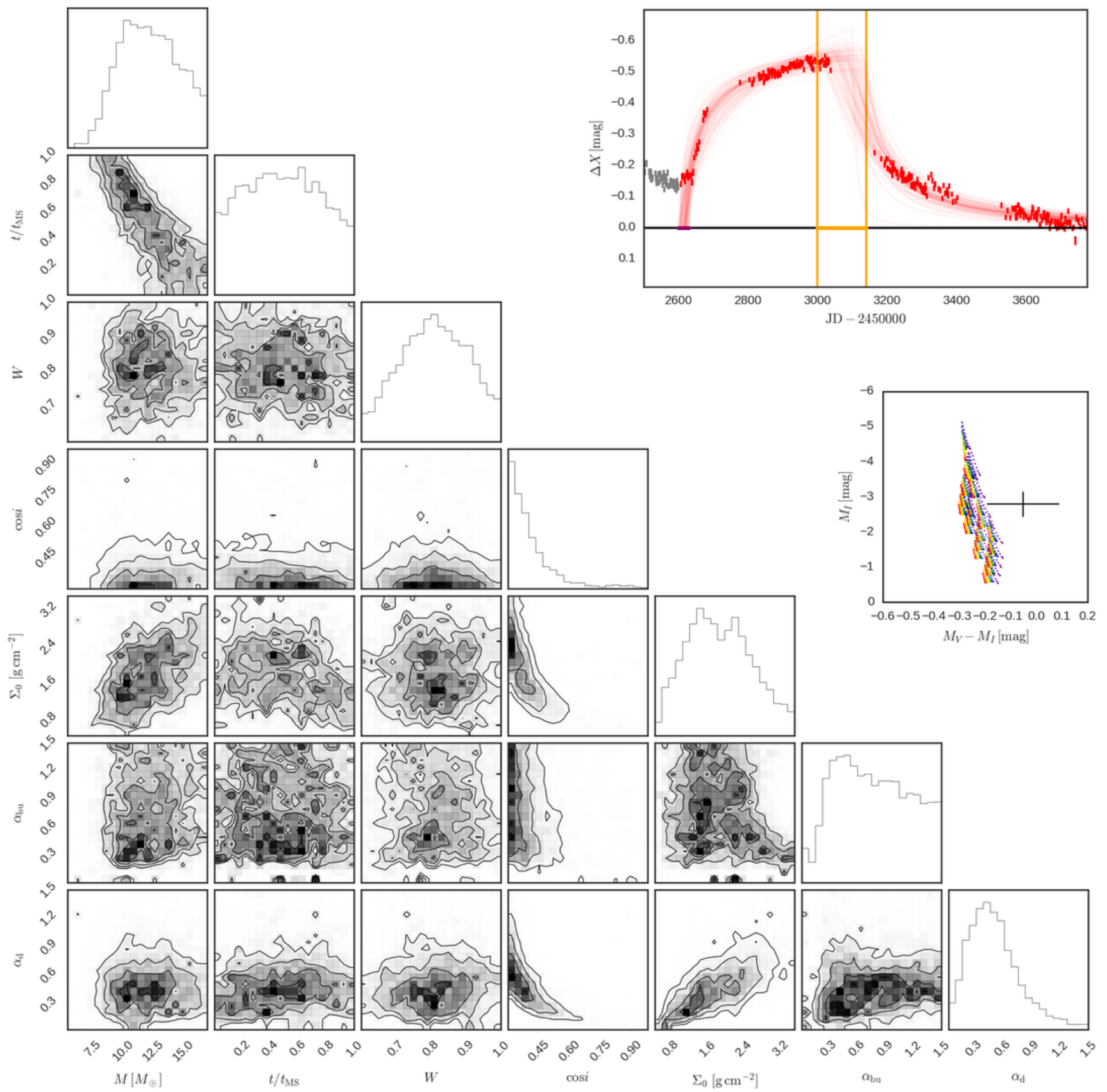

Figure C33. Same as Fig. 8 for SMC_SC4 175272 and bump ID 01. 


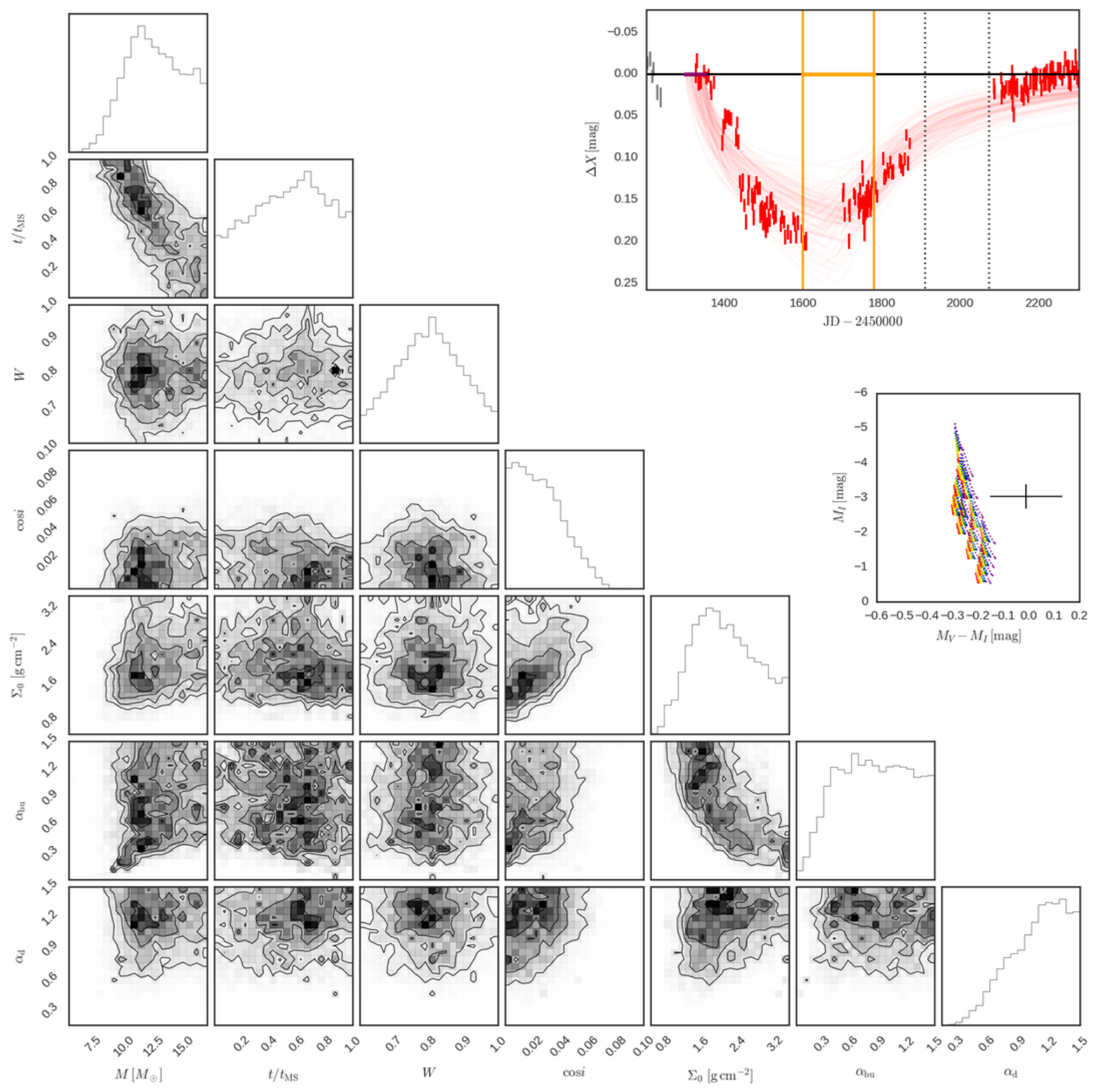

Figure C34. Same as Fig. 8 for SMC_SC4 179053 and bump ID 01. 


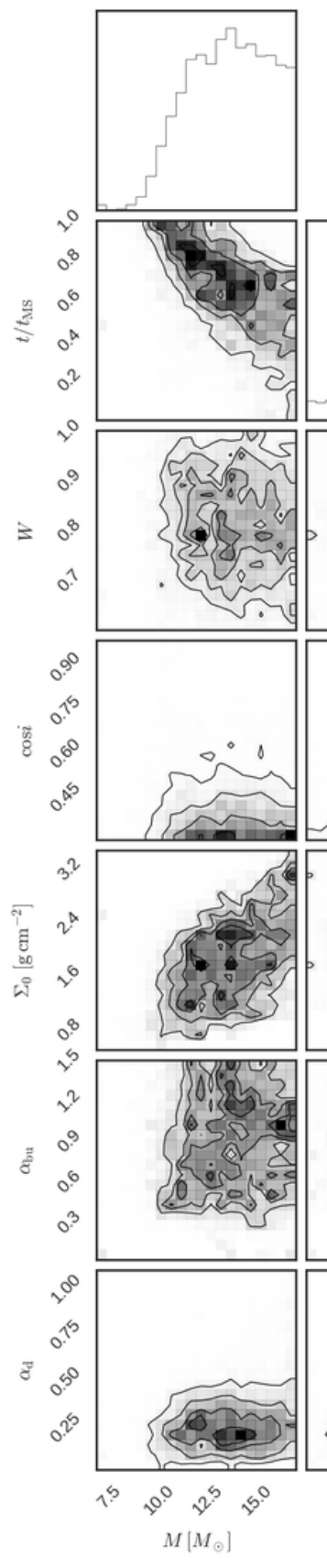

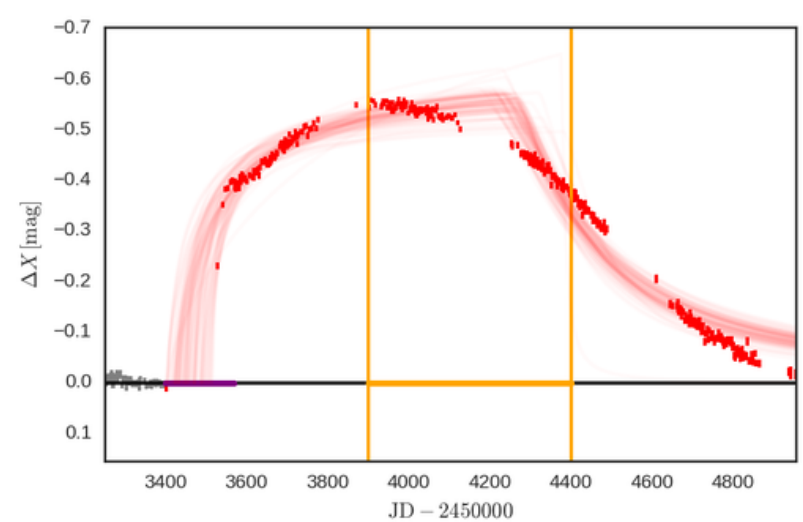

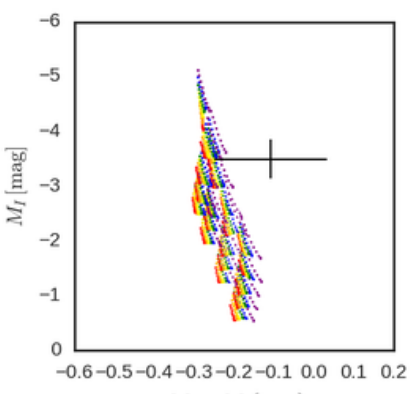

$$
M_{V}-M_{I}[\mathrm{mag}]
$$

Figure C35. Same as Fig. 8 for SMC_SC5 11453 and bump ID 01. 


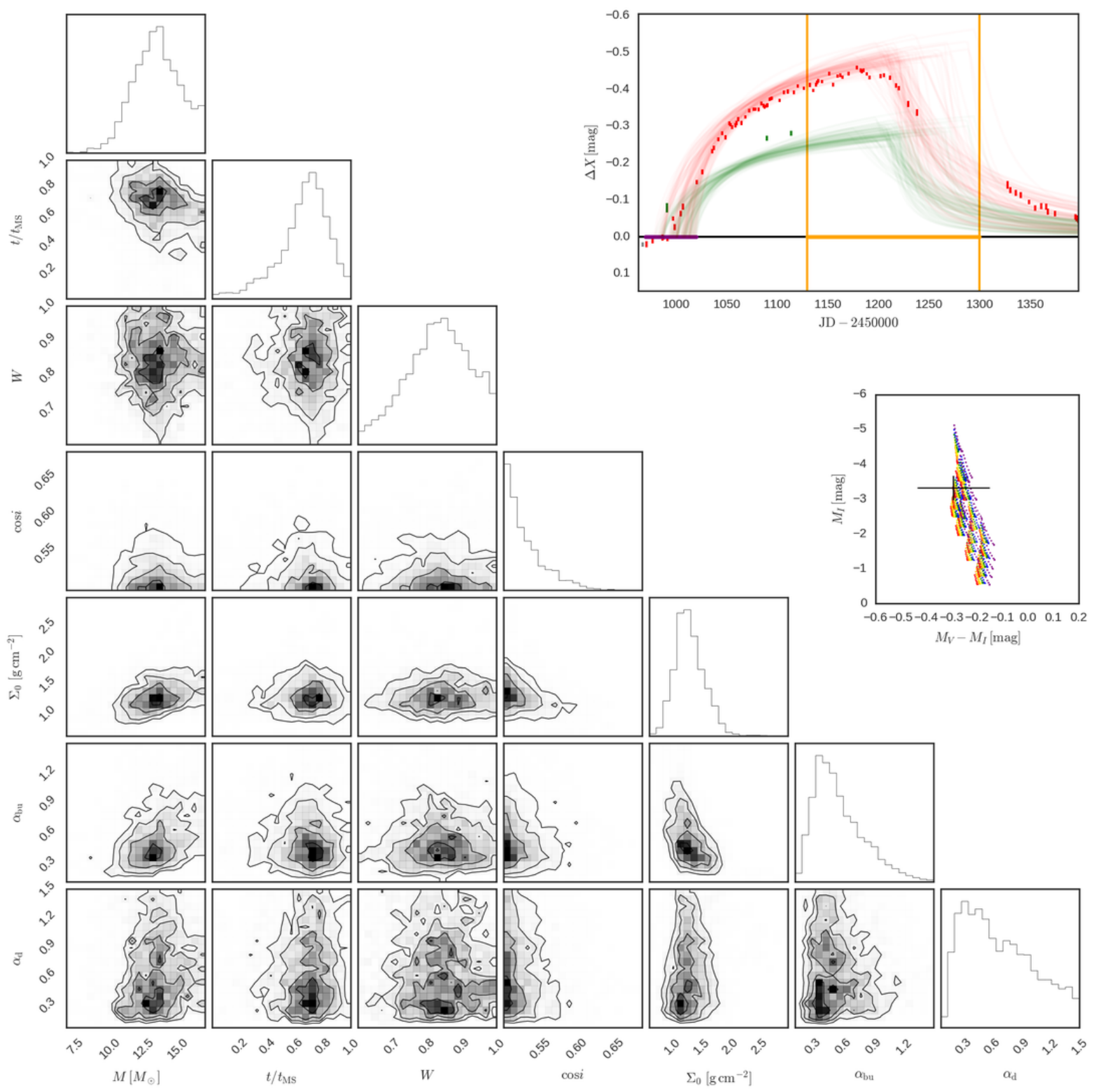

Figure C36. Same as Fig. 8 for SMC_SC5 21117 and bump ID 01. 

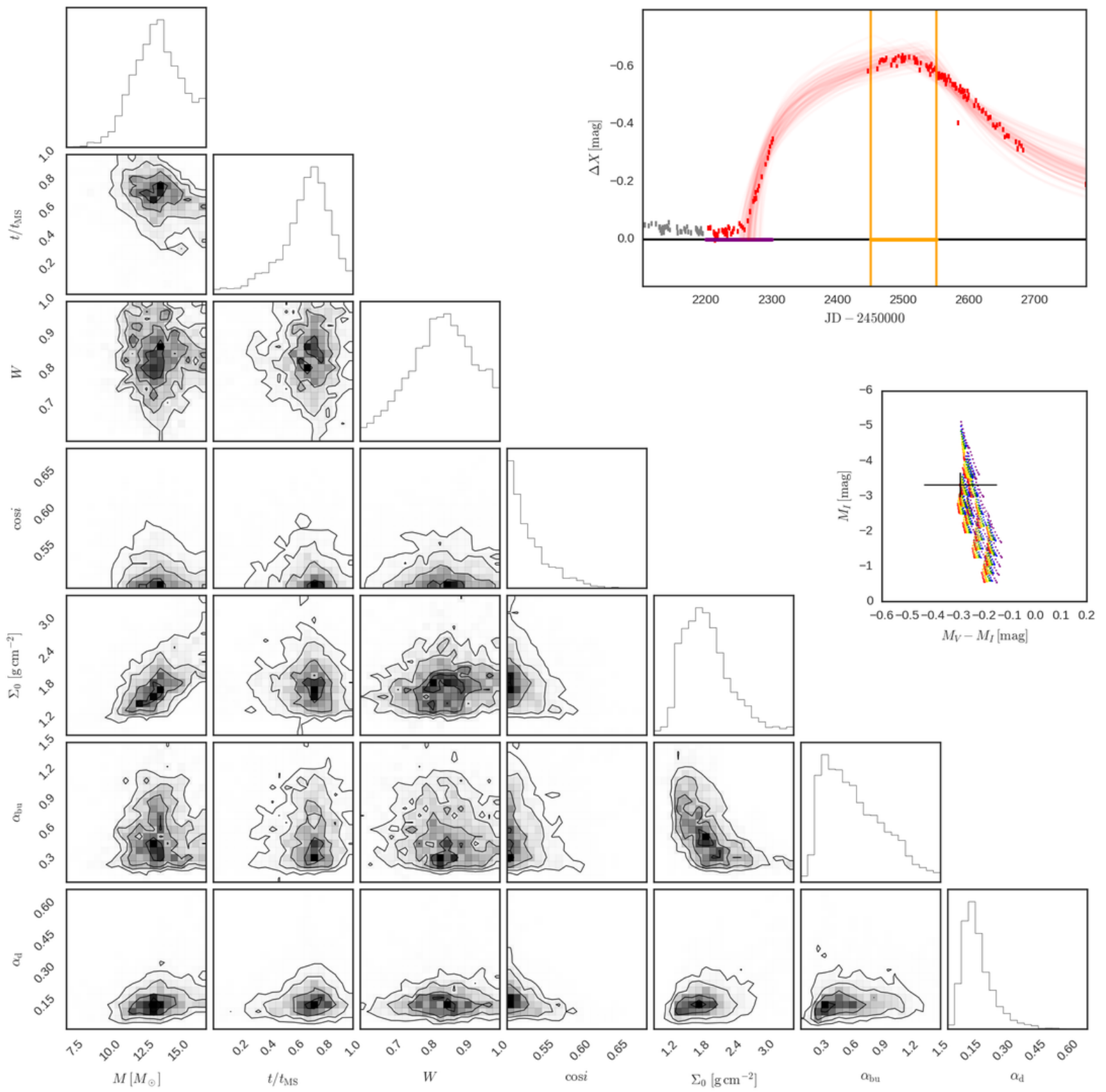

$$
M_{V}-M_{I}[\mathrm{mag}]
$$
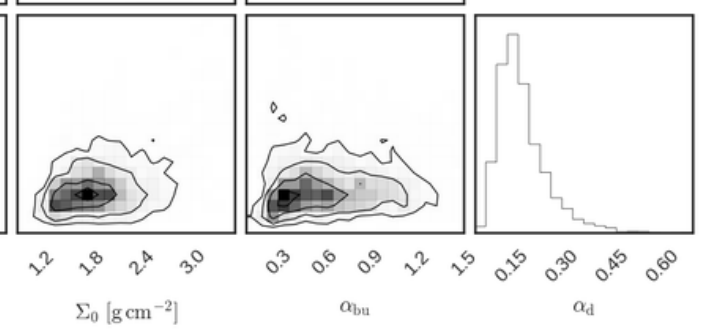

Figure C37. Same as Fig. 8 for SMC_SC5 21117 and bump ID 02. 


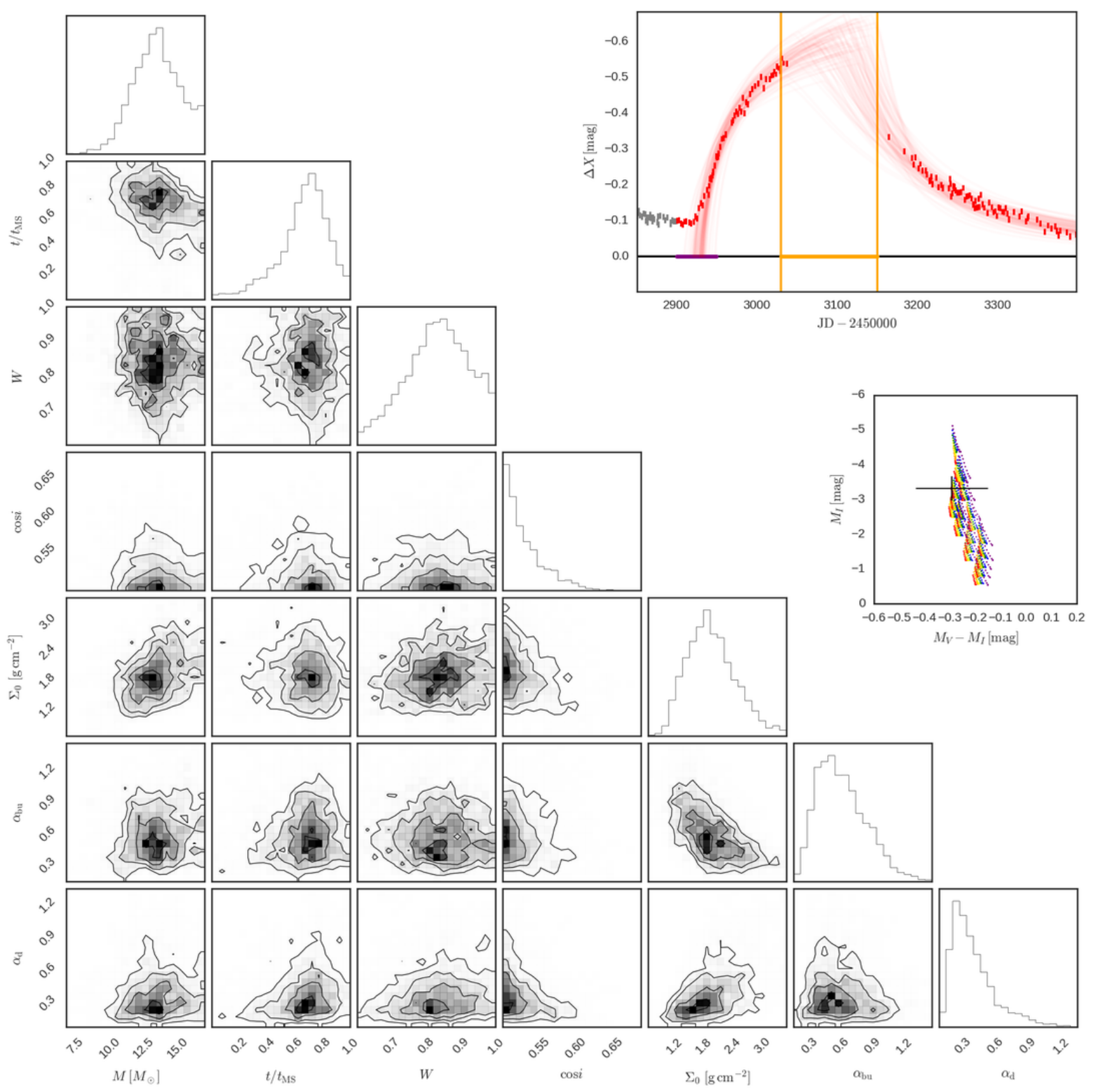

Figure C38. Same as Fig. 8 for SMC_SC5 21117 and bump ID 03 . 

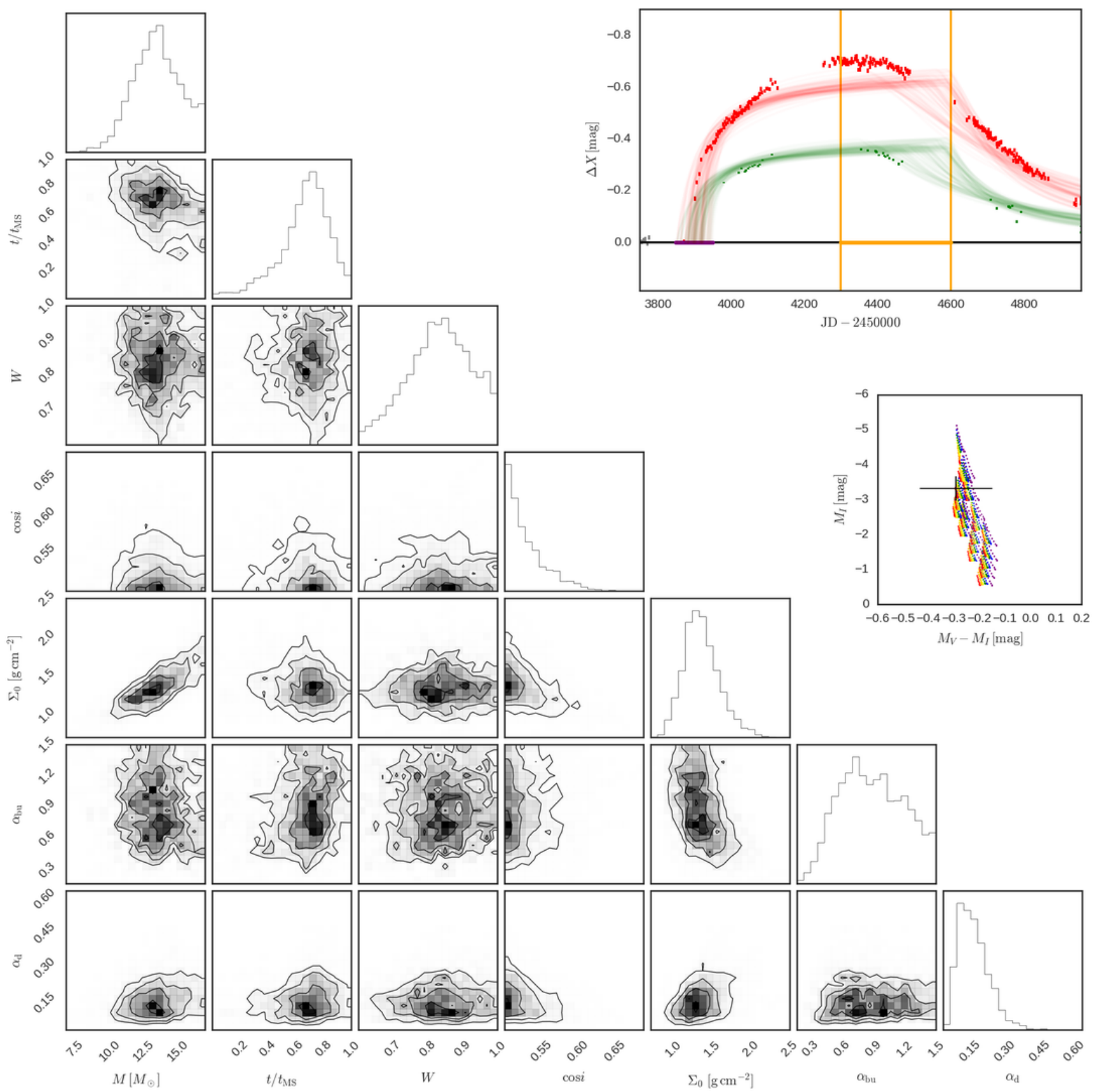

Figure C39. Same as Fig. 8 for SMC_SC5 21117 and bump ID 04. 


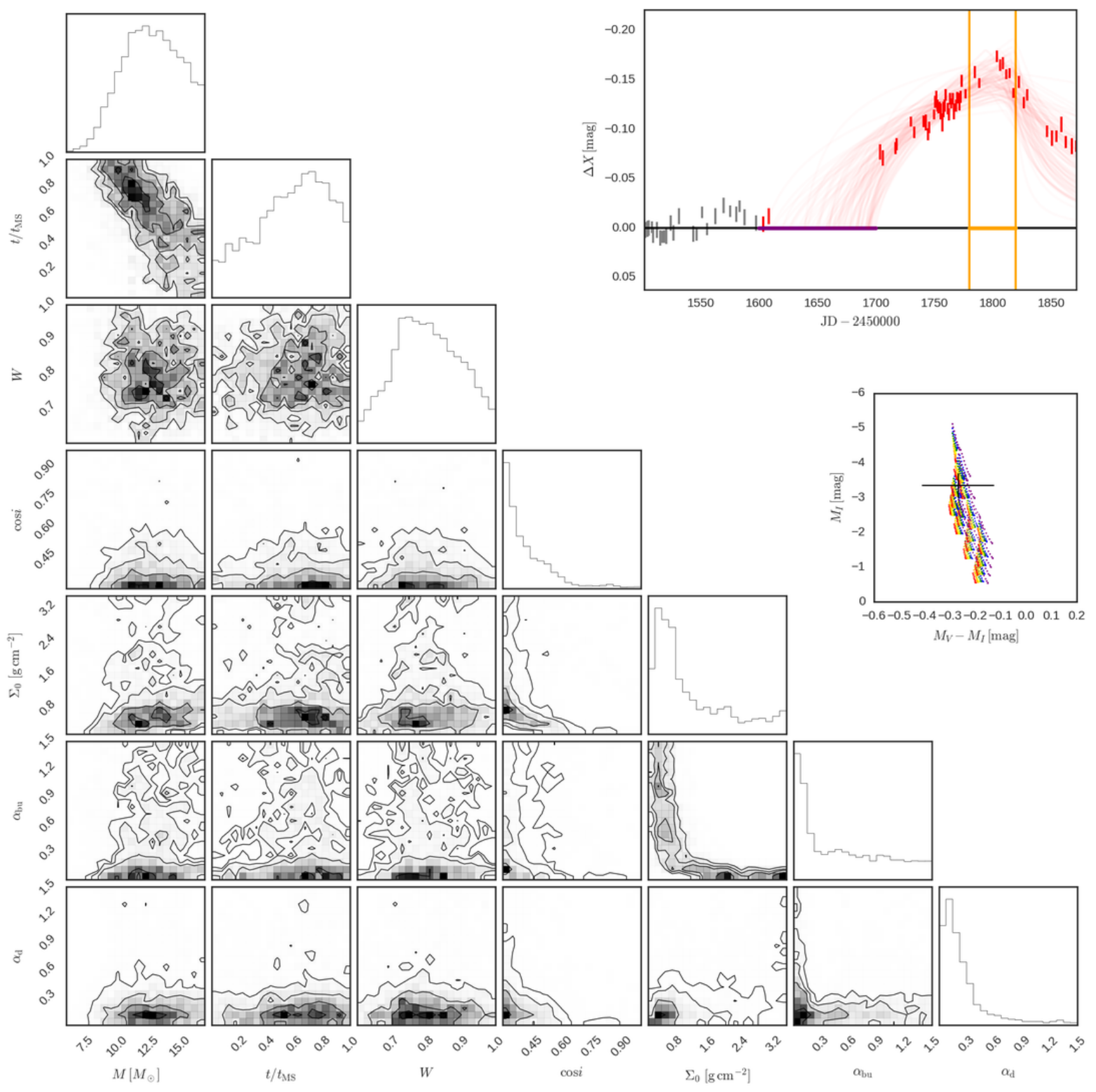

Figure C40. Same as Fig. 8 for SMC_SC5 21134 and bump ID 01. 

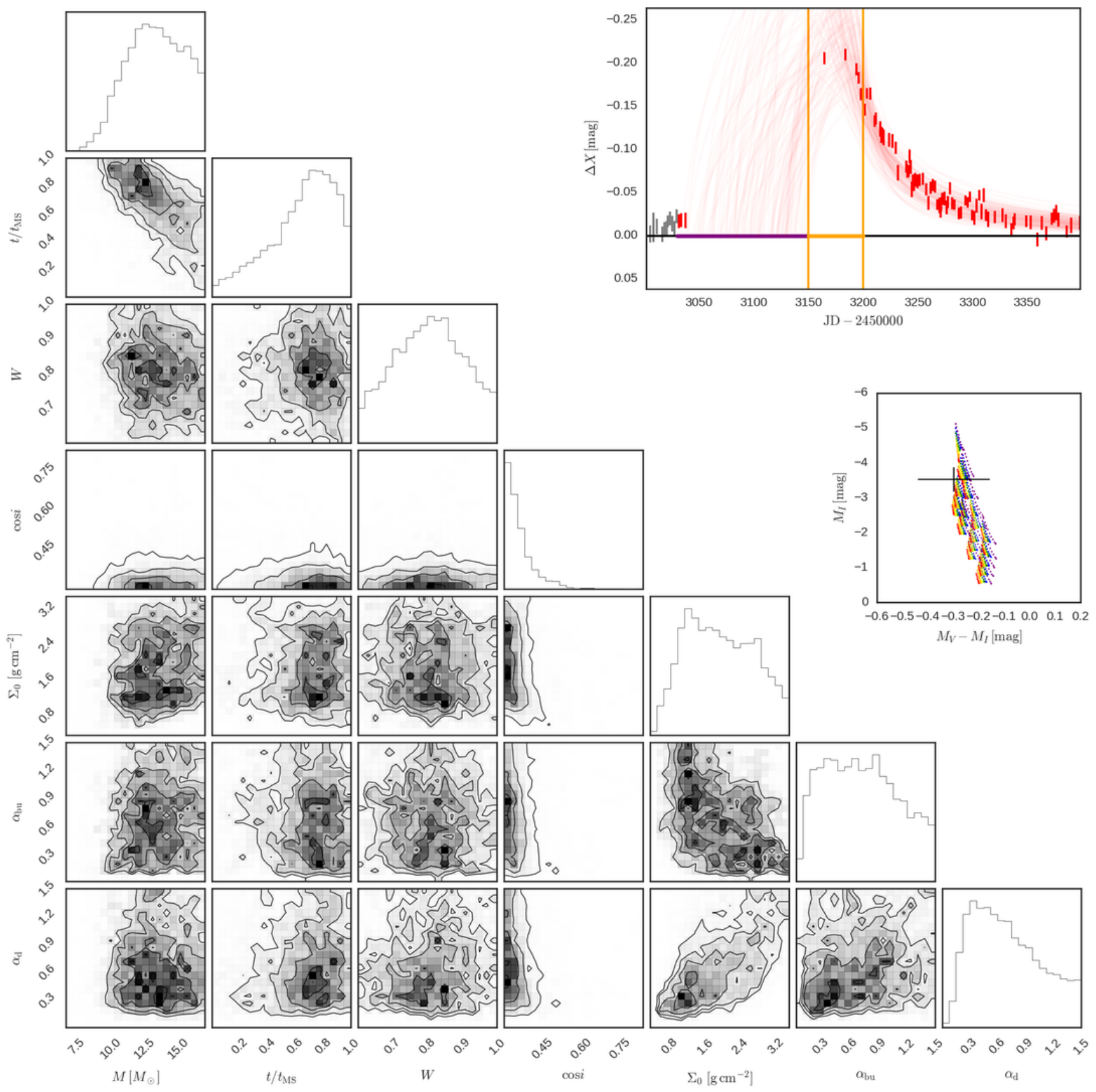

Figure C41. Same as Fig. 8 for SMC_SC5 32377 and bump ID 01. 


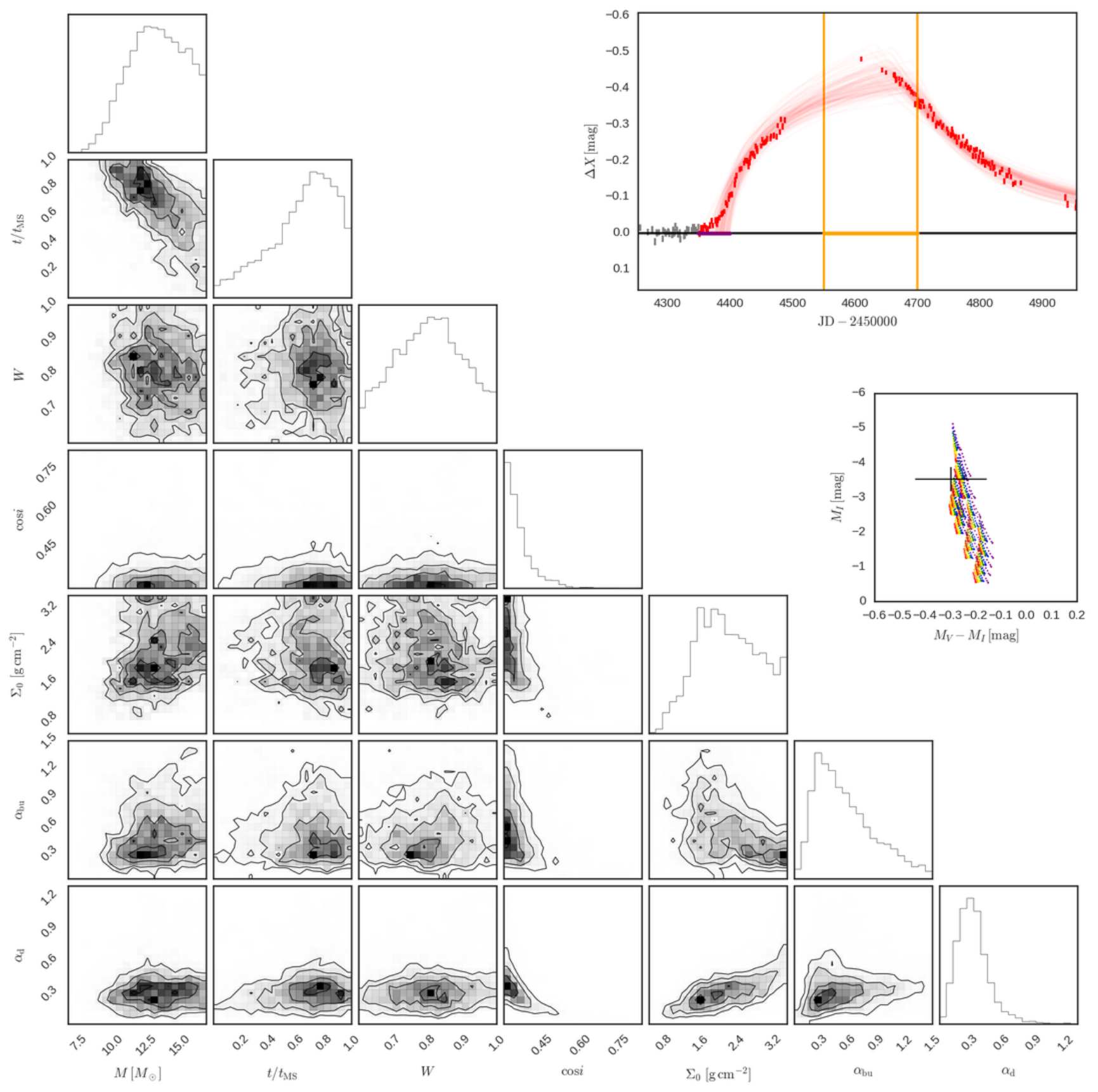

Figure C42. Same as Fig. 8 for SMC_SC5 32377 and bump ID 02. 


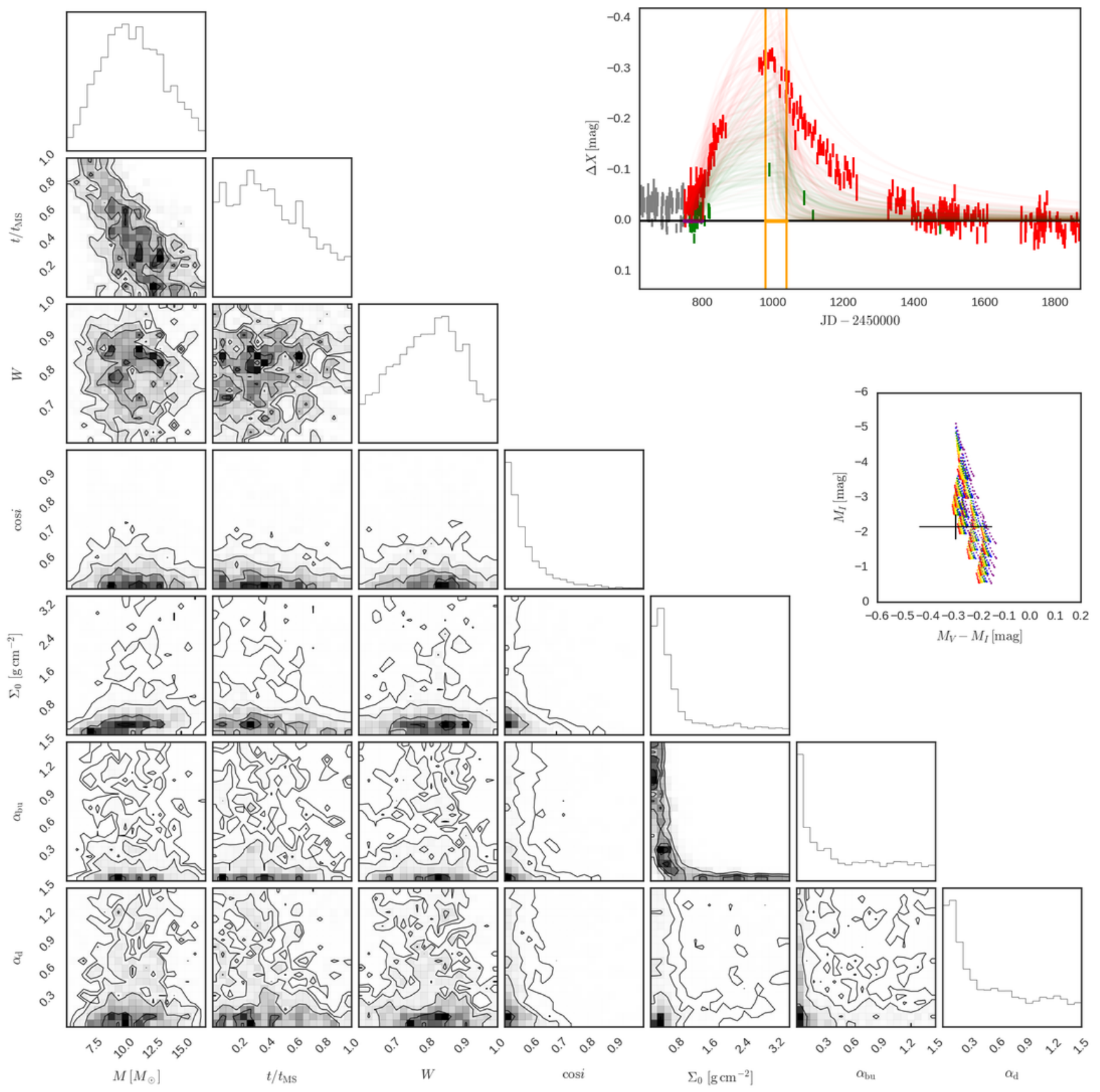

Figure C43. Same as Fig. 8 for SMC_SC5 43650 and bump ID 01. 


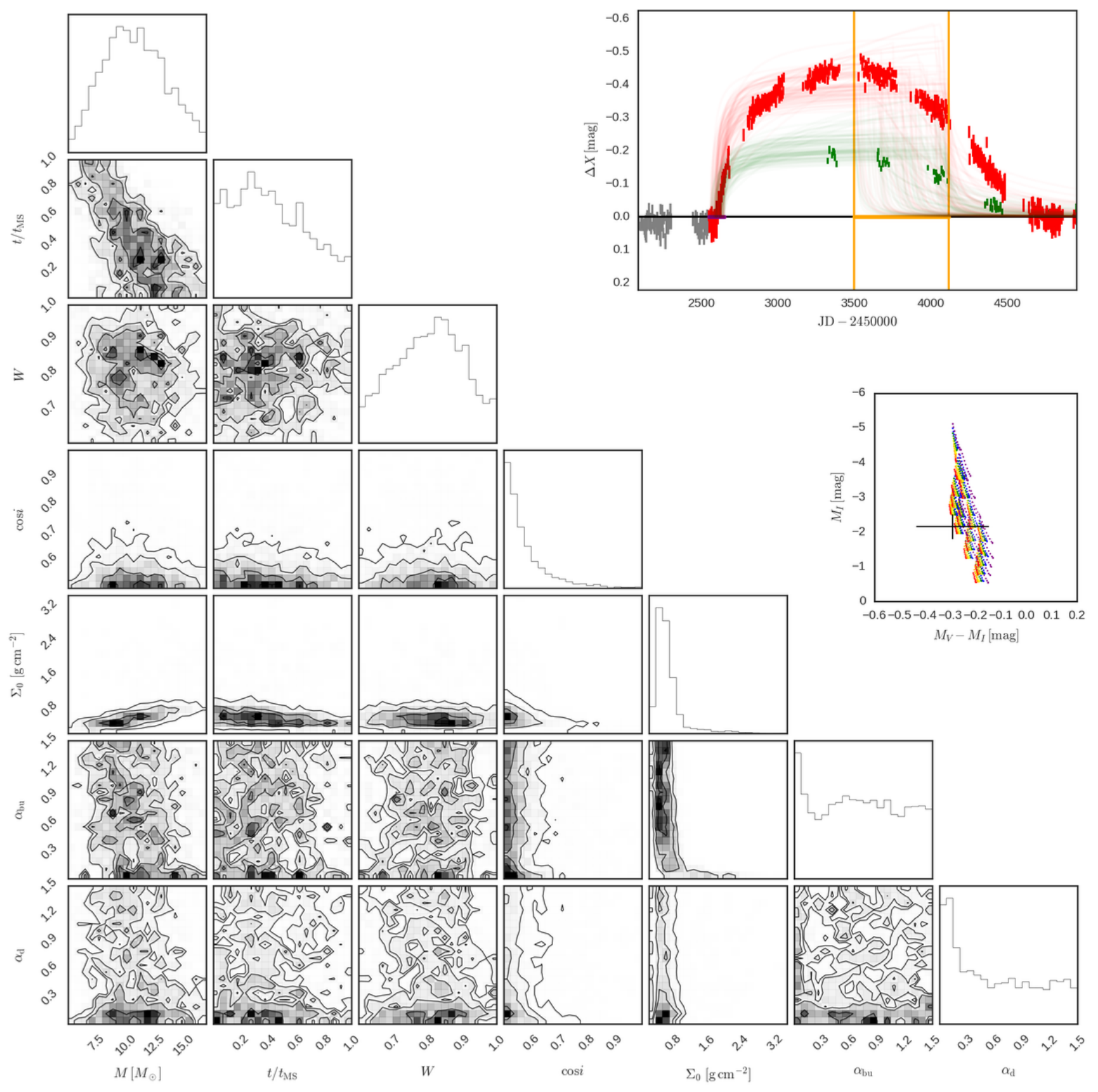

Figure C44. Same as Fig. 8 for SMC_SC5 43650 and bump ID 02. 

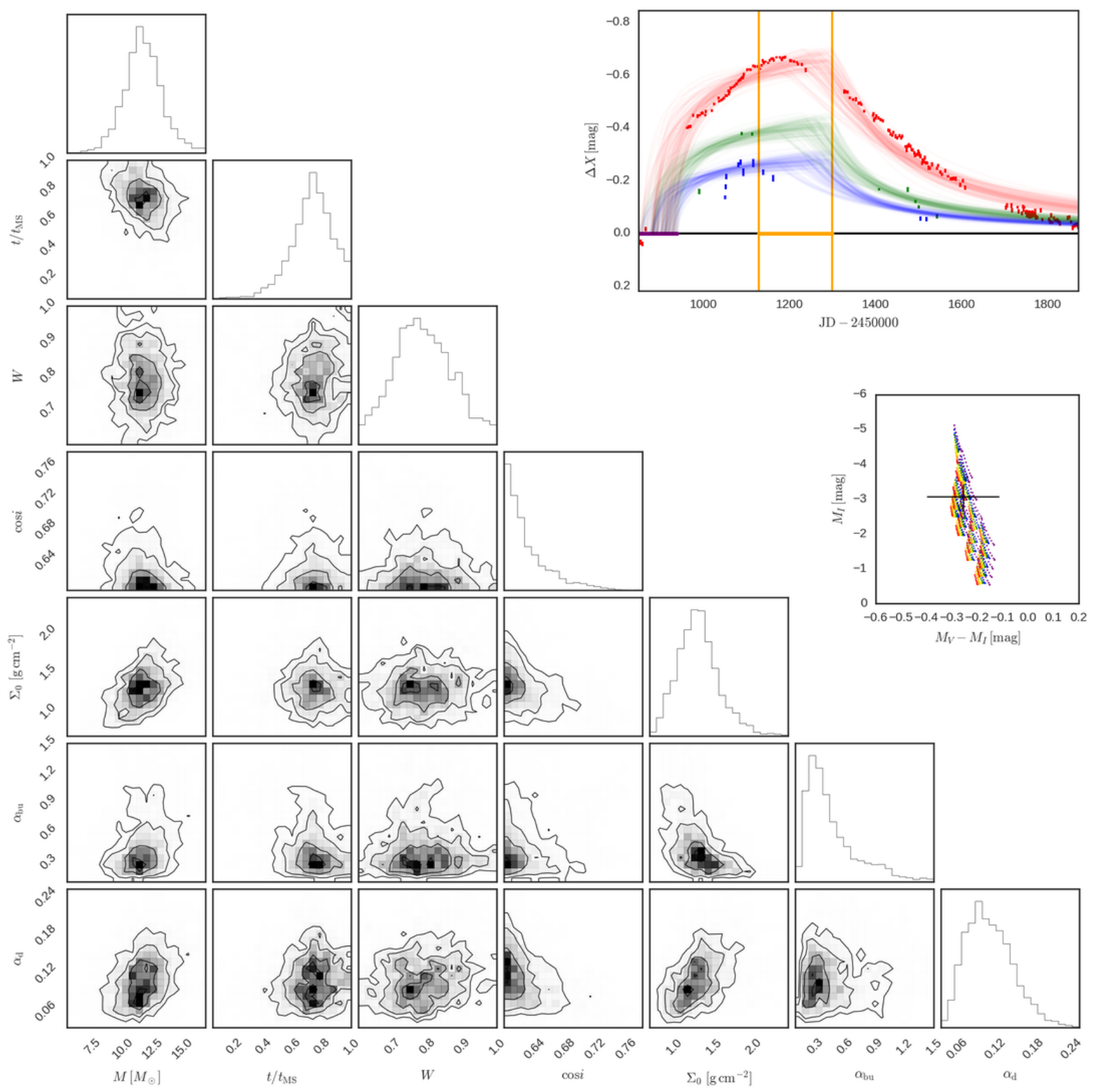

Figure C45. Same as Fig. 8 for SMC_SC5 54851 and bump ID 01. 


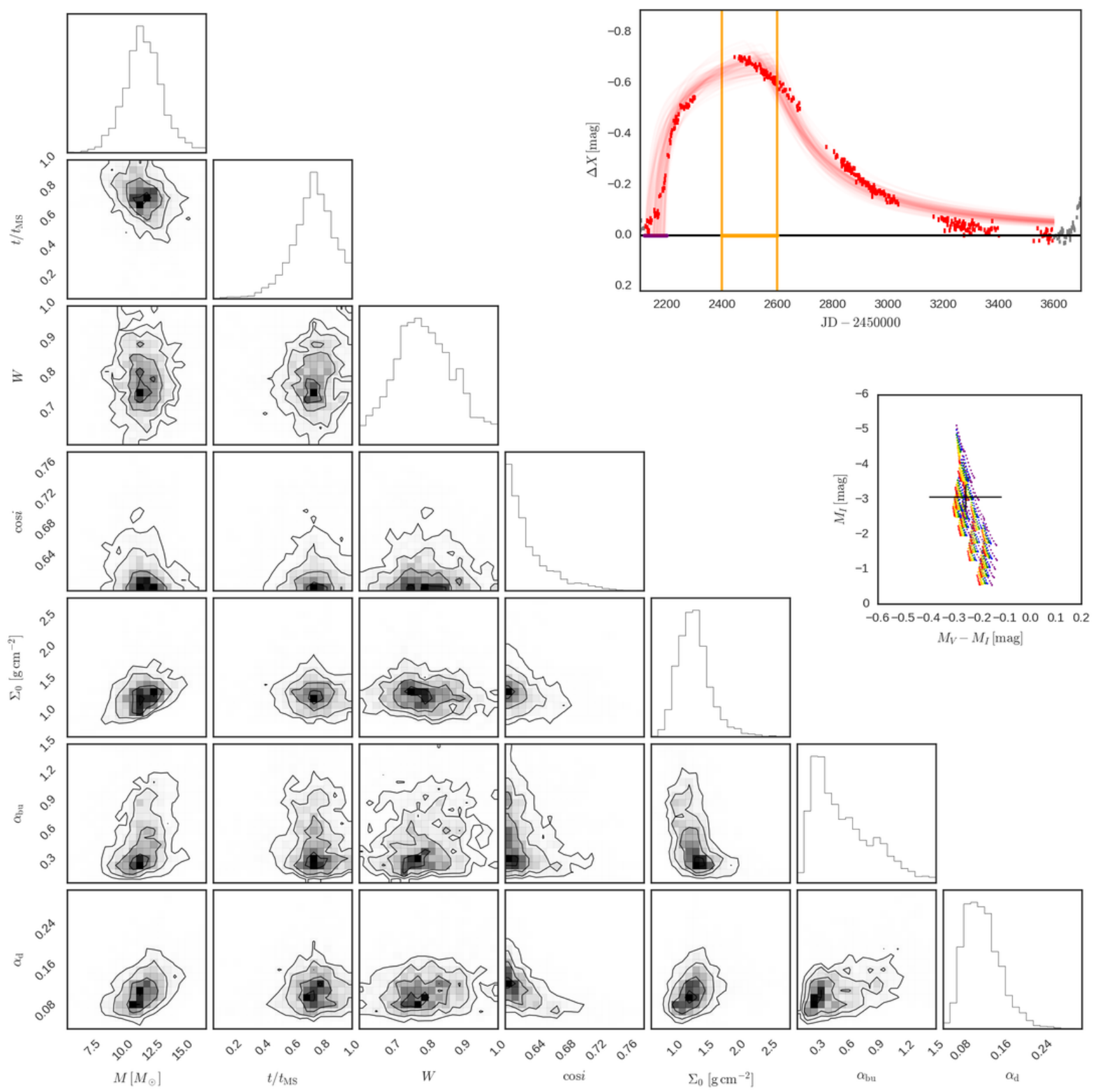

Figure C46. Same as Fig. 8 for SMC_SC5 54851 and bump ID 02. 

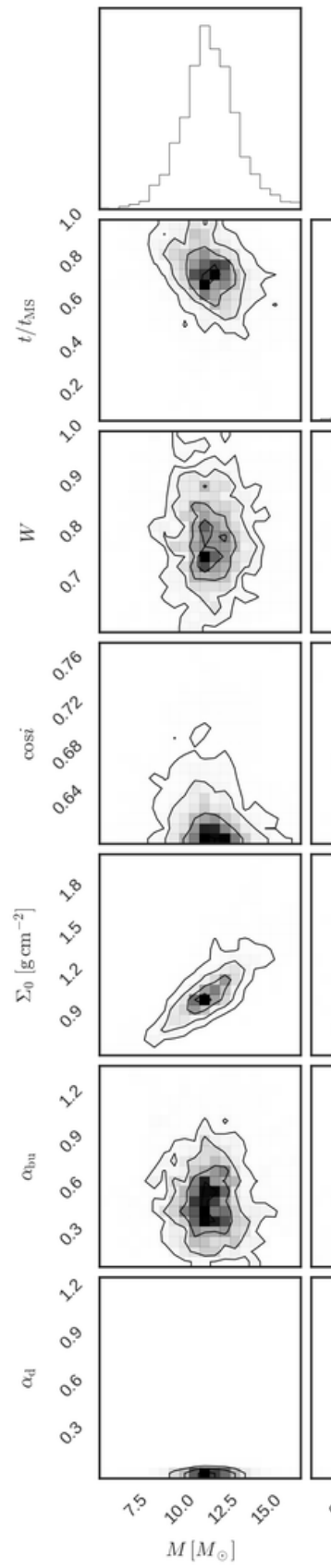
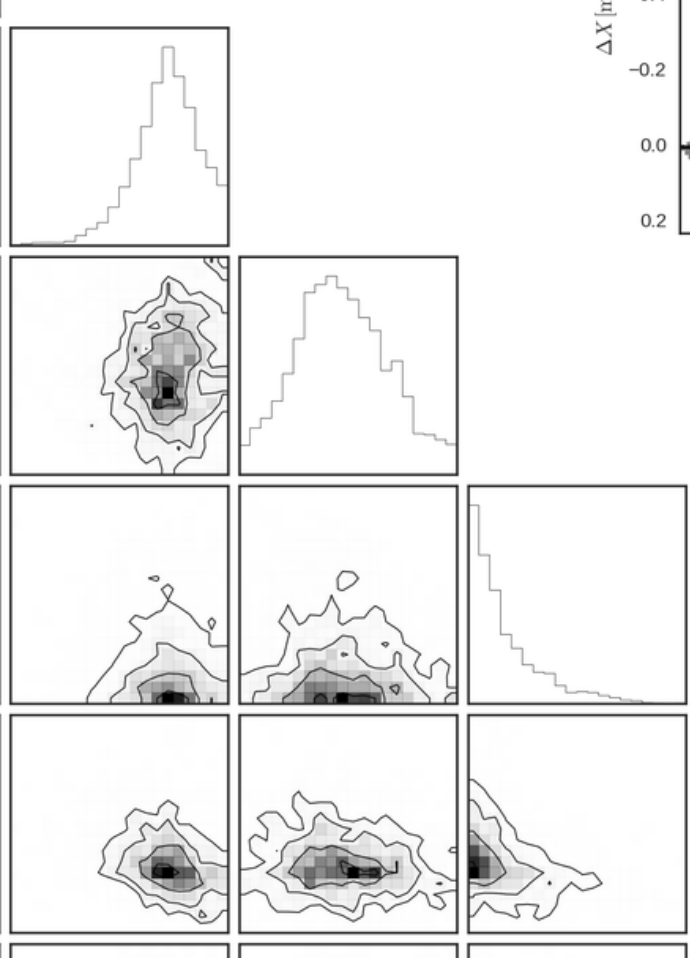
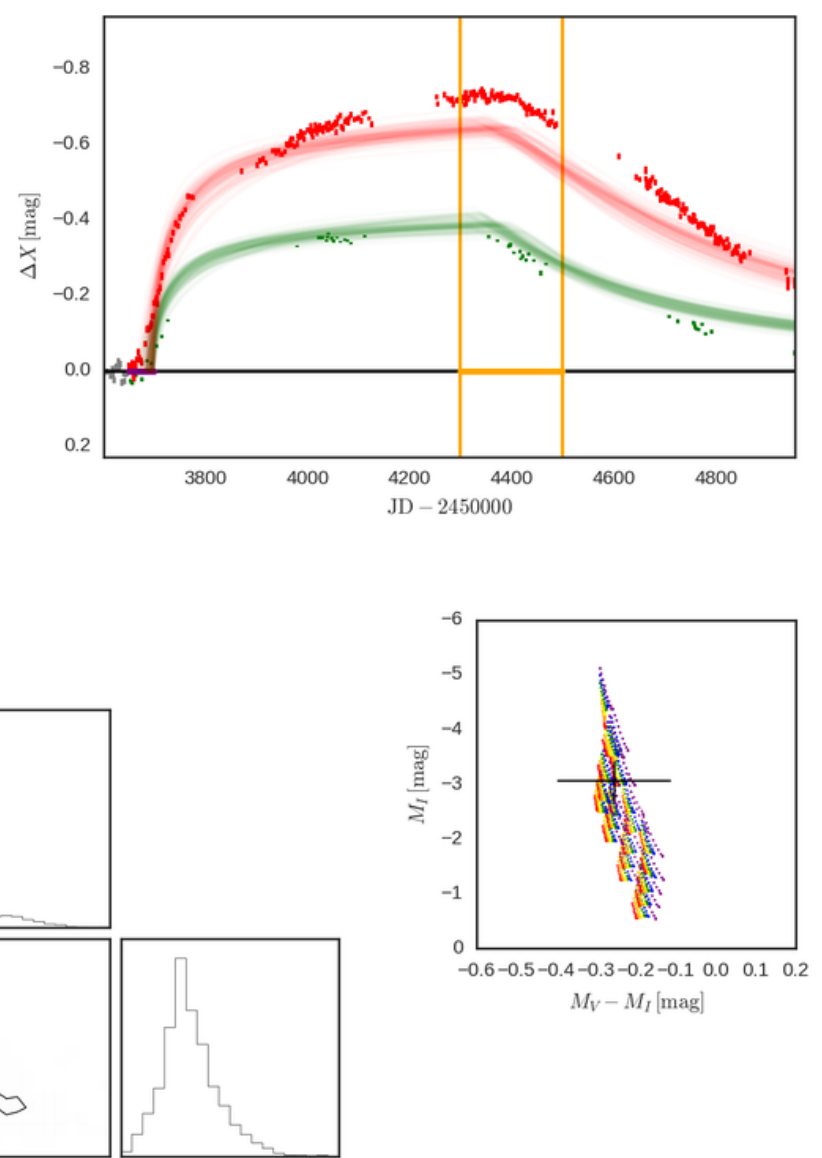

$$
M_{V}-M_{I}[\mathrm{mag}]
$$
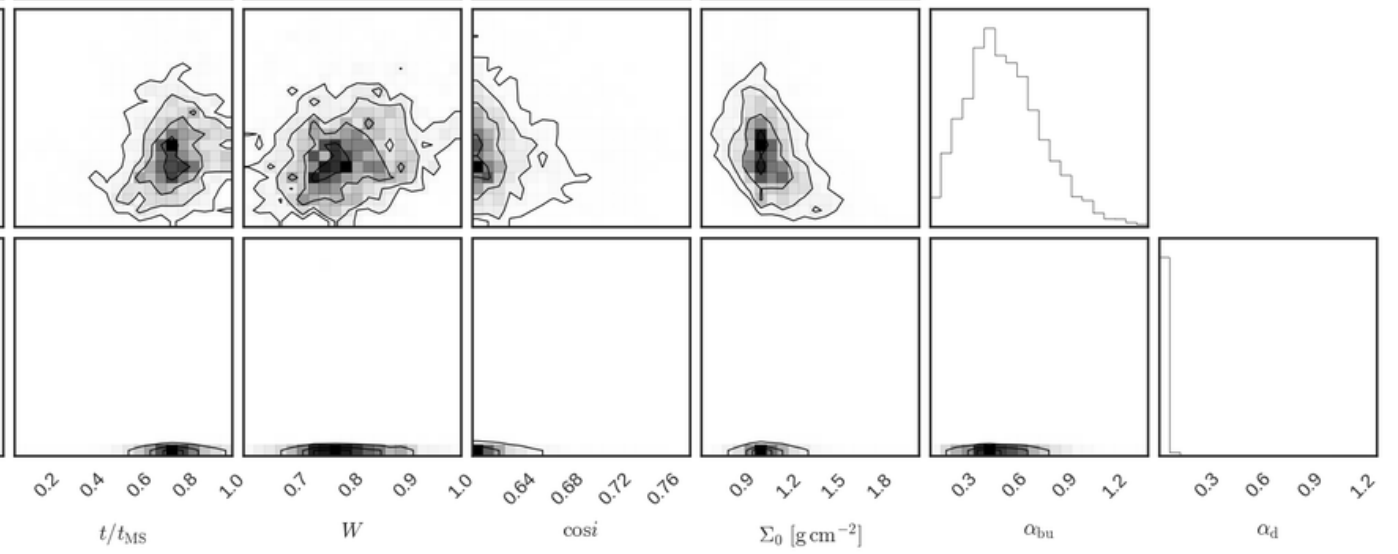

Figure C47. Same as Fig. 8 for SMC_SC5 54851 and bump ID 03. 


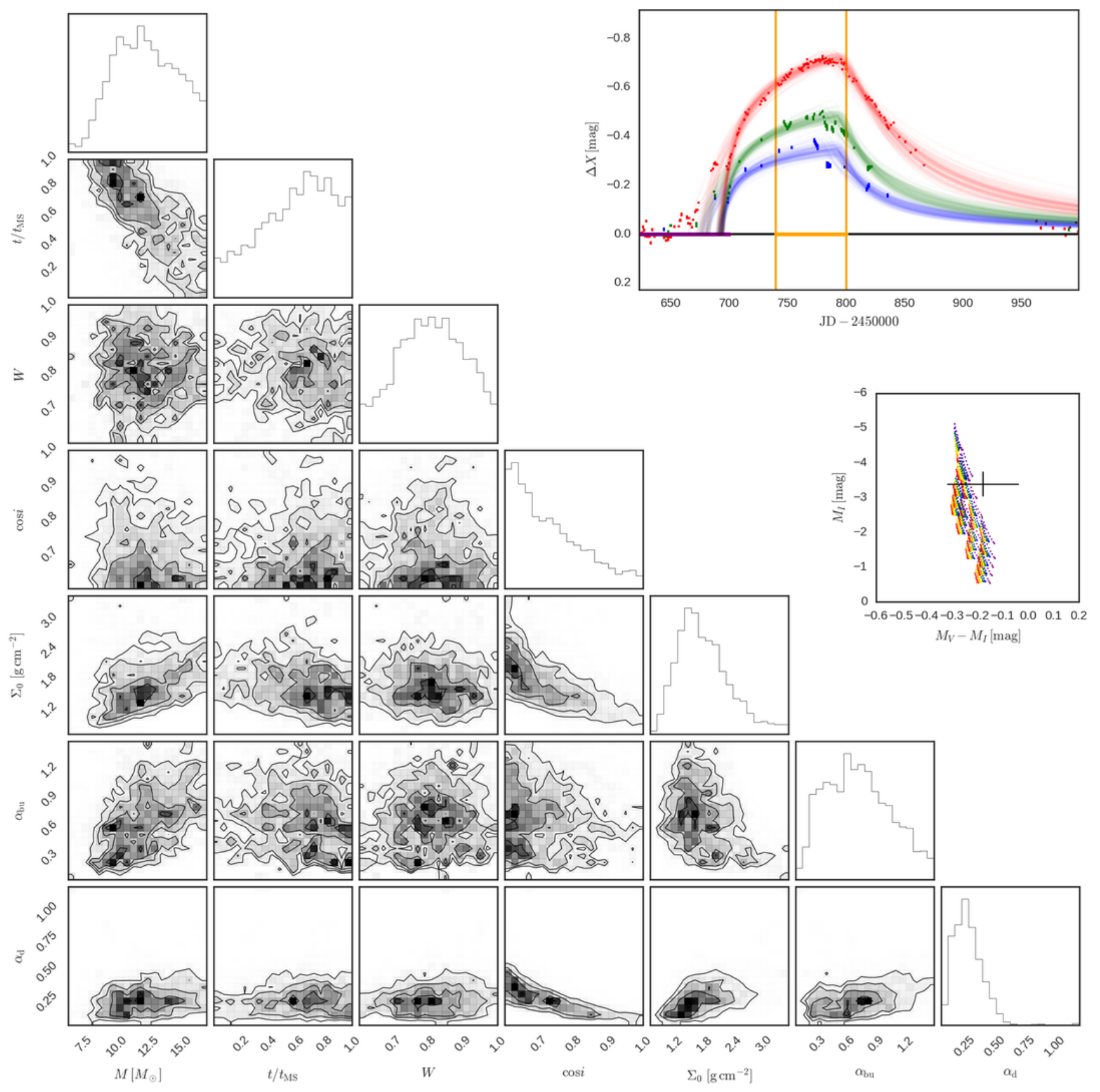

Figure C48. Same as Fig. 8 for SMC_SC5 65500 and bump ID 01. 


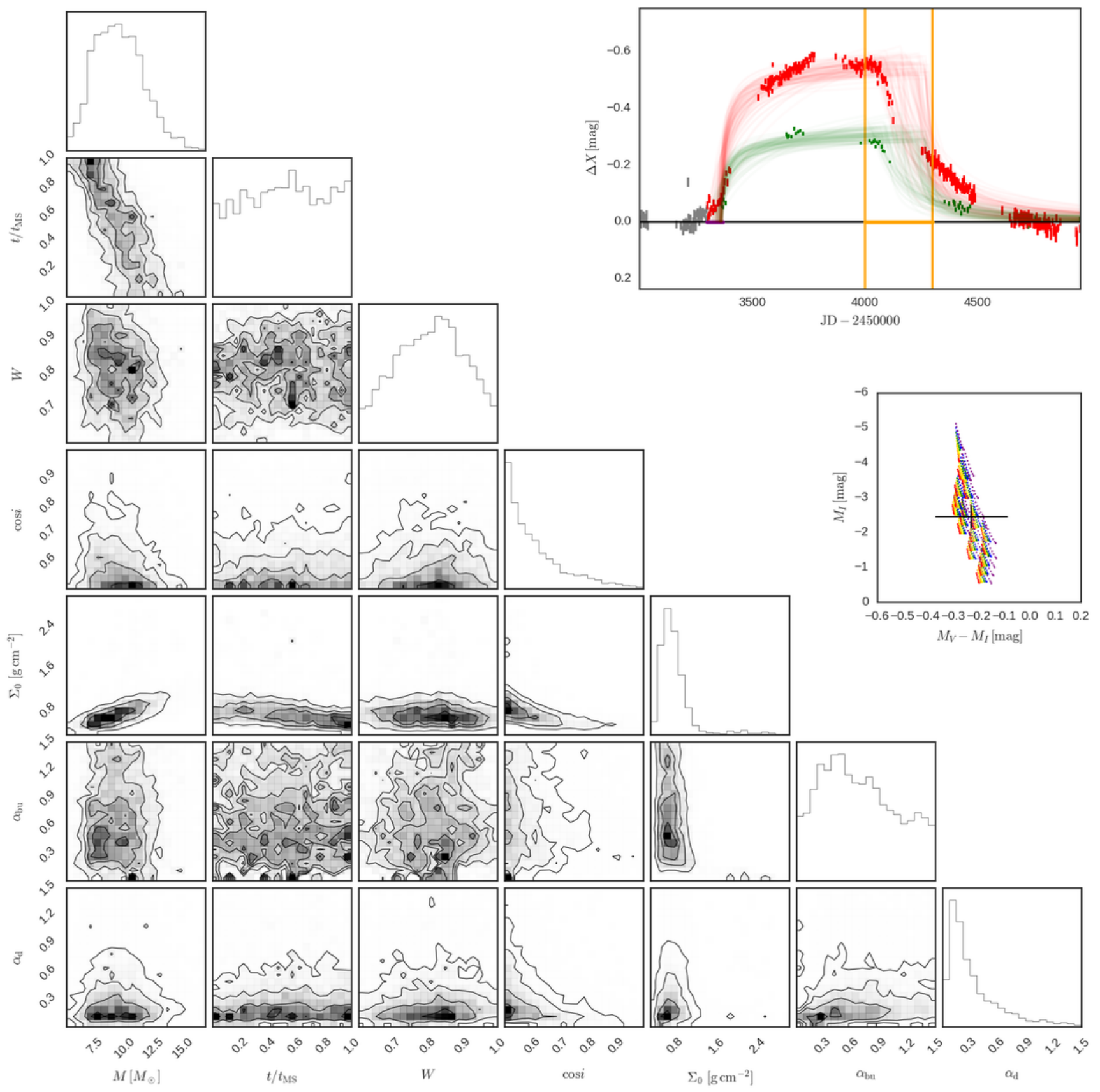

Figure C49. Same as Fig. 8 for SMC_SC5 129535 and bump ID 01. 


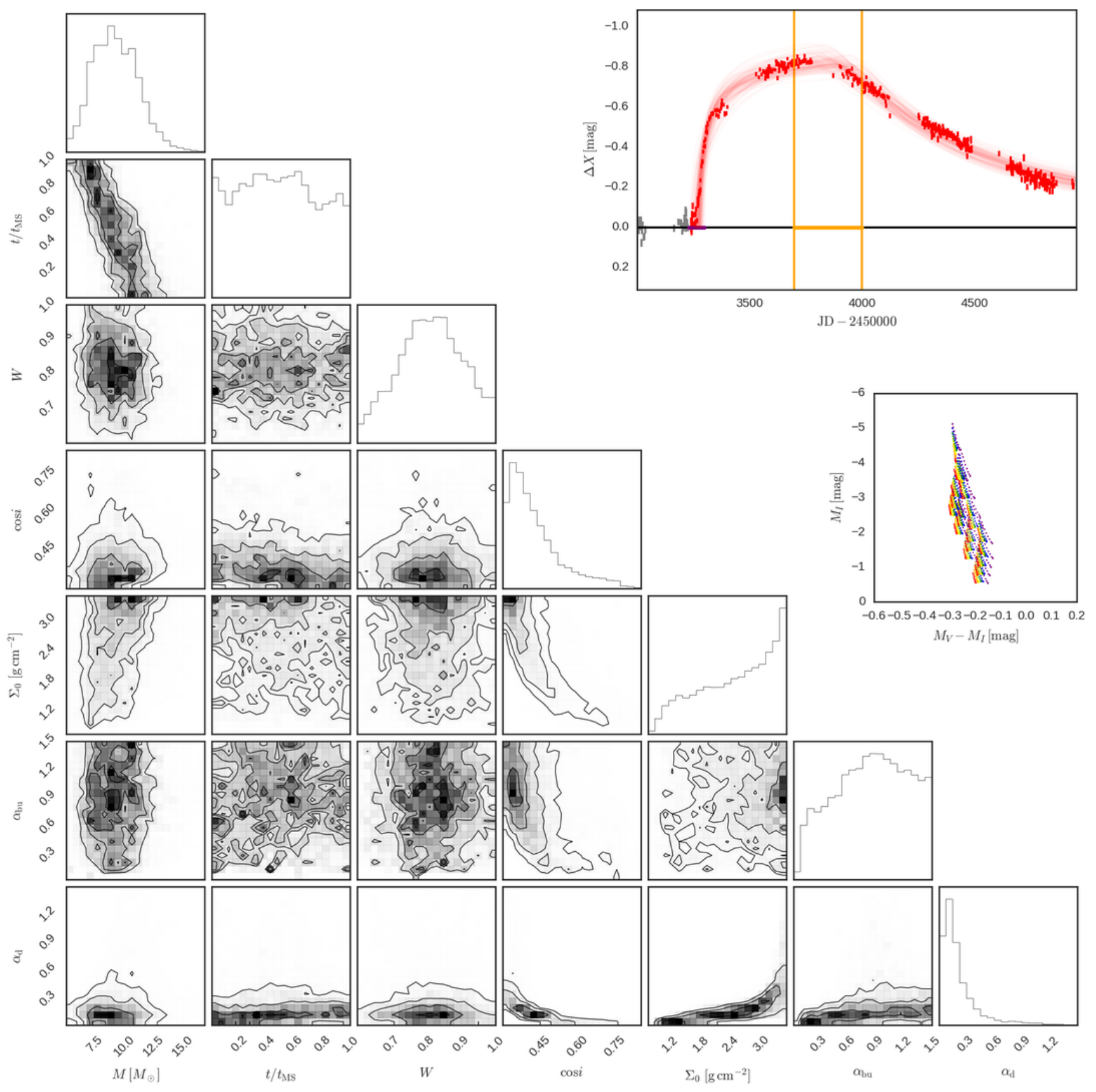

Figure C50. Same as Fig. 8 for SMC_SC5 145724 and bump ID 01. 


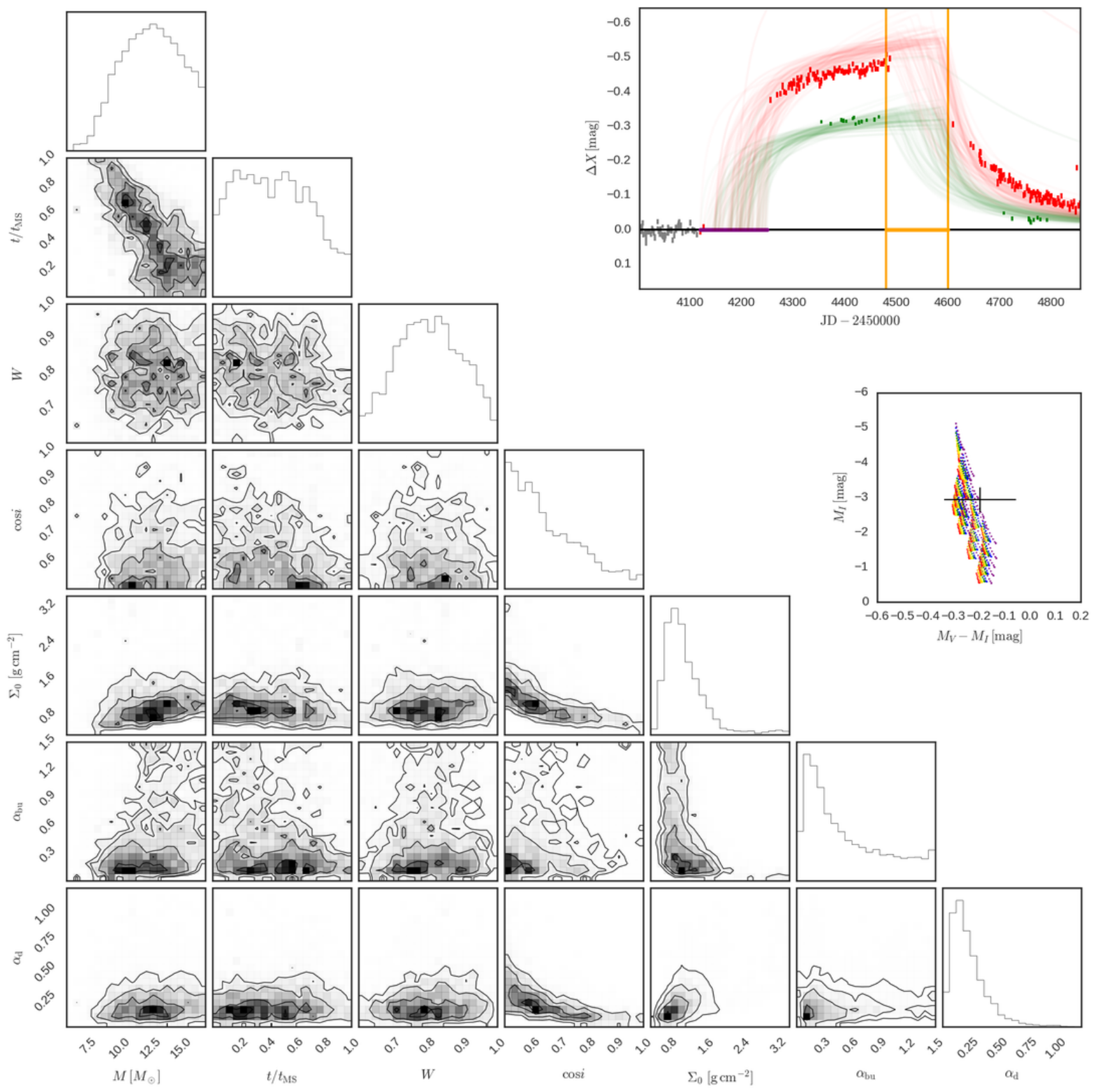

Figure C51. Same as Fig. 8 for SMC_SC5 180034 and bump ID 01. 


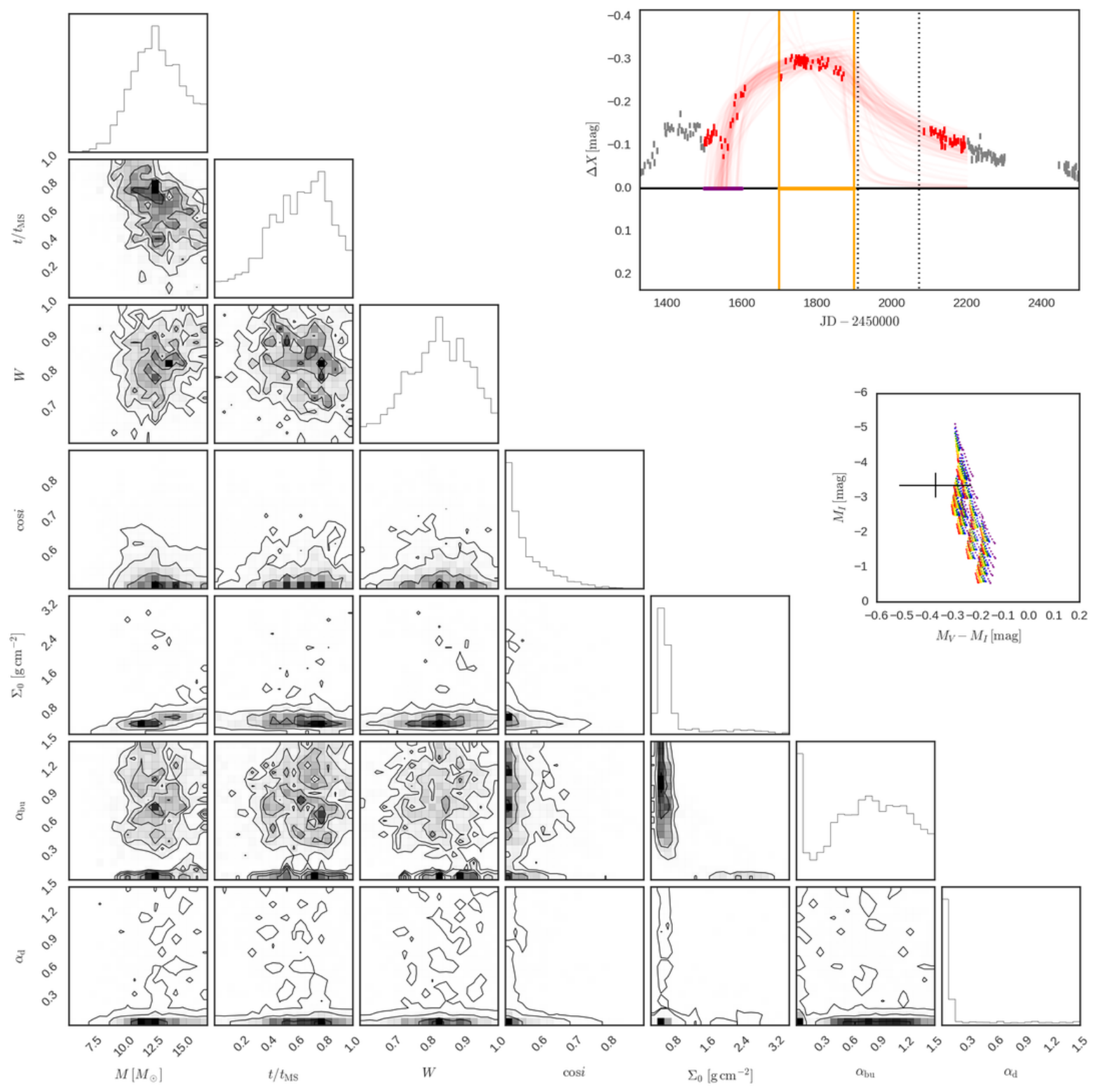

Figure C52. Same as Fig. 8 for SMC_SC5 260841 and bump ID 01. 

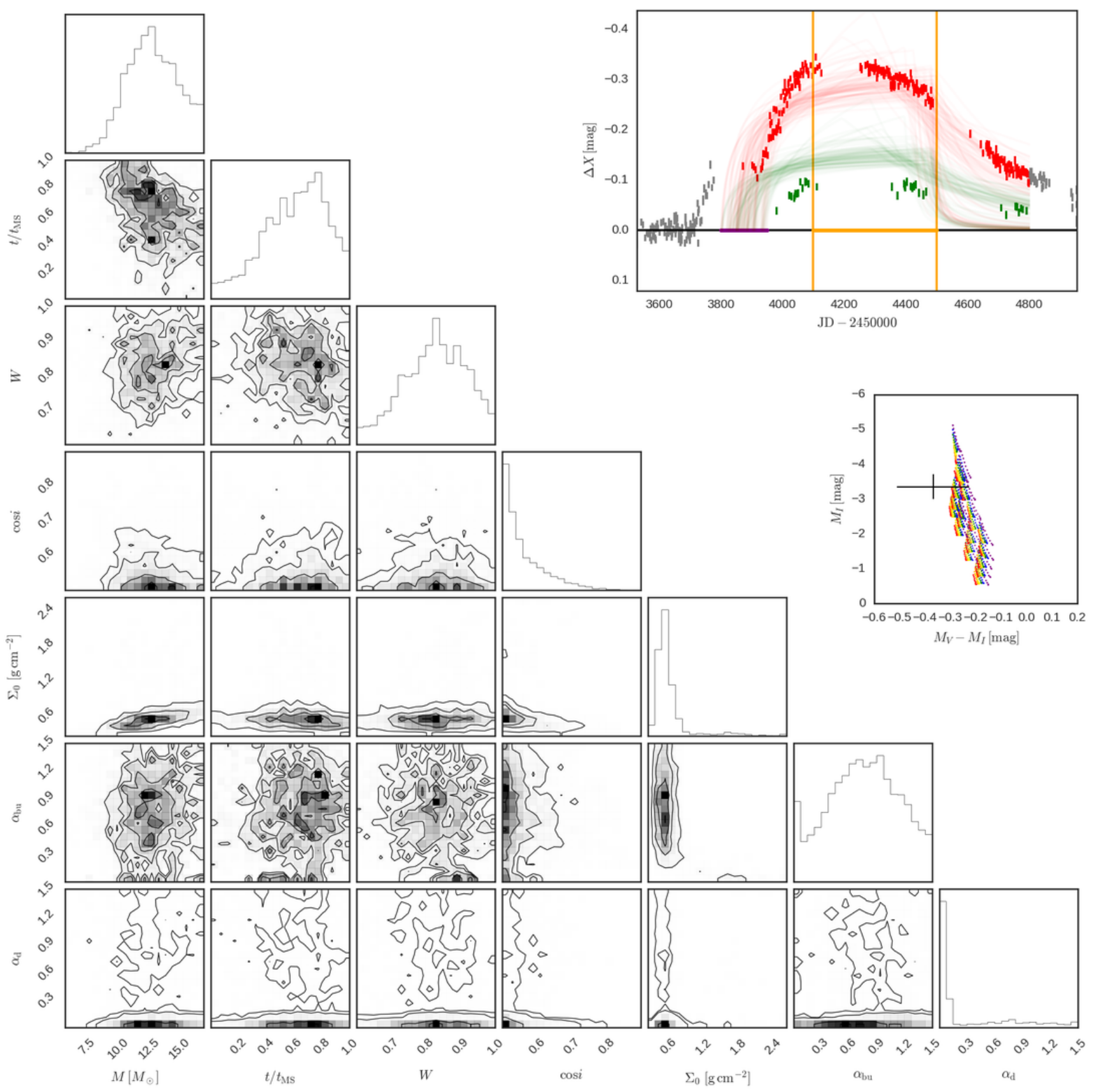

Figure C53. Same as Fig. 8 for SMC_SC5 260841 and bump ID 02. 


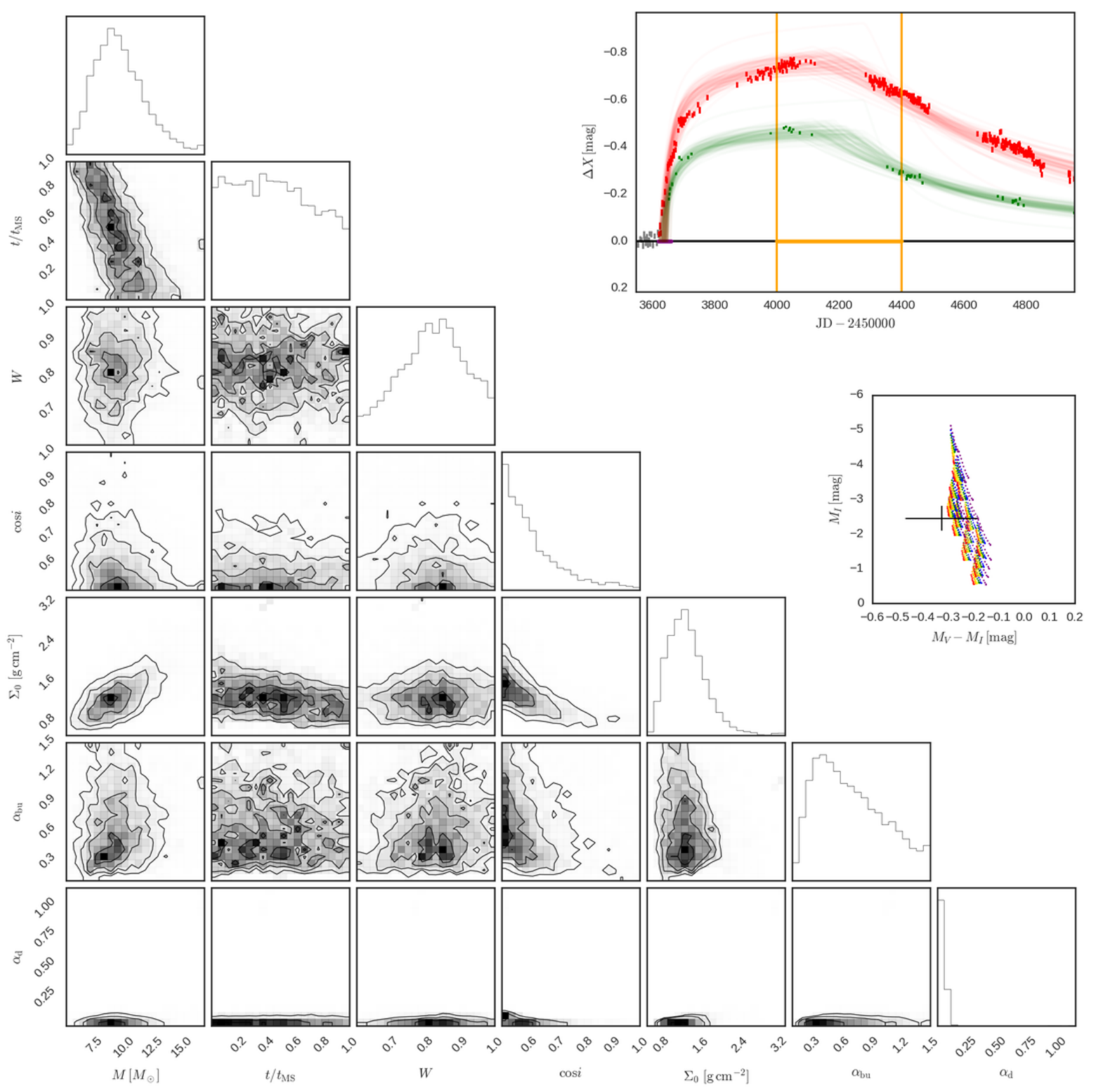

Figure C54. Same as Fig. 8 for SMC_SC5 260957 and bump ID 01. 


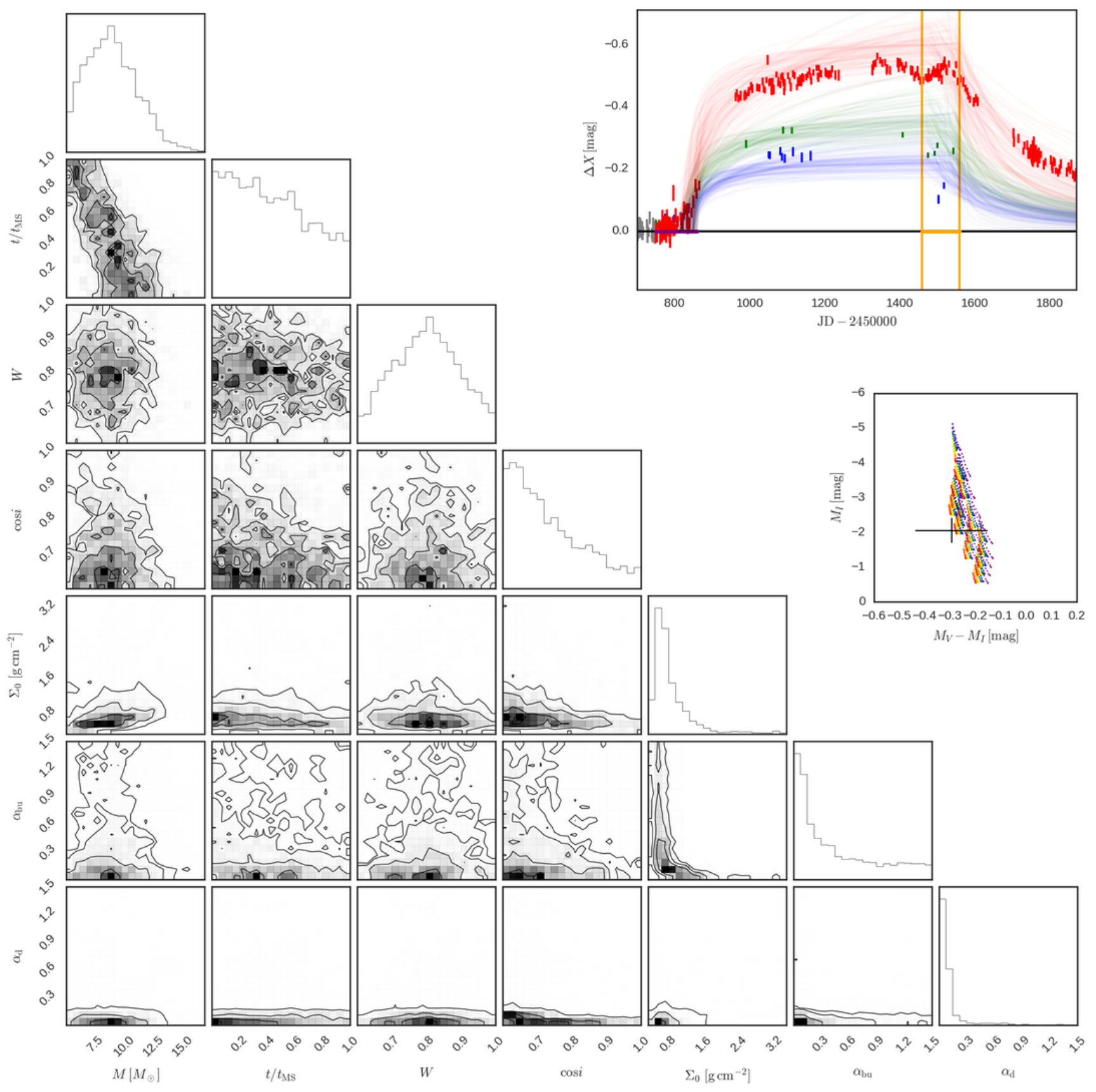

Figure C55. Same as Fig. 8 for SMC_SC5 266088 and bump ID 01. 


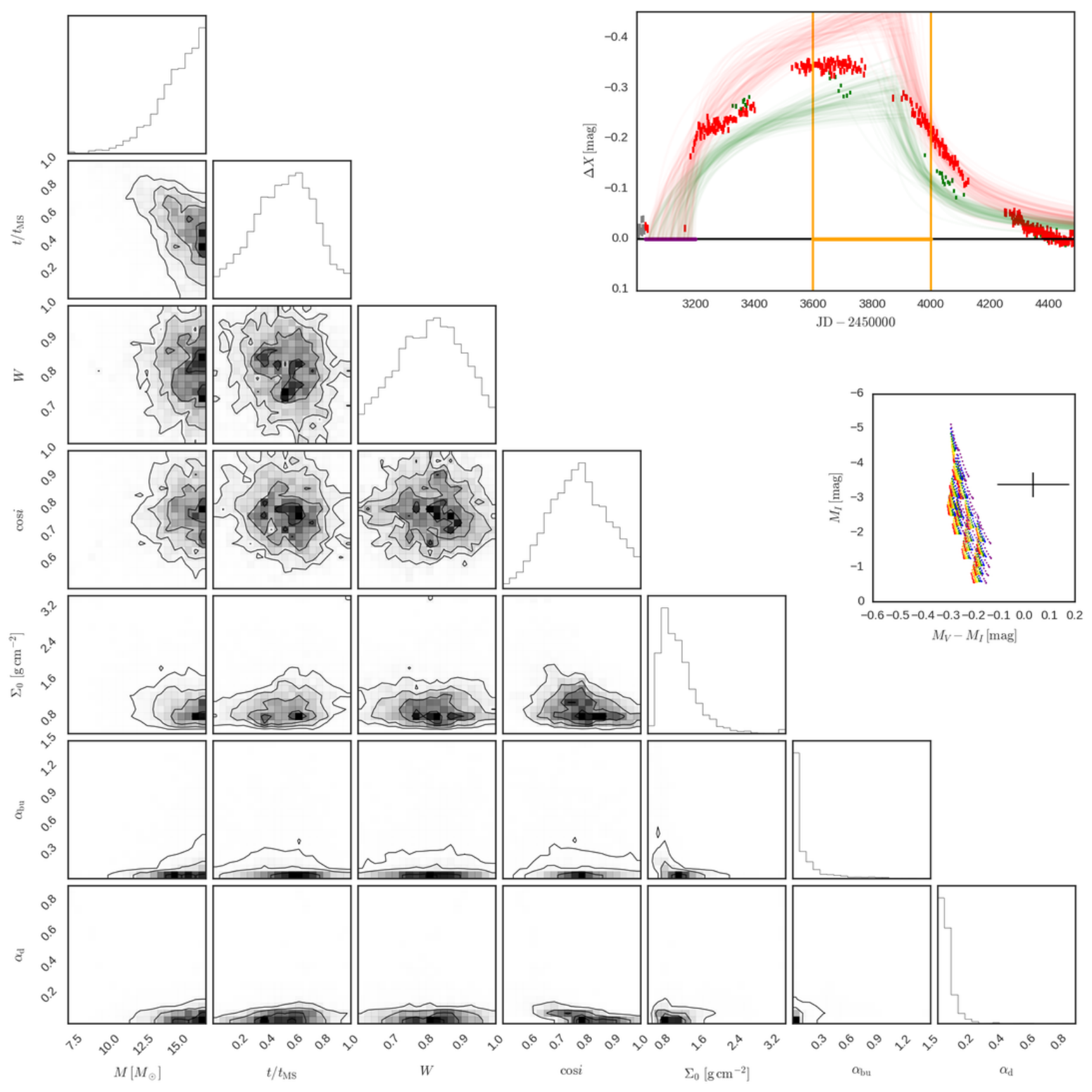

Figure C56. Same as Fig. 8 for SMC_SC5 276982 and bump ID 01. 


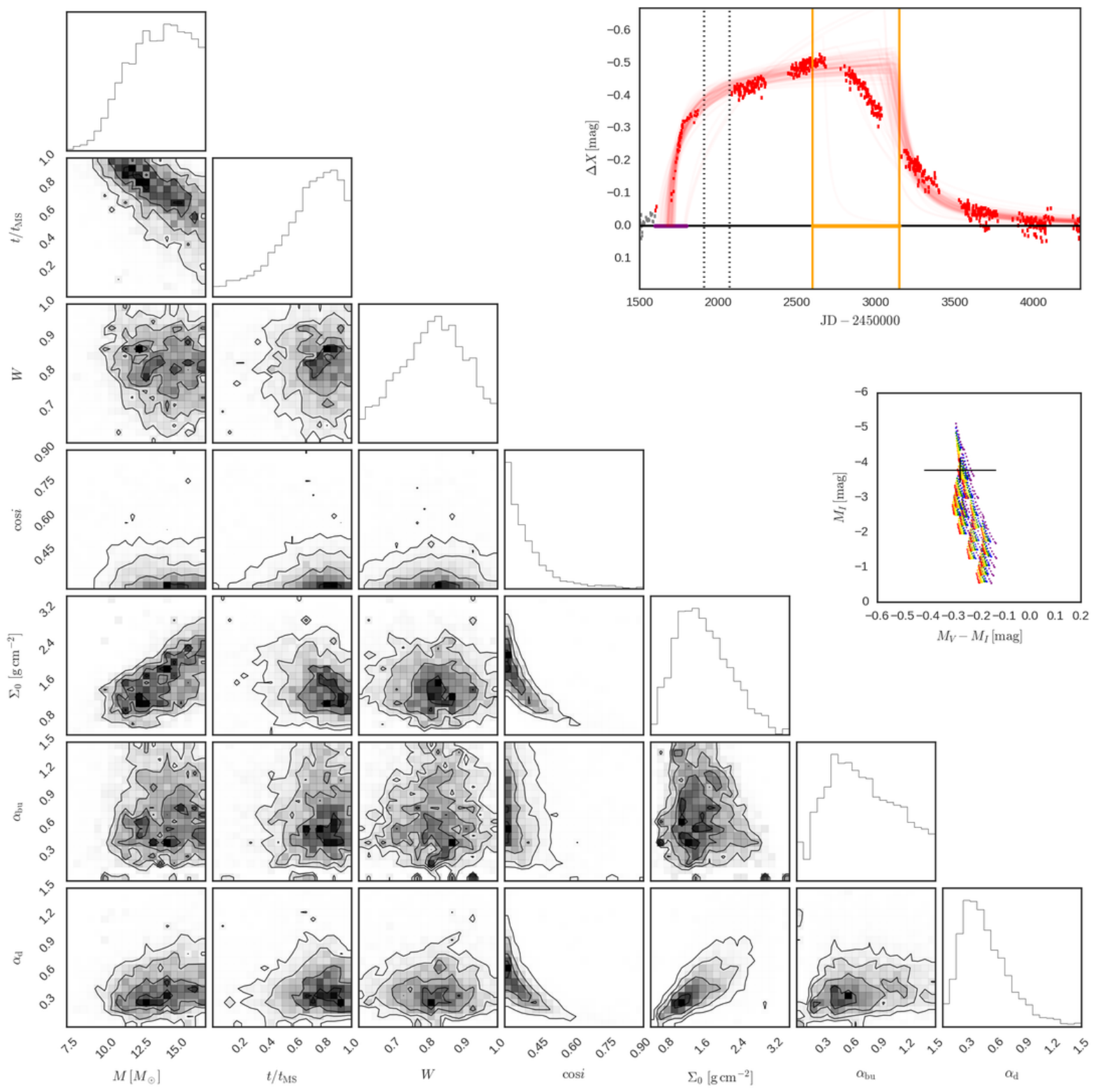

Figure C57. Same as Fig. 8 for SMC_SC5 282963 and bump ID 01. 


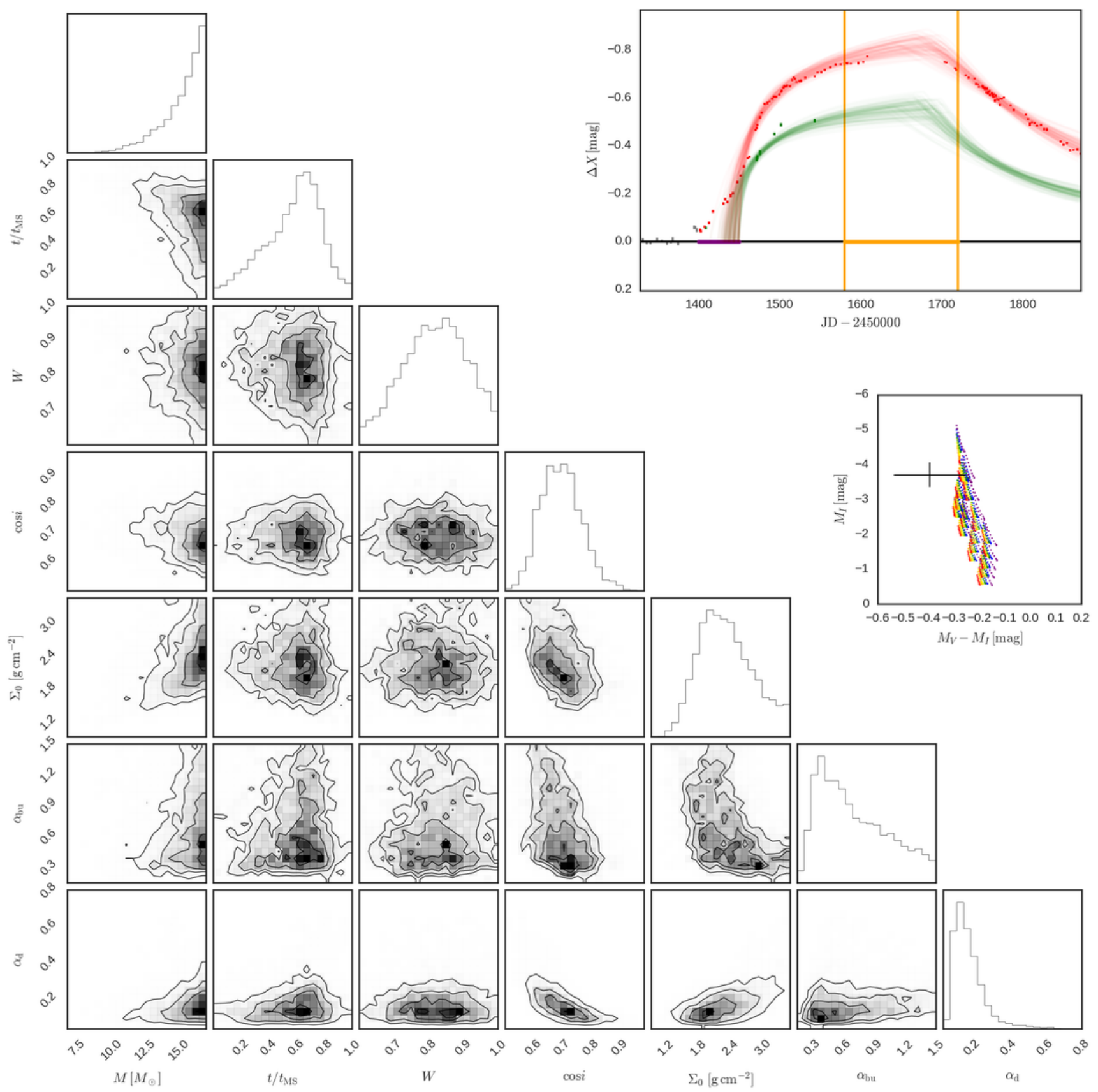

Figure C58. Same as Fig. 8 for SMC_SC6 11085 and bump ID 01. 

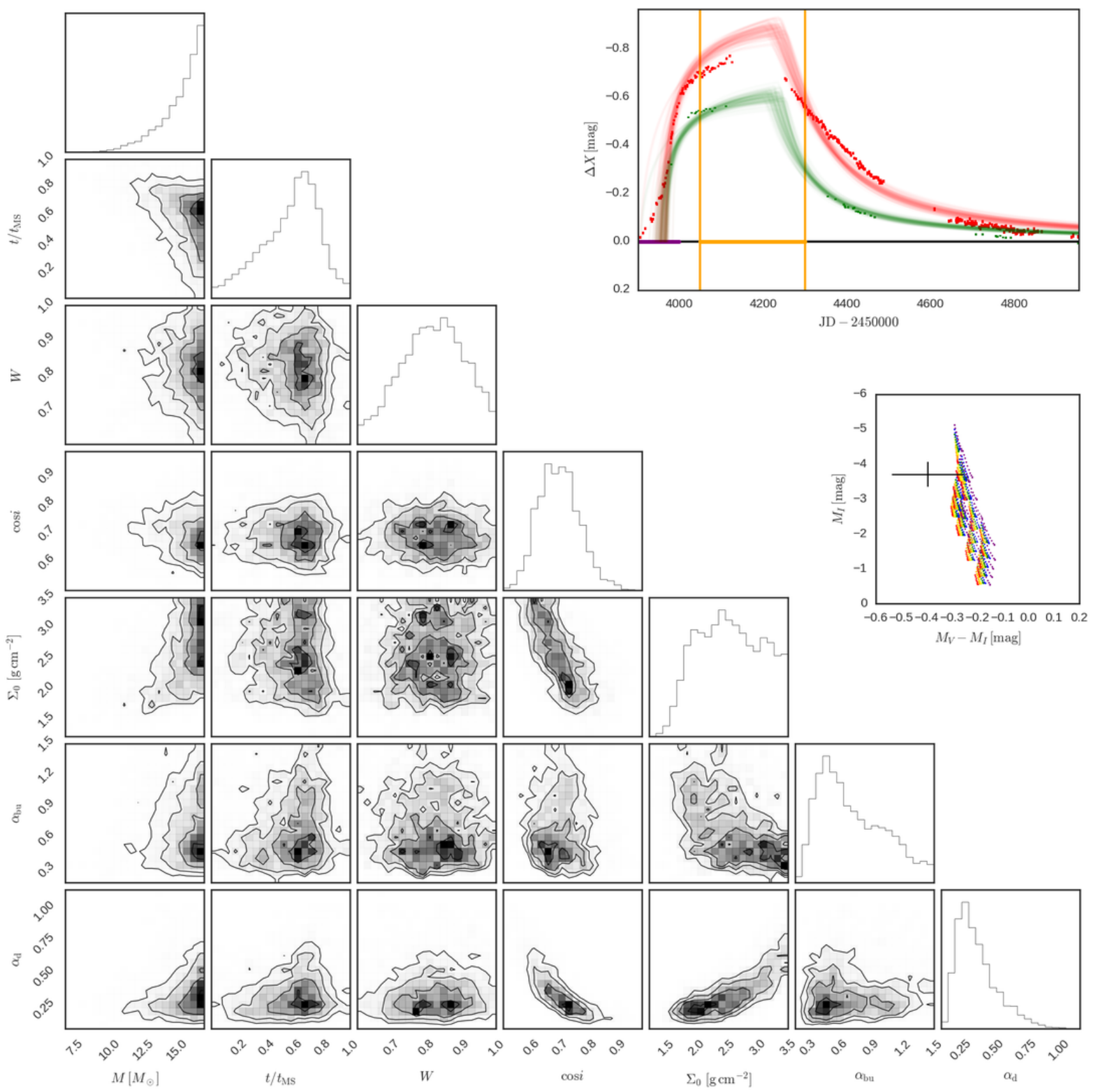

$$
M_{V}-M_{I}[\mathrm{mag}]
$$
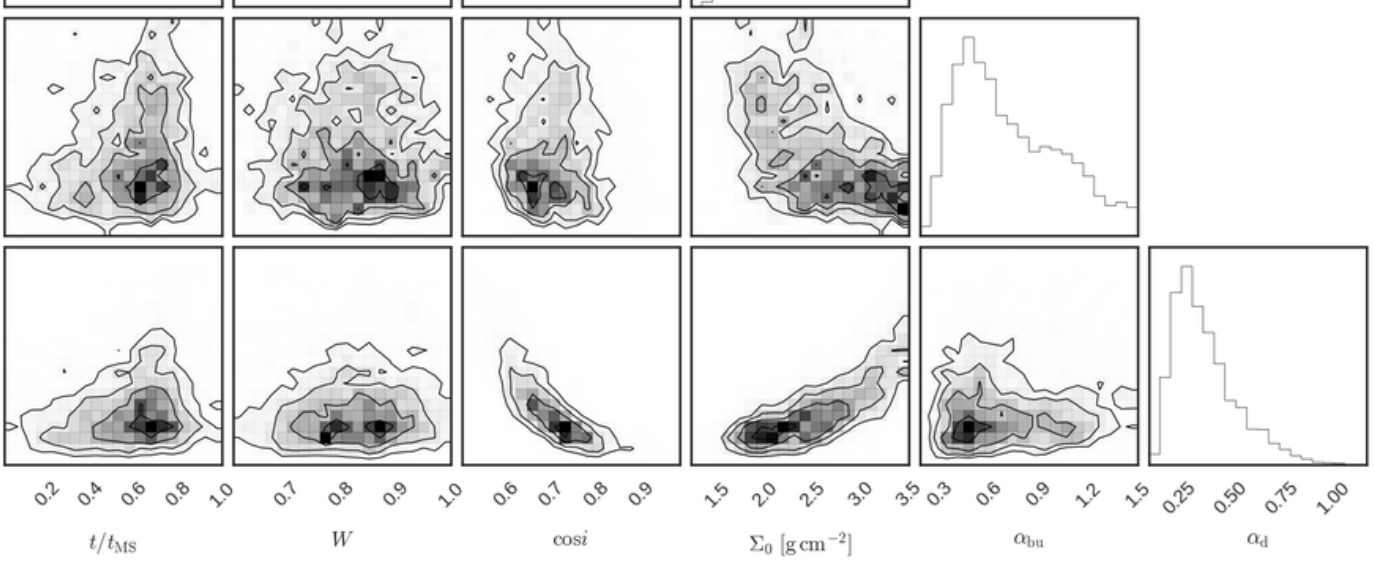

Figure C59. Same as Fig. 8 for SMC_SC6 11085 and bump ID 02. 


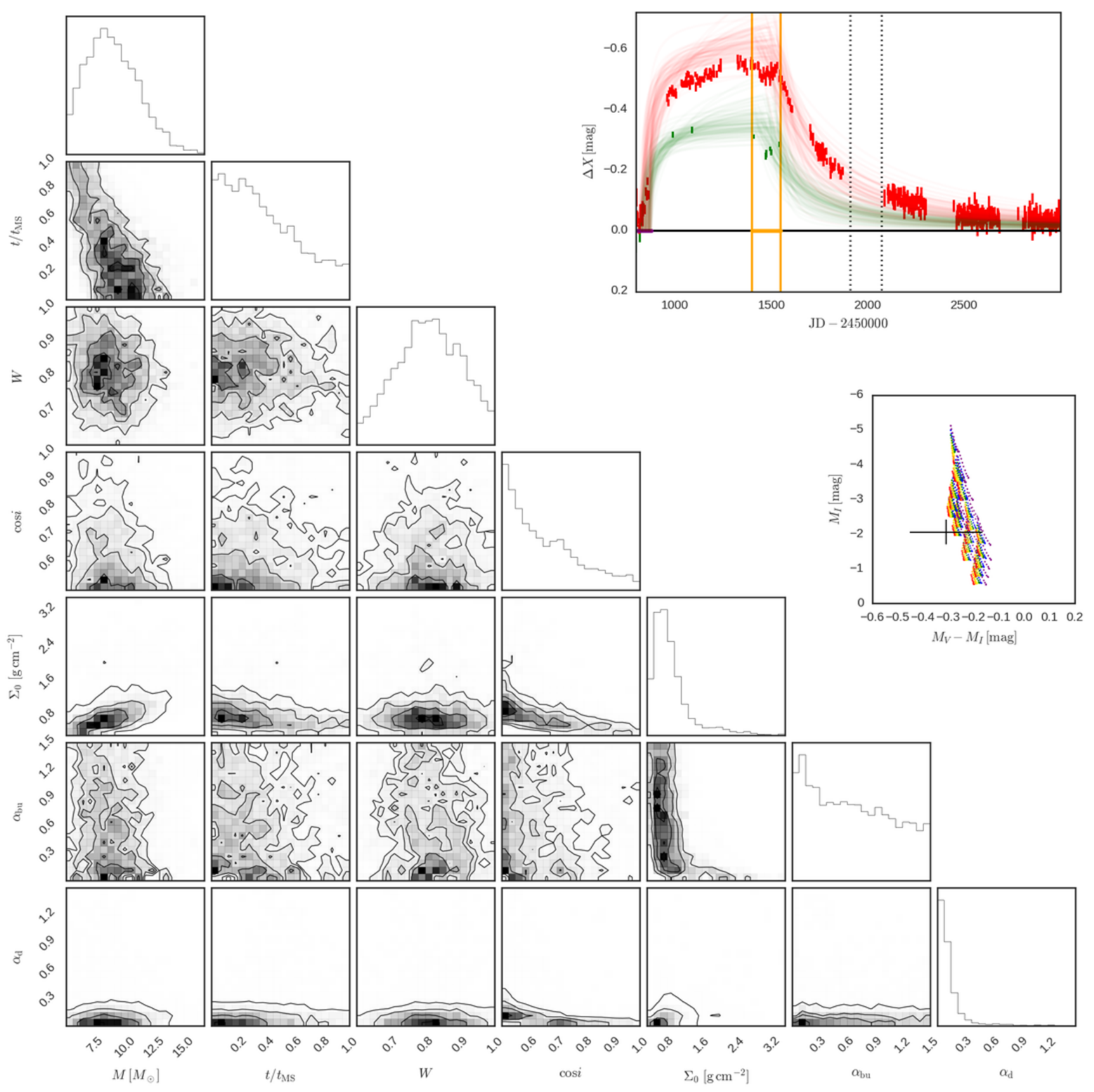

Figure C60. Same as Fig. 8 for SMC_SC6 17538 and bump ID 01 . 

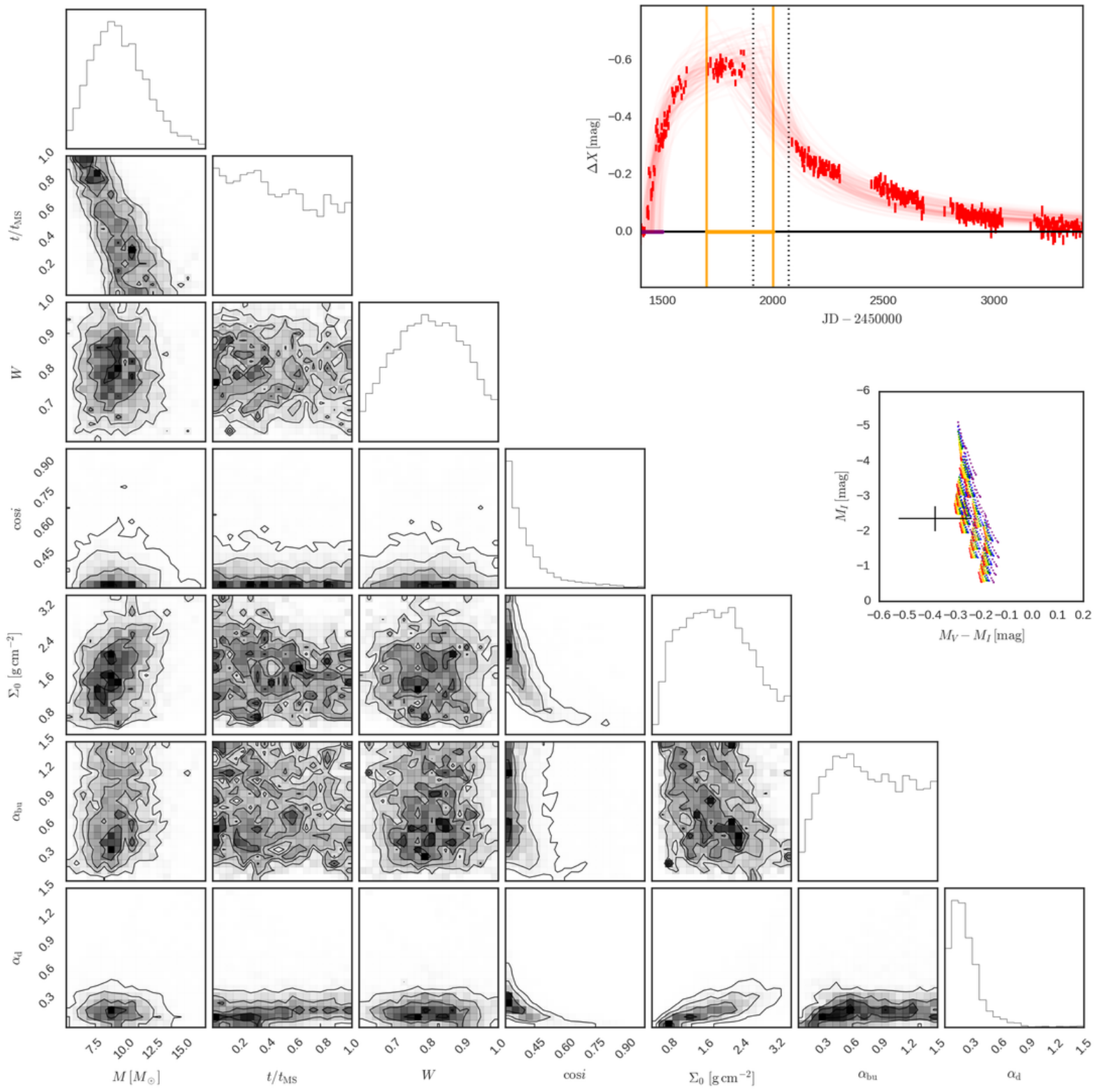

$$
M_{V}-M_{I}[\mathrm{mag}]
$$

Figure C61. Same as Fig. 8 for SMC_SC6 42440 and bump ID 01. 


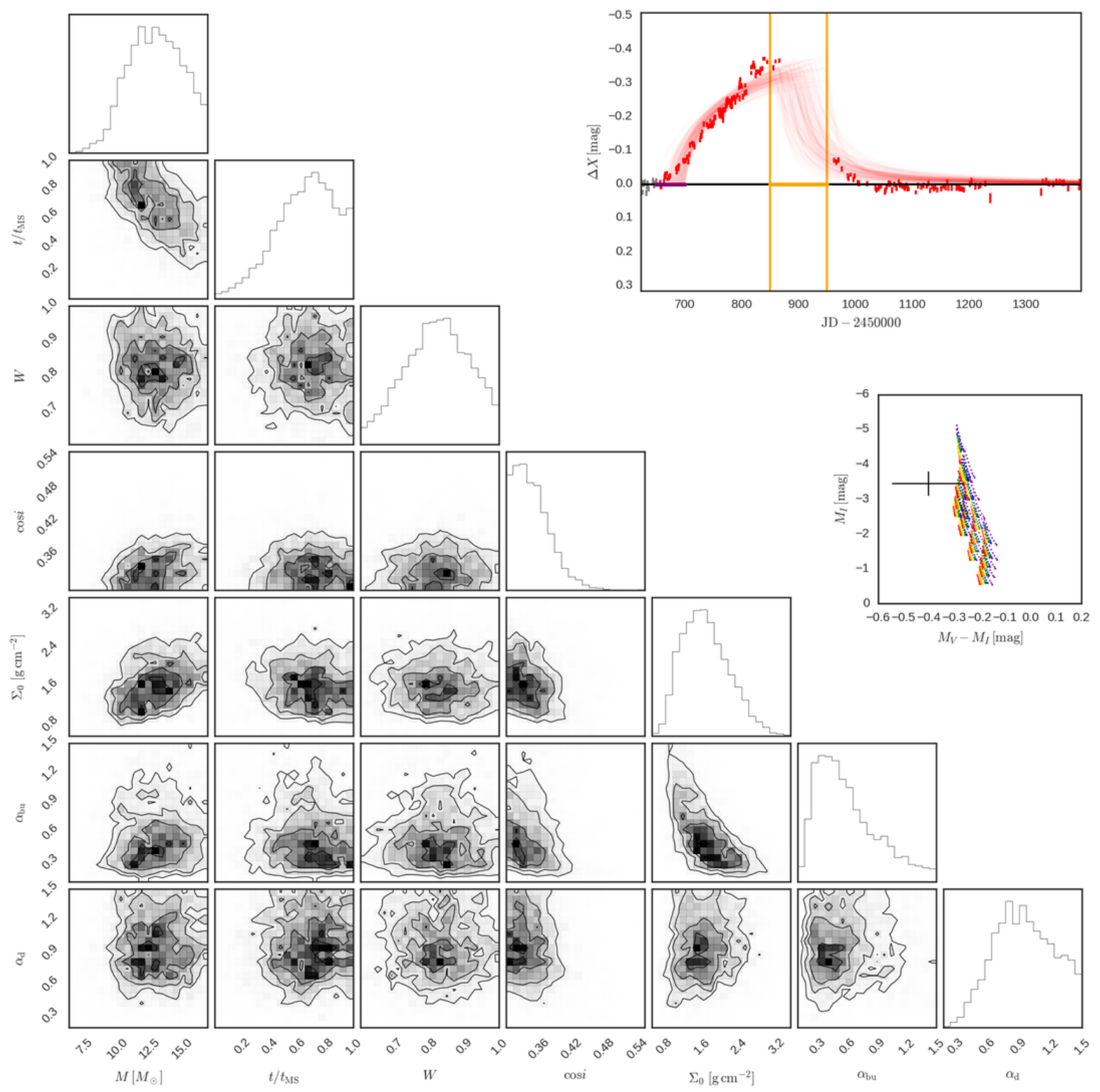

Figure C62. Same as Fig. 8 for SMC_SC6 99991 and bump ID 01. 

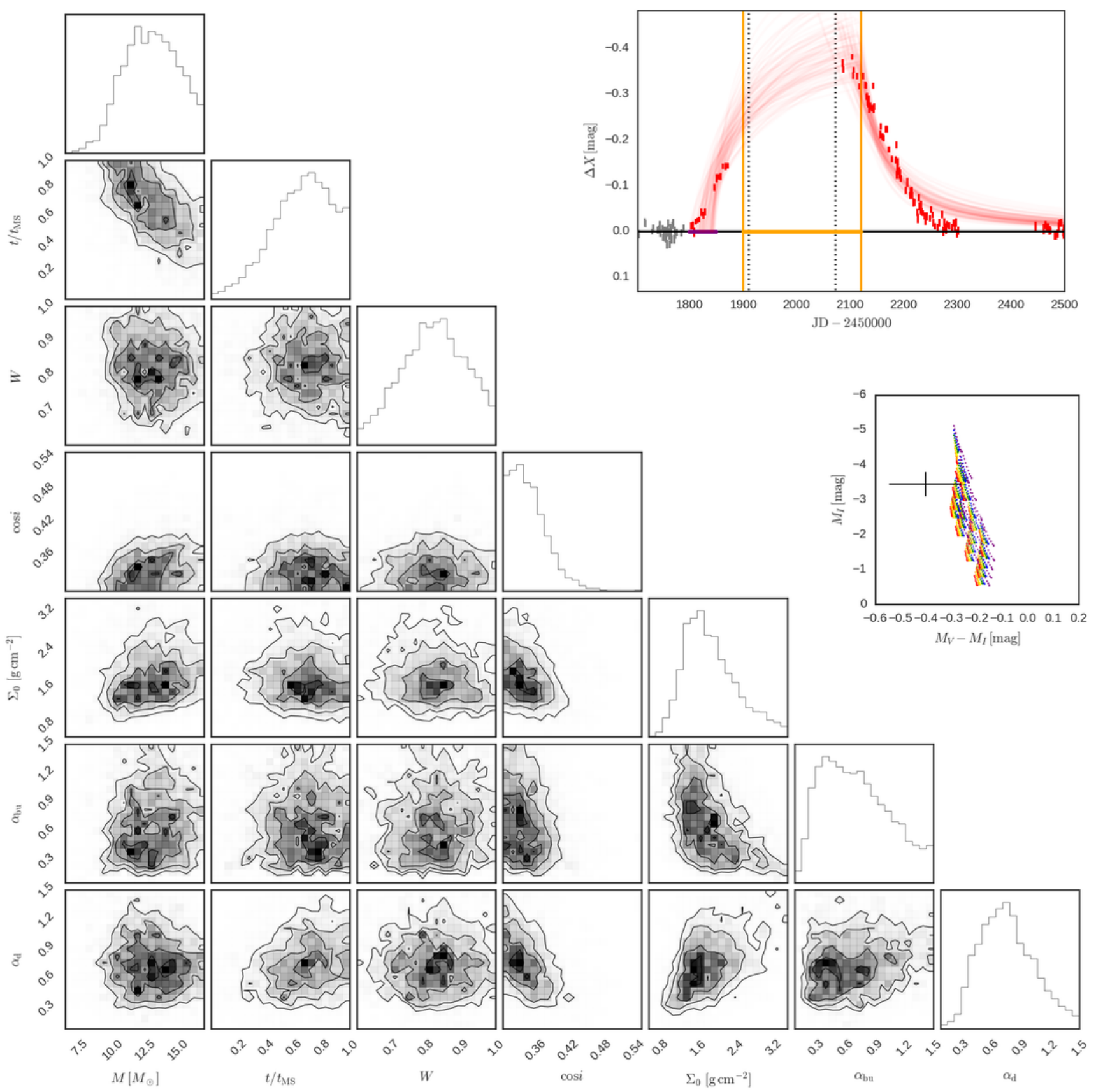

$$
M_{V}-M_{I}[\mathrm{mag}]
$$
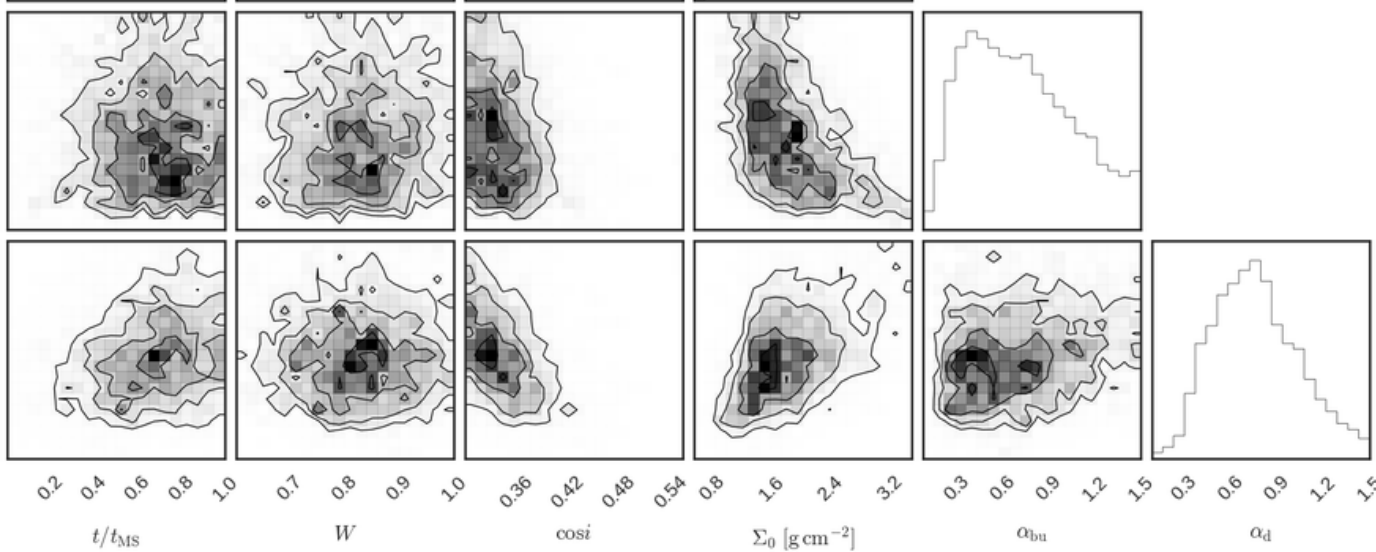

$0^{3} 0^{6} 0^{9} \gamma^{2} y^{5} 0^{3} \quad 0^{6} 0^{9} \gamma^{2} y^{5}$

Figure C63. Same as Fig. 8 for SMC_SC6 99991 and bump ID 02. 


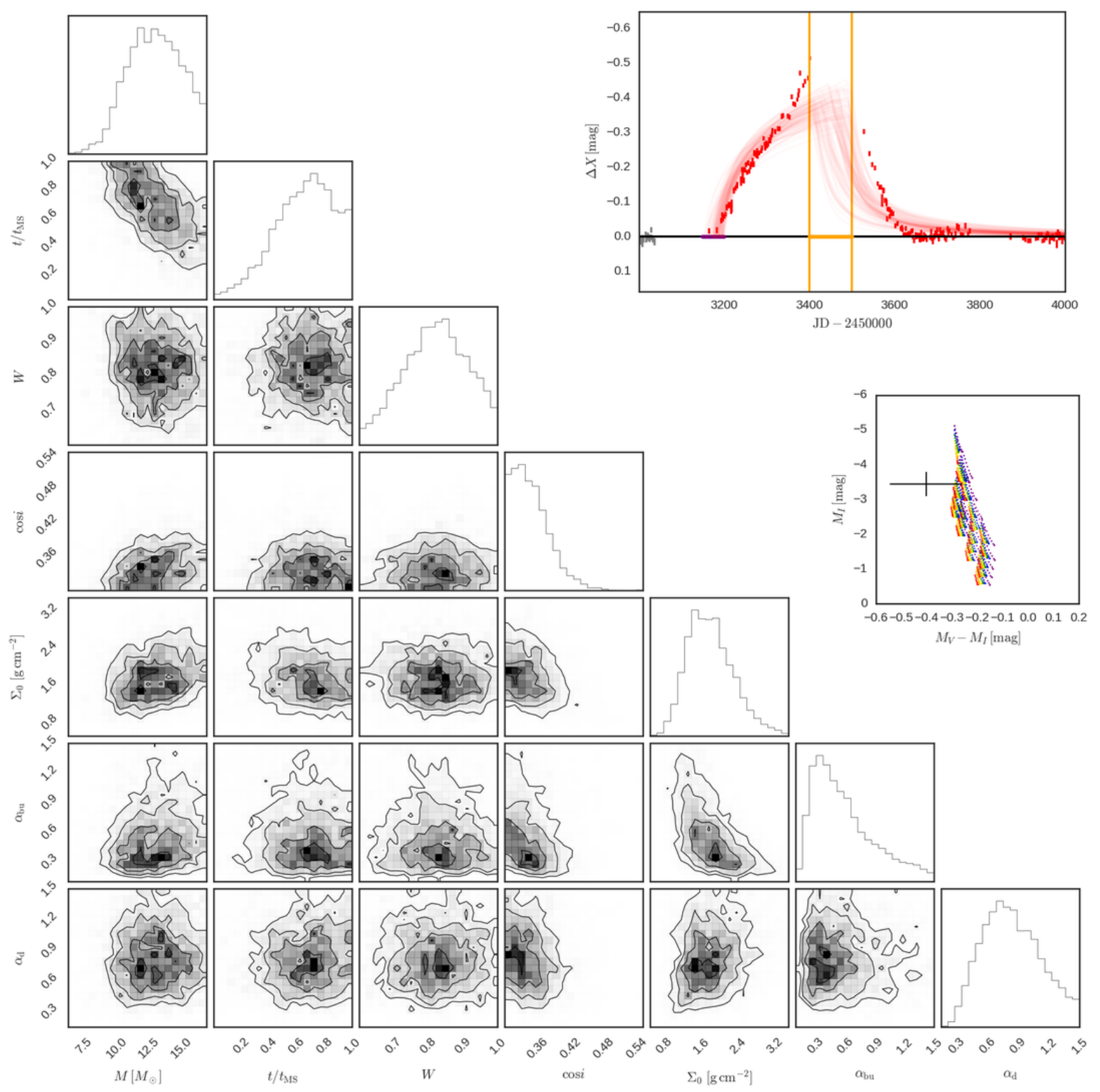

Figure C64. Same as Fig. 8 for SMC_SC6 99991 and bump ID 03. 

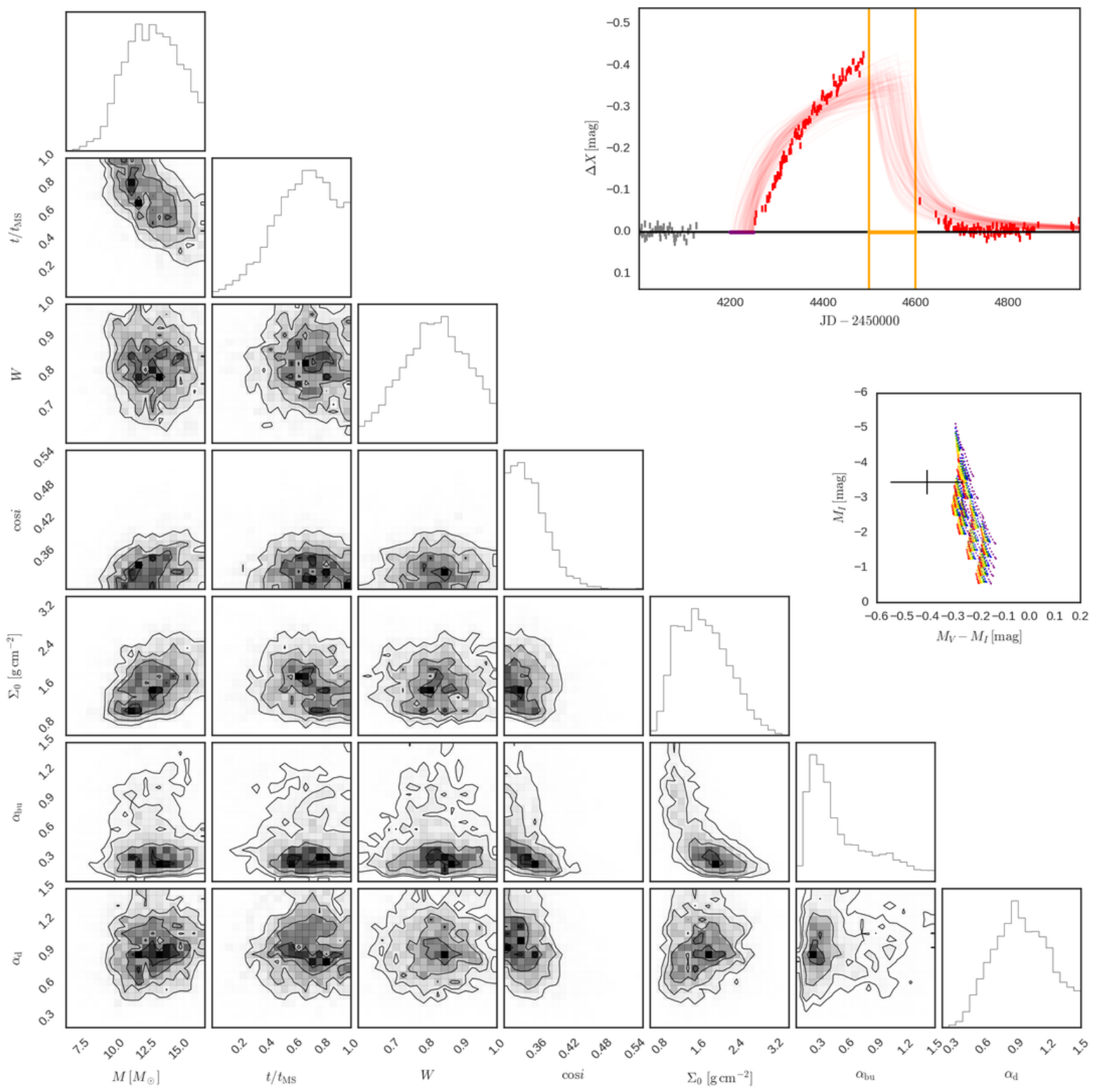

Figure C65. Same as Fig. 8 for SMC_SC6 99991 and bump ID 04 


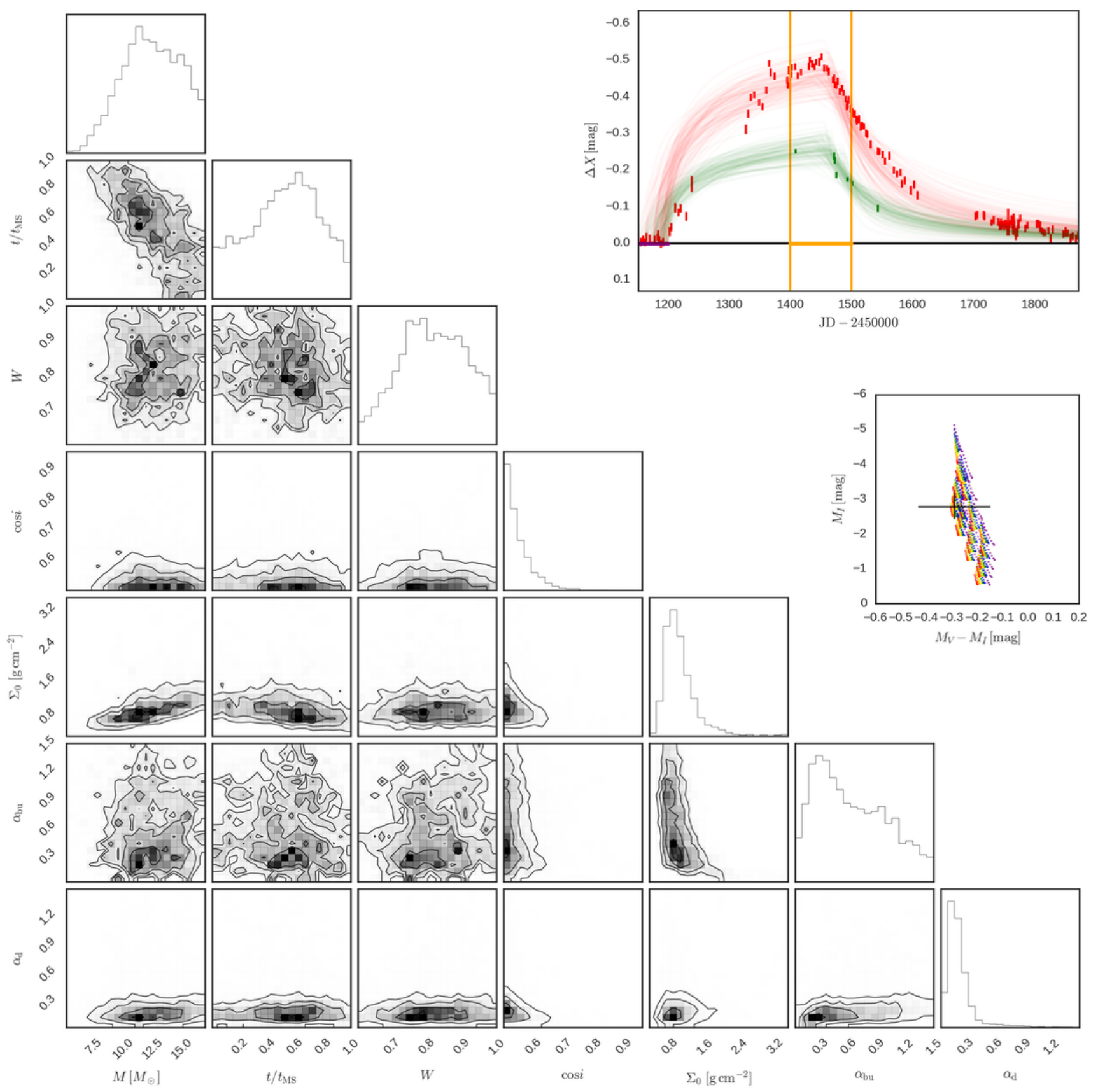

Figure C66. Same as Fig. 8 for SMC_SC6 105368 and bump ID 01. 


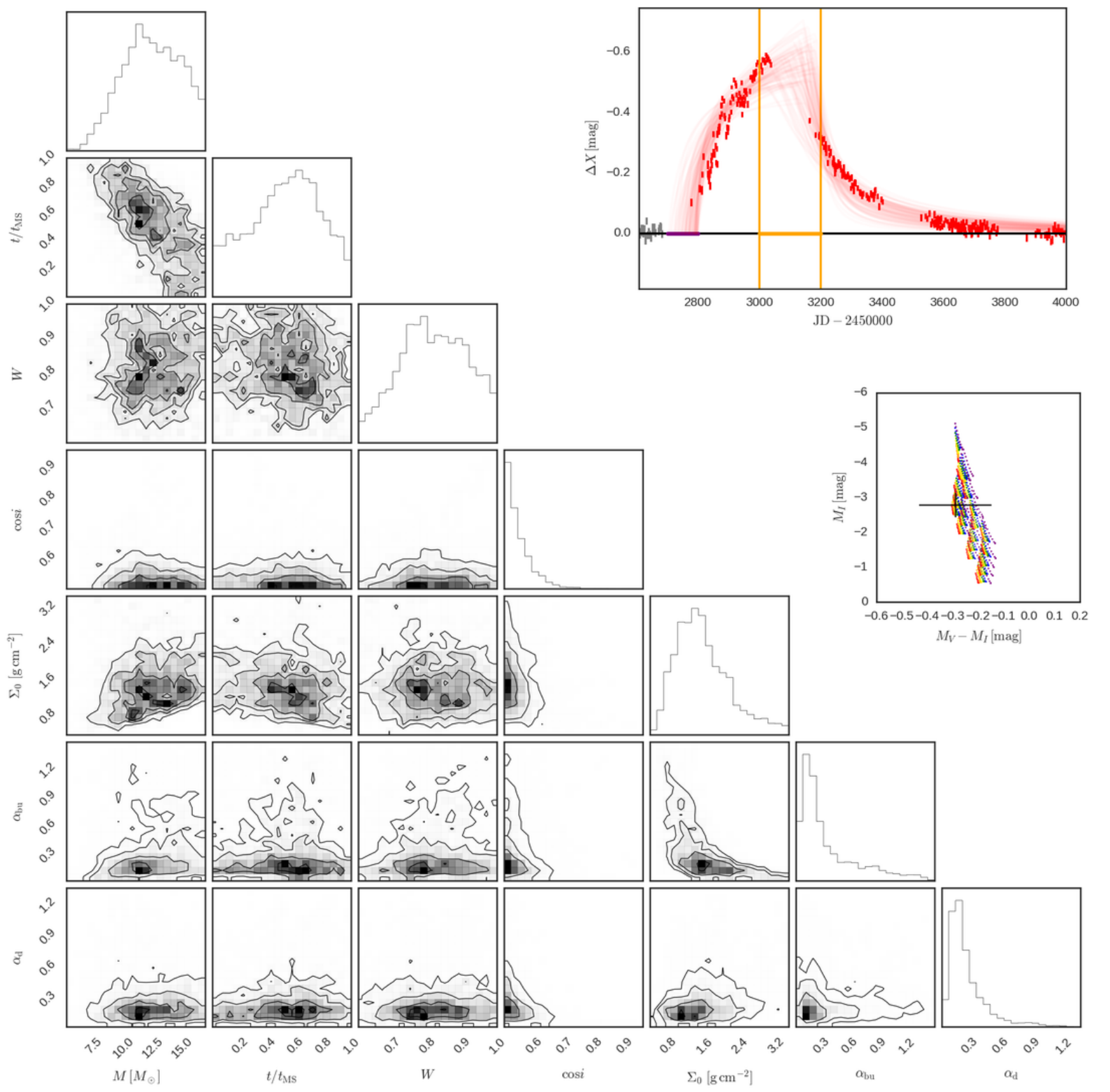

Figure C67. Same as Fig. 8 for SMC_SC6 105368 and bump ID 02. 


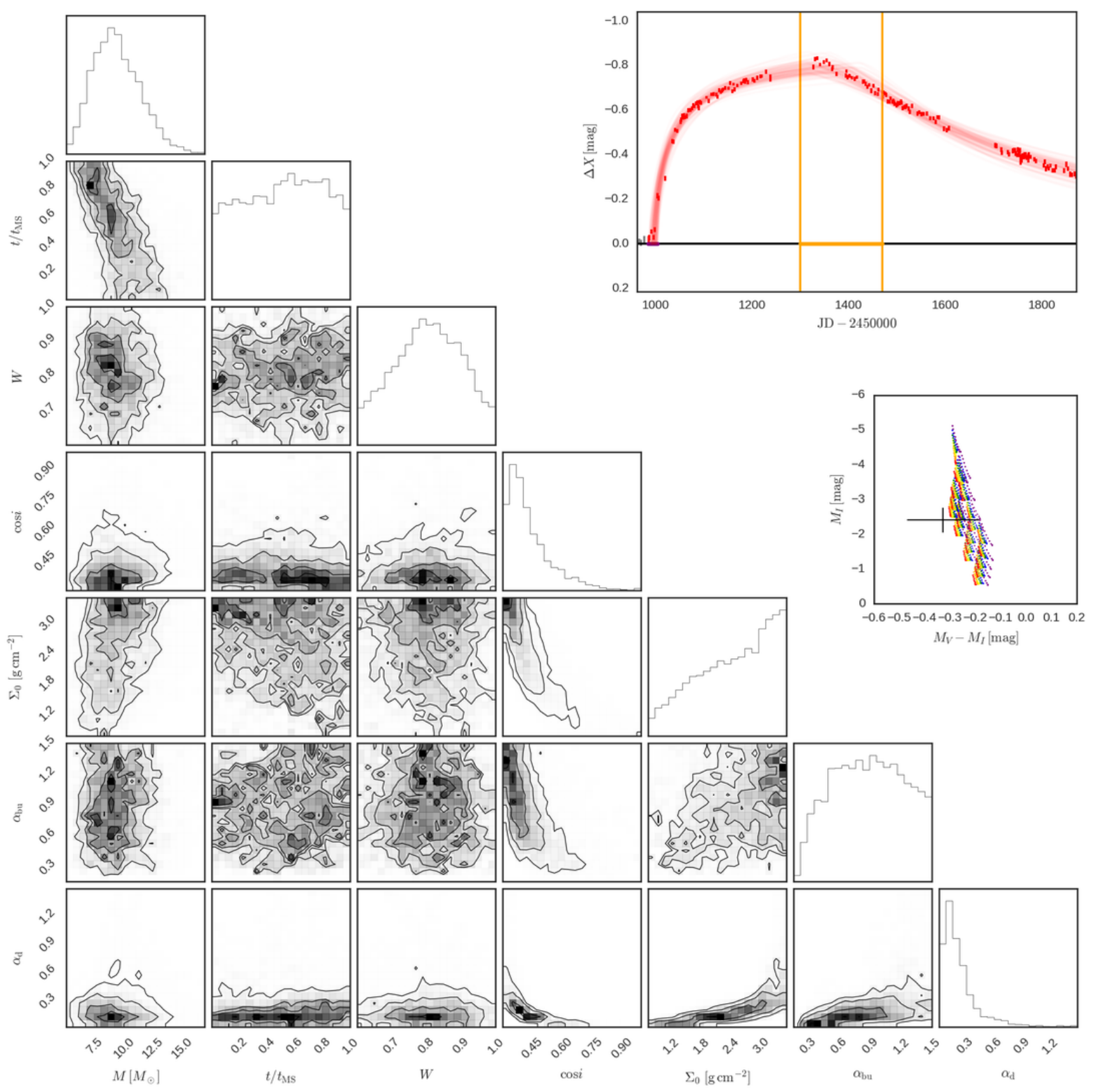

Figure C68. Same as Fig. 8 for SMC_SC6 116294 and bump ID 01. 


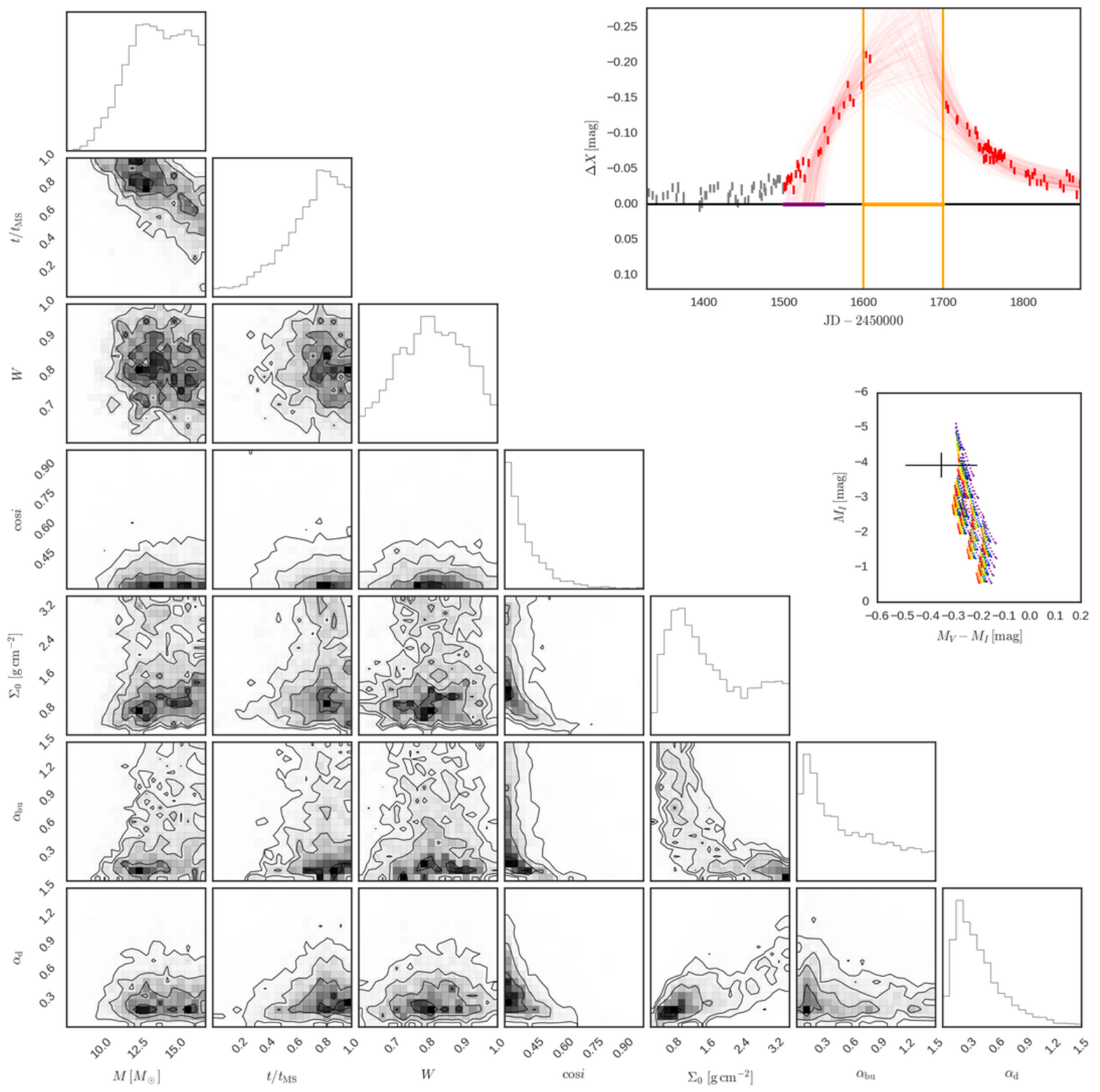

Figure C69. Same as Fig. 8 for SMC_SC6 199611 and bump ID 01. 


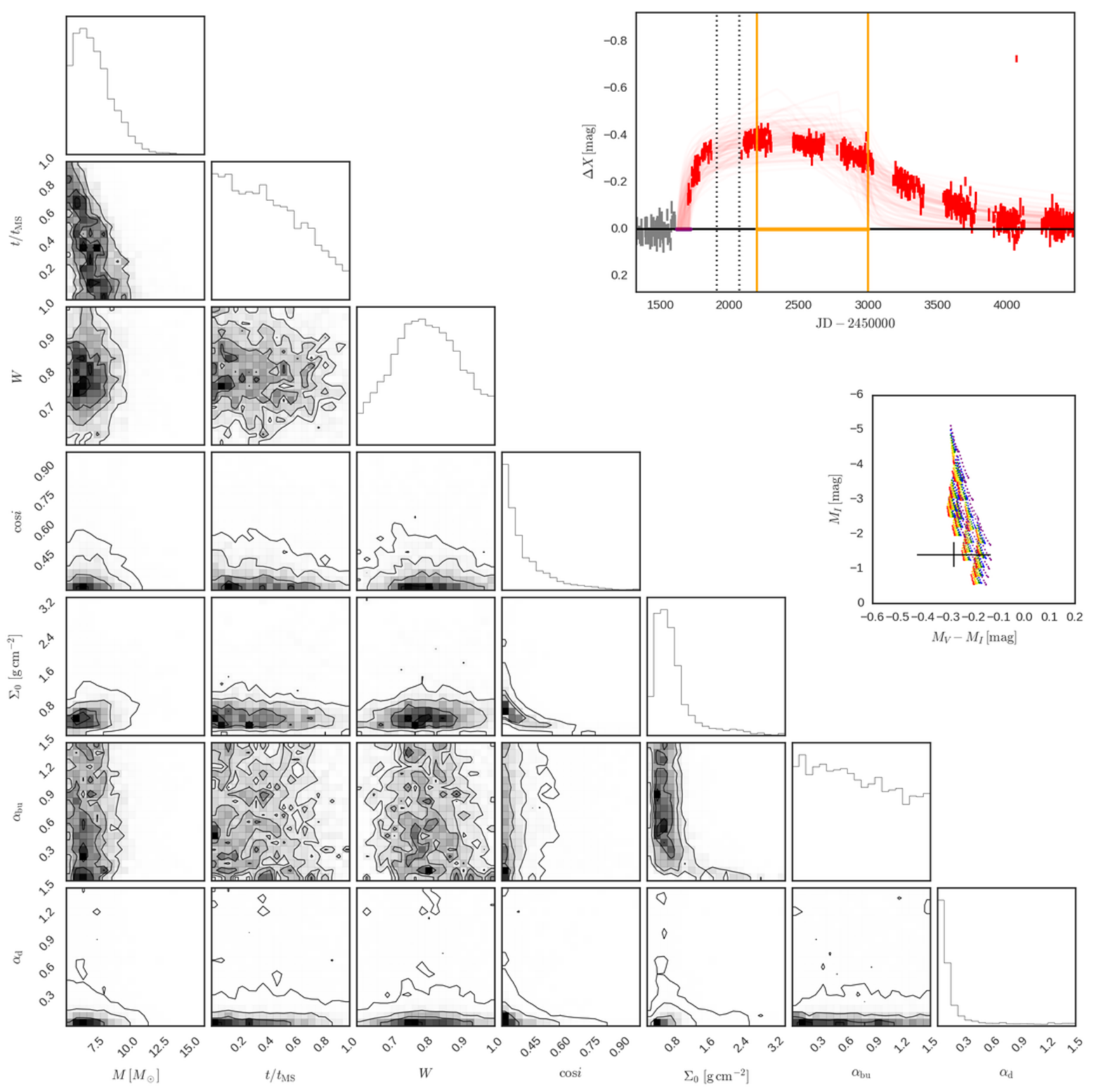

Figure C70. Same as Fig. 8 for SMC_SC6 272665 and bump ID 01. 


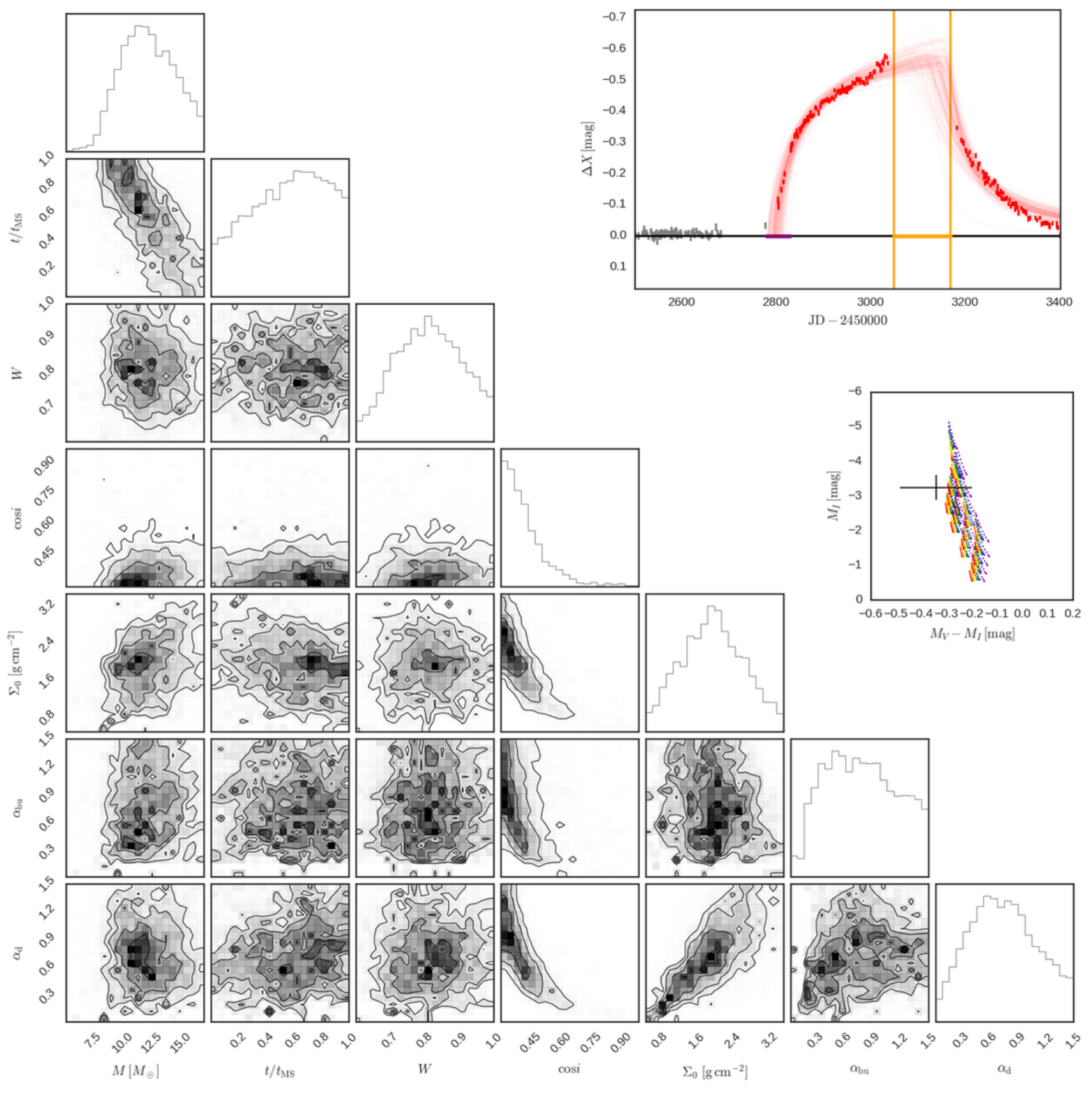

Figure C71. Same as Fig. 8 for SMC_SC7 57131 and bump ID 01 . 


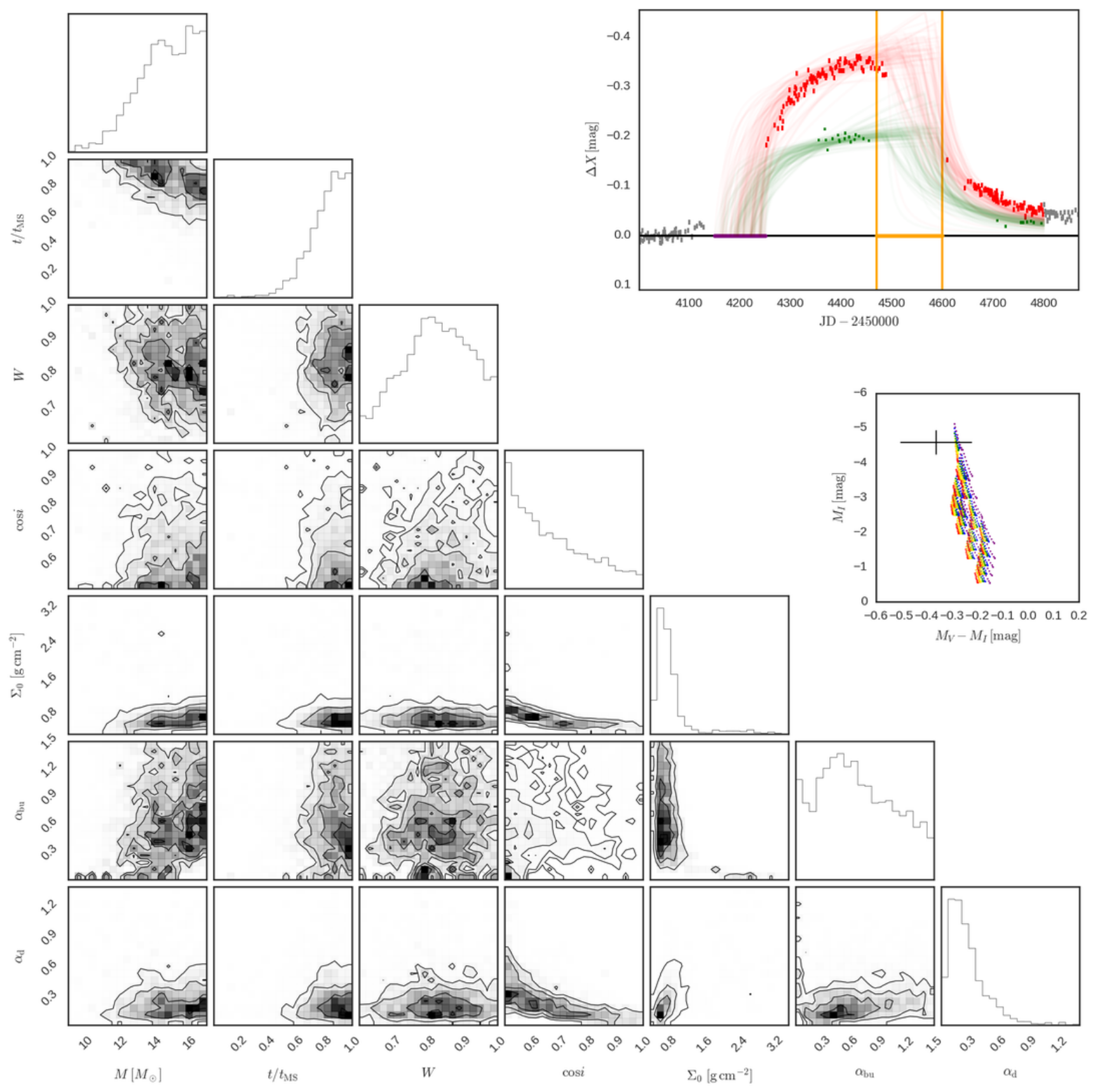

Figure C72. Same as Fig. 8 for SMC_SC8 183240 and bump ID 01. 


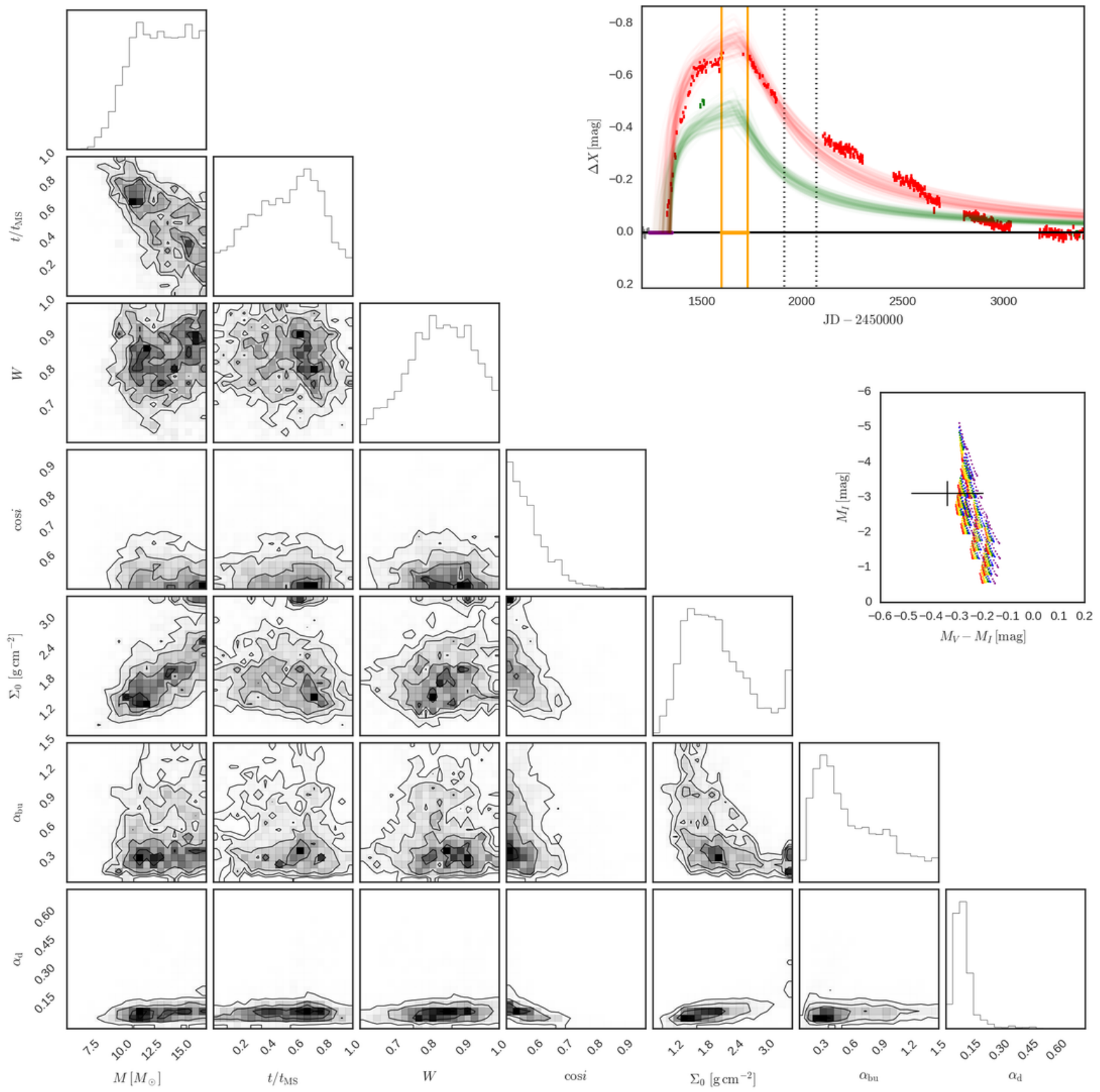

Figure C73. Same as Fig. 8 for SMC_SC9 105383 and bump ID 01. 


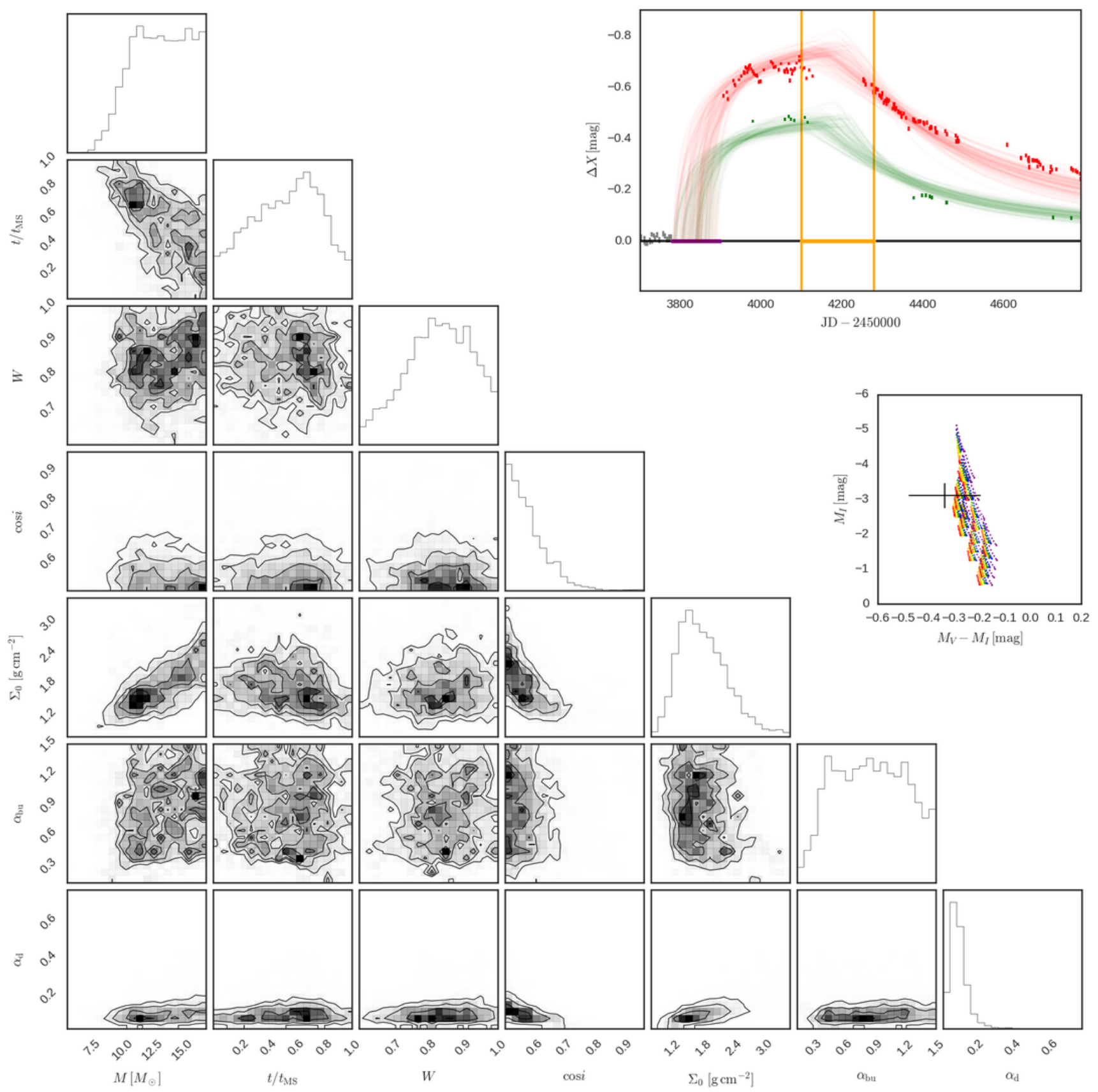

Figure C74. Same as Fig. 8 for SMC_SC9 105383 and bump ID 02. 


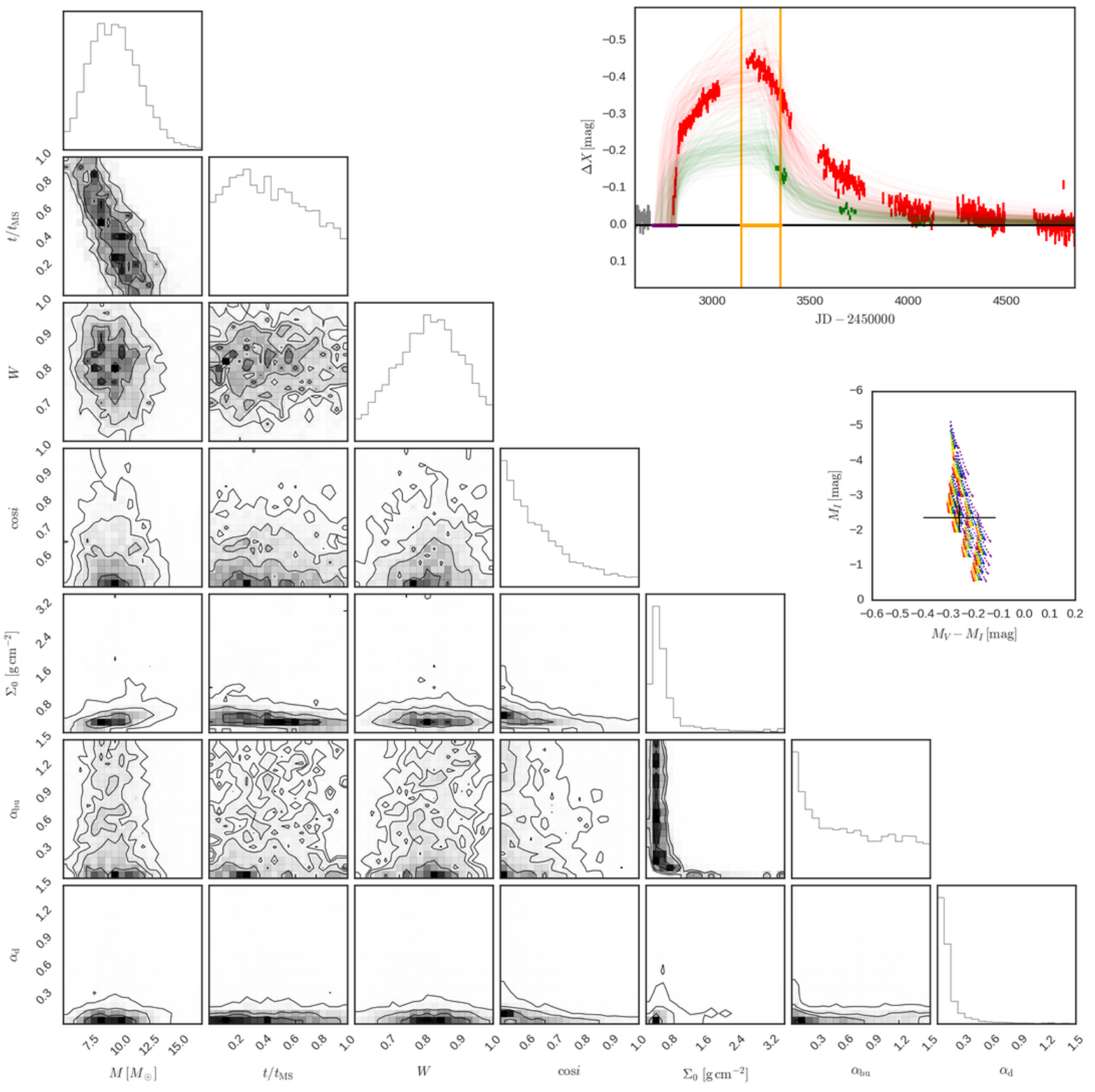

Figure C75. Same as Fig. 8 for SMC_SC9 168422 and bump ID 01. 


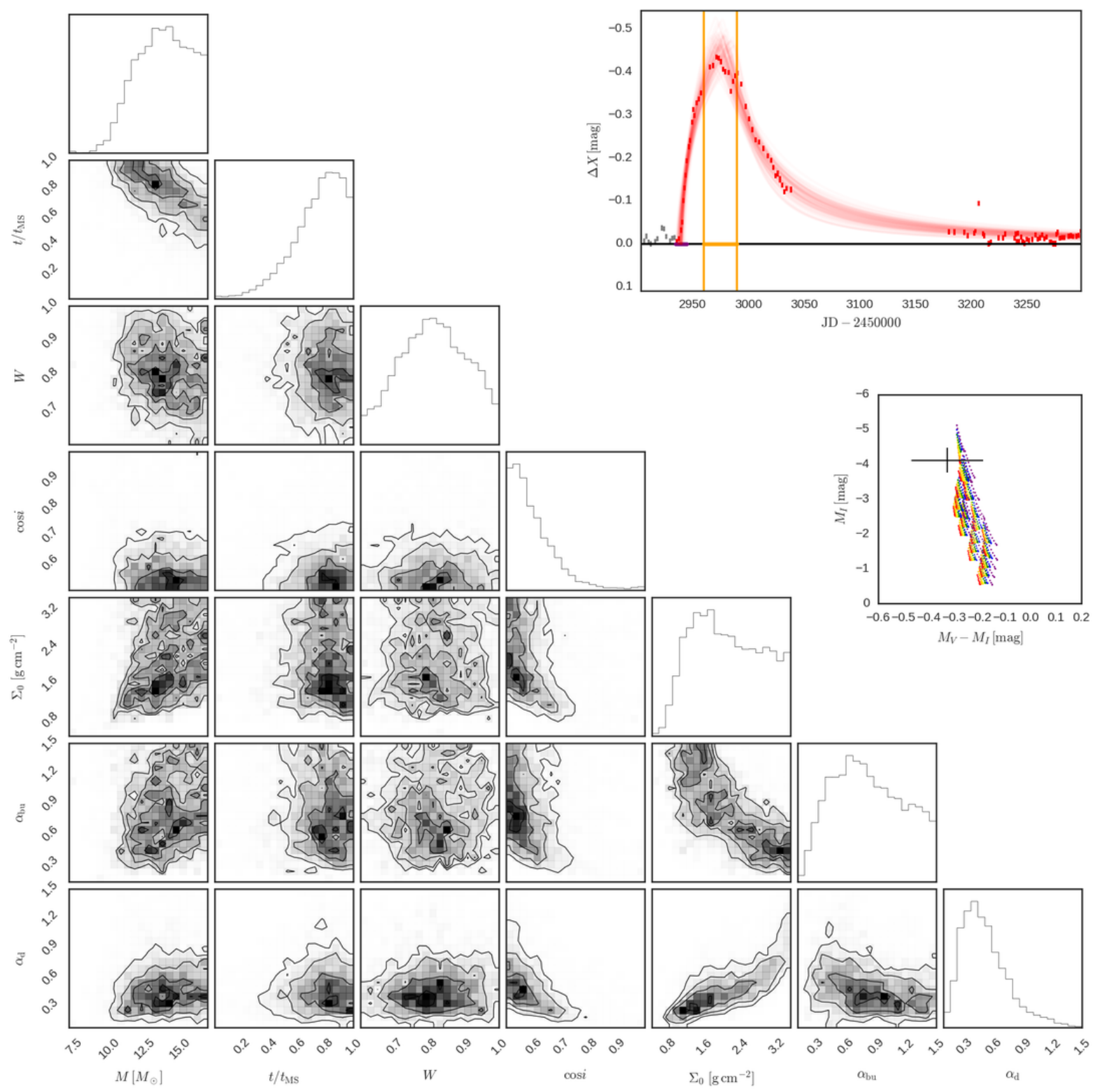

Figure C76. Same as Fig. 8 for SMC_SC10 8906 and bump ID 01. 

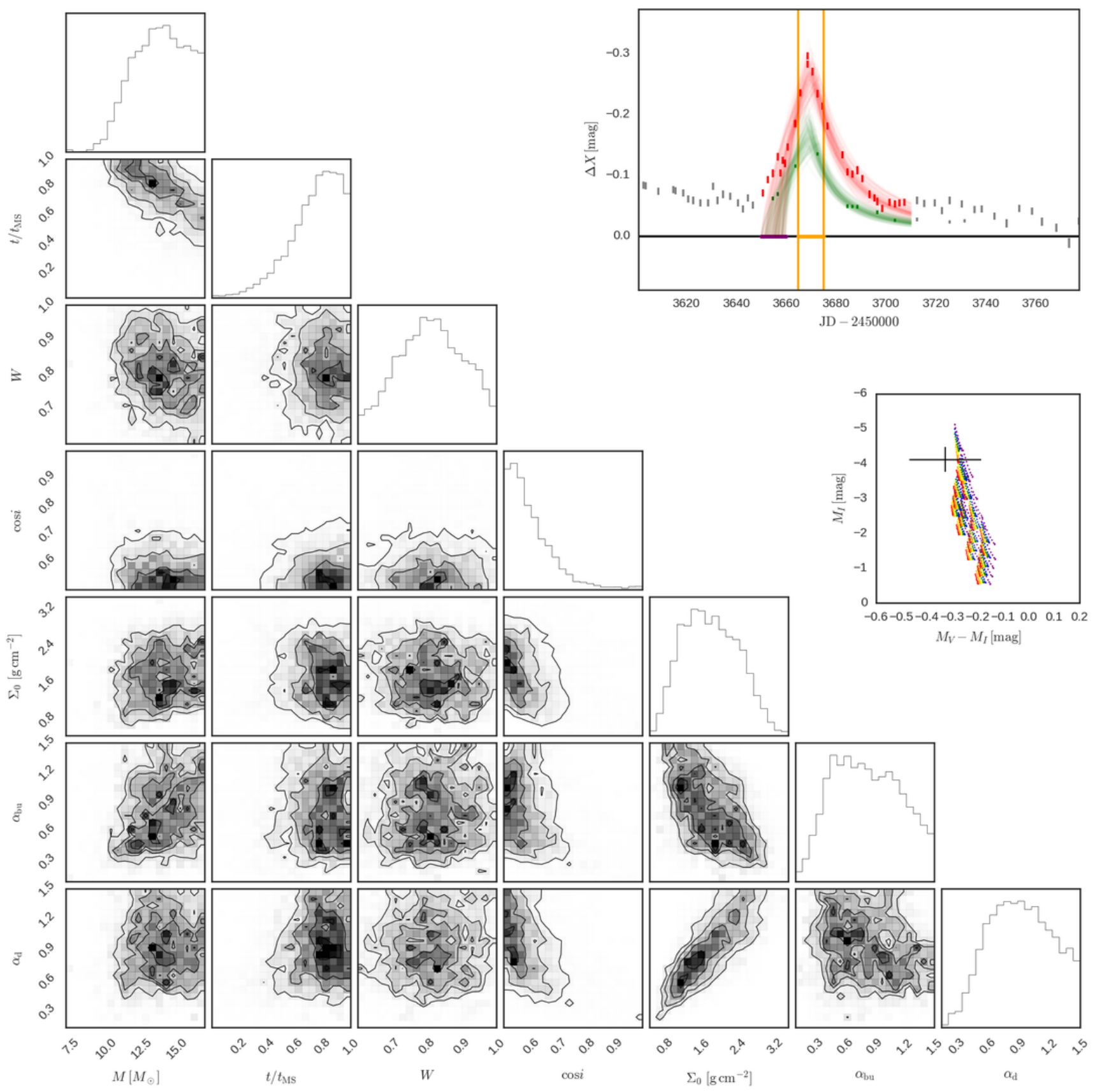

$$
M_{V}-M_{I}[\mathrm{mag}]
$$

Figure C77. Same as Fig. 8 for SMC_SC10 8906 and bump ID 02. 


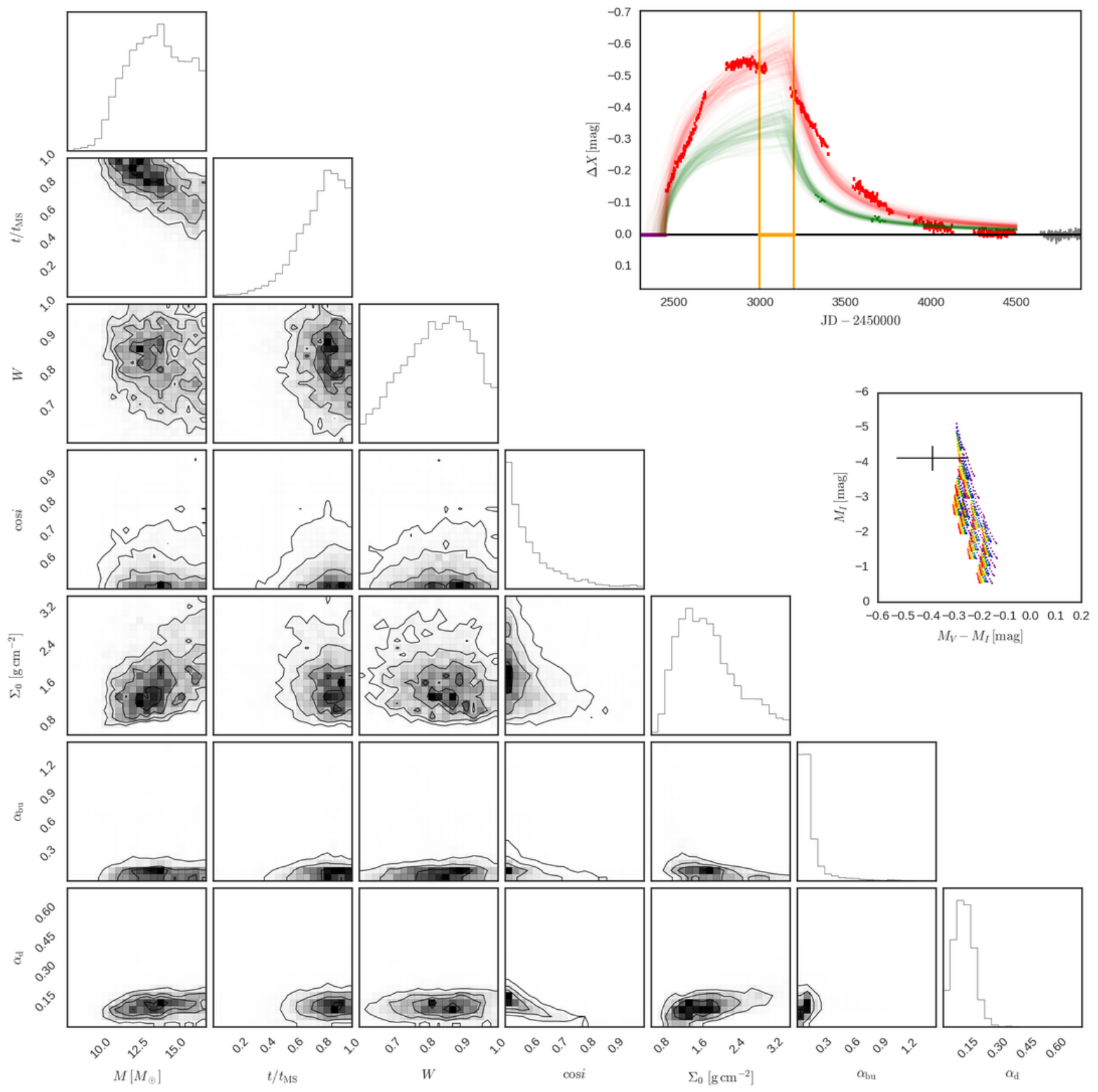

Figure C78. Same as Fig. 8 for SMC_SC11 28090 and bump ID 01. 


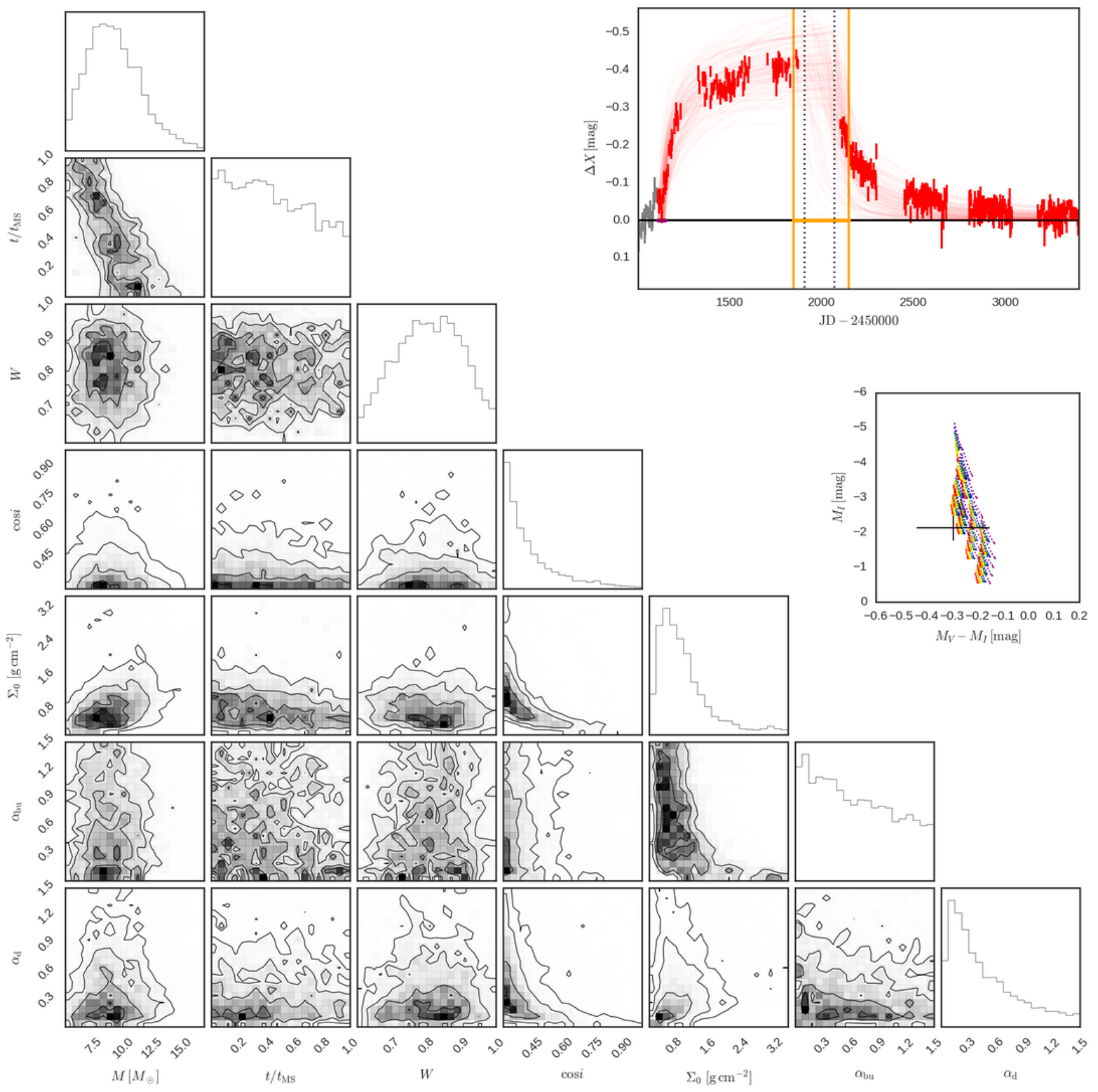

Figure C79. Same as Fig. 8 for SMC_SC11 46587 and bump ID 01. 
This paper has been typeset from a $\mathrm{T}_{\mathrm{E}} \mathrm{X} / \mathrm{L} \mathrm{T} \mathrm{E} \mathrm{X}$ file prepared by the author. 